\title{
SOUTHWEST REGION EXPERIMENT STATION (SWRES) 1990 REPORT
}

Prepared for

\author{
U.S. Department of Energy \\ Albuquerque Operations Office \\ P.O. Box 5400 \\ Albuquerque, New Mexico 87115 \\ DISCLAIMER
}

This report was prepared as an account of work sponsored by an agency of the United States Government. Neither the United States Government nor any agency thereof, nor any of their employees, makes any warranty, express or implied, or assumes any legal liability or responsibility for the accuracy, completeness, or usefulness of any information, apparatus, product, or process disclosed, or represents that its use would not infringe privately owned rights. Reference herein to any specific commercial product, process, or service by trade name, trademark, manufacturer, or otherwise does not necessarily constitute or imply its endorsement, recom' mendation, or favoring by the United States Government or any agency thereof. The views and opinions of authors expressed herein do not necessarily state or reflect those of the United States Government or any agency thereof.

$$
\text { Prepared by }
$$

\section{Southwest Technology Development Institute} P.O. Box 30001/3 SOL

Las Cruces, New Mexico 88003-0001 


\section{DISCLAIMER}

Portions of this document may be illegible

- electronic image products. Images are produced from the best available original document. 


\section{TABLE OF CONTENTS}

Section

Page

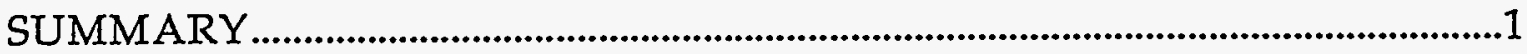

1.0 INTRODUCTION .........................................................................................................

2.0 Performance of the Grid Connected PV Systems at the SWRES......................1

2.1 Solar Insolation at the SWRES..........................................................................

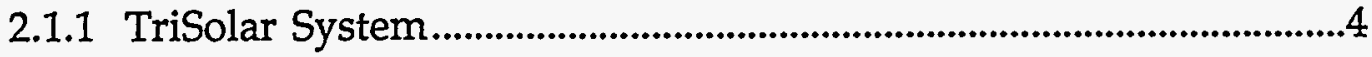

2.1.2 ARTU System ..............................................................................................

2.1.3 Solarex System.....................................................................................6

2.1.4 Arco Solar System........................................................................................7

2.1.5 Mobil Solar System.......................................................................................

2.2 PVFORM Analysis .............................................................................................9

2.2.1 PVFORM Comparison of SWRES vs. El Paso TMY Data .............10

2.2.2 PVFORM Comparison of Crystalline Systems..................................10

2.2.3 PVFORM Comparison of Amorphous Systems...............................12

2.2.4 Summary of PVFORM Analysis .......................................................14

Appendix A. 1990 Details on Downtime.................................................................

Appendix B. ARTU 1990 …..........................................................................

Appendix C. Solarex 1990 ...................................................................................

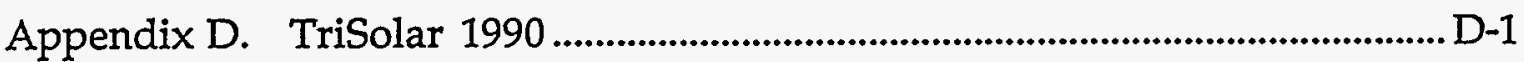

Appendix E. Mobil 1990............................................................................................ E-1 


\section{LIST OF TABLES AND FIGURES}

Table

Page

Table 1. Grid-Connected PV Systems at the SWRES............................................2

Table 2 System Characteristics........................................................................................2

Figure $\quad$ Page

Figure 1. 1990 Horizontal Insolation Comparison ...................................................

Figure 2. RES/ELP POA Insolation ......................................................................

Figure 3. TriSolar System AC Energy Production......................................................4

Figure 4. ARTU System AC Energy Production.....................................................6

Figure 5. Solarex System AC Energy Production. ......................................................7

Figure 6. MSEC System AC Energy Production...........................................................9

Figure 7. PVFORM Comparison of SWRES and El Paso TMY.........................10

Figure 8. SX System; PVFORM vs. Actual Energy Production ...........................11

Figure 9. TSC System; PVFORM vs. Actual Energy Production.........................11

Figure 10. MSEC System; PVFORM vs. Actual Energy Production....................12

Figure 11. ARTU System; PVFORM vs. Actual Energy Production...................12

Figure 12. CT System; PVFORM vs. Actual Energy Production...........................13

Figure 13. SOVONICS System; PVFORM vs. Actual Energy Production.........13

Figure 14. CHRONAR System; PVFORM vs. Actual Energy Production ........14 


\section{SUMMARY}

The Southwest Region Experiment Station (SWRES), operated by the Southwest Technology Development Institute, located on the New Mexico State University campus in Las Cruces, New Mexico, performed the following tasks in 1990. Many of these projects were funded in whole or in part by the U.S. Department of Energy's National Photovoltaic Program.

- Collected a complete year of weather data.

- Collected data from and prepared a report describing performance of the PV systems at the Southwest Region Experiment Station.

\subsection{INTRODUCTION}

Photovoltaic (PV) systems and components have been tested at the SWRES since 1981. The U.S. Department of Energy provided the initial funding for the .12 support structures on the site. Approximately $40 \mathrm{~kW}$ of PV was installed on the roofs of these support structures to study the performance of residential applications. A review of the performance of these systems during the decade of the 1980 's is included in Section 2 of this report.

\subsection{PERFORMANCE OF THE GRID CONNECTED PV SYSTEMS AT THE SWRES}

Eight grid-connected prototype photovoltaic systems were installed at the SWRES in 1981-82. These systems represented the state-of-the-art in residential PV systems when they were installed. They were designed by different companies and all of the component manufacturers of that time were represented. In 1984, a ninth prototype 
support structure with a Mobil Solar Energy Corporation ribbon type module was added at the SWRES. The characteristics for the four systems are given in Table 1.

In 1987 amorphous silicon (a-Si) modules became available commercially and these new technology systems were installed at the SWRES. Three a-Si systems, all less than $1 \mathrm{~kW}$, have been installed and operated at the SWRES in the last four years. These system characteristics are given in Table 2 .

Table 1

Grid-Connected PV Systems at the SWRES

\begin{tabular}{|l|c|c|c|c|l|}
\hline \multicolumn{1}{|c|}{ System } & $\begin{array}{c}\text { Area } \\
\left(\mathrm{m}^{2}\right)\end{array}$ & $\begin{array}{c}\text { Installed } \\
\text { Rating } \\
(\mathrm{kW})\end{array}$ & $\begin{array}{c}1988 / 1989 / 1990 \\
\text { Rating } \\
(\mathrm{kW})\end{array}$ & $\begin{array}{c}\text { Tilt } \\
\text { Angle }\left(^{\circ}\right)\end{array}$ & $\begin{array}{c}1990 \\
\text { PCS }\end{array}$ \\
\hline ARTU & 55.2 & 4.9 & $2.5 / 2.5^{*} / 2.2$ & 45 & Gemini \\
\hline Solarex & 70.2 & 5.1 & $3.9 / 4.2 / 3.9$ & 26 & APCC \\
\hline TriSolar & 58.0 & 5.3 & $4.9 / 4.7 / 4.4$ & 30 & Gemini \\
\hline MSEC & 53.0 & 3.9 & $3.7 / 3.7 / 3.4$ & 30 & APCC \\
\hline
\end{tabular}

*Configuration changed from original.

Table 2

System Characteristics

\begin{tabular}{|c|c|c|c|c|c|}
\hline $\begin{array}{c}\text { System } \\
\text { Name }\end{array}$ & $\begin{array}{c}\text { Installed } \\
\text { Rating (kW) }\end{array}$ & $\begin{array}{c}\text { Dec. } 1990 \\
\text { PCS }\end{array}$ & $\begin{array}{c}199046^{\circ} \\
\text { Max Power } \\
\text { Rating (kW) }\end{array}$ & Tilt & $\begin{array}{c}\text { Dec. 1990 } \\
\text { Area (m²) }\end{array}$ \\
\hline Chronar 2 & 0.72 & Teslaco & 0.617 & $25^{\circ}$ & 18.37125 \\
\hline Sovonics 1 & 1.15 & ROSS & 0.925 & $25^{\circ}$ & 23.0913281 \\
\hline OTP-101 & 0.943 & ROSS & 0.505 & $30^{\circ}$ & 14.8336 \\
\hline
\end{tabular}




\subsection{Solar Insolation at the SWRES}

The total horizontal solar insolation received in 1990 is shown in Figure 1.

Comparing this actual data to meteorological year (TMY) for El Paso produces the results shown in Figure 2.

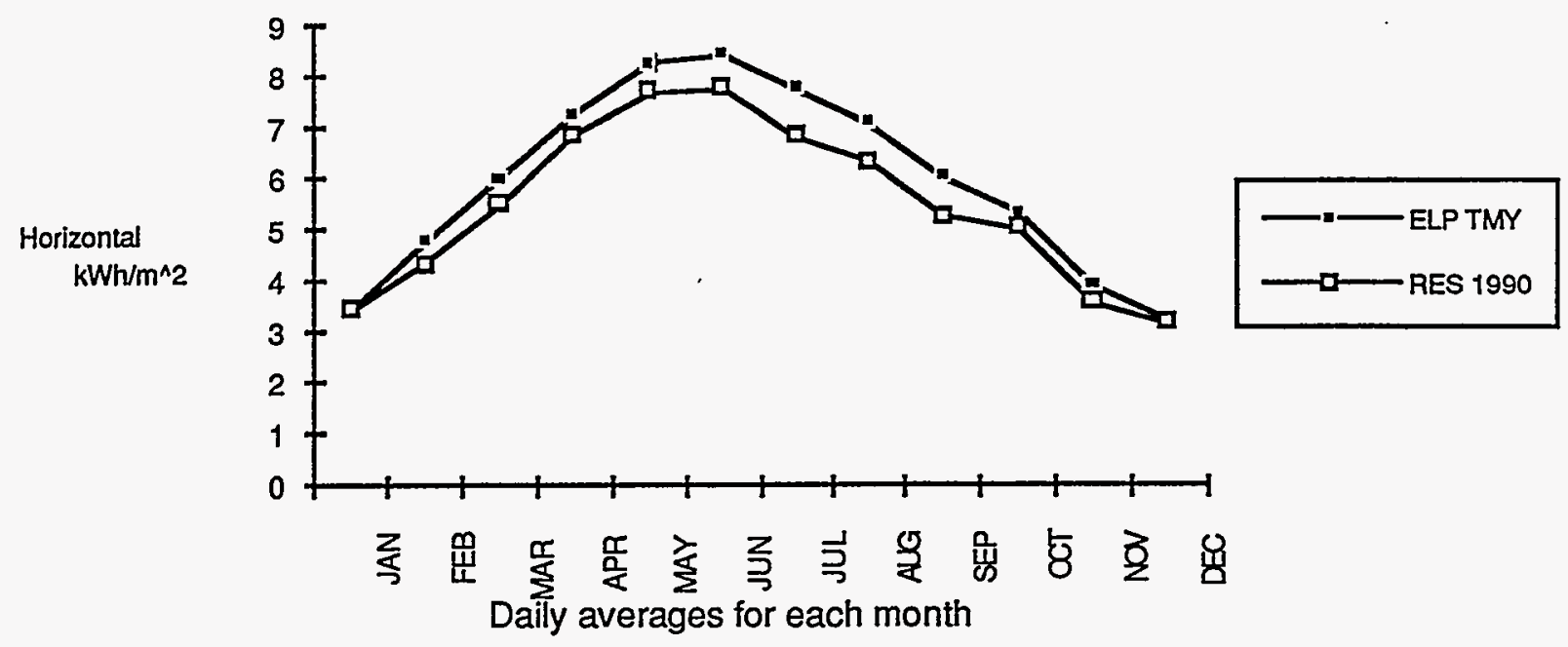

Figure 1. 1990 Horizontal Insolation Comparison

RES/ELP POA Insolation

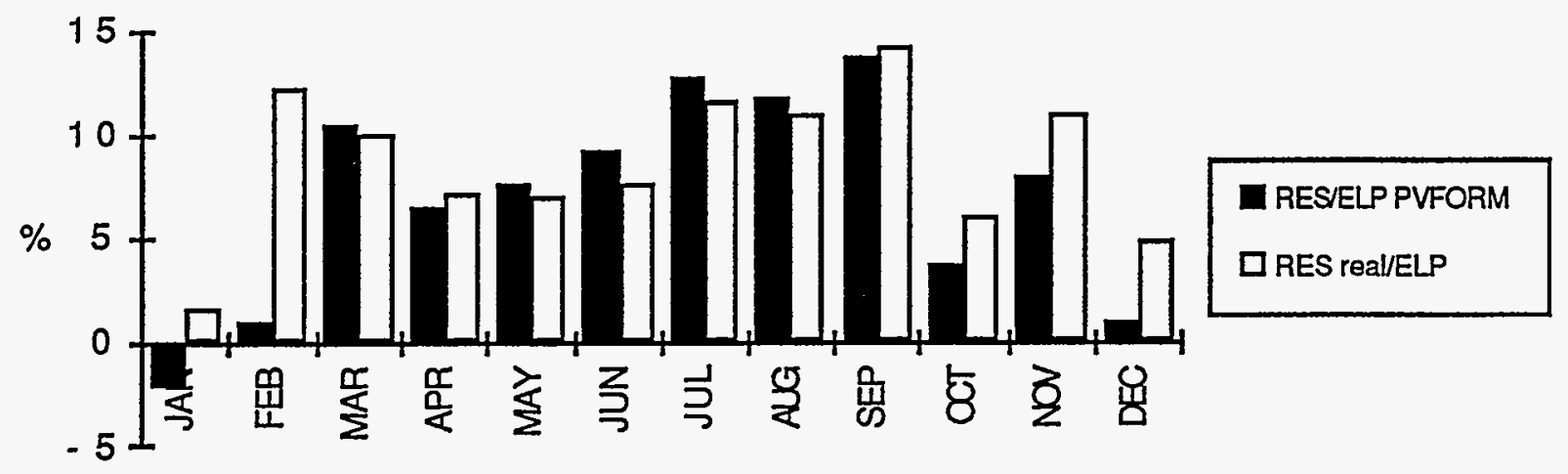

Percent Difference by Month

Figure 2. RES/ELP POA Insolation 


\subsubsection{TriSolar System}

The $5.3 \mathrm{~kW}$ system installed by TriSolar, Inc., in March 1981 was the first SWRES system to become operational. This system used Applied Solar Energy Corporation (ASEC) modules in an integral roof mount with the tilt angle fixed at $30^{\circ}$.

A Gemini/Omnion PCS was used. This system has been the most consistent performer at the SWRES. The energy production is shown in Figure 3.

This system produced $8518.01 \mathrm{kWh}$ of energy in 1990. The system rating determined from I-V curves taken in 1990 gives a value of $4.36 \mathrm{~kW}$ at standard test conditions $\left(1,000 \mathrm{~W} / \mathrm{m}^{2}, 25^{\circ} \mathrm{C}\right.$ module temperature). This is 17.8 percent lower than the original rating given in 1981. (Note: Over the years, the test equipment used to measure array power has improved significantly. The techniques did not change, but the different equipment may cause some variation in comparing readings made in 1990 and 1982.)

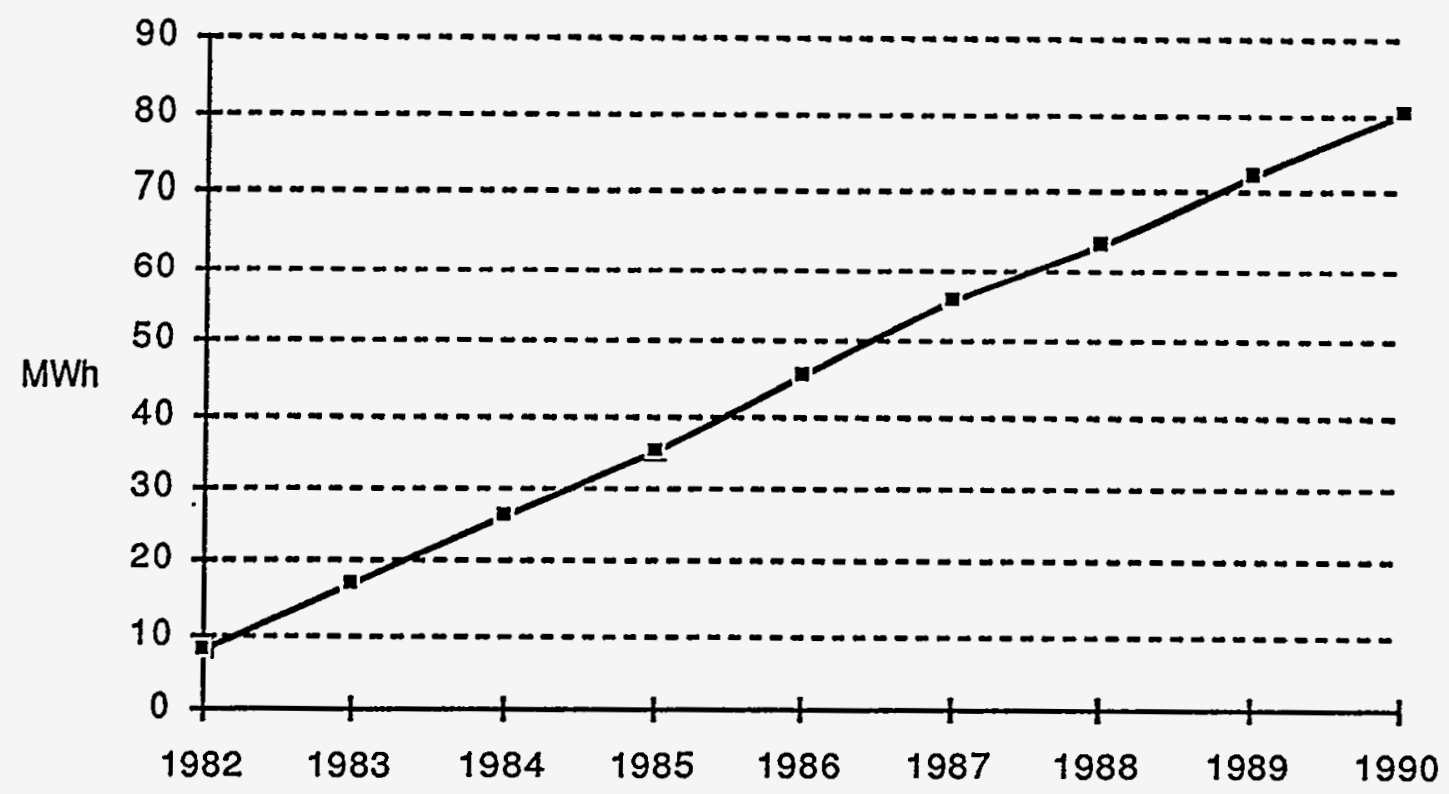

Figure 3. TriSolar System AC Energy Production. 
The reliability of the TriSolar system has been outstanding. There are only two recorded instances of unscheduled downtime. One instance was caused by a nearby lightning strike that burned the movistor on the output of the PCS, and the second instance was a fuse blown on the input of the PCS. Both problems were repaired in a few hours. There have been no hardware failures in the PCS or in the array. The modules are still producing at their original capability. The only visual change is a slight discoloration around the junction box.

\subsubsection{ARTU System}

The ARTU system started operation in July 1981. The ARTU system produced 4237 kWh of power in 1990. The energy production trends are shown in Figure 4. The system rating at standard test conditions is $2.2 \mathrm{~kW}$ based on I-V curves taken in December 1990. In 1985, one-third of this array was removed to support other testing. This caused the noticeable decrease in the annual production trend. 


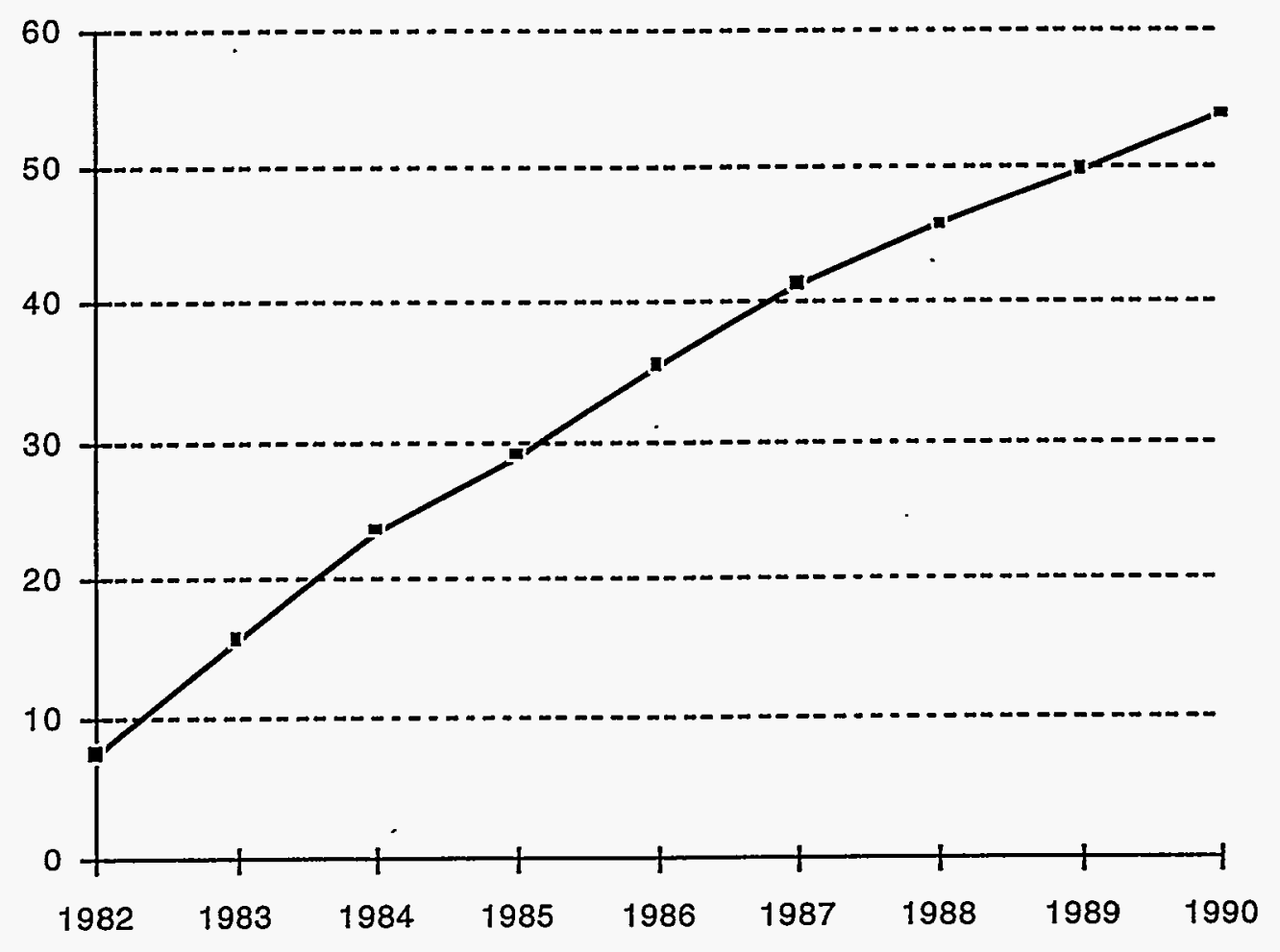

Figure 4. ARTU System AC Energy Production.

\subsubsection{Solarex System}

The Solarex system began operation in March 1981. This system used polycrystalline modules mounted in a low stand-off ( 1.75 inches) at a tilt angle of $26^{\circ}$. The energy production in 1990 was $7447 \mathrm{kWh}$, Figure 5. A $3.91 \mathrm{~kW}$ array rating was assigned in December 1990.

The system required maintenance several times in 1990 to correct loss of power in parts of the generating system. The most frequent failure was in the string connections. The connections corroded until the string was disconnected from the bus and not contributing any power. Corrections of these problems resulted in the higher 1989 rating. Also, the dc power relay in the inverter failed. This was a component related failure where the coil opened and would not close the contactor. 


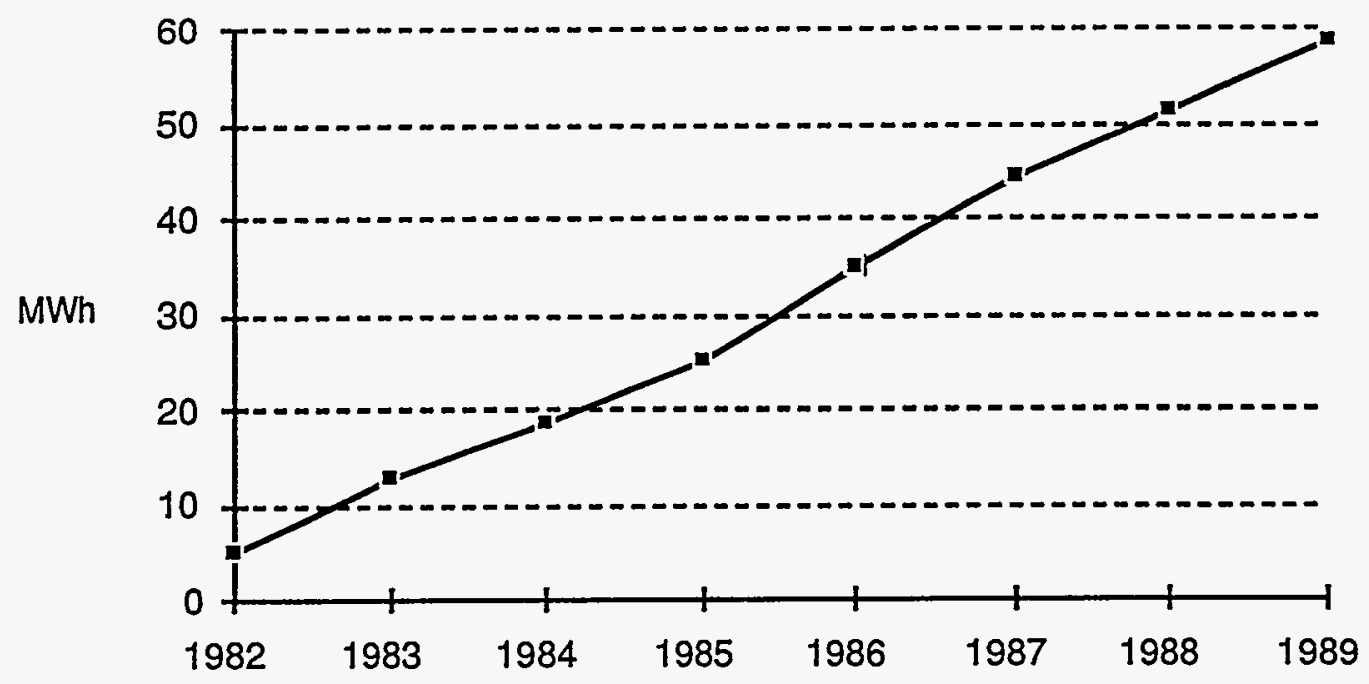

Figure 5. Solarex System AC Energy Production.

\subsubsection{Arco Solar System}

Arco Solar Corporation installed a $7.4 \mathrm{~kW}$ system at the SWRES and the initial production was in September 1989. The system used a Tedlar covered "batten and seam." module that was direct mounted to the $25^{\circ}$ tilt roof. The direct mount caused this array to operate at temperatures equivalent to the GE array-about $15^{\circ} \mathrm{C}$ higher than other arrays on the site. This higher temperature penalized the energy production by 7 to 8 percent over an equivalent array that operates $15^{\circ}$ cooler.

This system was dismantled in 1987 because of multiple shorts in the array. The Tedlar covering on the modules delaminated from the metal sheet used in the batten and seam construction. This allowed dust and moisture to invade, particularly in the corners of the modules, and electrical shorts occurred. Because of the lack of access to the direct mounted modules, it was almost impossible to 
maintain or repair the array. The poor experience with this array and the GE array caused the direct mount technique to be abandoned.

\subsubsection{Mobil Solar System}

This system started production in October 1983. The array uses the $4 \times 6$ foot ribbon modules produced by Mobil for large arrays. The array is an integral mount at $30^{\circ}$ tilt angle. The PCS is made by American Power Conversion Corporation (APCC).

This system has been reliable with only two instances of unscheduled downtime in the last seven years. Both were caused by PCS problems but these were repaired in a few hours. In 1990, the array produced $60 \mathrm{kWh}$ of energy. The energy production is given in Figure 6. A fuse failed in the ac side of the inverter for an unknown reason (the second failure within a few months), and a module was replaced at Mobil's request. The latest $\mathrm{I}-\mathrm{V}$ curves taken in 1990 show a system rating of $3.4 \mathrm{~kW}$.

There has been a significant browning of the MSEC modules used on this array. This can be correlated with the $2 \%$ percent/year drop in power since 1988. Several of these modules were returned to MSEC so they could analyze the apparent discoloration of the EVA encapsulent. 


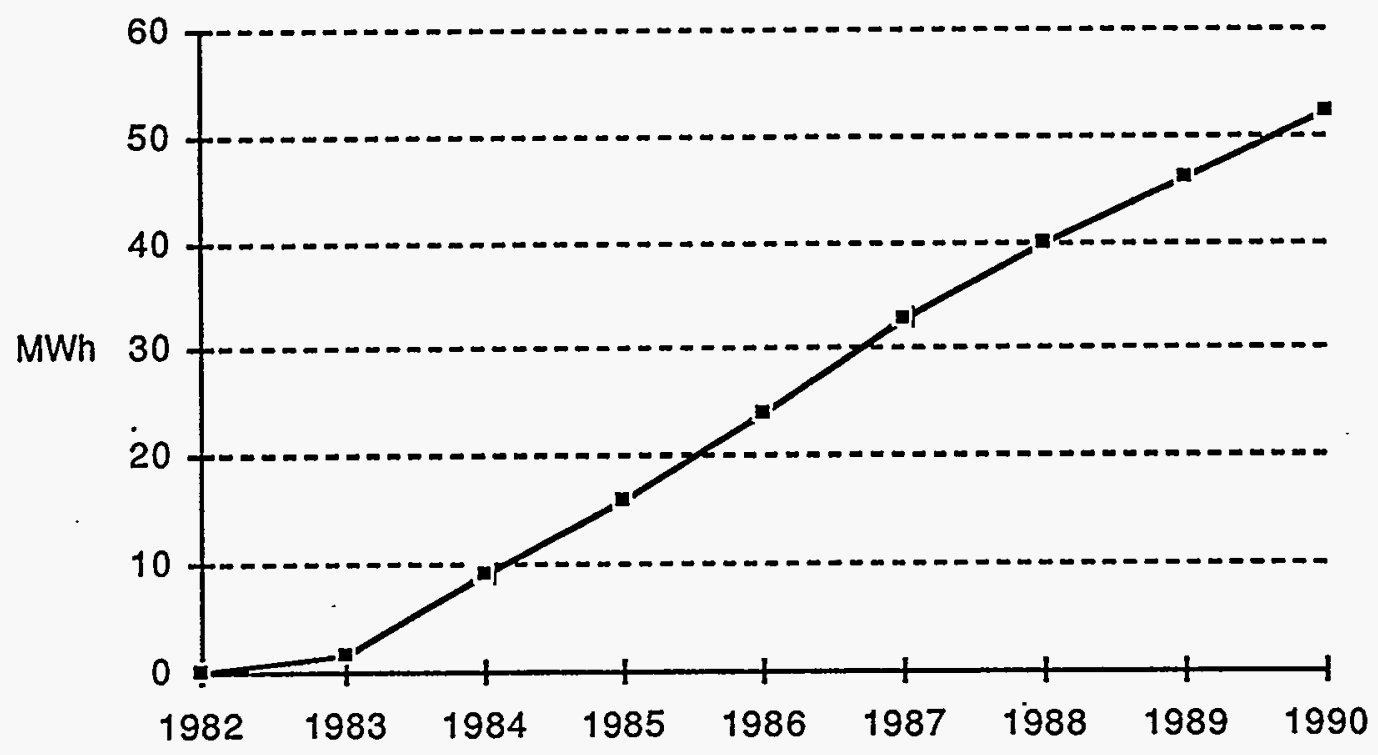

Figure 6. MSEC System AC Energy Production.

\subsection{PVFORM ANALYSIS}

PVFORM is a software package used to design and size PV systems. PVFORM uses a typical meteorological year (TMY) data file and PV system specifications to forecast monthly energy production. In past years PVFORM was analyzed using SWRES crystalline system specifications and El Paso TMY data. Results were favorable for the crystalline systems and PVFORM found to be fairly accurate. El Paso data was used because local weather data was unavailable. This year SWRES weather data is used in the analysis and the amorphous systems are included as well. The result is that PVFORM accurately forecasts energy production for crystalline systems, but is not accurate for amorphous systems. 


\subsubsection{PVFORM Comparison of SWRES vs El Paso TMY Data}

To clarify differences in SWRES and El Paso TMY data, PVFORM was run with each data set, using the same system specifications. A graph of the forecasted monthly energy production is shown in Figure 7. This shows there is no major discrepancies in the data sets, although real weather produces lower energy forecasts.

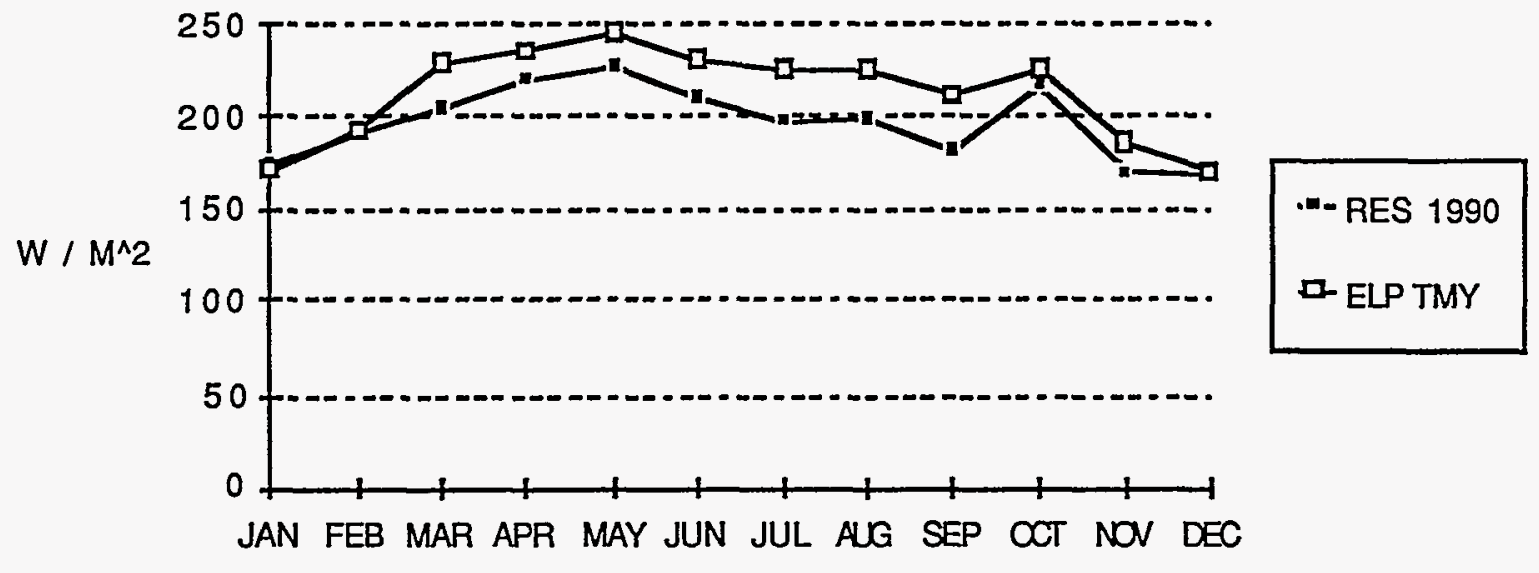

Figure 7. PVFORM Comparison of SWRES and El Paso TMY

\subsubsection{PVFORM Comparison of Crystalline Systems}

PVFORM was run with SWRES weather data using the specifications of the four crystalline systems on site. Graphs of the predicted versus actual energy production are shown below in Figures 8 through 11. The PVFORM energy production consistently follows the same trend as the actual energy production. The few differences, such as July for the MSEC system, are caused by system downtime for maintenance or repairs. This shows that PVFORM accurately (within $+5 \%$ ) forecasts energy production for crystalline systems. 


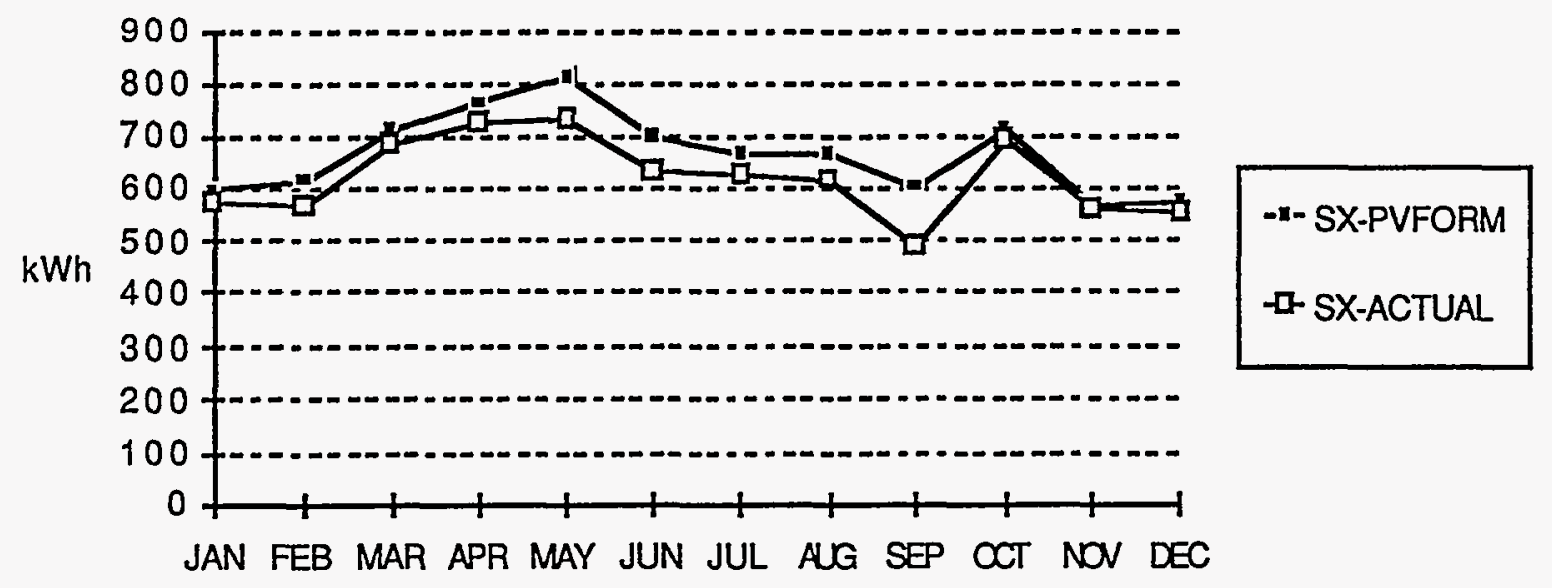

Figure 8. SX System; PVFORM vs. Actual Energy Production.

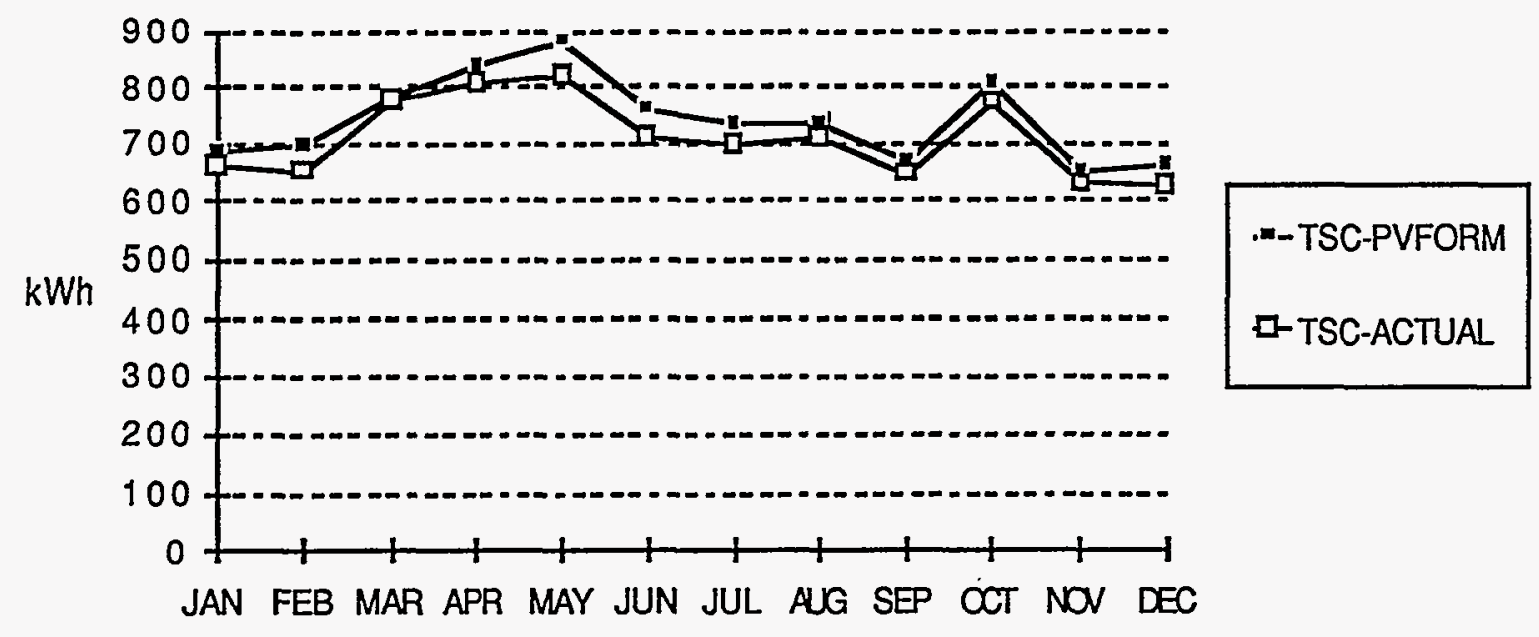

Figure 9. TSC System; PVFORM vs. Actual Energy Production. 


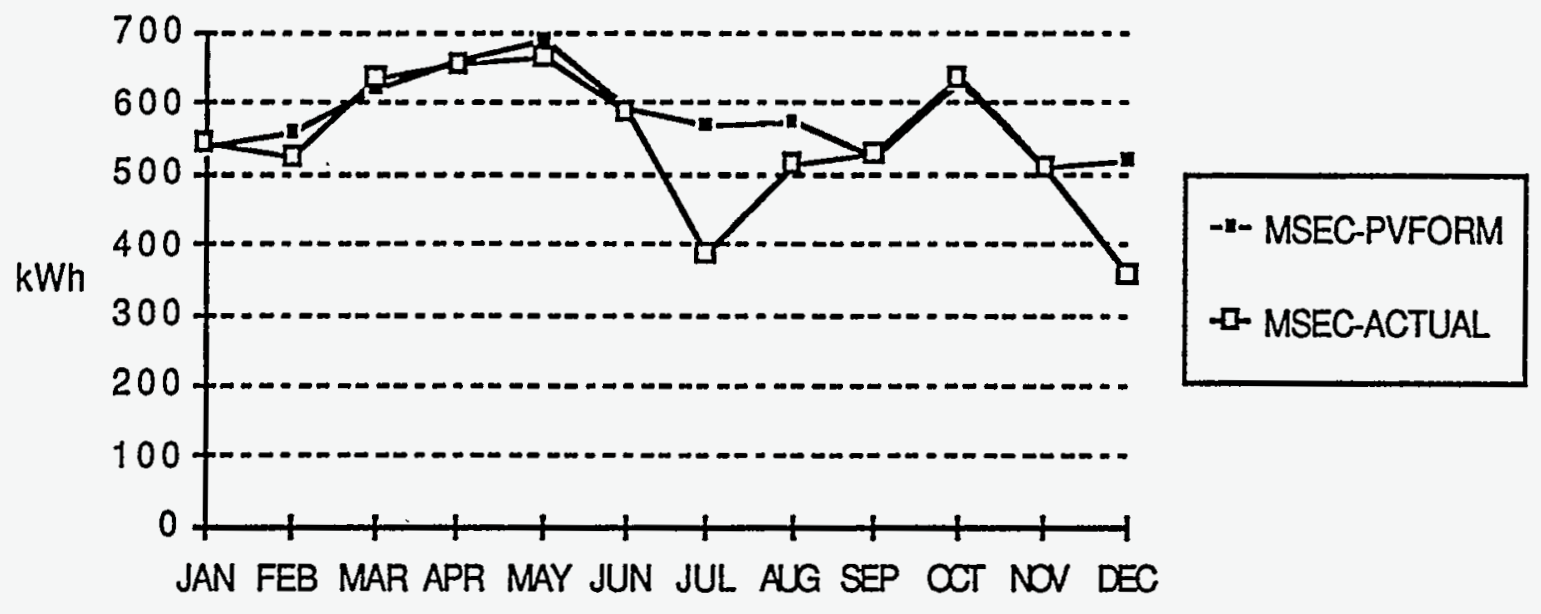

Figure 10. MSEC System; PVFORM vs. Actual Energy Production.

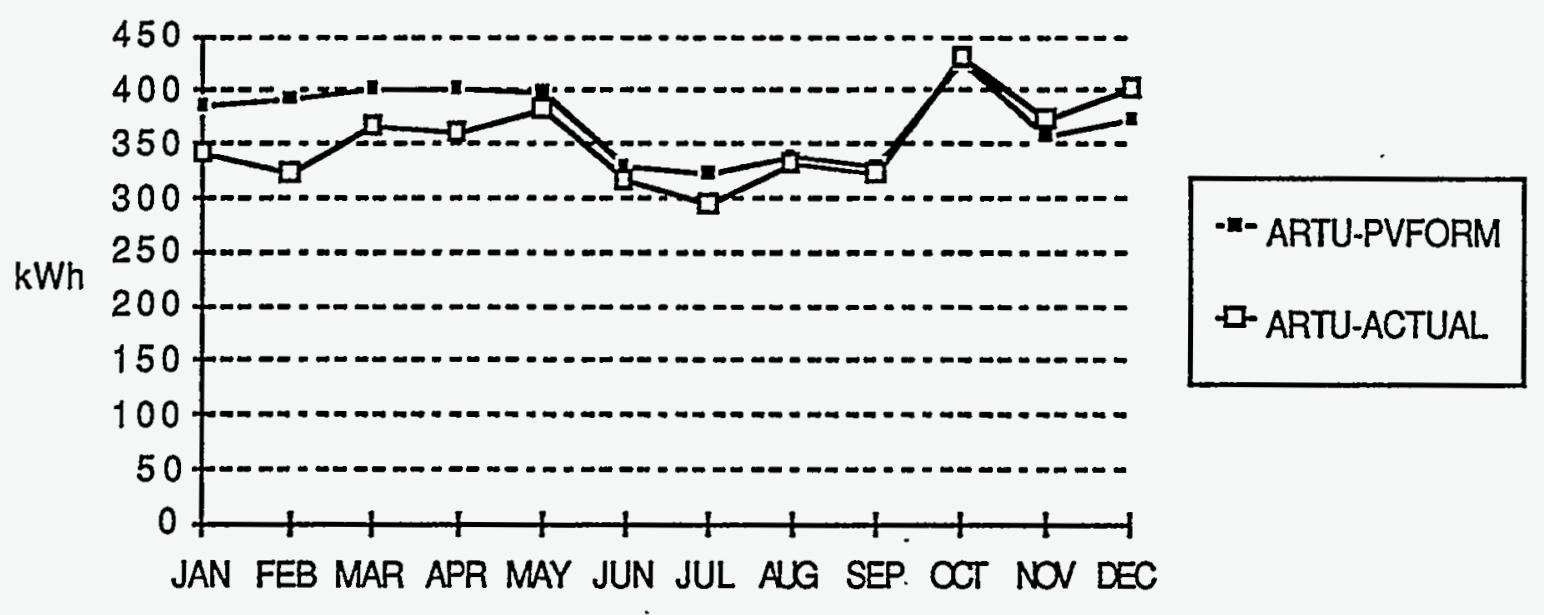

Figure 11. ARTU System; PVFORM vs. Actual Energy Production.

\subsubsection{PVFORM Comparison of Amorphous Systems}

PVFORM was run with SWRES weather data using the specifications of the three amorphous systems on site. The predicted energy production does not consistently follow the actual production trend (See figures 12 through 14). Notice how the data 
lines cross each other, which did not occur for the crystalline systems. PVFORM both over and under predicts energy production for amorphous systems by plus or minus five percent.

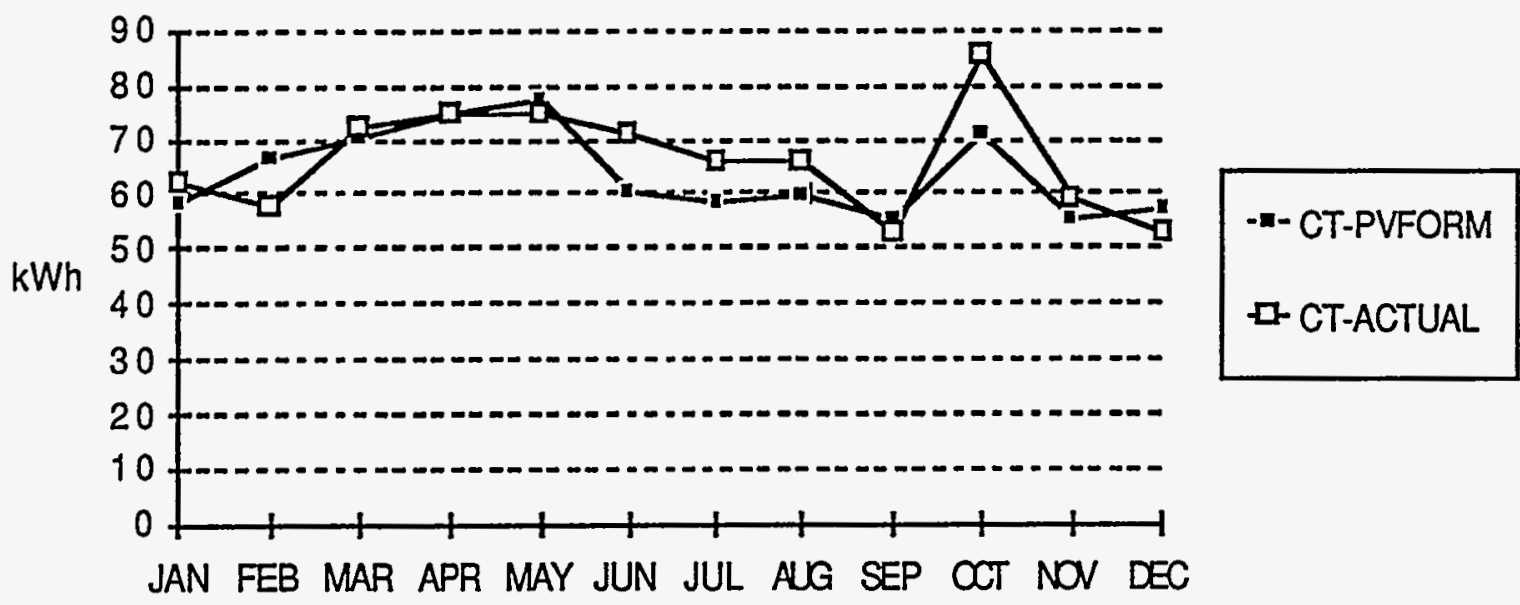

Figure 12. CT System; PVFORM vs. Actual Energy Production.

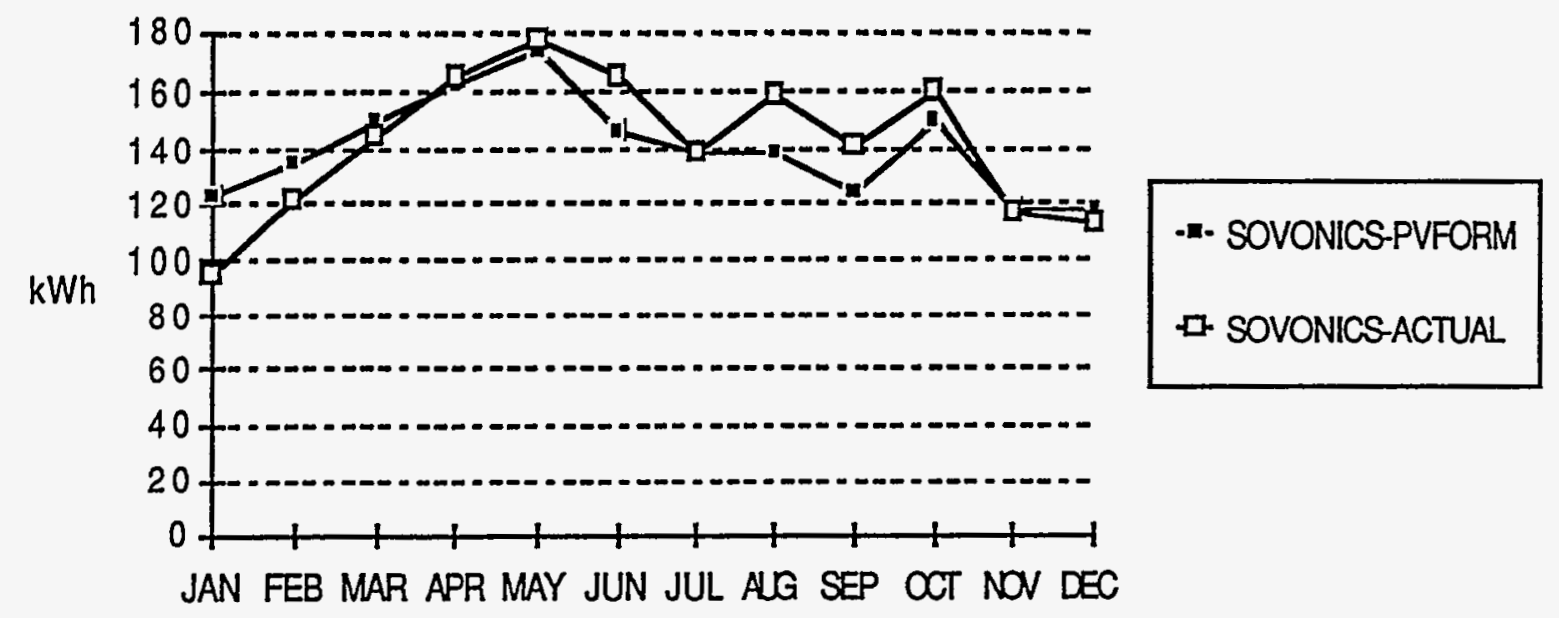

Figure 13. SOVONICS System; PVFORM vs. Actual Energy Production. 


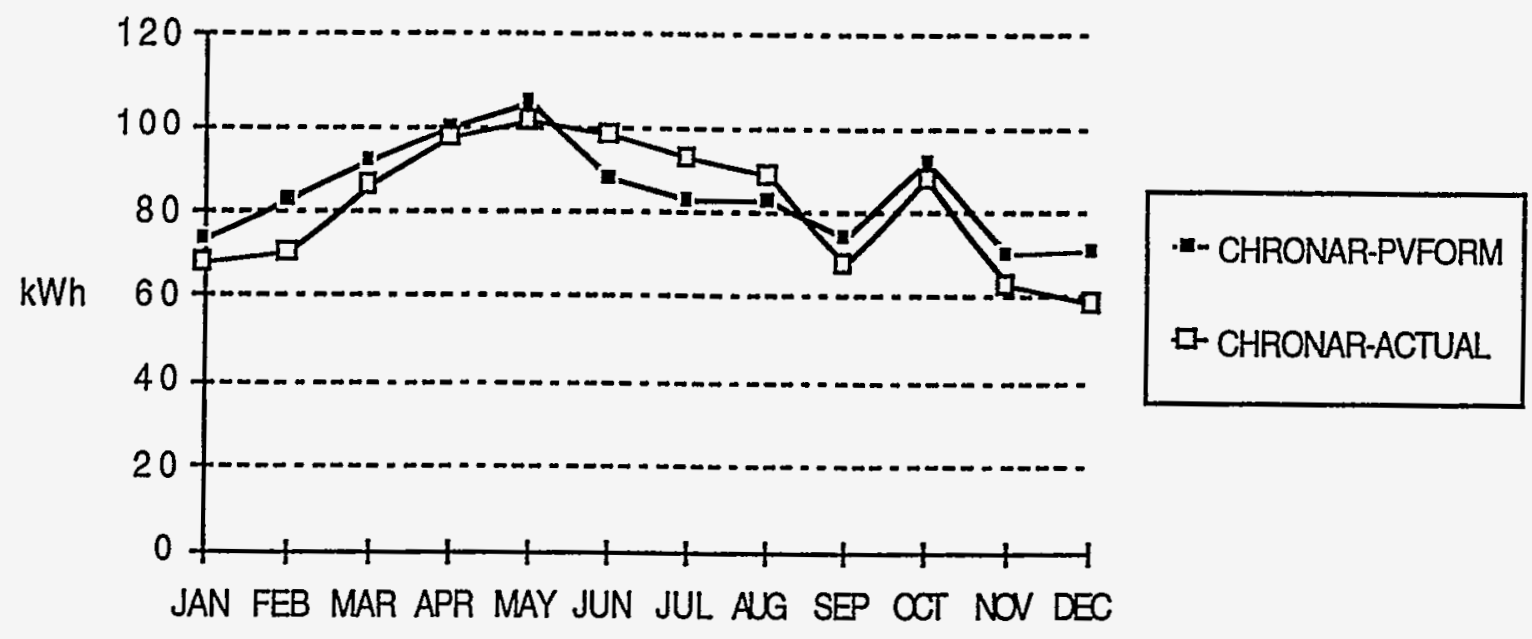

Figure 14. CHRONAR System; PVFORM vs. Actual Energy Production.

\subsubsection{Summary of PVFORM Analysis}

SWRES weather data was used to analyze PVFORM for both crystalline and amorphous systems this year. PVFORM accurately (within $+5 \%$ ) predicts energy production for crystalline PV systems. Amorphous systems are not as accurately predicted. The error factor doubled to $\pm 5 \%$. This indicates PVFORM needs modification to accurately forecast energy production for amorphous systems. 
APPENDIXA

1990 Details on Downtime

A-1 


\section{DETAILS ON DOWNTIME}

\begin{tabular}{|c|c|c|c|}
\hline SYSTEM & DATE & TIME & EXPLANATION \\
\hline \multirow[t]{2}{*}{ Sovonics } & 010490 & $1045-1120$ & New inverter breaker Switch. \\
\hline & $\begin{array}{l}072890 \\
073190\end{array}$ & $\begin{array}{l}1230 \\
1530\end{array}$ & Rewired Ross Inverter. \\
\hline \multirow[t]{2}{*}{ GE } & $\begin{array}{l}010490 \\
010990\end{array}$ & $\begin{array}{l}1450 \\
0900\end{array}$ & $\begin{array}{l}\text { Removed } 3 \text { bad modules and rewired } \\
\text { system for best power. }\end{array}$ \\
\hline & $\begin{array}{l}053090 \\
111290\end{array}$ & $\begin{array}{l}\text { 1508-Indef. } \\
\text { 1500-Indef. }\end{array}$ & $\begin{array}{l}\text { Removed inverter, returned to SNL. } \\
\text { Modules removed-moved to TEA } \\
\text { (module failures and no interest by } \\
\text { manufacturer) }\end{array}$ \\
\hline \multirow[t]{6}{*}{ Solarex } & 010990 & $0956-1123$ & $\begin{array}{l}\text { Replaced a Solarex module ITT/LL } 7821 \\
\text { with a Solarex module MIT 100-2967. }\end{array}$ \\
\hline & $\begin{array}{l}082090 \\
083090\end{array}$ & $\begin{array}{l}1515 \\
0730\end{array}$ & $\begin{array}{l}\text { Removed defective string switches. } \\
\text { Rewired everything directly to array. } \\
\text { Replaced all inadequate sized wires. }\end{array}$ \\
\hline & $\begin{array}{l}090790 \\
091090\end{array}$ & $\begin{array}{l}1000 \\
1600\end{array}$ & $\begin{array}{l}\text { Installed isolation transformer and } \\
\text { ground fault interrupter for testing. }\end{array}$ \\
\hline & 101890 & $1435-1505$ & $\begin{array}{l}\text { Modified ground fault interrupter } \\
\text { prototype. }\end{array}$ \\
\hline & 103090 & $0830-0930$ & $\begin{array}{l}\text { Installed ground fault interrupter for } \\
\text { testing. }\end{array}$ \\
\hline & 120390 & $1505-1520$ & $\begin{array}{l}\text { Photographed ground fault interrupter } \\
\text { prototype. }\end{array}$ \\
\hline \multirow[t]{5}{*}{ WST } & 013190 & $1200-1600$ & Soldered leads on Pyranometer. \\
\hline & $\begin{array}{l}020790 \\
020890\end{array}$ & $\begin{array}{l}0900 \\
1630\end{array}$ & Blown Fuse. Replaced both Fuses. \\
\hline & $\begin{array}{l}021690 \\
021990\end{array}$ & $\stackrel{?}{i} 100$ & $\begin{array}{l}\text { Array \& Inverter breaker found off. } \\
\text { Turned back on. }\end{array}$ \\
\hline & 042390 & $1500-1530$ & Re-routed Pyranometer cable. \\
\hline & $\begin{array}{l}081490 \\
031991\end{array}$ & $\begin{array}{l}0100 \\
1425\end{array}$ & $\begin{array}{l}\text { Removed inverter. Sent back to manu- } \\
\text { facturer for repair. Reinstalled. }\end{array}$ \\
\hline \multirow[t]{2}{*}{ Mobile } & $\begin{array}{l}022090 \\
030590\end{array}$ & $\stackrel{?}{0} 820$ & $\begin{array}{l}\text { Array \& Inverter breaker found off. } \\
\text { Turned back on. }\end{array}$ \\
\hline & $\begin{array}{l}121990 \\
010291\end{array}$ & $\stackrel{?}{?}$ & System found off. Turned back on. \\
\hline
\end{tabular}


1990 DETAILS ON DOWNTIME

\begin{tabular}{|c|c|c|c|}
\hline SYSTEM & DATE & TIME & EXPLANATION \\
\hline \multirow[t]{7}{*}{ TEA } & $\begin{array}{l}042690 \\
042790\end{array}$ & 1415 & Remount battery switch. \\
\hline & 052190 & 0915-1030 & Installed fuse protector. \\
\hline & $\begin{array}{l}070990 \\
071690\end{array}$ & $\begin{array}{l}0700 \\
1430\end{array}$ & Installed Ni-H2 Batteries \\
\hline & 081590 & $1100-1345$ & $\begin{array}{l}\text { Wired } 2 \text { additional batteries } \\
\text { (Trojan L-16's) in parallel } \\
\text { with existing system. }\end{array}$ \\
\hline & $\begin{array}{l}100390 \\
100890\end{array}$ & $\begin{array}{l}0825 \\
0730\end{array}$ & Equalized batteries. \\
\hline & 112190 & $1105-1715$ & $\begin{array}{l}\text { Installed } 30 \text { Solarex SA-20 } \\
\text { modules. }\end{array}$ \\
\hline & 112690 & $0800-0900$ & $\begin{array}{l}\text { Combined old Sovonics } \\
\text { array with new Solarex } \\
\text { array. }\end{array}$ \\
\hline \multirow[t]{3}{*}{ Trisolar } & 050890 & 0830 & $\begin{array}{l}\text { Condensation inside Pyro } \\
\text { glass dome. }\end{array}$ \\
\hline & $\begin{array}{l}053190 \\
060190\end{array}$ & $\begin{array}{l}1500 \\
1000\end{array}$ & $\begin{array}{l}\text { System found off. Turned } \\
\text { back on. }\end{array}$ \\
\hline & 071790 & 0830-1015 & Tester work (Steve Durand). \\
\hline \multirow[t]{5}{*}{ CT/OT } & $\begin{array}{l}062690 \\
070390\end{array}$ & $\begin{array}{l}0800 \\
1730\end{array}$ & $\begin{array}{l}\text { Scheduled down time (Dug } \\
\text { trenches for tracker). }\end{array}$ \\
\hline & $\begin{array}{l}070890 \\
071290\end{array}$ & $\begin{array}{l}1500 \\
1123\end{array}$ & $\begin{array}{l}\text { Testing ground fault } \\
\text { interrupter. }\end{array}$ \\
\hline & 071790 & $1015-1040$ & Tester work (Steve Durand). \\
\hline & $\begin{array}{l}072890 \\
092090\end{array}$ & $\begin{array}{l}1230 \\
0920\end{array}$ & $\begin{array}{l}\text { Removed inverter. Sent } \\
\text { back to manufacturer for } \\
\text { repairs. Reinstalled. }\end{array}$ \\
\hline & $\begin{array}{l}101290 \\
102290\end{array}$ & $\begin{array}{l}0100 \\
0815\end{array}$ & Blown inverter fuse. \\
\hline
\end{tabular}


APPENDLX B

ARTU 1990

B-1 


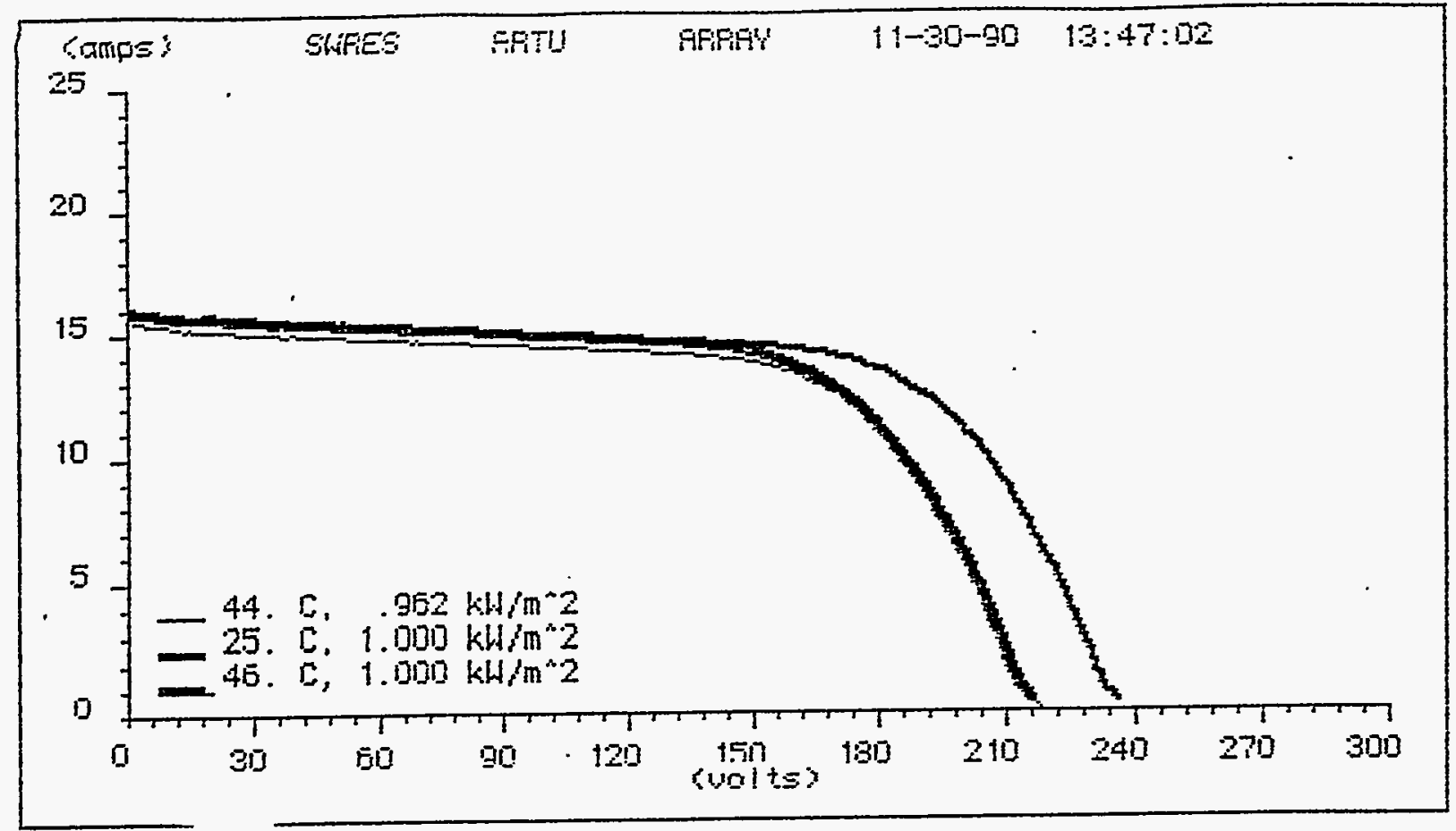

Cell Temperature ....... 44.000 deg $C$

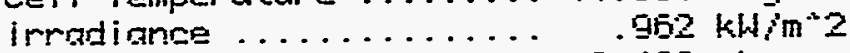

Series Resi Đtanice ....... 2. 100 ohms

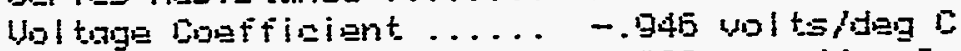

Curpent coeffimient ..... . O09 ampsideg 5

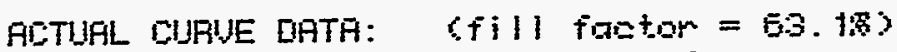

Short aimatit aurrent .... 15.5 amps

Gpen circuit val tage ..... 213.7 wolts

Max poumer cturpent. ...... 1:.2.2 amps

Mox puwer val toge ...... tE t. . wol t:

Hox poun ........... 2135.3 matt.5

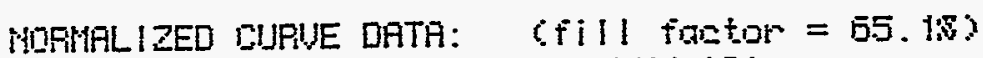

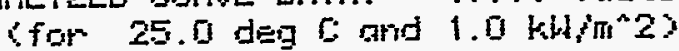

Max pourar cimpent. ...... 13.5 amps

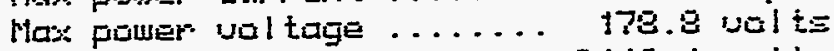

Hax power ............. 24413. 1 wgtts

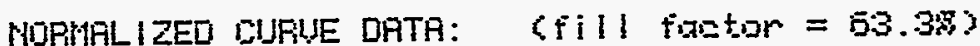

ifar 40.0 deg 5 and $1.0 \mathrm{kH} / \mathrm{m}^{\wedge} 23$

Max power curment ...... 13.4 ampE

Max pouer uol tage ....... 164.4 volts

Max pousar ........... 219.5.8 mats 


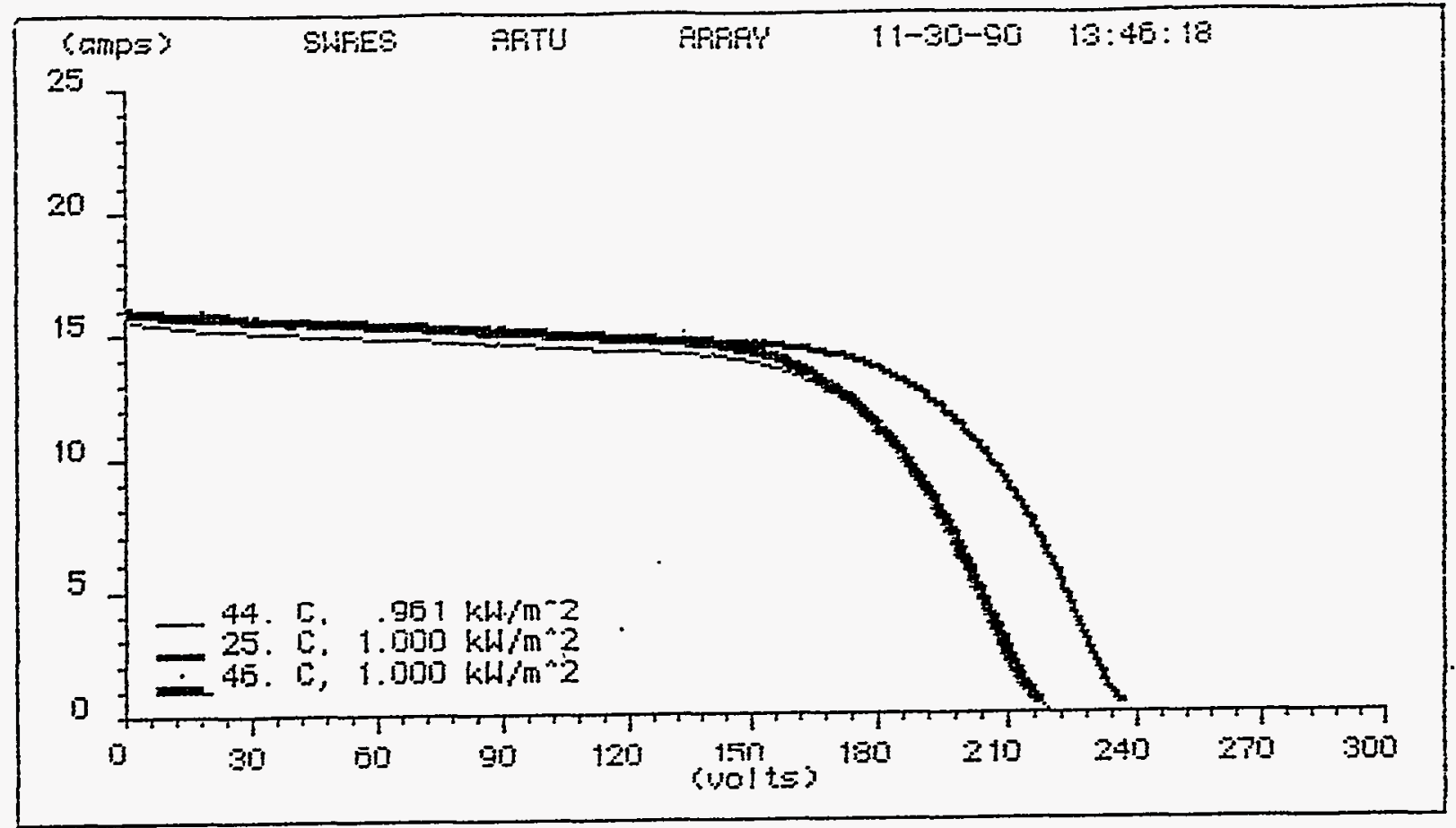

Cel I Temperature .........

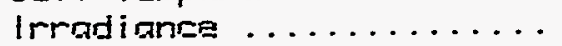

Seri iss Resistance ........

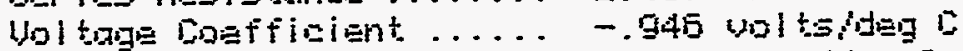

Cumpent coefficient ..... . ong ams: ideg $\mathrm{c}$

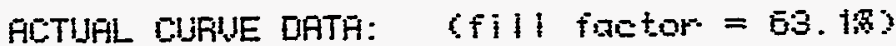

Short airodit currant. ... 15.5 amps Oper circuit voltage ..... 219.0 wolts Mres pouner current...... 13.0 amps Hox pouler ugl tage ....... tE. t: 2 wolt: Hax pumar ........... 2143.1 matis

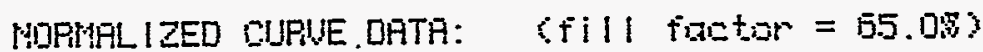
for 25.0 deg C and $1.0 \mathrm{kH}+\mathrm{m}^{* 2} \mathrm{z}$

Mate power aturent ....... 13.5 smps

Max puwer val tage ...... i. ig. g wal t:

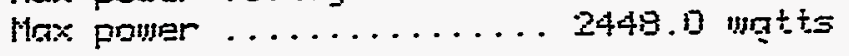

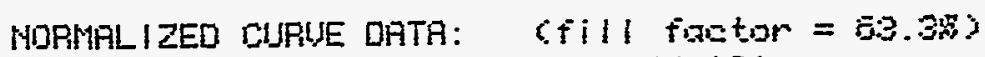
rich 46.0 deg $\left[\right.$ ard $1.0 \mathrm{kh} / \mathrm{m}^{*} 2$ )

Max pouser surnent ....... 13.5 amps Mox pourar ugl tage ....... 162.0 volts Mox punar ............ 2205.7 untts 


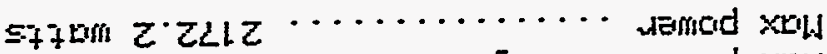

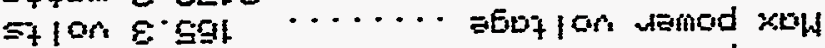

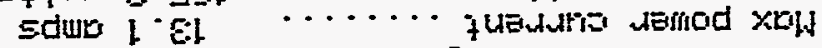
(Z.)

(स2 $29=$ waq

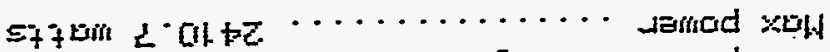

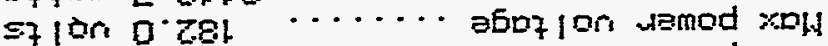

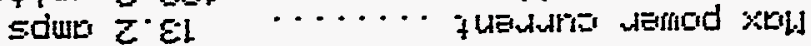

(C.

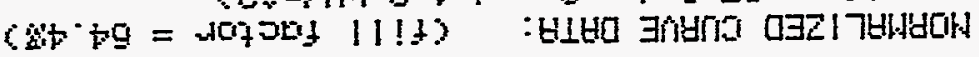

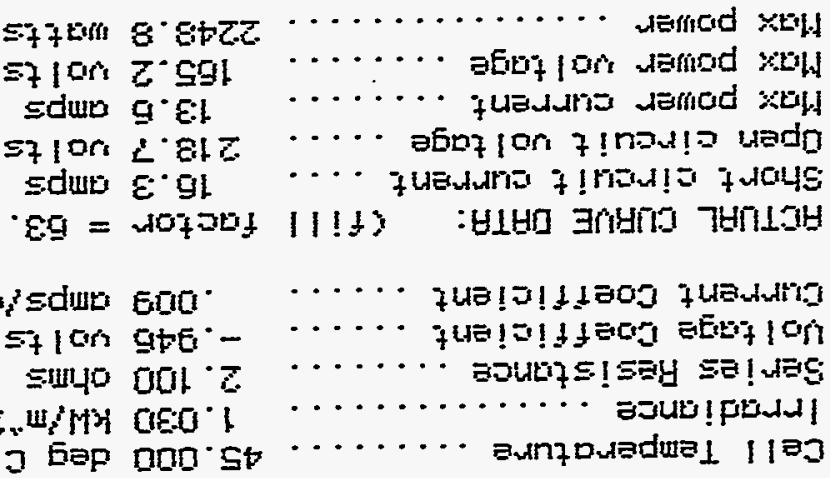

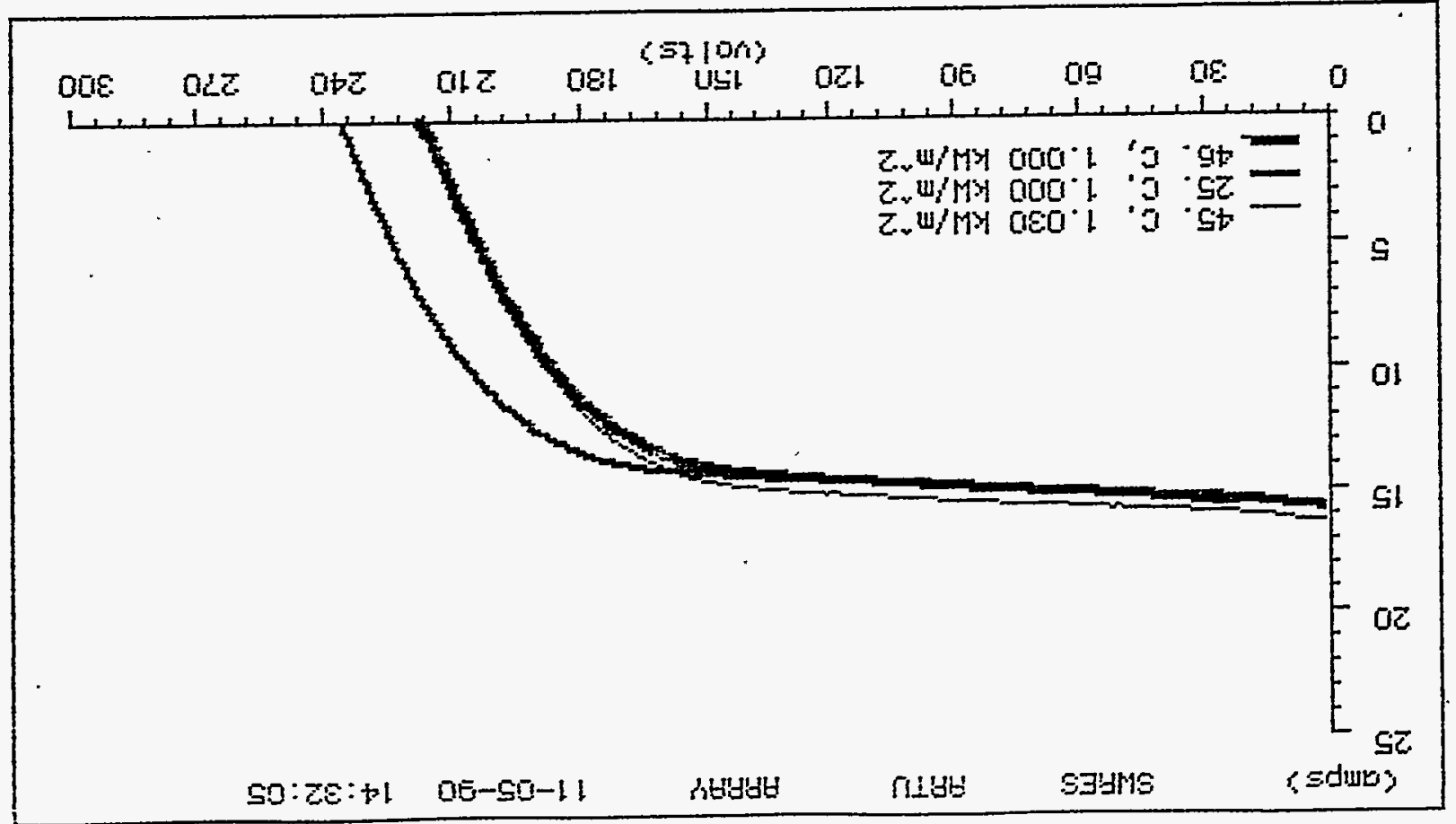




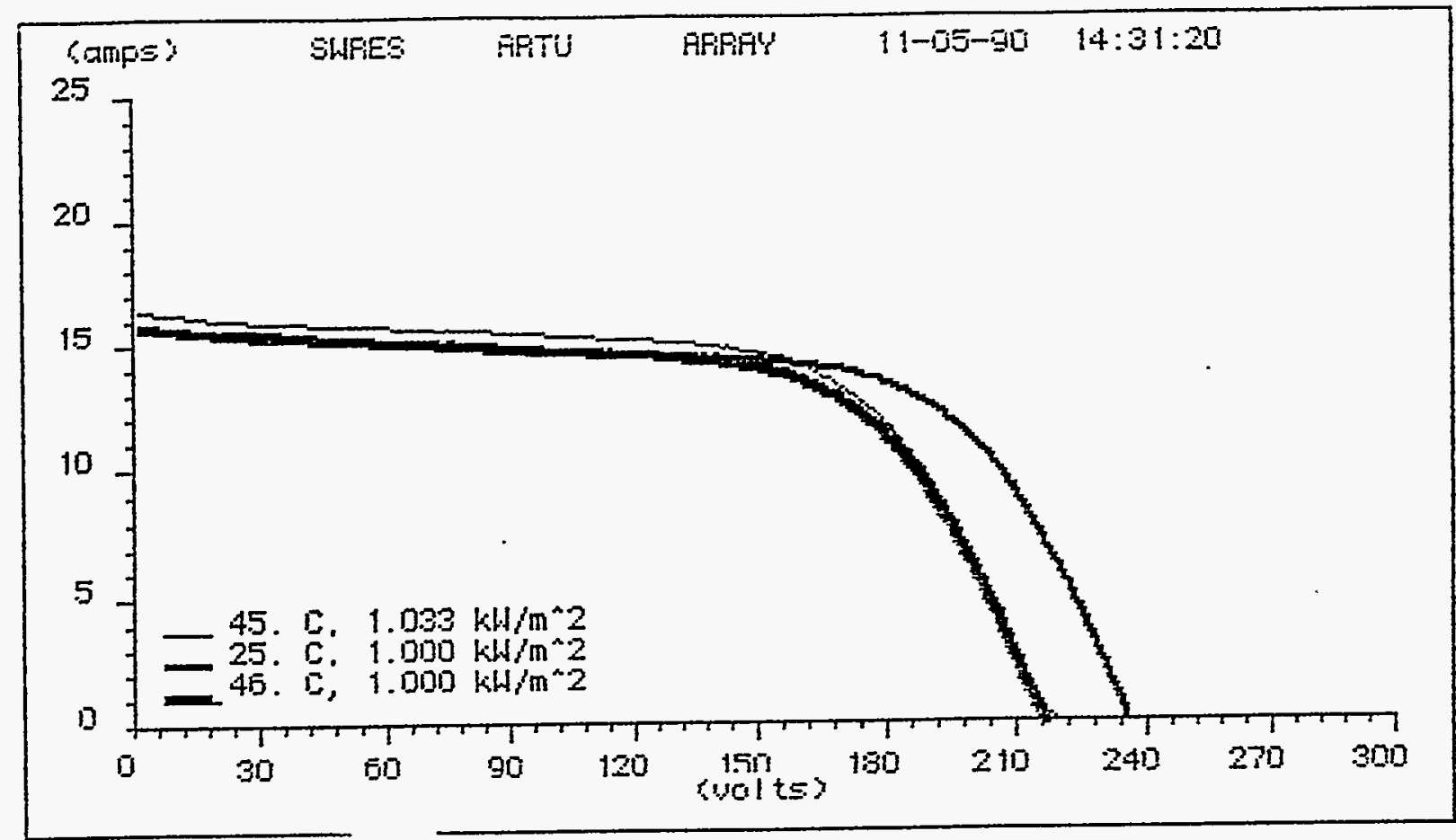

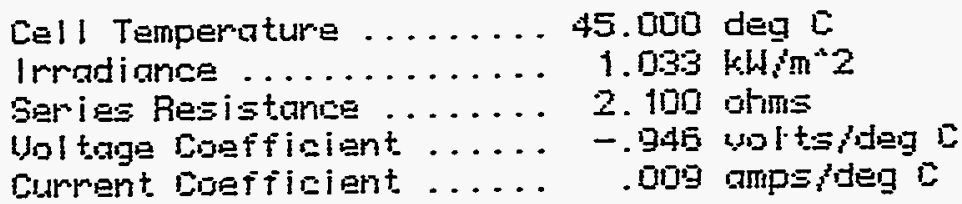

ACTUAL CURUE LATA: (fill faetor = 63.03)

Short circuit aurment. ... 15.4 smps

Upen circuit woltage .... 219.0 wolts

Max poyer current. ...... 13.7 amps

Max power vol tage ....... 16.5 .2 vo! ts

Max pounar ........... 2258.7 ungt:s

NORMALIZED CIURUE DATH: Gill faetor $=64.48 \%$.

(for 25.0 deg 0 and $1.0 \mathrm{kH} / \mathrm{m}^{*} \mathrm{z}$ )

Max pouser asrrent ...... 13.3 amps

Ma* power vol tage ....... 130.9 val ts

Max pouer ............2415.1 watts

MDFMALIZED DURUE DATA: (fill faotor = 62.78)

(for 40.0 deg $C$ and $1.0 \mathrm{kH} / \mathrm{m}^{\mathrm{n}} 2$ )

Max pouer aurrent ...... 13.2 amps

Max power voltage ...... 1E5.4 wolts

Max powar .......... 2175.5 untts 


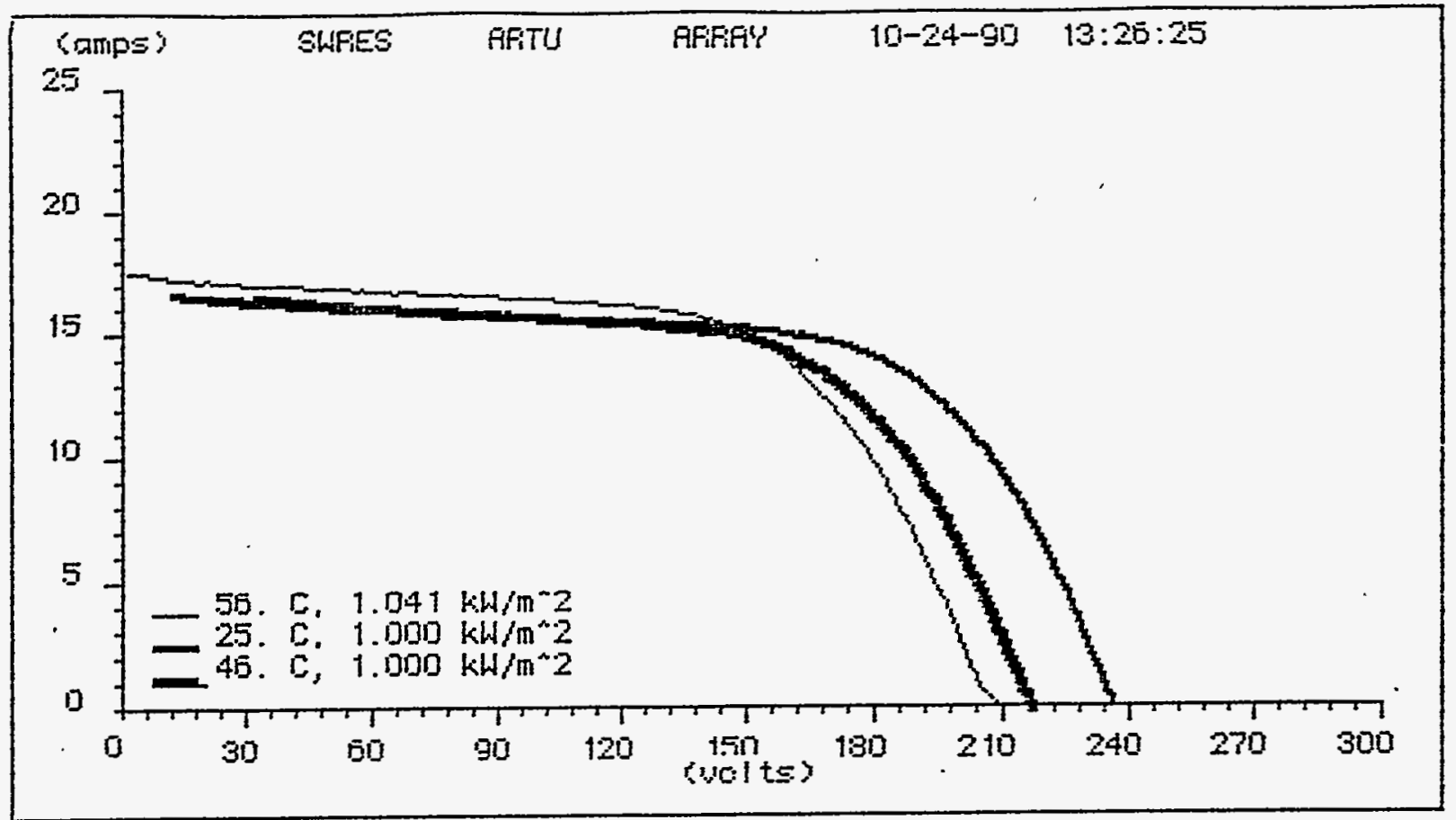

\footnotetext{
Lell Temperature ........ SE. 000 dèg $C$

Irradiance ........... $1.041 \mathrm{kH}^{2} \mathrm{~m}^{2} \mathrm{z}$

Series Resistance ....... 2.100 otims

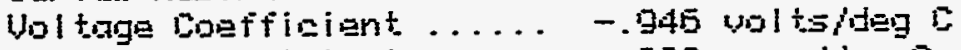

Eur.ment Coeficiant ..... . . Do9 ampsideg $\mathrm{c}$

ACTUHL CURUE DATA: $\quad$ fill fGEtor $=61.13 \mathrm{~s}$

Short cirauit current. ... 17.5 amps Open Eireuit wol tage ..... 209.0 volts Max pouser surrant....... 14.3 amps Max power voltage ...... 150. n volts Hax powar ........... 2242.5 watts

MDFIALLIZEO CUPUE DFTA: CIIII faCtOH = 63.5B ffor 25.0 deg [ ard $1.0 \mathrm{kH} / \mathrm{m}+2 \mathrm{~s}$

Max pouner current. ...... 14.0 amp:s

Max power vol tage ....... 131.3 volts

Max power ........... 25:33.4 mạtts

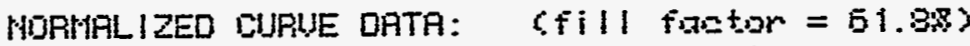

6ior $40.0 \mathrm{deg} C$ and $1.0 \mathrm{kH} / \mathrm{m}^{\wedge} \mathrm{Z}$ )

Max pouler cirrrant....... 14.2 amps

Mox power vol tage ....... 151.1 volts

Max pouler ........... 2283.4 watts
} 


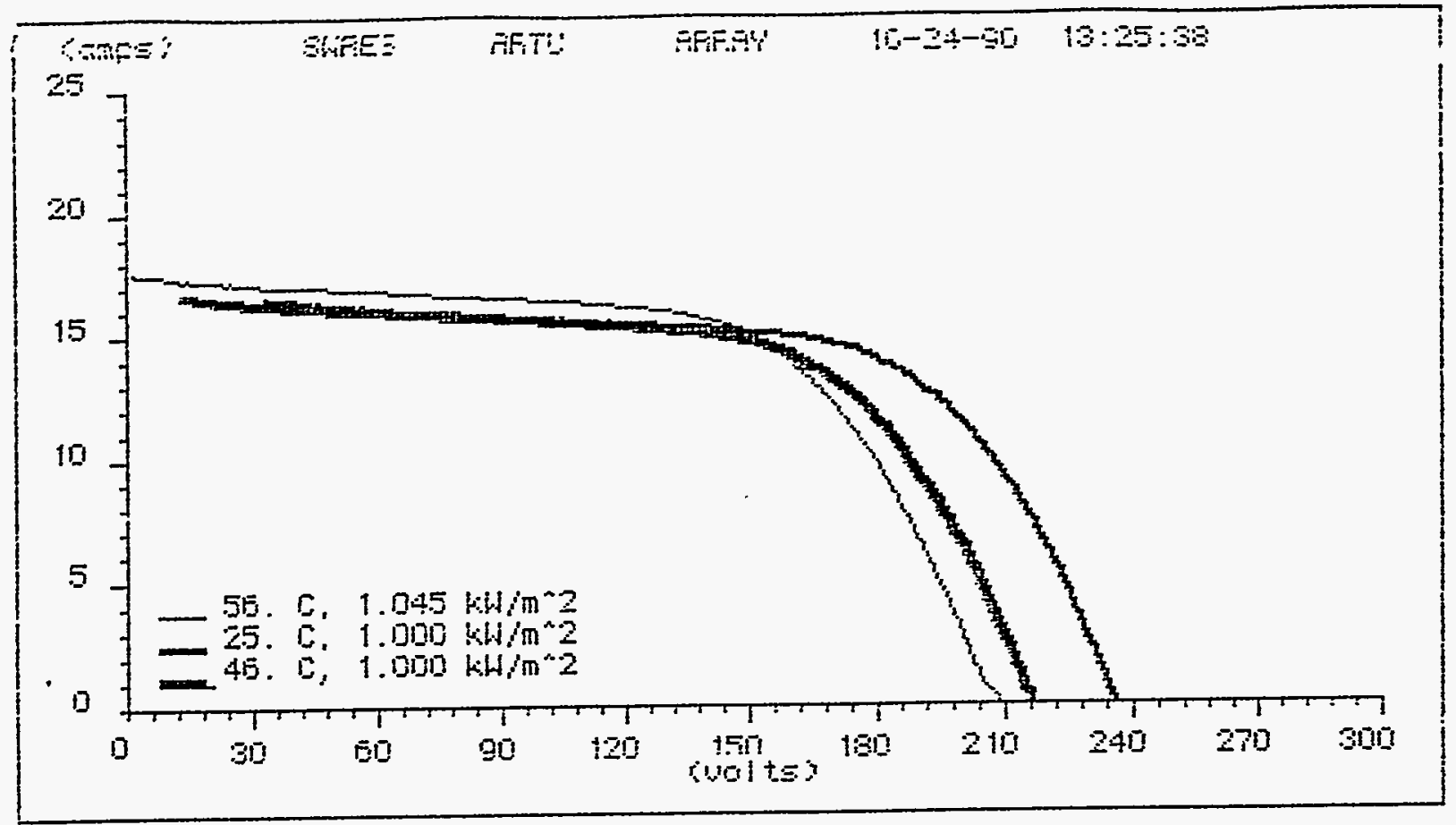

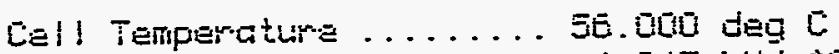

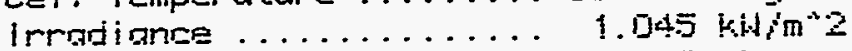

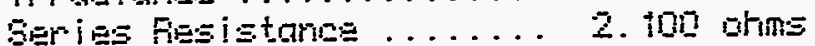

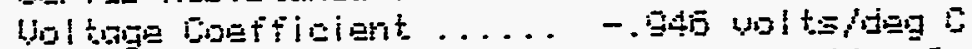

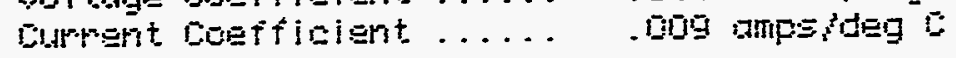

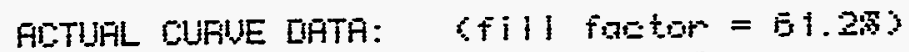

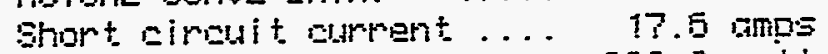
Gper circuit voltage ..... 209.2 wolts Hoy power cturrent. ....... 14.8 smps

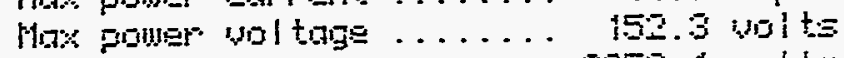
Mox poum ........... 225] 1 mat.

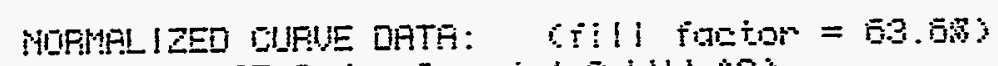

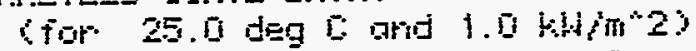

Mox poumer altisent. ...... 14.2 amps

hax power uol tage ....... 179. 1 vol t:

Hax pouner . . . . . . . . . .

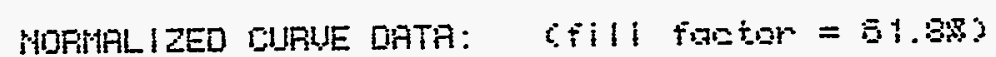

(for 40.0 deg $C$ artd $\left.1.04 \mathrm{H}^{2} \mathrm{~m}^{\wedge} \mathrm{z}\right)$

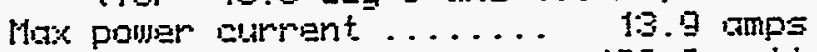

figx pous voltoge ....... 153.5 volts

Max pouler ............. 2278.4 unatss
} 


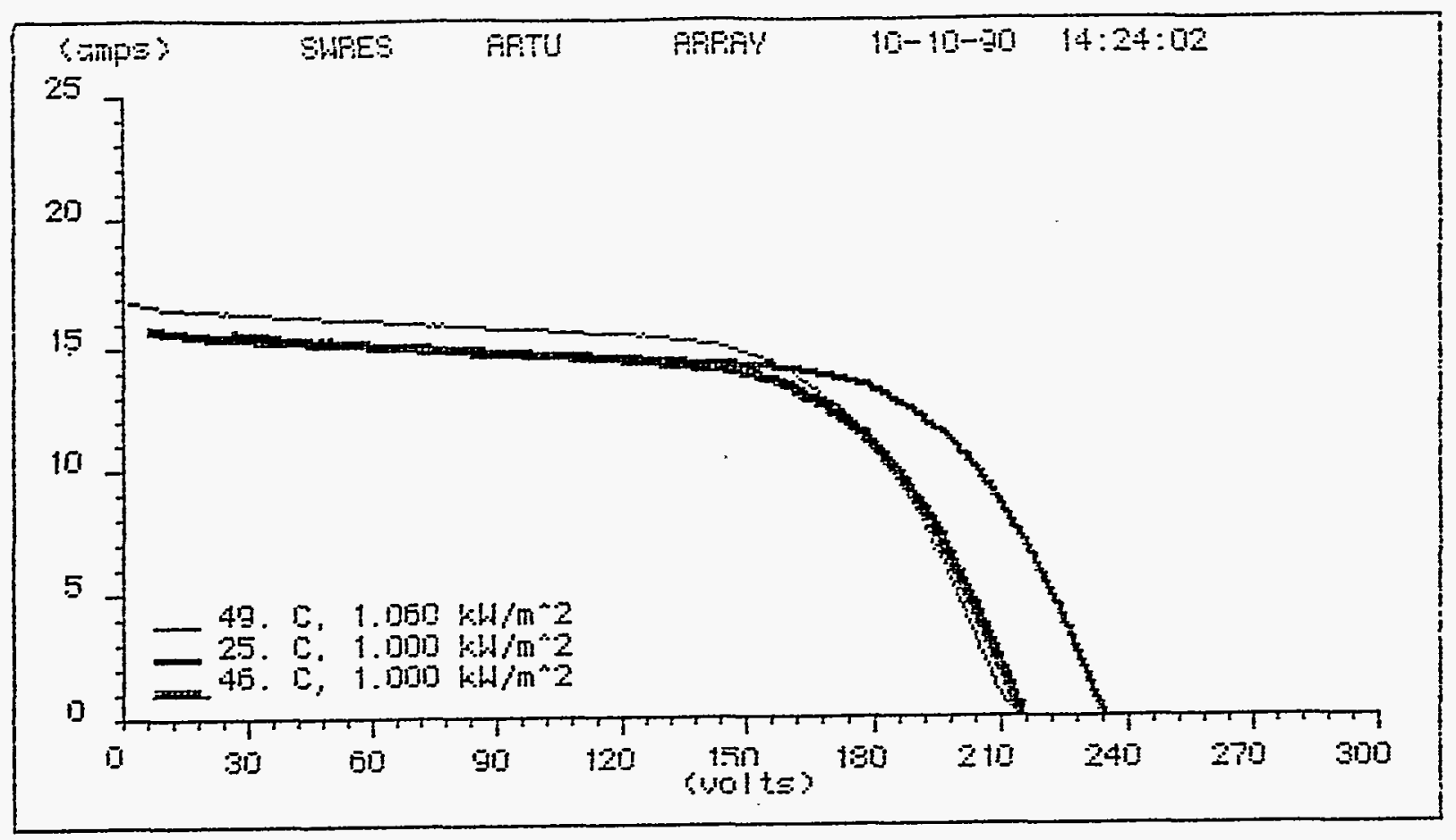

Cal! Temperature .......4.49.000 deg $c$

Irradianse ........... 1.050 khim²

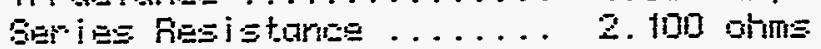

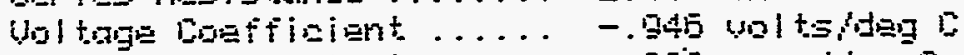

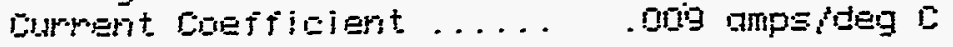

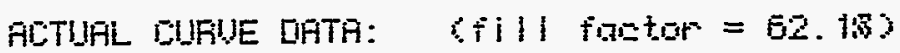

Short ciratit atrmert.... 15.9 amp:

Gpen circuit vol tage ..... 214.2 vol to

Max power arment. ...... 14.:3 amps

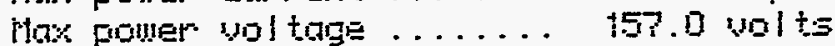

Max pumar .......... . 2242.4 mot.t.s

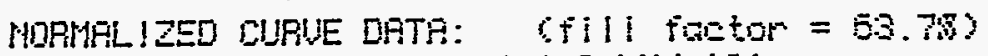

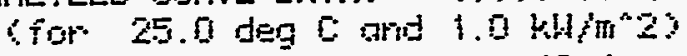

Max gumer aurrent. . . . . . . 13.1 amps

May puiler vol tage ...... 182.2 val te

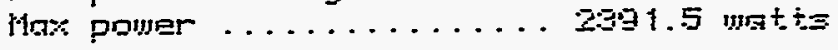

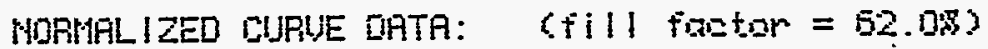

ifor 40.0 deg $C$ arid $1.044\left(m^{*} 2\right)$

Max pumar girront ...... 13.3 amps

Max poiner wol toge ....... 181.9 volts

Max pounar .......... 2153.3 ugtts 


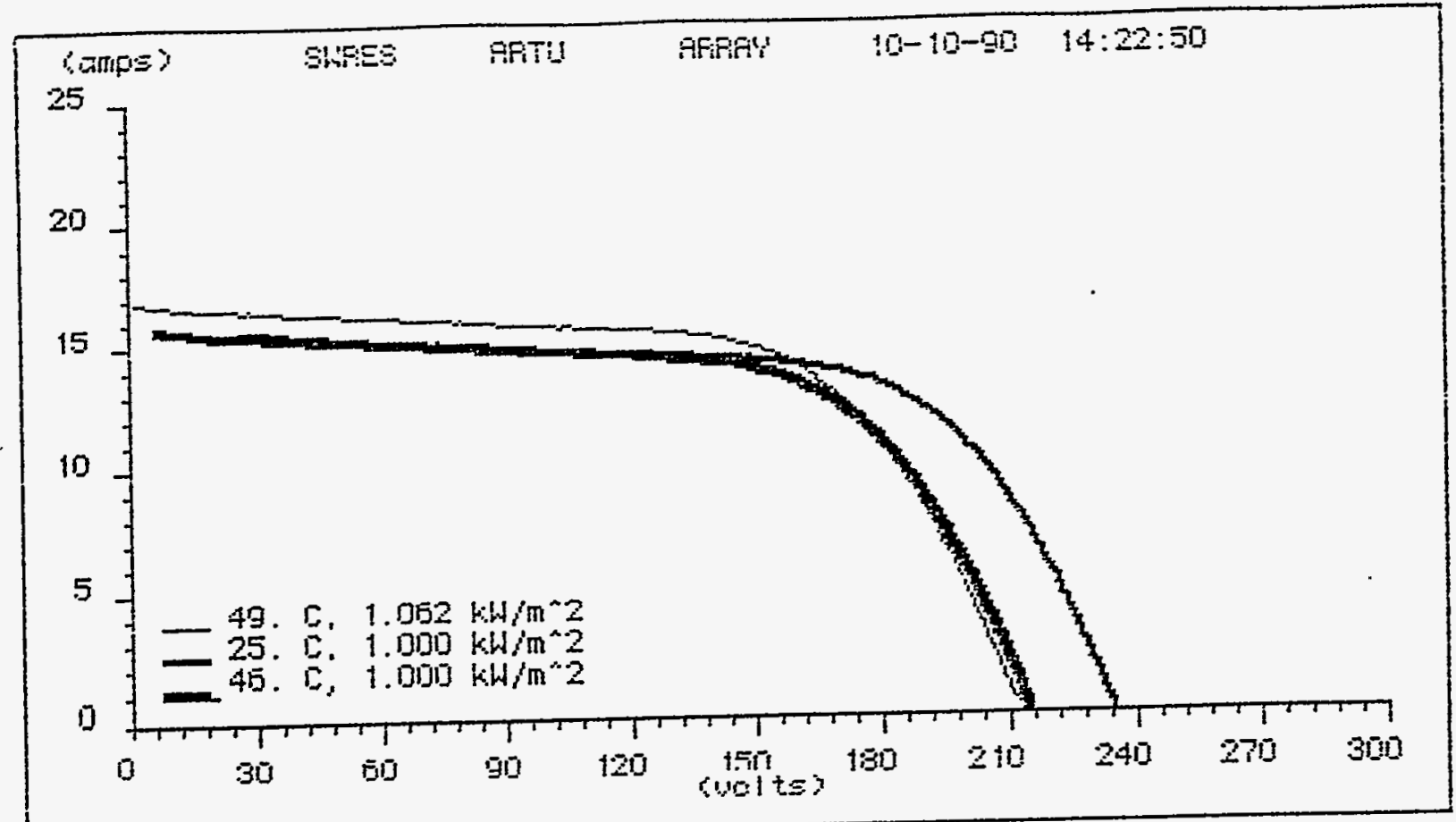

La!! Temperature .......49.000 jeg $E$

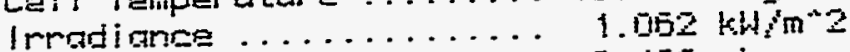

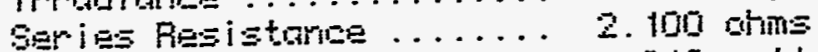

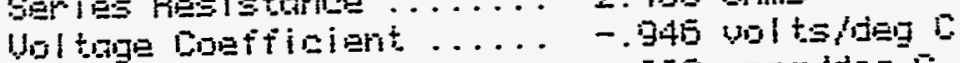

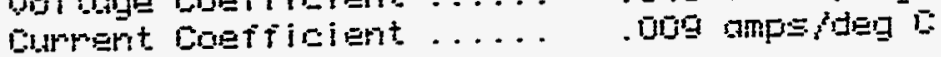

FCTUAL CURUE DATA: GIIll faEtor = 62.2S

Short airouit current.... 15.9 amps

Den Eireuit voltage .... 214.3 volts

Hax power current....... 14. 1 amps

Mox funder vol toge ...... 159.4 wol t.

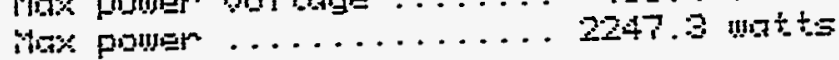

HDFMLLIEED CURUE DATF: GIIll fROTOR = 53.7\%

(for 25.0 dog Cond $1.0 \mathrm{kH} / \mathrm{m}+2 \mathrm{~s}$

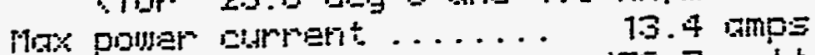

Max power vol tage ....... 178.7 wolts

Max pomer ...........2391.0 matts

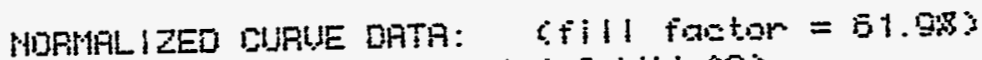

(for 40.0 deg 0 and $1.0 \mathrm{kH} / \mathrm{m}^{2} 2$ )

Mas power aturent. ...... 13.1 anps

Max power yol tage ....... 154.3 volts

Mox pomer ........... 2151.4 yntt: 
SWRES ARTU ARRA 10-03-90 13:14:23

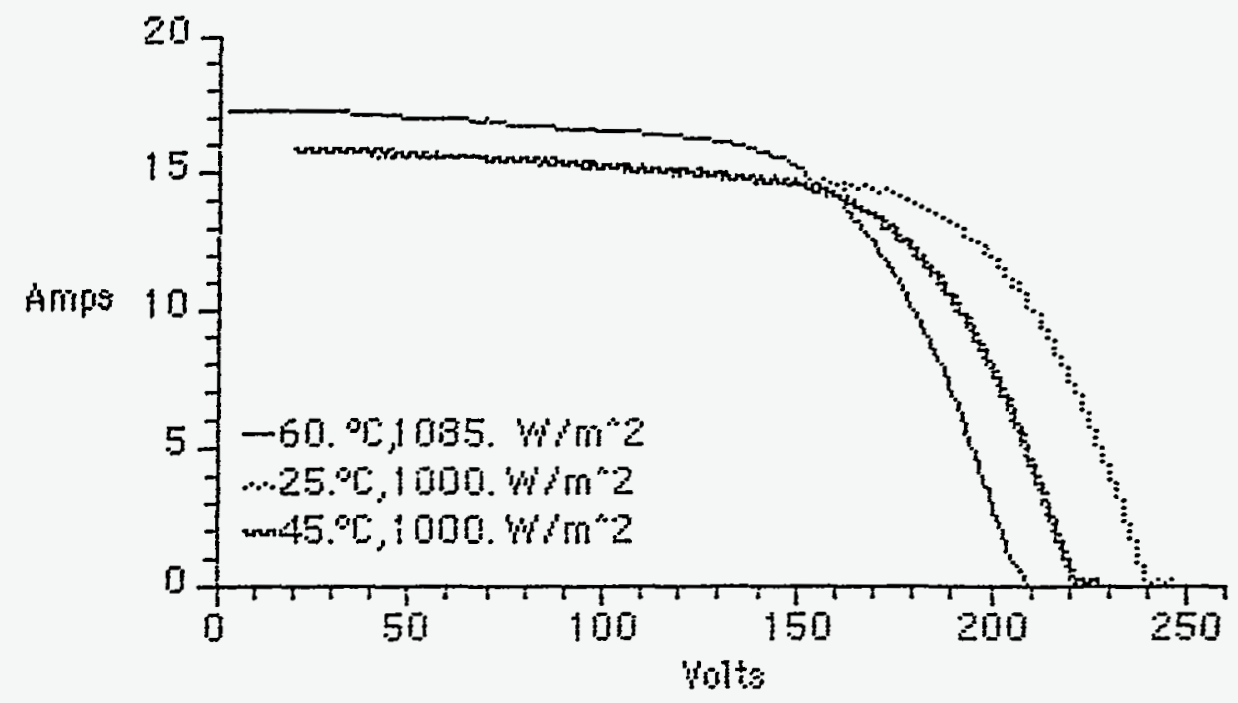

Mudule Ternperature

6U.8 $0 \mathrm{C}$

Insulation

1005.0 in/m 2

Series Resistance

2.100 ninms

Yoituge Coefficient.

-0.946 wits

Current Coeficient

0.009 ampsio

ACTUAL CURUE CATA: TfIII factor $=62.7 \%$

Short Cirouit Eurrent ............ 17.3 amps

Open Circuit lutiage .............. 209.5 volts

Max Power Current ............... 15.0 amps

Hax Fower viltane ................. 151.2 units

Max Fower .......................... 2289.1 4yitis

NORMALIZEOCURUE DATA: (fill factü $=65.4$ T: ( $25^{\circ} \mathrm{L}$ and $\left.1000 \mathrm{~m} / \mathrm{m}^{2} 2\right)$

Max Power Current $15.7 \quad$ amps

Max Power 'yoltage ................. 18.5.0 yolts

Max Fower ............................ 2514.4 Watts

BORMALISEO CURUE DATA: (fill factor = 6.5.7 8 ) (450 and 1000 44: $\left./ \mathrm{m}^{2} 2\right)$

Max Püwer Current 13.5 amps

Max Power Wiltage ................. 168.5 woits

Max Fower

2279.7 matts




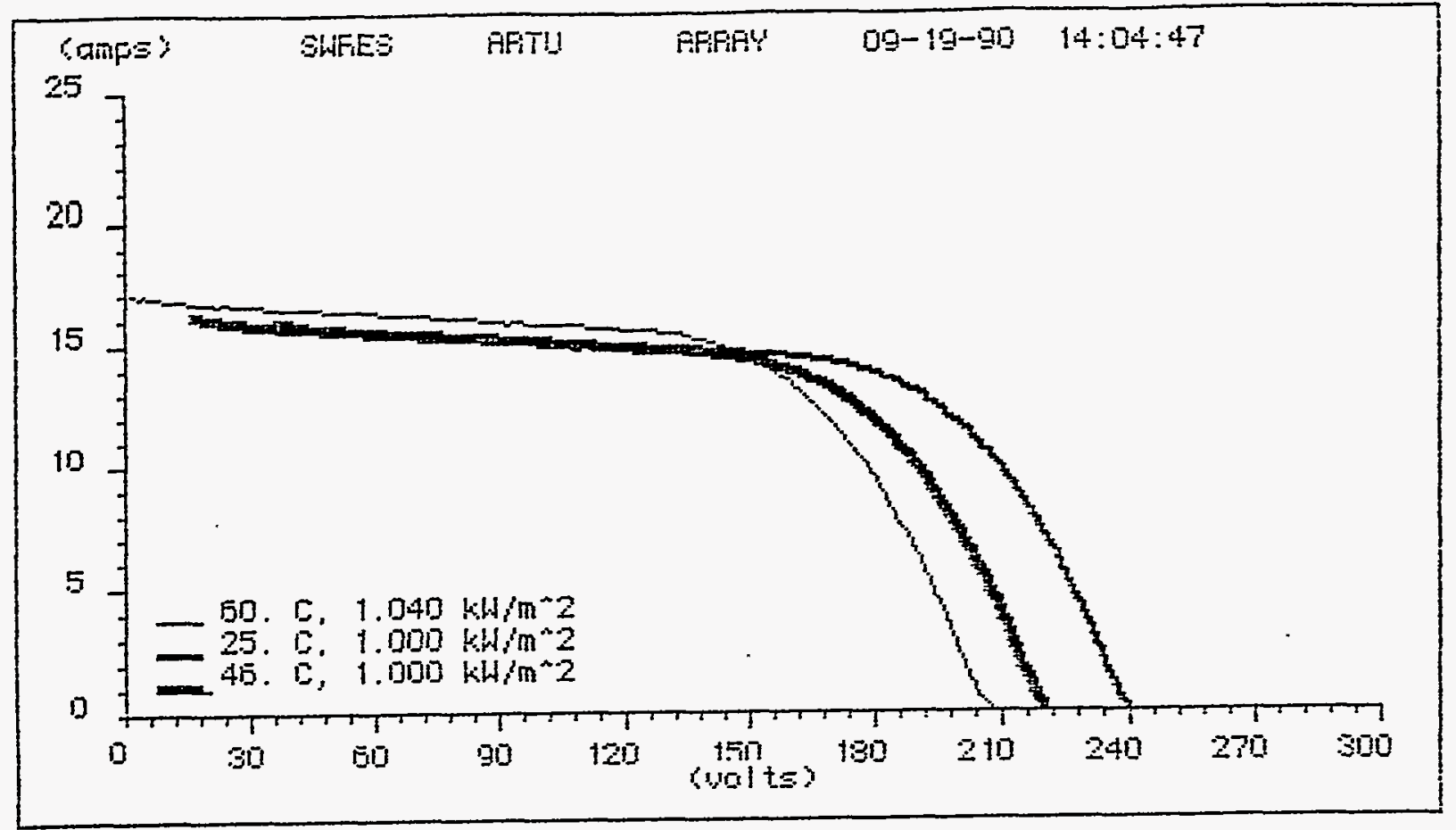

Cel i Temperature ........ EO. DUO deg $c$

Irradiance ............ $1.040 \mathrm{kH} \mathrm{m}^{* 2}$

Sories Resistance ...... 2. 100 bhims

Ualt.

Durment. Coefficient. .... . Dug ampeideg $\mathrm{C}$

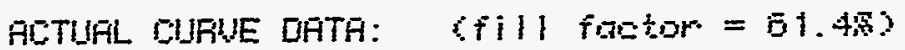

Ghort Biratit sument. ... 17.n amps

Gper circuit uel tage ..... 208.3 uol t:

Max poijer current ....... 14.3 amps

Max promer vol toge ...... 152.3 yol t:

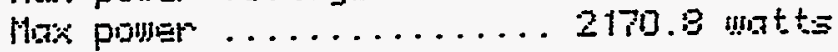

HIDRTHLIZED CIJPUE DATA: (till faEtor $=64$. IN)

Cfor 25.9 deg [ and 1.0 Khy 2 ?

May pouser arirrent...... 13.9 amps

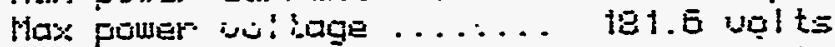

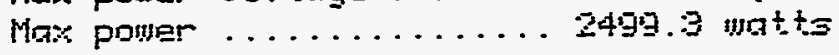

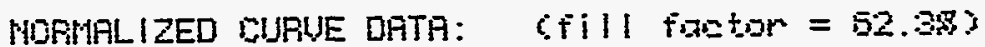

(for $40.0 \mathrm{deg} \mathrm{L}$ ard $\left.1.0 \mathrm{kH} / \mathrm{m}^{\circ} \mathrm{z}\right)$

Hox power gurment. ...... 13.5 amps

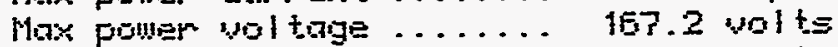

Max pouner .......... 2253.9 wit.t.s 


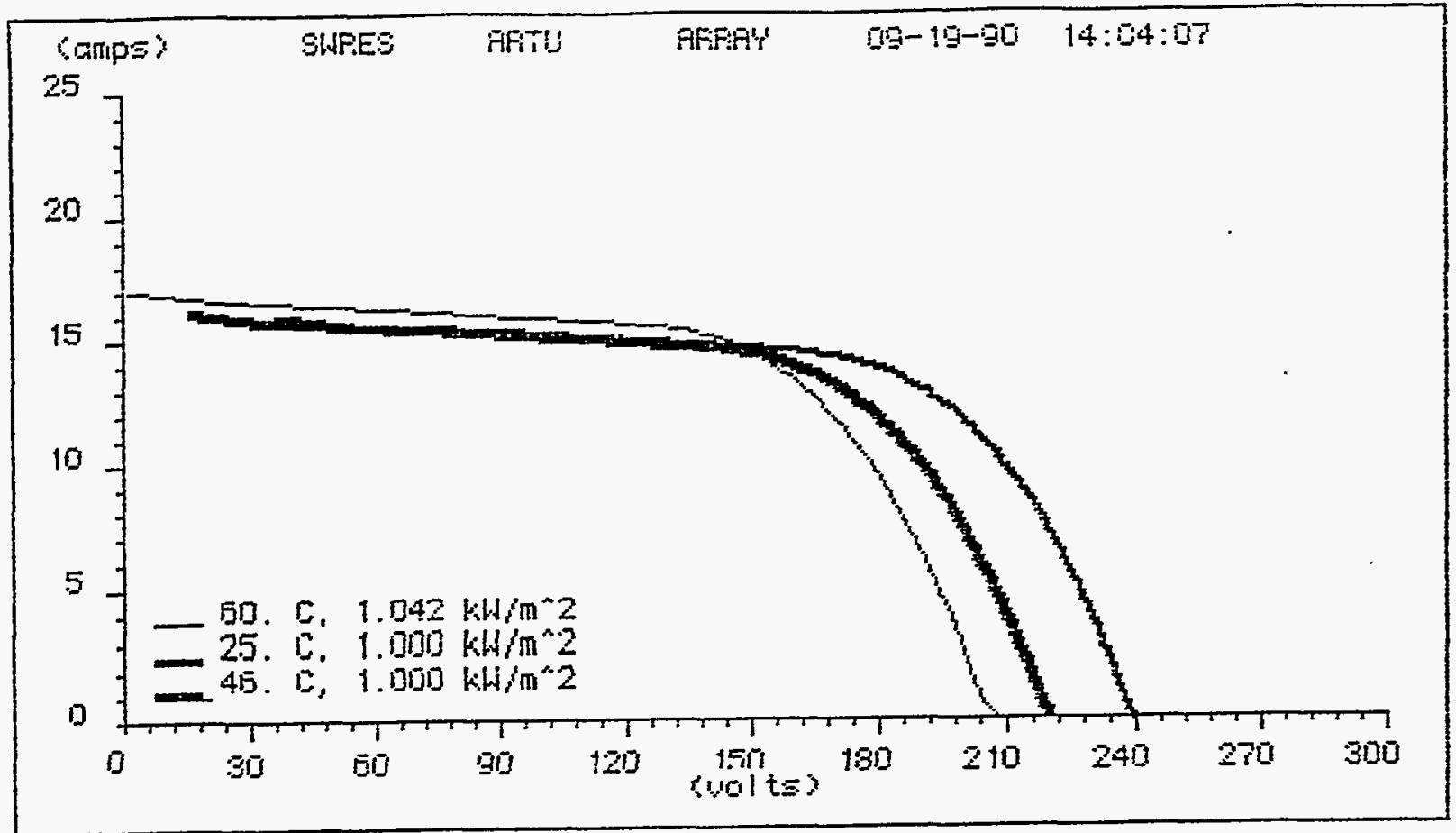

Eell Temperature ....... 00.000 deg $\mathrm{C}$ Irradiance ............ $1.042 \mathrm{kH} / \mathrm{m}^{2} 2$ Series Resi tance $_{\text {. . . . . . 2. }}$ 200 ohms Ual toge Doefficiant. . . . . . g45 uoltsidag 0 current coefijeiont..... . Dog ampsideg 0

ACTUAL OUAUE EATA: (TIll factor $=61.3 \%$

Ghort airatit adrrent. ... 17.0 amps Upen eireuit vol tage .... 208.3 volts Max pumer gurrent. ...... 14.3 amps Hax pouler vol tage ....... 152.3 volts

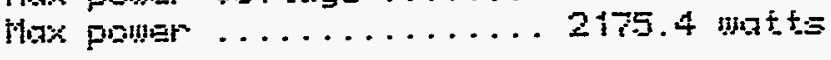
MDFHALIZEO DURUE DATA: (fill factor $=54.13$ ) ifor $25.0 \mathrm{deg} \mathrm{C}$ and $1.0 \mathrm{kH} / \mathrm{m}^{*} \mathrm{2}$ ?

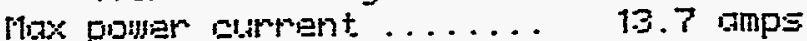
Max pewer val tage ....... 182. Mrox power ........... 2505.0 watts

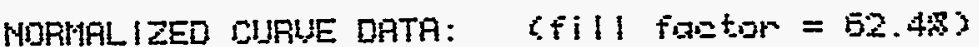
(ror 40.0 deg $C$ and $1.0 \mathrm{kH} / \mathrm{m}^{\circ} \mathrm{2}$ )

Max power aurrent. ...... 13.7 amps

Max pourer vol tage ....... 1E4.9 wolts

Mox powar ........... 2259.0 matt.s 


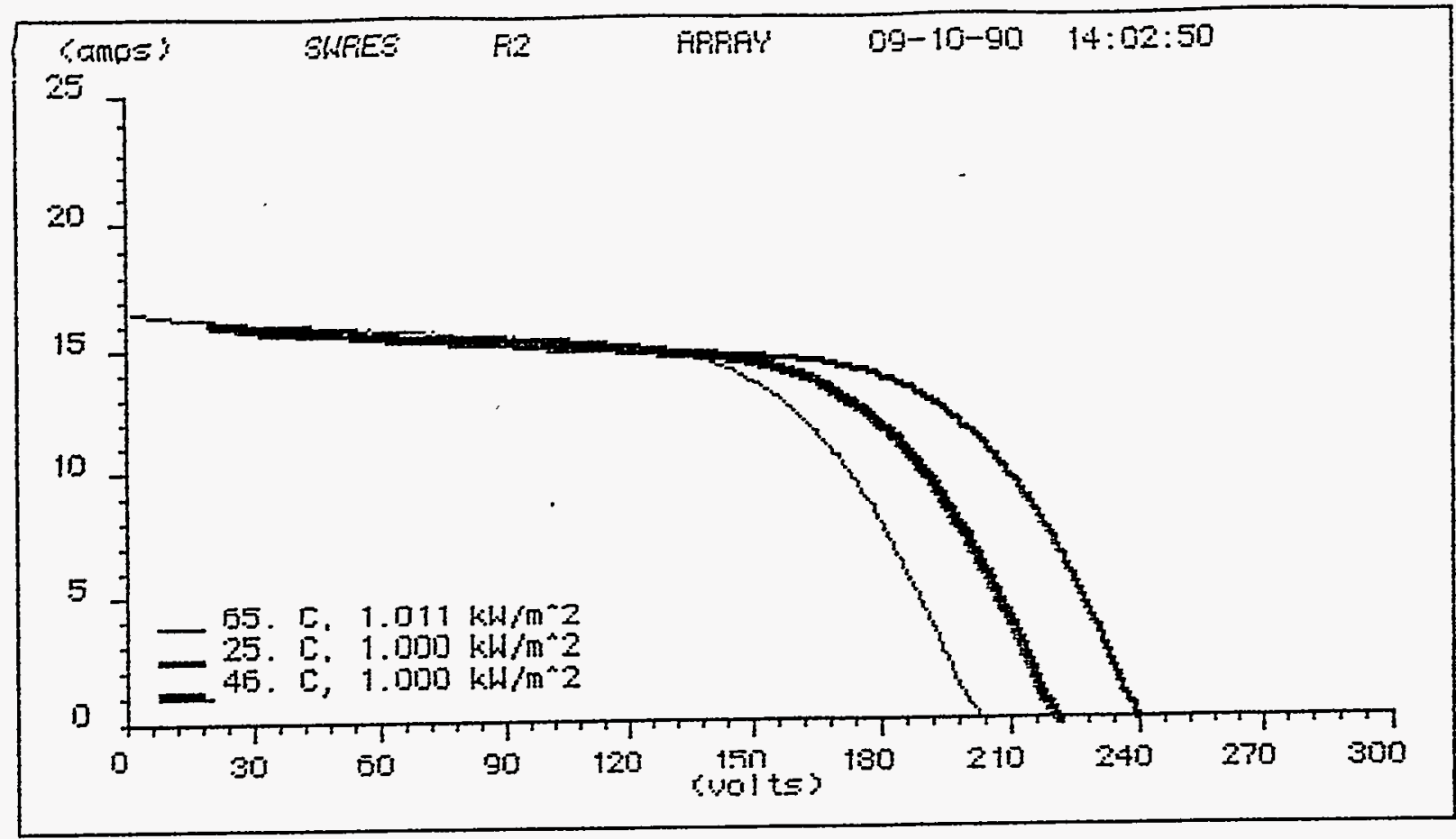

Cell Temperature ........ E5. DUU deg $\mathrm{C}$

irradi nnce ........... $1.011 \mathrm{kH} \mathrm{hm}^{2} \mathrm{z}$

Ser i

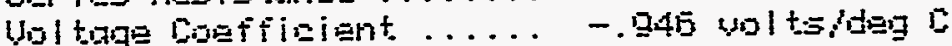

Durrent Coefficiant ..... . Dug ampsideg $\mathrm{E}$

FCTUAL CURUE DHTA: (fill fagtor = BD.QS3

Short airauit current. . . 15.5 amps

Uperi circuit voltage .... 203.5 volts

Max poufer numpent....... $1.3 .9 \mathrm{amps}$

Max poujer vol toge ...... 147.7 wolts

Hax poum ............ 2045. 1 matts

NDPHALIZED CURUE DATA: Gill factor $=64.3 \%$

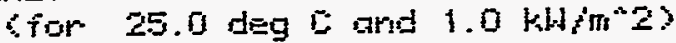

Max pourer current ....... 13.5 amps

Max power val tage ....... 183.1 vol t:

Mrax power ............ 2495.5 mats

HORMAL IZEO CURUE DATA: CFIll fAEtor $=52.5 \%$

(jor $46 . \bar{d}$ deg $C$ and $\left.1.0 \mathrm{kH}, \mathrm{m}^{20} \mathrm{z}\right)$

Mox pouner astrent ...... 13.7 amps

Max pownor vist toge ....... 154.0 volts

Max pouser ........... 2251,2 yot:s 


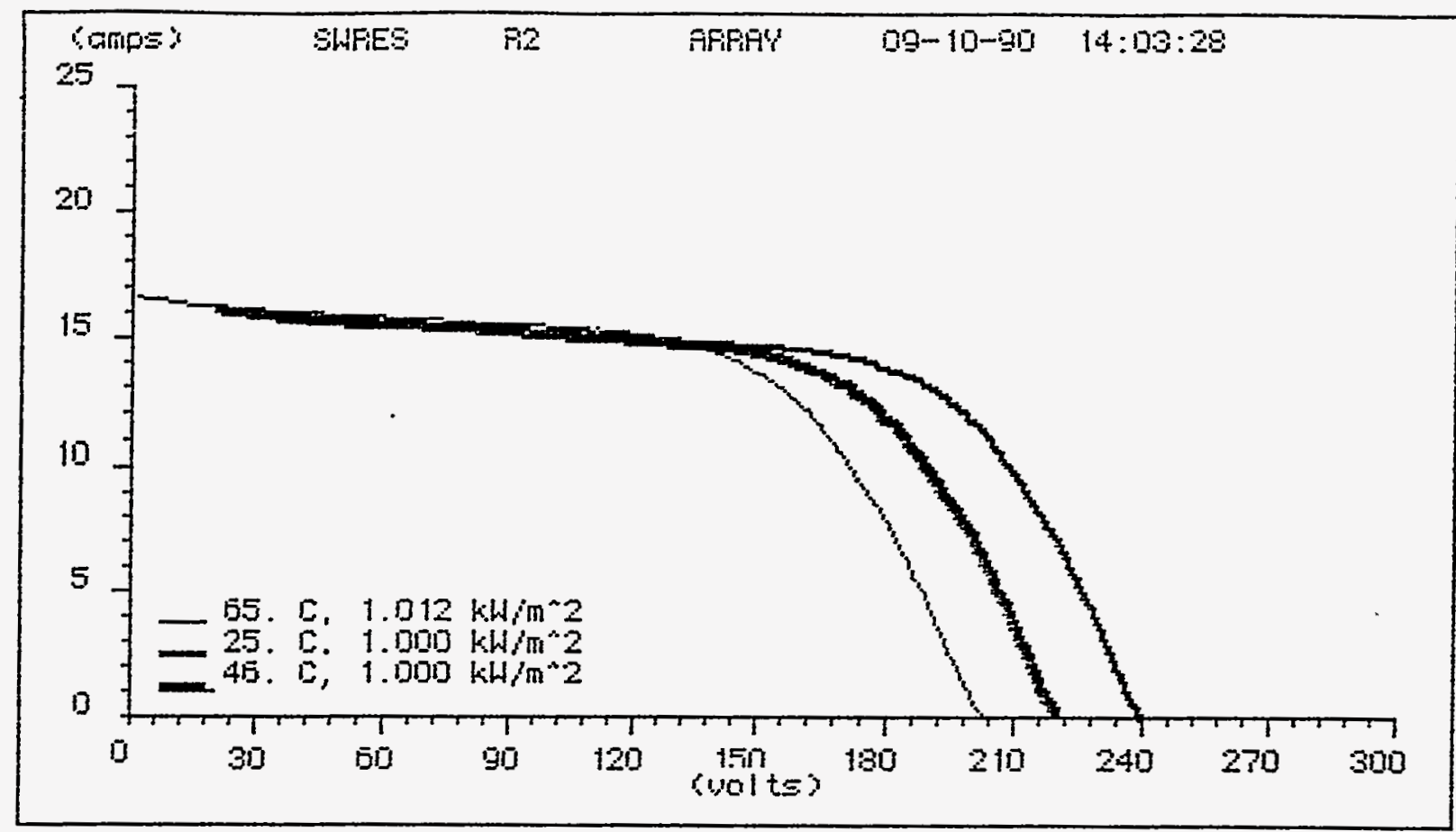

Cell Temperature ....... Es. OUU deg $c$

Irradiance ............ $1.012 k \mathrm{kln} \mathrm{m}^{2} \mathrm{z}$

Series Resistarice ....... 2. 100 ohms

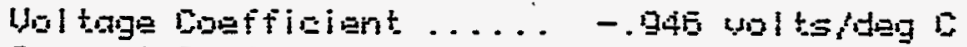

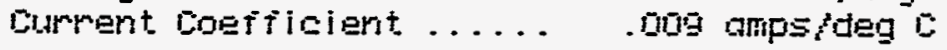

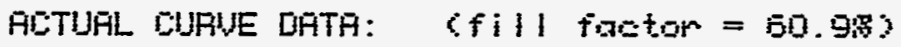

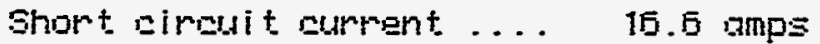

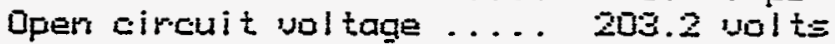

Hax pouner current ....... 1:.3 anps

Max pouer yol tage ....... 148.8 waits

Max pouner ........... 2052.4 unt

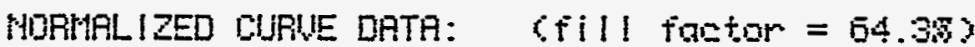

(for 25.0 deg 5 arid $1.0 \mathrm{kH} \mathrm{H}^{* 2} \mathrm{z}$

Ma\% pouser atrpent....... 13:7 amps

Max power val tage ....... $191 . \overline{\text { g valts }}$

Mrax pouser ............2439.8 motts

MORHAL IZED BURUE DHTA: CFIII fantom = $52.5 \mathrm{~s}$

Siar $\$ 6.0$ deg 0 and $1.0 \mathrm{kH} / \mathrm{m}^{2} \mathrm{Z}$ )

Hax pouter cturrent....... 13.5 amps

Max pouner vol tage ....... 156.4 volts

Mox pumer ........... 2251.2 migt:s 

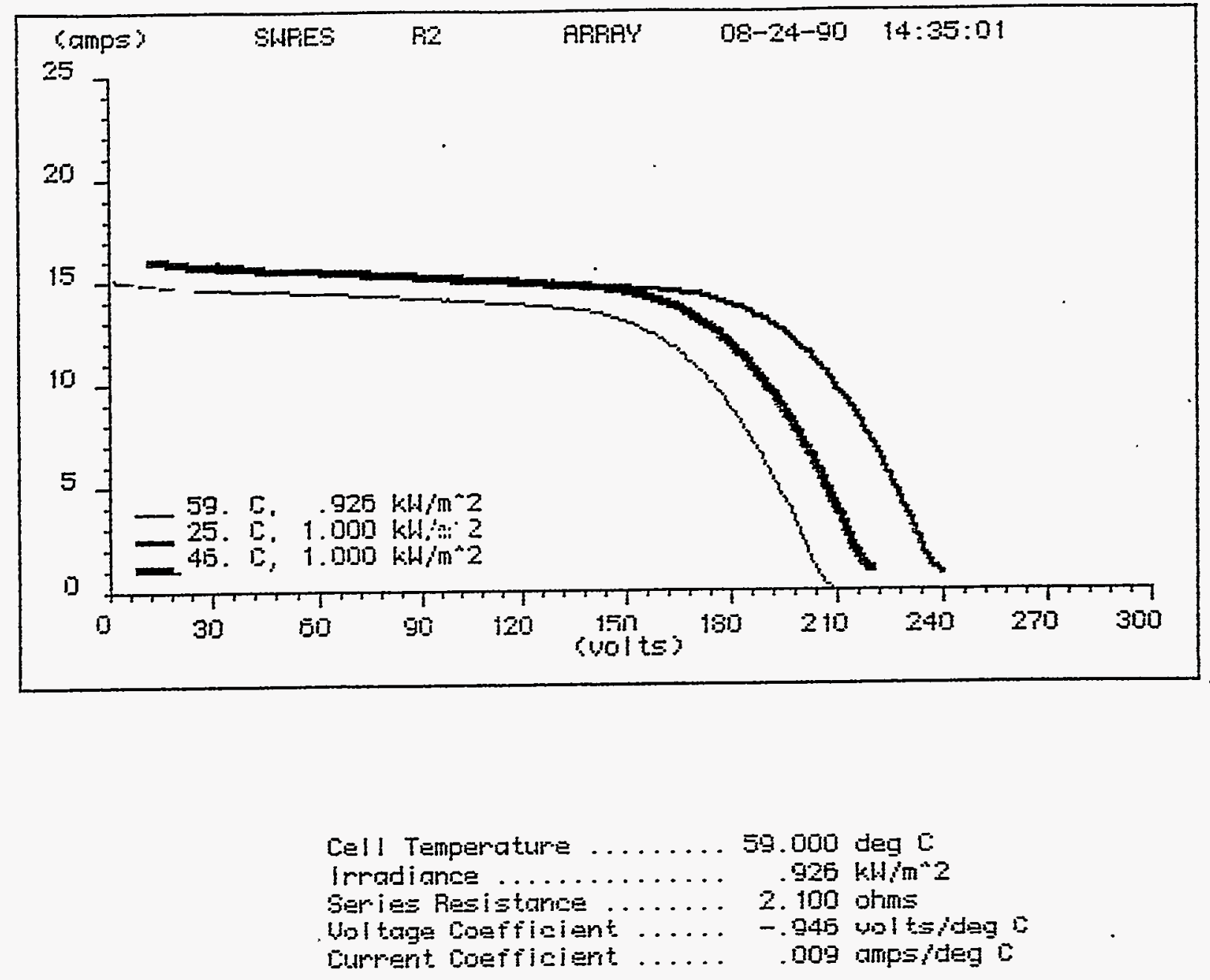

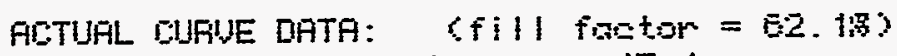

Short airouit ourrent ... 15.1 umps Dpen circuit voltage .... 209.7 vol $t \equiv$ Hox pouner current. ...... 12.5 emps Hax pouer vol thge $\therefore \ldots \ldots$. 155.9 volts Max power ............ 1954.5 mint

HORIALLIZED CURUE DATA: Sfill factor $=65.58 \mathrm{y}$ (for $25.0 \mathrm{~d}=9 \mathrm{C}$ and $1.0 \mathrm{kH} / \mathrm{m}$ "2)

Max pourar gurront ...... 13.7 amps

Ma\% power vol tage ....... 182. 5 vol te

Max pouer . . . . . . . . 250:3 wot.ts

MORHALIZED CURUE DATA: (fill faotor $=53.9 \%$

Sfor 40.0 deg $\left[\right.$ and $1.0 \mathrm{kH} / \mathrm{m}^{2} \mathrm{z}$ )

Max pourer aurrent ....... 13.5 amps

Max pouer voltage ...... 165.9 volt

Max pouser ........... 2259.8 matts 


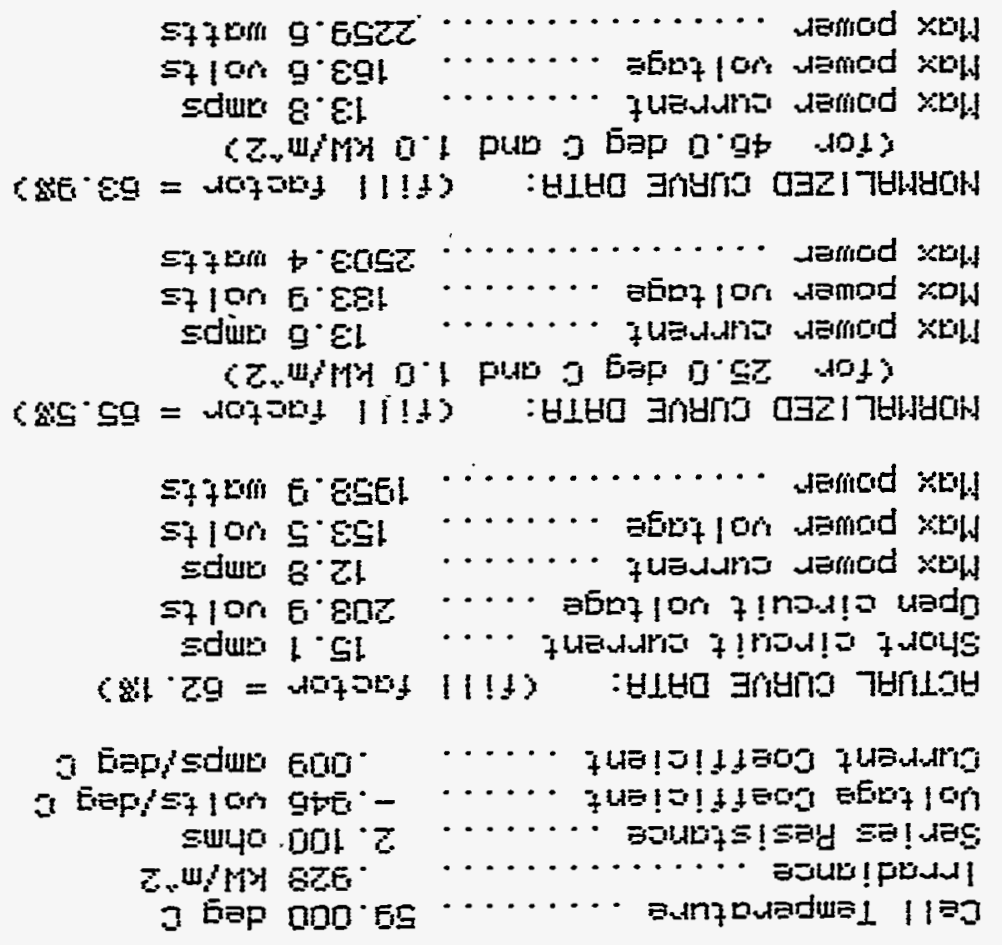

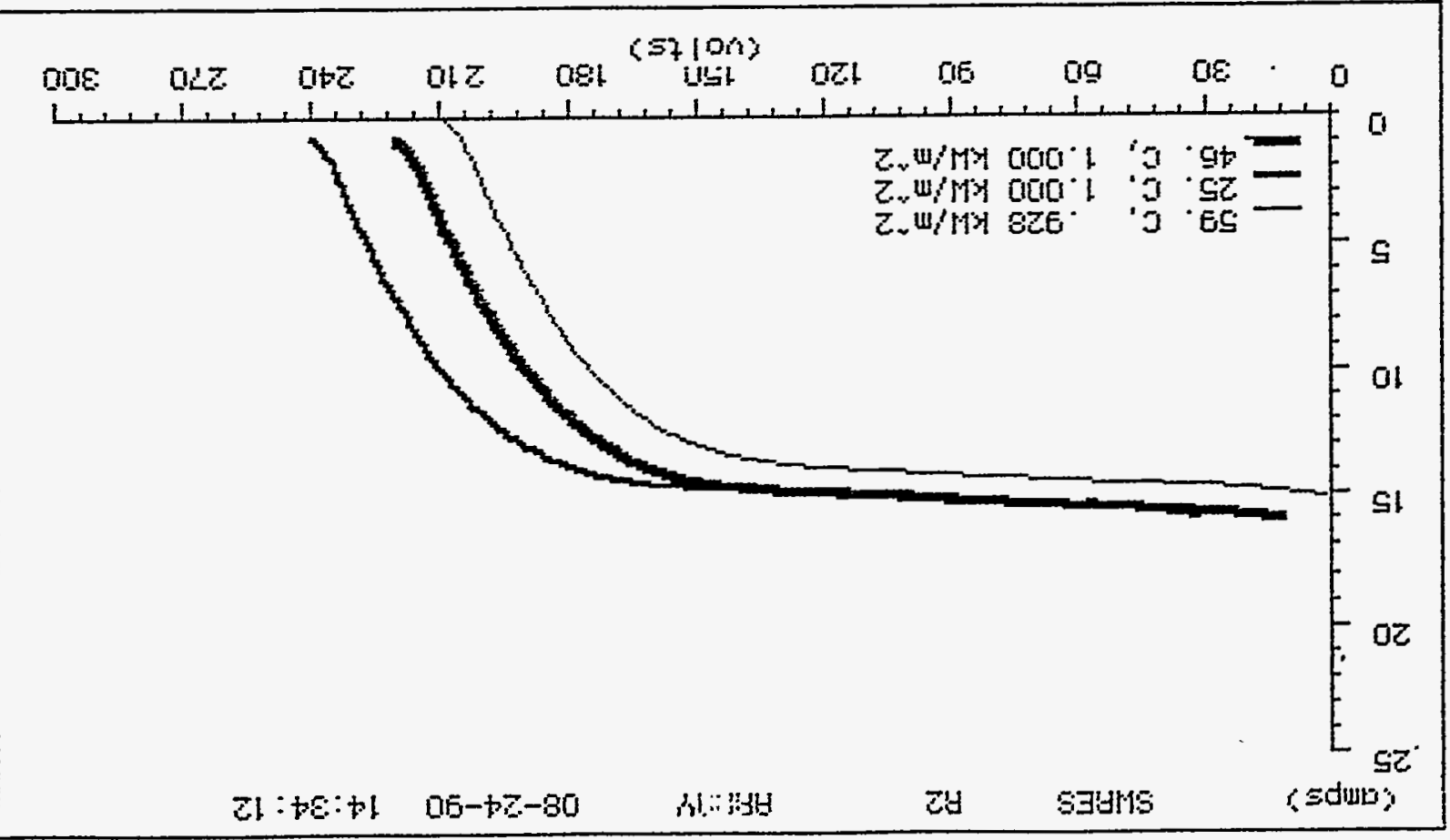




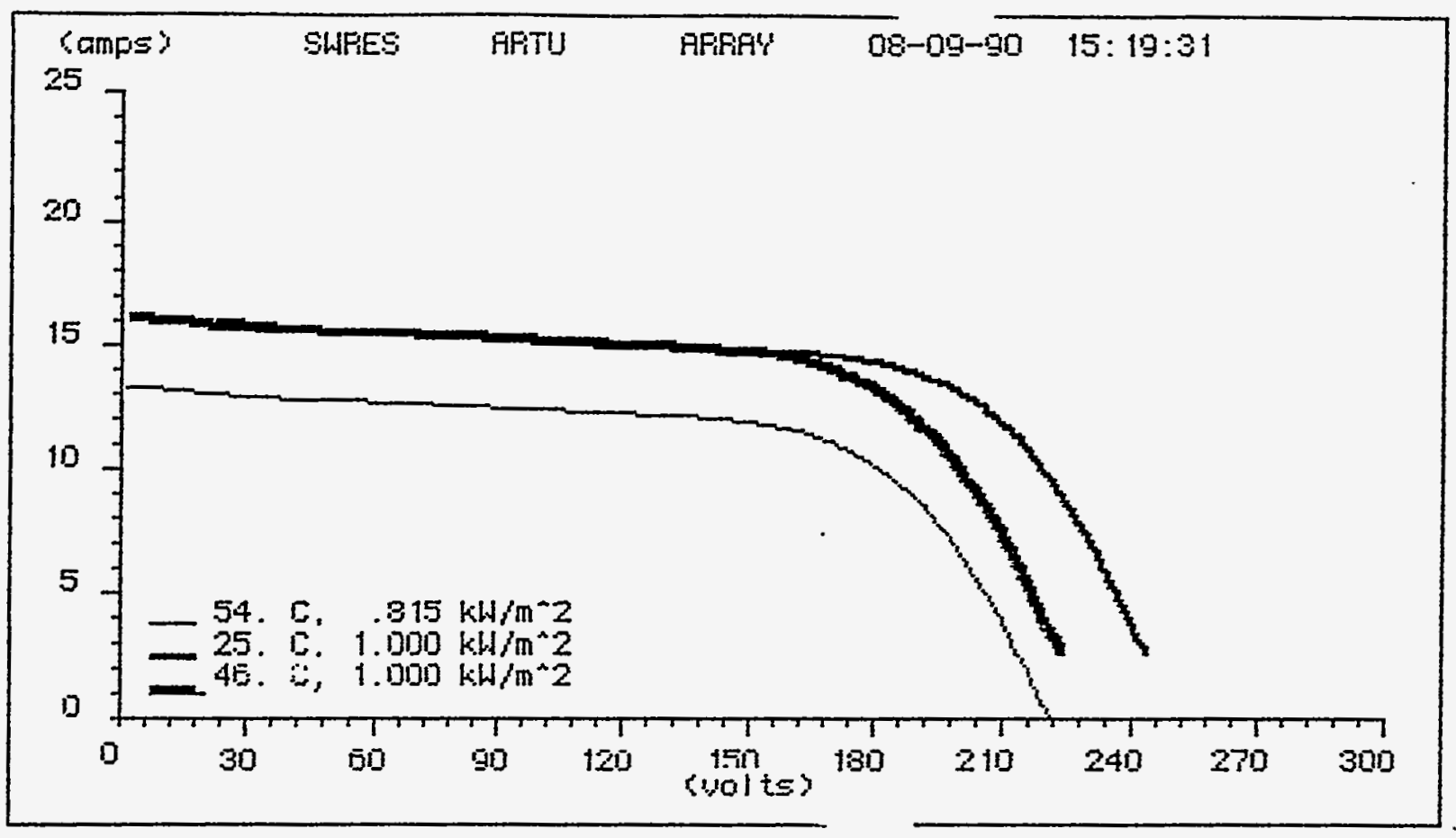

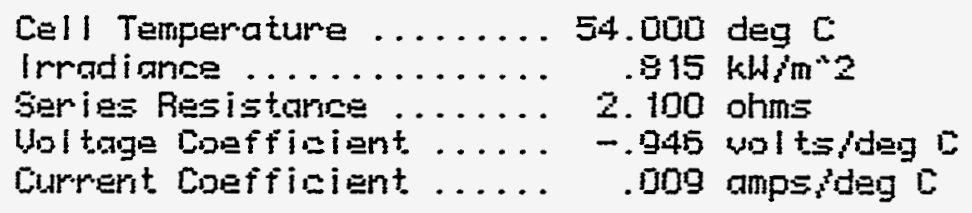

ACTUAL CURUE DATA: (fill fretor $=64.0 \mathrm{~g}$ )

Short aircuit aturgent. ... 13.3 amps.

Dpen eircuit wol tage .... 221.3 volts

Mrox power current ....... 11.2 amps

Max power voltage ....... 168.8 volts

Mox pourar ............ 1884.9 matts

HORMILIZED DURUE DATA: (

(for 25.0 deg [ and $1.0 \mathrm{kH} / \mathrm{m}^{*} 2$ )

Max pourer aurrent....... 13.5 amps

Mas power val tage ....... 194.0 valt:

Hox power ........... 2545.5 matts

MORMFLIZED DUAUE DATA: (tIll faAtor $=55.5 \%$ )

Sior 40.0 deg $C$ and $1.0 \mathrm{kw} / \mathrm{m}^{\circ} \mathrm{2}$ )

Max pouser aurrent ....... 13.9 amps

Max power voltage ....... 173.7 volts

Maxx piuser ............ 2408.1 usatts 


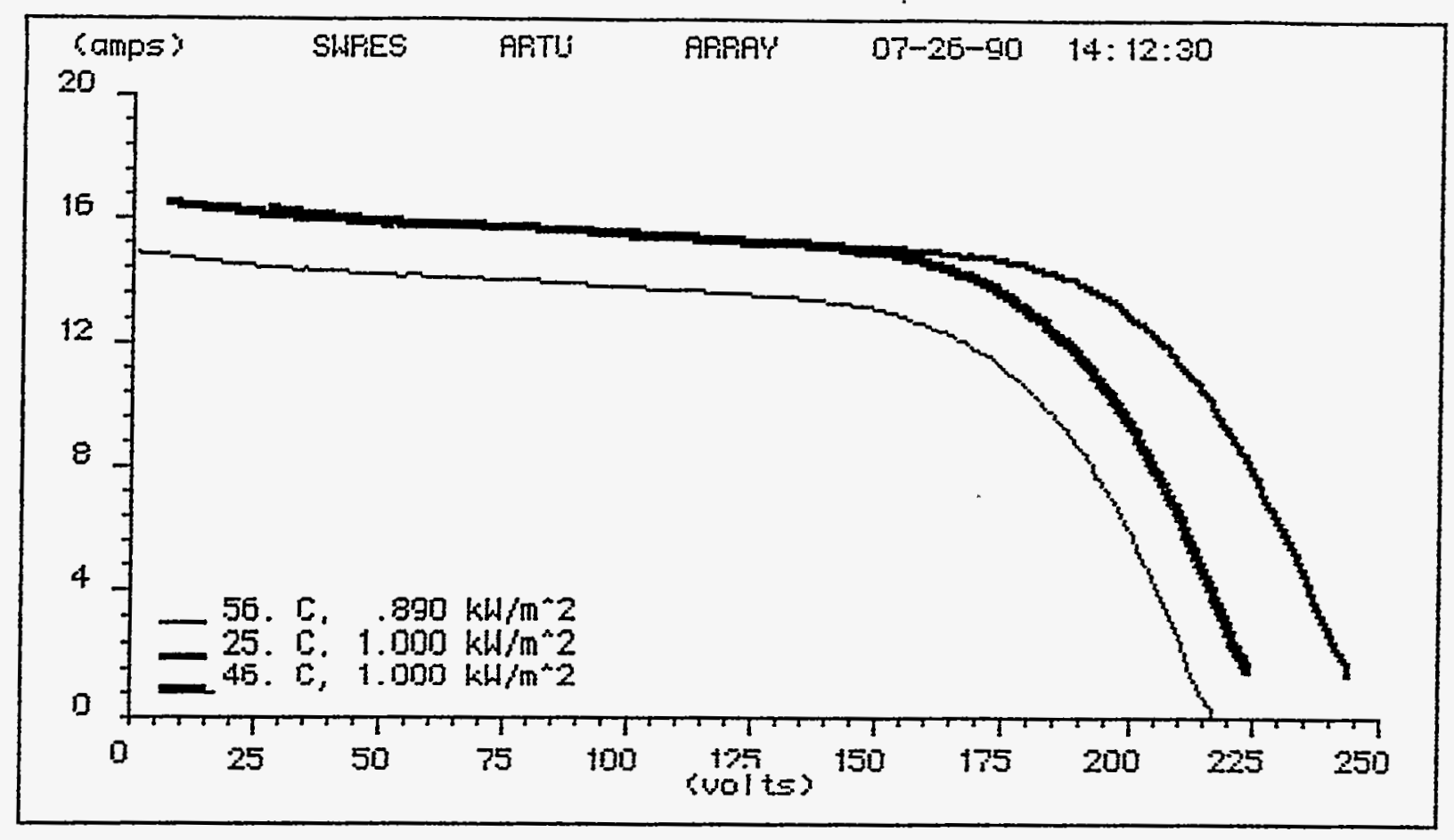

Cell Temperature .......55.1300 deg C

Irradiance .......... .890 $\mathrm{kW} / \mathrm{mm}^{\circ 2}$

Saries Resistance ........ 2. 100 ohms

Woltage coeficient ..... - .940 woltsideg $c$

Cumpent Coefficient ..... . .009 amps/deg $c$

FITTURL CIJPUE DRTA: (fill figetor $=62.93$ )

Short circuit current .... 14.9 amps

Dpen circuit voltage .... 217.1 volts

Max pouer current. ...... 12.4 amps

Max puiner uol tage ....... 154.1 wolts

Max pouser ...........2027.3 watts

NORMALIZED CURUE DATA: (fill faEtor $=65.38$ )

Sfor 25.0 deg $C$ and $1.0 \mathrm{~km} / \mathrm{m}^{2} 2$ )

Mas pawer current....... 13.9 amps

Hax pouner voltage ....... 190.2 volts

Max power ............ 2541.9 watts

HORMALIZED CURUE DATA: (Till factor $=54.58$ )

(for $46.0 \mathrm{deg} C$ and $1.0 \mathrm{kH} / \mathrm{m}^{-2} 2$ )

Max power cumpent....... 14.1 amps

Max poufer woltage ....... 169.9 volts

Max pawer ........... 2394.8 watts 


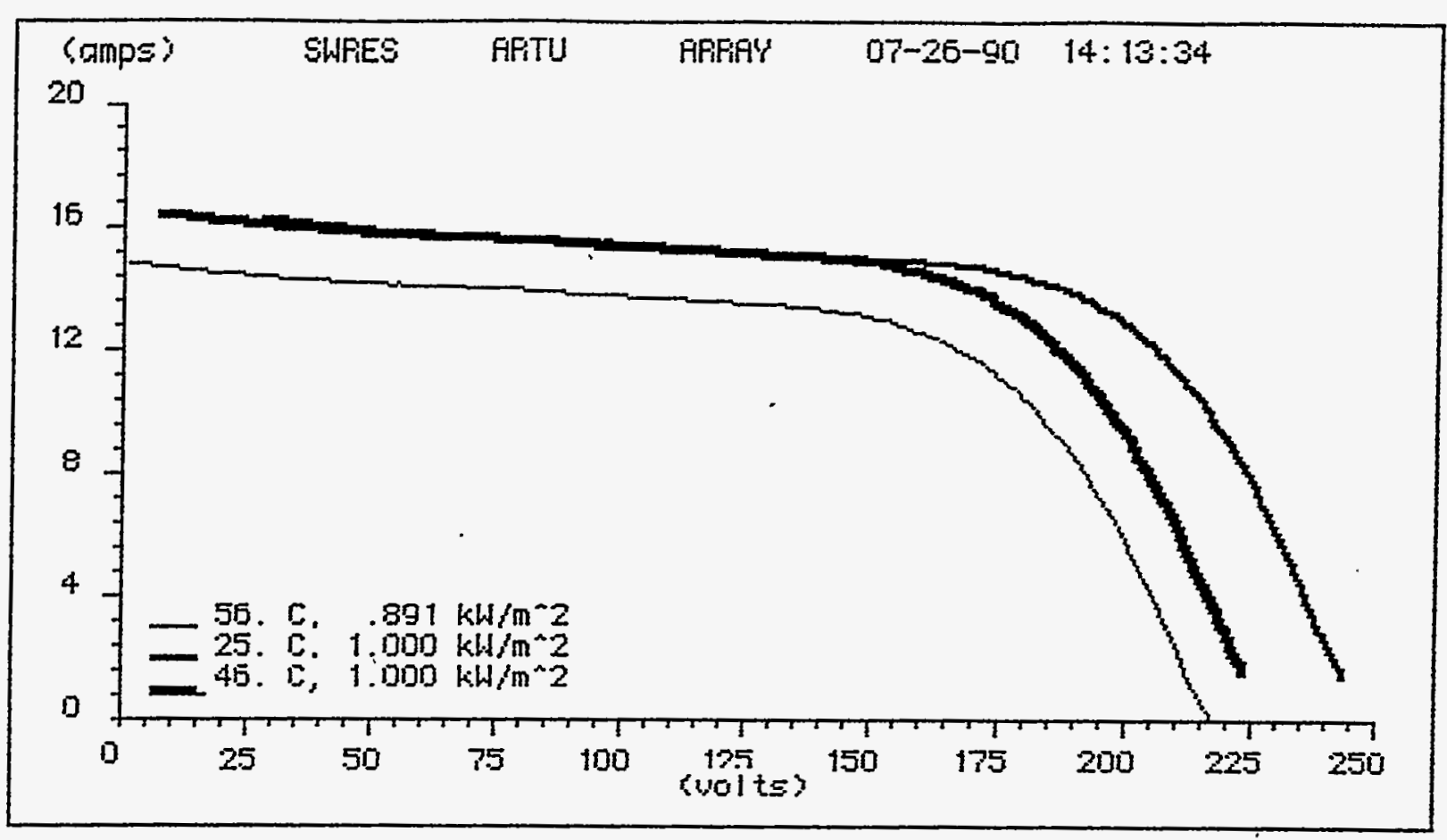

Dell Temperature ....... 55.000 deg $\mathrm{C}$

Irradianoe ........... . $391 \mathrm{kill} / \mathrm{m}^{\prime 2} 2$

Series Risistanco ....... 2. 100 ohms

Vol tage coeficiant ..... - - 940 voltsideg $c$

Current Coefficient. ..... . Dog ampsideg 5

ACTIAL CIJPUE DRTA: (fill factor $=62.98$ )

Short circuit current .... 14.3 amps

Dpen cirrsit ultage .... 215.9 ults

Max power current ...... 12.3 amp:

Max pouser voltage ....... 154. 1 walts

Max pomer ...........2024.5 whtts

MOAMALIZED CURJE DATH: ifill factor $=6 E .38 \mathrm{~s}$

Sfor 25.0 deg $C$ and $1.0 \mathrm{kH} / \mathrm{m}^{\wedge} \mathrm{z}$ )

Max power curment ....... 13.9 amps

Max pouser yol tage ....... 190.2 uplts

Max power ........... 2534.7 watts

MORMALIZED DURVE DATA: Efill factor $=64.83$ ?

(for $46.0 \mathrm{deg} C$ and $1.0 \mathrm{kH} / \mathrm{m}^{2} 2$ )

Max pouser surpart ....... 14.1 amps

Mox poiser upl tage ....... 159.9 uol ts

Max power ...........2383.4 watts 


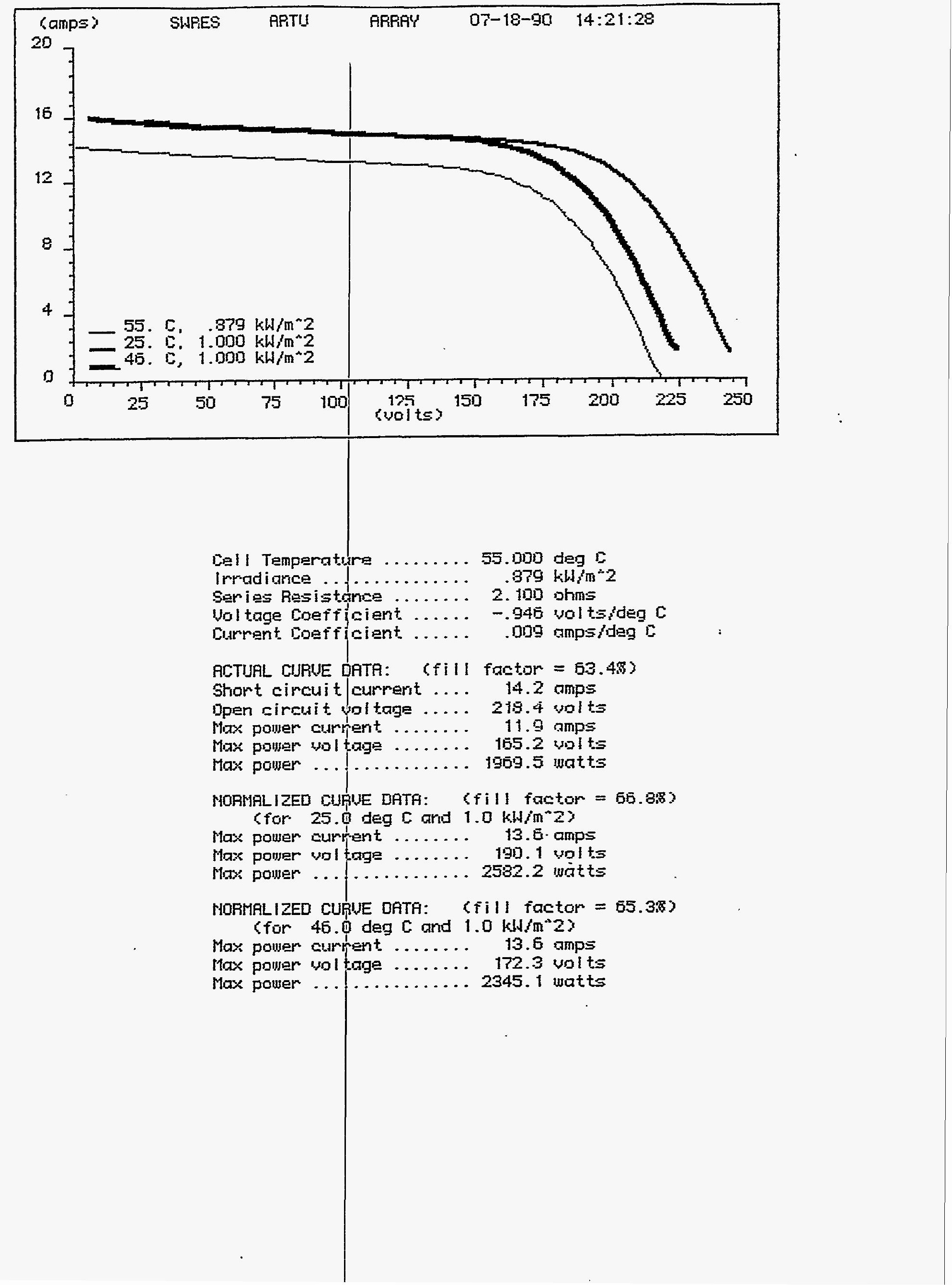




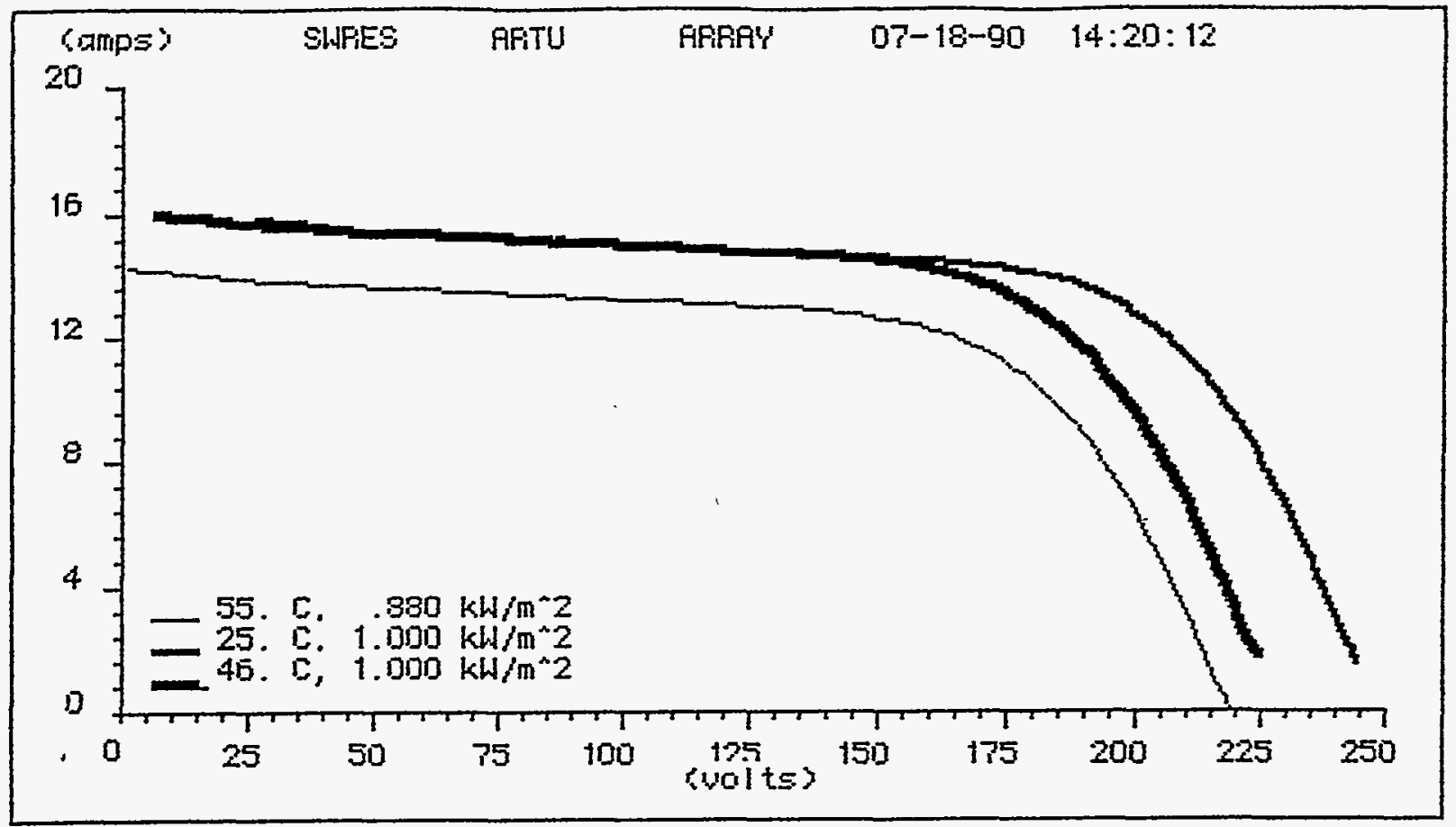

Dell Temperature .......55. Don deg $\mathrm{C}$

Irroidiance ............ . $830 \mathrm{kH} \mathrm{km}^{\circ 2}$

Sories Resistanca ....... 2. 110 sims

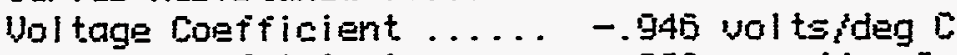

Cumpant coefficient ..... . . D09 ampsideg $\mathrm{C}$

RCTUAL CIURUE DRTA: (fill factor $=63.6 \%$ )

Short circuit current.... 14.3 amps

Dpen sircuit vol trage ..... 219.0 volts

Max power cumpent....... 11.9 amps

Max poun sol tagge ....... 155.4 wolts

Max power ........... 1983.6 matts

MORHALIZED DURUE DATA: (fill faEtor = ED. 98 3

(for $25.0 \mathrm{deg}\left[\mathrm{and} 1.0 \mathrm{kH} / \mathrm{m}^{\circ} \mathrm{2}\right.$ )

Max power current ....... 13.E amps

Max pouner vol tage ....... 191.3 yolts

Hax power ........... 2595.7 watts

NORMALIZED CURUE DATA: (Till factor $=5.5 .43$ ?

(for 46.0 deg $\mathrm{C}$ and $1.0 \mathrm{kH} / \mathrm{m}^{2} 2$ )

Mas power current ....... 13.7 amps

Max pouser yol tage ...... 172.2 wolts

Max power ............. 235E.4 watts 

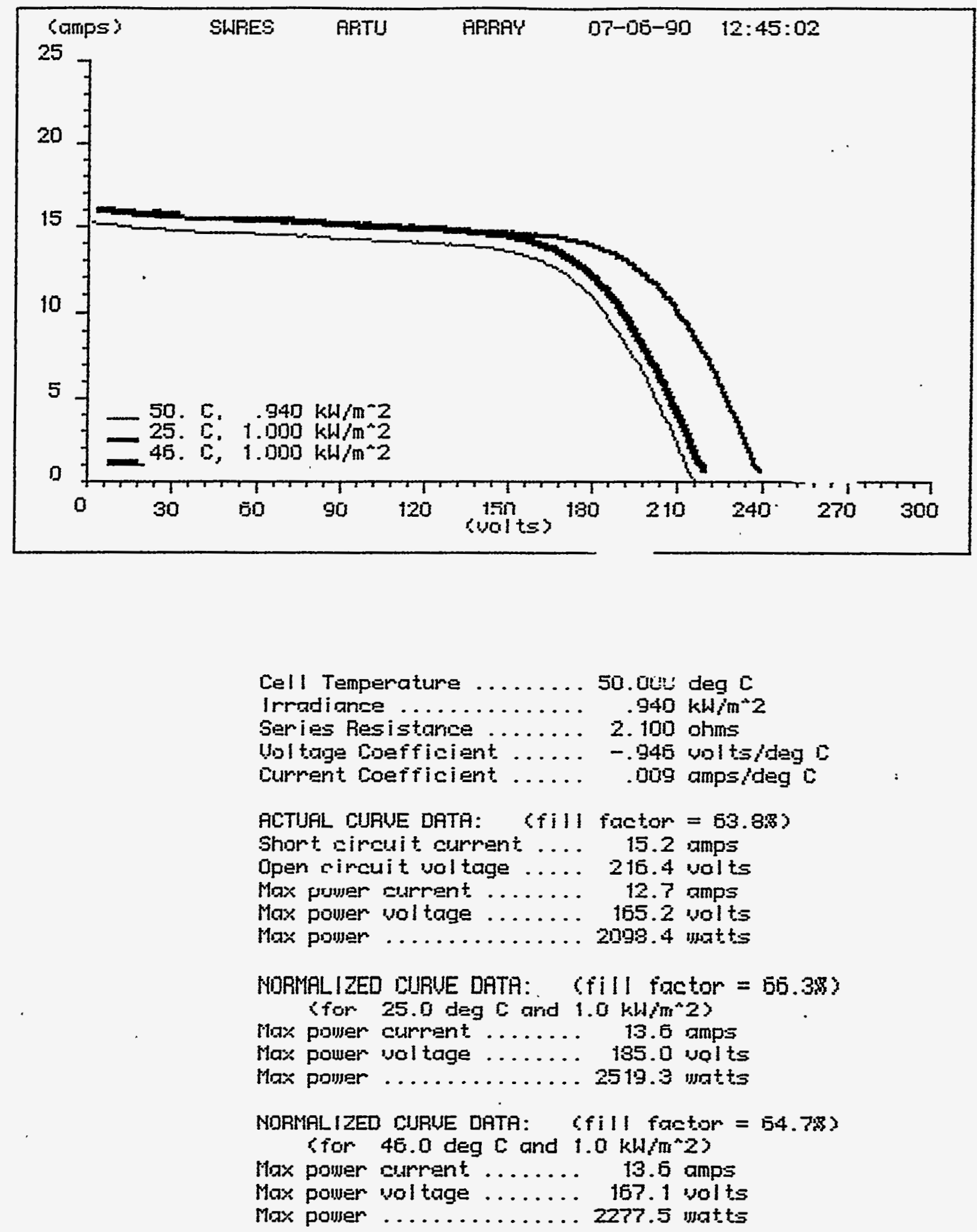


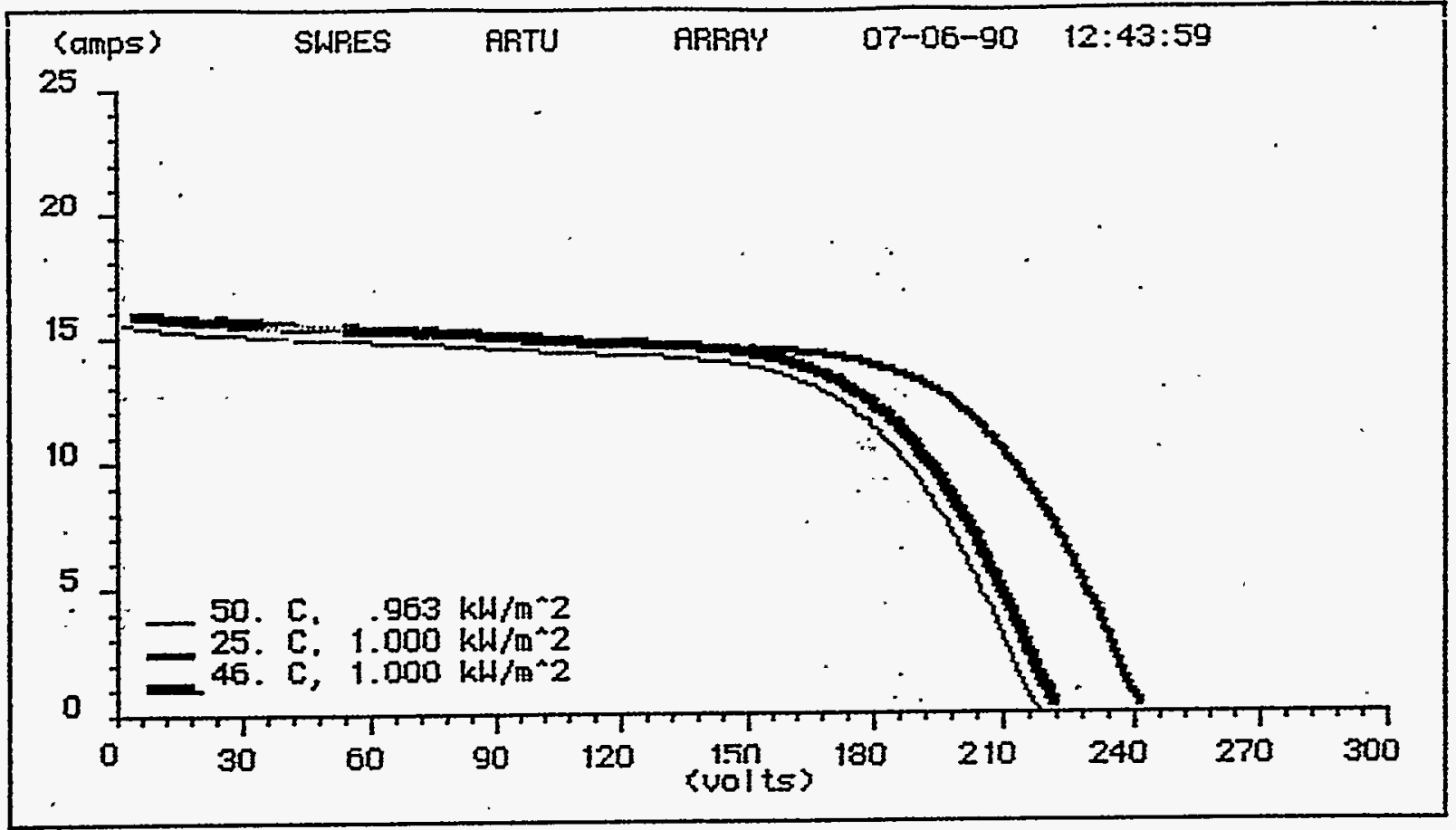

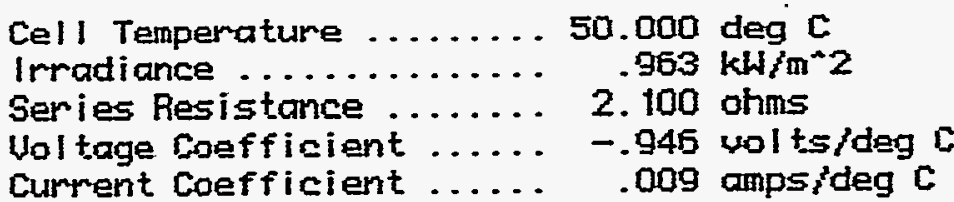

ACTUAL CURUE DATA: : (fill factor $=63.98$ )

Short circuit current .... 15.5 amps

Open circuit vol tage .... 218.0 volts

Max power current ....... 13.1 amps

Max power vol tage ....... 165.2 volts

Max poyser ............ 2157.9 matts

NORMALIZED CURUE DATA: ( $\mathrm{fi} / 1$ factor $=65.18$ )

(for 25.0 deg $C$ and $1.0 \mathrm{kH} / \mathrm{m}^{\wedge} 2$ )

Max pouver currment ....... 13.4 amps

Has power jol tage ........ 188.1 volts

Max pomer ............ 2525.2 matts

NORMALIZED COURUE DATA: “ (fill famtor $=54.5 \%)$

(for 46.0 deg $C$ and $1.0 \mathrm{kH} / \mathrm{m}^{\wedge} 2$ )

Max pouser curment ....... :13.6 amps

Max power vol tage ...... 167.8 volts

Max power ............ 2285.9 matts 


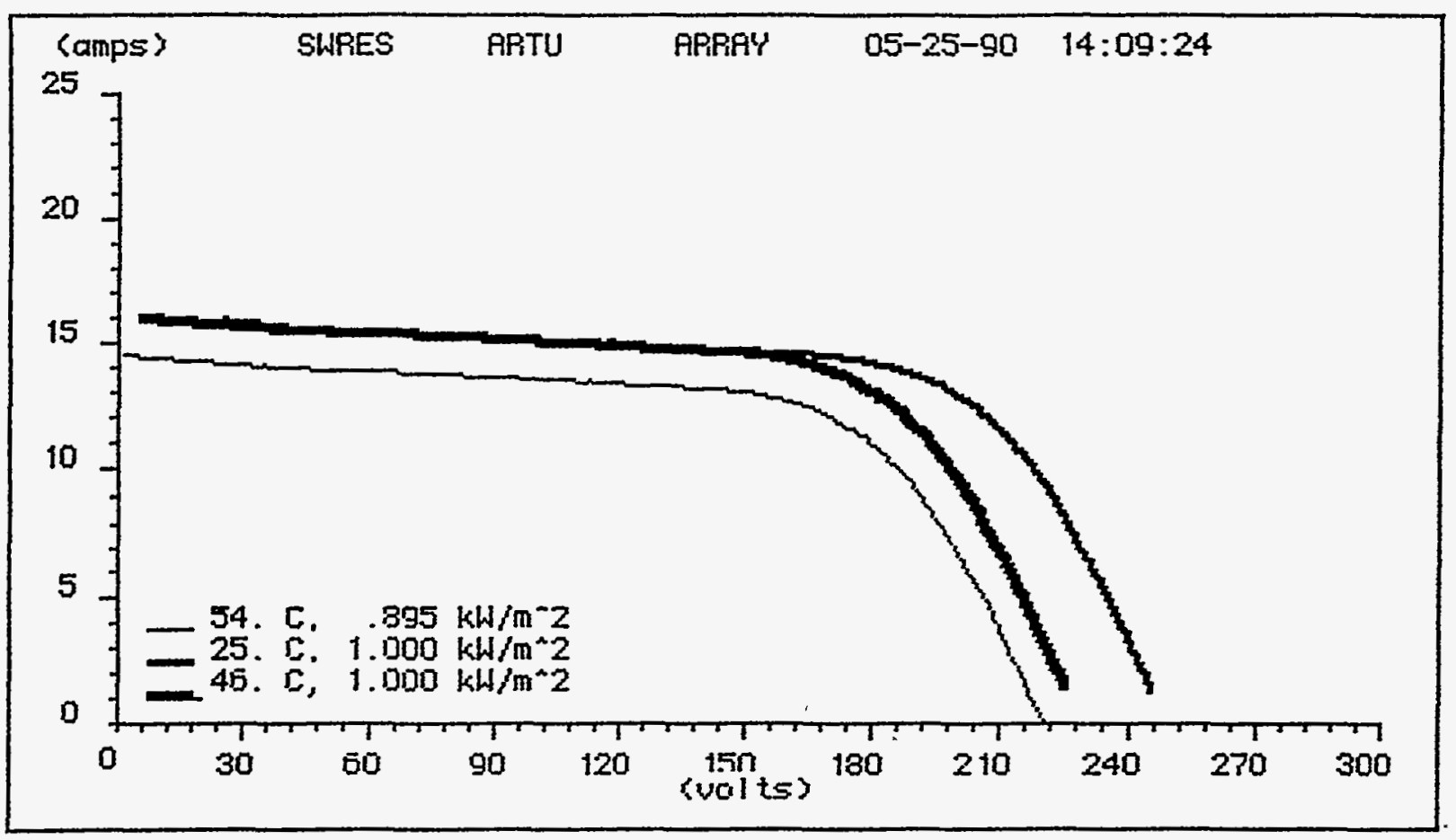

Cell Temperature .......54.000 deg c

Irradiance ............ .895 kW/m²

Series Resistance ....... 2. 100 ohms

Uoltsige Coafficiant ...... - -.945 wolts/dag c . :

Cumpent Coefficient ..... . .009 ampsfodeg $c$

ACTUAL CURUE DATA: (fill factor $=63.98$ )

Short circuit current .... 14.5 anps

Open circuit wal tage ..... 220.2 valts

Max poujer Eurrent ...... 12.2 amps

Max power voltage ....... 167.6 volts

Max pouser ............2042.8 matts

MORMALIZEO CURUE DATR: (fill factor $=67.0 \%$ )

Sfor 25.0 deg $C$ and $1.0 \mathrm{kH} / \mathrm{m}^{\wedge} 2$ )

Max pouser current ....... 13.6 amps

Max power vol tage ....... 192.0 valts

Max power ...........2513.8 matts

NDRMALIZED CIJRUE DATA: (fill fantor = 65.58)

Sior $4 E .0$ deg $C$ and $\left.1.0 \mathrm{~kW} / \mathrm{m}^{-2} \mathrm{z}\right)$

Max pawer current ...... 13.7 amps

Max power voltage ...... 172.9 volts

Max power ........... 2373.2 unatts 


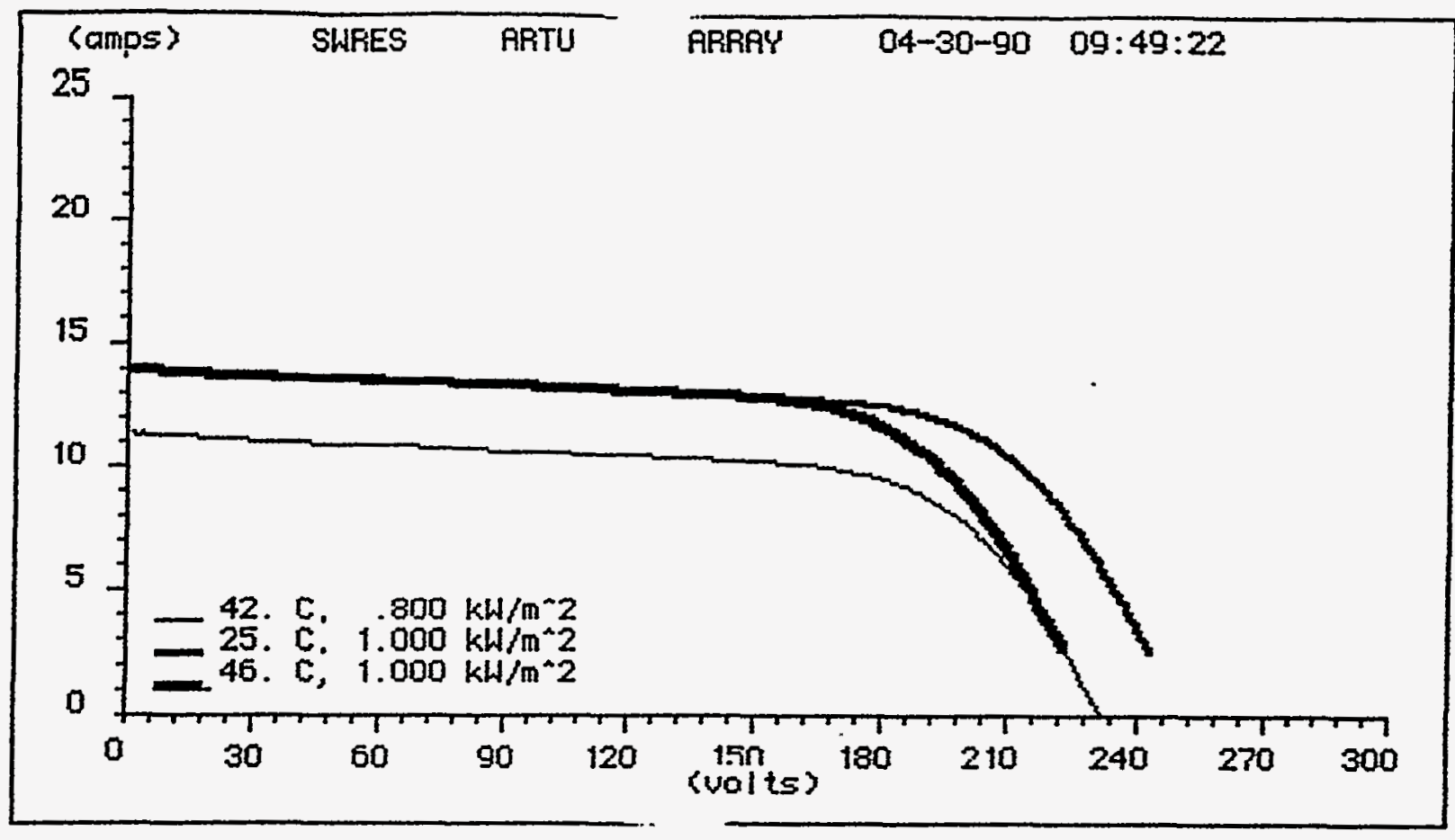

Cell Temperature

Irradiance

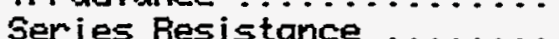

Voltuge Coefficient ..... -.945 unlts/deg C

Current Coefficient ..... .009 amps/deg $c$

RCTUAL CURIJE DATA: (fill factor $=65.6 \%$ )

Short circuit current .... 11.3 amps

open circuit val tage ..... 231.4 volts

Max power current. ...... 9.4 amps

Max power vol tage ....... 182.8 volts

Max powar ............ 1720.2 watts

MORMALIZED CURUE DRTA: ( $i$ ill factor $=68.98$ )

(for 25.0 deg $\mathrm{C}$ and $1.0 \mathrm{~kW} / \mathrm{m}^{\wedge} 2$ )

Max pouner current ....... 12.1 amps

Max power val tage ....... 193.3 volts

Max power ............ 2331.1 watts

NORMALIZED CIJRUE DATA: (fill factor $=67.6 \%$ )

(for $40.0 \mathrm{deg} C$ and $1.0 \mathrm{~kW} / \mathrm{m}^{\wedge} 2$ )

Max power current ....... 12.1 amps

Max power vol tage ....... 176.5 vol ts

Max power ............ 2129.7 watts 


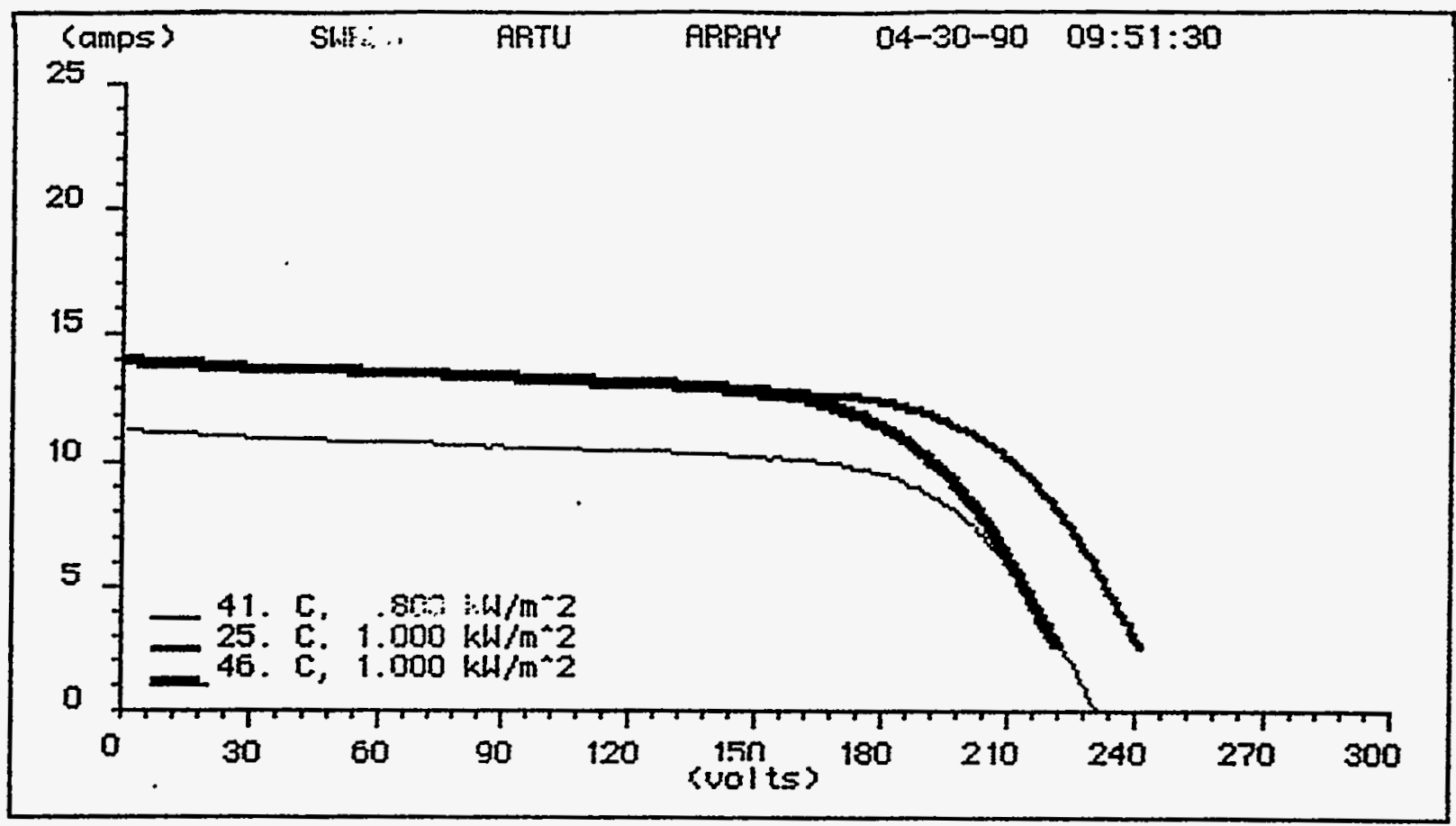

Cell Temperature ........41.000 deg C

Irradiance ........... . $803 \mathrm{kH} / \mathrm{m}^{\wedge} \mathrm{z}$

Series Resistance ....... 2.100 ohms

Uoltage Donfficient ..... - -.945 volts/deg C

Current coefficient ..... . .009 ampsideg $c$

ACTUAL CURUE DATA: (fill factor $=65.68$ )

Short circui t current. ... 11.4 anps

Open circuit val tage ..... 231.0 valts

Max power current. ....... 9.5 amps

Max power voltage ....... 181.6 volts

Max pouser ........... 1720.1 watts

NORMALIZED CURUE DATA: (fill factor $=68.88$ ) (for 25.0 deg $C$ and $1.0 \mathrm{~kW} / \mathrm{m}^{\wedge} \mathrm{Z}$ )

Max power current. ...... 12.1 amps

Max power vol tage ....... 191.3 volts

Max poujer ............. 2311.0 ujatts

NORMALIZED DURUE DATA: 〈fill factor $=67.5 \$$

(for $46.0 \mathrm{deg} C$ and $1.0 \mathrm{kH} / \mathrm{m}^{\wedge} 2$ )

Max power current....... 12.1 amps

Max power vol tage ....... 174.5 volts

Max power ............ 2109.1 watts 


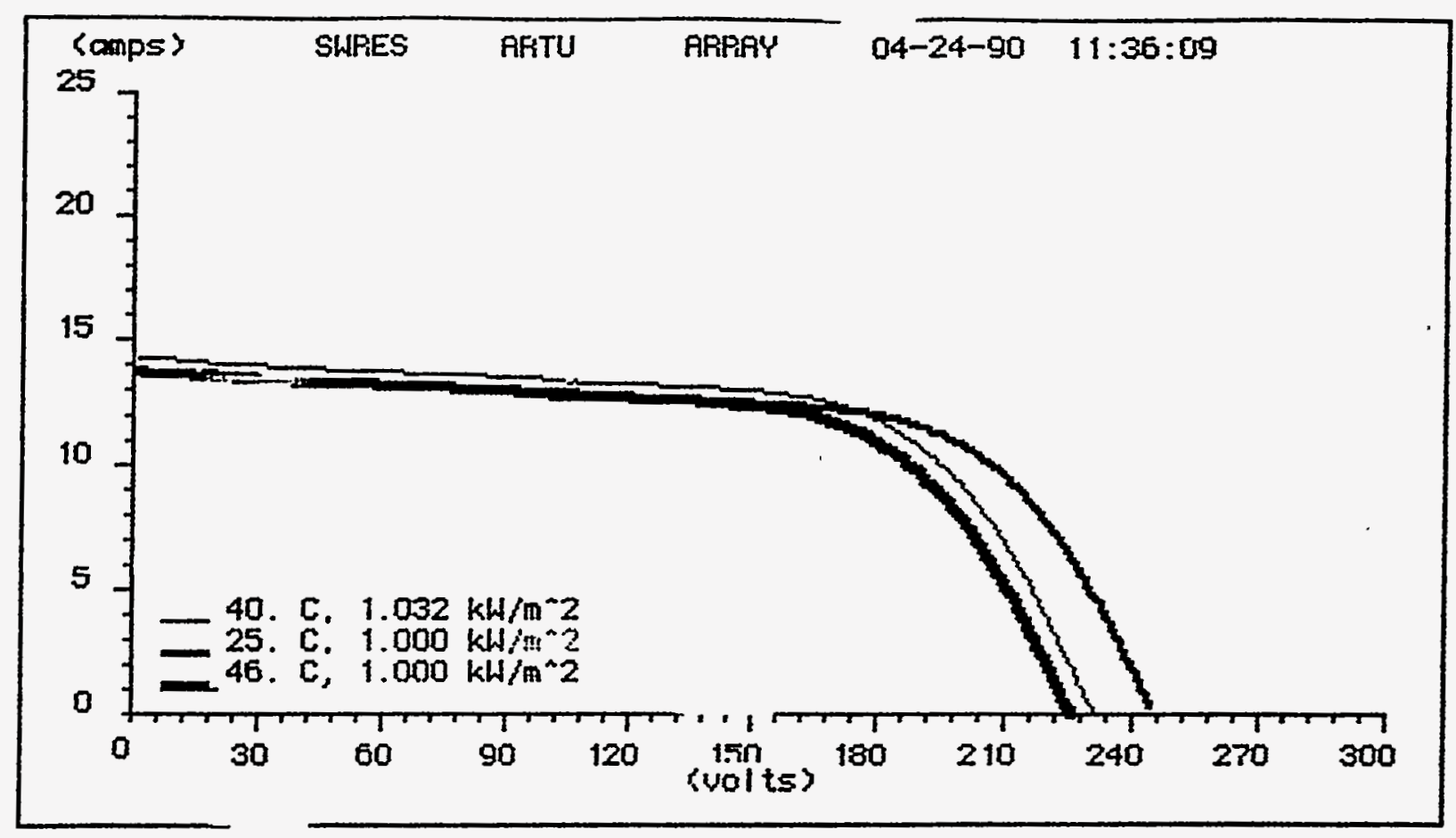

Cell Temperature ...... 40.000 der r.

Irradiance ........... $1.032 \mathrm{kWrm}^{2} \mathrm{Z}$

Series Resistance ....... 2.100 ohms

Voltage coefficient ..... -.946 volts/deg $c$

Cumment Coefficient ..... .009 amps/deg $C$

ACTUAL CURUE DATA: (fill factor $=64.88$ )

Short circuit current .... 14.3 amps

Open circuit voltage .... 231.3 volts

Max power current ....... 12.3 amps

Max power vol tage ....... 174.6 volts

Max pouser ............ 2139.0 watts

MORMALIZED CURUE DATA: (fill factor $=65.6 \%$ )

(for $25.0 \mathrm{deg} C$ and $1.0 \mathrm{kH} / \mathrm{m}^{\wedge} 2$ )

Max power current ....... 11.7 amps

Max power val tage ....... 190.0 volts

Max power ........... 2219.3 watts

NORMAL IZED CURUE OATA: (fill factor $=64.08$ )

(for $46.0 \mathrm{deg} C$ and $1.0 \mathrm{kH} / \mathrm{m}^{\wedge} 2$ )

Max pouser current ....... 11.9 amps

Max power voltage ....... 169.8 volts

Max power ............2013.0 watts 


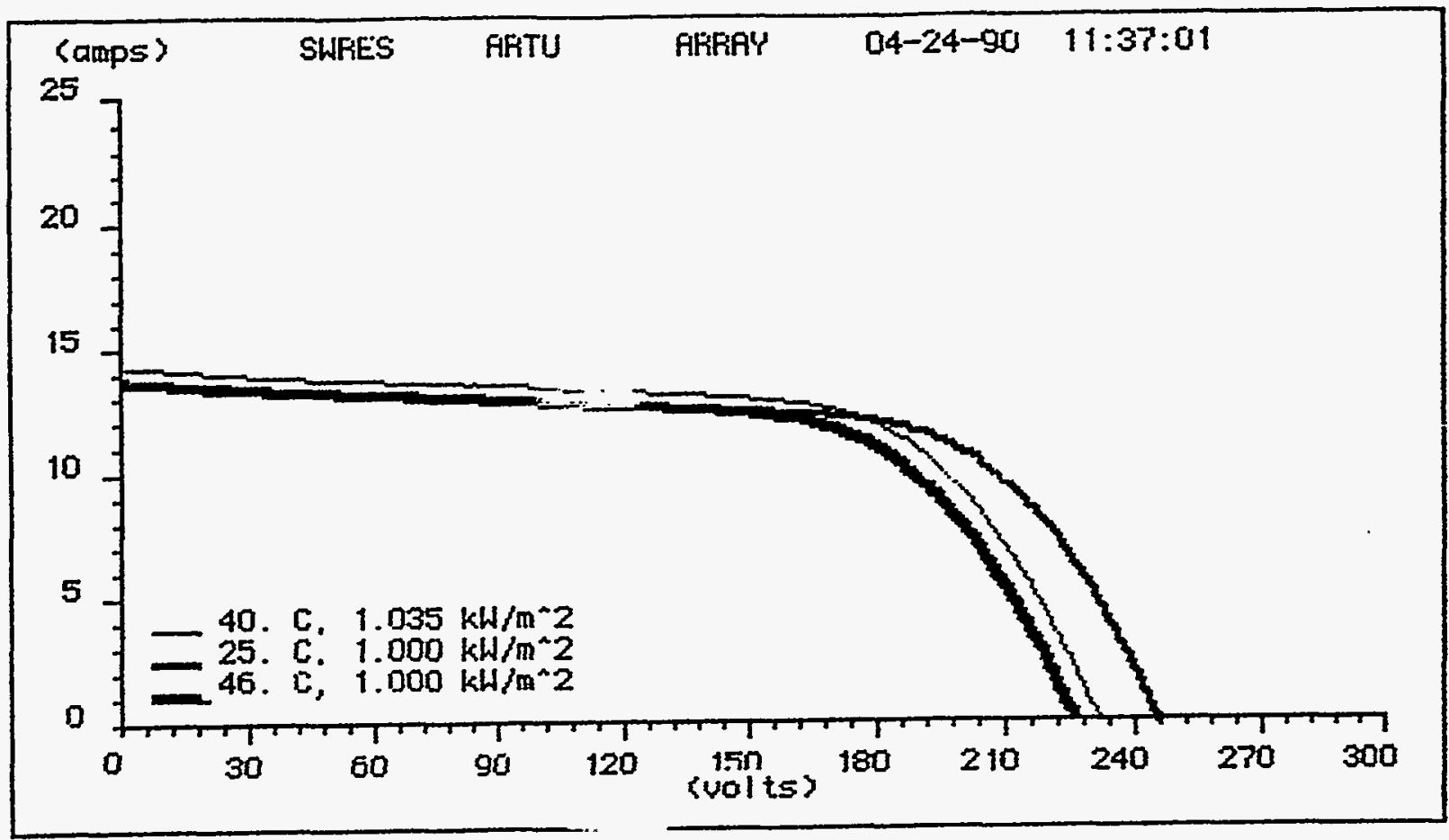

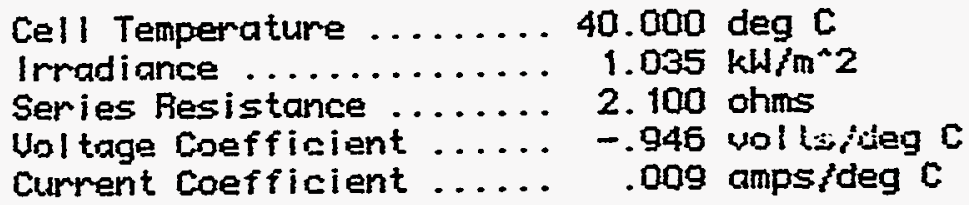

ACTUAL CURUE DATA: (fill factor $=64.98$ )

Short circuit current. ... 14.3 amps apen circuit voltage .... 231.6 valts Max pouser gurrent. ...... 12.2 ampro Max powe: woltage ....... 175.8 volts Max power ........... 2142.8 watts

MORMALIZED CURUE DATA: ( $\mathrm{fill}$ factor $=65.7 \%$ ) (for $25.0 \mathrm{deg} C$ and $1.0 \mathrm{~kW} / \mathrm{m}^{\circ} 2$ )

Max power current ....... 11.6 amps Max power vol tage ....... 191.2 volts Max power ...........2215.0 watts

NORMFLIZED CURUE DATA: ( $\mathrm{fill}$ factor $=64.18$ ) (for 46.0 deg $C$ and $1.0 \mathrm{kH} / \mathrm{m}^{\circ} 2$ )

Max power current ....... 11.8 amps

Max power vol tage ....... 131.0 volts Max power ...........2010.8 wat 


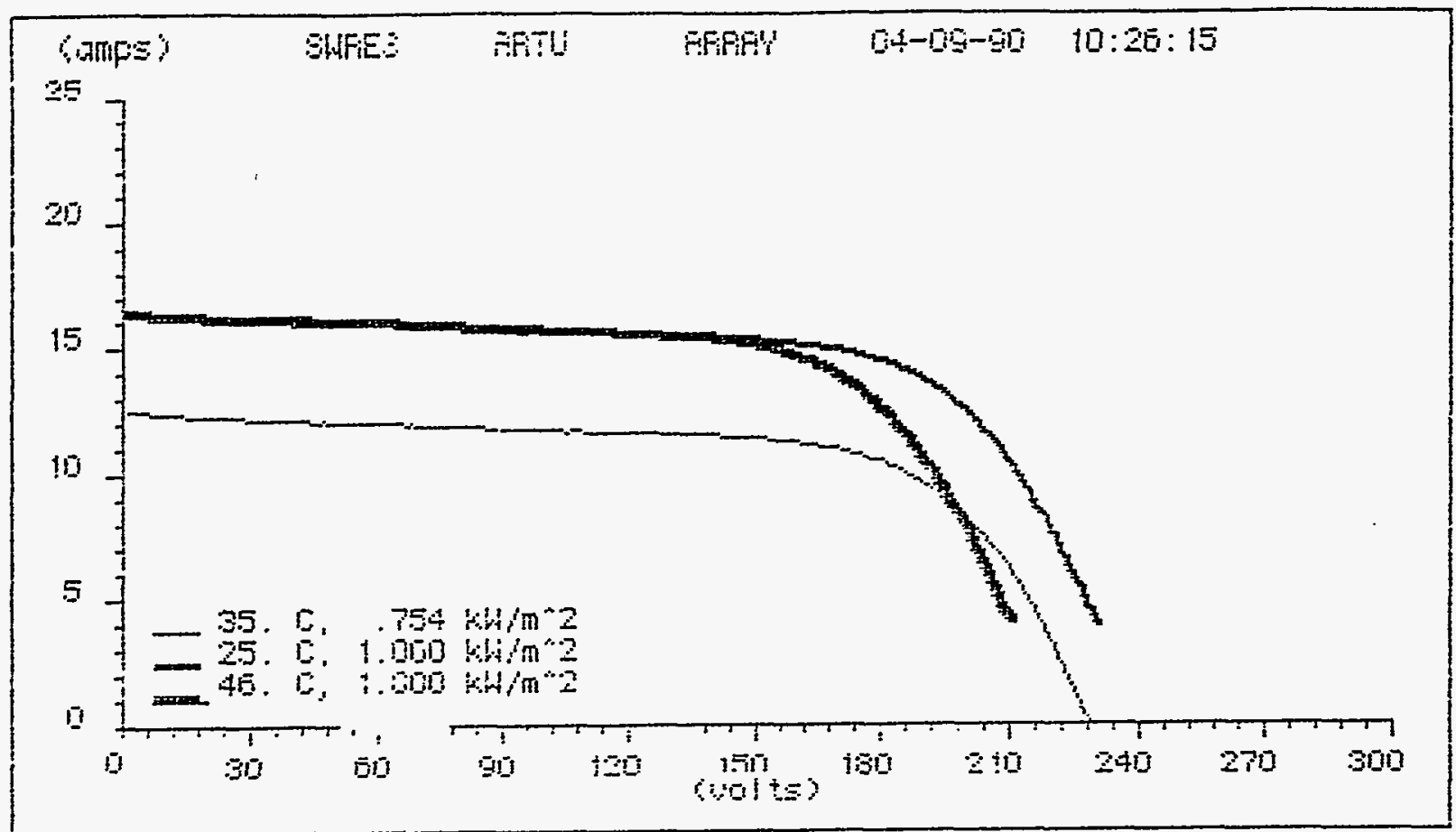

Del! Tempenature . . . . . . irmadiance ............

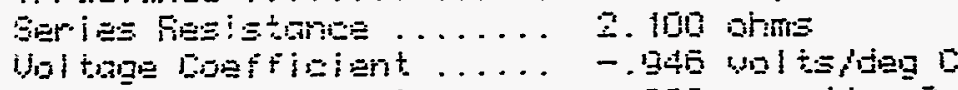

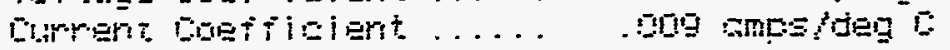

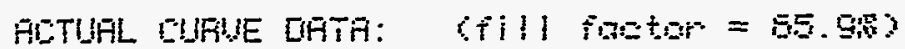

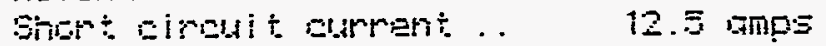
Lper: cirouit wol tage ... . 229.0 woits Moxe power current. ...... 10.5 amps

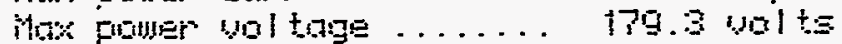
Mox pouner ........... 1000.0 matts

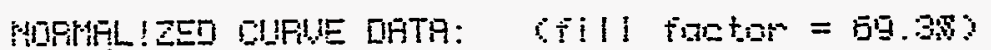

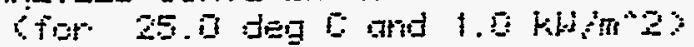

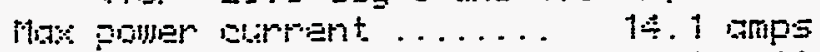
hox pouter so! tage ...... 190.3 volts tho pomer ........... 2525.0 watts

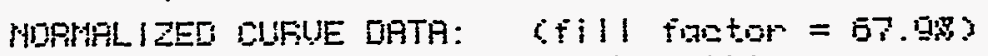
Gror 40.0 deg 0 and $1.0 \mathrm{kH} \mathrm{m}^{\circ} \mathrm{2}$ ?

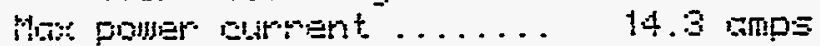
Mox fromer vol toge ....... 185.9 wolts hax pomer ........... 2379.0 131.t.5 

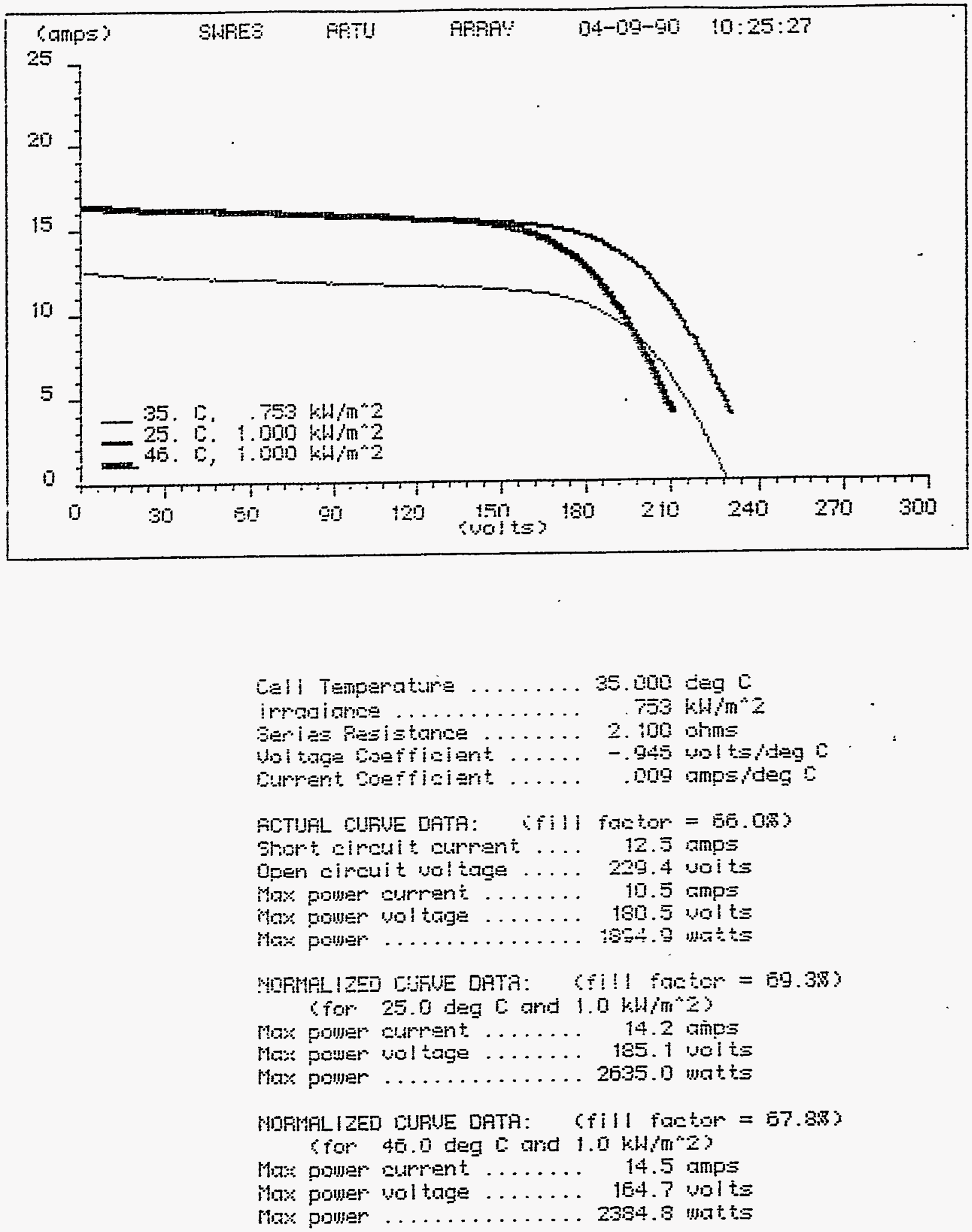


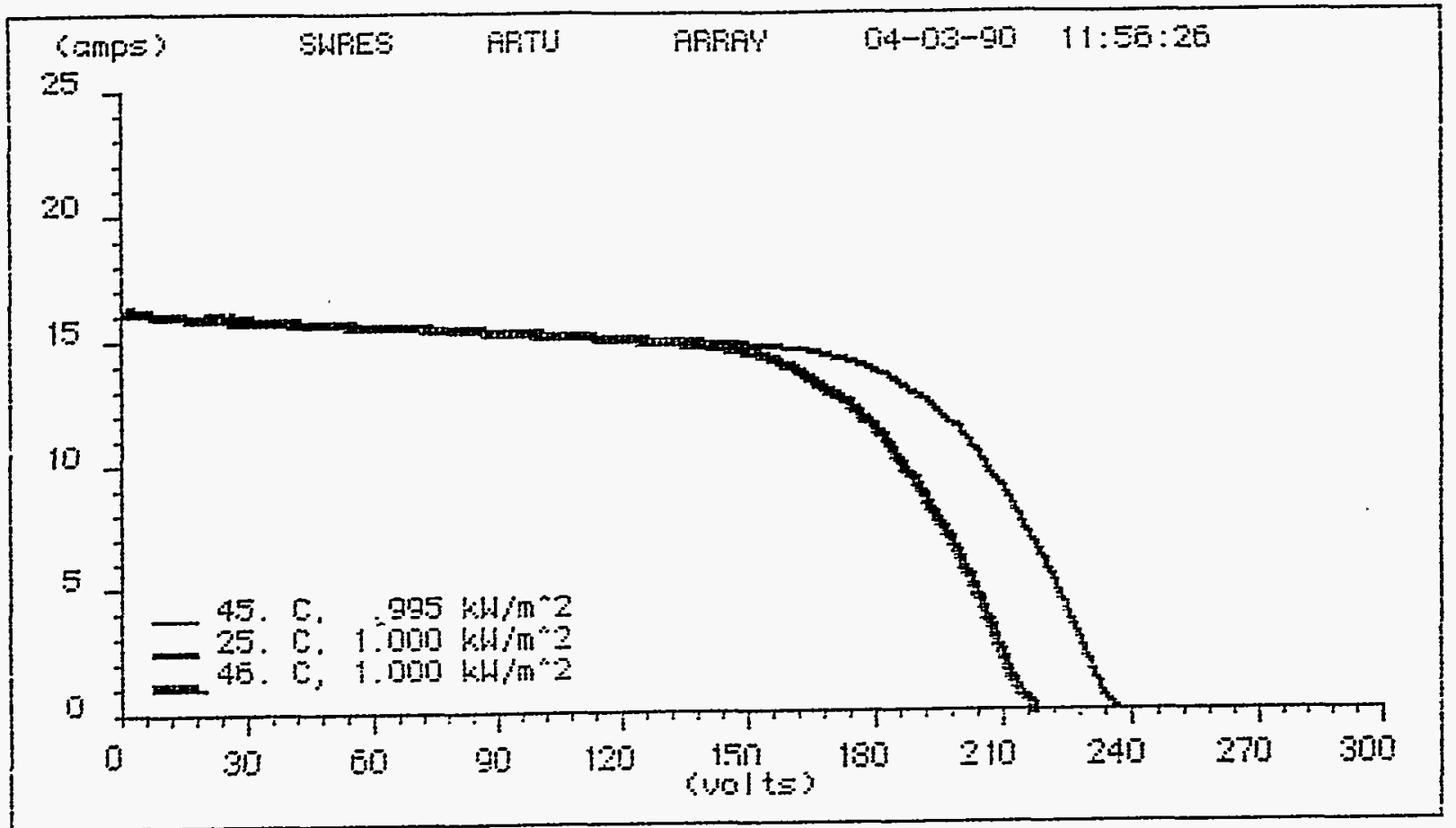

Cel! Temperature ......45.000 deg $c$

Irradiance .......... .995 $\mathrm{kH} / \mathrm{in}^{2} \mathrm{Z}$

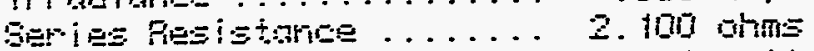

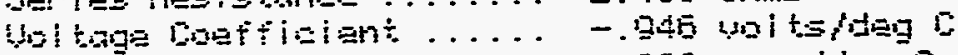

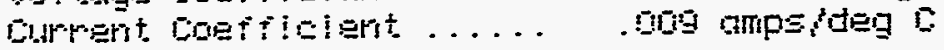

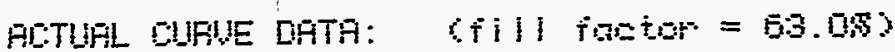

Short cirouit Diryert. ... 15.2 amps

Guen eirauit voltage .... 2te.1 valts

Hos: power etirrent. ...... 13.7 rmps

Max pouler voltage ...... to. . wolts

Max poum . . . . . . . . 201.5 watts

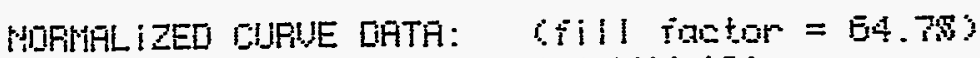

(for 25.0 deg 5 arod $\left.1.0 \mathrm{kH} / m^{*} 2\right)$

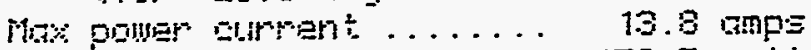

Mas pouer jol tage ....... 179.7 volts

Mas pourer ........... 2478.5 watts

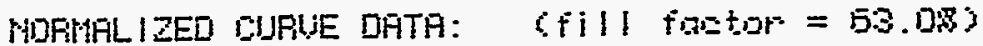

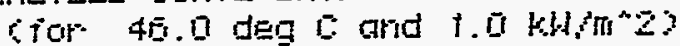

Hox poust aurment. ...... 13.3 amps

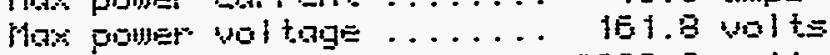

Mox pomer ........... 2230.6 motts 


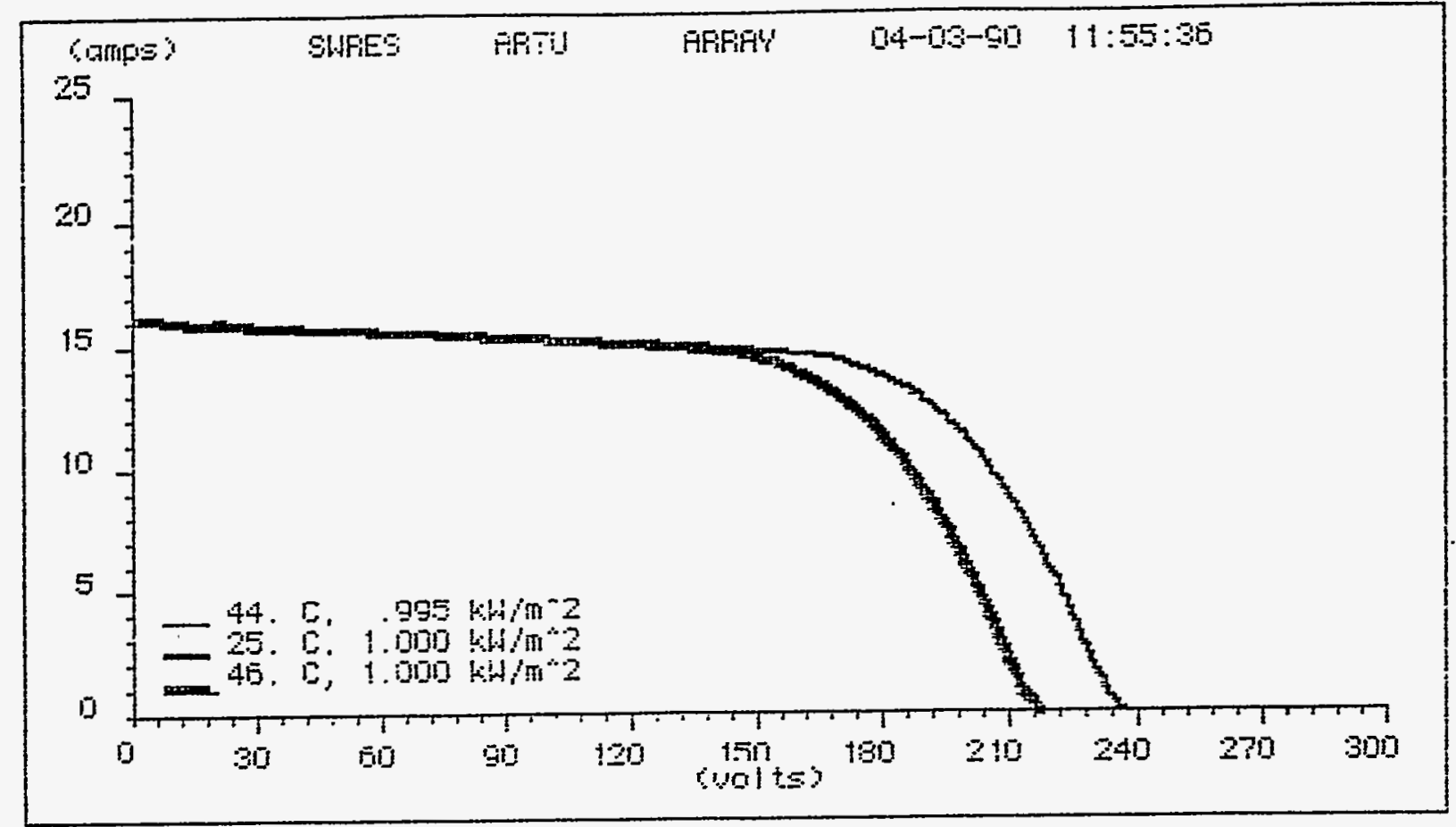

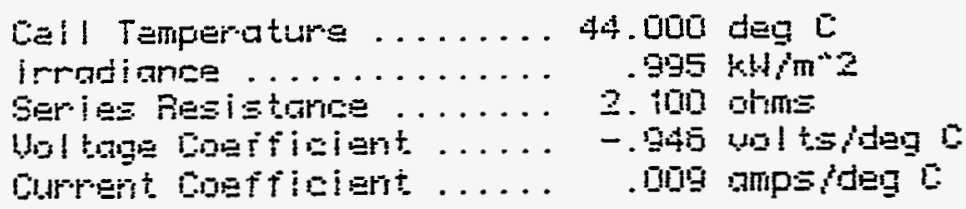

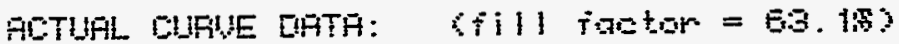

Short cirouit current ... 15.2 umps

Gper cireui : vol tage .... 2 2 :.E volt.

Mos power atrrent ....... 13.9 rimps

tow poulder valtage ...... the t.7 volts

Max pomer . . . . . . . 2235. o unt ts

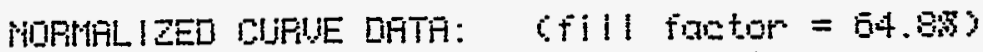

6ror 250 deg o ond $1.0 \mathrm{kH} / \mathrm{m}^{\circ} 2$ ?

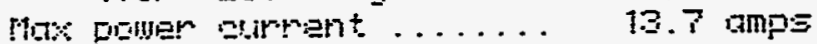

Ma火 pewer val tage ....... 179.9 vol $t=$

Hax pouper ...........2470.5 watts

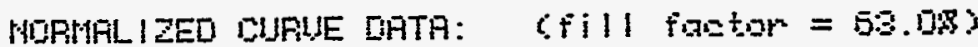

stor 40.0 deg $[$ and $1.0 \mathrm{kH} / \mathrm{m} 2 \mathrm{z}$

Max pouser aurrent ....... 13.9 umps

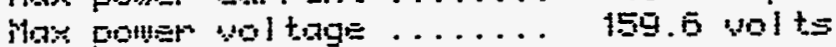

Mase pourer . . . . . . . . 2221.7 watts 


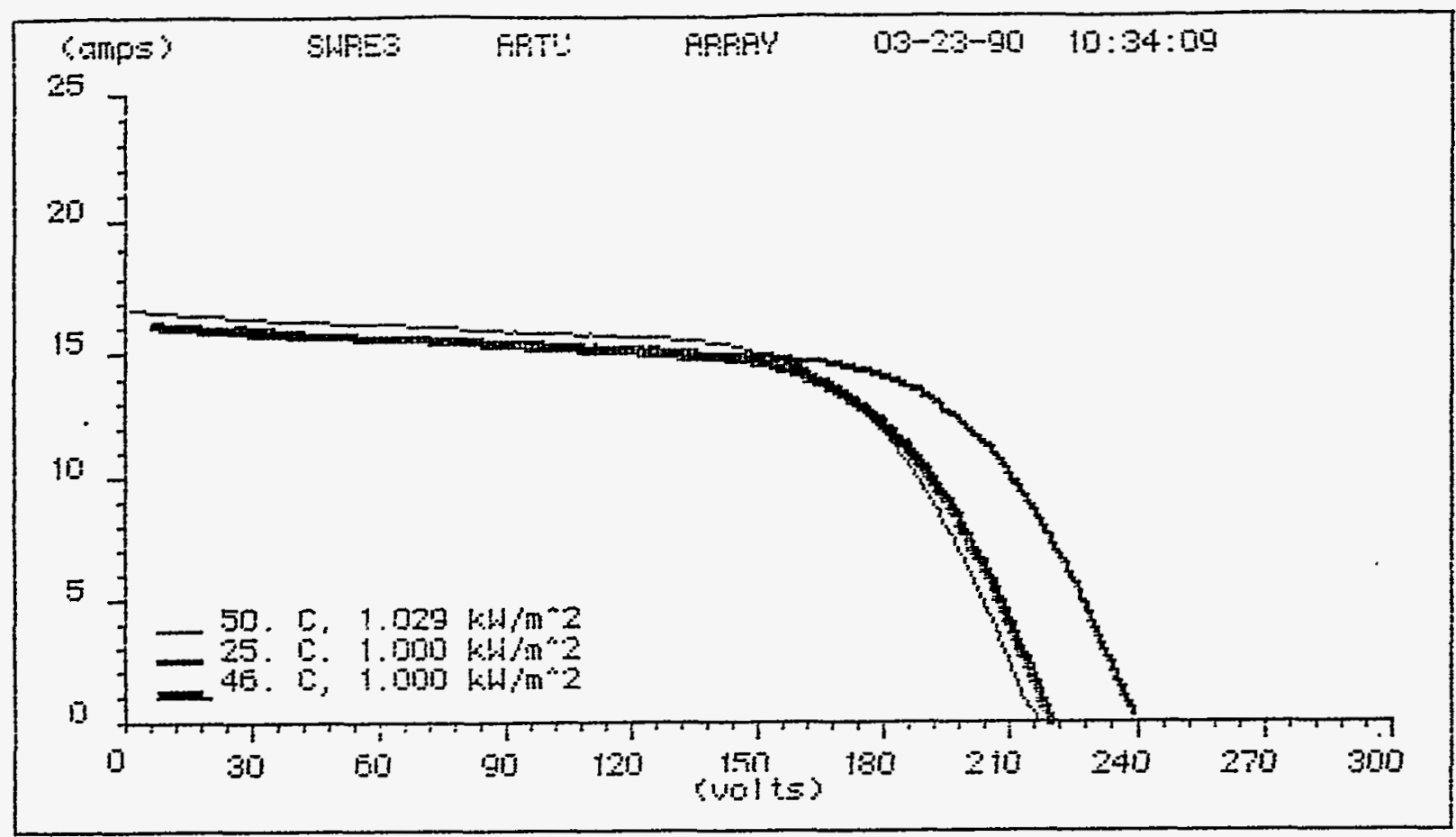

Ce:! Temperoture ....... 50.000 deg 0

Impriaroe .......... 1.02g $44 m^{2} 2$

Ser i Ee Fes juance ...... 2. 100 ohms

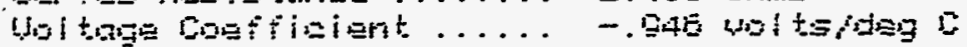

Durrent boeficient ..... . Dog smperdeg 0

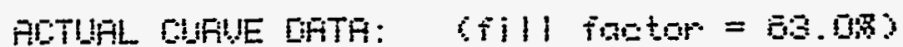

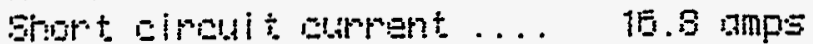

Gen cimoul t vol tage .... 217.7 volt

Mrax power ctirrent ....... $14.3 \mathrm{amp}=$

Has pourer wol toge . . . . . 150.5 wolts

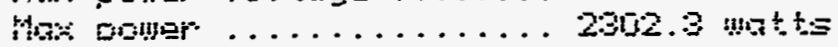

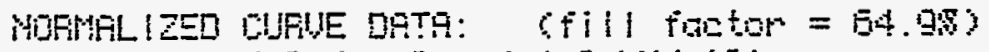

Cfer 250 deg 0 ond 1.0 th $m^{2} 2$ ?

Max poumer atrment. ...... 13.8 rmpse

llax peuer vol tage ...... 183.3 volts

Max power ........... 25:37.? motts

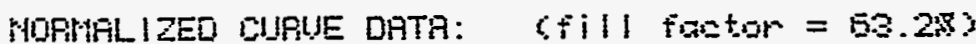

rior" 40.0 deg o and $1.0 \mathrm{kH}$ (m)

Mase poumer gurrent ...... 13.3 smps

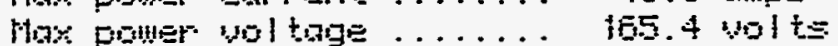

Mod poiner .......... 2286.0 matt:s 

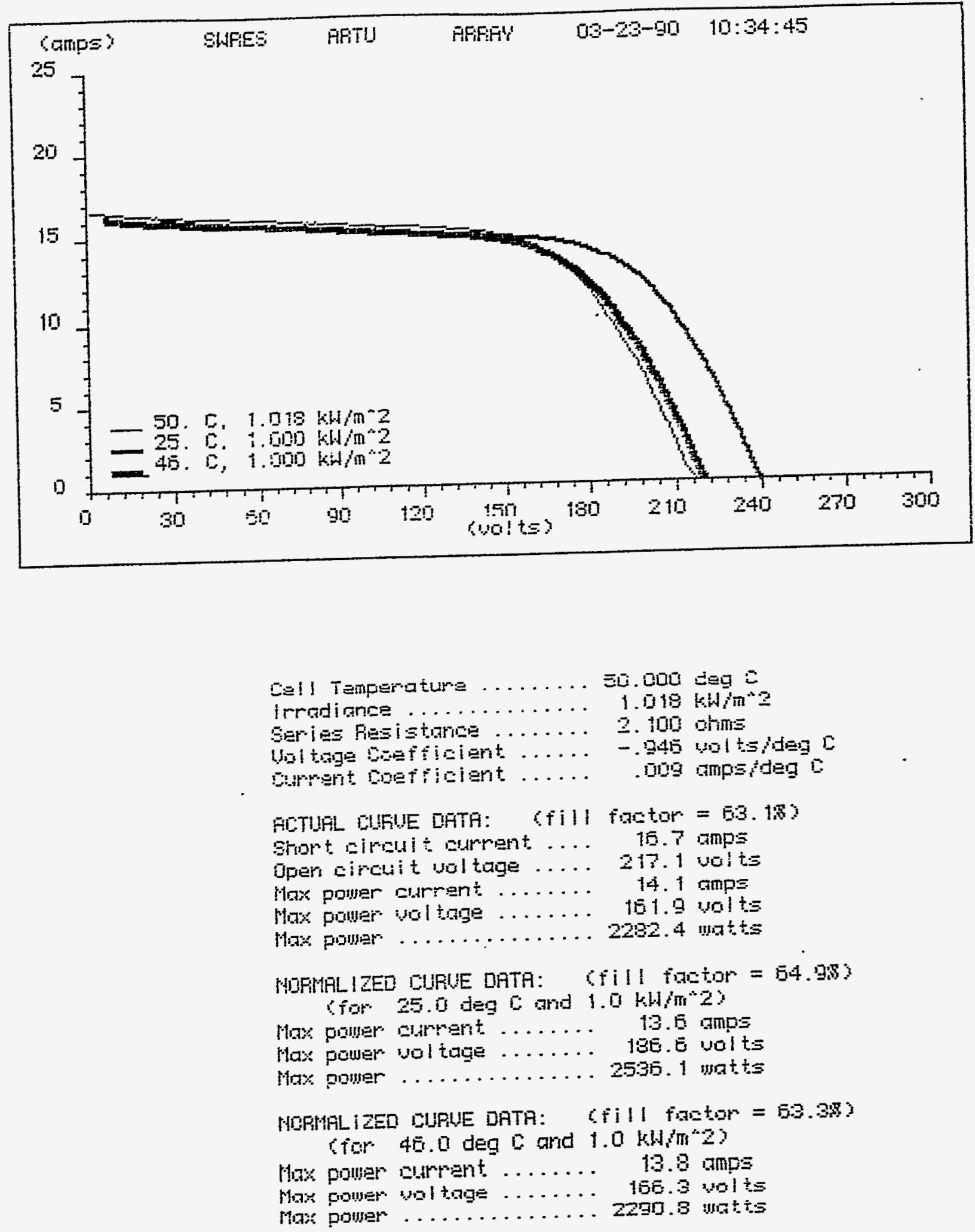


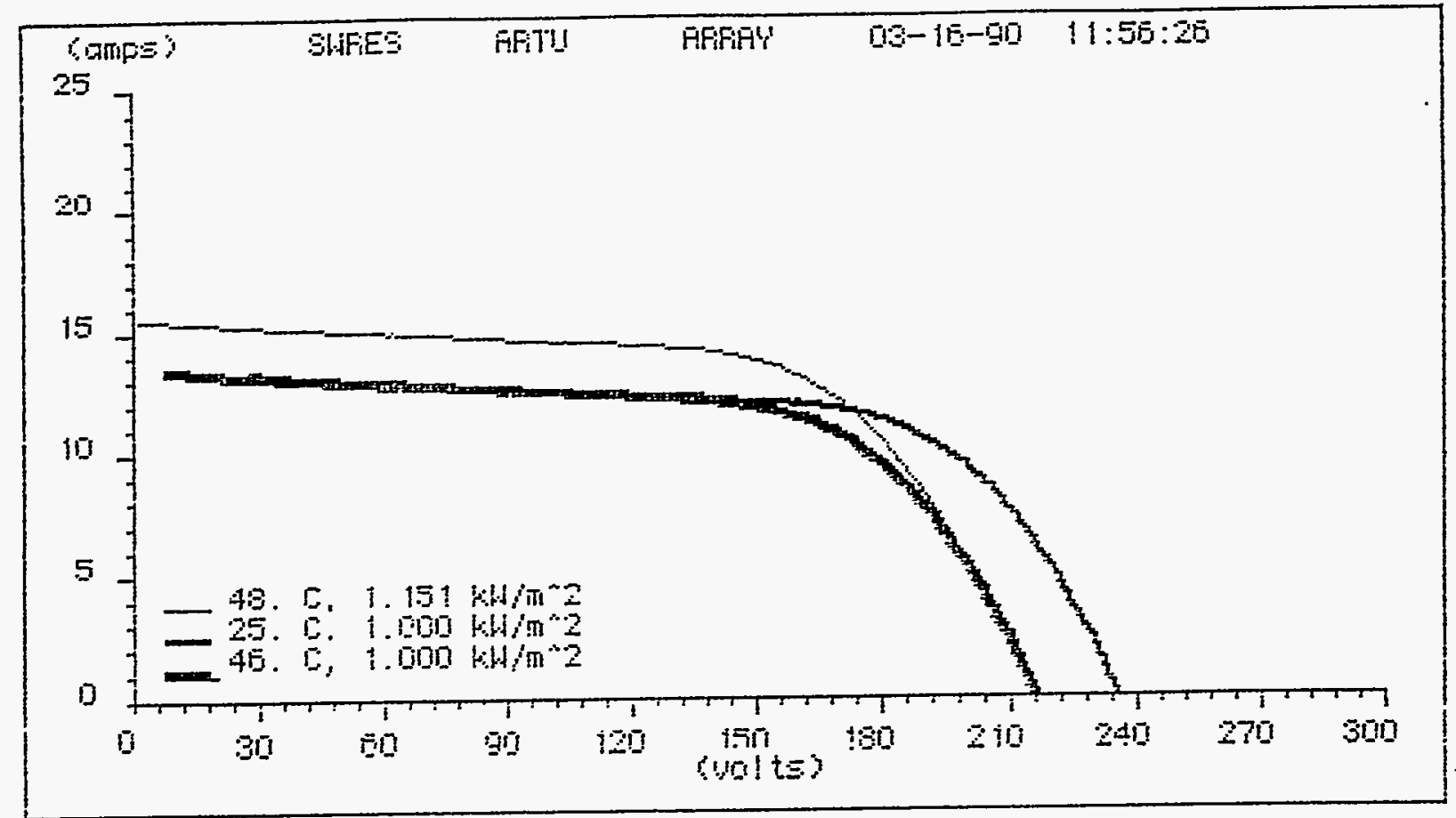

Cell Temperature ...... 48. Dü das C

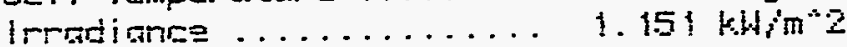

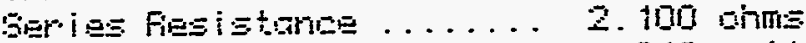

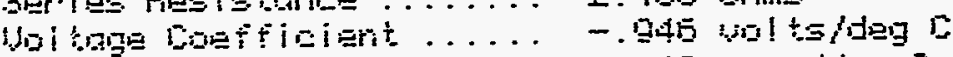

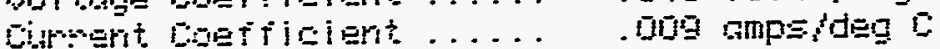

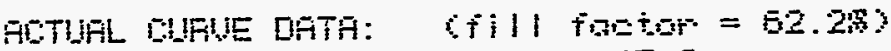

Bhort almolit burront ... 15.6 gmps

Goer Eircuit vol tage .... 217.4 volts

Mro Eomer atirment. ...... 13 . smps

Mox pouler woi toge ...... 157.0 volts

Mox Fumbro. . . . . . . 2184.2 m.

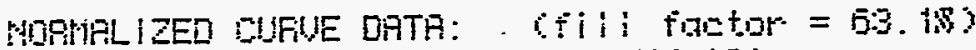

(ior 25.0 deg 0 and $1.0 \mathrm{kH} / \mathrm{m} 2 \mathrm{~s}$

Ma: pouser asrrent ...... 11.3 ghps

Hax pomer vol tage ....... 181. t wolts

Nax pouer ........... 2052.7 matts

HDFIALIZEO OUFUE DATA: 6fill faOtor $=51.33$

ctor 40.0 deg 0 and 1.0 this $\pi^{\circ} 22$

Hax pouper aurrent. ...... 11.3 amps

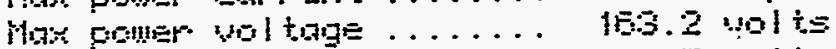

Max pourer .......... 1951.7 matts 


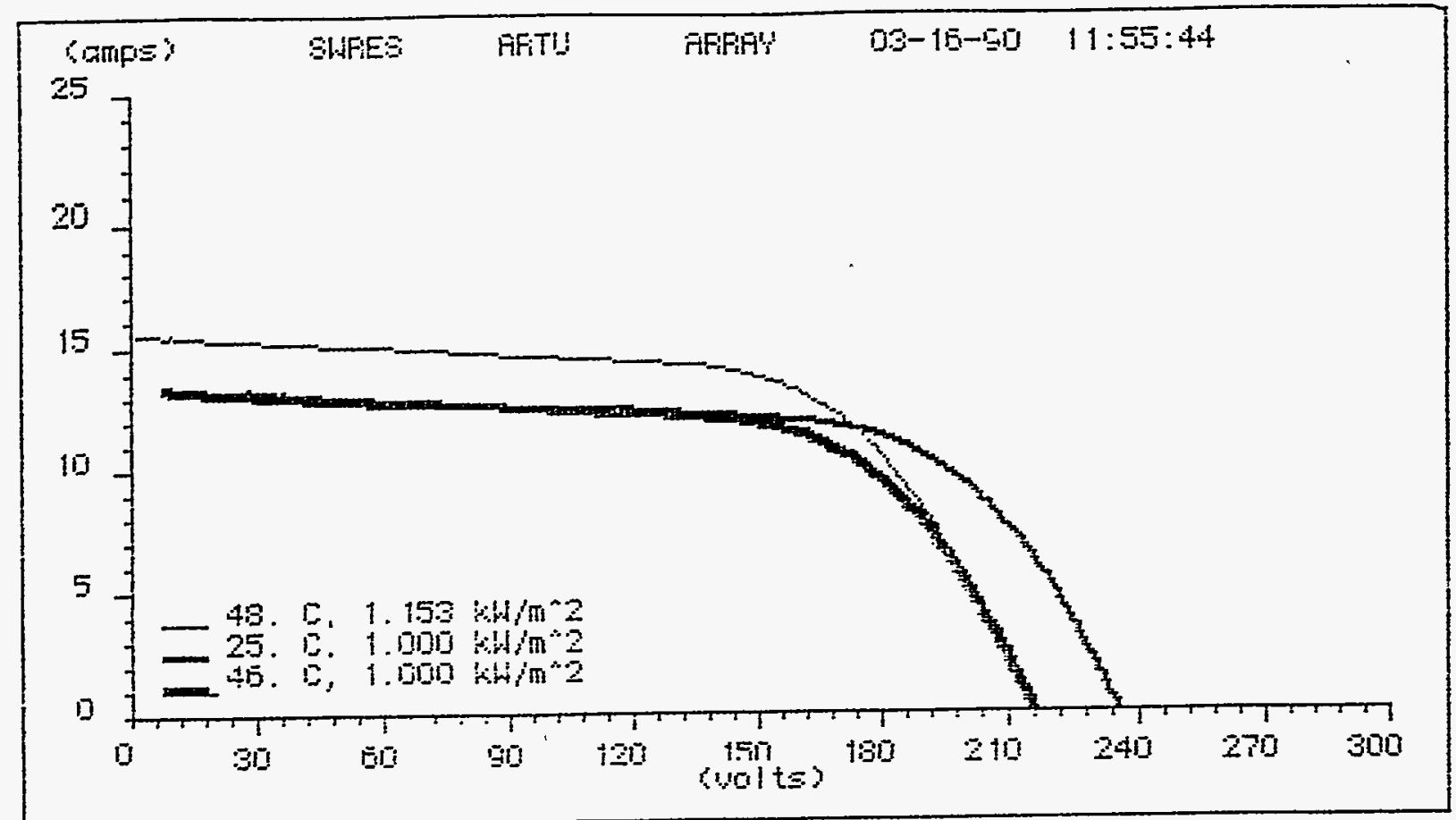

$\therefore$ I! Temerature .......4.000 deg $c$

irrariance ........... $1.150 \mathrm{kH}^{2} \mathrm{~m}^{2} \mathrm{z}$

Geries Fesistarice ....... 2. ton ohms

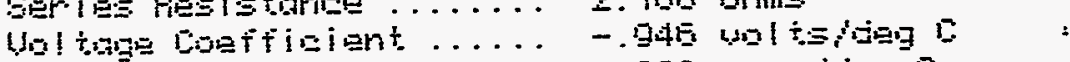

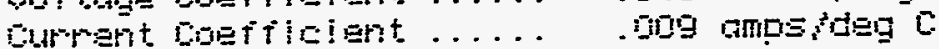

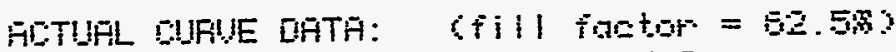

Short. airouit ourtent. . . 15.5 amps

Gpen circuit ugitage .... 210.5 uolte

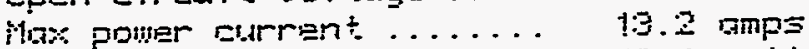

Max puner valtage ...... 159.4 volts

Hox pusmer ...........2105.4 matts

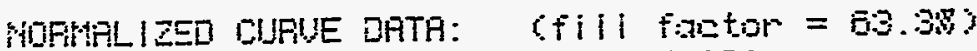

ion 25.0 deg 0 and $1.0 \mathrm{kH} / \mathrm{m}^{*} \mathrm{z}$

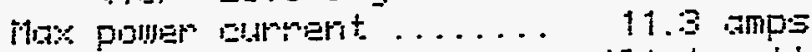

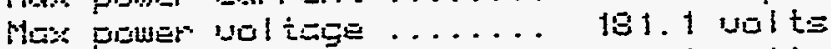

Mrow power ............ 2040.1 mot

HDRIILLIZED OUFUE DATA: GIII frotor $=51.5 \%$ ?

Gror 40.0 deg $C$ and $1.0 \mathrm{kH} / \mathrm{m}^{*} 2$ ?

Max Enwer athrent . . . . 11.5 amps

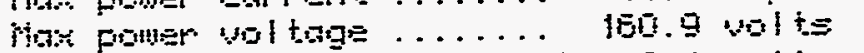

Mox pourer .......... 1846. 1 matts 


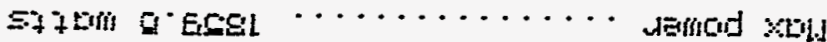

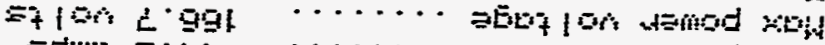

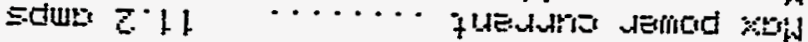

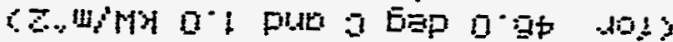

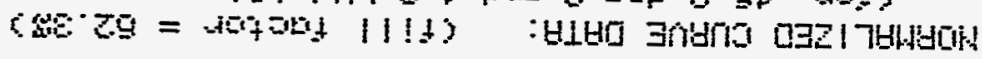

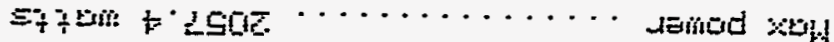

Glor s.

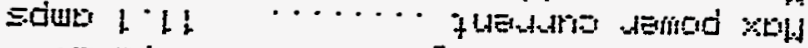

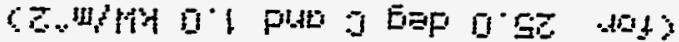

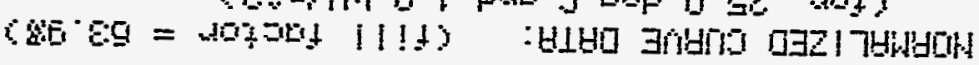
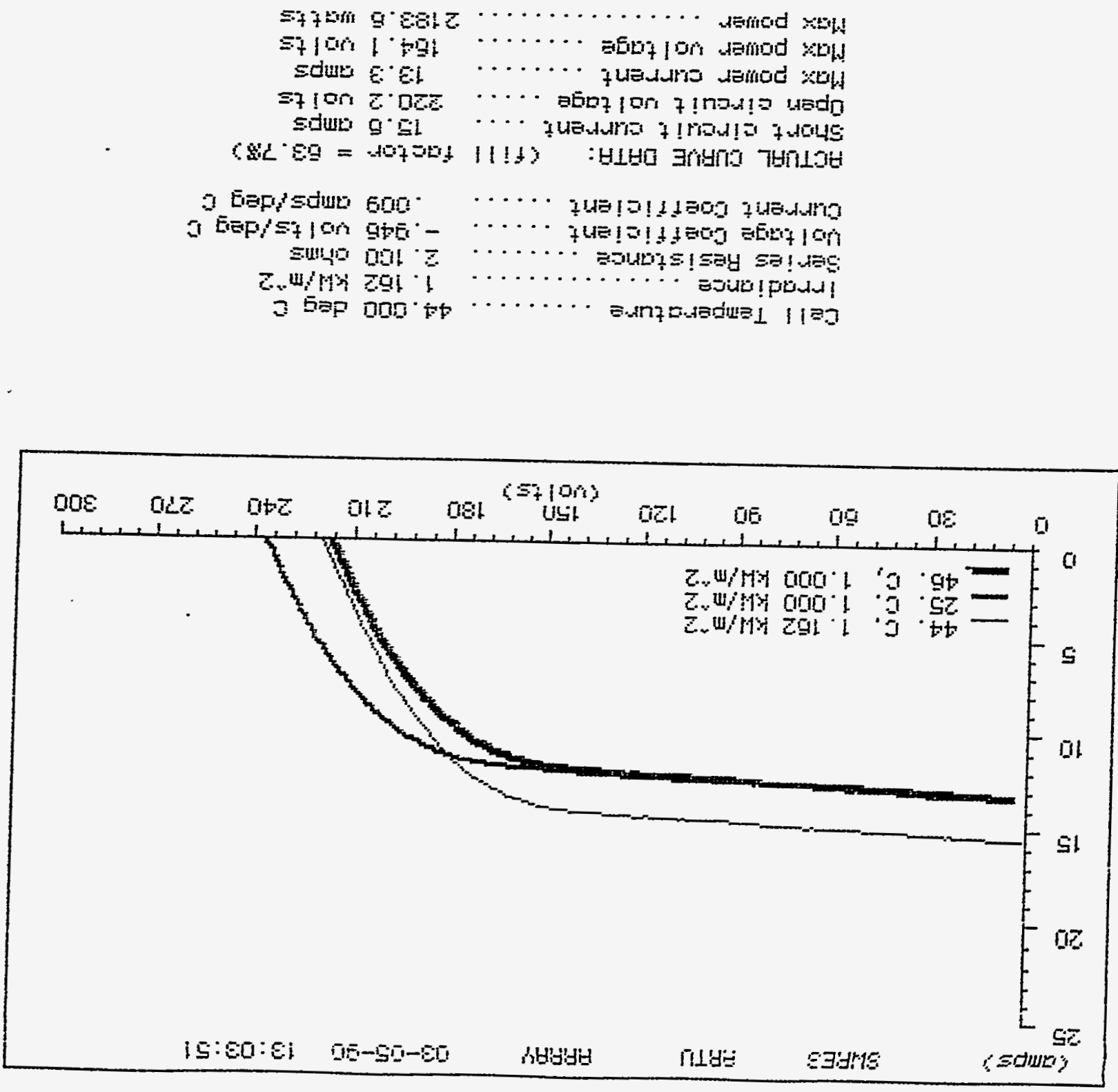


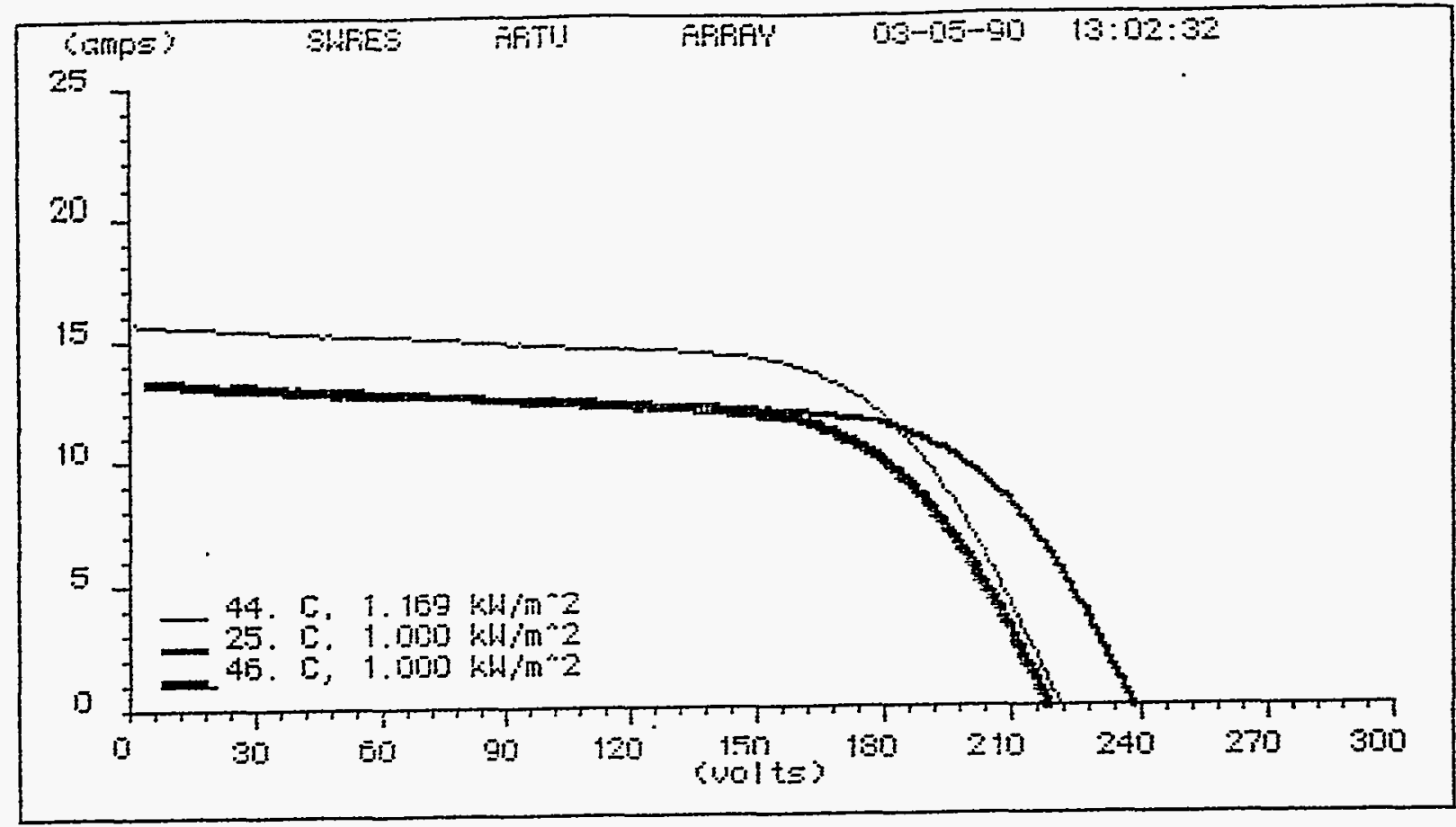

Le!l Temperature .......44. ULU dag $L$

Irradiance ........... 1. $159 \mathrm{kH} \mathrm{k}^{-2}$

Ser is: Resigtarue ....... 2. 100 ntams

Ual t.

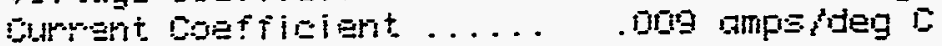

ACTUAL OUFIE EATA: GTIll factor = $83 . \mathrm{BS}$

Short airouit aurrent. ... 15.7 amps

Deen circuit voltage .... 221.3 volt

Mos pomer current. ...... 13.3 amps

Hox fouler voltoge ....... 150.4 volts

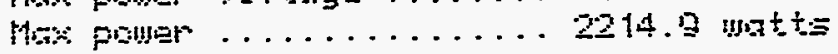

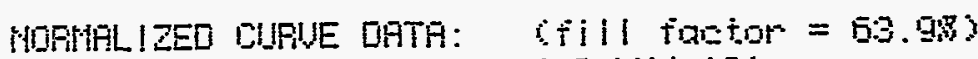

for $25.0 \mathrm{~d}=9 \mathrm{C}$ ard $1.0 \mathrm{kh}+2 \mathrm{~m}$

Mox pouser ithari. . . . . 11.2 amps

Ma. pinjer vol tage ...... 134.8 volts

Hax power ........... 2074.9 wotts

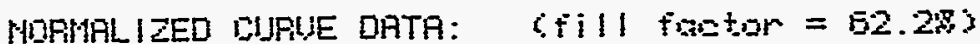

Sior 40.0 deg 0 and $f .0 \% H / m \% 2$ )

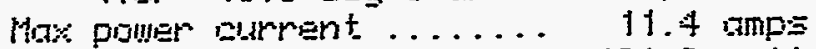

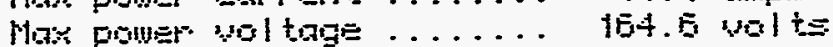

Max pumin ............ 1873.8 watts 


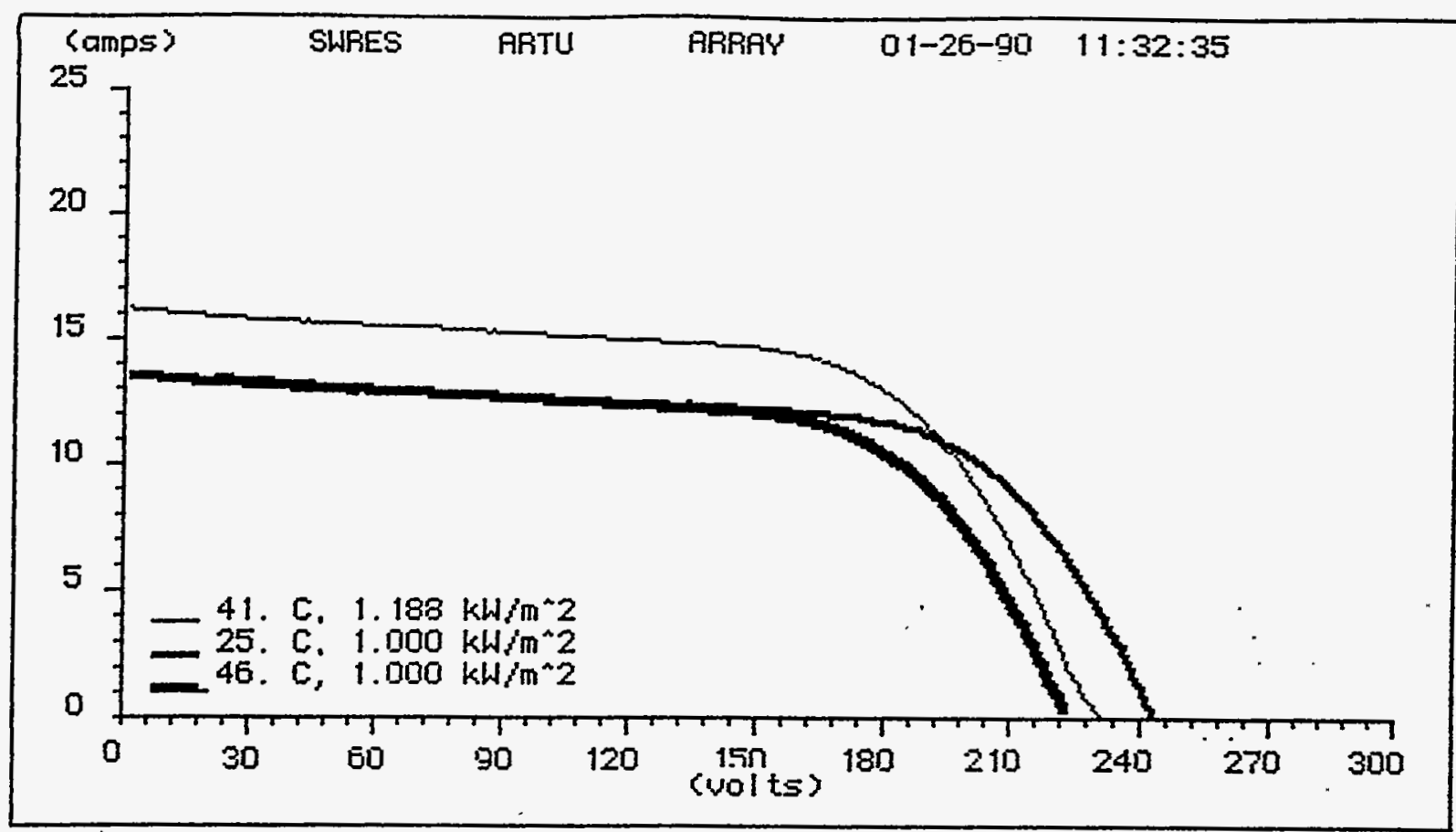

Cel I Temperature ....... 41.000 deg

Irradiance ............ 1. $133 \mathrm{kH}^{2} \mathrm{~m}^{2}$

Serias Resistance ....... 2. 100 ohms

Uol tage Coefficient ...... -.946 val tsideg $C$

Durrent Doefficiant ..... . Dug amps/deg $C$

AITIUAL CURUE DATH: (fill factor $=63.28$ )

Shor.t circuit curment .... 16.2 amps

Dpen sircuit usltage ..... 231.0 volts

Max pouer current ....... 13.7 amps

Max power vol tage ....... 172.3 volts

Max power ...........2 2365.3 watts

MOFMALIZEO CURUE DATA: $\quad$ TIII factor $=63.083$ (for 25.0 deg $\mathrm{D}$ and $1.0 \mathrm{~kb} / \mathrm{m}^{4} \mathrm{2}$ )

Max power current ...... 11.4 amps

Max power wal tage ....... 188.3 volts

Max power ............2 2143.5 watts

NORMALIZED CURUE DATA: (fill factor $=61.38$ ?

(for 40.0 deg $C$ and $1.0 \mathrm{kh} / \mathrm{m}^{*} 2$ )

Max power current ....... 11.5 amps

Max pouser vol tage ....... 168.2 volts

Max power ............ 1939.6 watts 


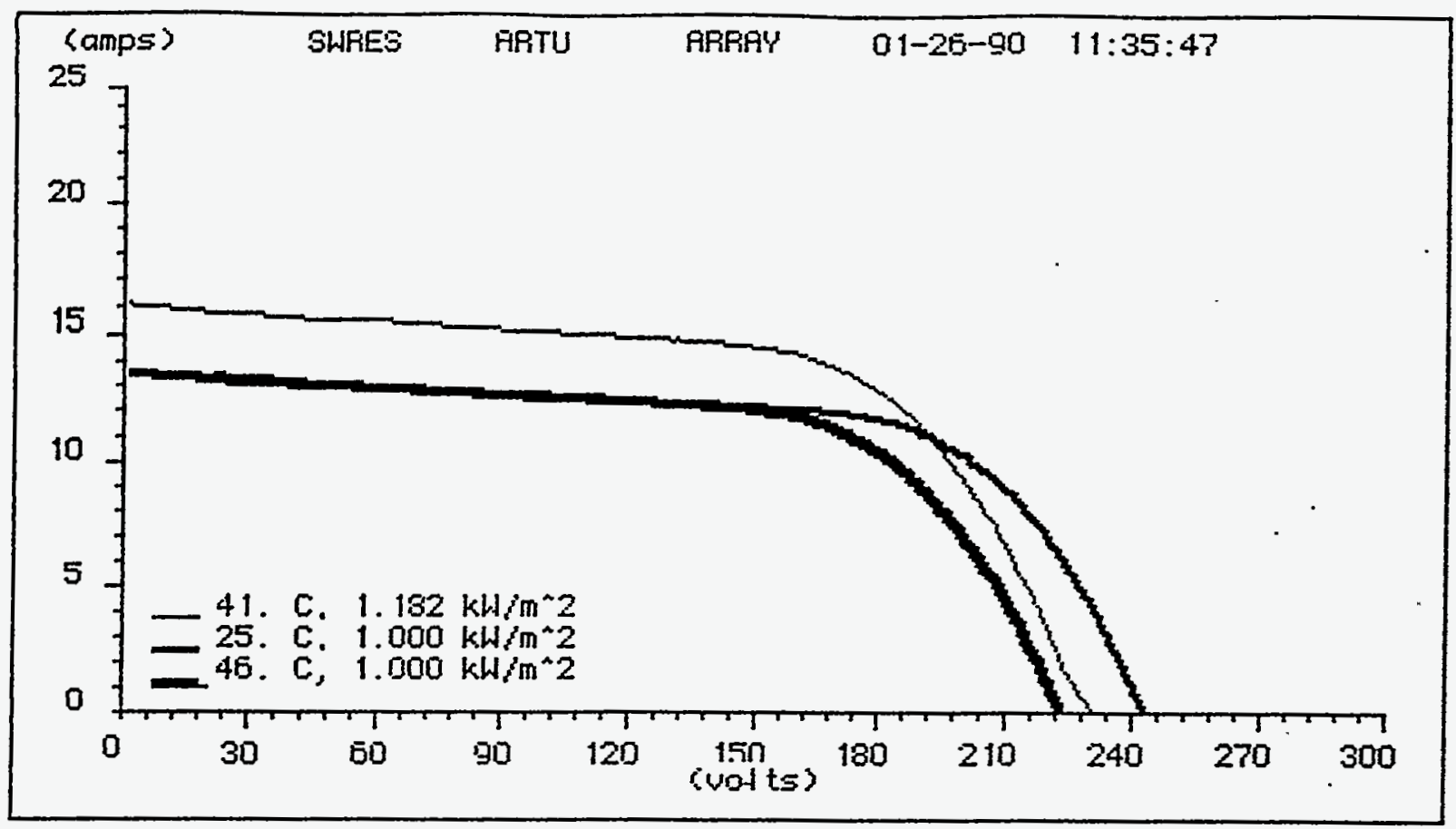

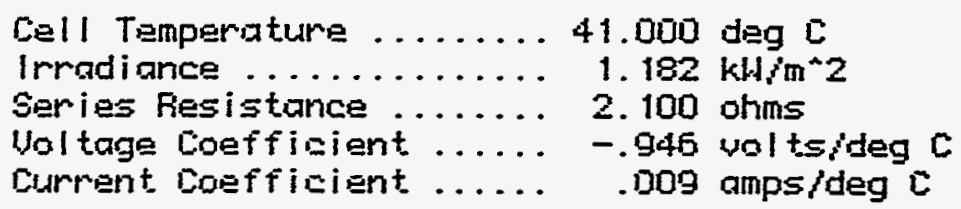

ACTUAL CUFUE DATA: (fill frietor $=53.28$ )

Short circuit current .... 16.1 amps Open eirouit woltage ..... 230.4 volts Max pouler current ....... 13.7 amps Max power vol tage ....... 171.2 wolts Max power ........... 2342.5 watts

MORIALIZED CURUE DATA: (fill factor $=63.18\rangle$ (for 25.0 deg $C$ and $1.0 \mathrm{kH} / \mathrm{m}^{2} 2$ )

Max power eurrent ....... 11.4 amps Max power vol tage ....... 187.0 volts Max power ............ 2134.8 watts

MORMALIZED CURUE DATA: (fill factor $=61.3 \%$ )

(for $46.0 \mathrm{deg} C$ and $1.0 \mathrm{kH} / \mathrm{m}^{\wedge} 2$ )

Max power current ....... 11.6 amps

Max posver voltage ....... 165.8 wolts

Max pouler ............ 1930.0 watts. 
APPENDIXC

Solarex 1990

C-1 


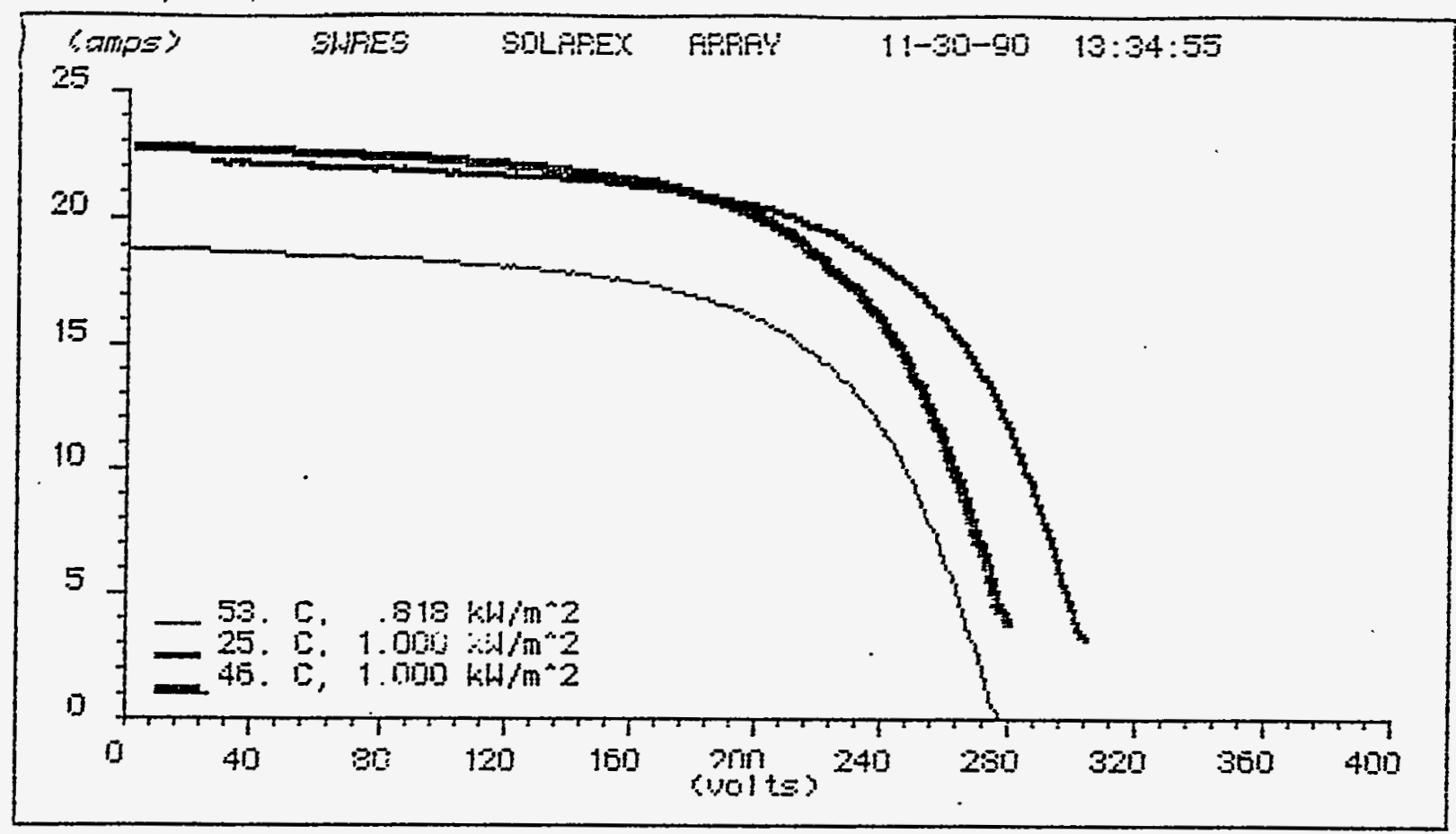

Lell Temperature ....... 53.000 deg $C$

Irradiance ........... .819 kh/m²

Ser ias Fesistunce ...... 1.700 ohms

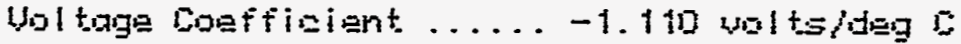

Current Doefilaient ..... .027 amperdeg 0

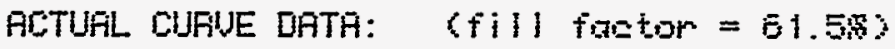

Short. cirault aurrant ... 19.9 amps

Gpen circuit woltage .... 278.0 volts

Hox poumer asurent. ...... 15.4 amps

Max power woltage ....... 209.9 wolts

Max pourar . . . . . . . . 3234.7 wat to

NDPMALIZED CIJPUE DATA: Gill foEtor $=5.5 .38 \mathrm{y}$

(for 25.0 deg o arud $1.0 \mathrm{~km} \mathrm{~m}^{\circ} 2$ )

Max pouser aurrent ....... 19.7 amps

Max pouer vol tage ....... 235.4 volts

How pouner . . . ....... $4401.0 \mathrm{mat} .5$

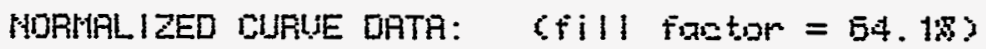

(ior 46.0 deg 2 and $1.0 \mathrm{kN} / \mathrm{m}^{2} 2$ )

Mox poulder current: ...... 19.1 amps

Max pouper voltage ....... 214.3 volts

Max pourer ........... 4095.3 watts 


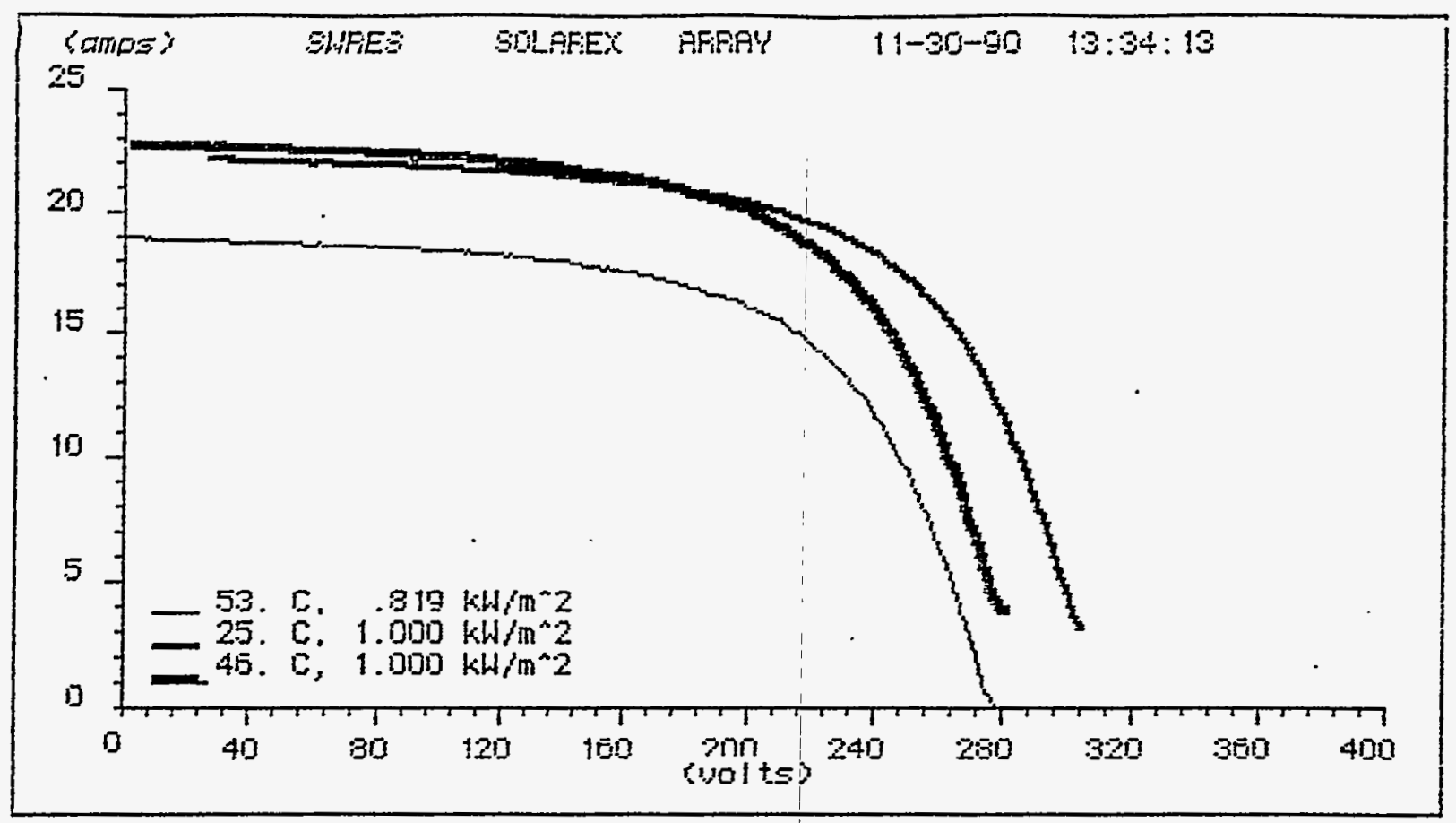

Cell Temperature ........ 53.0üu deg $c$ irradiance ........... .819 khim Series Resistance ........ 1.700 otms

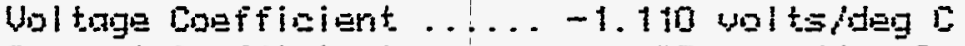
Current Doeficient ..... .02? ampeideg 0

ACTUAL CURVE IATA: 〈fill faCtor $=61.48$ ?

Short circuit current ... 19.0 amps Gpen eircuit vol tage .... 273.5 volts Max pomer current ....... 15.5 amps Max power woltage ....... 200.5 volts Max powar ............ 3239.4 inatts MIPNALIZED CURUE DATA: (fill fJEtor = 55.28 ) Cfor 25.0 deg $C$ and $1.0 \mathrm{kH} / \mathrm{m}^{\circ} \mathrm{z}$ ?

Mox power aurpent ....... 18.4 amps Max power vol tage ....... 23e.3 volts Has power . . . . . . . . 4402.4 watts

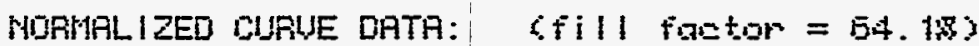
(for $40.0 \mathrm{deg}\left[\mathrm{C}\right.$ and $1.0 \mathrm{k} / \mathrm{h} / \mathrm{m}^{\circ} \mathrm{2}$ )

Max power gurrent. ...... 13.7 amps. Max pouser vol tage ....... 219.0 3olts Max power ...........4 4101.5 watts 

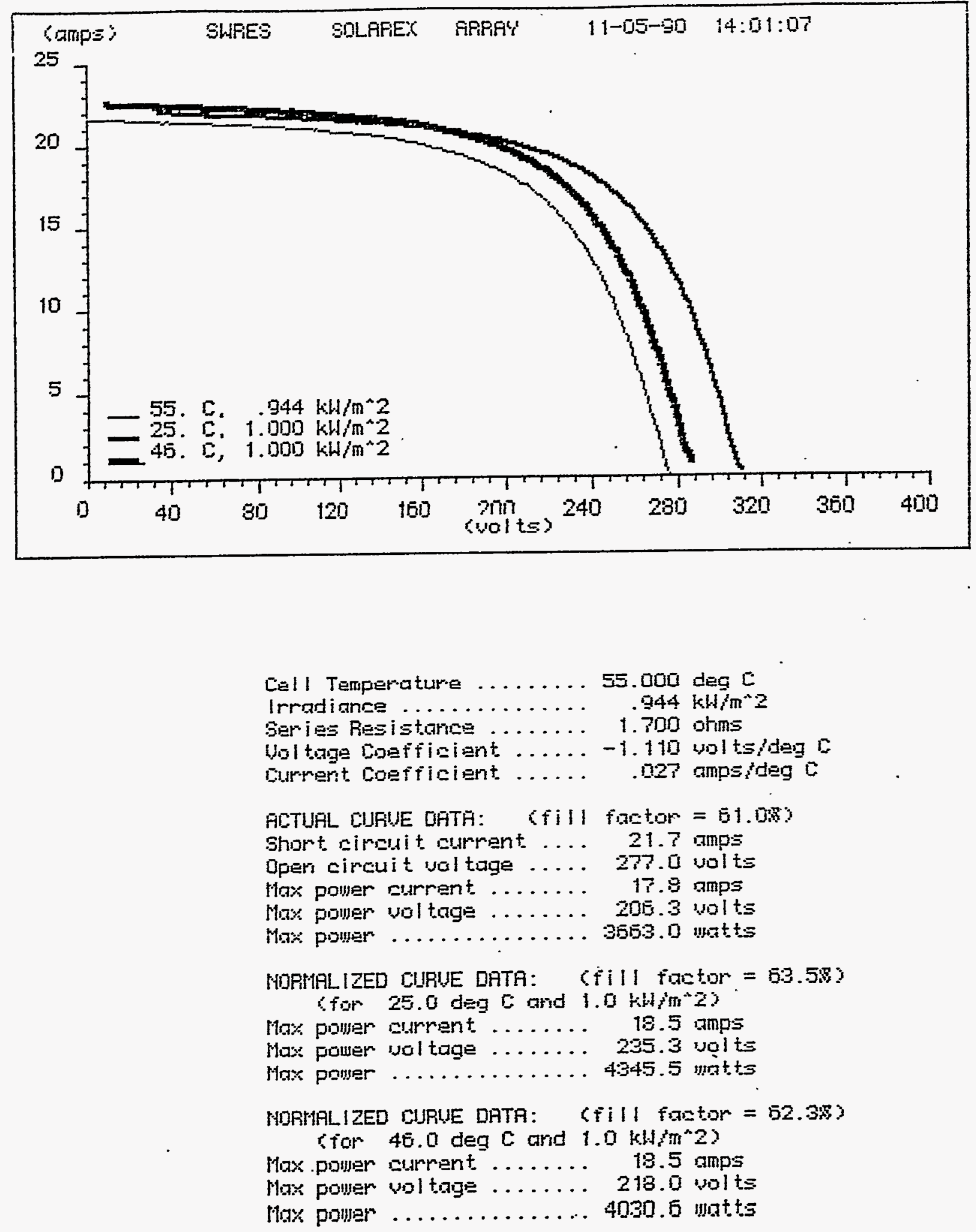


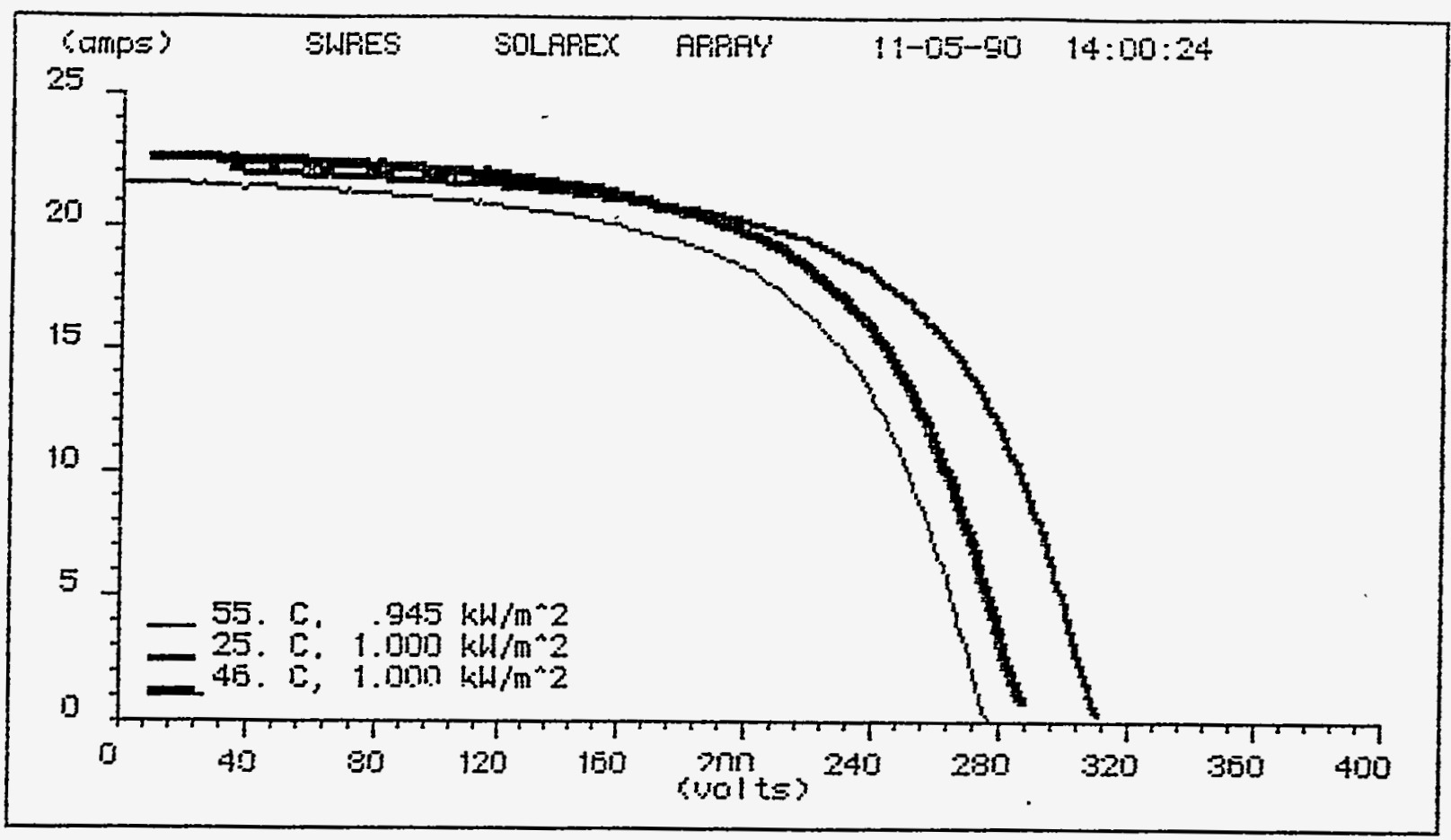

Cell Temperature ....... 55.000 deg $C$

Irradiance .......... .945 $\mathrm{kH} / \mathrm{m}^{\circ} 2$

Series Resistarice ....... 1.700 ohms

Uoltage cogeficiant ...... -1.110 woltsideg $c$

Cumment coefficiant ..... .027 nmpsideg $\mathrm{C}$

ACTUAL OURUE DHTA: \&IIII factor $=61.2 \mathrm{~m}$ )

Short cirouit current .... 21.7 amps

Open circuit vol tage .... 277.3 wolts

Max power curpent ....... 17.9 amps

Max power voltoge ...... 205.2 wolts

Mow pourer ............ 3575.5 unattos

HDFMALIZED CURUE OATA: (fill frator $=63.78$ )

\& for 25.0 deg o ond $1.0 \mathrm{kH} / \mathrm{m}^{\mathrm{n}} 2$ ?

Max pouser ourment....... 18.3 amps

Max power vol tage ...... 237.5 volts

Hax power ............ 4355.7 motts

HORMALIZED CIRUE DATA: CAIII faOtor $=62.4 \%$ ?

(for 40.0 deg $C$ and $1.0 \mathrm{kH}\left(\mathrm{m}^{2} \mathrm{Z}\right)$

Mox poujer current....... 18.9 amps

Max power vol tage ....... 213.5 vol ts

Mrox pouler .......... 4038.2 upatts 


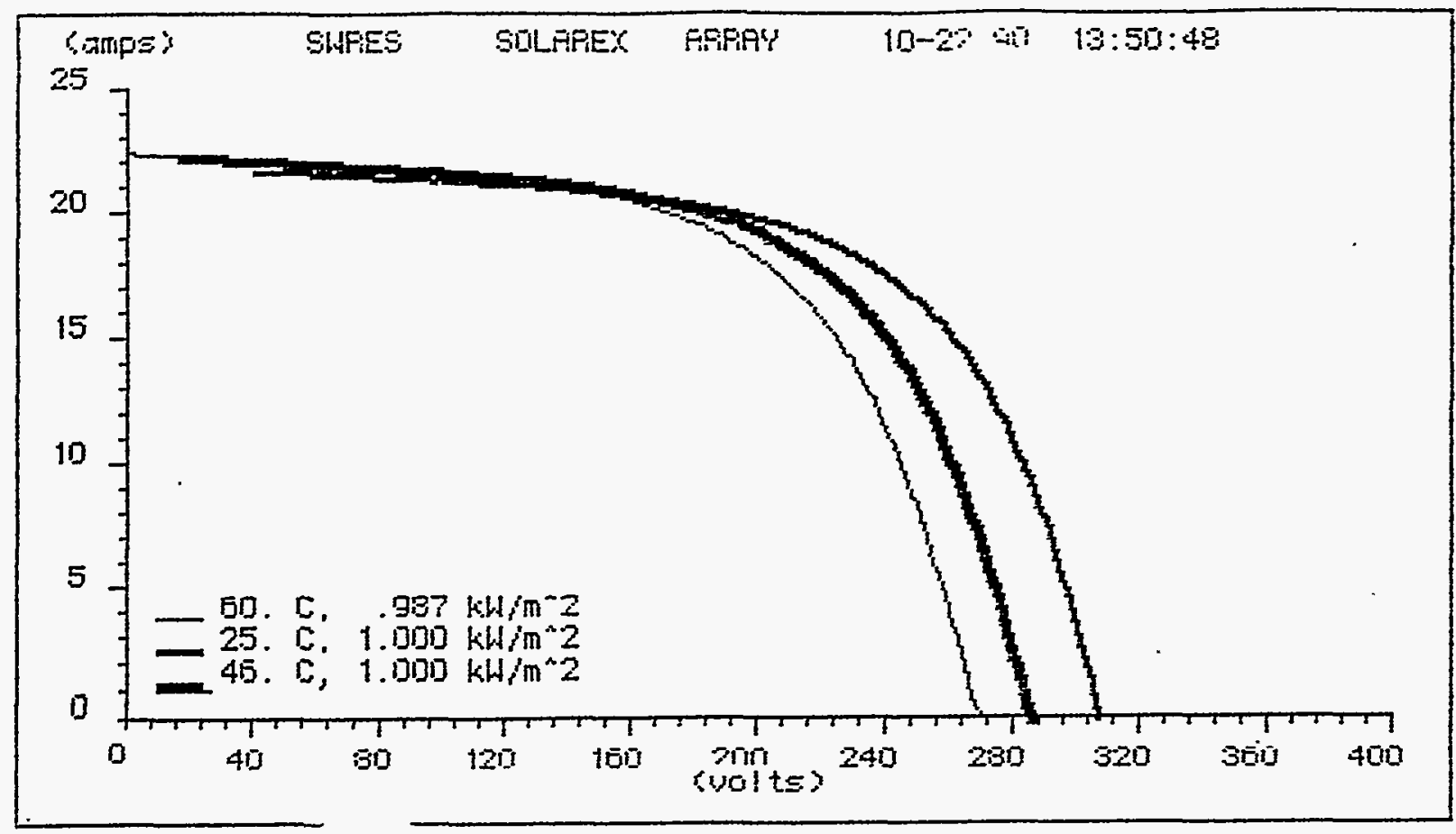

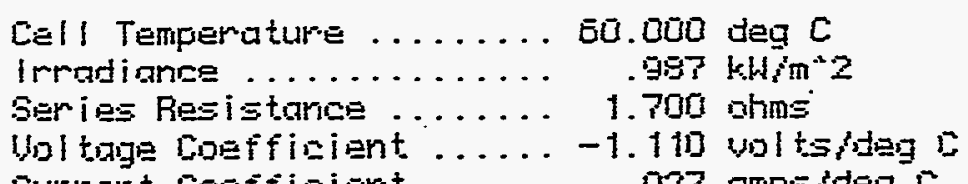

f Durment coefficient. ..... .027 ampsideg 5

FCTUAL CURUE DATA: \{fill fGotor $=60.3 \%$ ?

Shomt cimedit current .... 22.3 rmps Open eireuit vol tage .... 270.4 valts Mlax power rusrent. ...... 19.2 amps Mox pouer vol tage ....... 200.4 volts Hax pour ............. 3545.1 watts

HORMPLIZED CUPUE DATA: CFIll faOtor = 52.8\%

(for $25.0 \mathrm{deg}\left[\mathrm{o}\right.$ and $1.0 \mathrm{kH} / \mathrm{m}^{2} 2$ )

Max pourer aturrent ...... 18.1 amps

Max power wol tage ....... 233.3 volts

Max pomer ........... 4225.0 untts

HDRMALIZED DURUE DATA: CAIII TBotor $=.51 .4 B$ )

(for $46.0 \mathrm{deg} C$ and $1.0 \mathrm{kH}\left(\mathrm{m}^{\circ} 2\right)$

Max pouper aurrant ....... 18.1 amps

Max pouer woltage ....... 210.1 volts

Mox pourer ............ 3911.3 ungts 


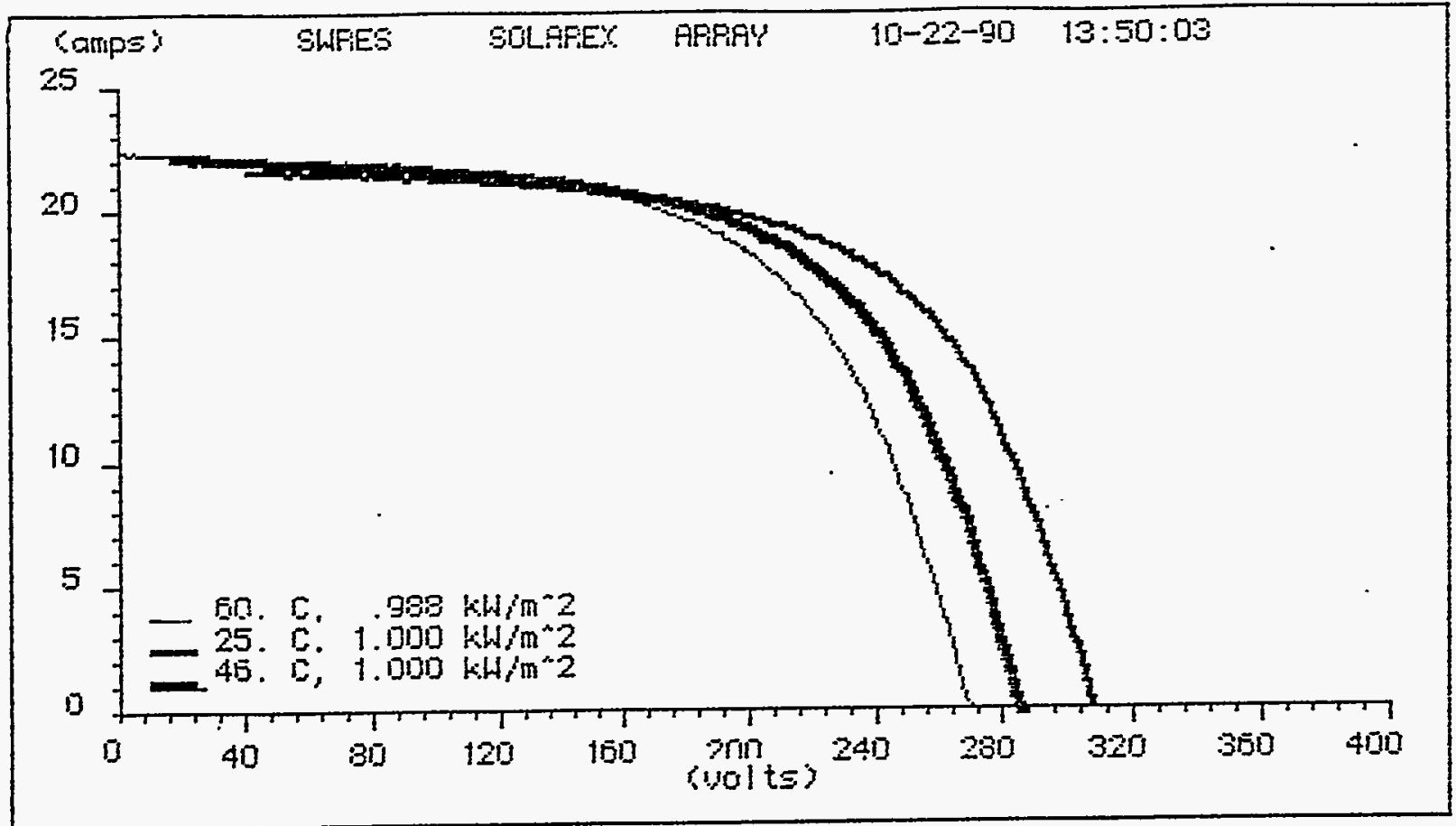

Cell Temperature ....... 60.000 deg $c$

Irradiance ............ .989 $\mathrm{kH} / \mathrm{m} \mathrm{m}^{* 2}$

Ser i Es Resistance ....... 1.700 ohms

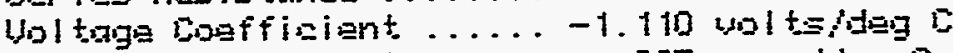

Durment coeficient ..... . OZP ampsideg $\mathrm{C}$.

ACTUHL CUAUE DATH: (fill fGEtor $=80.38\rangle$

Bhont aimeuit armant.... 22.4 amps

Open cireuit ual tage ..... 271.0 salts

Max poufer current....... 18.4 amps

Max pujer vol tage ....... 19g.6 wolts

How poumar ........... 3554.8 ugatts

MDEKRLIZED CURUE DATA: (fill factor $=62.8 \%$ )

Gor 25.0 deg $\mathrm{L}$ ard $1.0 \mathrm{kH} \mathrm{m}^{\wedge} 2$ ?

Max power alurrent. ...... 13.3 amp:

Max pouer wal tage ....... 231.3 volts

Max power ............ 4233.2 watts

MORMALIZED DURUE DATA: Cfill figfor $=51.483$

fior 40.0 deg $\mathrm{C}$ and $1.0 \mathrm{kH} / \mathrm{m}^{2} \mathrm{Z}$ )

Max pouser current ....... 18.3 amps

Max power vol tage ....... 214.4 volts

Max pouner .............. 3920.4 uatts 


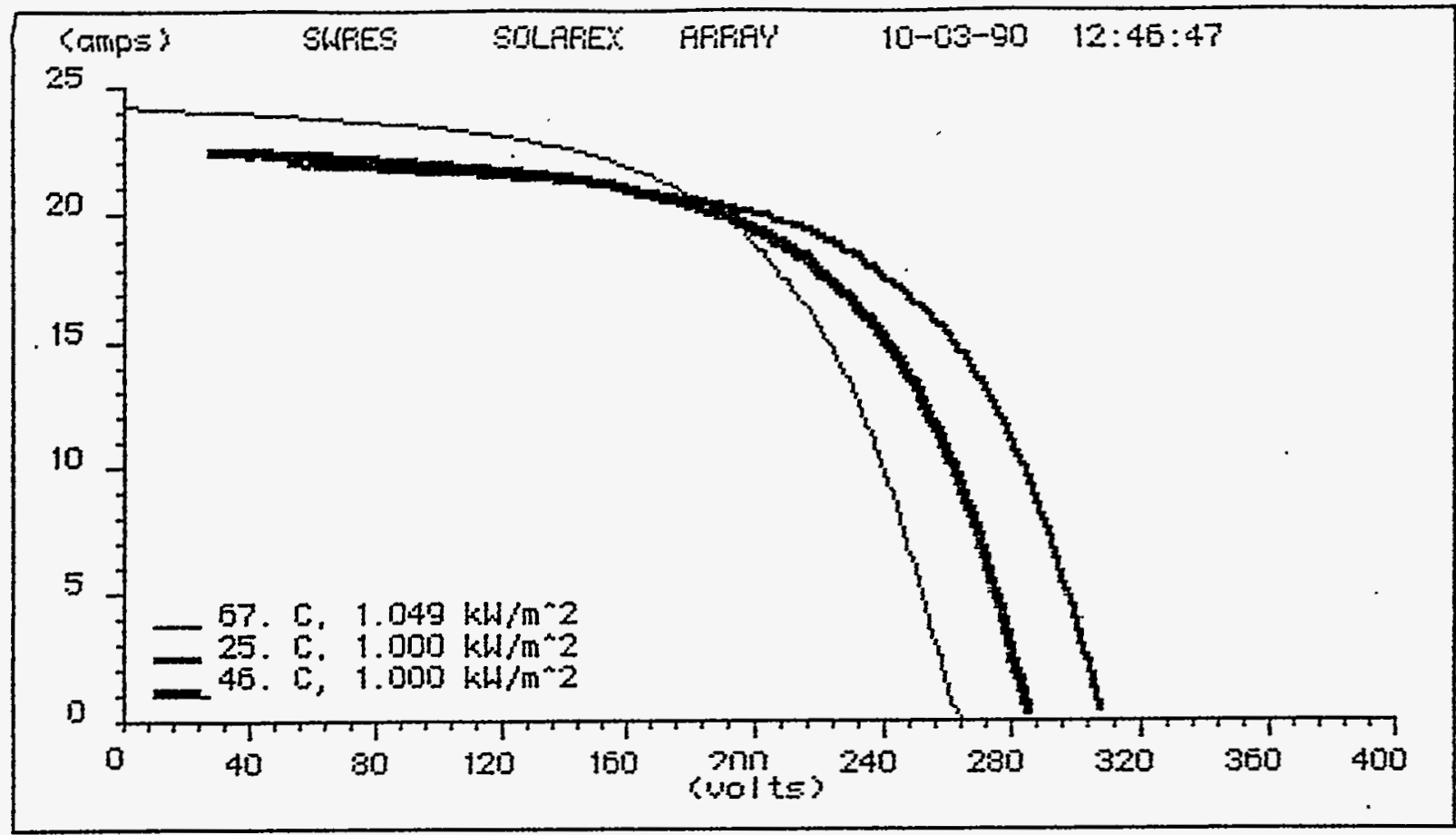

Cell Temperature ........E?.000 deg $\mathrm{C}$

Irradiance ........... $1.049 \mathrm{kH}^{\mathrm{m} \mathrm{m}^{-2} \mathrm{Z}}$

Series Resistarice ....... 1.700 ohms

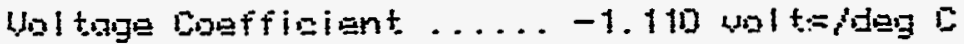

Curpent coefficient ..... . .027 ampsideg $\mathrm{C}$

ACTUAL CIURE DATA: GIll factor $=59.0 \mathrm{O}$ )

Short airauit adrrant. ... 24.2 smps

Uper eircuit val tage ..... 2E4:5 valts

Max poujer current. ...... 19.3 gmpE

Max pouer vol tage ....... 195.7 vol t.

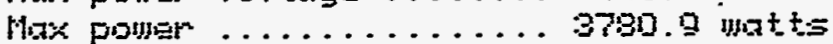

HDFHAL IZED CIJPUE DATA: (fill factor $=61.4 \%$ )

Sfor 25.0 deg 5 and $1.0 \mathrm{kH} / \mathrm{m}^{2} 2 \mathrm{~s}$

ligx poujer stmpent ....... 18.2 amps

Max puluer val tage ....... 234.4 val t=

Max power ...........4 4250.0 whtts

MORIAL IZED CIJUE DATA: (fill footor $=60.18$ )

(for $40.0 \mathrm{deg} E$ and $1.0 \mathrm{kH} / \mathrm{m}^{\circ} \mathrm{2}$ )

lax pouner current. . . . . . 18.4 amps

Max pouser vol toge ....... 213.7 volts

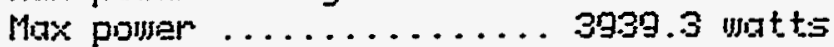




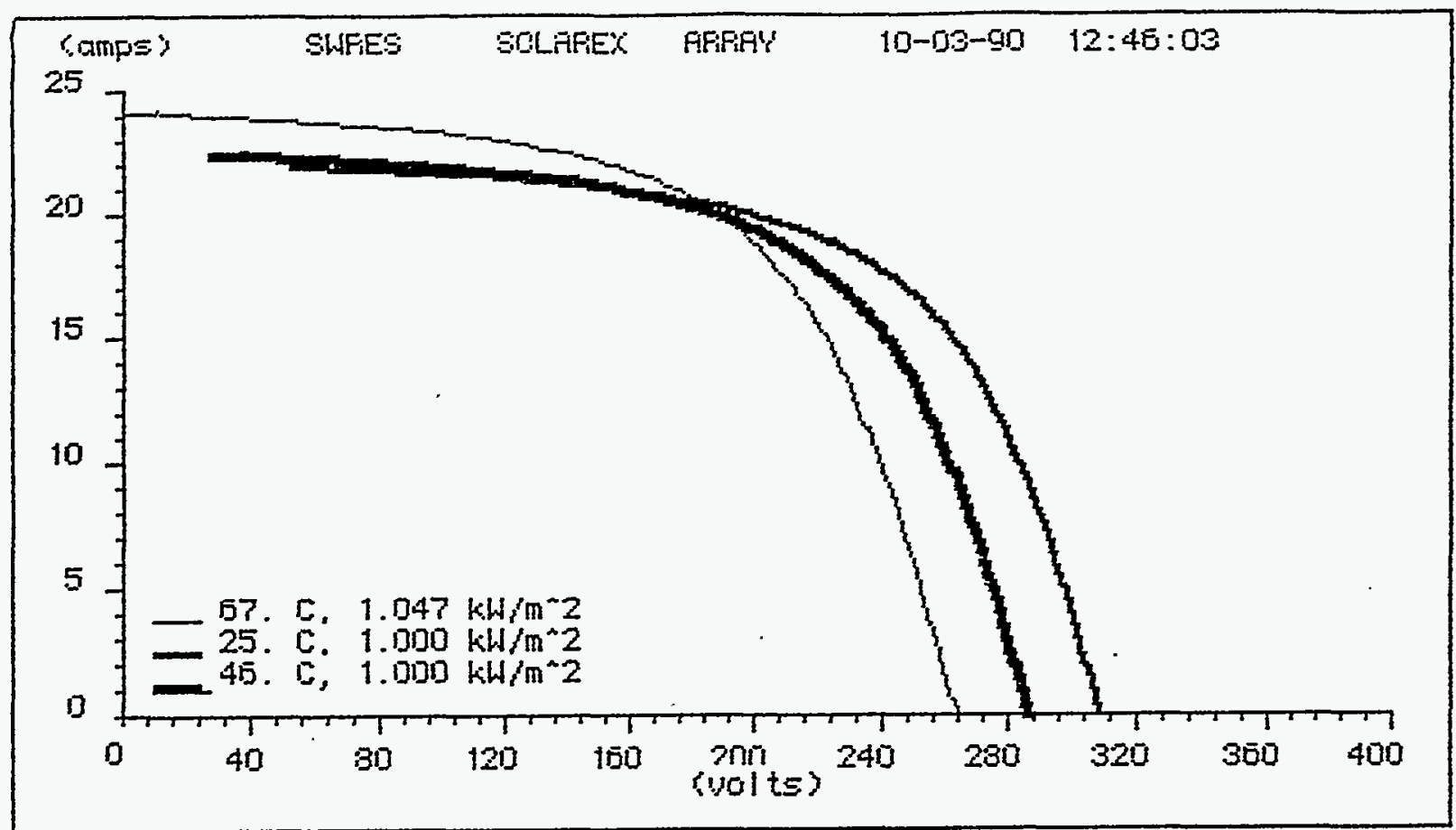

Cell Temperature ....... E7.000 deg $C$ Irradiance ............ $1.047 \mathrm{kH} / \mathrm{m}^{\circ} \mathrm{z}$ Series Resi $=$ tance ....... 1.700 ohms

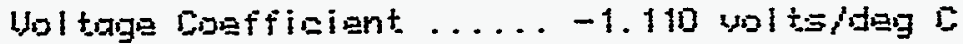
Current Coefficient ..... .027 ampsideg $\mathrm{C}$

ACTUAL CURUE DATA: \&IIll faetor $=58.78$ )

Short cirsuit current ... 24.3 umps Gpen circuit valtage ..... 265.0 volts Max pouper current ....... 19.3 amps Max pouer vol tage ....... 195.9 volts

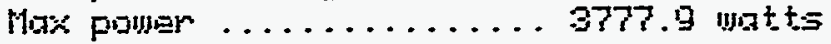

HORNALIZED CUPIJE DATA: CFill factor $=51.2 \%$ ? Sfor $25.0 \mathrm{~d}=9 \mathrm{C}$ and $1.0 \mathrm{kH} / \mathrm{m}^{*} \mathrm{z}$ ?

Thax pouser current $\therefore \ldots \ldots$. 18.2 amps Hax power vol tage..$\ldots \ldots .234 .3$ vol ts Max pouper . . . . . . . 4258.0 udt ts

MDPMALIZED CURUE DATA: (fill figstor $=59.7 \mathrm{k}$ ) (ior. 46.0 deg $C$ and $1.0 \mathrm{kh} / \mathrm{m}^{\wedge} \mathrm{2}$ )

Max pouper suprent ....... 18.8 amps

Max pomer voltage ....... 210.1 volts Max power ............ 3940.4 ugatts 

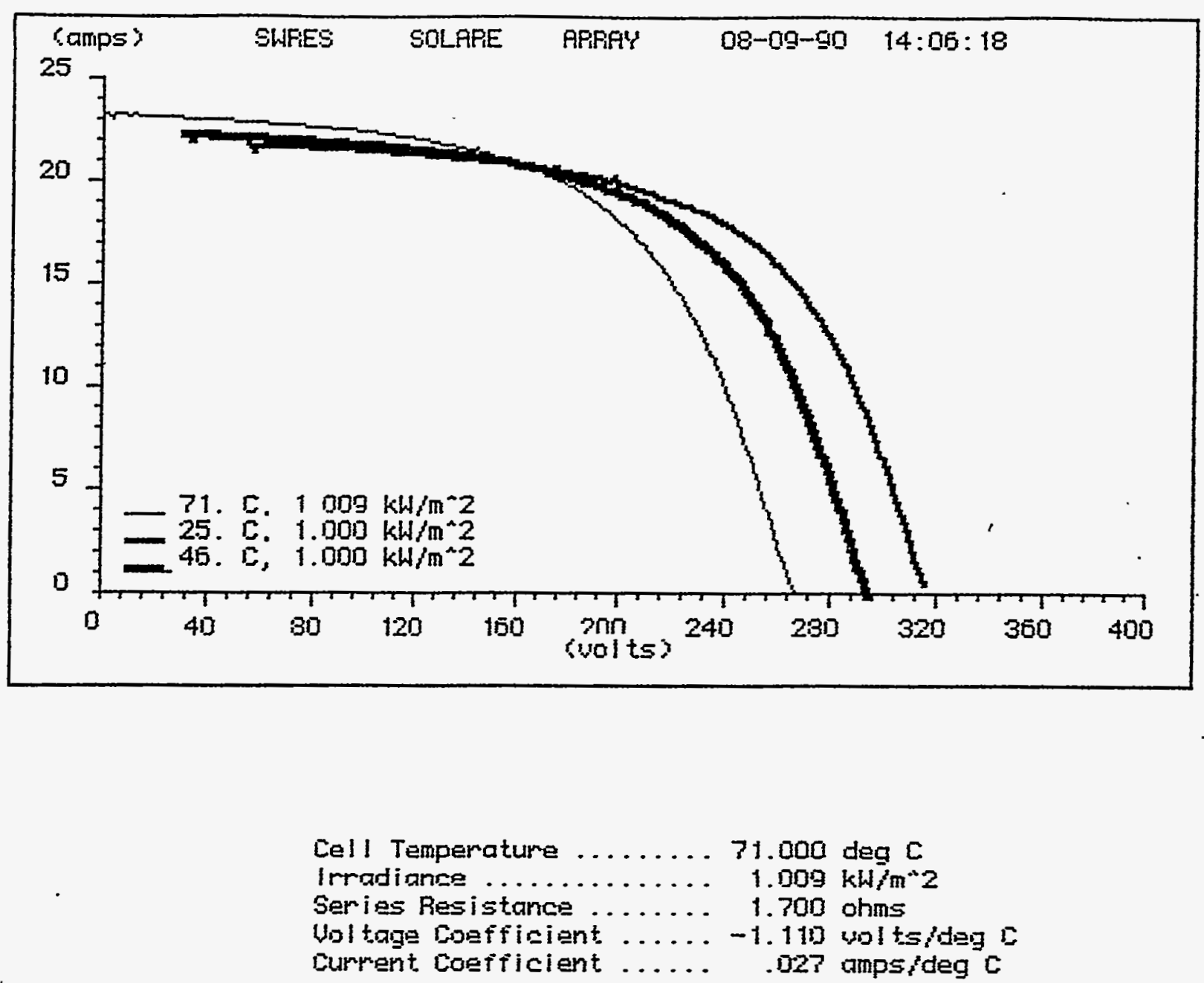

ACTUAL CURUE DATA: (fill factor $=58.18$ )

Short circuit current . ... 23.3 amps

Open circuit woltage ..... 268.5 volts

Max pouser surrent ....... 19.7 amps

Max pouser vol tage ....... 194.5 volts

Maxx pouser ........... 3529.9 matt=

MOPMAL IZED CURUE DATA: (fill factor $=61.2 \%)$

(for 25.0 deg $\left[\right.$ and $1.0 \mathrm{kH} / \mathrm{m}^{2} \mathrm{2}$ )

Max pouser gumpent ....... 13.1 amps

Max power vol tage ....... 237.5 volts

Max pouer ........... 430.5.5 matts

MORMALIZED CURUE DATA: (fill fugtor $=59.8 \mathrm{~s}\rangle$

(for $46.0 \mathrm{deg} C$ and $\left.1.0 \mathrm{k \omega} / \mathrm{m}^{\wedge} \mathrm{2}\right)$

Max poimer current....... 18.5 amps

Mar pomer voltage ...... $215 . ?$ volts

Max pourer ............ 3989.2 urgtts 


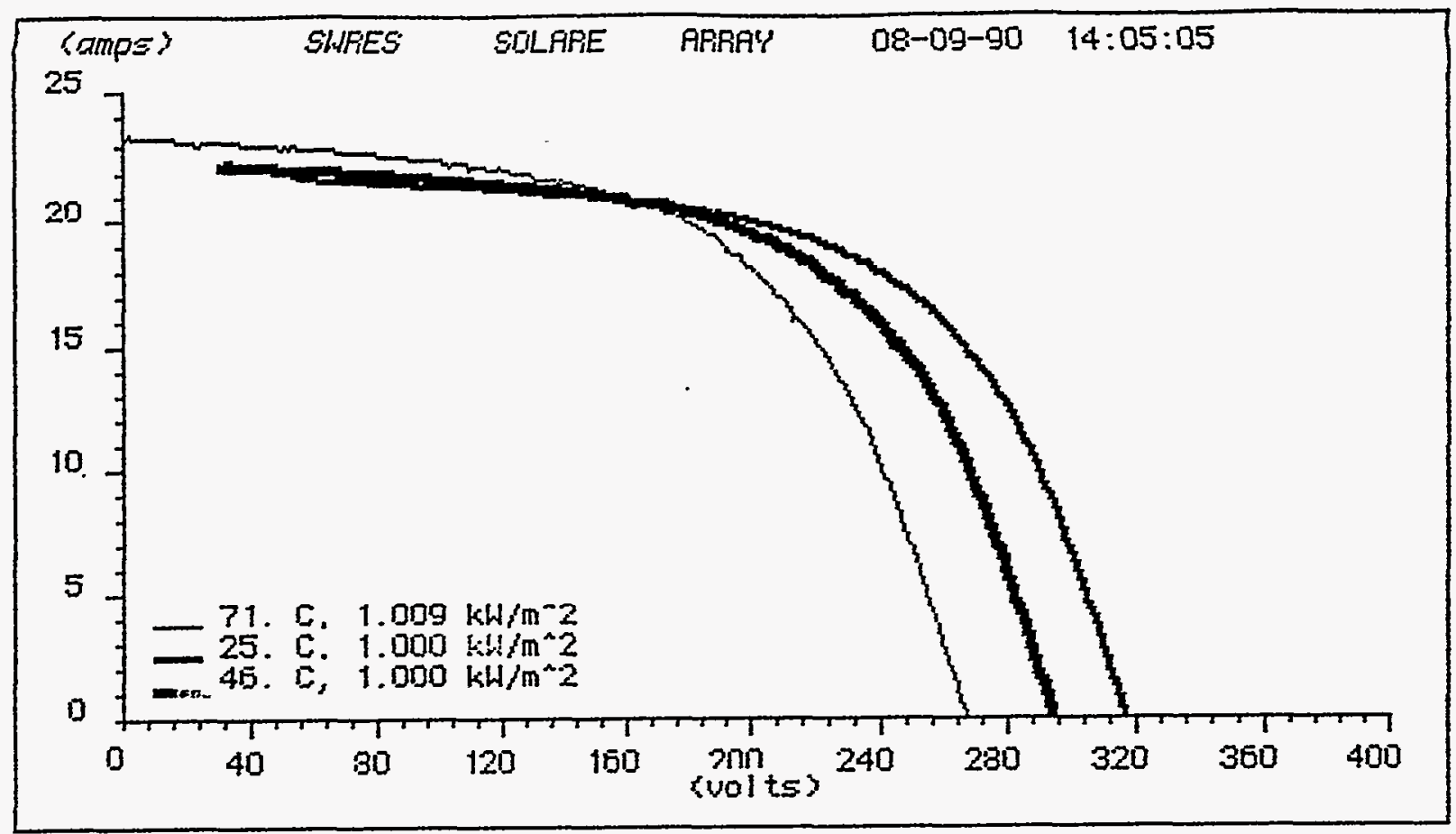

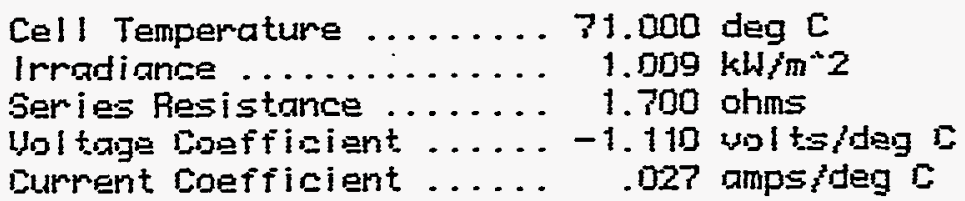

ACTUAL CURUE DATA: ( $f$ ill factor $=50.08$ )

Short cirsuit current .... 23.4 amps

upen circuit voltage .... 268.5 volts

Max power surrent. ....... 18.5 amps

Max pouner vol tage ....... 195.7 vol ts

Hax pamer ............. 3538.1 matts

NDRMALIZED CURUE DATA: (fill factor = 51. 13 )

(for $25.0 \mathrm{deg} C$ and $1.0 \mathrm{kH} / \mathrm{m}^{2} \mathrm{z}$ )

Max pouser cusrent. ...... 18.2 amps

Max power vol tage ....... 236.5 valts

Max pouser ........... 4311.0 untts

NORMRLIZED CURUE DATA: (fill fastor $=59.8 \mathrm{~s}$ )

(for 40.0 deg $C$ and $1.0 \mathrm{kH} / \pi^{\wedge} Z$ ?

Max power current....... 18.4 amps

Max pomer vol tage ........ 217.9 volts

Max power ............ 3999.3 watts 


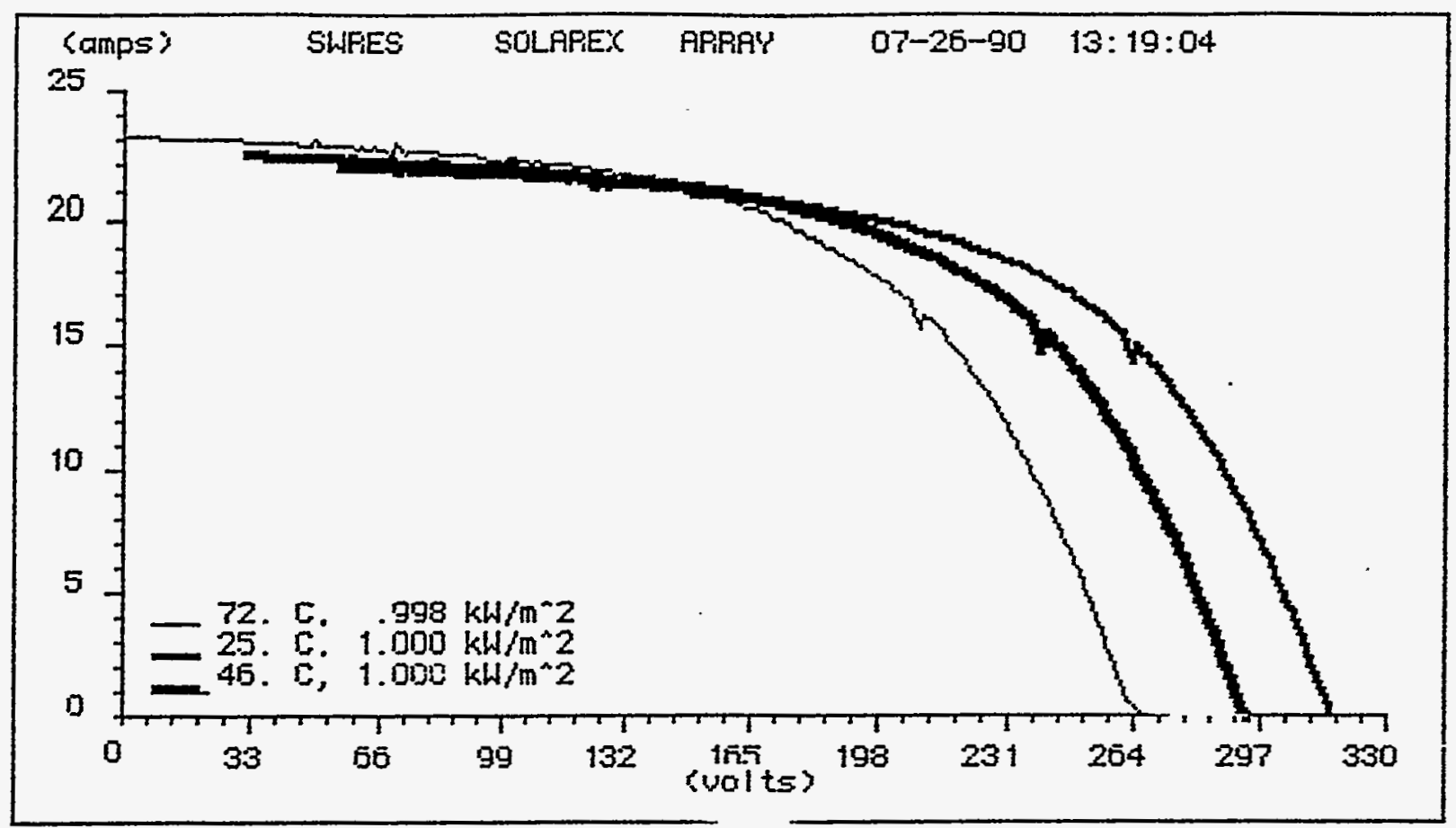

Dell Temperature ....... 72.000 deg $\mathrm{L}$

Irradiance ............ . $998 \mathrm{kH} / \mathrm{m}^{2} \mathrm{z}$

Sarias Resistanca ....... 1.700 ohms

Voltage Coefficient ......-1. 110 voltsfieg $c$

Durrent Doefficient. ..... . .027 amps/deg $C$

RETURL DIJRUE DRTR: (fill factor $=57.58$ )

Shomt cimcuit current .... 23.1 amps

Dpen cirauit ultage .... 250.0 volts

Max power curment ....... 18.4 amps

Mox pouser usl tiage ...... 192.2 wolts

Max power ............ 3540. 1 matts

MORMALIZED CURIE DATA: (fill faEton = $61.0 \%$

(for $25.0 \mathrm{deg} \mathrm{C}$ and $1.0 \mathrm{kH} / \mathrm{m}^{\wedge} \mathrm{z}$ )

Max power current ....... 13.1 amps

Max pouner unltage ....... 235.9 volts

Max power .......... 4280.2 matts

NORMALIZED CURUE DATA: (fill factor $=59.5 \%$

(for $46.0 \mathrm{deg} C$ and $1.0 \mathrm{kH} / \mathrm{m}^{-2} \mathrm{~s}$ )

Max power curment ....... 18.5 mmps

Max pourer vol tage ....... 214.0 volts

Max power........... 3961.0 watts 


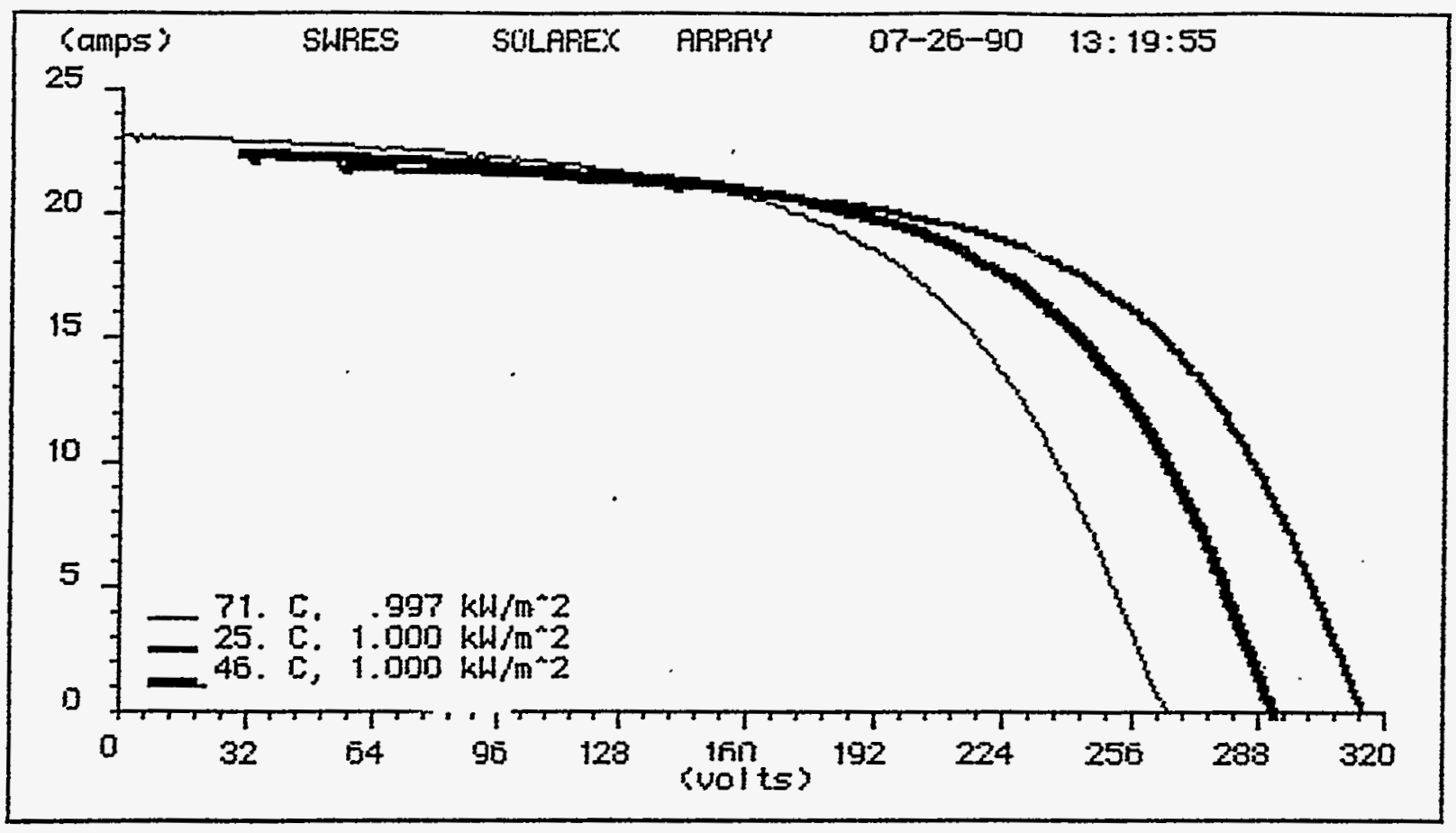

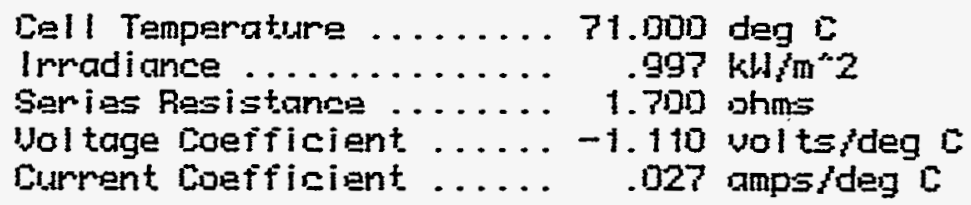

RCTURL LURUE DATR: (fill factor $=57.7 \%$ )

Shomt circuit current .... 23.1 amps

Dpen circuit vol tage .... 25.9.9 volts

Max power curment ...... 18.4 amps

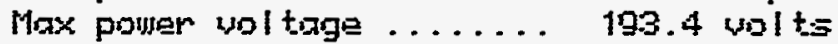

Max power .............3548.2 watts

NORMALIZED CURUE DATA: (fill factor = 01.13 )

(for 25.0 deg $C$ and $1.0 \mathrm{kH} / \mathrm{m}^{-2}$ )

Max power current ....... 18. 1 anps

Max pouer voltage ....... 235.9 unlts

Max power ........... 4274.9 watts

MORMALIZED CURUE DATA: (fill factor $=59.6 \% 3$

(for 46.0 deg $C$ and $1.0 \mathrm{kH} / \mathrm{m}^{-2}$ )

Max power current ....... 18.5 amps

Max poner voltage ....... 213.9 wolts

Max power ............. 3956.3 watts 


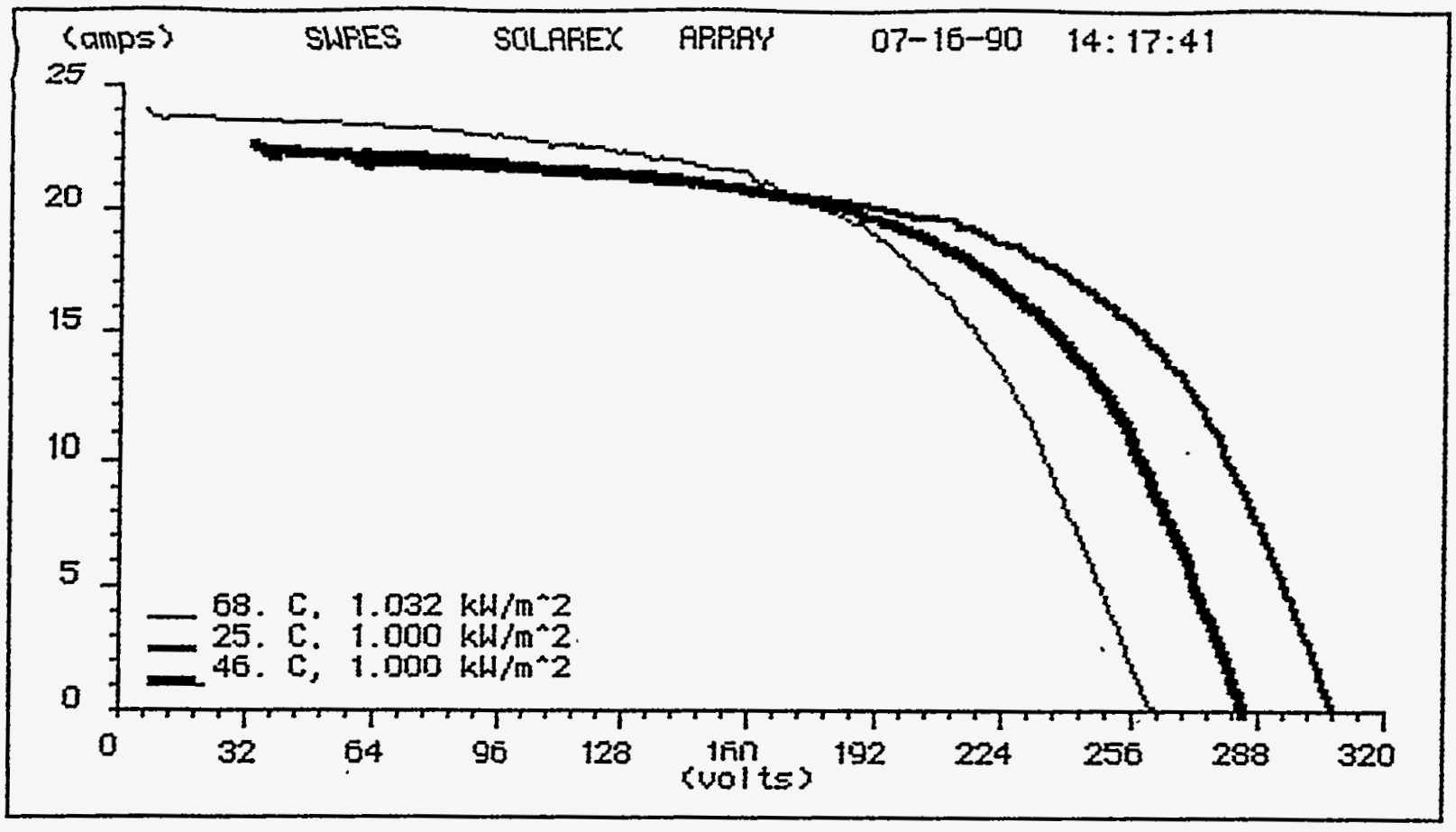

Cell Temperature ....... 68.000 deg $\mathrm{C}$

Irpadiance ........... $1.032 \mathrm{~kW} / \mathrm{m}^{*} 2$

Series Resistanas ....... 1.70n ohms

Vol tage Coefficient ......-1.110 vol ts ideg $c$

Cumpent Coeficicient ..... . .027 amps/deg $C$

ACTURL CURUE DATR: (fIII factor $=57.68$ )

Shout circuit current .... 24.0 amps

Dpen sircuit voltage .... 252.1 uolts

Max power current ....... 19.3 amps

Moxx pouer usl tage ....... 197.8 wolts

Max power ............ 3628.1 watts

MORKALLIZED CURUE DATA: \{fill factor $=60.6 \%$

(for 25.0 deg $C$ and $1.0 \mathrm{kH} / \mathrm{m}^{-2}$ )

Max power current ....... 18.5 amps

Max pouner voltage ...... 227.4 volts

Max power ............ 4204.8. watts

NORMALIZED CURUE DATA: (fill factor $=58.98$ )

(for 46.0 deg $C$ and $1.0 \mathrm{kH} / \mathrm{m}^{\circ} 2$ )

Max power curment ....... 19.0 amps

Max pouer uol tage ....... 203.2 volts

Max power ............ 3868.2 watts 

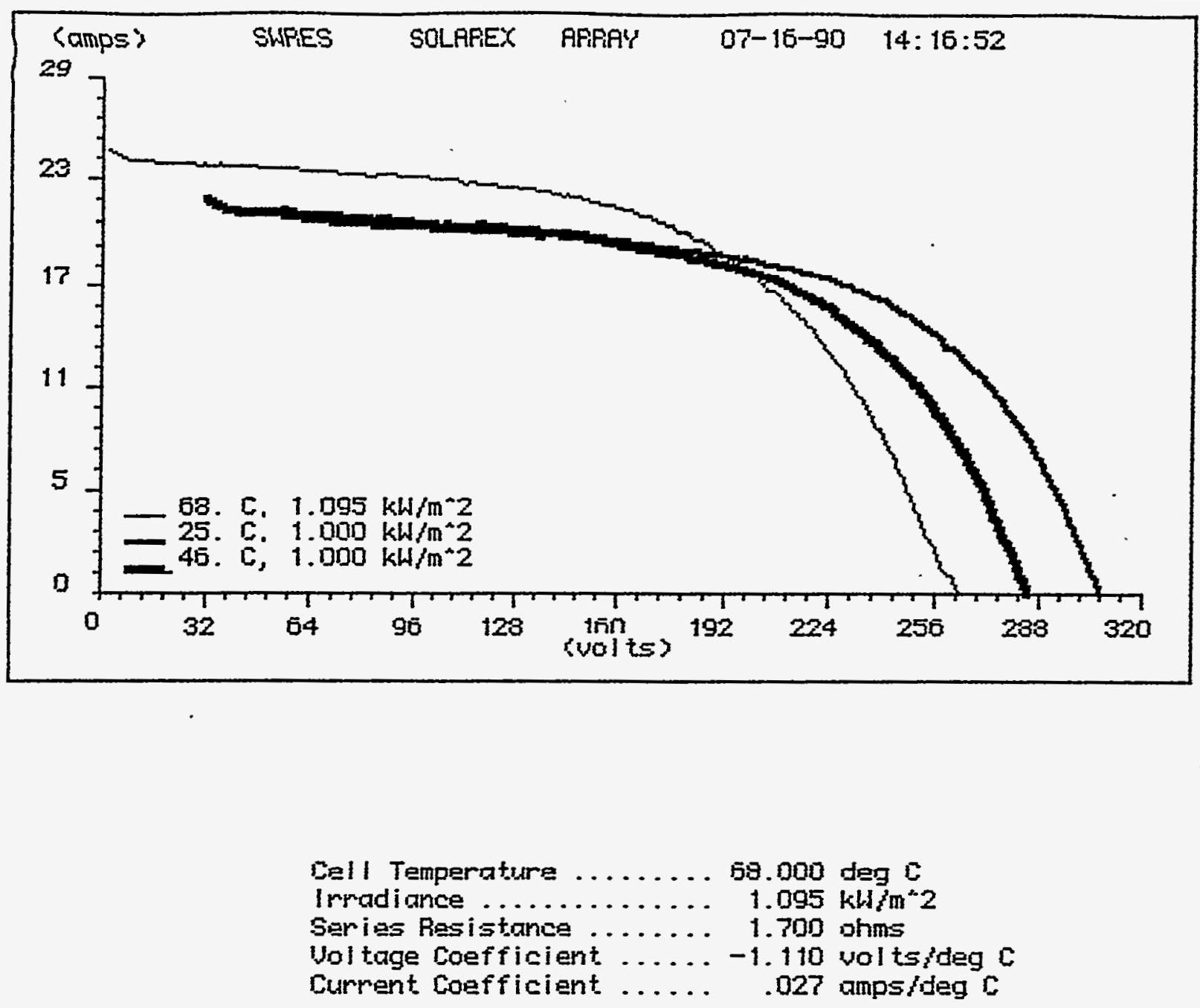

RCTURL CIJUE DATF: (fill factor $=56.58$ j

Short circuit current .... 25. 7 amps

Dpen sircuit voltrage .... 253.1 volts

Max power cumpent ....... 20.5 amps

Max pouser voltage ....... 135.3 wolts

Max power ............ 3815.0 unatts

MORMALIZED CURUE DATA: \{fill factor $=58.3 \%$

(for $25.0 \mathrm{deg} C$ and $1.0 \mathrm{~kW} / \mathrm{m}^{-2} \mathrm{z}$ )

Max power current ....... 13.1 amps

Max power uol tage ....... 230.3 volts

Max power ........... 4161.6 watts

NORMALIZED CURUE DATA: (fill factor $=57.37$ )

(for 46.0 deg $C$ and $1.0 \mathrm{kH} / \mathrm{m}^{\circ} 2$ )

Mas power current....... 18.4 amps

Max pourer vol tage ....... 208.9 volts

Max power ............. 3332.8 watts 


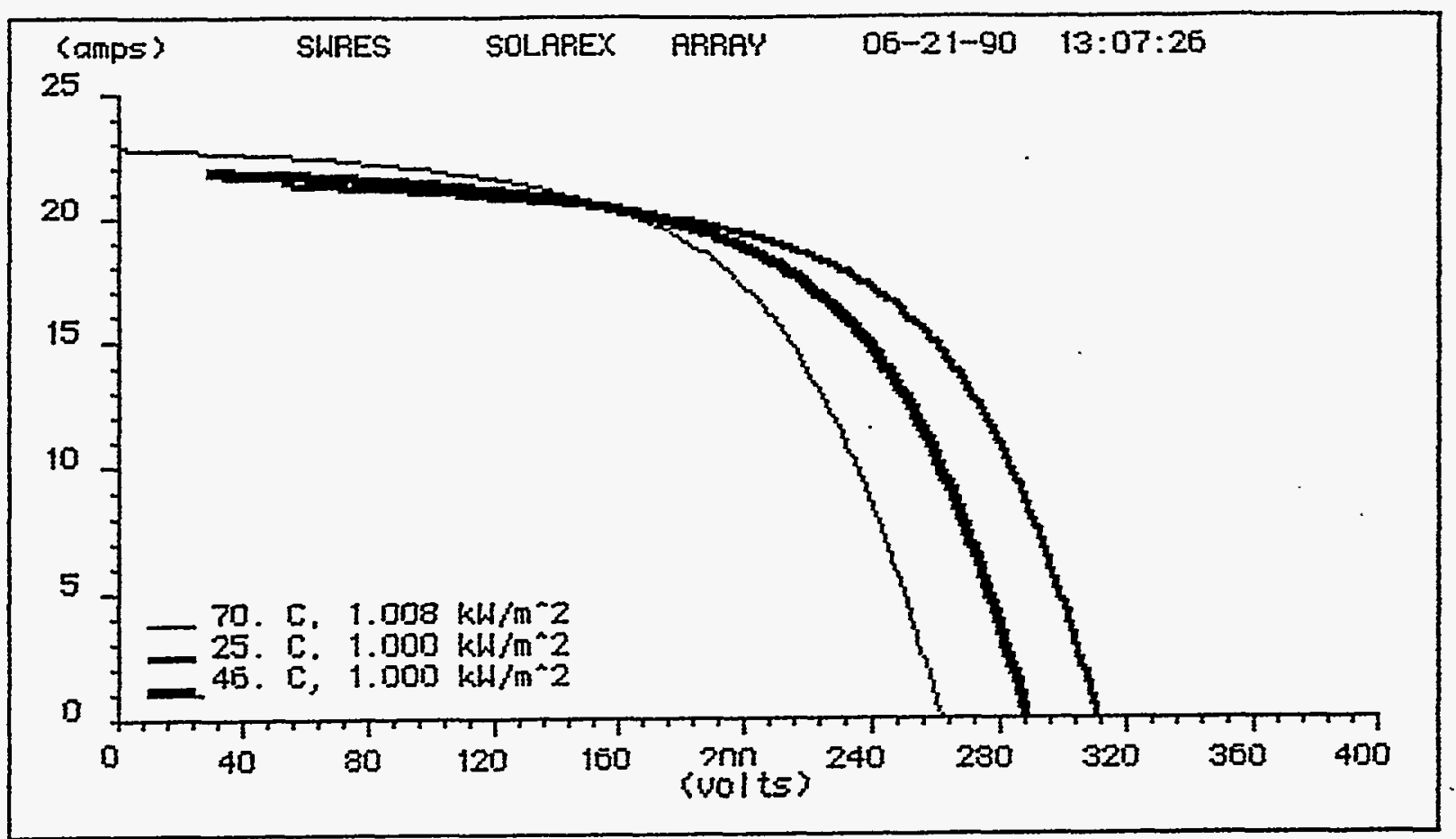

Cell Temperature ....... 70.000 deg $c$

Irradiance ............. $1.008 \mathrm{kH} / \mathrm{m}^{2} 2$

Series Resistance ....... 1.700 ohms

Ualtage colefficient ..... -1.110 wolts/dag $c$

Current Coefficient ..... .027 ampsideg $\mathrm{C}$

ACTUAL CURUE DATA: (fill factor $=53.0 \%$ )

Short circuit curpent. ... 22.9 amps

Open circuit val tage .... 262.9 volts

Max power gurrent. ....... 19.5 amps

Max power vol tage ....... 187.5 volts

Max poiner ........... $3481.9 \mathrm{motts}$

MORMALIZEO CIURUE DATA: ( $\mathrm{fill}$ factor $=51.08$ )

(for 25.0 deg $C$ and $1.0 \mathrm{kH} / \mathrm{m}^{\wedge} 2$ )

Max power current ...... 17.3 amps

Max power vol tage ........ 231.5 volts

Max pouser ........... 4128.0 unatts

NORMALIZED CJURE DATA: ( $\mathrm{fill}$ factor $=59.78$ )

(for $46.0 \mathrm{deg} C$ and $1.0 \mathrm{~kW}^{-2} \mathrm{~m}^{-2}$ )

Max power current ....... 17.7 amps

Max power voltage ....... 215.5 volts

Max powjer ...........3824.9 watts 

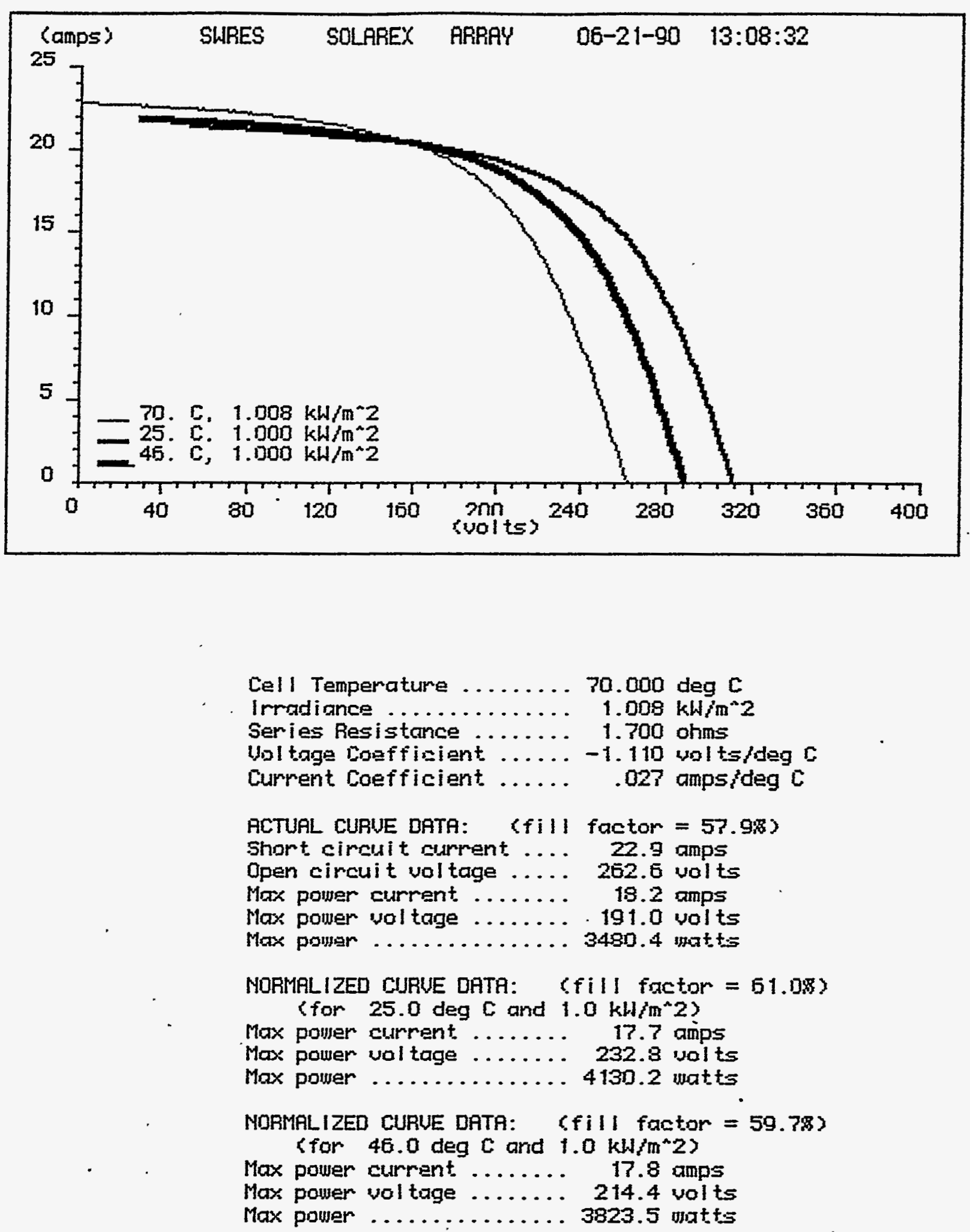


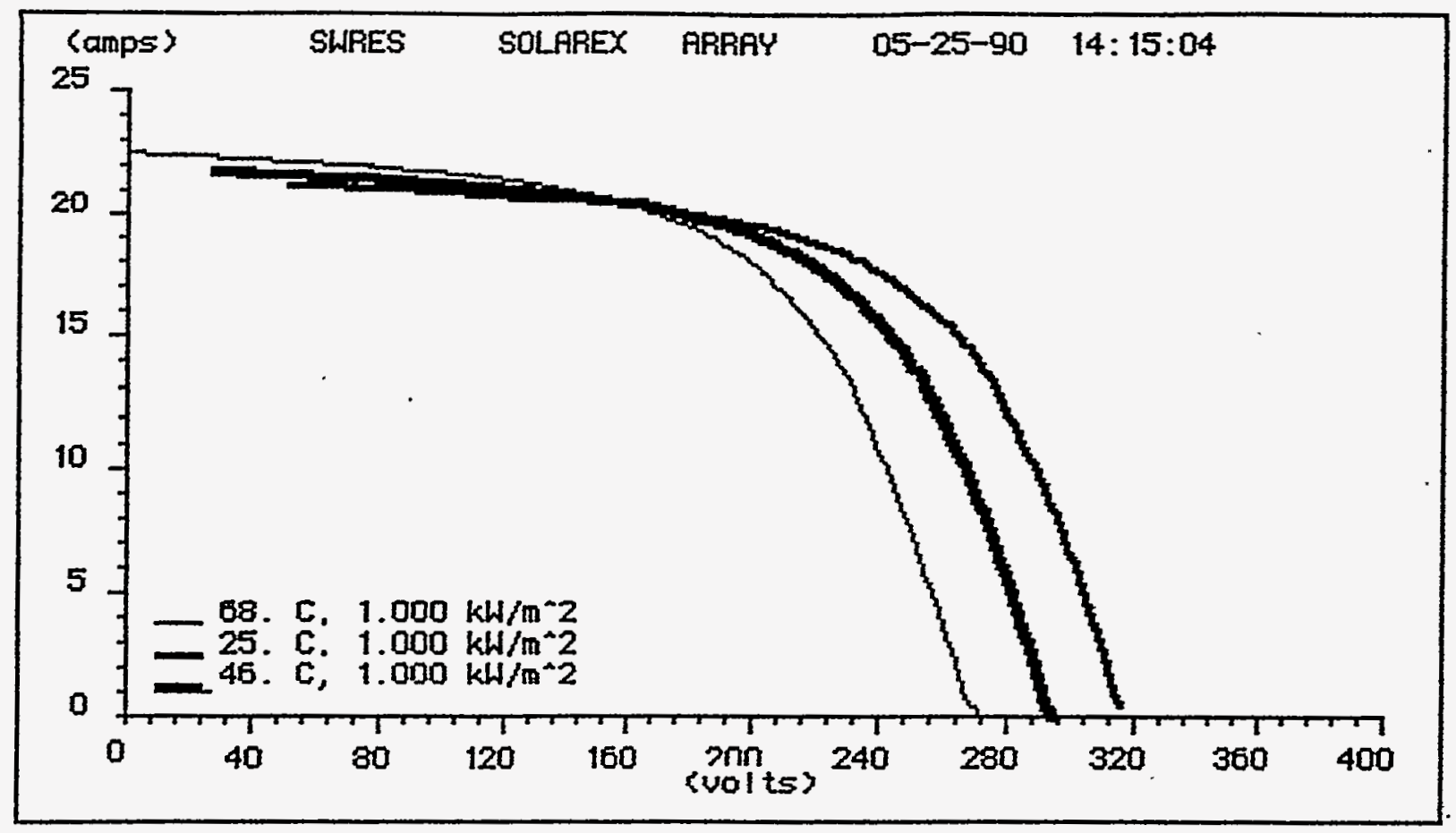

Cell. Temperature ......68.000 deg $c$

Irradiance ........... $1.000 \mathrm{~kW} / \mathrm{m}^{*} 2$

Series Resistance ....... 1.700 ohms

Uoltage coefficient...... -1.110 wolts/deg $c$

Current Coefficient ..... .027 amps/deg $c$

ACTUAL CURUE DATA: (fill factor $=58.78$ )

Short circuit curment .... 22.5 amps

Open circuit val tage .... 271.7 volts

Max power current....... 18.1 amps

Max power vol tage ....... 198.2 volts

Max power ............ 3585.3 matts

NORMALIZED CURUE DATA: ( $\mathrm{fi}$ I factor $=61.78$ )

(for $25.0 \mathrm{deg} C$ and $1.0 \mathrm{~kW} / \mathrm{m}^{\circ} 2$ )

Max power current ....... 17.8 amps

Max power valtage ....... 237.4 volts

Max power ............ 4227.0 watts

NORMALIZED CURUE OATA: (fill factor $=60.3 \%$ )

(for $45.0 \mathrm{deg} C$ and $1.0 \mathrm{~kW} / \mathrm{m}^{-2}$ )

Max power current ....... 17.8 amps

Max power vol tage ....... 220.0 volts

Max power ............ 3921.0 matts 

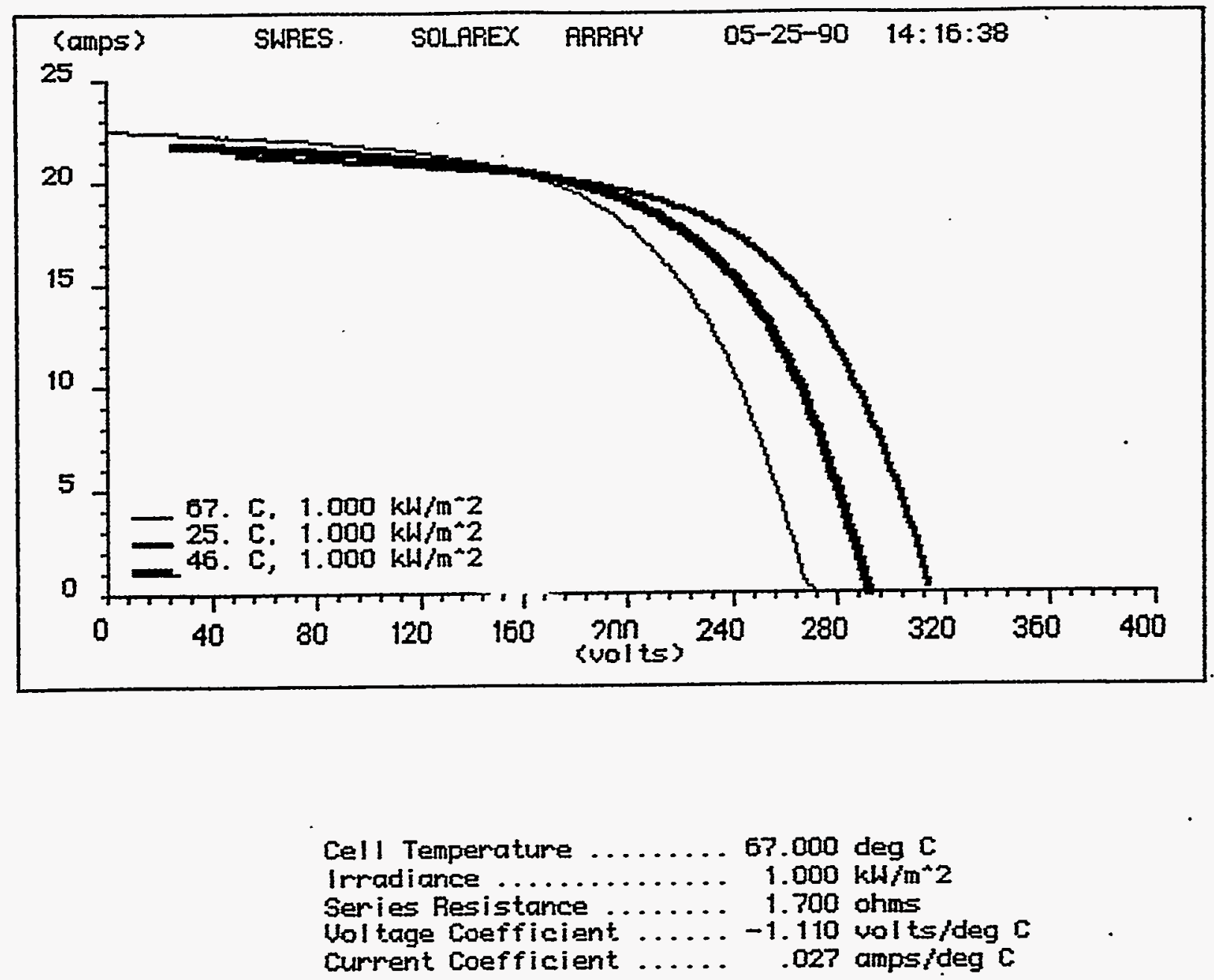

ACTURL CURUE DATA: (fill factor $=58.68$ )

Short circuit current .... 22.5 ramps Open circuit vol tage ..... 271.4 valts Max power current ....... 18.4 amps Max pourer vol tage ....... 194.5 volts Max pomer ............. 3575.5 matts

NORMALIZED CURUE DATA: ( $\mathrm{fi} / 1$ factor $=61.5 \%$ ) (for 25.0 deg $C$ and $1.0 \mathrm{kH} / \mathrm{m}^{\wedge} \mathrm{2}$ ) max poiver current ....... 17.9 amps Max power val tage ....... 235.0 volts Max power ........... 4201.2 watts

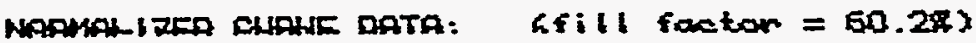

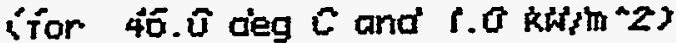
Max power current ...... 17.8 amps Max power voltage ...... 218.8 volts Max power ............ 3897.0 matts 


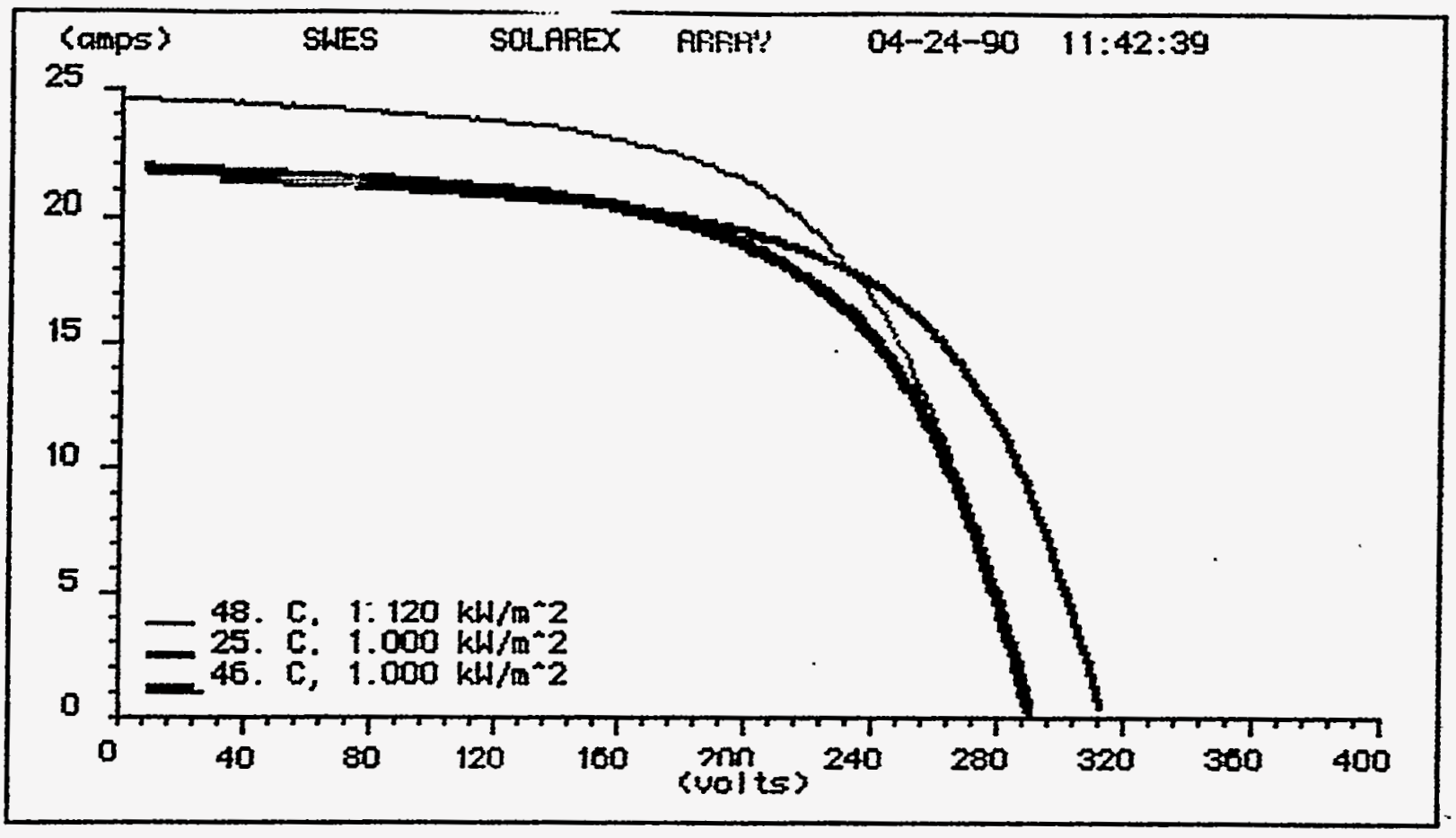

Cell Temperature .......448.000 deg C

Irradiance ............ $1.120 \mathrm{kH} / \mathrm{ma}^{\wedge} 2$

Series Resistance ....... 1.700 ohms

Voltage Coefficient ..... -1.110 volts/deg c

Current Coefficient ..... .02? amps/deg $C$

ACTUAL CURUE DATA: (fill factor $=60.4 \%$ )

Short circuit current .... 24.6 anps

Opan circuit voltage ..... 291.1 valts

Max power current. ...... 20.1 amps

Max power valtage ....... 215.6 valts

Max power ........... 4332.0 watts

NORMFLIZED CURUE DRTA: (fill factor $=60.9 \%$ )

(for 25.0 deg $C$ and $1.0 \mathrm{~kW} / \mathrm{m}{ }^{\wedge} 2$ )

Max power current ....... 17.9 anps

Max pourer val tage ....... 234.9 valts

Max power ............ 4203.4 watts

MORMALIZED CURUE OATA: (fill factor $=59.4$ )

(for $46.0 \mathrm{deg} C$ and $1.0 \mathrm{kH} / \mathrm{m}^{\wedge} 2$ )

Max power current ....... 17.8 amps

Max power vol tage ........ 217.? volts

Max pomer ............ 3884.9 watts 


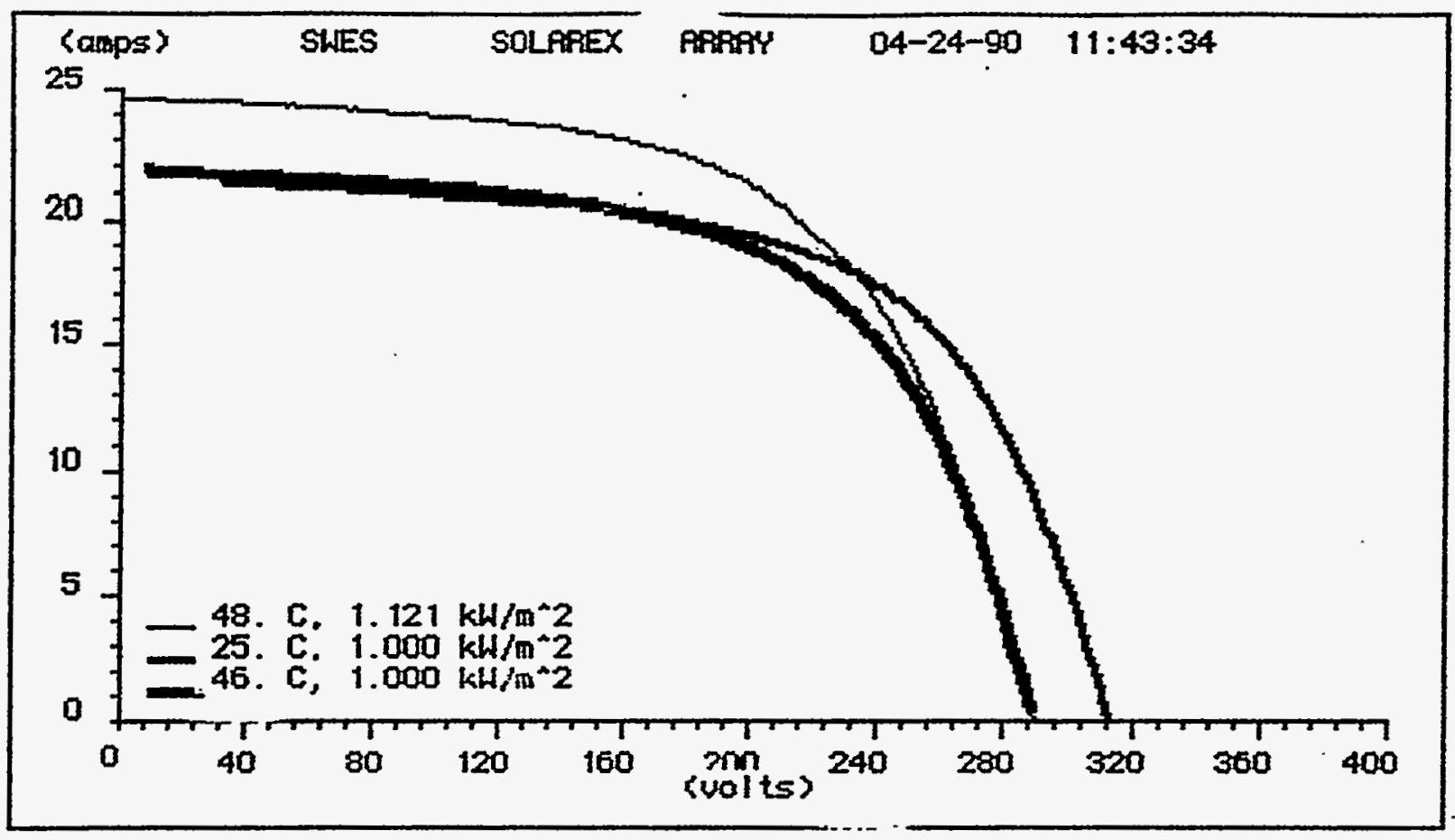

\footnotetext{
Cell Temperature .......48.000 deg $\mathrm{C}$

Irradiance ............ $1.121 \mathrm{kH} / \mathrm{b}^{\wedge} 2$

Series Resistance ....... 1.700 ohmre.

Voltage Coefficient ......-1.110 volts/den c

Cumpent Coefficient ..... .027 amps/deg C

ACTUFL CURUE DATA: (fill factor $=60.35$ )

Short circuit current .... 24.7 amps

Open cimcuit valtage ..... 290.6 volts

Max power current....... 20.0 amps

Max power vol tage ....... 215.6 volts

Max power ...........4323.4 watts

NORMALIZED CURUE DATA: (fill factor $=60.7 \%$ )

(for 25.0 deg $C$ ard $1.0 \mathrm{kH} / \mathrm{h}^{\wedge} 2$ )

Max power current ....... 17.6 anps

Max power val tage ....... 237.2 valts

Max power ............44186.0 watts

NORMALIZED CURUE DATA: (fill factor $=59.38$ )

(for $46.0 \mathrm{deg} C$ and $1.0 \mathrm{kH} / \mathrm{ha}^{\wedge} 2$ )

Max power current ....... 17.8 amps

Max power voltage ....... 217.8 volts

Max power ............ 3871.9 watts
} 

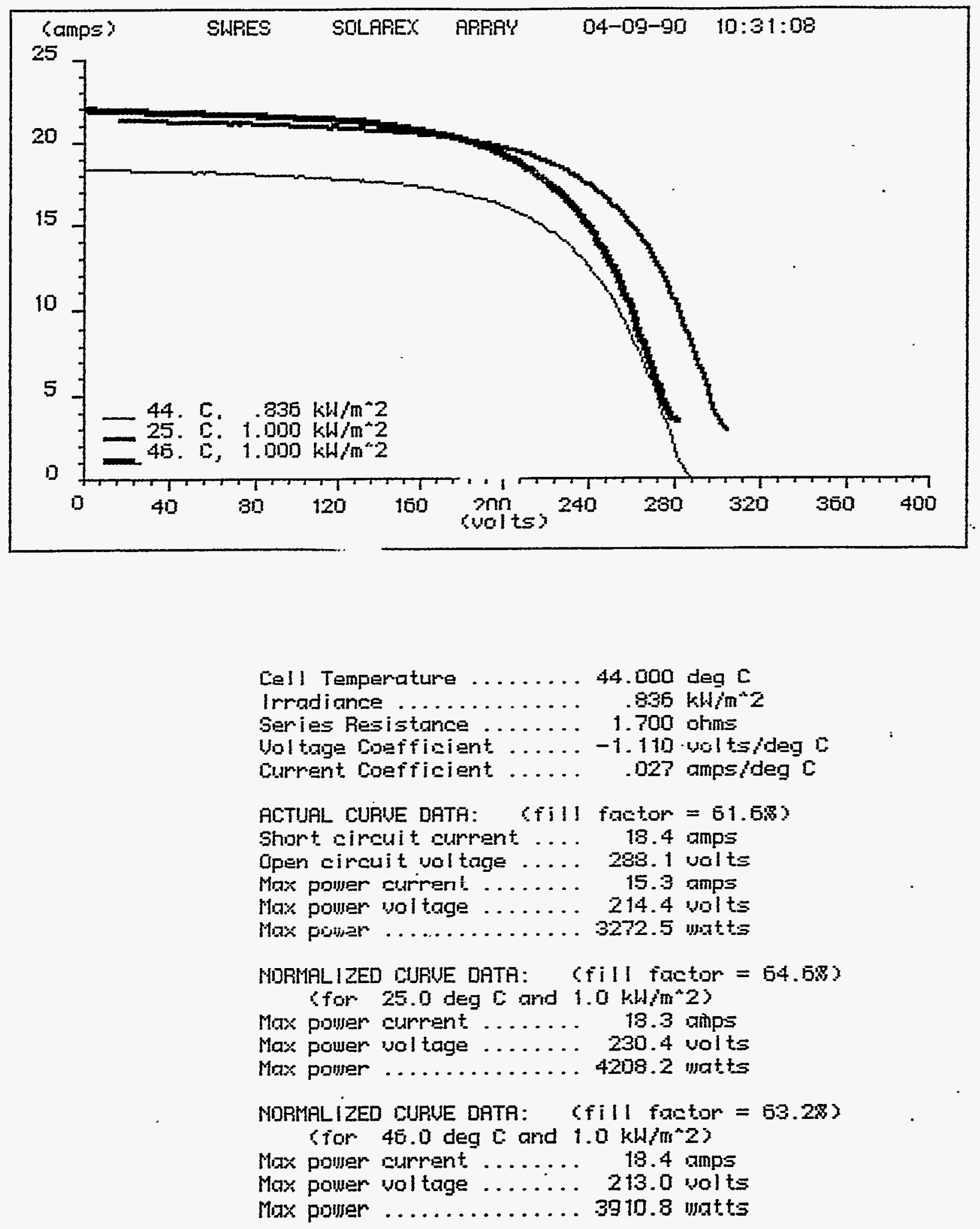


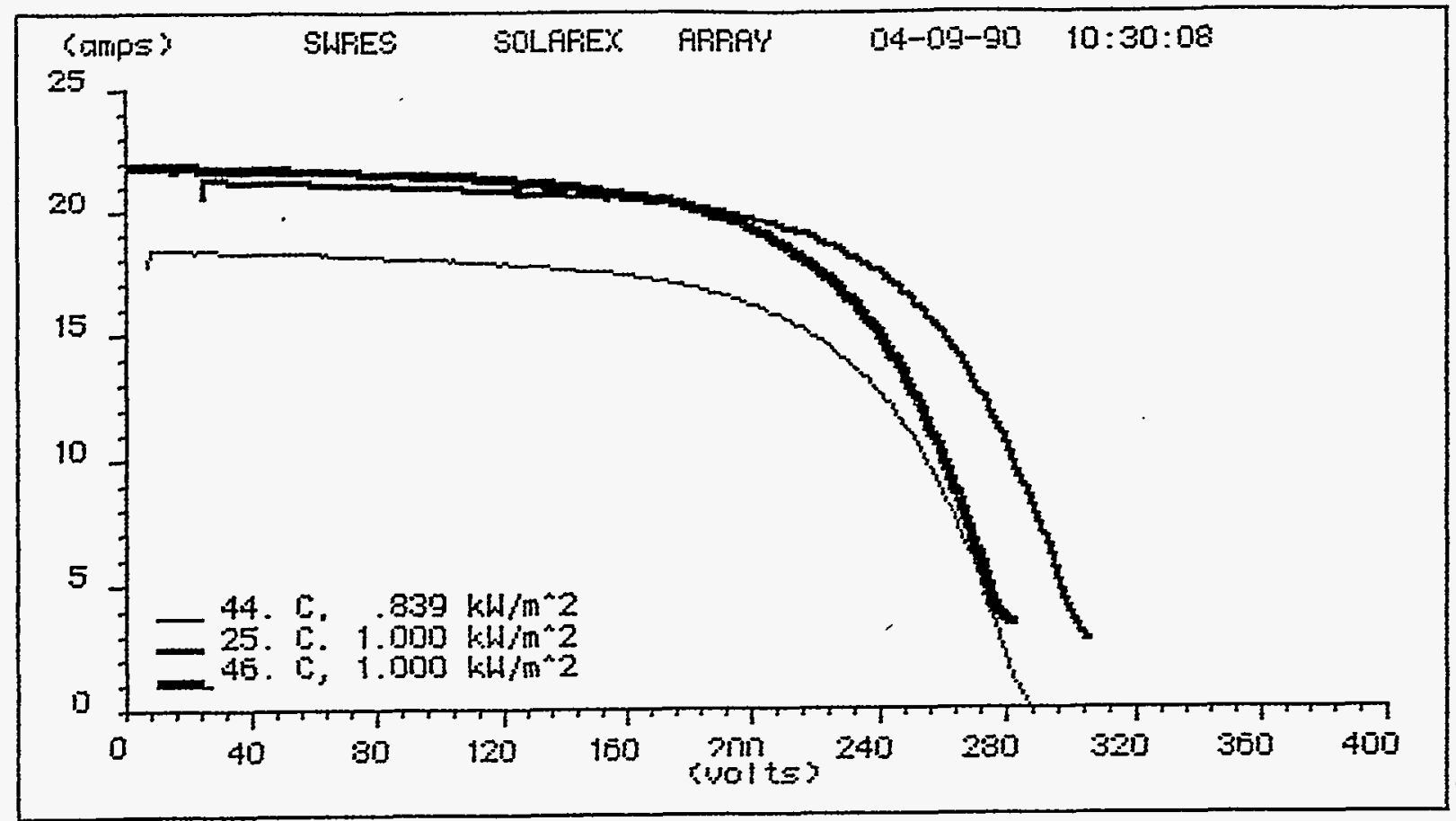

Cell Temperature .......44.000 deg $c$

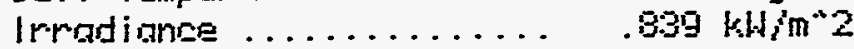

Series Resistance ....... 1.700 ohms

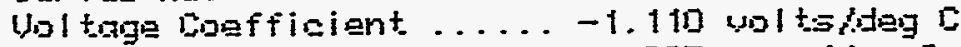

Durrent Coefficient ..... . . D2P amps ideg $C$

ACTUAL CUAUE DATA: STIII FAEtor = $51.8 \%$

Short cirsuit current.... 18.4 amp:

Open circuit val tage ..... 238.9 volts

Max pouser current. ...... 15.5 rmps

Max power yol tage ...... 212 . 1 is ts

Max poumar ........... 329?.7 mats

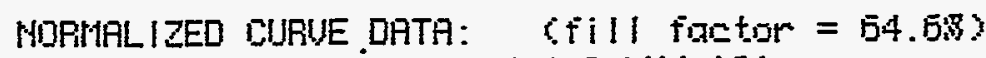

(for 25.0 deg $c$ arid $1.0 \mathrm{kH} / \mathrm{m}^{2} 2$ )

Max pouser "aurrent ....... 13.4 umps

Max power val tage ....... 223.2 val ts

Max pomer ............4 4205. 1 matts

MDRMALIZED CIJRUE DATA: (fill factor $=53.3 \mathrm{~s})$

tior 46.0 deg $c$ and $1.0 \mathrm{kH}\left(\mathrm{m}^{\circ} \mathrm{z}\right)$

Max pourer current....... 13.3 amps

Mox power valtage ....... 214.3 volts

Max power ............3911.5 untts 


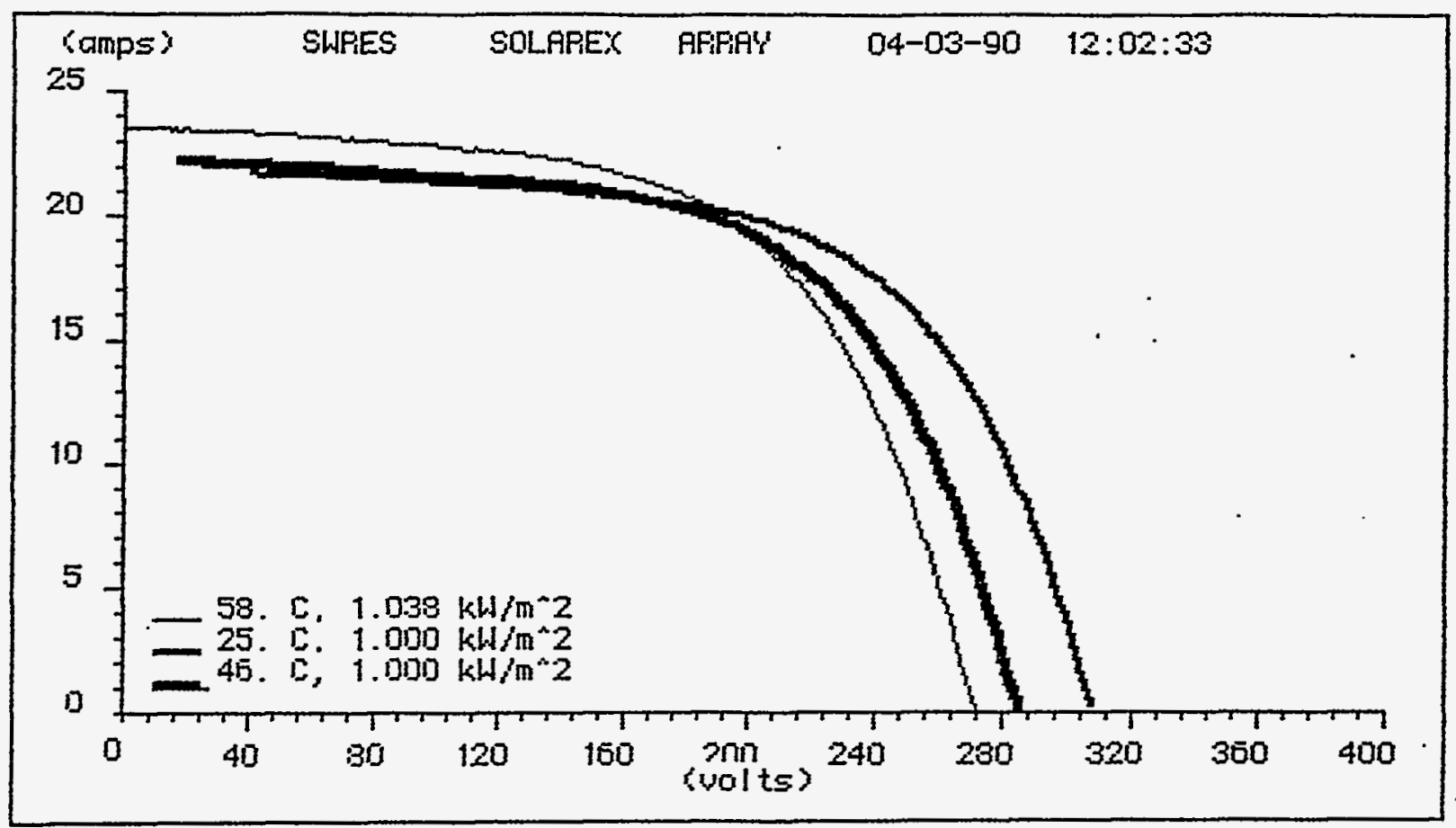

\footnotetext{
Cell Temperature ........53.000 deg $c$

Irradianse ........... 1.039 $\mathrm{kH} / \mathrm{m}^{\wedge} 2$

Series Resi

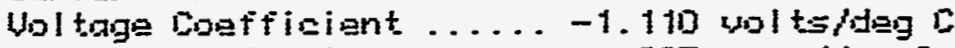

Cumpent coeficient ..... . .027 ampsideg $\mathrm{E}$

ACTUAL CURUE DATA: (fill faEtor = 50.08)

Short cirsuit surrent .... 23.6 anps

Opan circuit uol tage ..... 273.5 valts

Max power surrent ....... 19.2 amps

Max poujer voltage ....... 201.6 valts

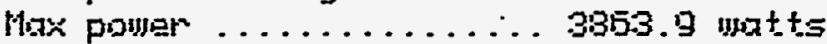

HDRIALL IZED CISRUE DATA: ifill factor $=62.133 \mathrm{y}$

(for 25.0 deg 5 and $1.0 \mathrm{kH} / \mathrm{m}^{2} \mathrm{Z}$ )

Max pauser current ....... 18.4 gmps

Max puwer sal tage ....... 230.5 sol ts

Mrax power ............4240.1 watts

MORMALIZED CIJRUE DATA: $\quad$ fill frotor $=60.43)$

ifor 40.0 deg $C$ and $1.0 \mathrm{kH} / \mathrm{m}^{2} \mathrm{Z}$ )

Max power currient. ....... 18.4 amps

Max powern vol tage ....... 212.2 wolts

Max pouser ............ 3907.7 watts
} 


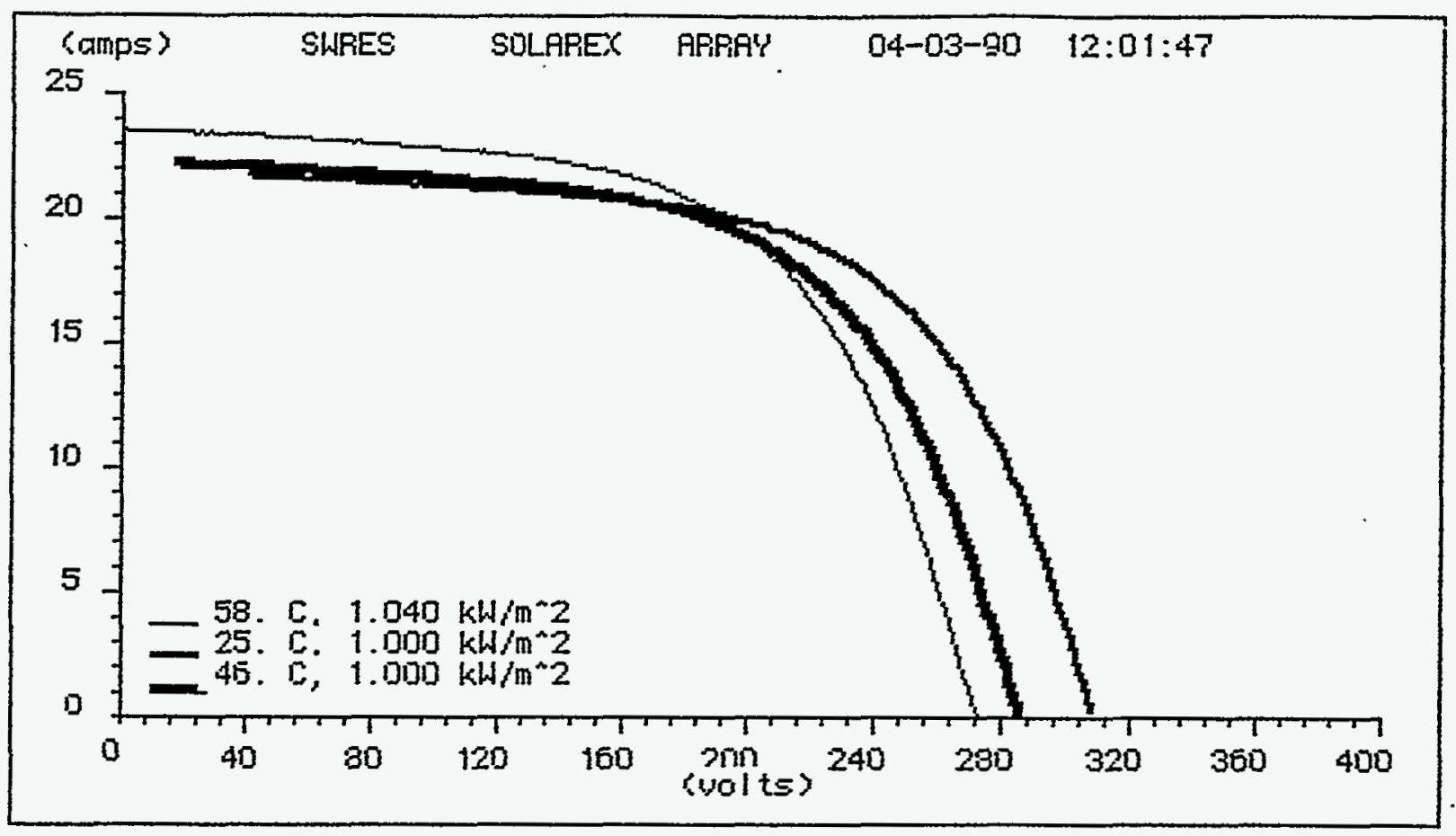




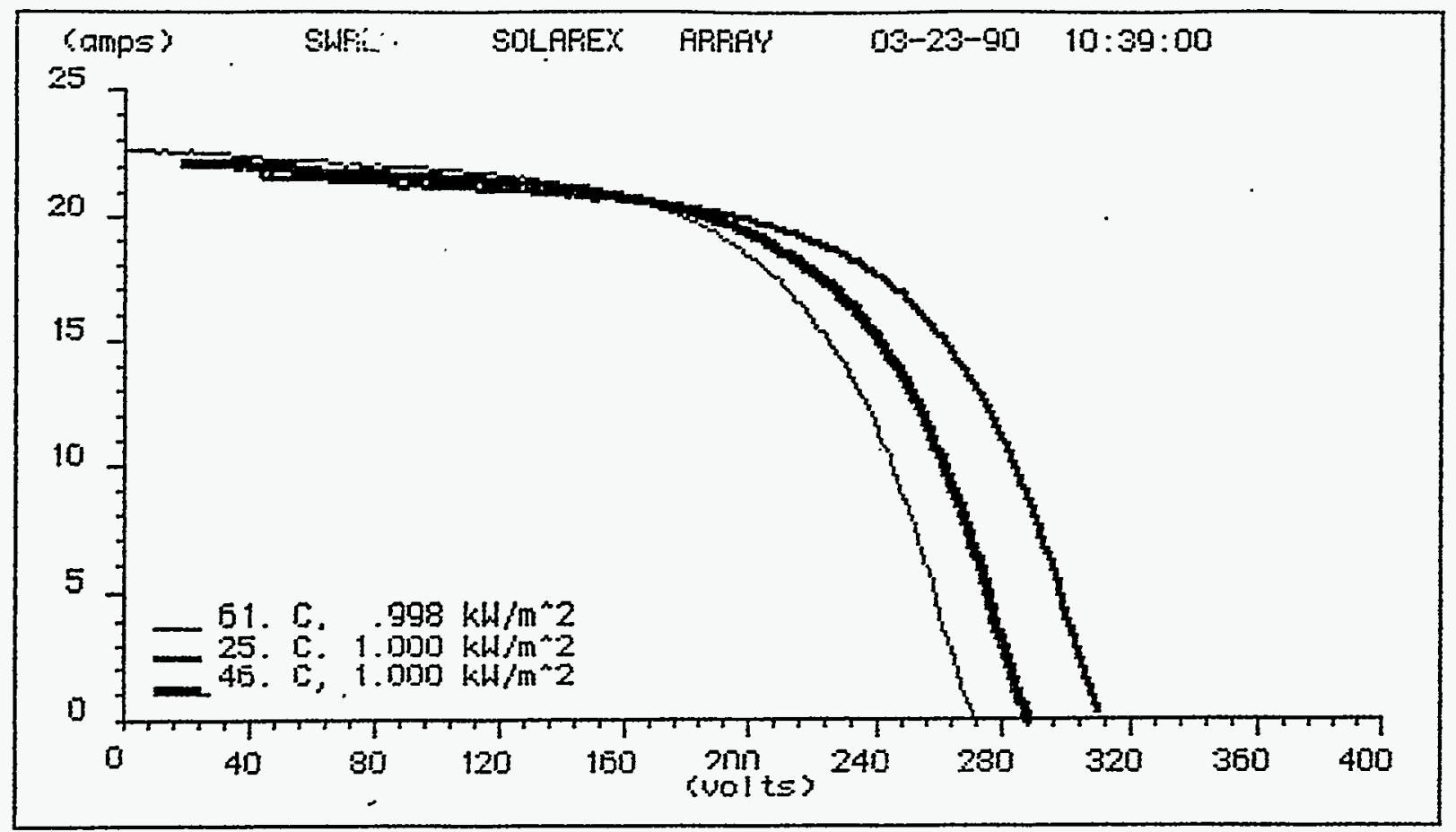

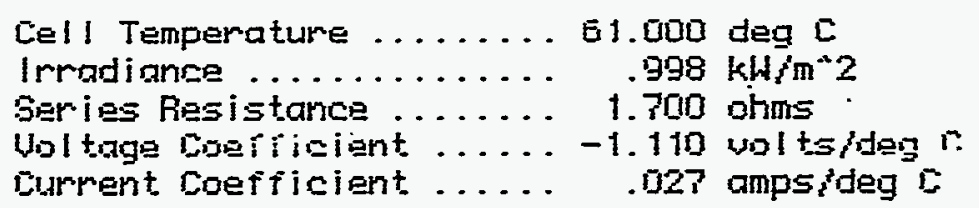

ACTURL CURUE DATA: (fill fuetor $=59.88$ )

Ghort circuit current .... 22.5 amps

Dpen circuit voltage .... 272.0 volts

Max pourer gurrent. ...... 18.7 amps

Max pouer vol tage ....... 195.9 volts

Max pomer ........... 3683.5 undts

NORHALIZED CIIRUE DATA: CFIll fRCtor $=62.3 \mathrm{~N} 2$

(for $25.0 \mathrm{deg} \mathrm{C}$ and $1.0 \mathrm{kH} / \mathrm{m}^{*} \mathrm{2}$ )

Max pourer curpent ....... 17.8 amps

Max power vol tage ....... 238.4 volts

Max pouser ........... 4239.4 watts

NORTALIZED CURUE DATA: (till faotor = 51.DS)

(for $40.0 \mathrm{deg} C$ and $1.0 \mathrm{kh} / \mathrm{m}^{*} 2 \mathrm{z}$

Max pouner current....... 18.3 amps

Hax power voltage ....... 214.1 volts

Max pouser ........... 3929.4 unatts 


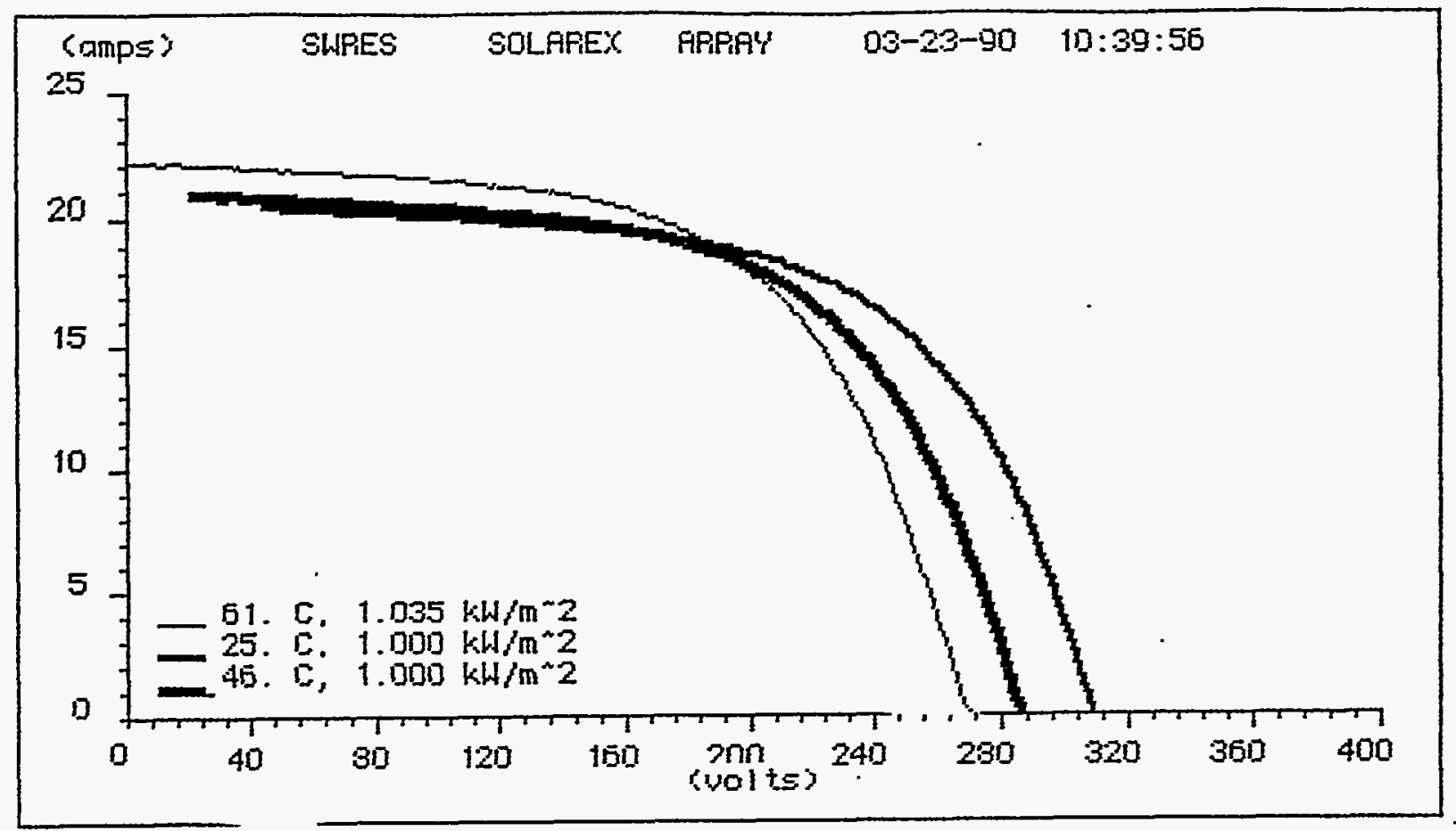

Cell Temperature ....... 01.000 deg $C$

Irradiance .......... $1.035 \mathrm{kH} / \mathrm{m}^{2-2}$

Series Resistance ....... 1.700 ohms

Uoltage coefficient...... -1.110 woltsidog 5

Curment coefficient ..... .02? ampsideg 5

ACTUAL CURUE DATA: (fill faEtor $=60.2 \%$ )

Short sirouit curpent ... 22.2 umps

Open circuit voltage .... 270.3 volts

Max pouner gurrent. ...... 19.2 nmps

Max pouner vol tage ....... 198.1 volts

Max poumer ........... 3502.5 matts

NORIALLIZED CURUE DATA: GIIII fGetor $=62.333$

(for 25.0 deg $C$ and $1.0 \mathrm{kH} / \mathrm{m}^{\circ} 2$ )

Max poiser gurrent ...... 17.2 amps

Max power sol tage ...... 232.? solts

Max pouser ............ 3995.3 unatts

MORMALIZED CURUE DATR: CAIII faEtor = 6D.9X

(for $46.0 \mathrm{deg} C$ and $1.0 \mathrm{~kW} / \mathrm{m}^{\wedge} 2$ )

Max pouser aurrent ....... 17.6 amps

Max power soltage ........ 209.6 wolts

Max poun ............ 3595.3 mats.s 


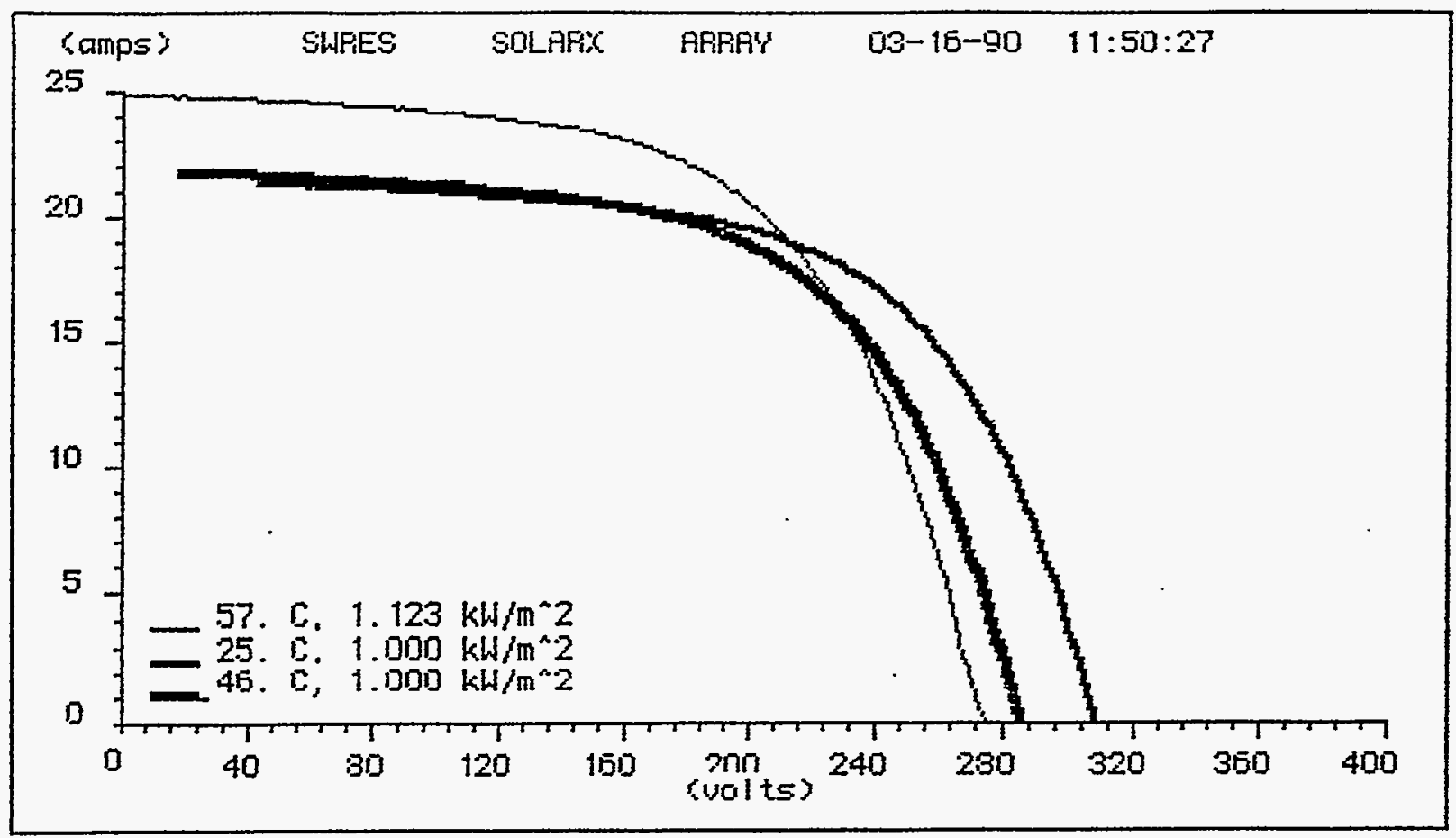

Cell Temperature .......57.000 deg $\mathrm{C}$

Irradianse .......... $1.123 \mathrm{kH} / \mathrm{m}^{22}$

Series Resistance ....... 1.700 ohms

Uoltage coufficiant ....... -1.110 wolts/dag c

curpent coeficient ..... . .027 ampsideg $c$

ACTUAL CURUE DRTA: (fill fGEtom $=59.7 \%$ ?

Short circuit current .... 24.9 amps

Dpen circuit voltage ..... 276.0 walts

Max pomer surrent...... 20.5 amps

Max poujer vol tage ....... 200.4 volts

Max pouner ...........4410.0 umatts

MIDPMALIZED DURUE DATA: (till factor $=51.18$ s

Sfor 25.0 deg $C$ and $1.0 \mathrm{kH} / \mathrm{m}^{*} 2$ ?

Max pouser aurrent....... 18.1 amps

Max power vol tage ....... 230.1 volts

Hax pomer ...........4 454.0 matts

NDRMALIZED CURUE DATA: (fill factor $=59.5 \mathrm{~s}$ )

Sfor 40.0 deg $C$ and $1.0 \mathrm{kH} / \mathrm{m}^{\circ} 2$ )

Max power atument. ....... 18.0 amps

Max pouer voltage ....... 213.0 volts

Max powjer ............ 3828.7 watts 

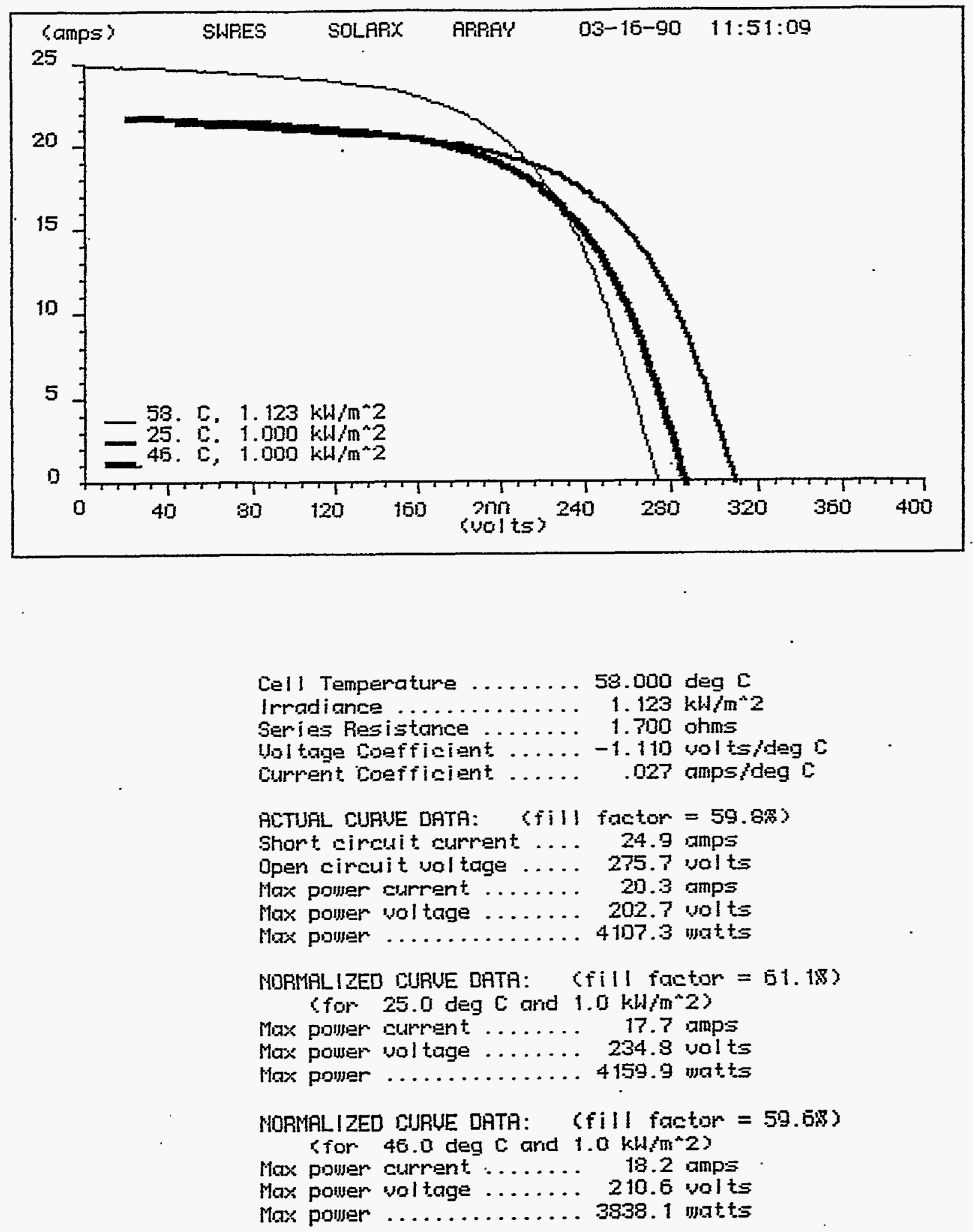


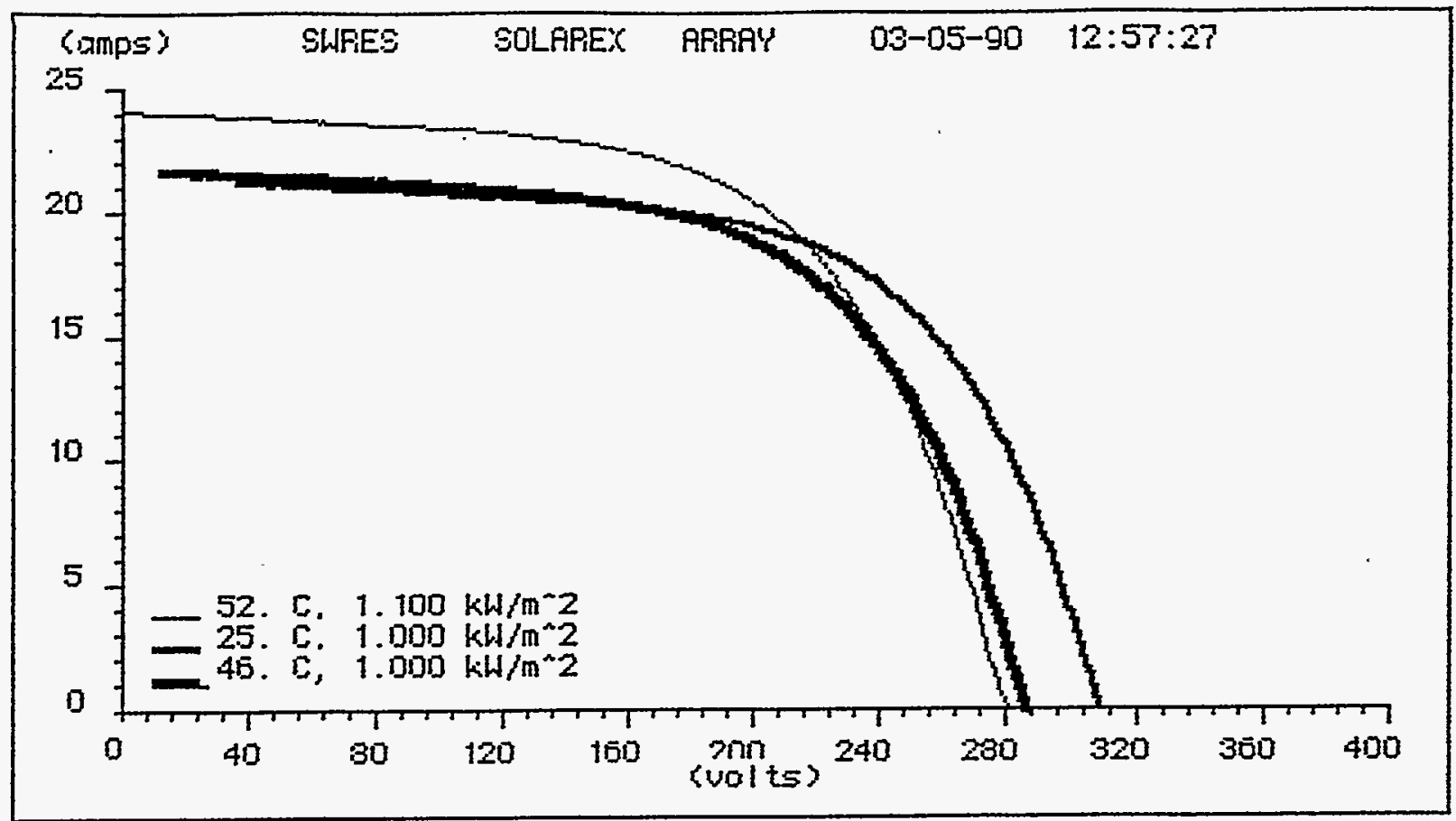

Cell Temperatume .......52.000 deg $c$

Irradiance ........... 1. ino $\mathrm{kH}_{\mathrm{im}} \mathrm{m}^{-2}$

Series Resistance ....... 1. PDO ohms

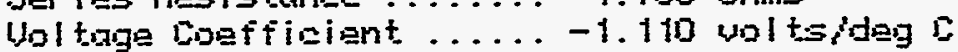

Current Coefficient..... . .027 ampsideg $\mathrm{C}$

ACTUHL CURUE DATA: (fill fGEtor = 50.33

Short cirouit cumpent.... 24.1 amps

Dpen circuit val tage .... 282.3 volts

Max ppomer current. ...... 19.3 amps

Max power vol tage ....... 206.3 volts

Max pouser ...........4 4104.4 mat.ts

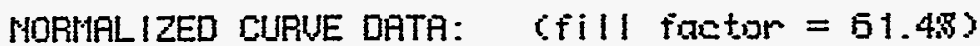

(for $25.0 \mathrm{deg}$ is ard $1.0 \mathrm{kH} / \mathrm{m}^{\star 2} \mathrm{z}$ ?

Max power currant ....... 17.3 amps

Max power vol tage ....... 232.9 uol ts

Max pouser ...........4439.4 mátts

NIDRMAL IZED CIJRUE DATA: (fill fastor $=59.93$ )

Sior 40.0 deg $C$ and $1.0 \mathrm{~kW} / \mathrm{m}^{*} 2$ )

Max poujer current...... 18.3 amps

Max power wal tage ....... 208. ? volts

Max pouner .............3817.1 unats 


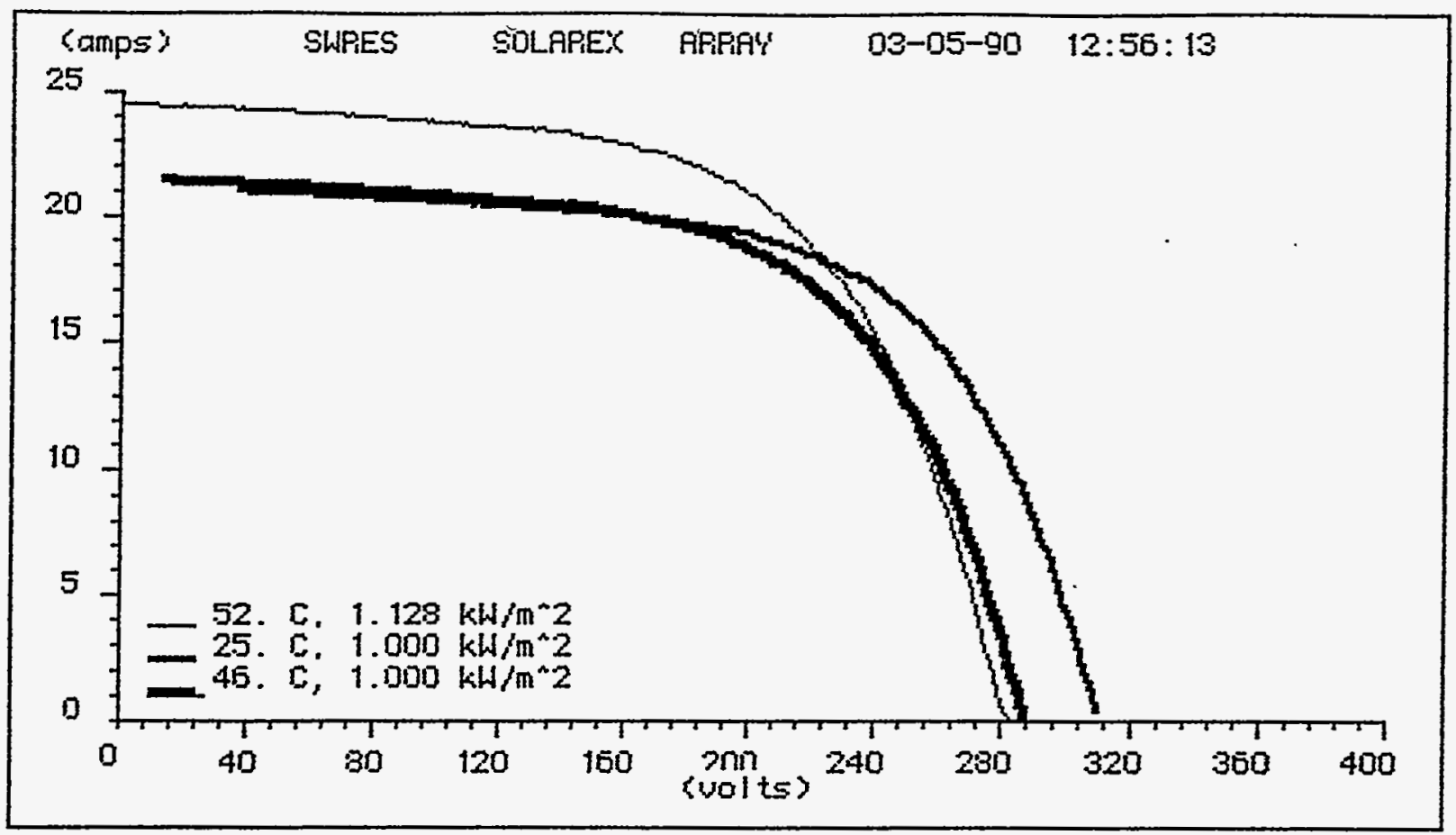

Cell Temperature .......52.000 deg $\mathrm{C}$

Irradiance .......... $1.128 \mathrm{kH} / \mathrm{m}^{* 2}$

Series Resistance ....... 1.700 ohms

Woltage coafficient ...... -1.110 wolts/deg c

Current Coefficient ..... .027 ampsideg $c$

ACTURL CURUE DATA: (fill fretor $=60.43$ )

Short circuit current. ... 24.5 amps

Open circuit voltage .... 284.2 volts

Max power current ...... 20.1 amps

Max pourer vol tage ....... 209.3 volts

Max pomar ........... 4212.2 matts

MDPMALIZED CURUE DATA: (fill factor $=51.3 \mathrm{~s}$

(for $25.0 \mathrm{deg}\left[0\right.$ and $1.0 \mathrm{kH} / \mathrm{m}^{\wedge} 2$ )

lox pouser current ....... 17.7 amps

Max pomer valtage ....... 234.1 valts

Max power .......... 4141.8 watts

NDRMALIZED CURUE DATA: (fill faOtor $=59.83$ )

(for $40.0 \mathrm{deg} C$ and $1.0 \mathrm{~kW} / \mathrm{m}^{2} 2$ )

Max poiser current ........ 17.9 amps

Mas power voltage ....... 213.2 volts

Max poiner ........... 3825.1 watts 


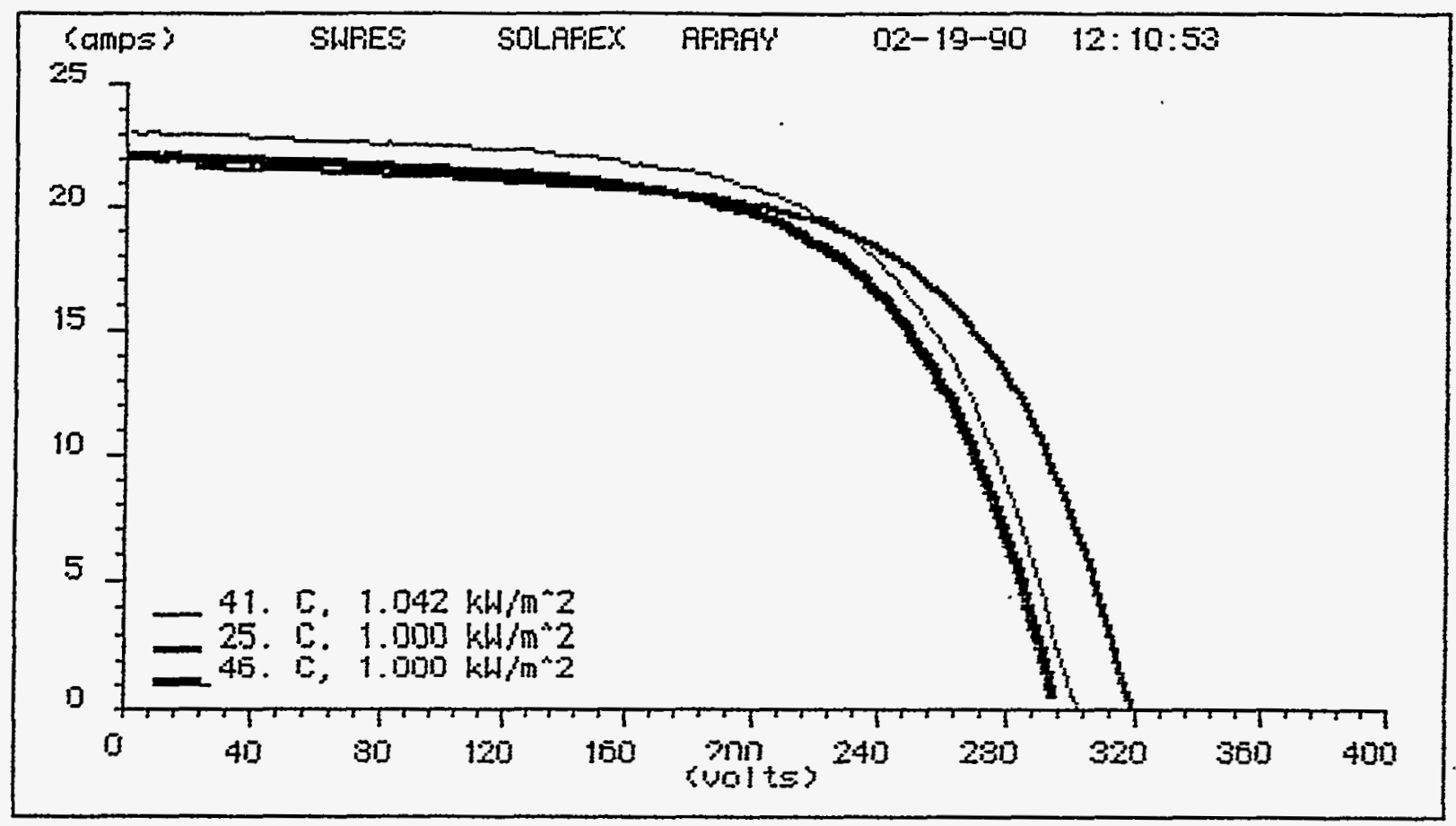

\footnotetext{
Cell Temperature .......41.000 deg $C$

irradiance .......... $1.042 \mathrm{kH}^{\circ} \mathrm{m}^{\circ 2}$

Series Resistance ....... 1.700 ohme

Woltage coefficiant .....-1.110 woltsideg c

Current Coefficient ..... . .027 ampsideg $\mathrm{C}$

ACTUAL CUAUE DATA: Sfill factor $=62.2 \%$ s

Short cirouit aurrent. ... 23.1 anps.

Open circuit voltage .... 302.8 ualts

Max pouper current....... 19.3 amps

Mox power uol tage ....... 225.0 volts

Hax pouer ........... 434?.0 motts

MGPMALIZED CIJPUE DATA: (Aill fantor $=62.78$ )

(for 25.0 deg $D$ and $1.0 \mathrm{kH} / \mathrm{m}^{n} \mathrm{z}$ )

Max pomer current. ....... 13.0 amps

Max power ual tage ....... 245.Q ualta

Max pouner ........... 4404.7 matts

NDRNALIZED DURUE DATA: (fill fortor $=51.48$ )

(for $4 \overline{0} .0$ deg $C$ and $1.0 \mathrm{~kW} / \mathrm{m}^{2} 2$ )

Max powar aurrent ....... 18.5 amps

Max power vol tage ....... 220.8 volts:

Max pounar ...........4089.2 matts
} 


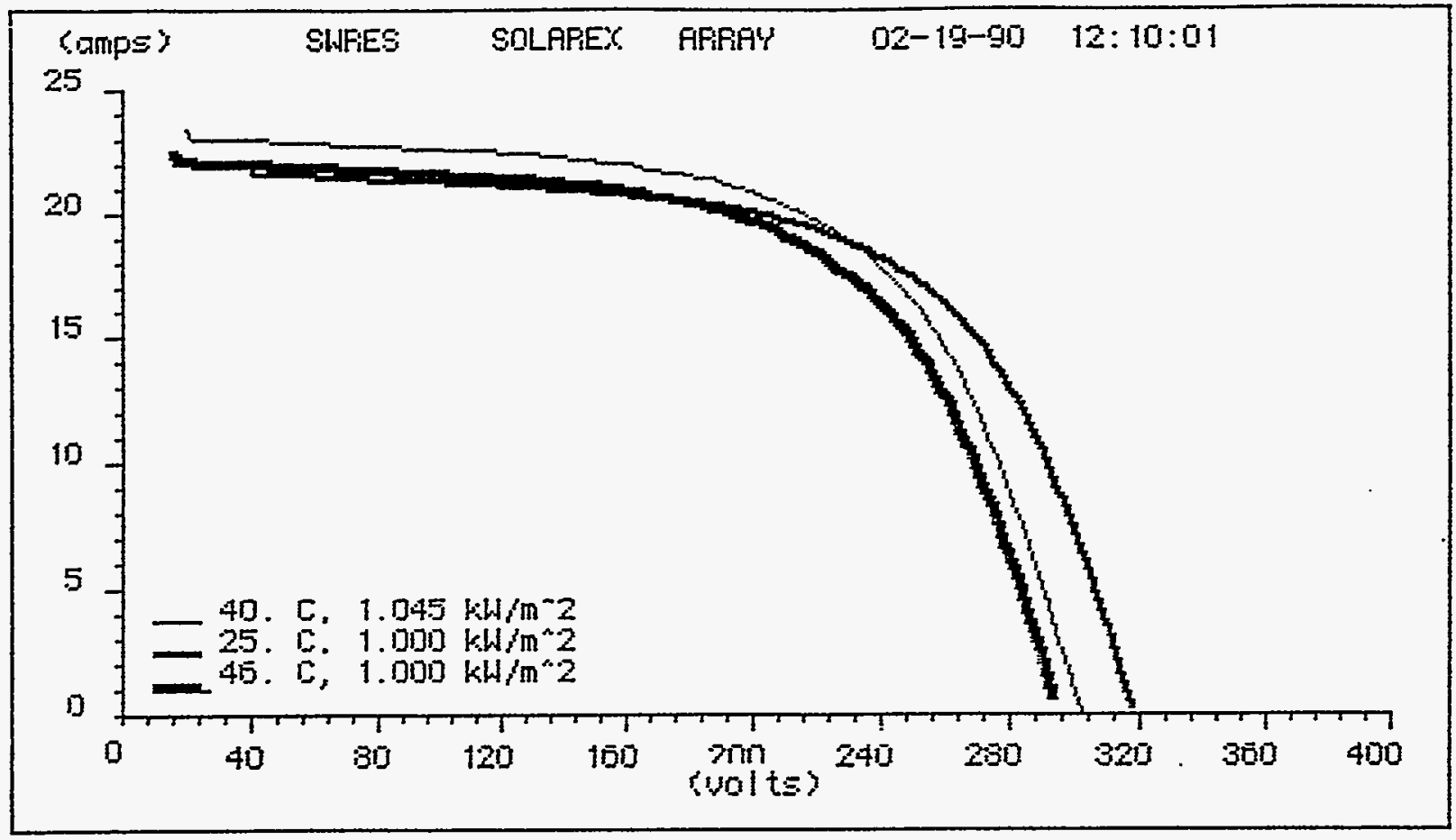

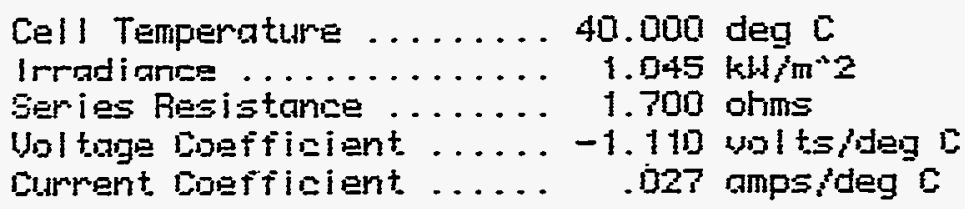

ACTUAL CURUE DATA: (fill factor $=51.48$ )

Short gircuit curpont ... 23.4 amps Open eircuit vol tage .... 302.9 volts Max pouer gurrent. ....... 19.1 amps Max pouer woltage ...... 227.3 volts Max pourer ........... 4349.0 matts

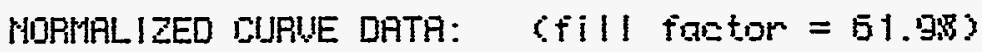

(for $25.0 \mathrm{deg}$ c and $1.0 \mathrm{kH} / \mathrm{m}^{2} \mathrm{z}$ ?

Mox pouper current ....... 18.3 amp:

Max power vol tage ....... 239.3 valts

Max pomer ............ 4391. 1 watts

NORHALIZED CIJRUE DATA: CFill faOtor = 50.5\%)

(for 40.0 deg $C$ and $\left.1.0 \mathrm{kH}, \mathrm{m}^{*} \mathrm{Z}\right)$

Max pouser gurrent....... 13.3 umps

Max pouer voltage ...... 222. 1 ,olts

Mox pioger .......... 4060. 1 watts 


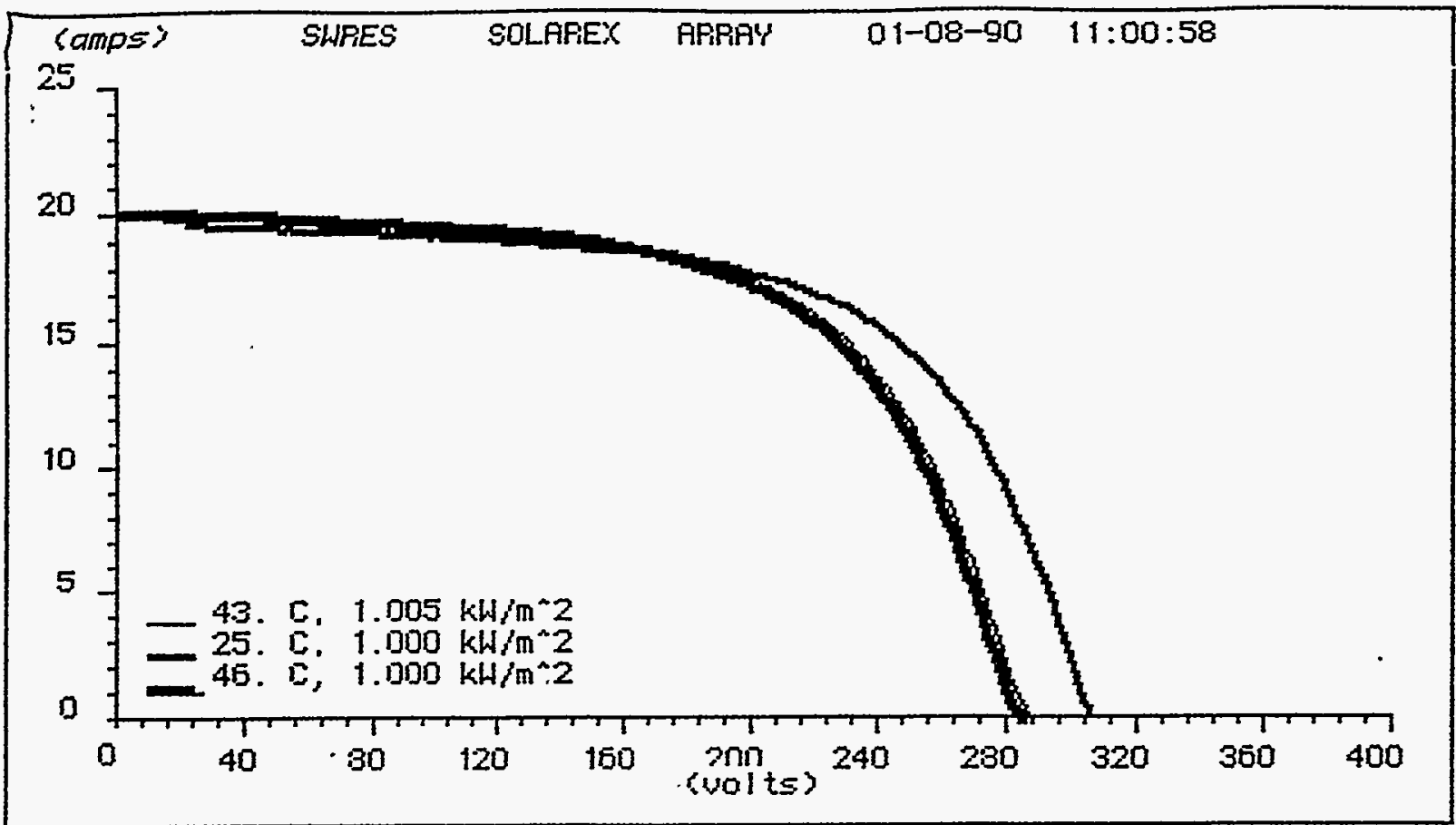

\footnotetext{
Cell Temperature .......43.000 deg $C$

Irradiance ............ $1.005 \mathrm{kHim}{ }^{* 2}$

Series Resistance ........ 1.700 ohms

Uoltage coufficient ......-1.110 wolts/oge $c$

Current Coeficient ..... .027 ampsideg $\mathrm{C}$

ACTURL CURVE DATA: (fill frotor $=61.38$ )

Short circuit gurrent. ... 20.2 amps

Open circuit valtage ..... 287. 7 volts

Max poiner current ...... 15.5 amps

Max power vol tage ....... 215.6 volts

Max power ........... 3550.1 watts

NORMALIZED CURUE DATA: (fill factor $=62.68 \mathrm{~s}$

(for 25.0 deg $C$ and $1.0 \mathrm{khl} / \mathrm{m}^{\wedge} \mathrm{Z}$ )

Max pourar surrent...... 15.5 amps

Max power val tage ....... 229.6 volts

Max power ............ 3782.3 watts

MORMALIZED SIJRUE DATA: Cfill factor $=61.1 \mathrm{k}$ ?

\{for $4 \overline{0.0}$ deg $C$ ard $1.0 \mathrm{~kW} / \mathrm{m}^{\wedge} 2$ ?

Max poujer current ....... 15.4 amps

Max power vol tage ....... 213.5 volts

Max pouner ............. 3501.5 watts
} 

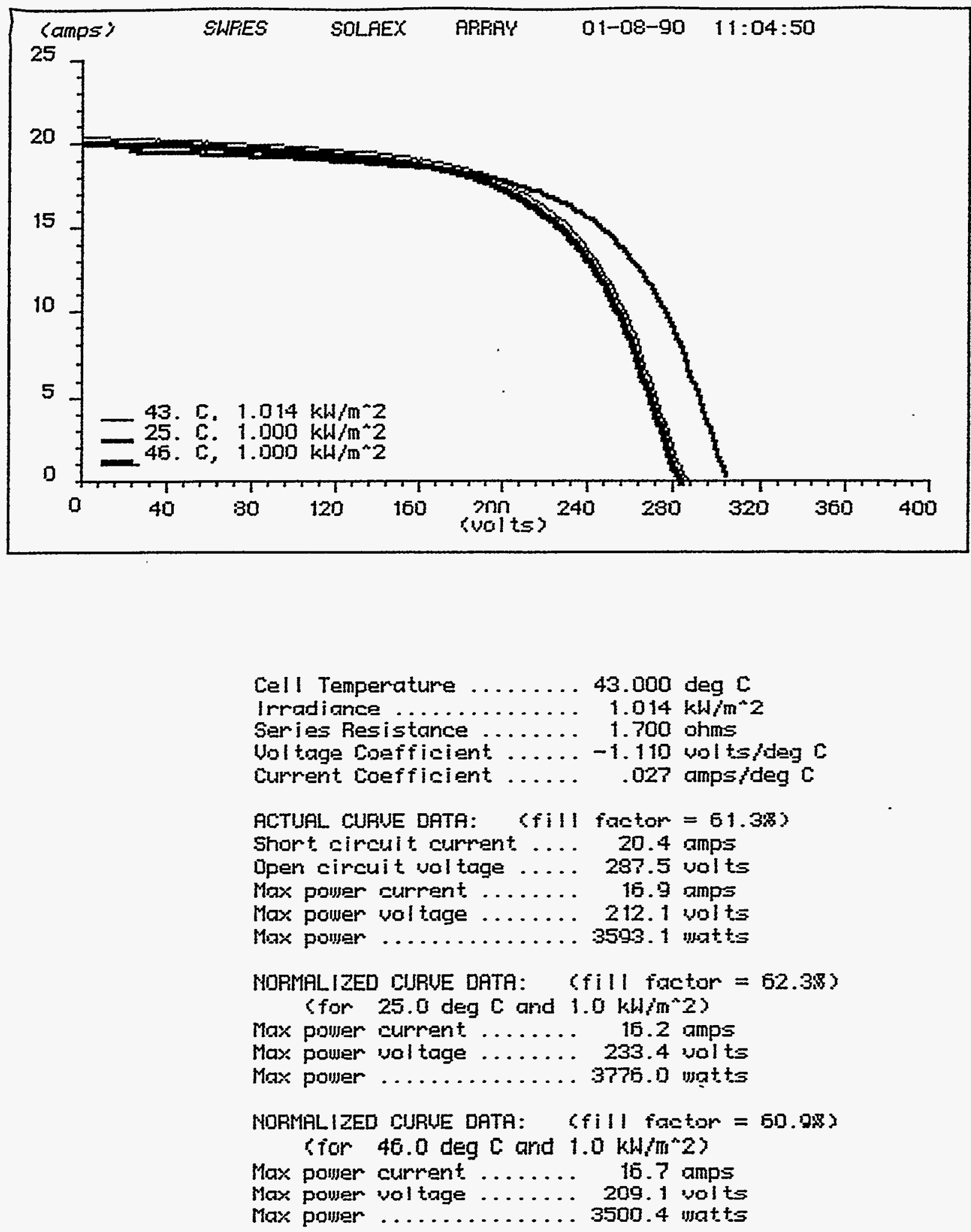


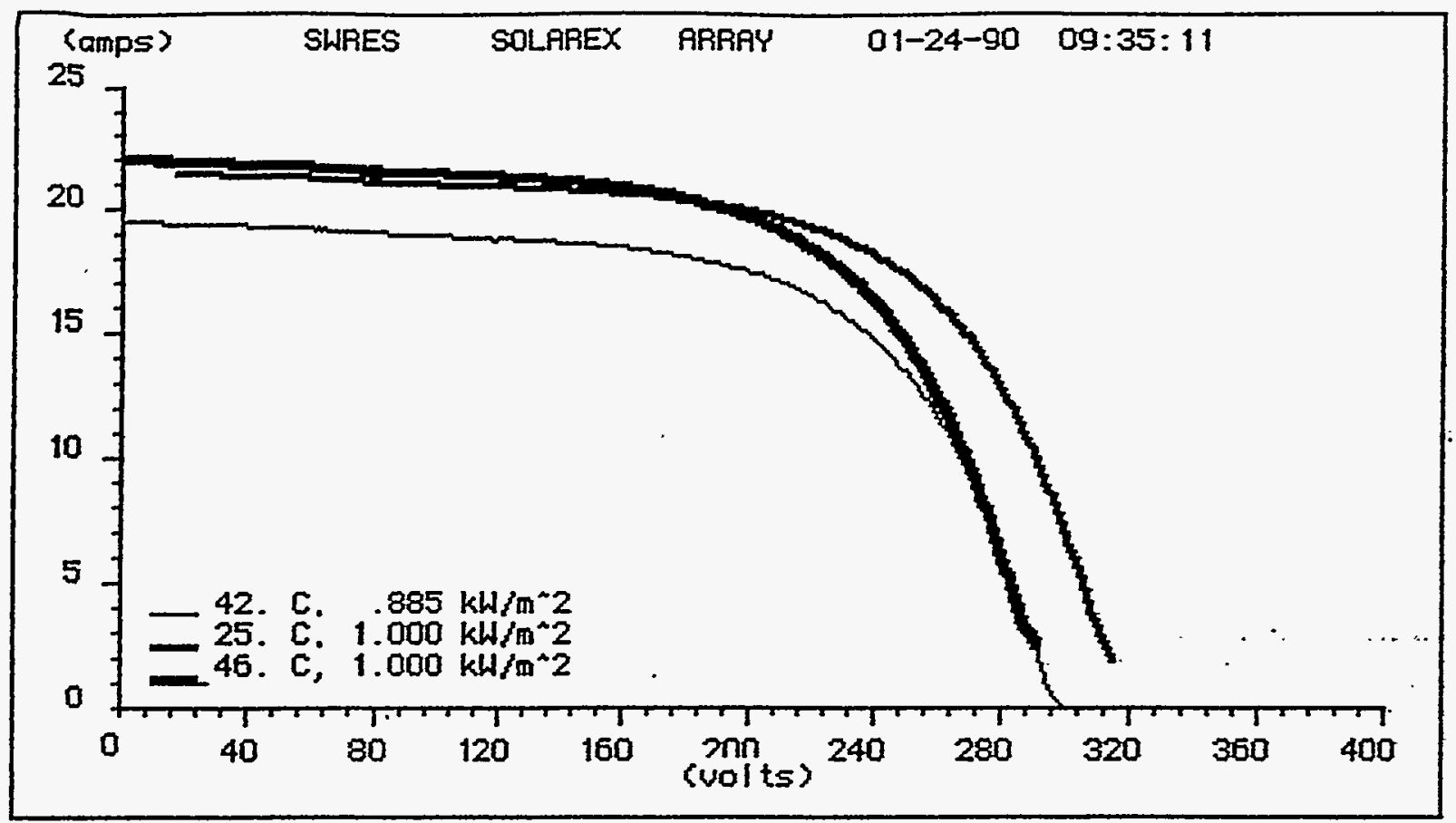

Cell Temperature .......42.000 deg C

Irradiance .......... .885 $\mathrm{kH} / \mathrm{m}^{\wedge} 2$

Series Resistance ........ 1.700 ohms

Voltage Coefficient ..... -1.110 volts/deg $C$

Current Coefficient ...... .027 amps/deg $C$

ACTUAL CURUE DATA: ( $\mathrm{f} i l$ ) factor $=62.38$ )

Short circuit current .... 19.5 amps

Open circuit voltage ..... 298.8 volts

Max power current ........ 16.3 amps

Max power vol tage ....... 222.7 volts

Max pomer ........... 3629.4 watts

MORMALIZED CURUE DATA: ( $\mathrm{fi}$ ll factor $=64.68$ )

(for $25.0 \mathrm{deg} C$ and $1.0 \mathrm{kH} / \mathrm{m}^{\circ} 2$ )

llax power current ....... 18.1 amps

Max power voltage ....... 240.4 volts

Max power ........... 4362.4 watts

NORMALIZED CURUE DATA: (fill factor $=63.3 \%$ )

$$
\text { (for } 46.0 \mathrm{deg} C \text { and } 1.0 \mathrm{kH} / \mathrm{m}^{\wedge} 2 \text { ) }
$$

Max power current ...... 18.4 amps.

Max power vol tage ....... 220.7 volts

Max power ........... 4062.3 watts 


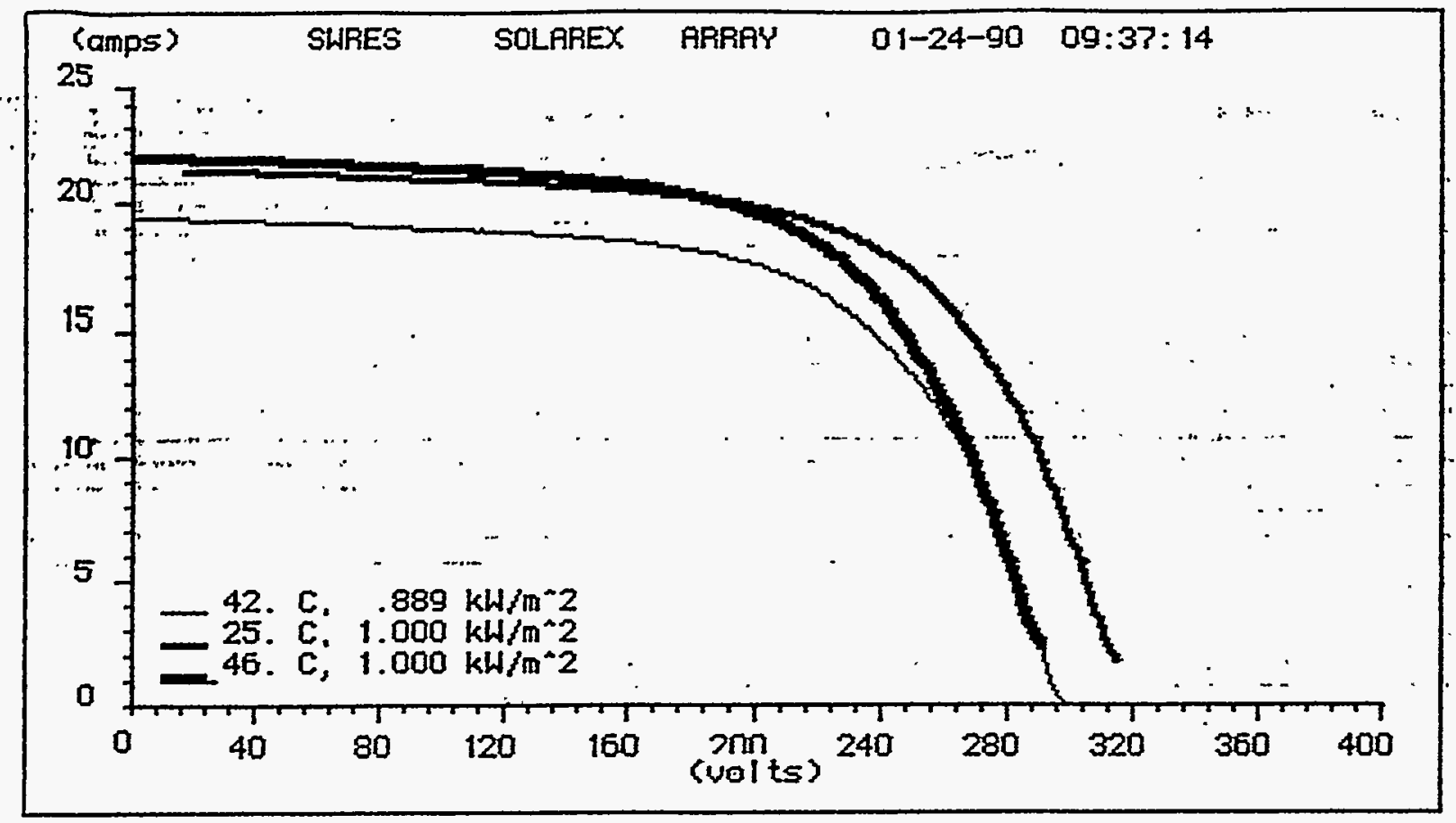

Cell Temperature

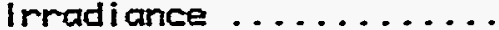

Series Resistance ..........

Voltage Coefficient ....... -1.110 volts/deg $c$

Current Coefficient ..... .027 amps/deg $C$

ACTUAL CURUE DATA: (fill factor $=62.3 \%$ )

Short circuit current :... 19.4 amps

Open circuit yol tage ..... 298.8 volts

Max power current .........: 16.3 amps::

Max power vol tage :....... 221.6 volts

Max power ............ 3608. 1 watts

MORMALIZED CURUE DATA: (fill factor $=64.58$ )

(for 25.0 deg $C$ and $1.0 \mathrm{~kW} / \mathrm{m}^{\circ} 2$ )

Max power current ....... 17.9 amps

Max power vol tage ....... 240.8 volts

Max power ............4315.9 watts

MORMALIZED CURUE DATA: (fi) f factor $=63.28$ )

(for 46.0 deg $C$ and $1.0 \mathrm{kH} / \mathrm{m}^{2} 2$ )

Max power current ....... 18.2 amps

Max power voltage ....... 220.9 volts

Max power ............4021.4 watts 
APPENDIX D

TriSolar 1990

D-1 


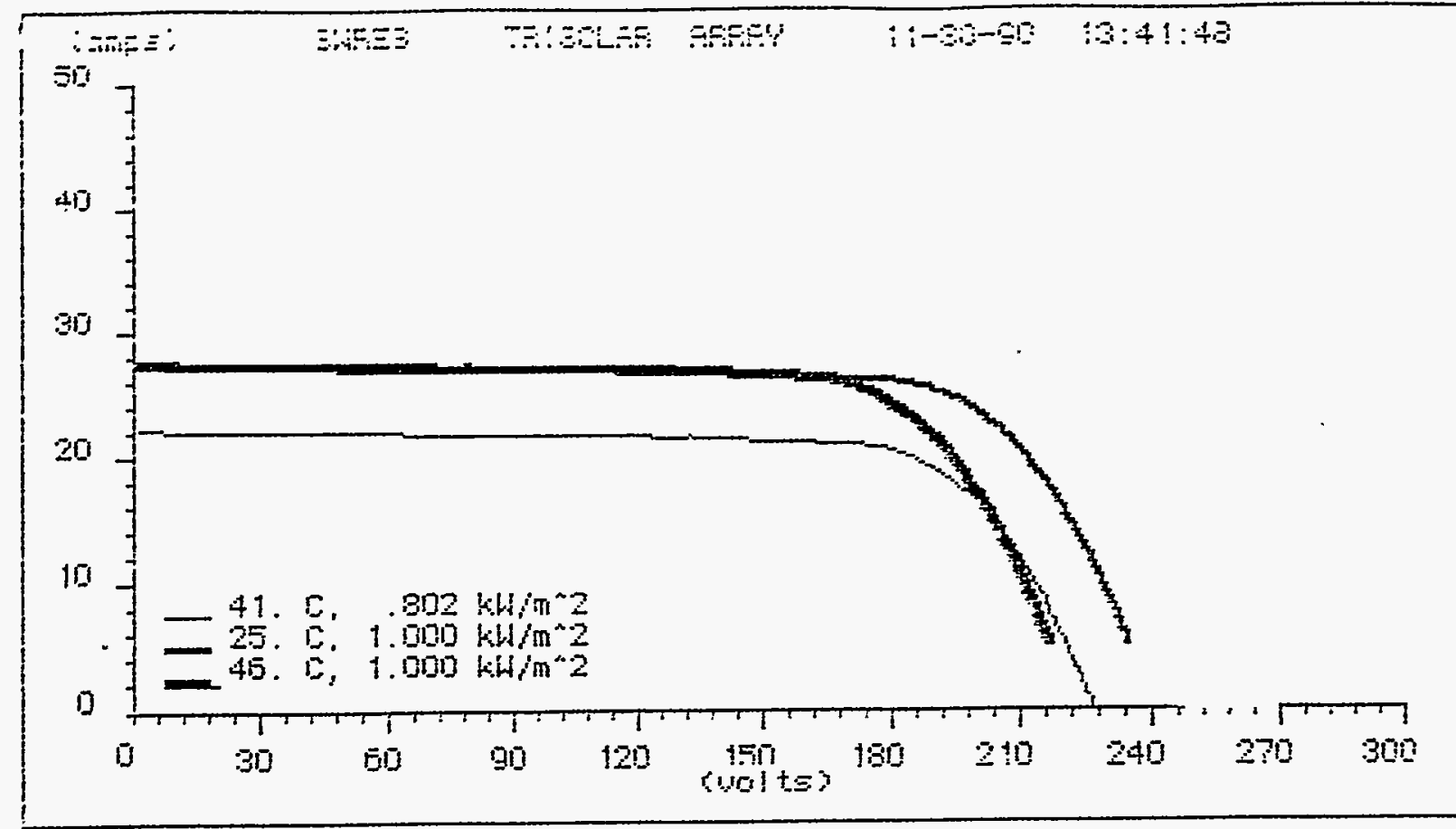

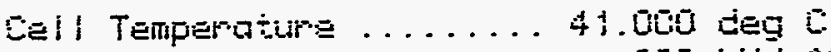

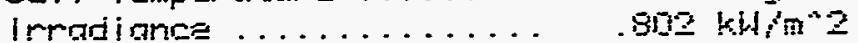

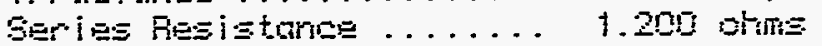

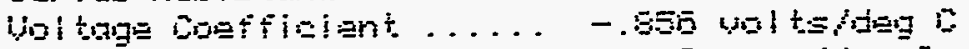

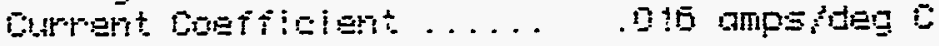

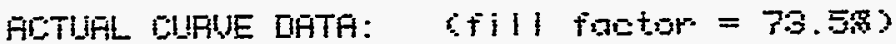

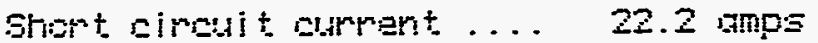

Gper Eireuit val tage ..... 22E. E volts

Max pouer mumpent ...... 20.5 amps

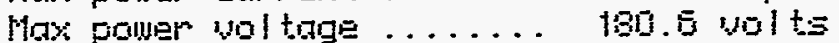

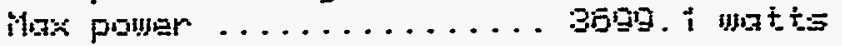

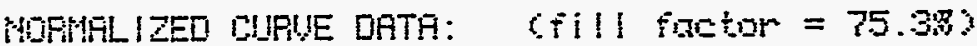

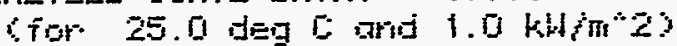

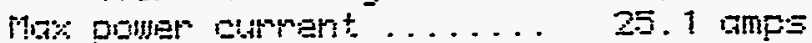

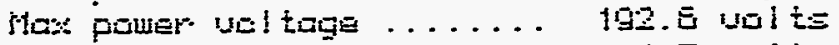

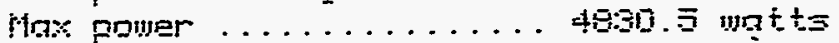

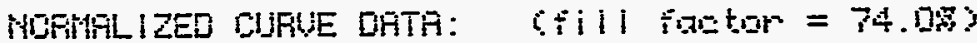

rfor $4 \overline{0} .0$ deg $\left[\right.$ and $1.0 k 4 m^{*} 2$

Mak pouler aurpent ....... 25.5 amps

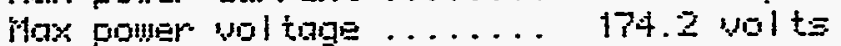

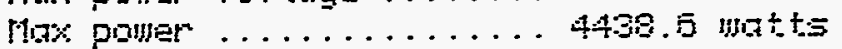




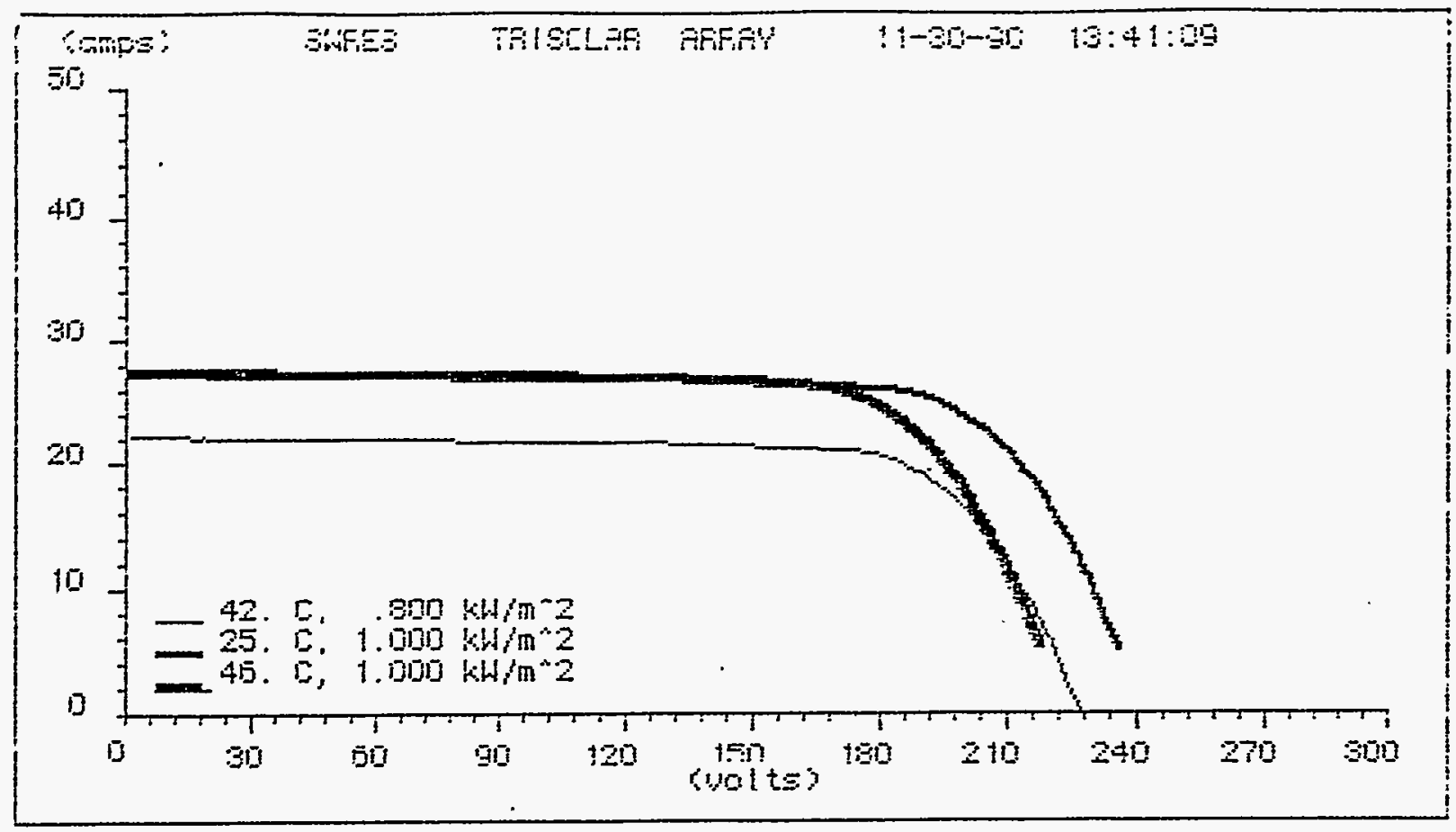

Ceil Temperature $\ldots \ldots \ldots \ldots$

Irrodiance .............

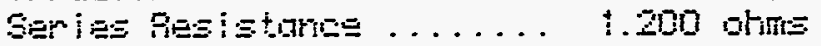

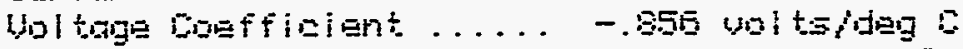

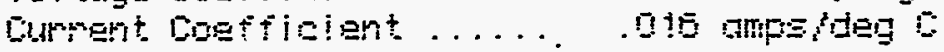

FCTUFL DURUE EATA: GIII fagtor $=73.483$

Shont airatit aurmant . . . 22.3 amps

Dpen cirouit wol tage .... 220.9 wolts

Hox pouer current. ...... 20.3 amps

Mro pourer wo!tage . . . . . 192.8 wolt:

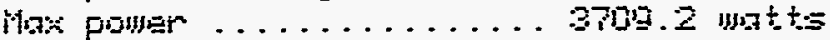

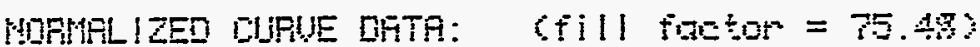

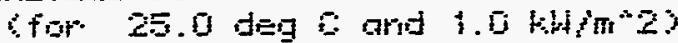

Max pouper aument ...... 25. 4 amps

Mog poubr vol tage ...... 192.2 jol to

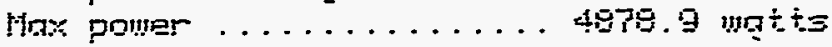

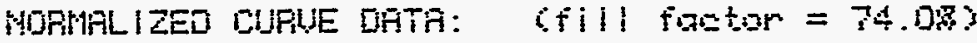

sfor 40.0 deg $C$ and $1.0 \mathrm{kH} / \mathrm{m}+2$ ?

Max pouper atirrent. ...... 25.3 smps

Max puyer vol toge ...... 173.8 volts.

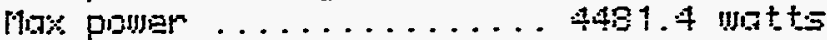



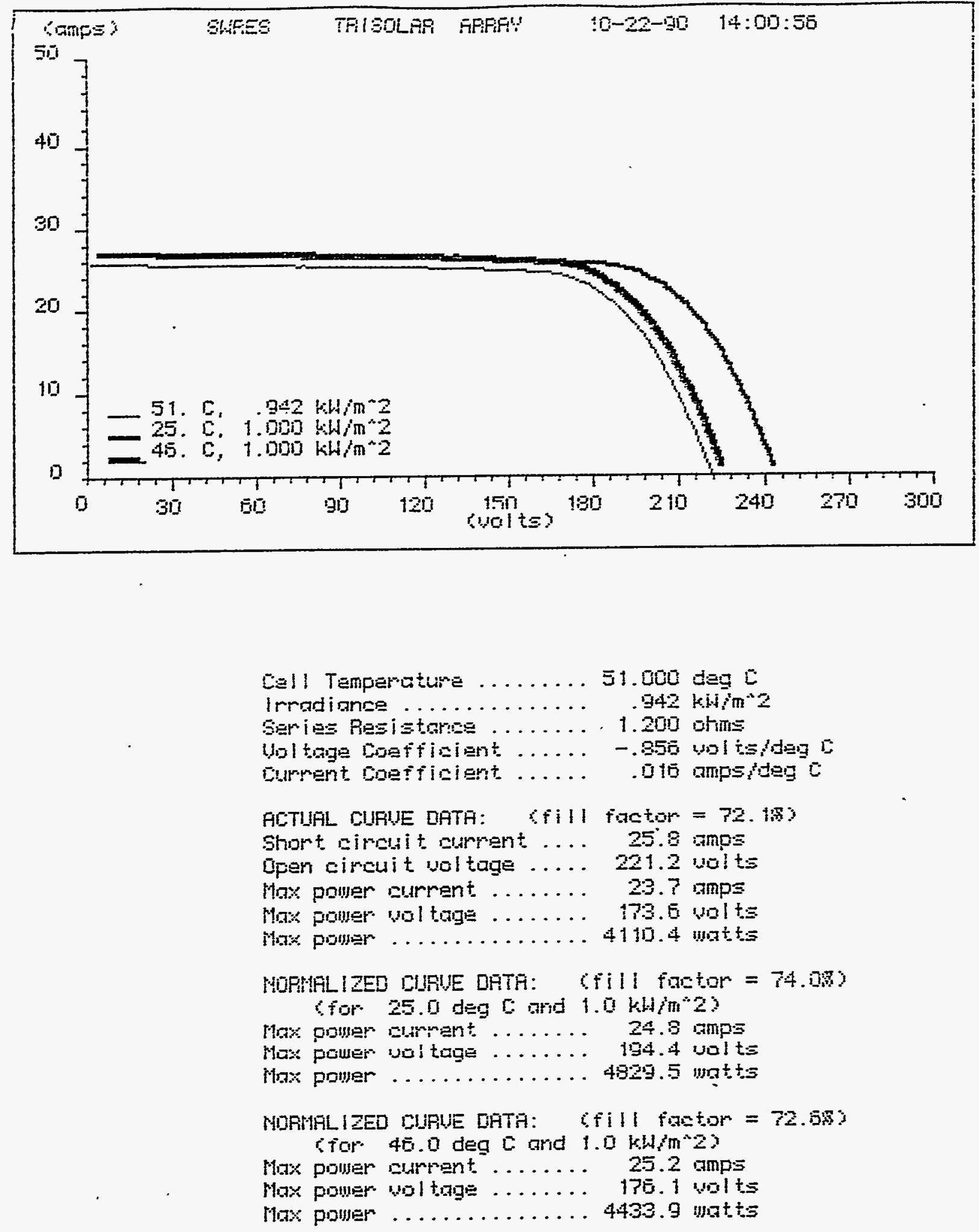


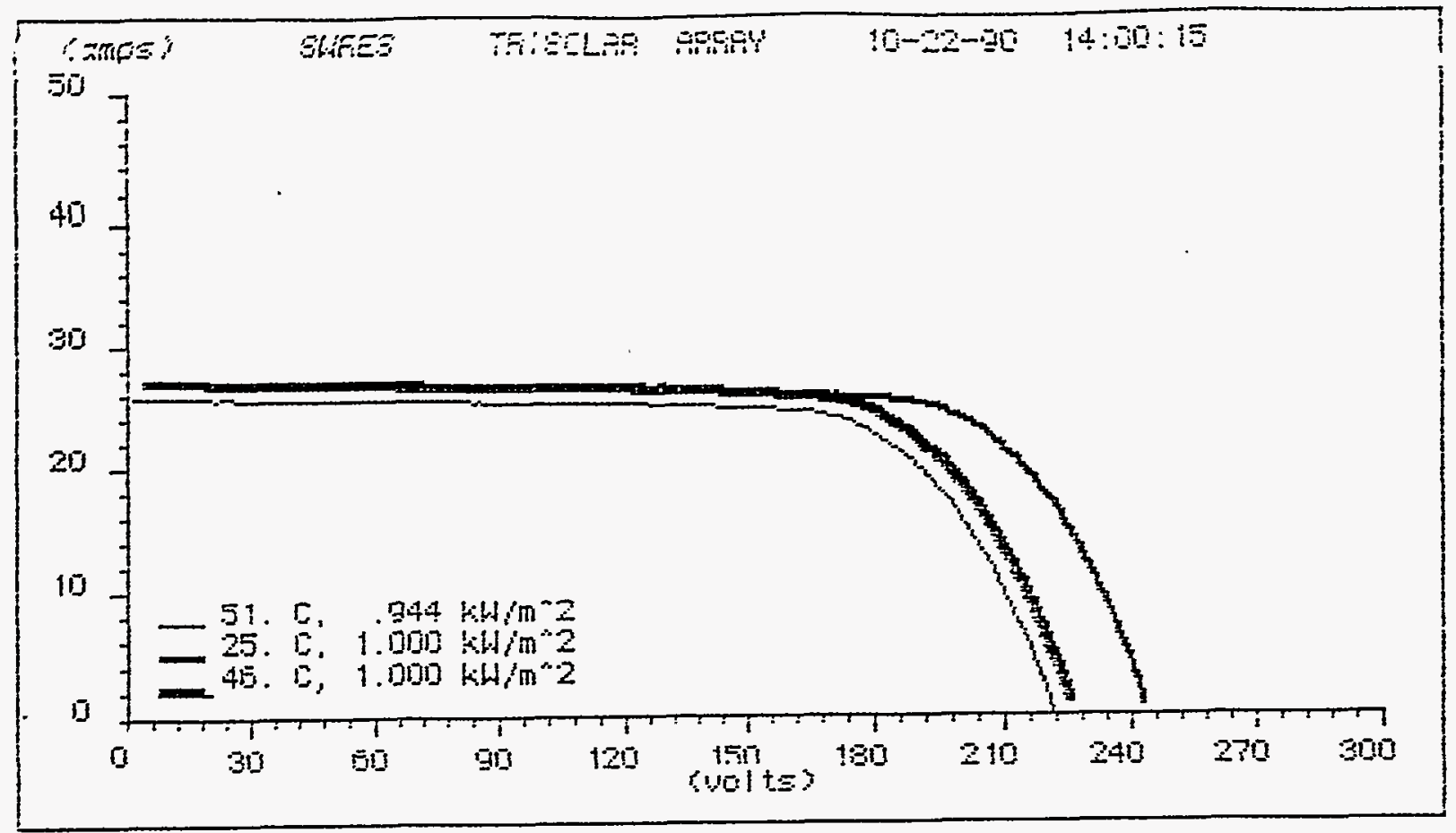

\footnotetext{
Cai! Temperature ....... \$1. DuU dea $C$

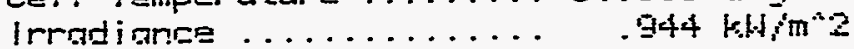

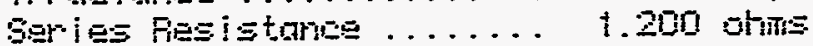

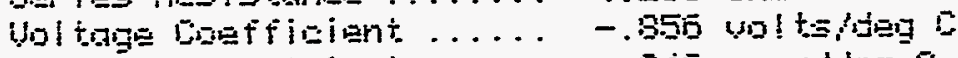

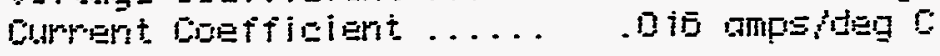

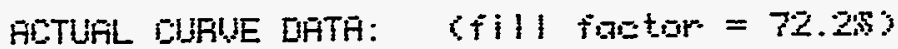

Short aimadit curpent .... 25.8 anfs

Open circuit vol tage ..... 221:5 volts

Max pouner aurment ....... 23.5 amps

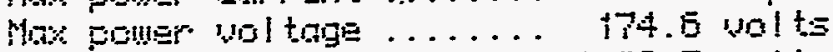

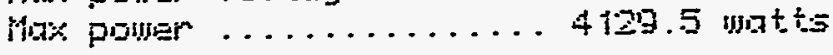

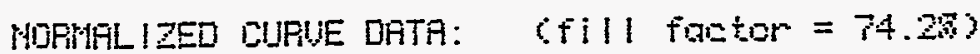

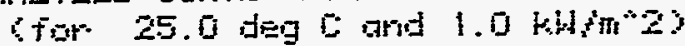

Max pouner strment. . . . . . 24.9 gmps

Max puluer wal tage ...... 194.4 val ts

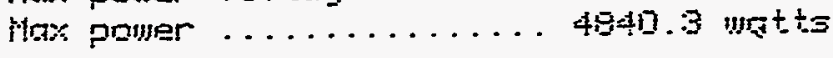

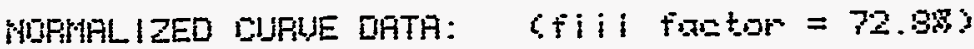

rior 45.0 deg $C$ and $1.046 m^{2} 2$;

Max puuer aumont ...... 25.1 ump:

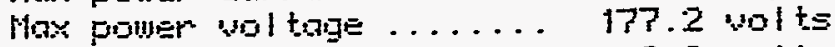

Max pouler ........... 4445.3 watts
} 

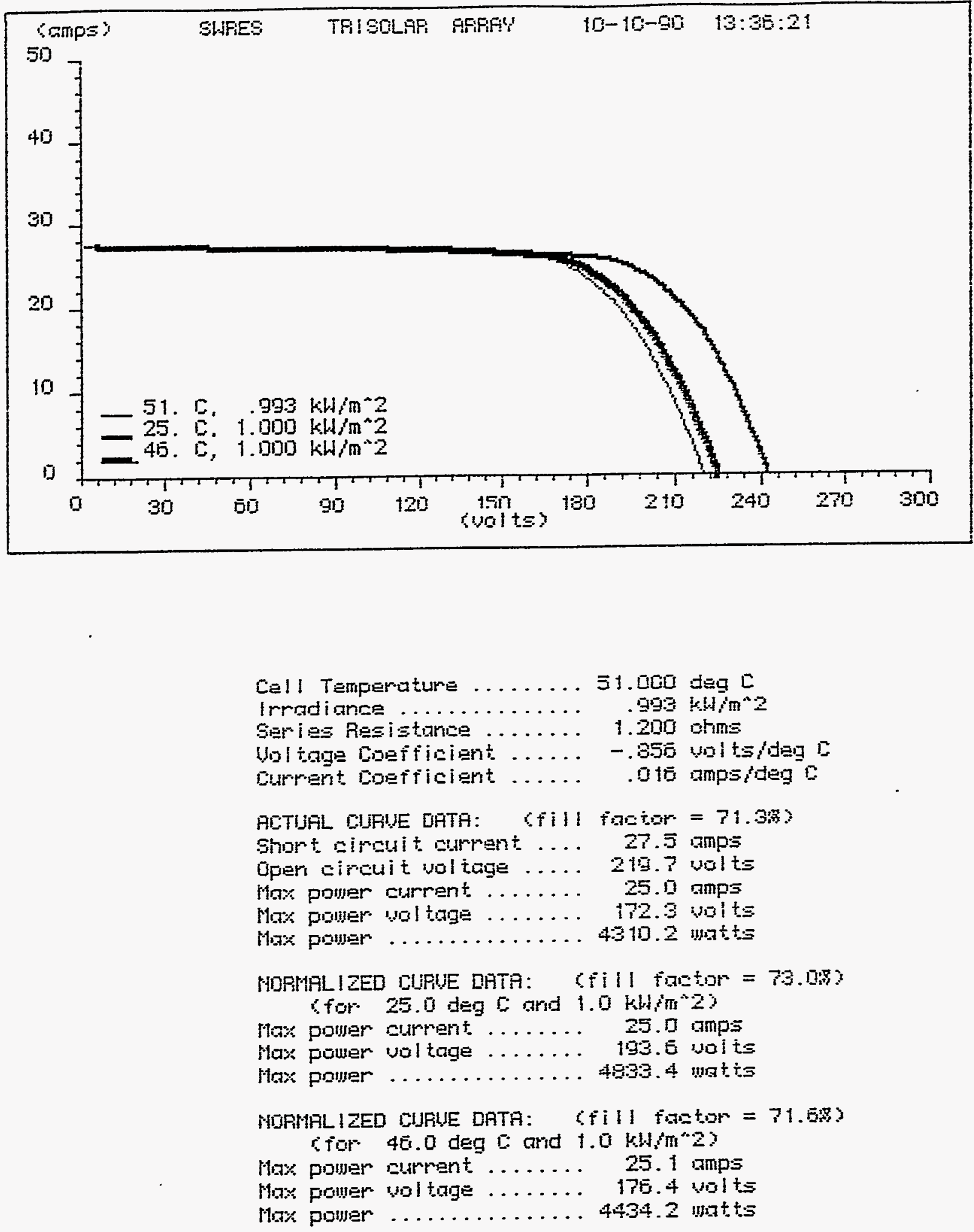


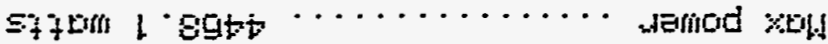
St

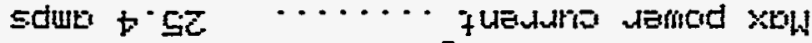

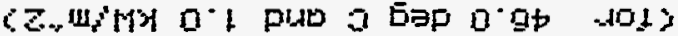

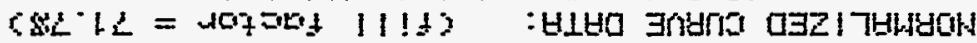

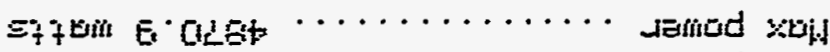

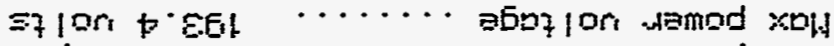

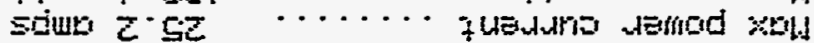

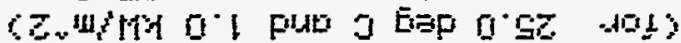

(S) $\theta_{2}=$ do

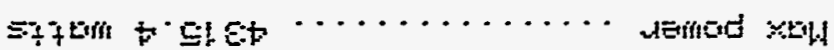

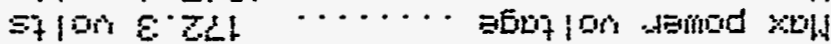

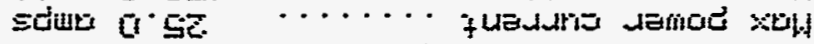

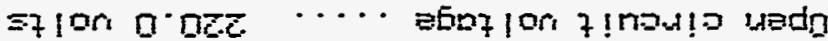
डdwe $5.22 \quad \cdots \cdots$; the cact $12=$ dofos! $111+3$ :

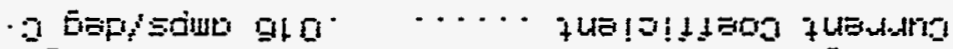
I Eiars

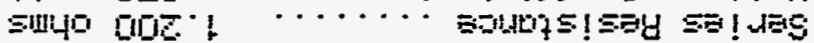

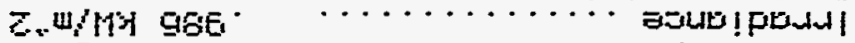

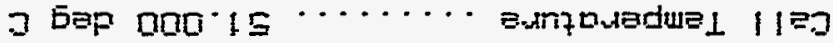

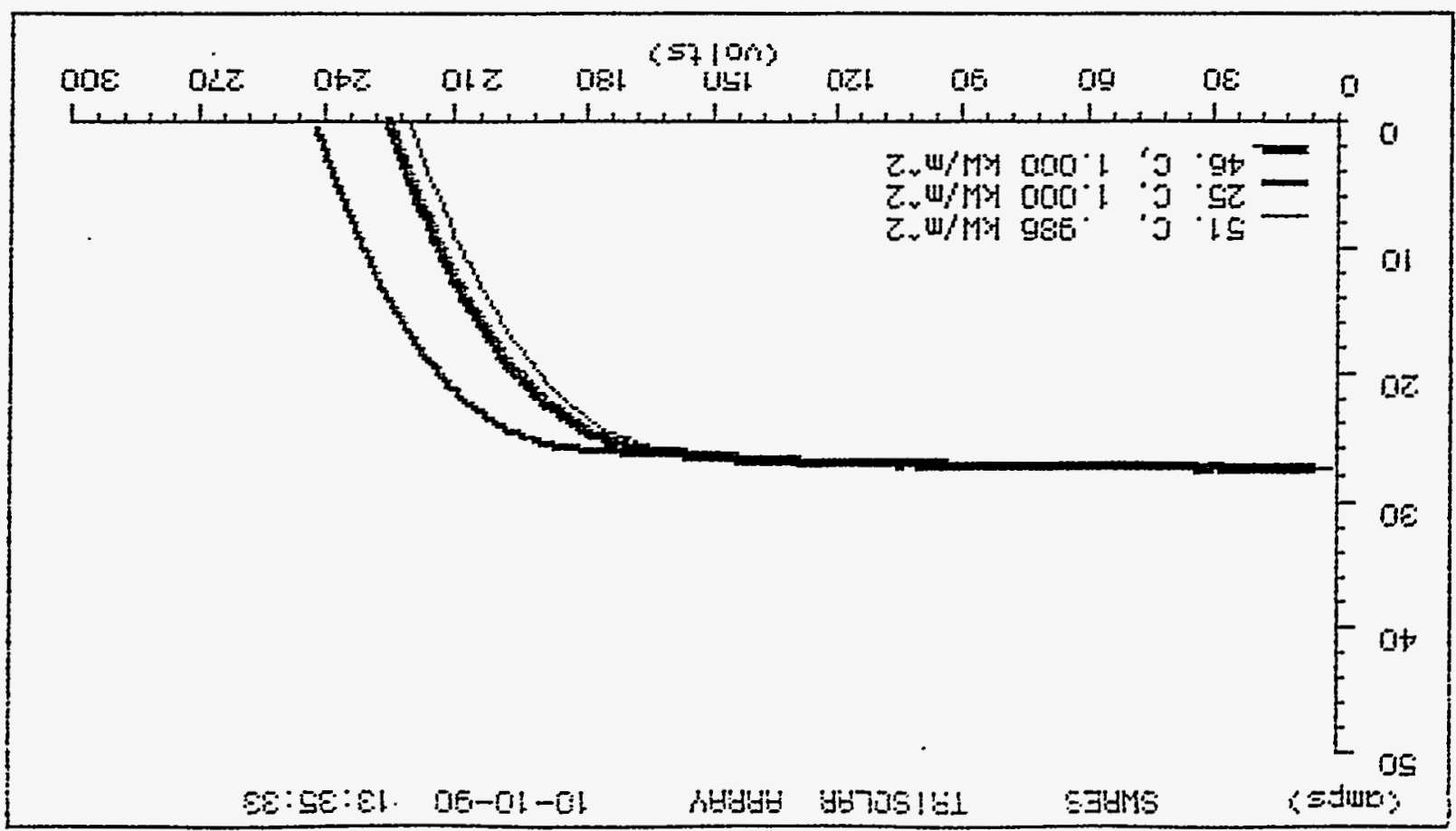




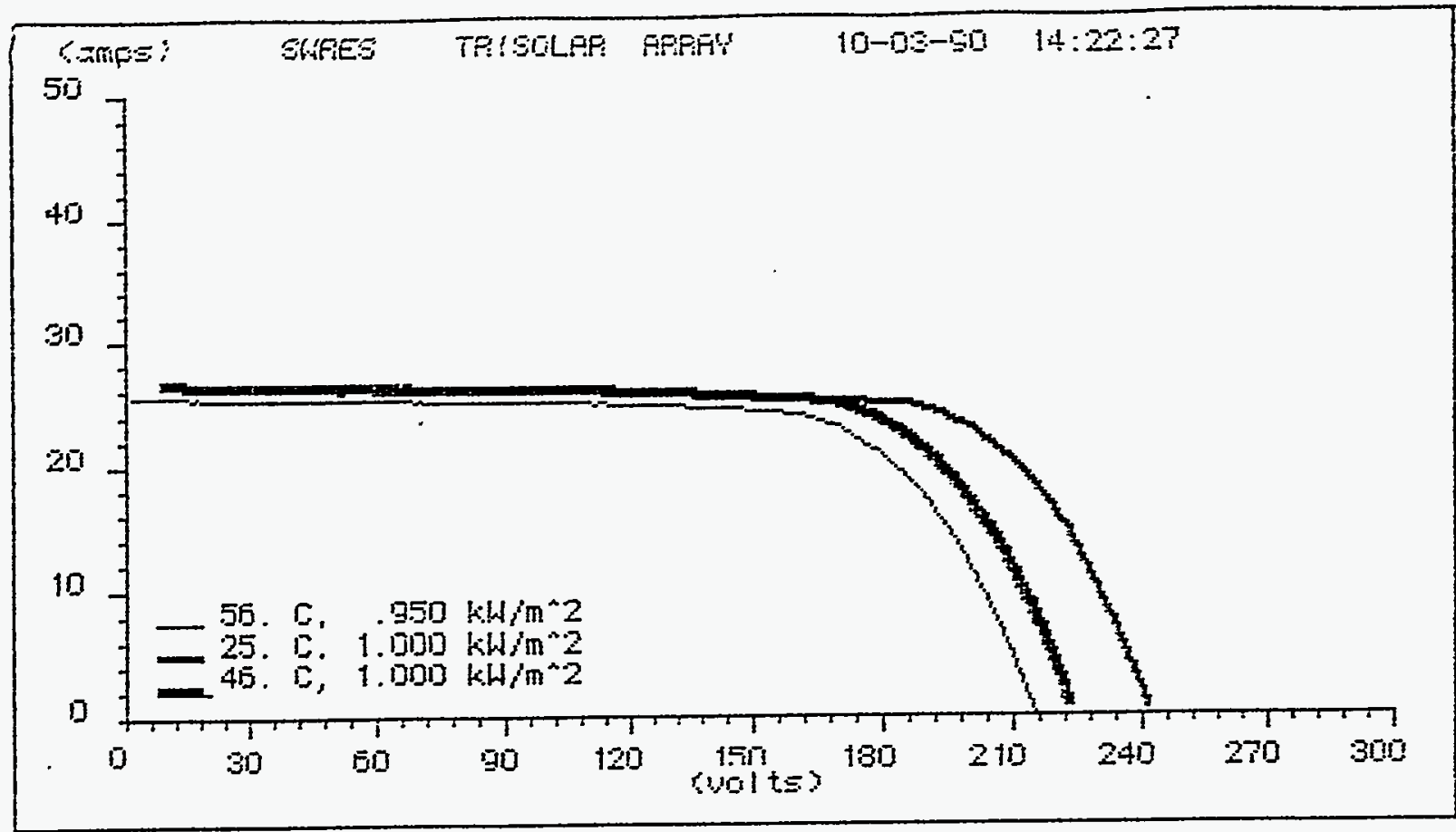

Cell Temperature ....... $3 \overline{0}$. Dư deg $匚$

lrradiance ........... . $95 \mathrm{j} \mathrm{kHim} 2$

Series Resistance ....... 1.200 ohms

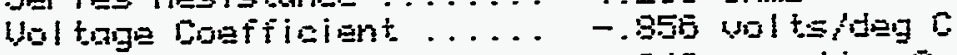

Dirrant coefficient. .... . D to ampeideg

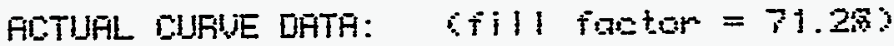

Snort aingdit adrant .... 25.5 amps

Dper cireuit voltage . 2i5.2 volts

Max pouer surrent. ...... 23.3 amps

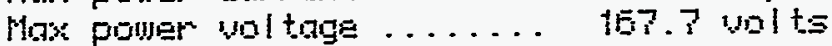

Hor. Fumer .......... 3911.2 wat:

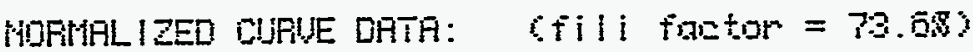

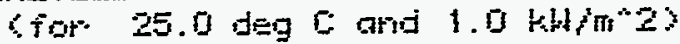

Max pumer aurpent ....... 24.2 amps

Max puwer vol tage ....... 193.3 salts

Max pomer ........... 4557.5 motts

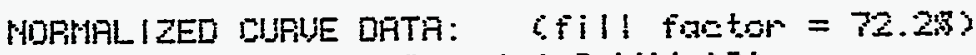
Sior $40.0 \mathrm{deg} C$ ard $1.0 \mathrm{kH},\left(\mathrm{m}^{\circ} \mathrm{Z}\right)$

Max poujer atmmt ...... 24.5 amps

Hax pouser wol tage ...... 174.9 volts

Mase pomer ........... 4233.9 matts 


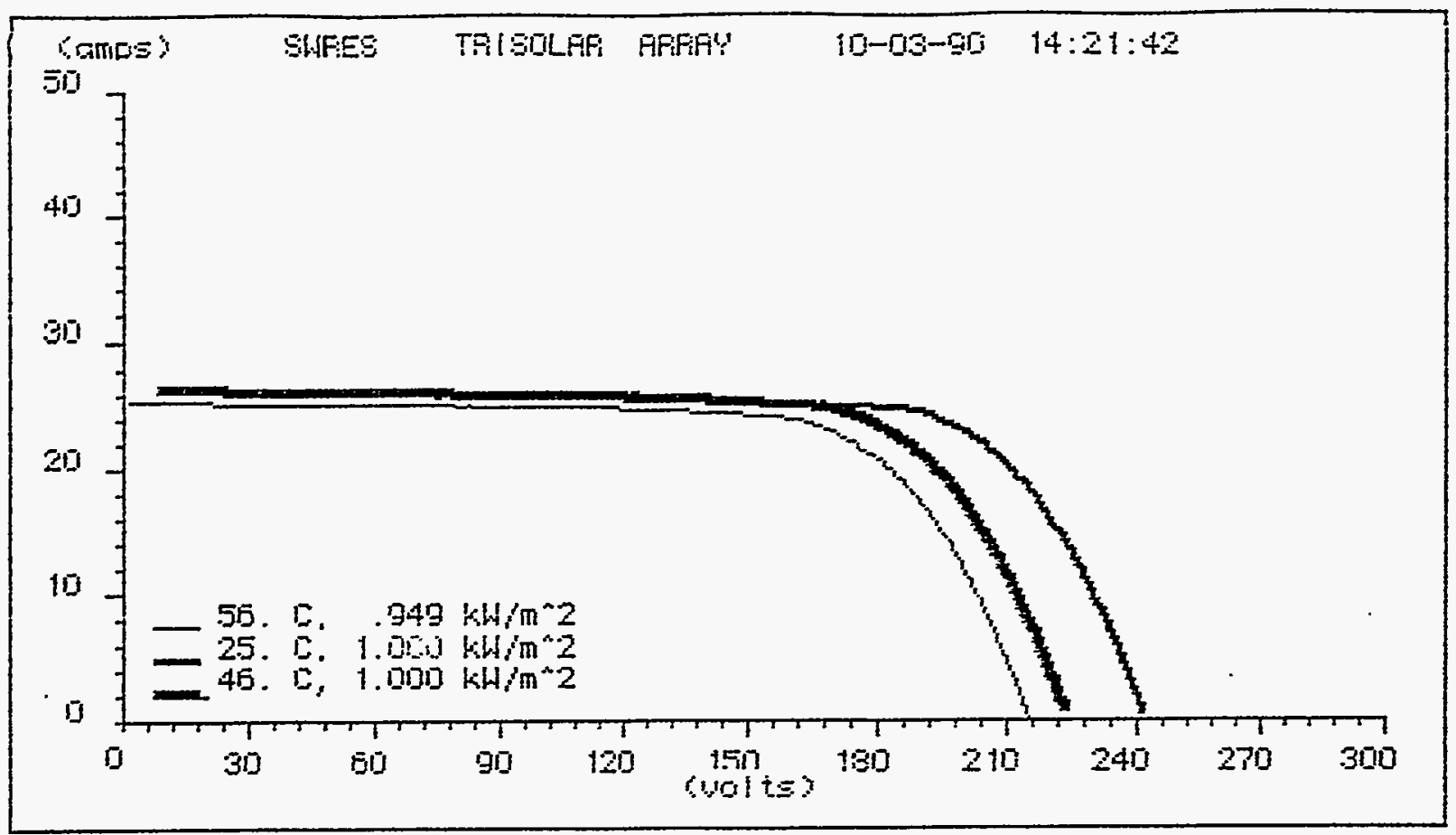

Cell Temperature ....... SE.C00 deg C

Irradiance .......... .949 kH/m²

Series Resistarice ....... 1.200 ohms

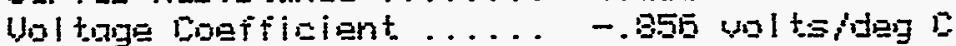

Durrent Doefficient ..... . Di5 empsideg 0

ACTUAL CIURUE DATA: (fill factor $=71.33$ )

Short citruit ourrant. ... 25.5 amps

Upen circuit voltage .... 215.3 volts

Mex pouer surrent. . . . . . 23.5 mps

Max pouner voltage ...... 156.4 volts

Max pouser ............ 3020.5 mutts

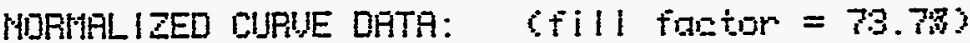

(for 25.0 deg 0 and $1.0 \mathrm{kH} / \mathrm{m} \times 2$ )

Max pomers curment. ...... 24.4 anps

Max puwer val tage ....... 191.9 jal $t s$

Mrop prwer ............ 4597.0 watts

MOPHALIZED DURUE DATA: GIII fGEtor $=72.2 \mathrm{~s})$

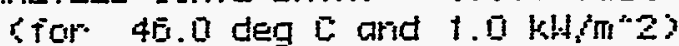

Max poumer cturent. ...... 24.9 amps

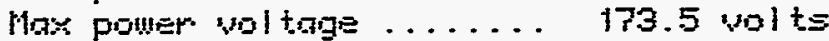

Mox pourer ........... 4297.8 ujatts 


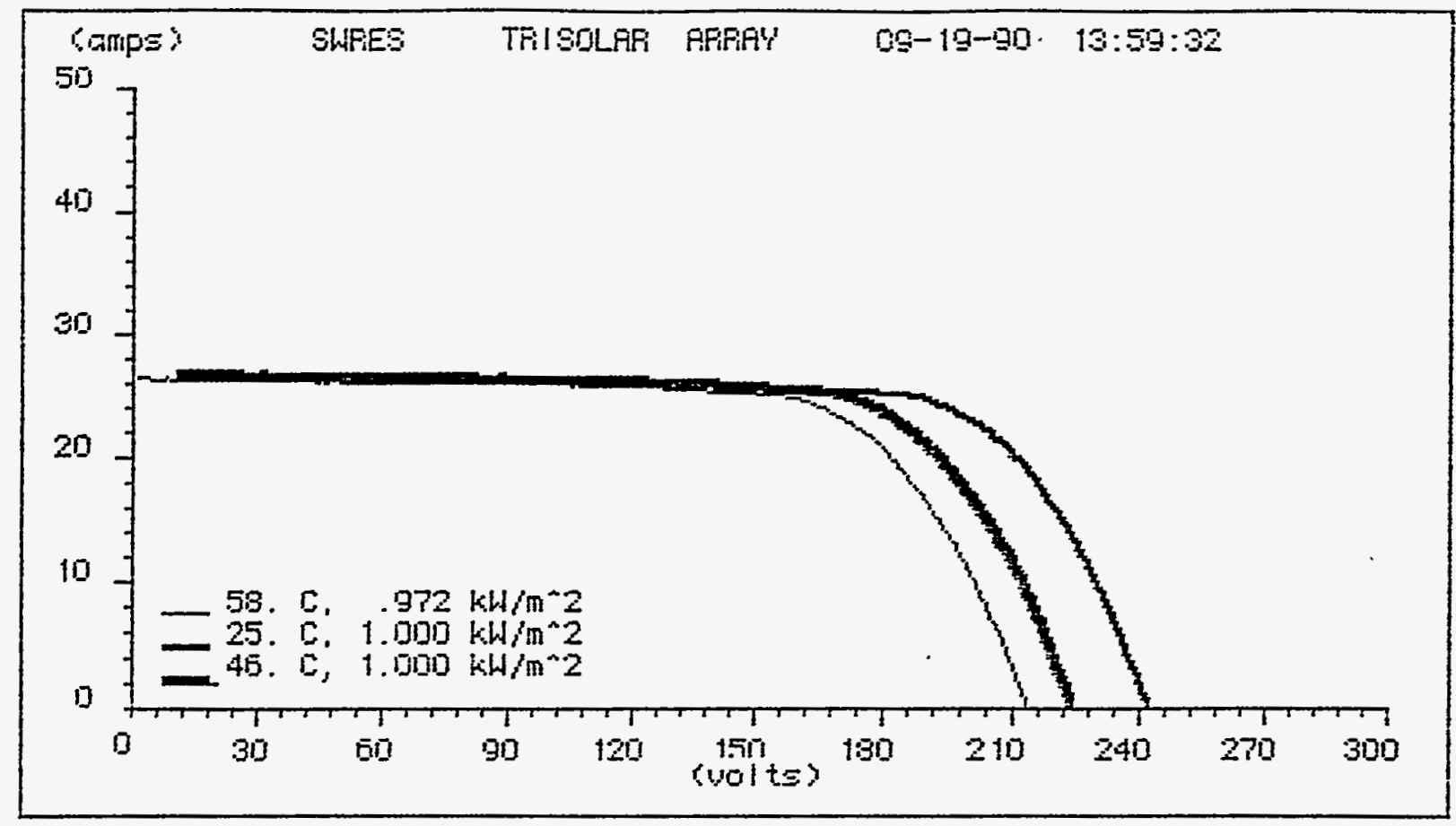

Cell Temperature ........ 33.000 deg $\mathrm{L}$

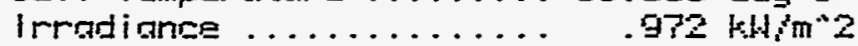

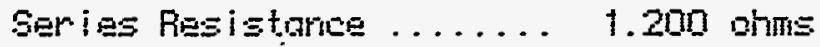

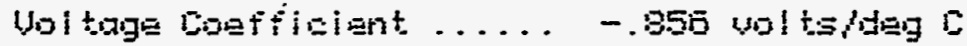

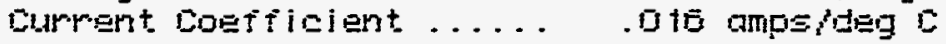

FCTUAL CURUE DATA: (Till factor $=70.8 \% 3$

Shart cimault asment. .... 25. 4 amps

Upen cireuit ualtage ..... 213.4 wo!ts

Max power curment. ...... 24. 1 amps

Max pguer voltage ....... IE. 4 volts

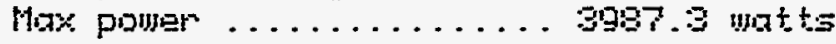

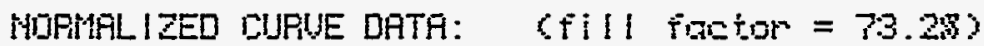

(for 25.0 deg 0 and $1.0 \mathrm{kH} / \mathrm{m}^{\wedge} 2$ )

Max poner ouppent. ...... 24.5 amps

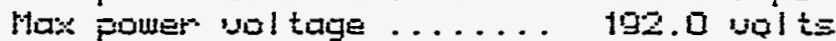

Mrs power ...........4.708. I matts

HDFMALIZED DURUE DATA: fill fantor = 71.3B?

Sior 4 E. 0 deg $C$ ard $1.0 \mathrm{kH} / \mathrm{m}^{*} 2$ )

Max pouser current. . . . . . 24.7 amps

Mex pomer sol t.3ge ...... 175.0 walts

liax pobser ...........4.477.9 watts 


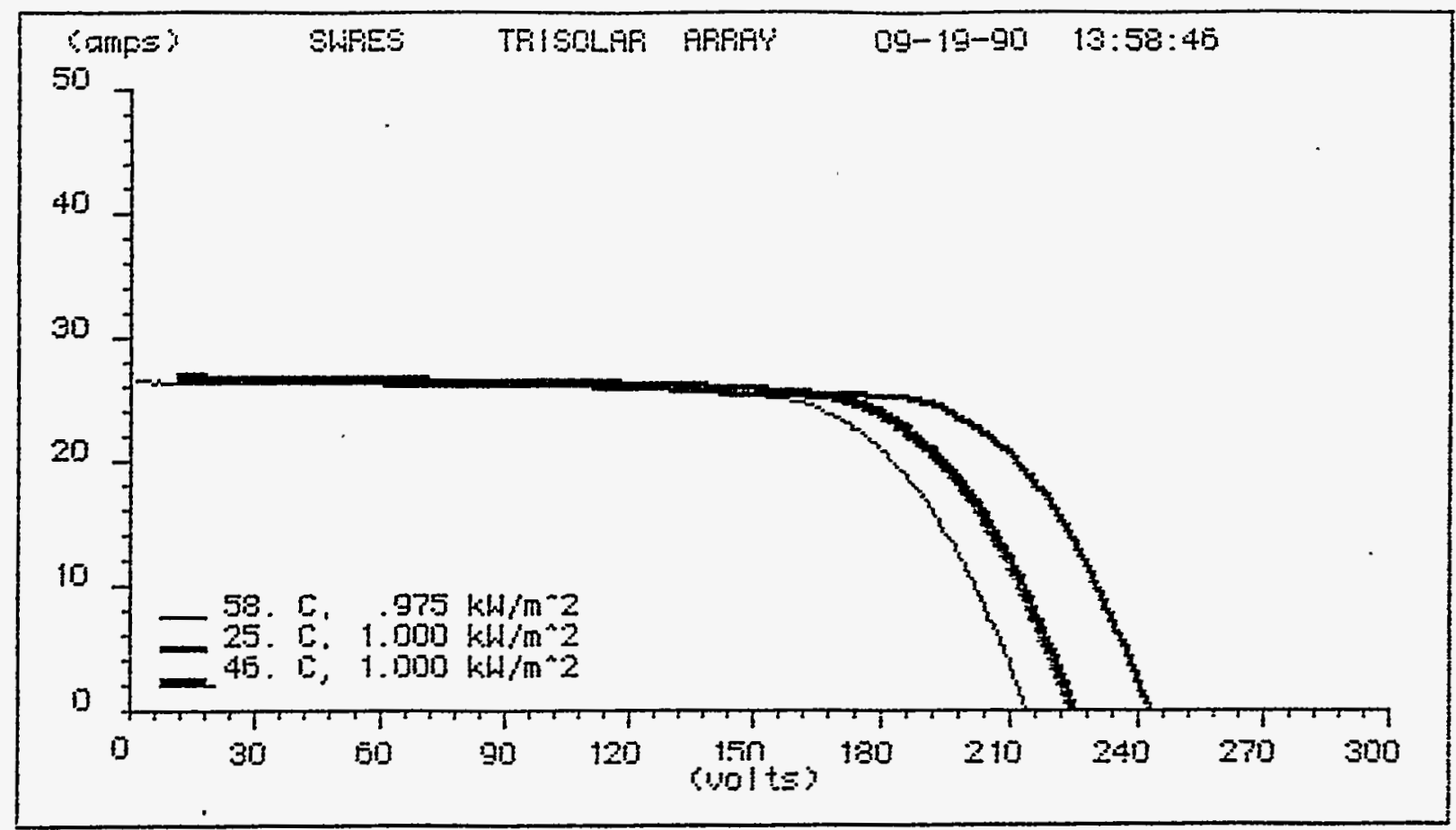

Cell Temperature ........ SE. Quad deg $L$

Irradiance ........... .975 kM/m²

Sen ies Resistance ....... 1.200 ohms

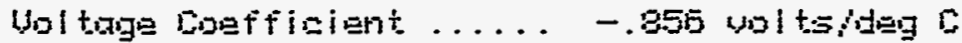

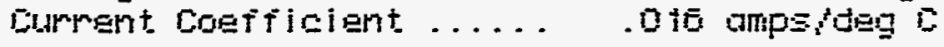

AITUHL CIJALE DATA:

Short cirauit aurnent. ... 25.5 smp:

Upen Eircuit vol tage ..... 213.9 volts

Max power cturment. ...... 24.2 amps

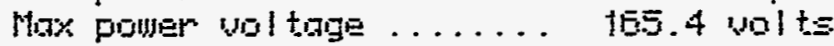

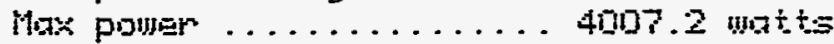

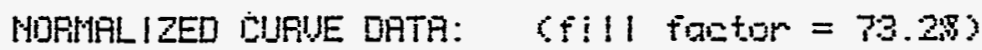

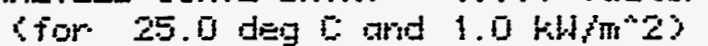

Max pourer aturrent ...... 34.4 amps

Max power vol tage ....... 193.4 volts

Hax power ...........4 4715.4 wat $t=$

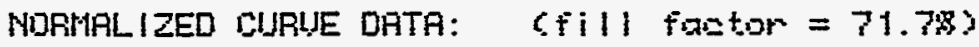

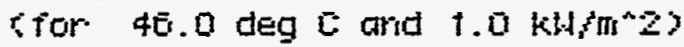

Max poujer currant. ...... 24.7 amps

Max power sol tage ....... 175. 1 vol ts

Max poumer ........... 4.327.2 ugtts 


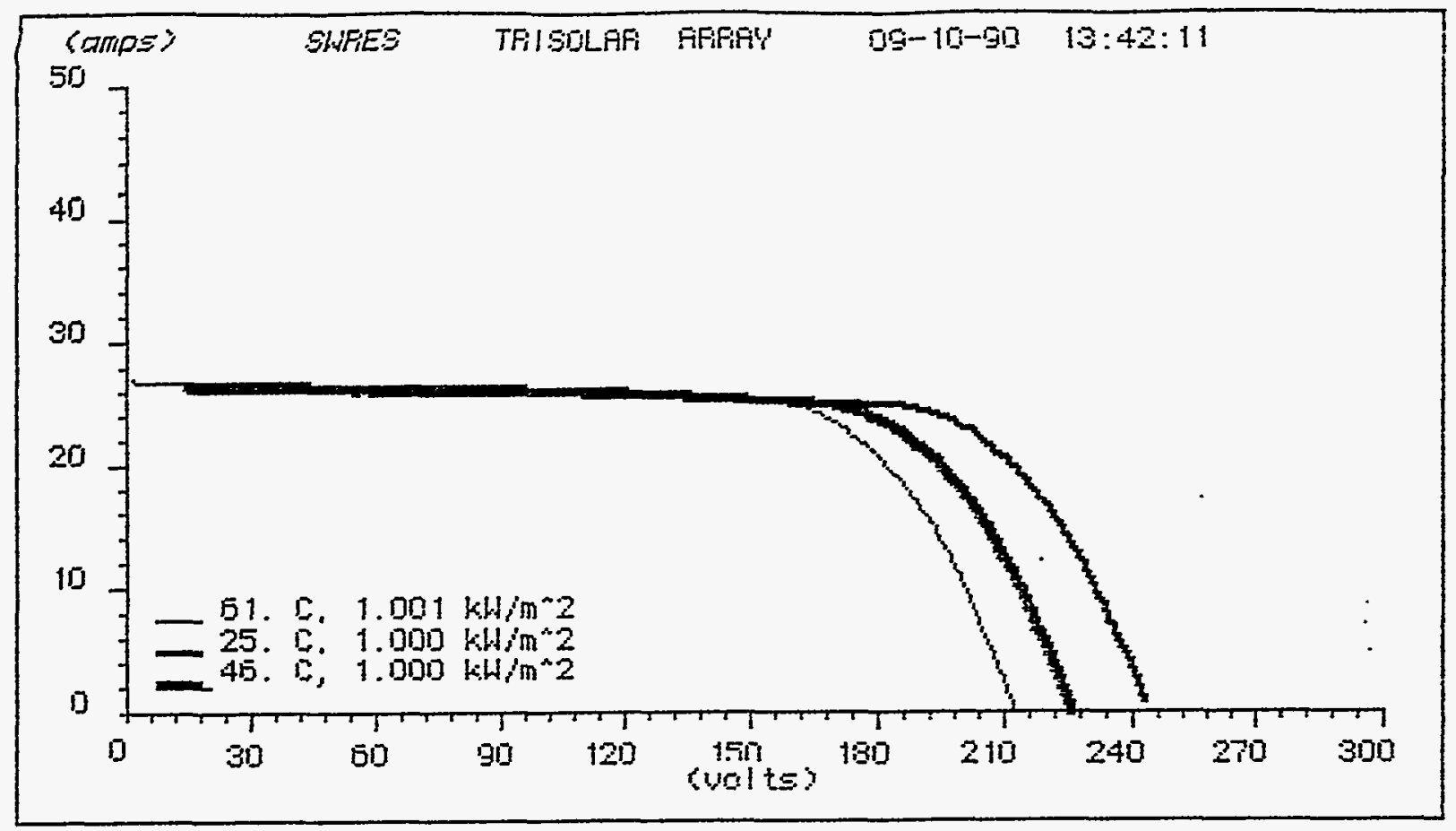

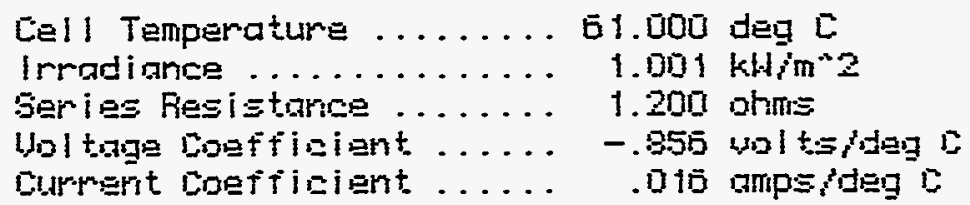

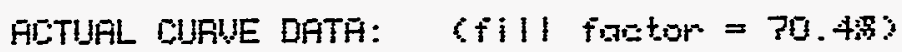

Short circuit current ... 25.9 umps Upen eircuit wol tage .... 212.5 volte Max poijer current . . . . . 24.5 nmps Max pouner voltuge ....... 104. I wolts Mow poumar ........... 4025.0 matt=

HORMALIZED DURUE DATA: ifill fantor $=73.0 \%$

(for 25.0 deg [0 and $1.0 \mathrm{kH} / \mathrm{m}^{*} 2 \mathrm{y}$

Mox pouler aturgent. ..... 24.1 amps

Max power uol tage ....... 194.4 jolt $=$

Max pouner . . . . . . . 4585.7 wntts

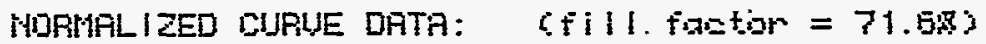

ifor 40.0 deg $C$ and $1.0 \mathrm{kH}\left(\mathrm{m}^{\circ} \mathrm{2}\right)$

Max pouser aurpent. ...... 24.3 amps

Max pouner voltage ....... 177.2 wolts

Max poun .......... 4302.6 matts 


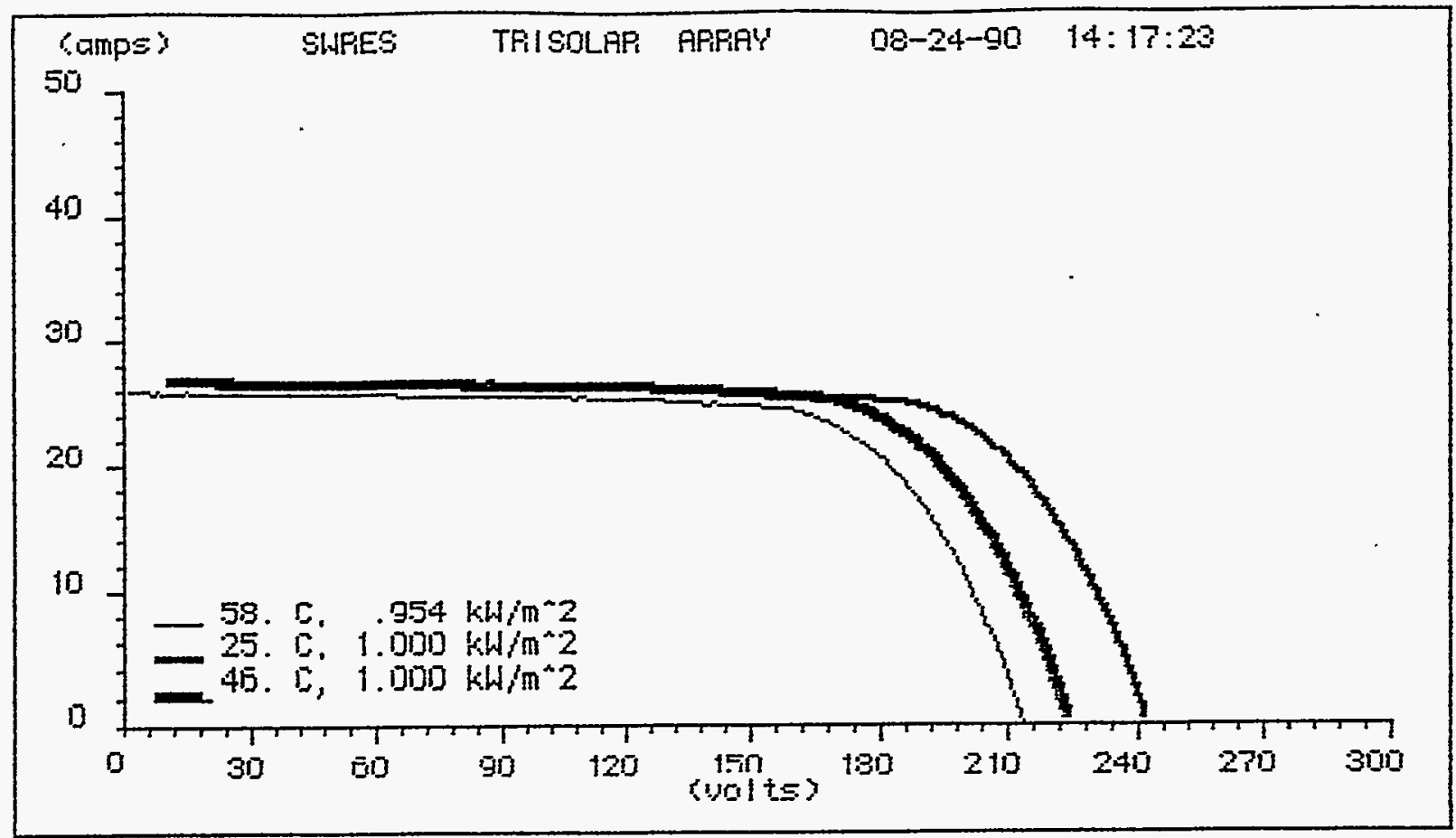

\footnotetext{
Cel I Temperature ....... 53.000 deg $c$

Irradianee .......... .954 $\mathrm{kH} / \mathrm{m}^{2} 2$

SEr i es Resi $\equiv$ tance ....... 1.200 chm!

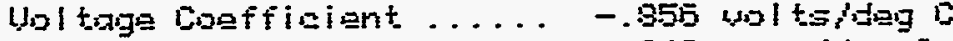

Eumrent Doefficient ..... . Dto ampsideg $\mathrm{E}$

AETUAL CURUE DATA: $\quad$ OHII fuEtor $=71.0 \mathrm{~g}$

Short ciradit cumpent .... 25.9 amps

Uperi cimcuit valtage .... 213.5 volts

Max pouser Eurrent ...... 2:.8 amps

Max power wal tage ....... 155.4 wolts

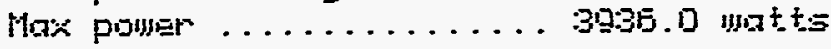

MDRHALIZED DUPUE DATA: ifill fantor $=73.5 \mathrm{~B}$

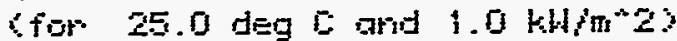

Max pouper aurment ...... 24.5 amps

Max power vol tage ....... 192.3 voilt:

Max power ...........4 4725. 1 matts

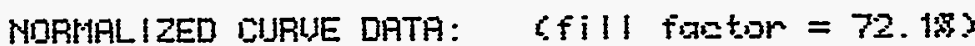

ifor 40.0 deg $C$ ard $t .0 \mathrm{kH} / \mathrm{m}^{*} \mathrm{Z}$ )

Hox pouser cirrent ...... 24.9 anps

Max power usl tage ....... 174.4 wolts

Max pouler ........... 4.3.34.3 נ⿲丨丨
} 


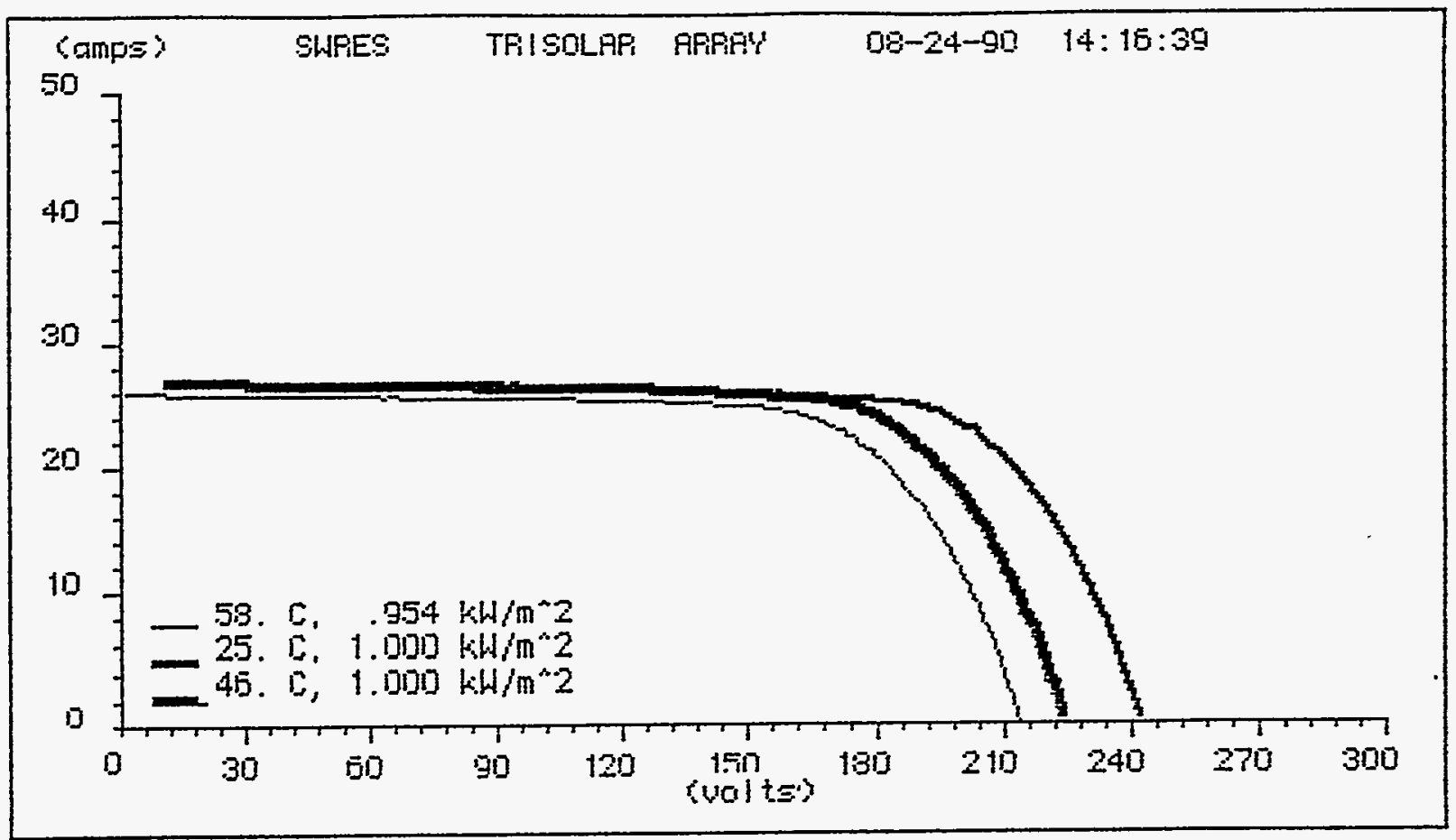

Cell Temperature ........58.000 deg $c$

Irmadiance ........... . $954 \mathrm{kH} / \mathrm{m}^{2} \mathrm{z}$

Ser i Es Ris istance ....... 1.200 ohms

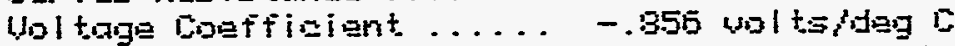

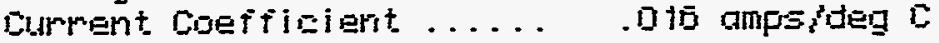

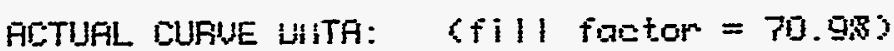

Ghort circuit cumpent. ... 25.0 amps

Open circuit vol tage .... 214.0 volts

Max pouer current ....... 23.7 amps

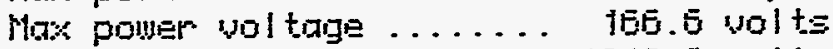

Max pumar ........... 3943.9 ynatts

MORHALIZEO CIUPUE DATA: CFill foofor $=73.483$

ffor 25.0 deg 0 orid $1.0 \mathrm{kH} / \mathrm{m}^{\wedge} 2$ ?

Max poujer aurrent ....... 24.5 amps

Max power vol tage ....... 192.3 ujlts

Max power ........... 4331.3 motts

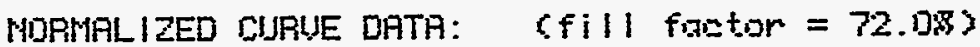

(fior 40.0 deg $c$ and $1.0 \mathrm{kH} / \mathrm{m}^{\wedge} 2$ )

Max power aurrent ....... 24.7 amps

Max power wol tage ....... 175.5 wolts

Max power .......... 4342.7 unatts 


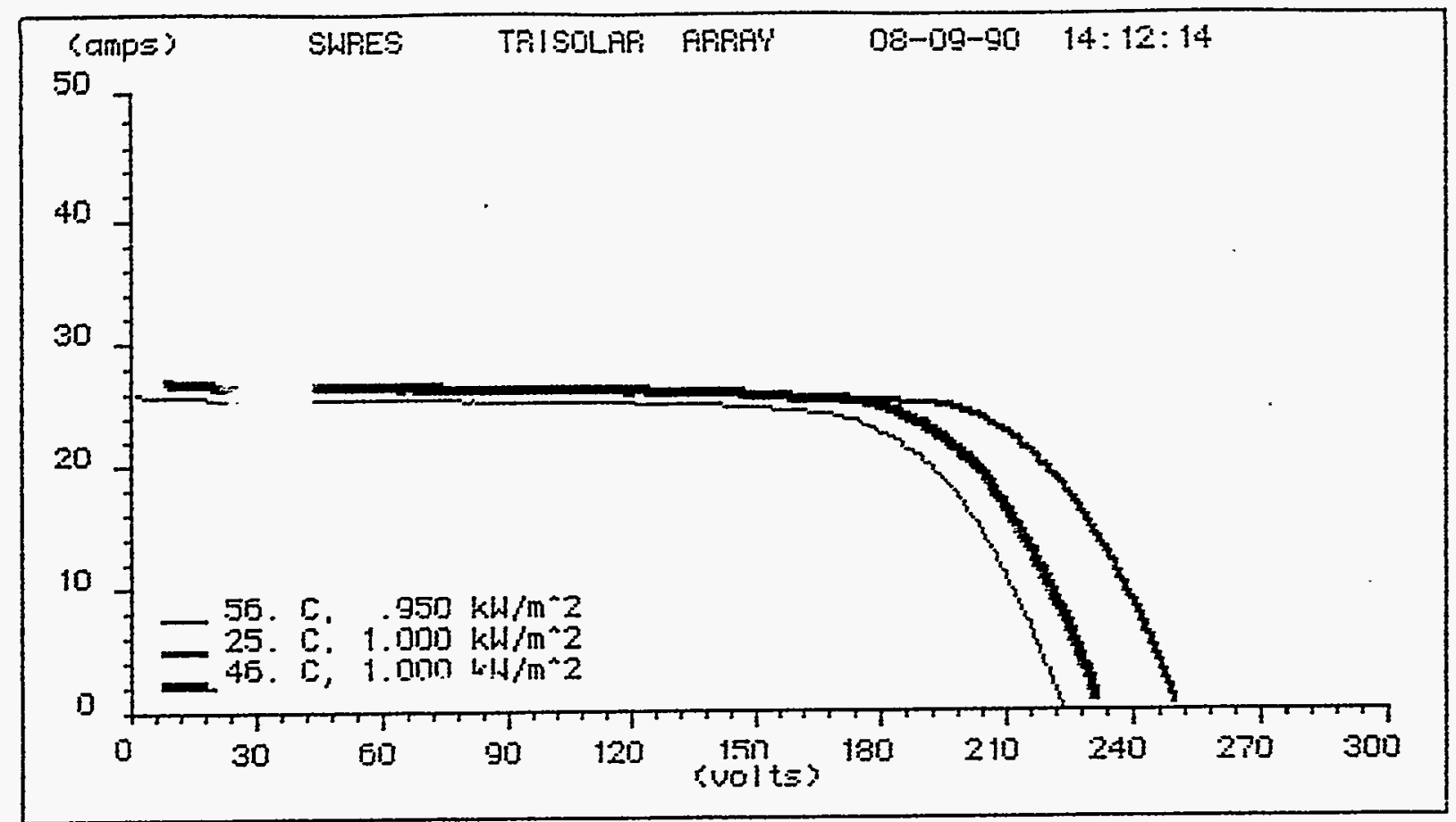

\footnotetext{
Eell Temperature ....... 56.000 deg [ Irradiance ........... . $950 \mathrm{kH} / \mathrm{m}^{2} \mathrm{z}$ Series Resistance ...... t.200 ohms Uol tage coefficient ..... -.855 wolts/dag c Current coefficient ..... . Dto ampsideg $\mathrm{D}$

ACTUAL CURUE DATA: (fill factor $=71.2 \mathrm{~T}$ ) Short simauit aurrent ... 25.0 amps Open circuit uol tage .... 223.4 wolt Max power cturrent ....... 23.5 smps Max power vo! toge ....... 174.6 volt.s Max pouner .......... 4127.3 matss

MOPIALIZED CURUE DATA: Gfill factor $=73.48 \%$ (for 25.0 deg 0 ard $1.0 \mathrm{kH} / \mathrm{m}^{*} 2$ ?

Max pours gurrent ....... 24.5 amps

Max power vol tage ....... 200. 1 vol ts Mrox power ........... 4902.3 matts

HOPMFLIZED CUPUE DATA: (fill faEtor $=72.13$ ) (ior 40.0 deg $C$ and $1.0 \mathrm{kH} / \mathrm{m}^{\wedge} 2$ )

Max poujer gurpent ....... 24.3 amps Max power vol tage ........ 181.7 nol ts Max pouner ........... 4514.5 matts
} 


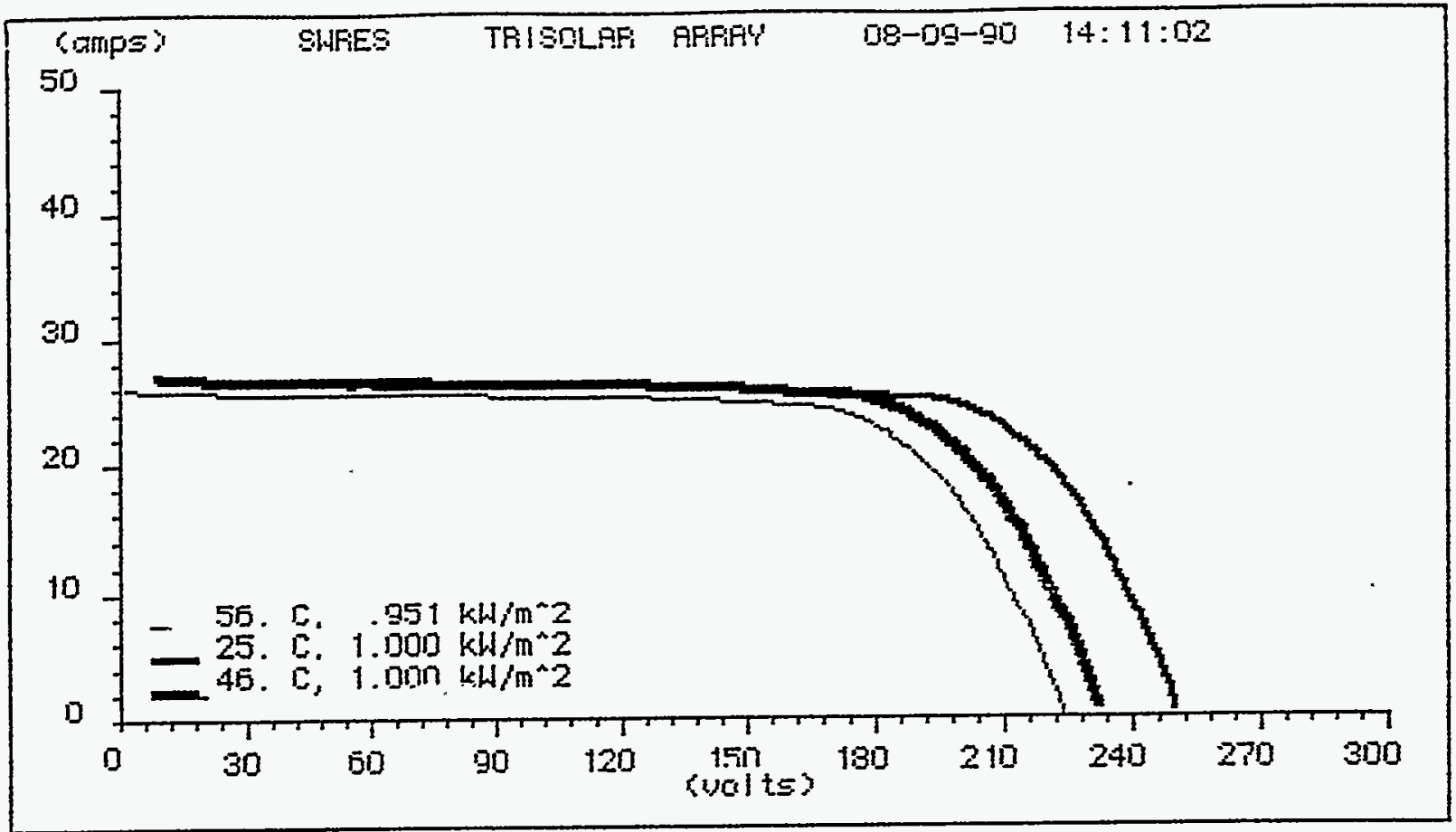

Lél Temperature ........

Irradiance ............ . $951 \mathrm{kH} / \mathrm{m}^{2} \mathrm{z}^{2}$

Series Resistanie ....... t.200 otm -

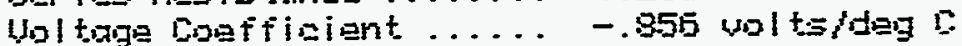

Cumpent Doefficient..... . Dib ampsideg $\mathrm{C}$

ACTUAL CURUE DATA: - (Aill faotor $=71.4 \%$ )

Short airouit current. . . 25.0 amps

Gpen circuit valtage ..... 223.3 volts

Max pouer current. ...... 23.4 amps

Max pouser vol tage ....... 175.9 volts

Max pouner ...........4 447.7 matts

WDPIALIZED CURUE DATA: (Fill factor $=73.63$ )

(for 25.0 deg $\left[\right.$ and $1.0 \mathrm{kH} / \mathrm{m}^{\circ} 2$ )

Max pouser current. ...... 24.3 smps

Max puwer vol tage ...... 202.5 jol ts

Max power ............4 4914.2 matts

MIPHALIZED CURUE DATA: (fill fagtor $=72.3 \times 3$

(ior $40.0 \mathrm{deg} C$ and $1.0 \mathrm{kH} / \mathrm{m}^{\wedge} \mathrm{2}$ )

Max pouser current ....... 24.5 anps

Max poujer vol toge ....... 184. 1 volts

Max pouser ............ 4531.4 uatts 


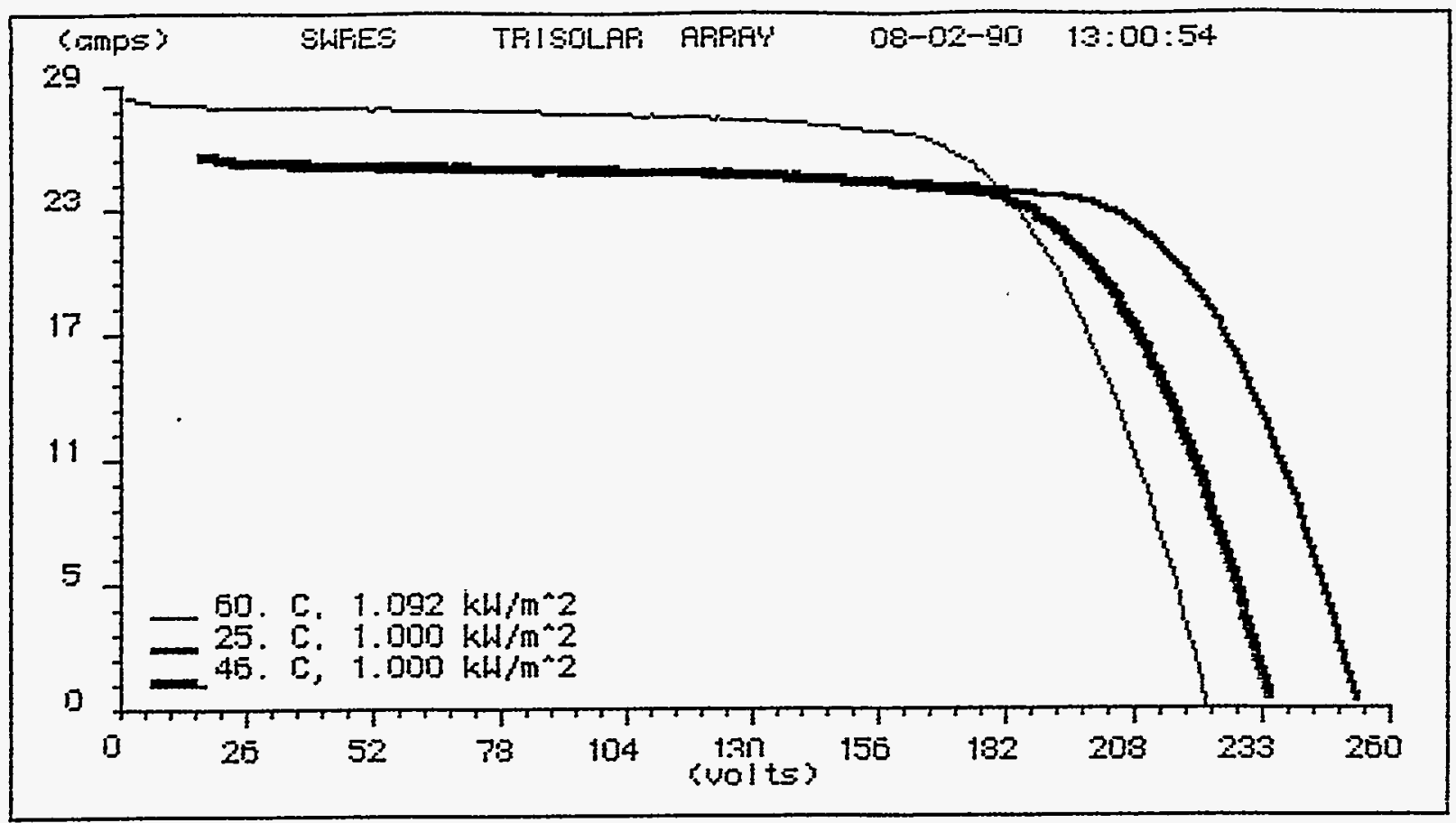

\footnotetext{
Cell Temperature ...... 50.000 deg L

Irradiance ........... $1.092 \mathrm{kH} / \mathrm{m} \% 2$

Series Resistance ....... 1.200 ohms

Voltage coeficient ..... - .850 volts $/ \mathrm{deg} c$

Current Coefficient ..... . Dis ampsideg $c$

RCTUAL DURUE DATA: (fIII factor $=70.13$ ?

Shomt eireuit current .... 29.4 amps

Jpen sircuit vol tage .... 222.5 volts

Max power current ...... 26.3 amps

Max poumar ual trage ....... 171.2 uolt:s

Max power ........... 4584. 1 matts

NORMALIZED CURUE DATA: (fill factor $=72.13$ )

(fior $25.0 \mathrm{deg} C$ and $1.0 \mathrm{kH} / \mathrm{m}^{2} 2$ )

Max pouter current ...... 24.2 omps

Max pomer voltrge ...... 201.3 volts

Max pouser.......... 4873.0 watts

MORMALIZED CURUE DATA: (fill factor = 70.7X)

(for 46.0 deg $C$ and $1.0 \mathrm{kH} / \mathrm{m}^{2} 2$ )

Max poiver current. ...... 24. 1 amps

Max pouner woltage ....... 185.4 wolts

Max power ............ 4491.9 watts
} 

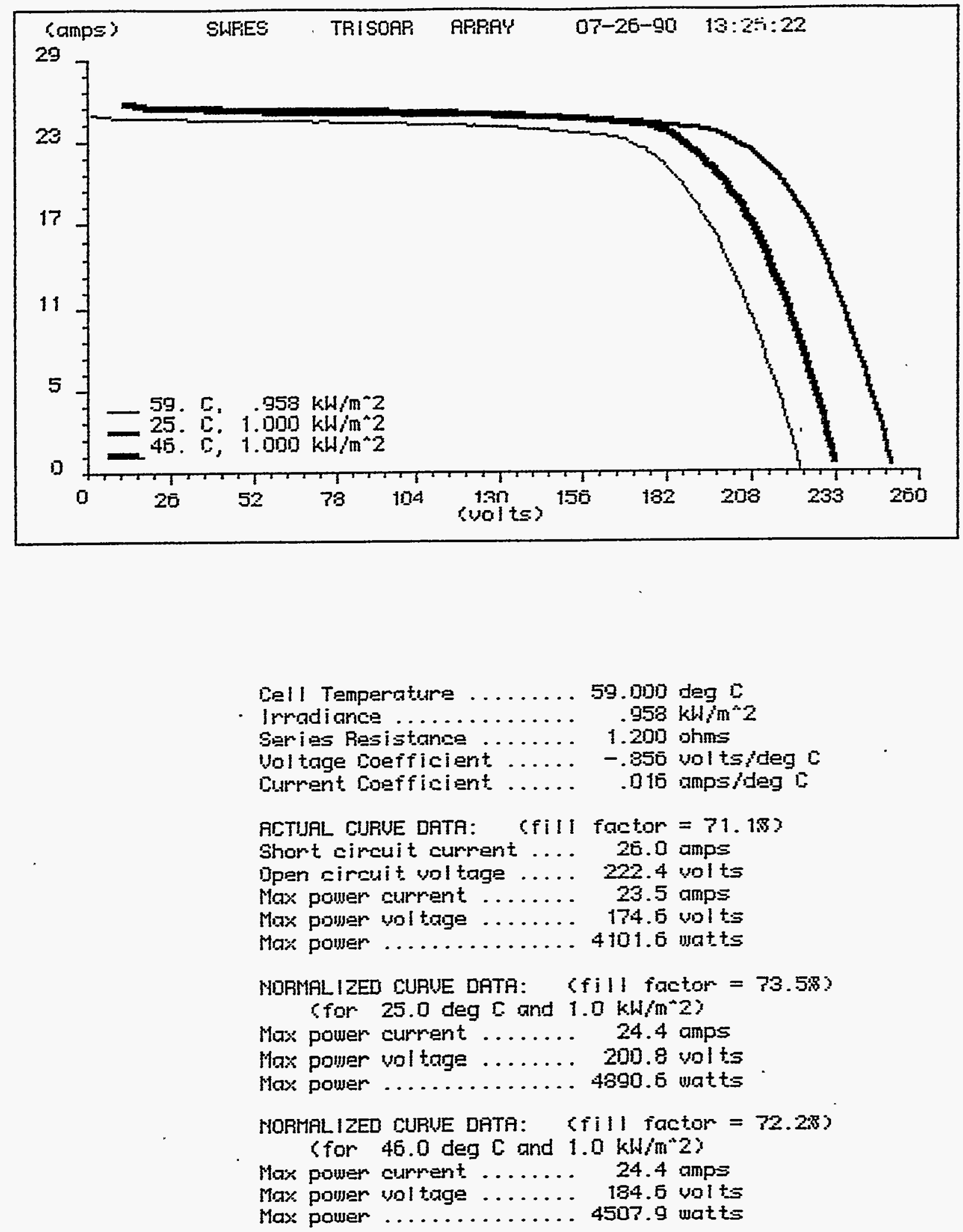


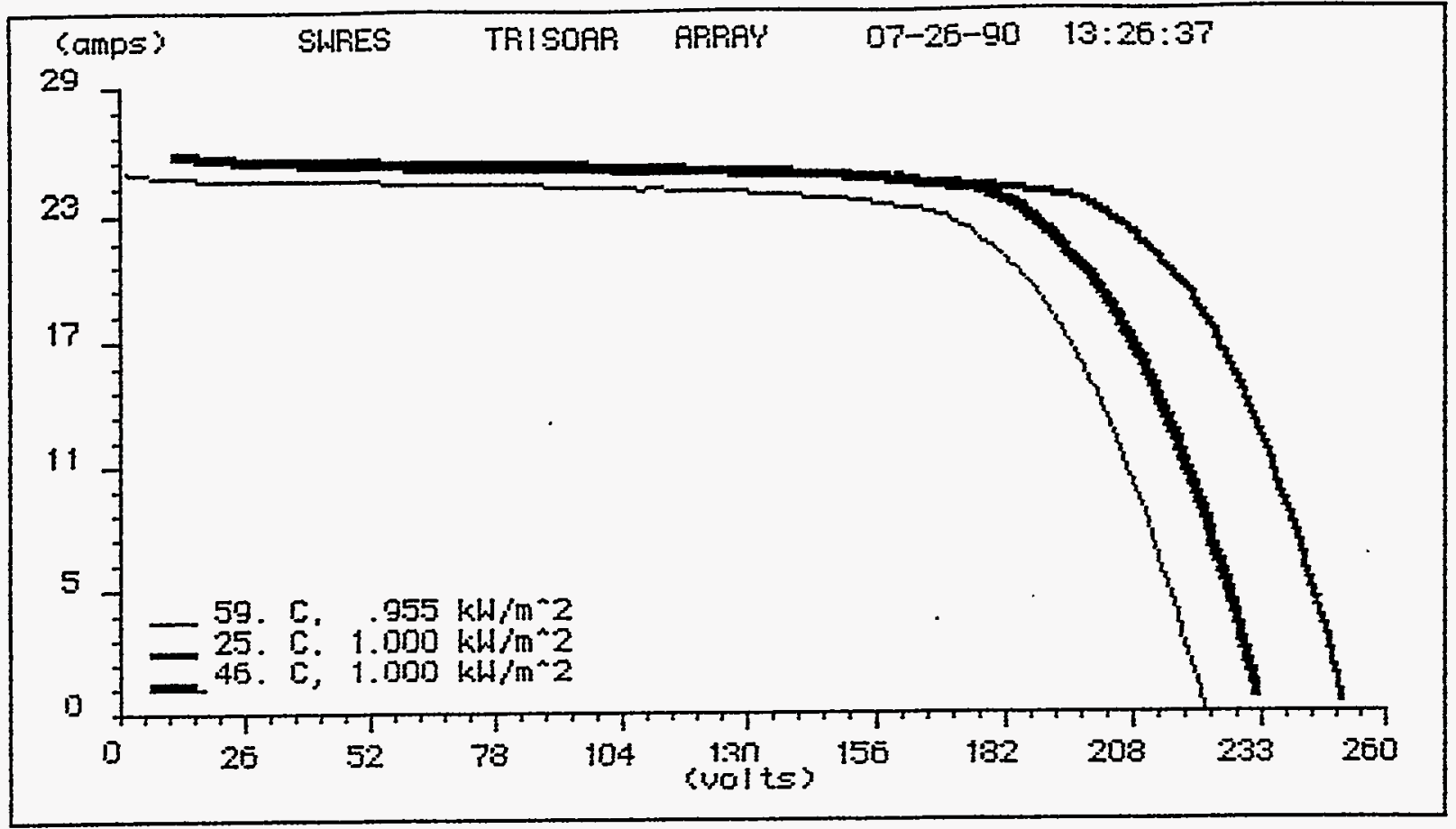

\footnotetext{
Cell Temperature .......59.000 deg D

Impradiance ............ $.955 \mathrm{kH} / \mathrm{m}^{\circ 2}$

Suries Resistanoe ...... 1.200 onms

Woltage coefficient ..... -.850 volts $/ \mathrm{deg} c$

Current Cogfficient ...... .015 umps/deg C

RCTURL CIJRUE DATA: (FIII faCtor $=71.13$ )

Short circuit current .... 25.9 amps

Dpen sircuit uol tage .... 222.1 volts

Max power curpent ....... 23.5 amps

Moxx powar wol tage ....... 173.5 wolts

Max power ........... 4087.8 watts

NORMALIZED CURIJE DATA: (AIII fuEtor $=73.5 \mathrm{~s}$ ?

(for $25.0 \mathrm{deg} \mathrm{L}$ and $1.0 \mathrm{kh} / \mathrm{m}^{\wedge} 2$ )

Max power current ....... 24.2 amps

Max pouner vol tage ...... 201.9 volts

Max power ........... 4838.5 matts

NORMALIZED CUFIJE DATA: (Till factor $=32.23\rangle$

(for $46.0 \mathrm{deg} C$ and $1.0 \mathrm{kN} / \mathrm{m}^{*} 2$ )

Max pouper cumpent. ....... 24.6 amps

Max pourer uoltage ....... 183.5 volt.s

Max power ............ 4506.3 watts
} 


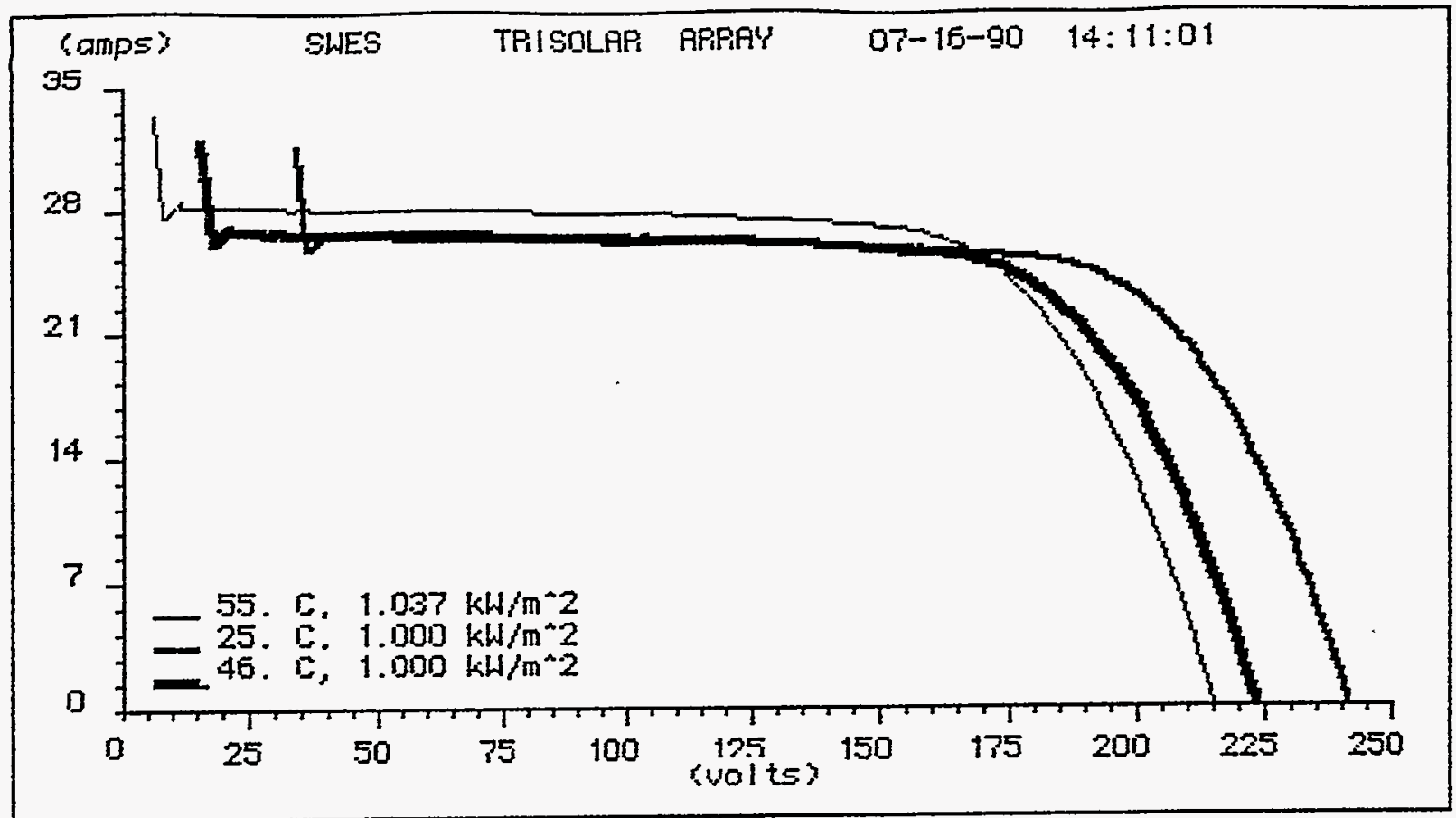

Sell Temperature .......5.5.0100 deg $\mathrm{D}$

Irradiance .......... $1.037 \mathrm{kH} / \mathrm{m}^{\wedge} \mathrm{Z}$

Saries Resistunias ....... 1.200 ohms

Valtage Coefficient ..... - - .855 sol tsideg I

Current coefficiant ..... . . D15 anpsideg $\mathrm{C}$

ACTURL CIURUE DATA: (fill fätor $=59.9 \%$ )

Shart circuit current .... 33.4 amps

Dpen cirruit unl tage .... 215.2 uplts

Max pouner current ....... 25.8 amps

Mox poinar ual taje ....... 155.4 us 1 t.s

Max polver ............ 4290.7 wotts

NORMALIZED CURUE DATA: (fill factor $=61.08$ ?

Sfor 25.0 deg $C$ ard $1.0 \mathrm{ks} / \mathrm{m}^{\wedge} 2$ )

Mas pawer current ....... 24.6 amps

Max pomer voltage ...... 190. Tolts

Max power ............ 4598.3 wgtts

HORMALIZED CURUE DRTA: \{

(for $46.0 \mathrm{deg} C$ and $1.0 \mathrm{kH} / \mathrm{m}^{*} 2$ )

Max power curment ...... 24.7 amps

Max polver usltage ....... 174.5 uplts

Max pawer ............ 4306.3 watts 

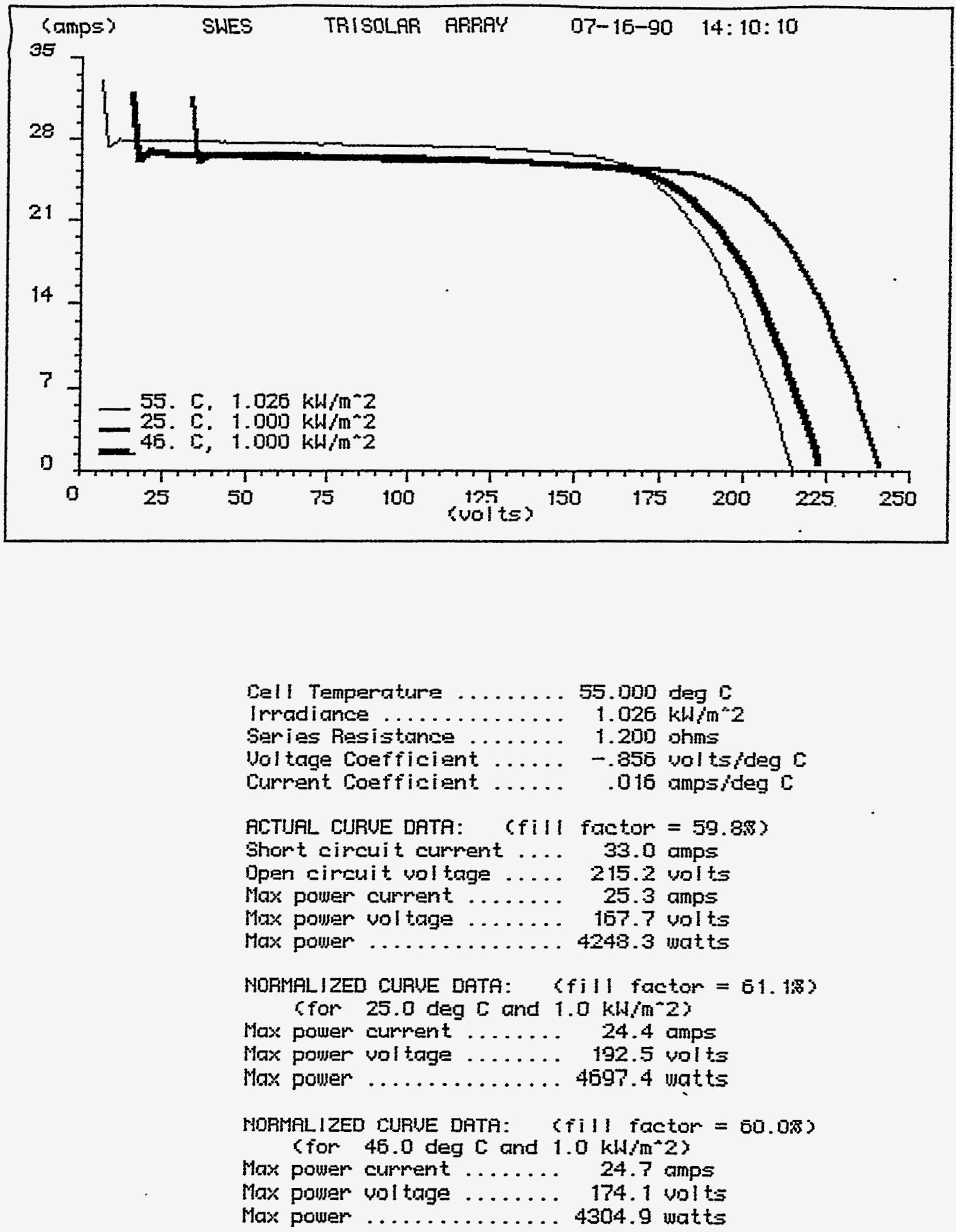


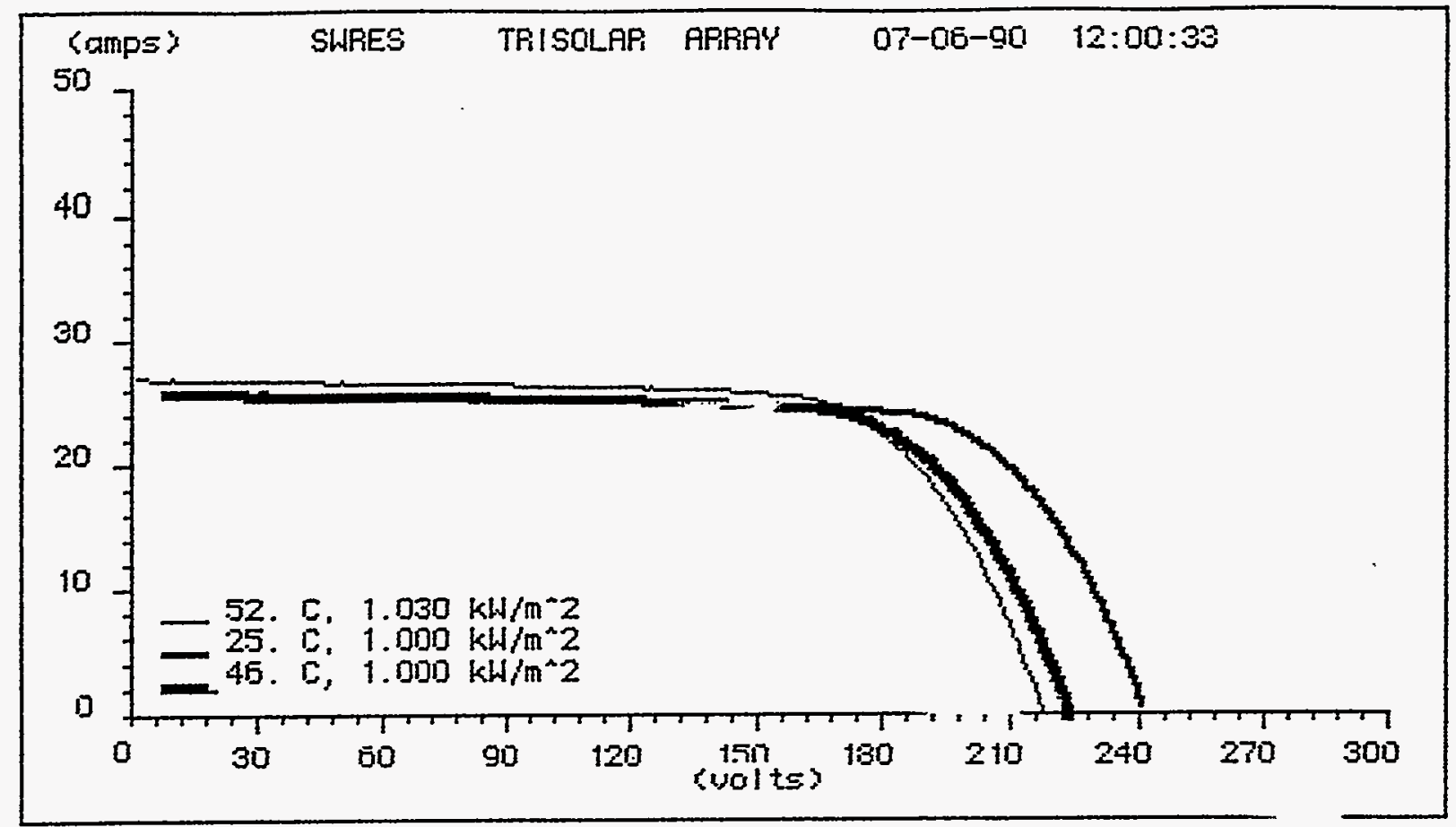

Cell Temperature ....... 52.000 deg C

Irradiance ........... 1.030 kh/ $/ \mathrm{m}^{\star 2}$

Series Resistance ....... 1.200 ohms

Uoltage coefficient ..... -.856 woltsideg $c$

Current coefficient. ..... .010 ampsideg $C$

ACTUAL CURUE DATA: (fill factor $=71.38$ )

Short circuit current .... 25.9 amps

Open circuit voltage .... 217.7 volts

Max pouser surrent ....... 24.4 amps

Max power vol tage ....... 171. 1 wolts

Max pourar ..........4 4175.3 matts

MDRMALIZED CIURUE DATA: (fill factor $=73.18$ )

Sfor 25.0 deg $C$ ard $1.0 \mathrm{kh} / \mathrm{m}^{2} 2$ ?

Max prower surrent. ...... 23.5 amps

Max power valtage ....... 193.3 volts

Max pouser ........... 4547. ? unatts

NORMALIZED CURUE DATA: (fill factor $=71.78$ )

(fior 46.0 deg $C$ and $1.0 \mathrm{kH} / \mathrm{m}^{\wedge} 2$ )

Max power current ...... 23.7 amps

Max power voltage ....... 176.1 volts

Max power .......... 4173.0 undts 

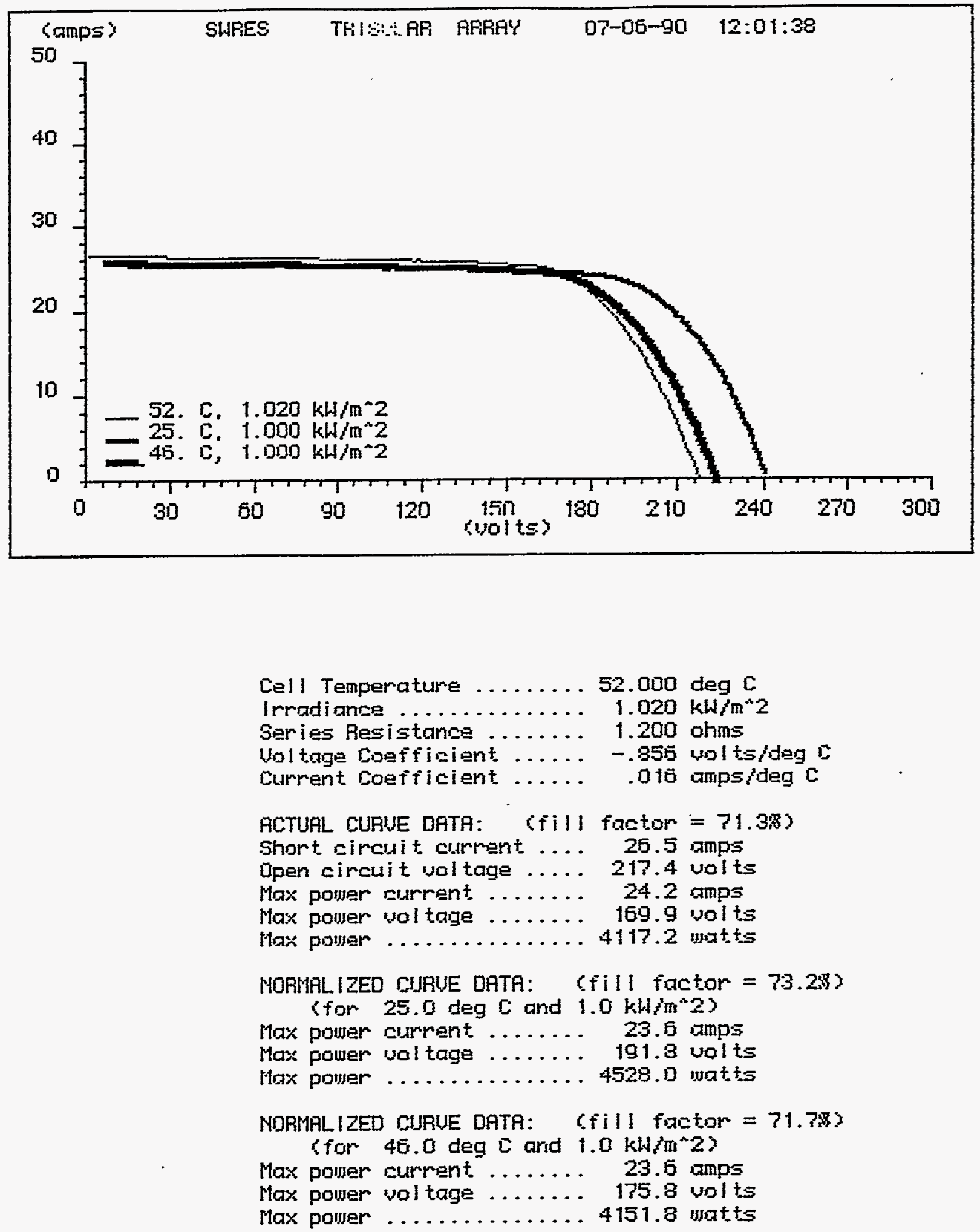


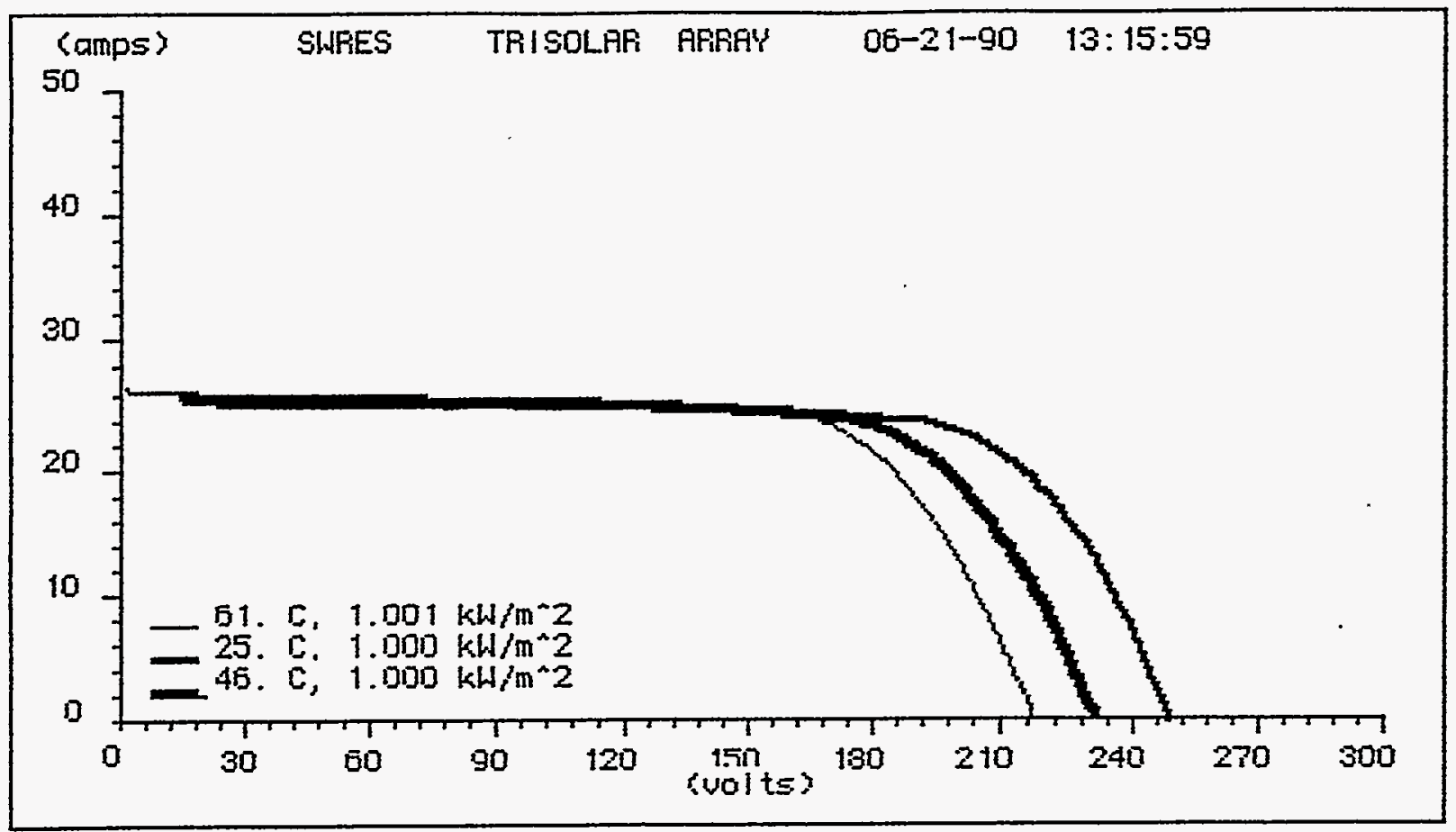

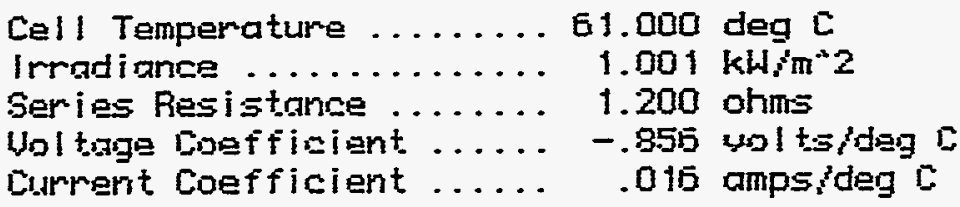

ACTUAL CURUE DATA: (fill factor $=70.38$ )

Short circuit aurrent .... 25.4 amps

Upen circuit vol tage ..... 210.9 jolts

Max poufer gurrent...... 23.7 rmps

Max pouner: vol tage ....... 170.1 wolts

Max pourer . . . . . . . 4027.3 ungtss

MDRMALIZED CIJRUE DATA: (fill fuEtor $=72.73$ )

(for 25.0 deg $C$ and $1.0 \mathrm{kH} / \mathrm{m}^{-2} 2$ ?

Max poiser current ....... 23.5 amps

Max pouer val tage ....... 198. 1 volts

Max power ............ 4657.0 matts

NORMRL IZED CIURUE DATA: (fill fantor $=71.38$ )

(for 40.0 deg $C$ and $1.0 \mathrm{kh} / \mathrm{m}^{\wedge} 2$ )

Max pouser cetrrent. ....... 23.8 amps

Max power voltage ....... 180.? volts

Max pouser ............ 4294.1 matts 


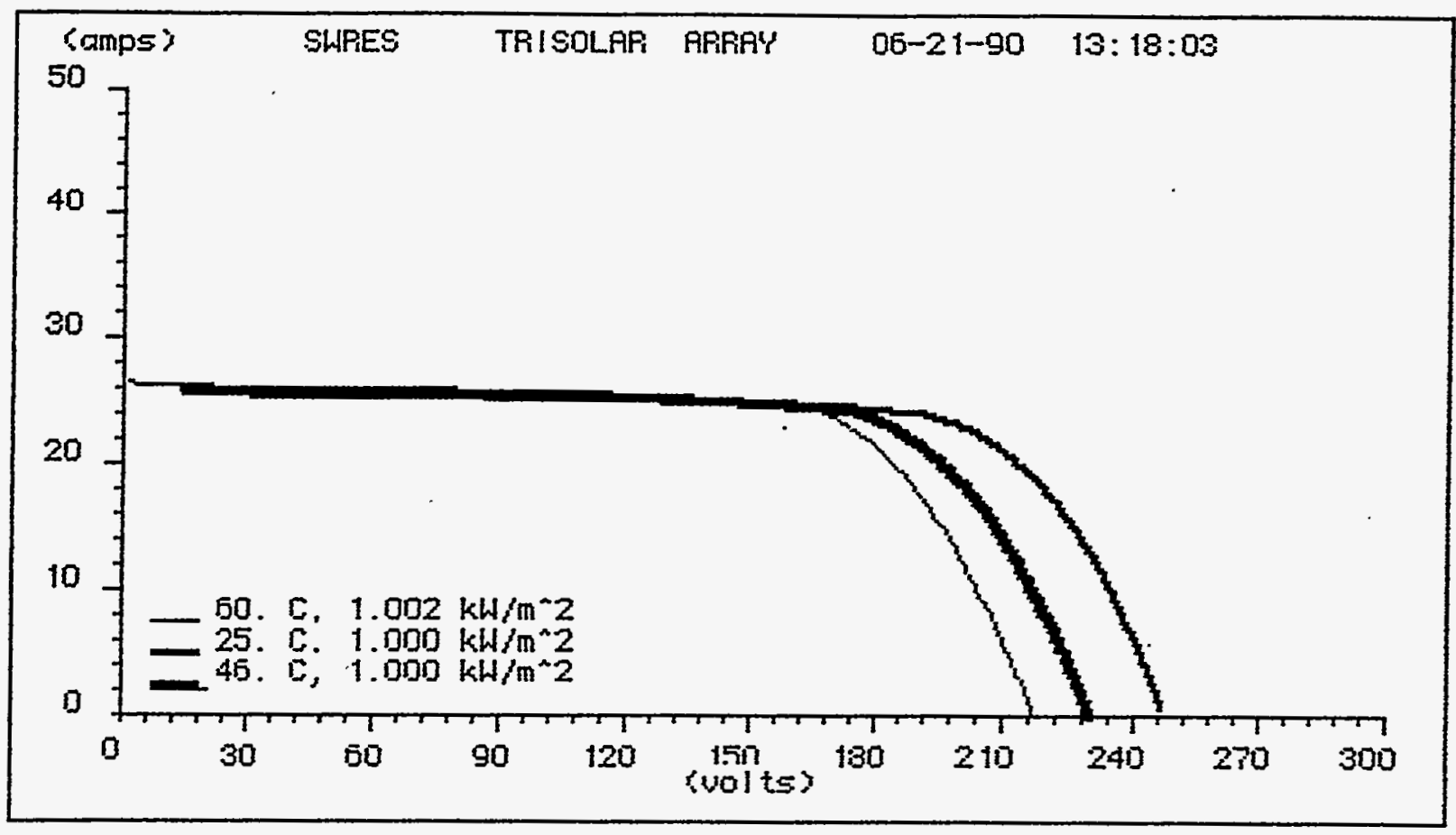

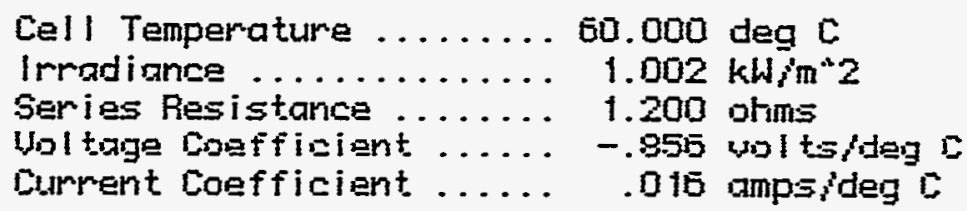

ACTUAL CURUE DATA: Sfill factor $=70.285$

Short circuil s.surant . . . $25.5 \mathrm{amps}$

Oper cimcuit voltage ..... 216.9 volts

Max pouser current ...... 24.0 rmps

Max power voltage ...... 167. 7 wolts

Max pousar ........... 4023.5 untts

NDRNALIZED CURUE DATA: ( $f$ ill factor $=72.6 \mathrm{~B}$ )

(for 25.0 deg $C$ and $1.0 \mathrm{kH} / \mathrm{m}^{\wedge} 2$ )

Max pouer current. ...... 23.6 amps

Max pawer val tage ....... ig?. 1 valts

Max pouner ............ 4549.7 ungtts

MORMRLIZED CURUE DATA: (fill fastor $=71.2 \times$ )

Sfor 46.0 deg $C$ and $\left.1.0 \mathrm{~km} / \mathrm{m}^{2} 2\right)$

Max power gurrent....... 23.7 amps

Max power vol tage ....... 180.0 volts

Max powar ........... 4275.5 watts 


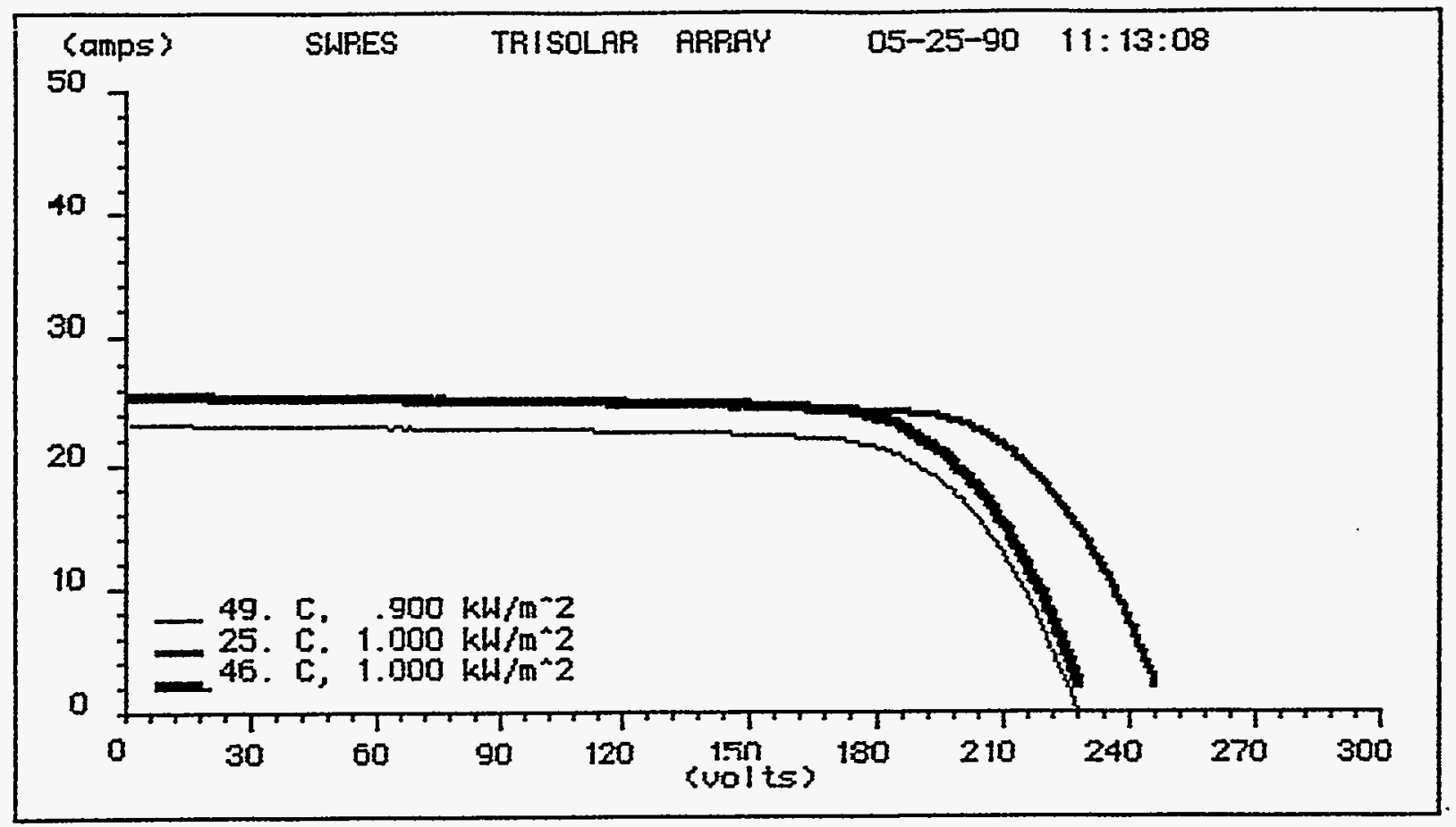

Cell Temperature .......49.000 deg C

Irradiance ........... .900 kW/ $/ \mathrm{m}^{\wedge} 2$

Series Resistance ....... 1.200 ohms

Wol tage coeffiriant...... -.855 wolts/ideg $c$

Current Coefficient ..... .016 ampsideg $C$

ACTUAL CURUE DATA: (fill factor $=72.4 \%$ )

Short circuit current .... 23.3 amps

Open circuit vol tage .... 227.6 volts

Max power current ....... 21.0 amps

Max power voltage ....... 183.0 volts

Max pourer ............. 3845.8 watts

NORMALIZED CURUE DATA: ( $\mathrm{fill}$ factor $=74.48$ )

(for 25.0 deg $C$ and $1.0 \mathrm{kH} / \mathrm{m}^{\wedge} 2$ )

Max power current ....... 23.2 amps

Max power val tage ....... 200.9 valts

Max power ............ 4559.9 watts

NORMALIZED CURUE DATA: (fill factor $=73.27$ )

(for 40.0 deg $C$ and $1.0 \mathrm{kH} / \mathrm{m}^{\wedge} 2$ )

Max power current ....... 23.6 amps

Max power voltage ....... 182.5 volts

Max power ........... 4299.3 watts 

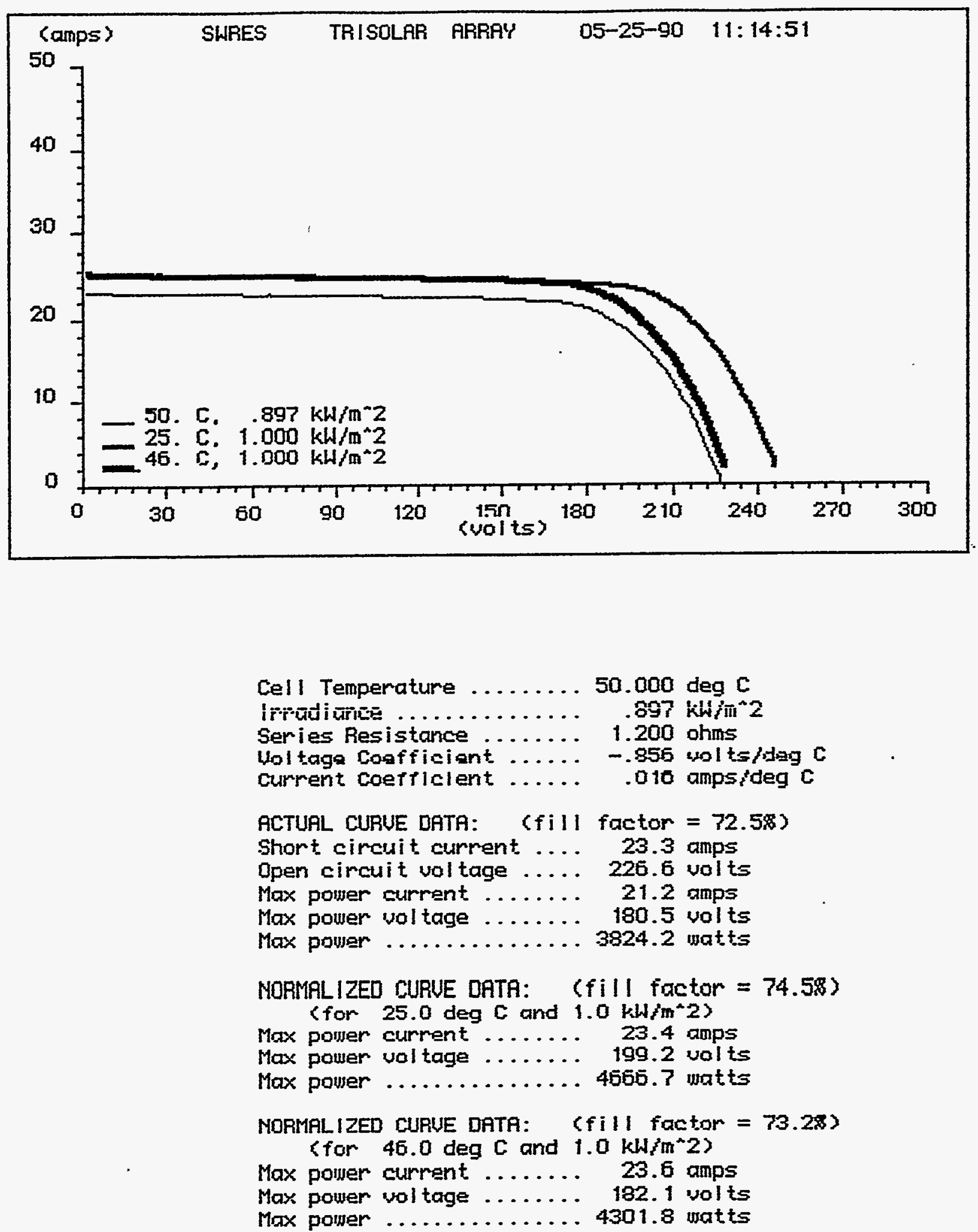


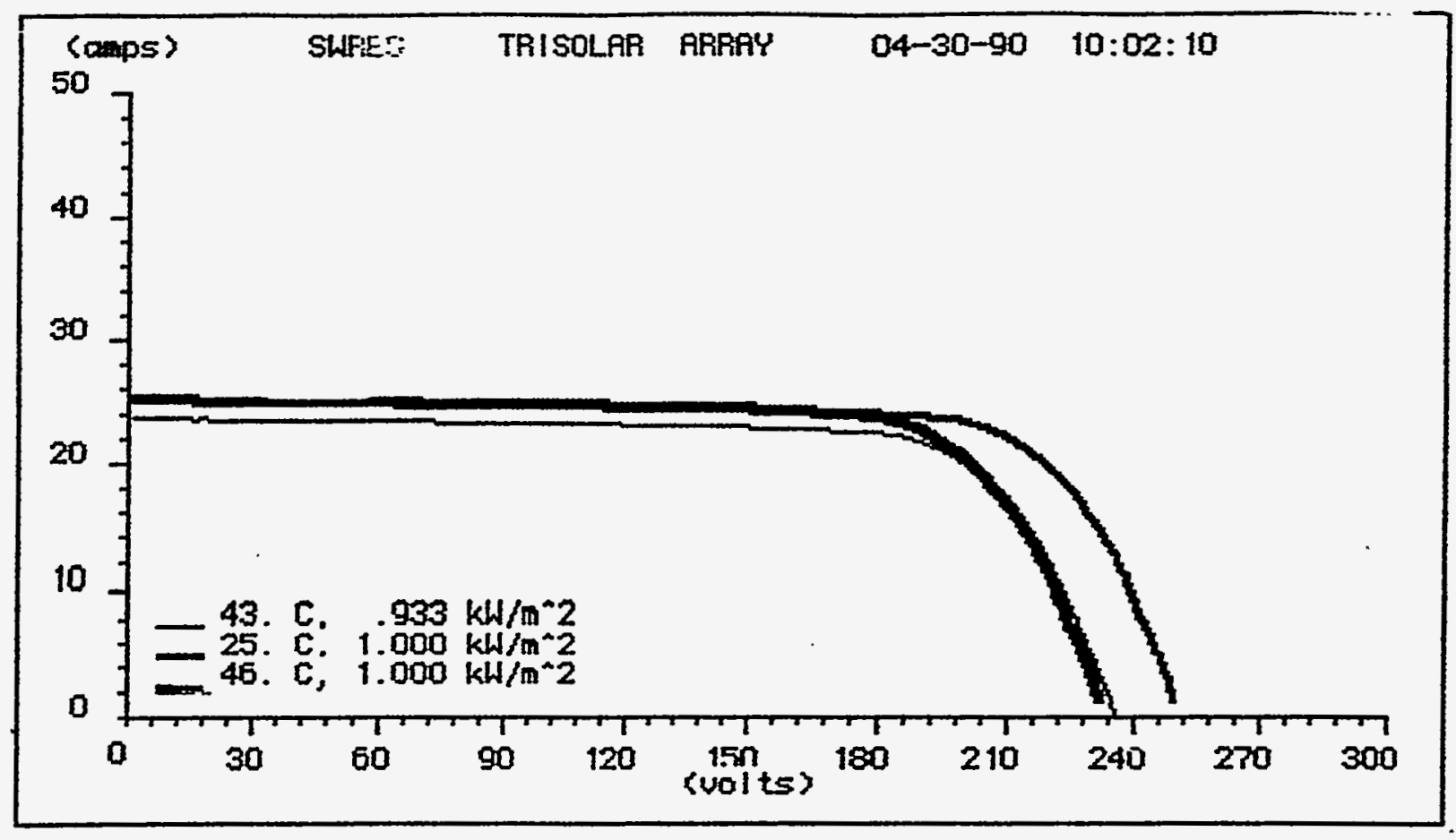

\footnotetext{
Cell Temperature ....... 43.000 deg $c$ Irradiance ............ $\quad .933 \mathrm{kN} / \mathrm{m}^{\circ} 2$

Series Resistance ....... 1.200 ohms

Wol tage Coefficient..... -.856 wolts/deg C

Current Coefficient ..... . .016 ampsideg $C$

ACTUAL CURUE DATA: ( $\mathrm{A}$ Ill factor $=73.38$ )

Short circuit current .... 23.9 amps

Open circuit vol tage ..... 235.4 volts

Max power current ....... 21.7 amps

Max power vol tage ....... 189.8 valts

Max power ............4119.5 ratts

NORMALIZED CURUE DATA: ( $\mathrm{f}$ Il factor $=74.7 \%$ )

(for $25.0 \mathrm{deg} C$ and $1.0 \mathrm{kH} / \mathrm{m}^{\wedge} 2$ )

Max power current ....... 23.1 amps

Max power val tage ....... 203.6 volts

Max power ............4704.6 watts

MORMALIZED CURUE DATA: (fill factor $=73.5 \%$ )

(for $46.0 \mathrm{deg} \mathrm{C}$ and $1.0 \mathrm{kH} / \mathrm{ma}^{\wedge} 2$ )

Max power current ....... 23.5 anps

Max powen voltage ....... 185.2 wolts

Max power ............ 4344.4 watts
} 


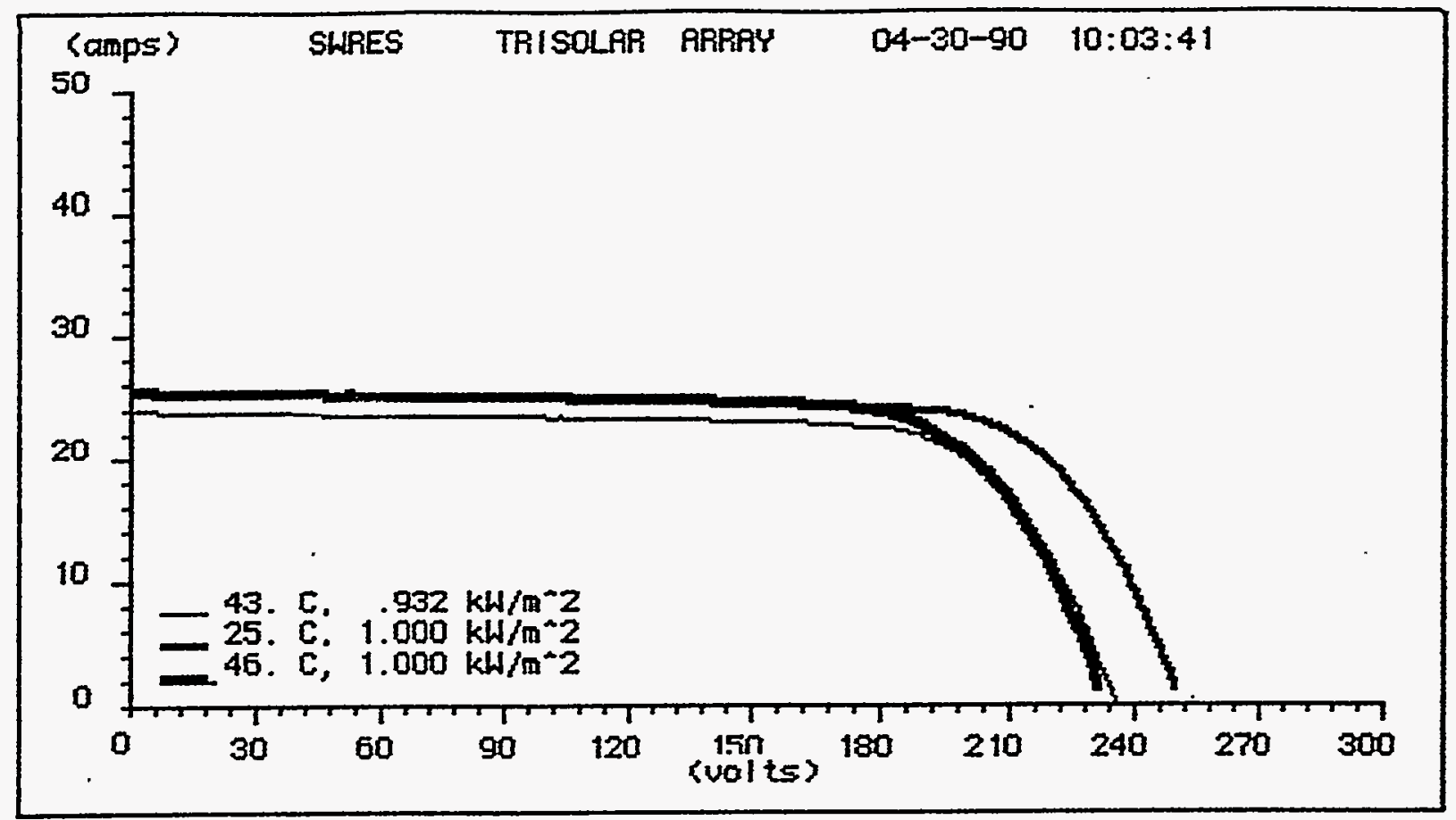

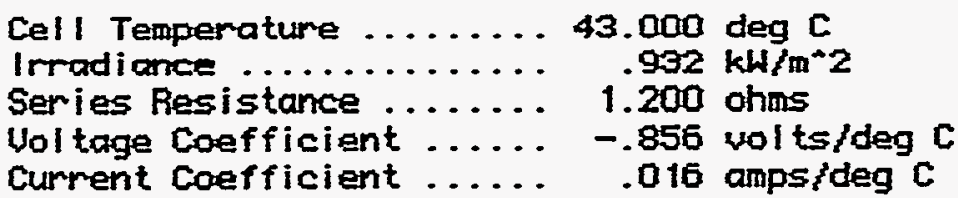

ACTUAL CURUE DATA: (fill factor $=73.18$ )

Short circuit current .... 24.0 amps

Open circuit val tage ..... 235.3 valts

Max power current ....... 21.9 amps

Max power vol tage ....... 188.7 vol ts

Max powper ........... 4128.1 watts

NORMAL IZED CURUE DRTA: (fill factor $=74.58$ )

(for $25.0 \mathrm{deg} C$ and $1.0 \mathrm{~kW} / \mathrm{m}^{\circ} 2$ )

Max power current ....... 23.3 amps

Max pomer vol tage ....... 202.3 volts

Max power ........... 4720.6 watts

MORMAL IZEO CURUE DATR: ( $\mathrm{f}$ ill factor $=73.2 \%$ )

(for $46.0 \mathrm{deg} C$ and $1.0 \mathrm{~kW} / \mathrm{m}^{\circ} 2$ )

Max power current ....... 23.4 amps

Max power vol tage ....... 186.3 volts

Max power ........... 4357.7 watts 


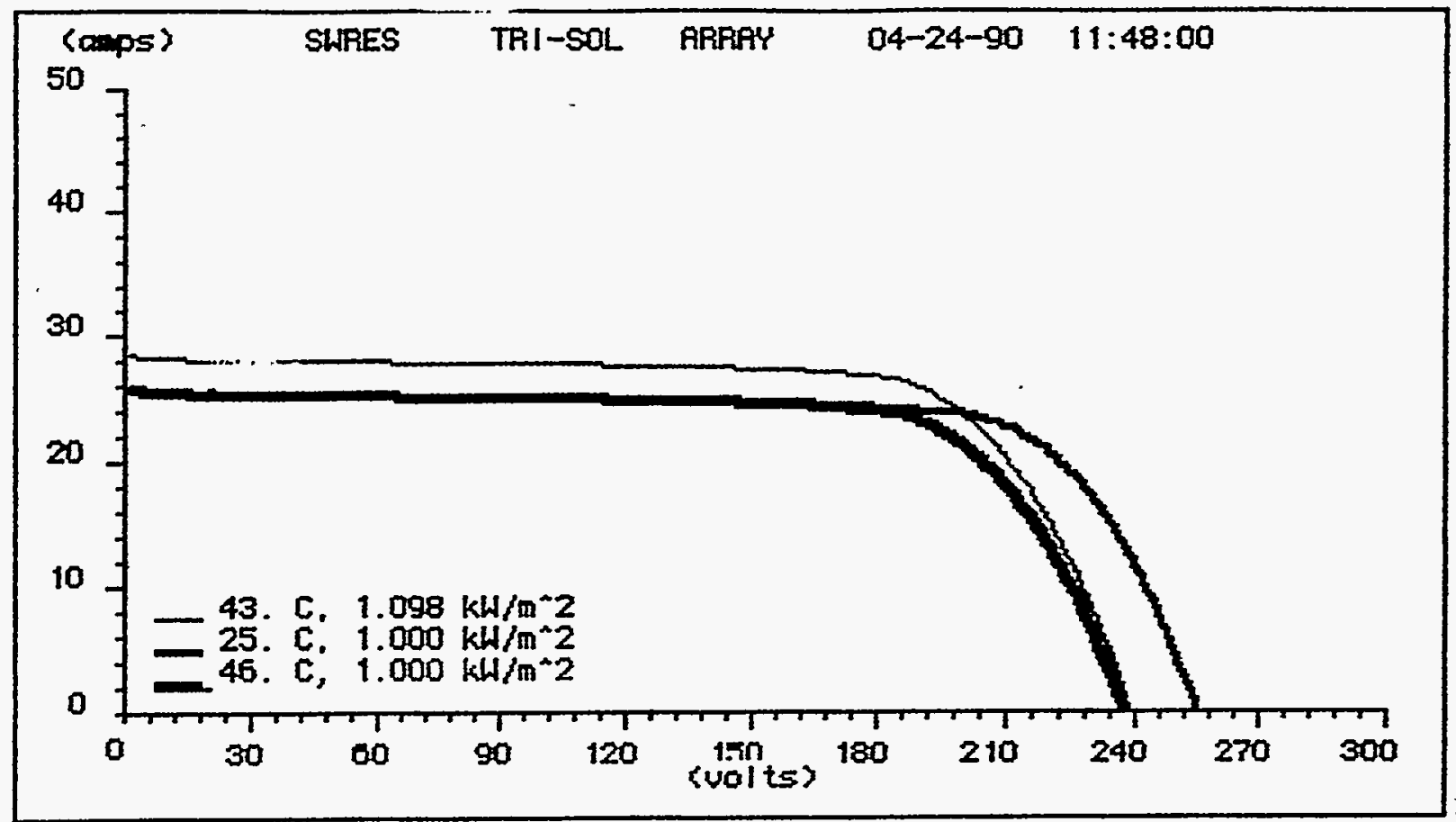

Cell Temperature .......43.000 deg $C$

Irradiance ........... $1.098 \mathrm{kH} / \mathrm{m}^{\wedge} 2$

Series Resistance ........ 1.200 ohins

Wol tage Coefficient ..... -.856 wolts/deg $C$

Current Coefficient ..... .016 amps/deg C

ACTUAL CURUE DATA: ( $\mathrm{fill}$ factor $=72.28$ )

Short circuit current .... 28.5 amps

Open circuit vol tage ..... 238.5 walts

Max power current ....... 25.8 amps

Max power vol tage ....... 189.8 volts

Max power ............ 4901.7 watts

NORMALIZED CURUE DATA: (fill factor $=73.08$ )

(for $25.0 \mathrm{deg} C$ and $1.0 \mathrm{kH} / \mathrm{m}^{\wedge} 2$ )

Max power current ....... 23.5 anps

Max power val tage ....... 205.1 volts

Max power ............ 4816.5 matts

MORTALIZED CURUE DATA: ( $\mathrm{fill}$ factor $=71.6 \%$ )

(for 46.0 deg $C$ and $1.0 \mathrm{~kW} / \mathrm{m}^{\wedge} 2$ )

Max power current ...... 23.8 anps

Max power val tage ....... 186.8 volts

Max power ............4441.7 watts 


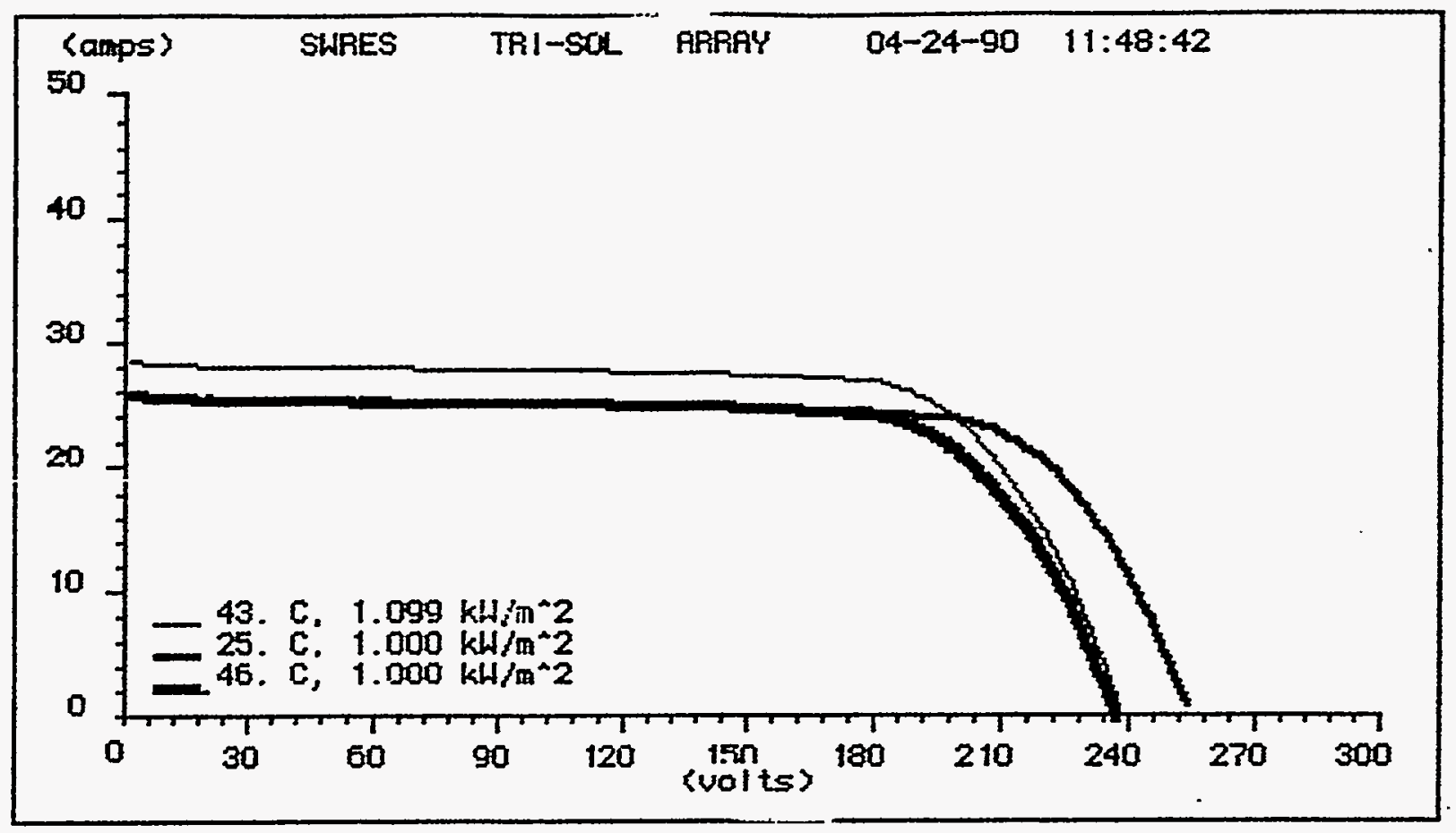

Cell Temperature ....... 43.000 deg $c$

Irradiance ............ $1.099 \mathrm{kH} / \mathrm{m}^{\wedge} 2$

Series Resistancs ....... 1.200 ohms

Voltage Coefficient ..... -.856 wolts/deg $c$

Current Coefficient ..... .016 amps/deg C

ACTUAL CURUE DATA: (fill factor $=72.3 \%$ )

Short circuit current ... 28.5 amps

Open circuit val tage ..... 237.9 volts

Max power current. ...... 26.0 anps

Max power vol tage ....... 188.7 volts

Max power ........... 4896.0 watts

NORMALIZED CURUE DATA: ( fill factor $=73.08$ )

(for 25.0 deg $C$ and $1.0 \mathrm{~kW} / \mathrm{m}^{\wedge} 2$ )

Max pooger current ....... 23.3 amps

Max pamer vol tage ....... 206.4 volts

Max power ............ 4803.9 watts

MORMALIZED CURUE DATA: (fill factor $=71.7 \%$ )

(for $46.0 \mathrm{deg} C$ and $1.0 \mathrm{kH} / \mathrm{s} \wedge 2$ )

Max power current ...... 23.6 amps

Max power voltage ....... 188.1 volts

Max power ...........4433.3 watts 


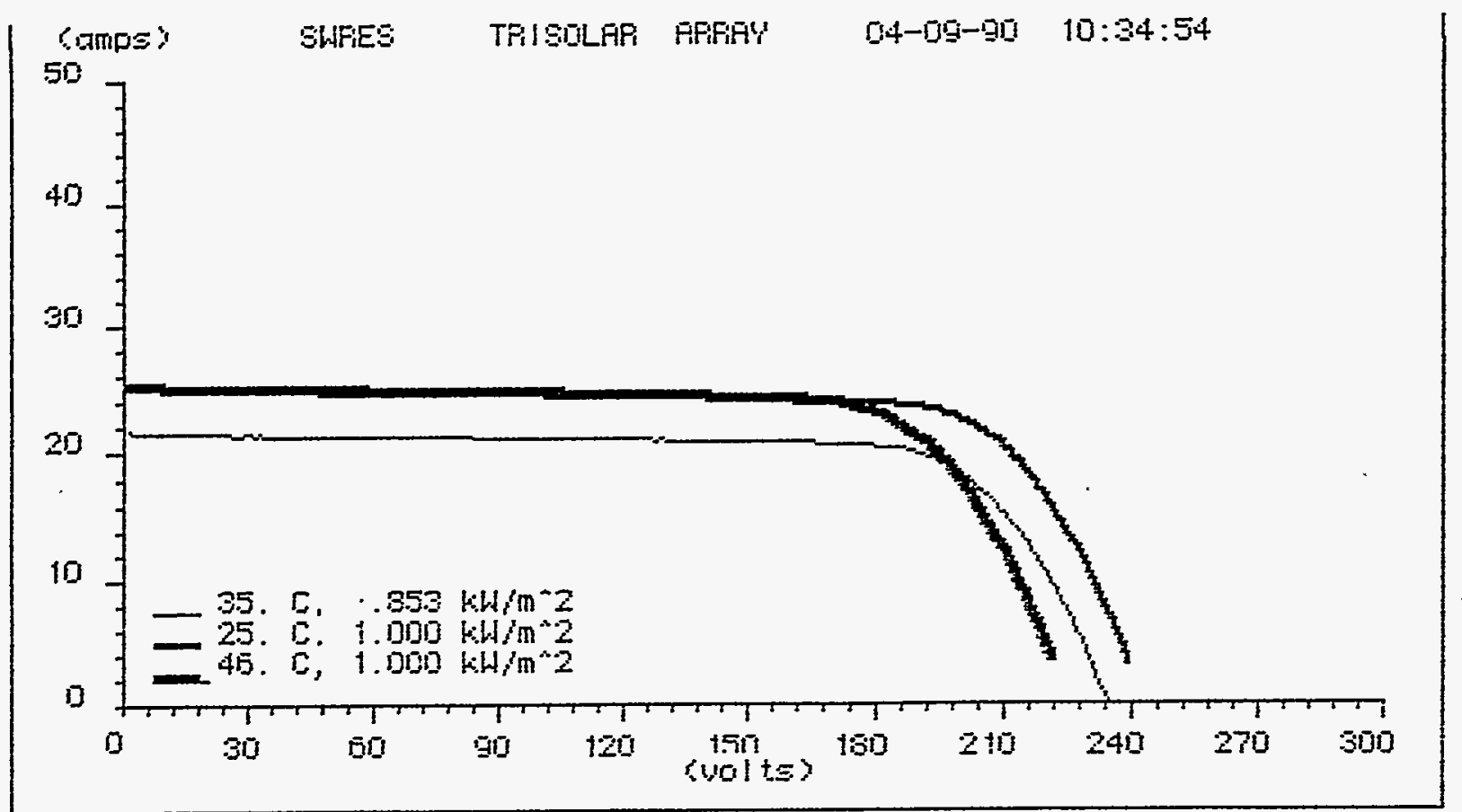

Cell Temperature ...... 35.000 ded $\mathrm{C}$

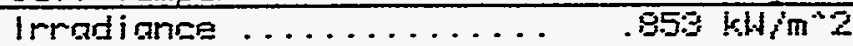

Series Resistanos ...... t.200 ohms

Woltage cogfficient. . . . . -.855 woltsideg 5

Current coefficient ..... . . 15 ampsideg $\mathrm{C}$

FCTUAL CIIRUE DATH: (fill factor $=74.48$ )

Short ciroult aurrent ... 21.6 umps

Open circuit jol tage .... 234.4 uolt:=

Max pouser current. ...... 19.9 amps

Max power wol tage ...... 189.8 volts

Max pumar ........... 2772. 1 matts

MOPHALIZED DURUE DATA: Cïll fuEtor $=75.6 \mathrm{y}$

(for 25.0 deg 5 and $1.0 \mathrm{kH} \mathrm{m}^{\circ} 2$ ?

Max pourer asurent. ...... 23.4 amps

Max Fower uol tage ....... 194. 1 uol ts

Max pouser ............ 4545. 1 matts

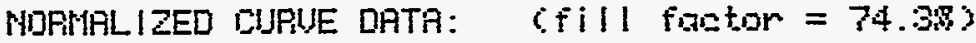

(for $4 \overline{1} .0$ deg 0 and $1.0 \mathrm{kH}, \mathrm{m}^{\circ} \mathrm{C}$ )

Max pouser currant ....... 23.5 amps

Max pouer wol toge ....... 173.2 volts

Mox pourer ............4 4185.0 matts 


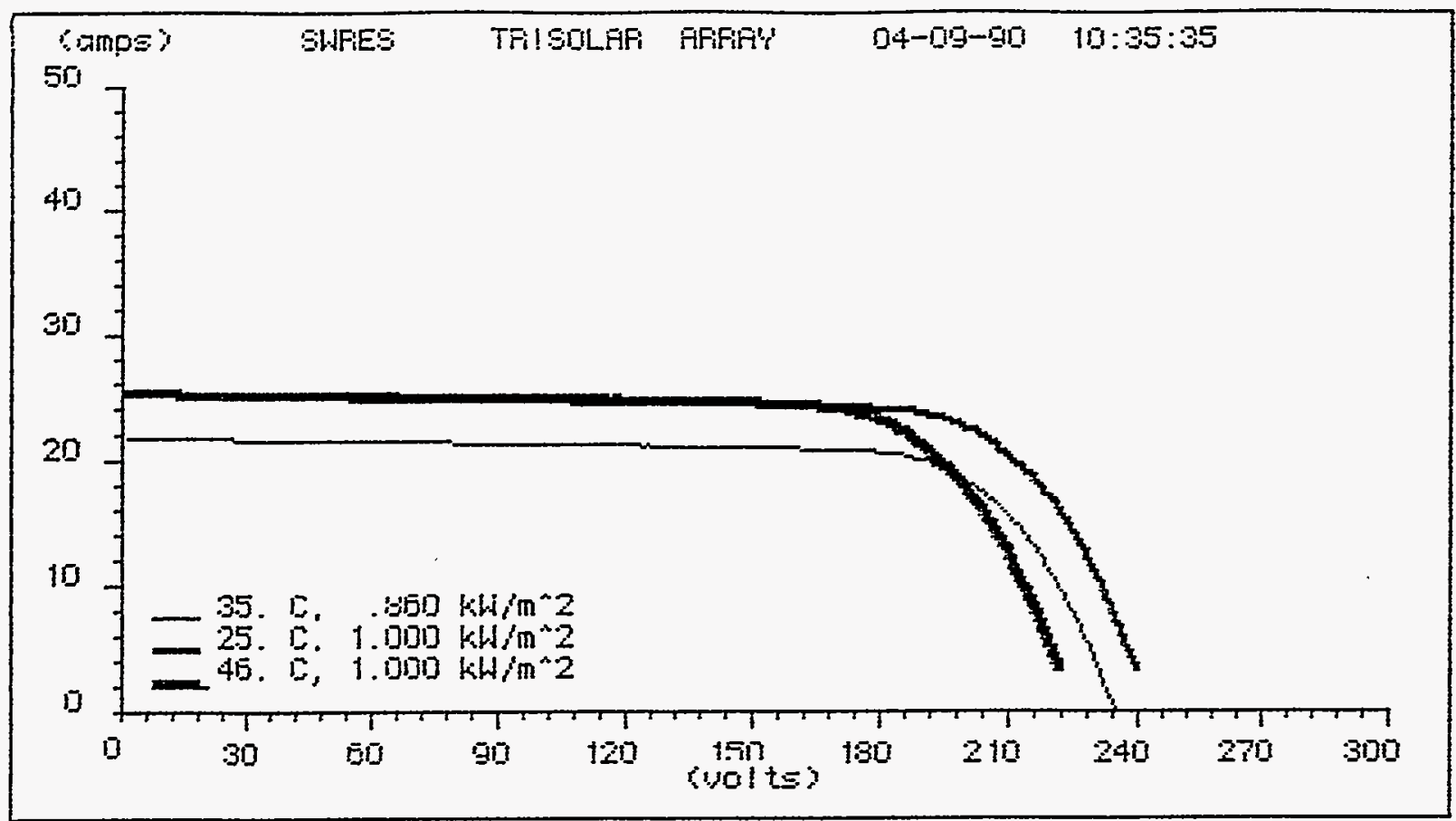

Ciel Temperatur.e ....... 35.000 deg $C$

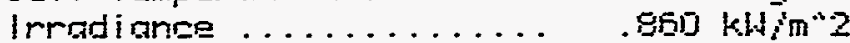

Ser.ies Resi $\equiv$ tanue ....... 1.20n ohms

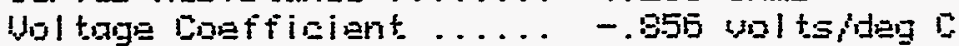

Eldrrent coefficient ..... . Dis ampsideg 5

HLTUFL DIIFUE DATA: 6fill fGEtor $=74.48 \%$

Short aimatit atrment.... 21.9 amps

Gjer circuit vol tage ..... 234.4 volts

Max power current ....... 20. 1 amps

Max pouler ugl toge ....... 189. B volts

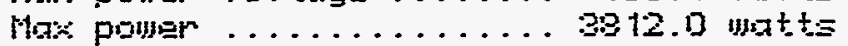

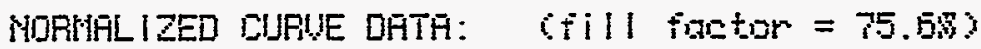

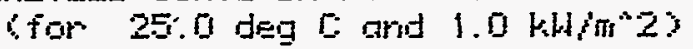

Mag\% pounar cirrent. ...... 2.3 .5 amps

Max pumer vol tage ....... 194.3 val t:

Max pouner ............ 45.58.5 watts

MOPMAL IZED LIJRUE DATA: (fill fätor $=74.35$

Sfor 46.0 deg $\left[\right.$ and $\left.1.0 \mathrm{kH} / \mathrm{m}^{2} \mathrm{z}\right)$

Haxe poumar currant...... 23.8 amps

Max powar wol tage ....... 175.9 vits

Max poujar ...........4 4193.2 matts 


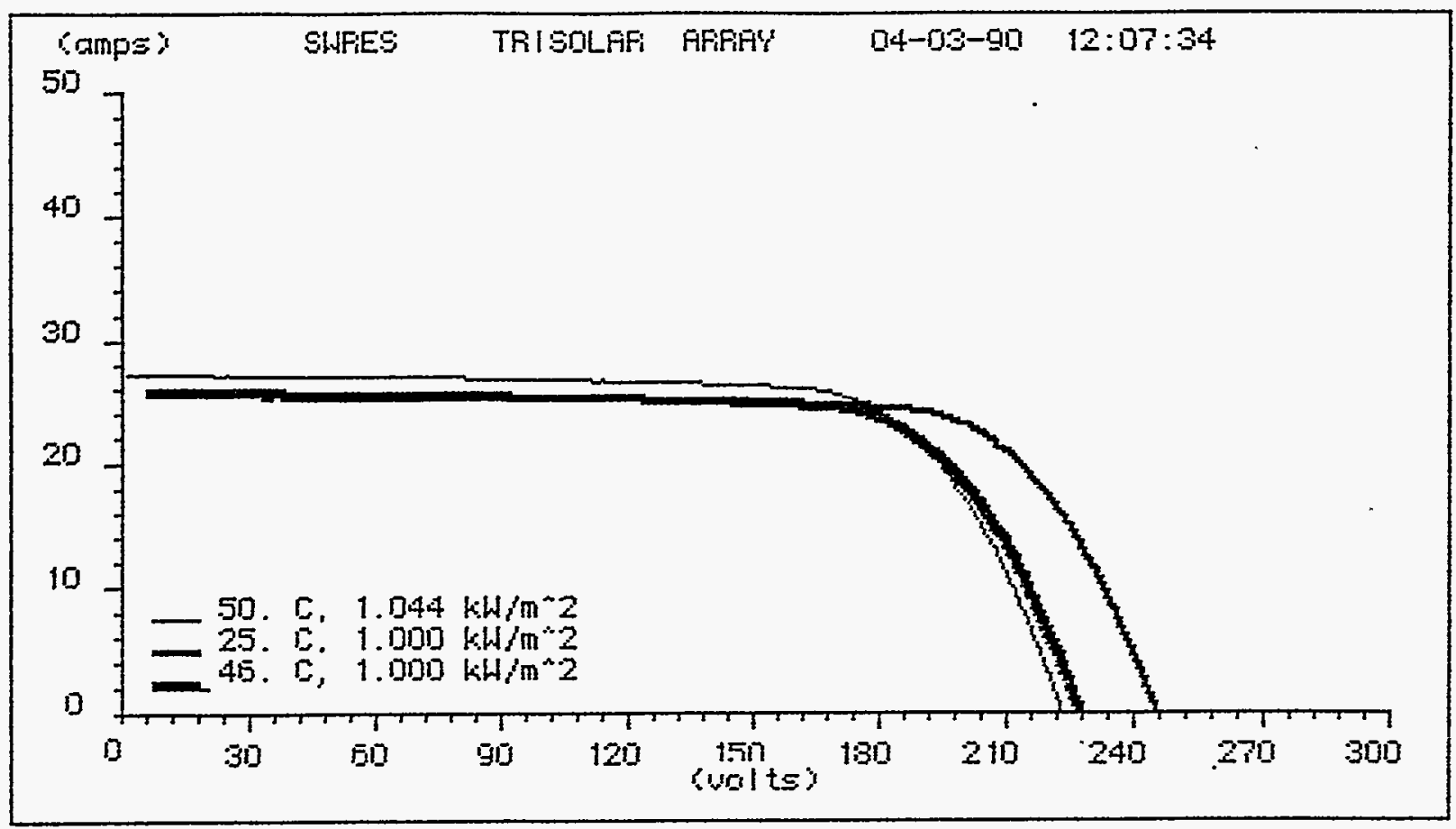

\footnotetext{
Cell Temperature ........ 5u. Uü deg [

Irradiance ........... $1.0144 \mathrm{kW/m} / \mathrm{h}$

Series Piesistance ....... 1.200 ohms

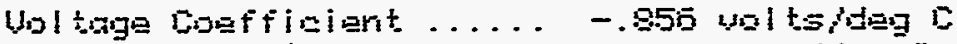

Curpant Doefificiert..... . Dis ampsideg $\mathrm{C}$

FETUAL DUAUE DATA: $\quad$ fill fGOtor = $71.3 \mathrm{~B}$

Short cirsuit ourrant .... 27.3 umps

Open cireuit vol tage ..... 223. 1 val

Max poujer current. ...... 24.9 amps

Max pouler unl tigge ....... 175. 3 volt.

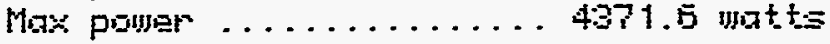

HDPHFL IZEO CIJPUE DHTA: $\quad$ fill faOtor = 73.43 ?

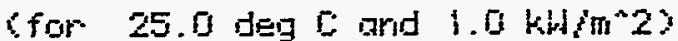

Max poumer aurrent. ...... 23.8 amps

Max pawer ual tage ....... 195.5 val ts

Max power ........... 45.54. 1 wat $t=$

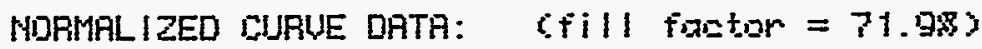

¿for $\$ \overline{0} .0 \mathrm{deg}\left[\mathrm{arnd} 1.0 \mathrm{kH} / \mathrm{m}^{\circ 2} \mathrm{z}\right.$

Max poujer current. ...... 23.7 amps

Max pouper viol toge ....... 180. 7 vol ts

Max piujer ..........44274.4 unatts
} 


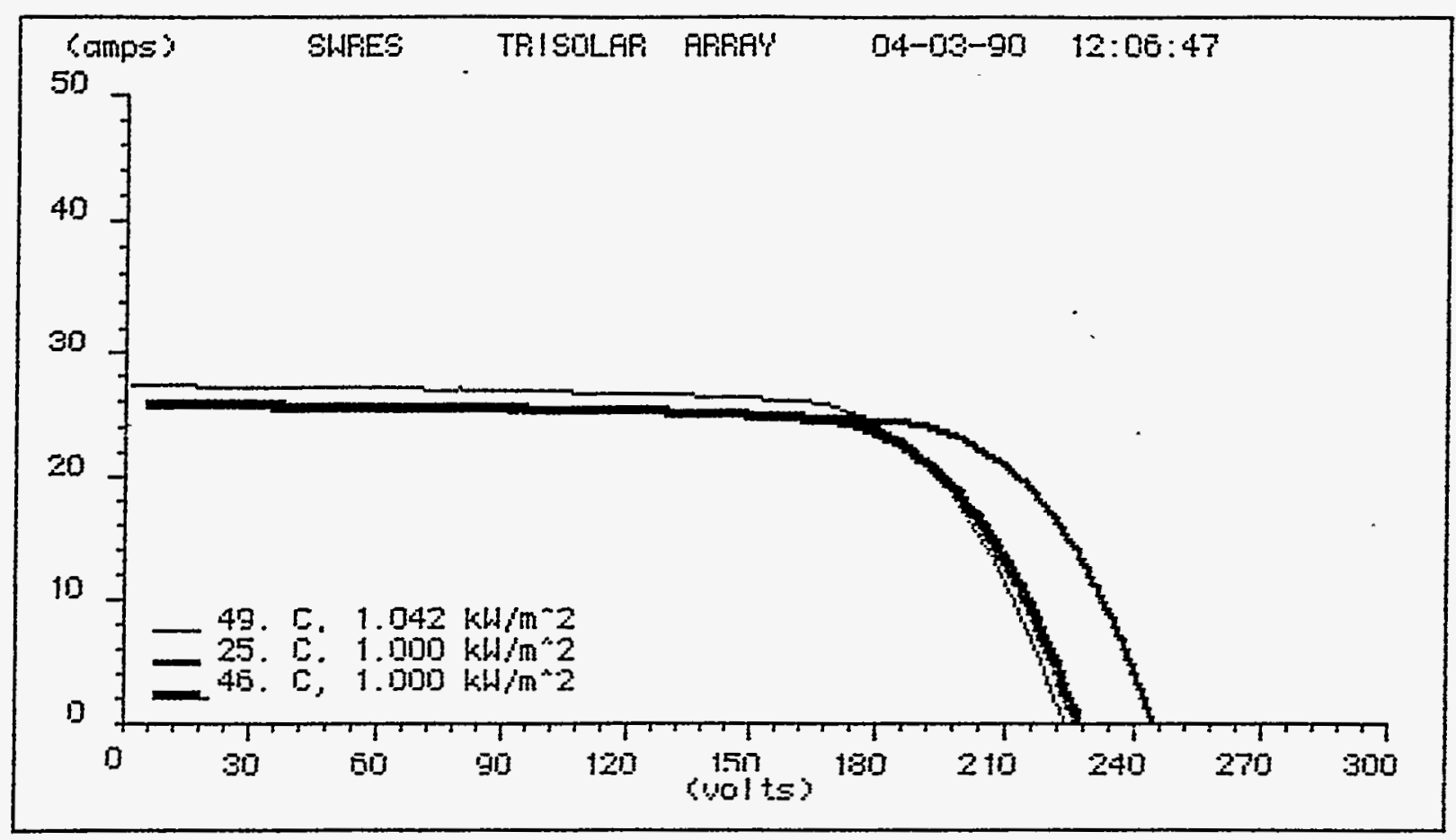

Cell Temperature .......49.0ua deg $\mathrm{C}$

Irradiance ........... $1.042 \mathrm{kH}^{2} \mathrm{~m}^{\mathrm{k}} \mathrm{z}$

Series Resistance ....... 1.200 ohns

Uoltage confinient ..... -.850 woltsideg c

Curment Doefficient. ..... . D15 ampsideg 5

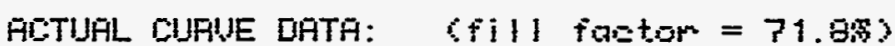

Short circult current .... 27.3 amps

Open circuit voltage .... 223.2 volts

Max pourer current ....... 24.7 amps

Max power voltage . . . . . 175.9 volts

Max power $\ldots \ldots \ldots \ldots \ldots \ldots$ 4358.9 matts

MOPHALIZED OUPUE DATA: CAill faOtor $=73.3 \mathrm{3}$

(for $25.0 \mathrm{deg}\left[\right.$ and $1.0 \mathrm{kH} / \mathrm{m}^{*} 2$ ?

Max pourer asmpent. ...... 23.5 smps

Max puwer val tage ....... 190.9 volts

Max pouer ........... 4539.5 unatts

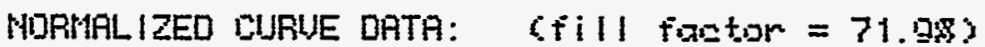

(ior 40.0 deg $C$ and $1.0 \mathrm{kh} / \mathrm{m}+2$ )

Max pourer currant....... 23.9 amps

Max pouser vol tuge ....... 178.5 wolts

Max poyer .......... 425.3.2 watts 


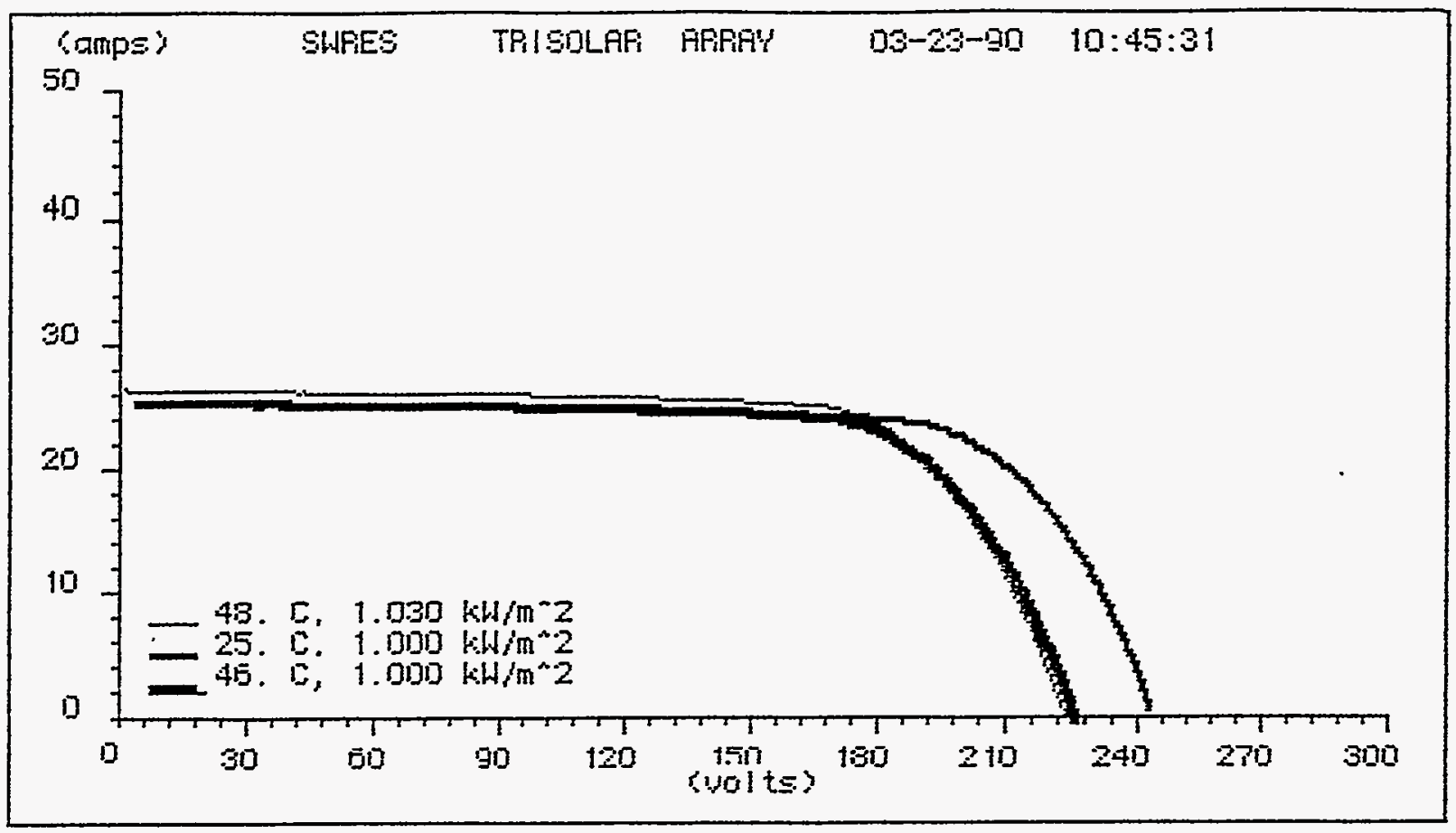

\footnotetext{
Cell Temperature ....... 4B. aũu deg [

Irradiance ............ $1.030 \mathrm{kH}^{2} \mathrm{~mm}^{\mathrm{2}}$

Series Resistance ....... 1.200 ohms

Woltage Doefficient ..... -.855 uoltsideg 8

Current Coefficient ..... . . to ampsideg $E$

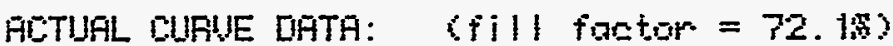

Short siradit current ... 25.4 umps

Open circuit uol tage .... 223.2 volts

Max pouer aurrent. ...... 24.2 amps

Mag pouer vol tage ....... 175.5 wolts

Max pomer ............ 4243.5 matts

HORMALIZED DURUE DATR: Cfill figofor $=73.5 \mathrm{~S}$ ?

for $25.0 \mathrm{deg} 0$ and $1.0 \mathrm{kH} / \mathrm{m}^{2} \mathrm{2}$ ?

Max pouper current. ...... 23.4 amps

Max pawer val tage ....... 194.5 vol te

Max power . . . . . . . . 4543.3 motts

MDRMALIZED DURUE DATA: $\quad$ fill faOton $=72.13$.

Sfor $4 \mathrm{E} .0 \mathrm{deg} \mathrm{C}$ and $\left.1.0 \mathrm{kH}, \mathrm{m}^{\circ} \mathrm{Z}\right)$

Hox pouer curpent...... 23.4 umps

Mox pouler vol tage ...... 178.5 volts

Max power .......... 4170.5 matts
} 


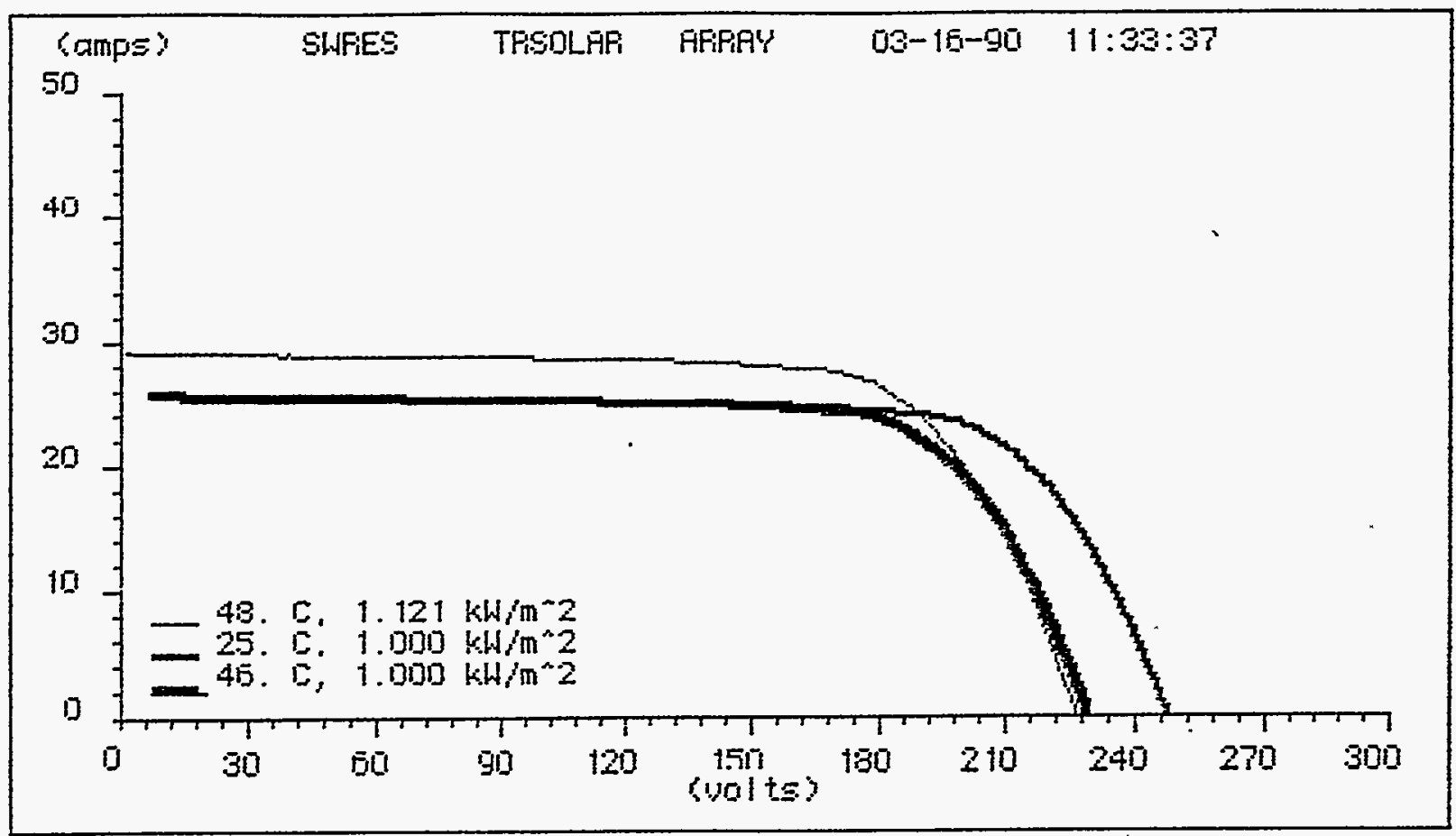

\footnotetext{
Cell Temperature .......43.000 deg $c$

Irradiance ............ $1.121 \mathrm{kH}^{\mathrm{A}} \mathrm{m}^{\mathrm{Z}}$

Series Resistance ....... 1.200 ohms

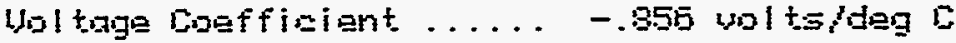

Current. Coefficient..... . oto ampsideg $\mathrm{C}$

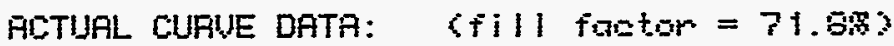

Short simouit current .... 29.2 amps upen cireuit val tage .... 220. E volt: Hax power current....... 25.8 amps Max pouner usl tage ...... 175.9 uolt.s

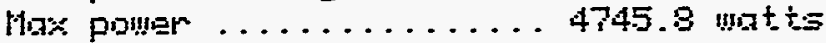

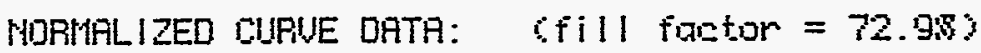

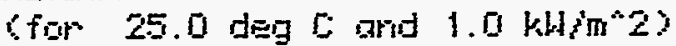

Ms\% poimer surmant ...... $23.5 \mathrm{mmps}$

Hax puwer val tage ....... 199. E val t.s

hax pouer ........... 4593.5 wat.ts

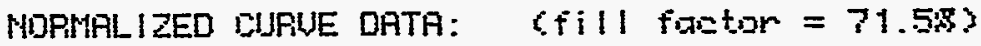

Sior 40.0 deg $\left[\right.$ and $1.0 \mathrm{kH} / \mathrm{m}^{\circ} \mathrm{2} \mathrm{y}$

Max power aurment ...... 23.3 anps

Max power usl tage ...... 181.3 volts

Max poumer ............ 4315.7 matts
} 

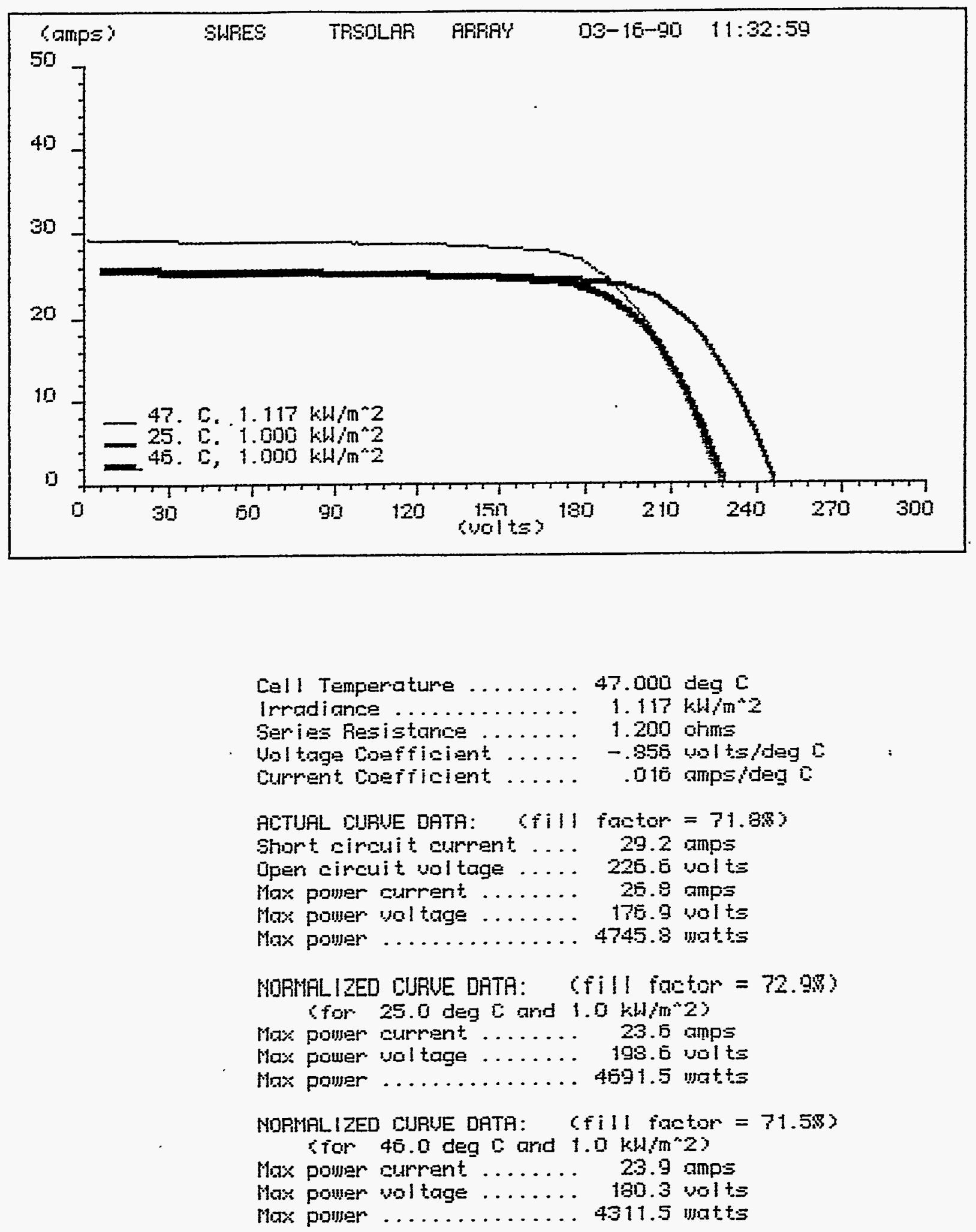


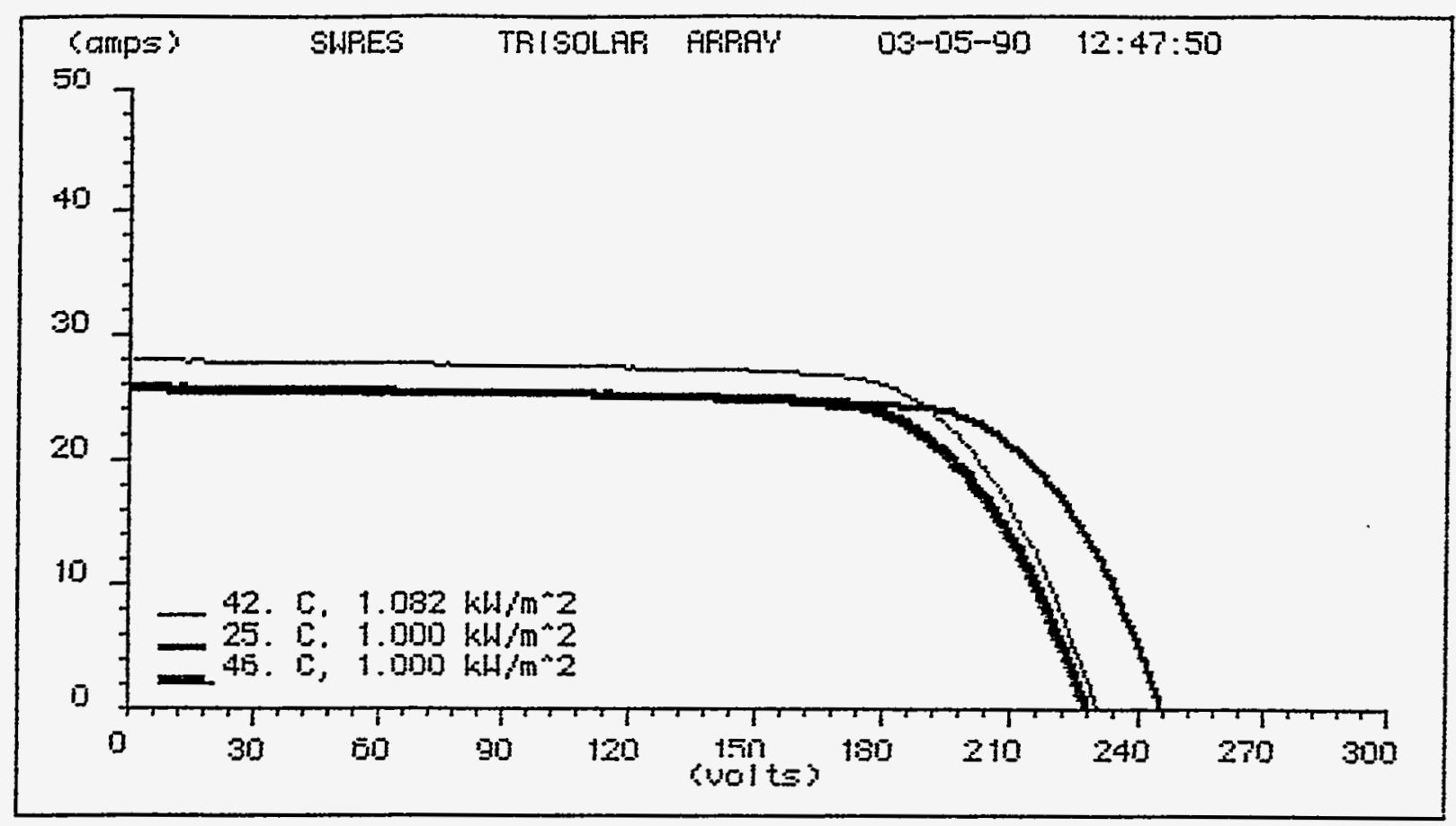

Cell Temperature ......442.000 deg $c$

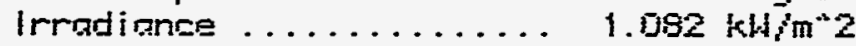

Series Resistance ....... 1.200 ohms

Uoltage coefficiant ..... - . 055 wolts/deg $D$

Current Coefficient. ..... .0 oto ampsideg 0

HCTUAL CUAUE DATA: (fill fBetor $=72.5 \mathrm{~s}$ )

Short cirouit curpent ... 23.1 amps

Opern circuit vol tage .... 230. 1 valts

Max pouser current ....... 25.0 amps

Max power vol tage ....... 160.5 wolts

Hox pomar ............ 4505.3 watts

NIORMALIZED CURUE DATR: (fill fortor $=73.48$ ?

(for 25.0 deg 0 and $1.0 \mathrm{khl} / \mathrm{m}^{2} 2$ ?

Max pouper current ....... 23.7 ramps

Max power sal tage ....... 19a. ? valt:

Max power ........... 4571.0 unats

NDPMALIZED CIUPUE DATA: (fill faEtor $=72.08\rangle$

(for 45.0 deg $C$ and $1.0 \mathrm{kH} / \mathrm{m}^{2} \mathrm{z}$ )

Max pouser surment. ...... 23.9 amps

Max pouner vol tage ....... 179.5 volts

Max poujer .......... 4290.7 unats 


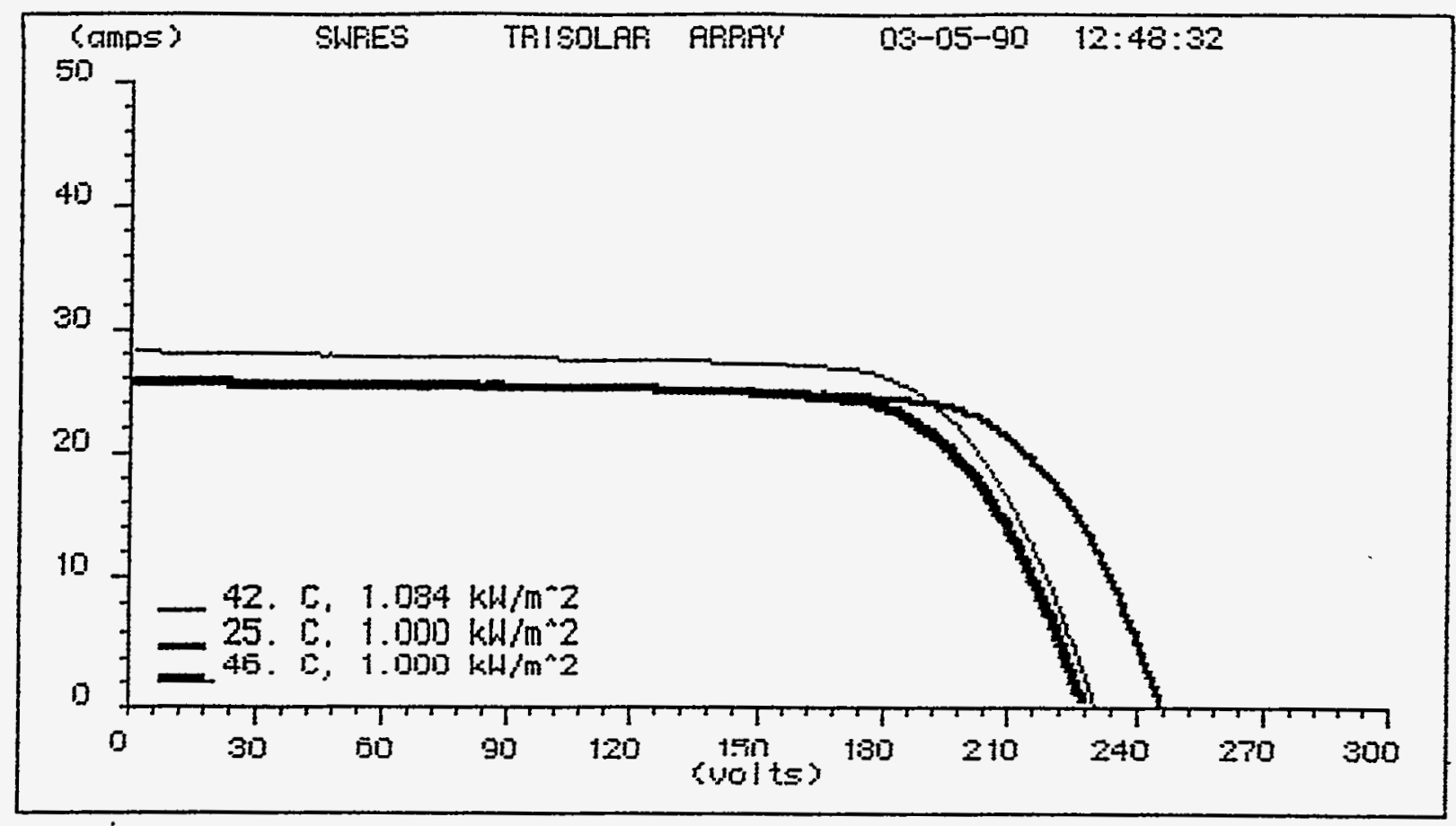

Cell Temperature ........42.000 deg c

Irradiance ........... $1.084 \mathrm{kH}^{2} \mathrm{k}$

Sieries Resistarice ...... 1.200 ohms

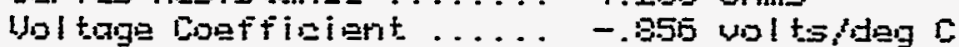

Curment Coefficient..... . Dto ampsideg $C$

ALTUAL QURUE LATA: (fill factor $=\vec{r} 2.7 \mathrm{~B}$ )

Short airauit surment .... 23.2 amps

Dpen circuit val tage ..... 230.4 volts

Max power current. ...... 25.0 amps

Max power voltage ....... 181.5 wolts

Mox pomar ........... 4722.5 unto

HDRTIRLIZED CUPUE DATA: (fill frotor $=73.5 \%$ ?

(for $25.0 \mathrm{deg} 0$ and $1.0 \mathrm{kH} / \mathrm{m}^{\mathrm{t}} \mathrm{z}$ )

Max pouner esurrent ....... 23.5 amps

Max power vol tage ....... 199.1 volts

Max power . . . . . . . . 4593.0 untts

NDRMRLIZED CUPIUE DATA: (fill footor $=72.18$ )

(for 40.0 deg $\left[\right.$ and $1.0 \mathrm{k} \mu / \mathrm{m}^{\wedge} 2$ )

Hax power ctarrent. ...... 23.9 amps

Mar poun vol tage ....... 180.8 volts

Max power ............ 4315.4 matts 


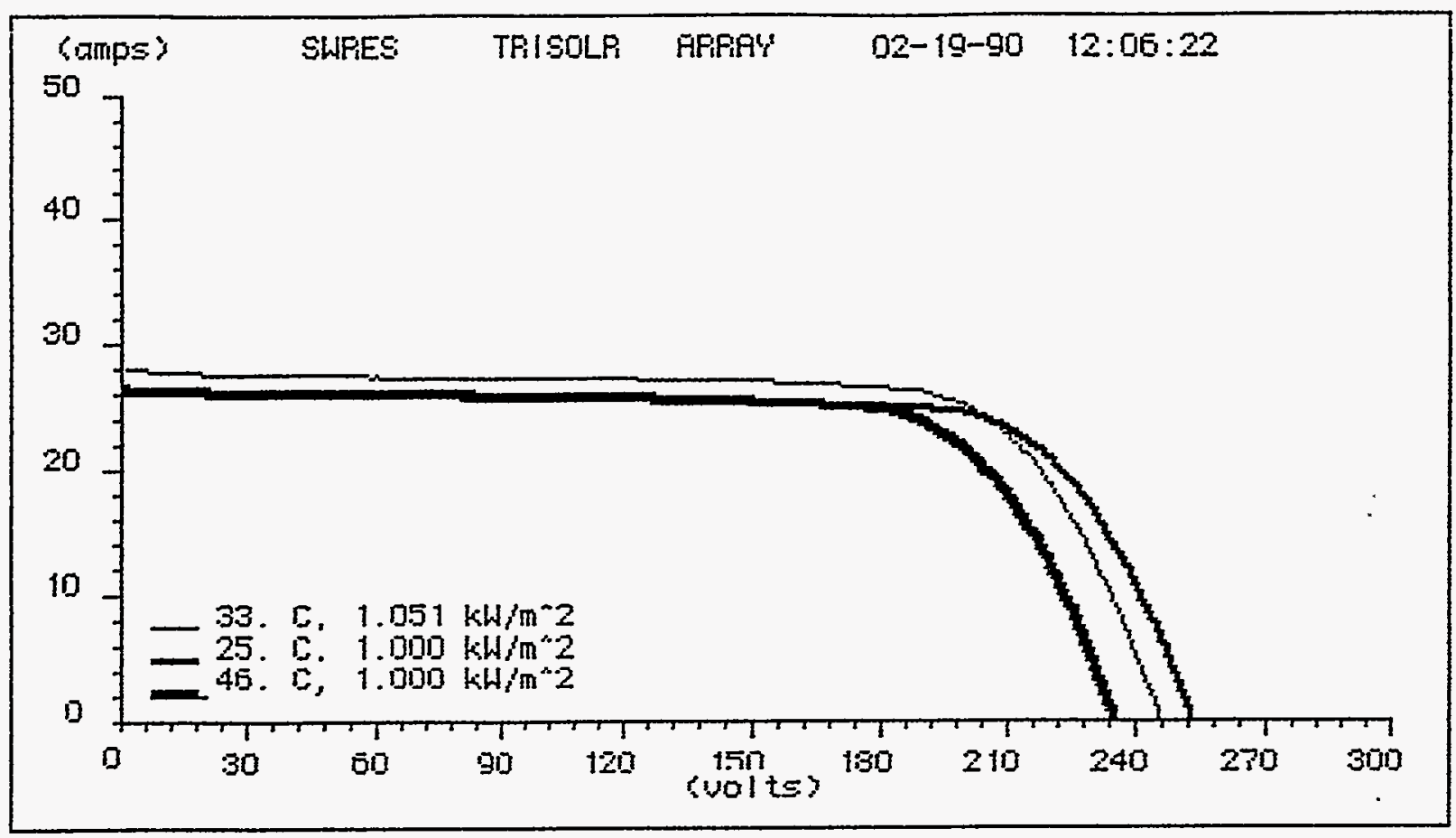

Cell Temperature .......33.000 deg $c$

Irradiance ............ $1.051 \mathrm{kH} / \mathrm{m}^{* 2}$

Series Resistance ....... 1.200 ohms

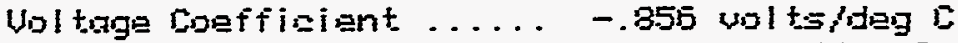

Current coefficient. .... . .016 ampsideg $c$

ACTUFL CURUE DATH: 〈fill fagtor $=72.9 \mathrm{~s}\rangle$

Short airouit currant .... 29.0 umps

Upen circuit uol tage .... 245.2 volts

Max poujer current. ....... 25.9 amps

Hax pouer sol tage ....... 194.5 wolts

Max pous . . . . . . . . 5015.0 matts

MDPMALIZED CURUE DATA: ifill factor $=73.2 \mathrm{3}$

(for 25.0 deg $c$ and $1.0 \mathrm{kH} / \mathrm{m}^{2} 2$ ?

Mox poujer ourrent...... 24.3 amps

Mase powam ual tage ....... 203.2 sal to

Max pruer ........... 49:35.5 untts

NORMALIZED OURUE DATA: (fill faotor $=71.935$

Sior 40.0 deg $C$ and $1.0 \mathrm{kH} / \mathrm{m}^{\circ} 2$ ?

Max pouser rurrent ...... 24.2 amps

Max power voltage ....... 183.3 volts

Max pouser ............4548.4 unatts 


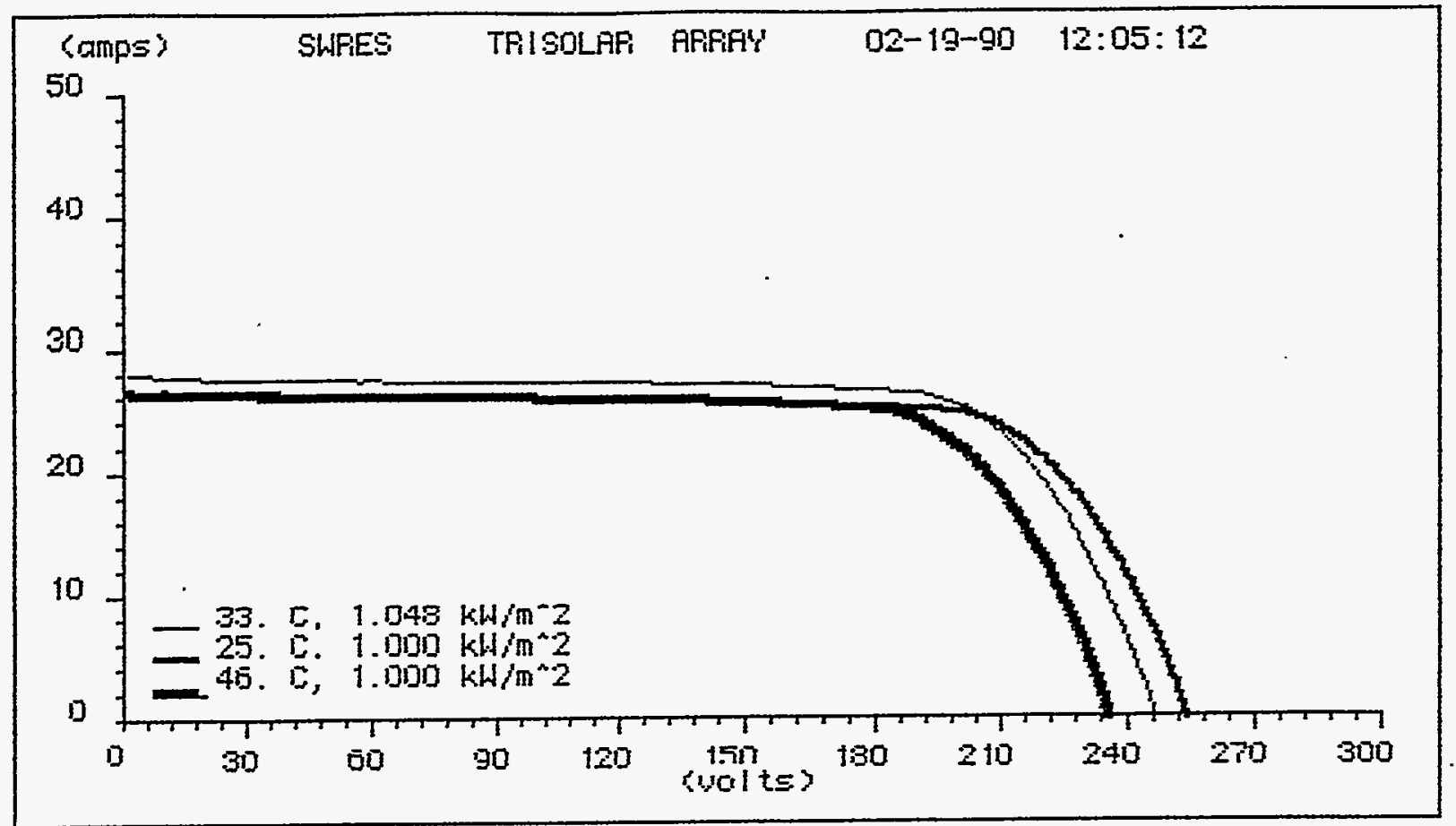

\footnotetext{
Cell Temperature ....... 33.000 deg $\mathrm{c}$

Irradiunce ........... $1.049 \mathrm{kH} / \mathrm{m}^{* 2}$

Semies Resistance ....... 1.200 ohms

Uoltage Doefficient ..... -.855 voltsideg L

Current Coefficient ..... .015 ampsideg $c$

ACTUAL CURUE DATA: Sfill factor = 73.0Bs

Short aircuit current ... 28.1 amps

Open eireuit vol tage ..... 246.1 wol t:

Max power current. ...... 25.5 mmps

Mak pouer vol toge ...... 196. 1 uglts

Max power ........... 5044.3 unatts

HDFMALIZED CIJRUE DATA: (fill factor $=73.23)$

Sfor 25.0 deg $C$ and $1.0 \mathrm{kH} / \mathrm{m}^{2} 2$ ?

Max pouner gurrent ...... 24.2 amps

Max power val tage ....... 205.E valt:

Max pouer ........... 4973.3 matts

MORMALIZED CURUE DATA: Gfill faotor $=71.9 \mathrm{M}$ )

Sior 40.0 deg $C$ and $1.0 \mathrm{kH} / \mathrm{m}^{2} \mathrm{z}$ )

Max pisurer curment ....... 24.4 umps

Max powen vol tage ....... 188.2 volts

Max pourar .......... 4583.6 matts
} 


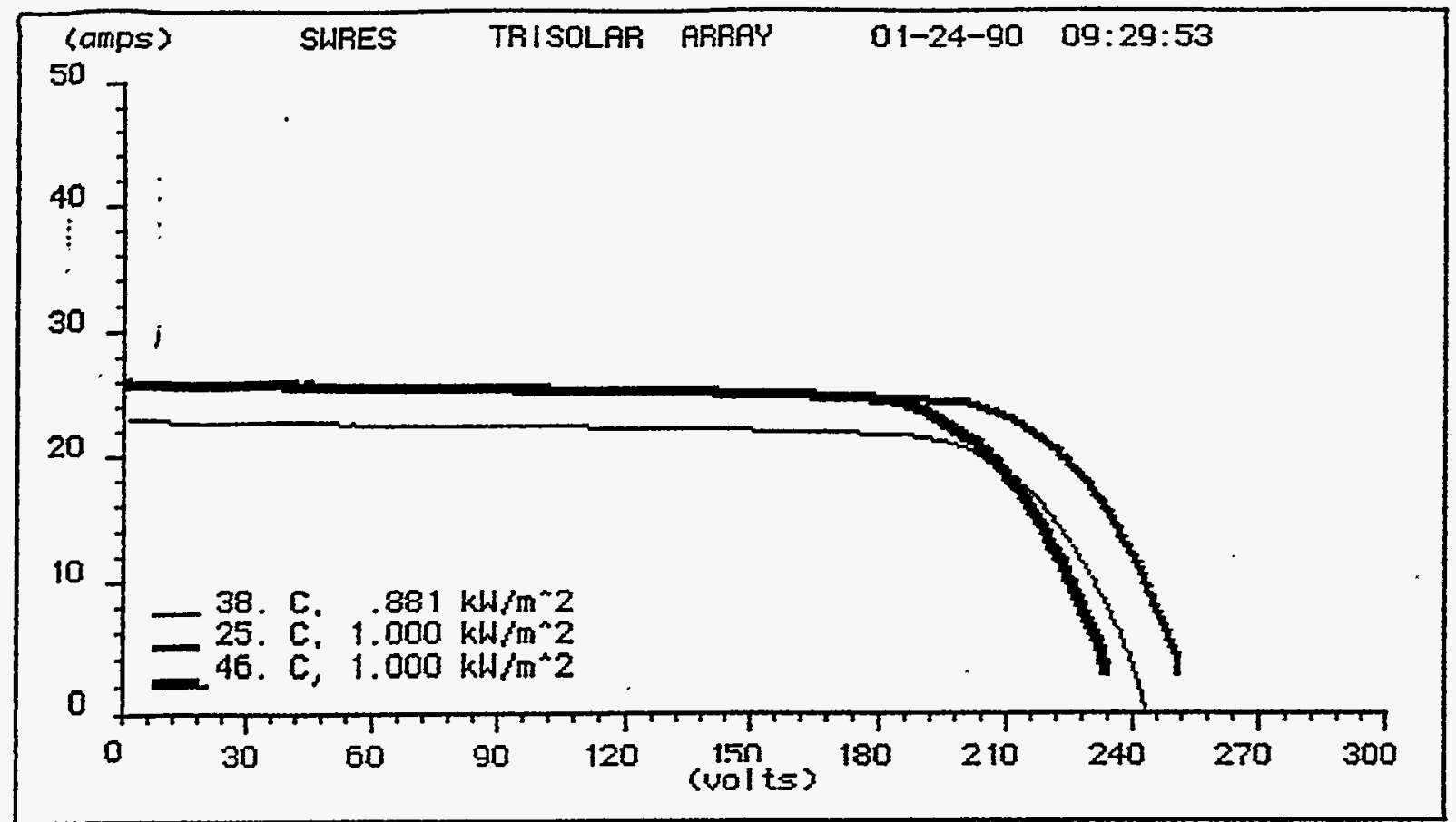

Cell Temperature ........ 38.000 deg c

Irradiance ........... .881 $\mathrm{kH} / \mathrm{m}^{\wedge} 2$

Series Resistance ....... 1.200 ohms

Uol tage Coefficient ..... -.856 uol tsideg $c$

Current Coefficient ..... . .016 amps/deg $C$

ACTUAL CURUE DATA: (fill factor $=73.9 \%$ )

Short circuit current .... 23.1 amps

Dpen circuit voltage ..... 243.0 volts

Max power current ....... 21.1 amps

Max power vol tage ....... 196.9 volts

Max power ...........4 4152.2 watts

NORMALIZED CURUE DATA: (fill factor $=75.38$ )

(for 25.0 deg $C$ and $1: 0 \mathrm{kH} / \mathrm{m}^{*} 2$ )

Max power current ....... 24.0 amps

Max power voltage ....... 204.5 volts

Max power ............ 4905. 1 watts

NORMALIZED CURUE DATA: (fill factor $=74.18$ )

(for 46.0 deg $C$ and $1.0 \mathrm{~kW} / \mathrm{m}^{\wedge} 2$ )

Max power current ....... 23.9 amps

Max power vol tage ....... 189.8 volts

Max power ........... 4536.3 watts 


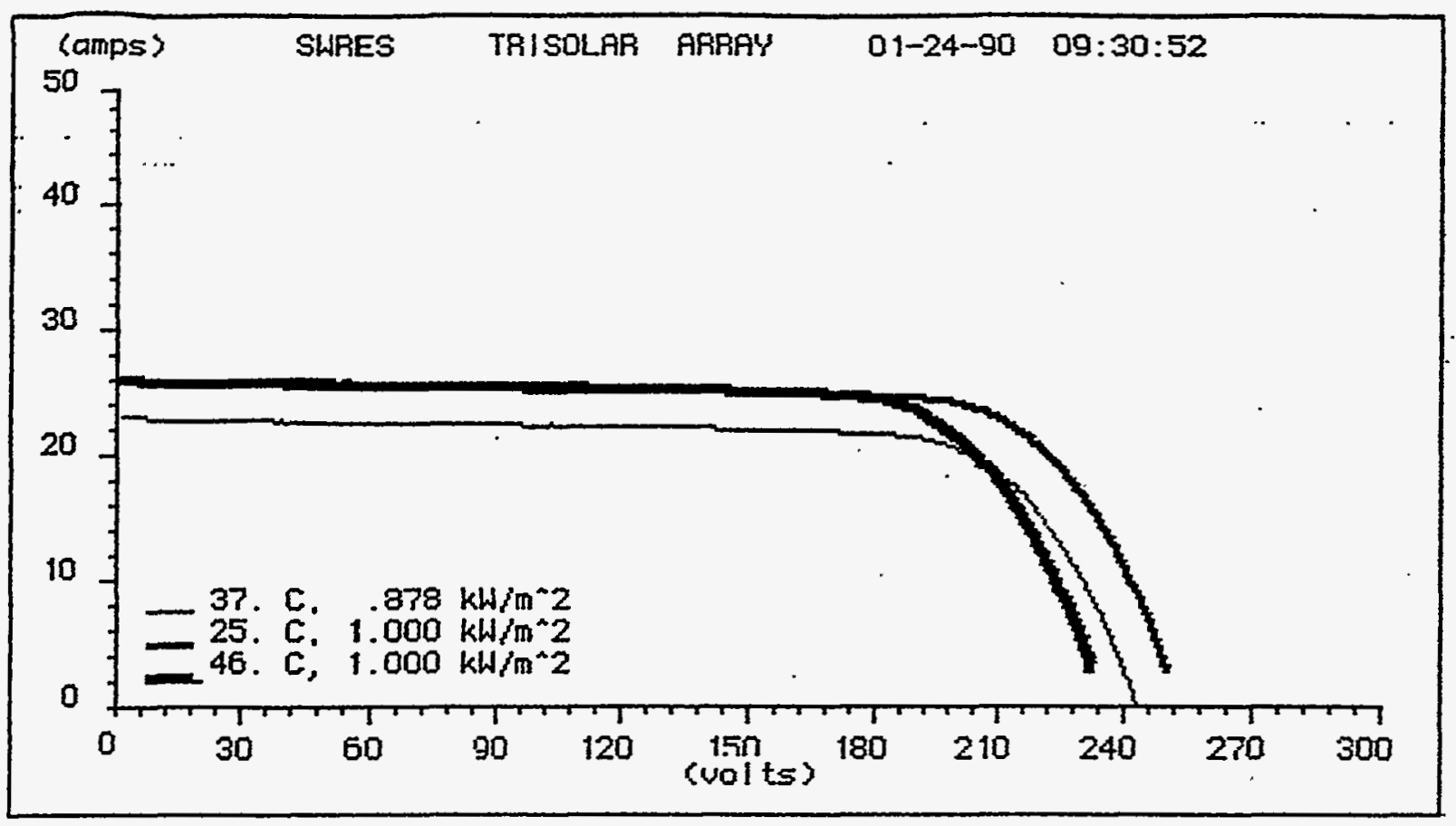

Cell Temperature ....... 37.000 deg C

Irradiance ............ .878 kh/m²

Series Resistance ....... 1.200 ohms

Vol tage Coefficient ..... -.856 volts/deg $C$

Current Coefficient ..... .010 amps/deg $\mathrm{C}$

ACTUAL CURUE DATA: (fill factor $=73.98$ )

Short circuit current ... 23.0 amps Open cirsuit yol tage ..... 242.? yol tis Max power current ....... 21.1 amps

Max power vol tage ....... 195.7 valts

Max power ............ 4131.2 watts

MORTALIZED CURUE DATA: ( $\mathrm{fill}$ factor $=75.2 \%$ )

(for 25.0 Jeg $c$ and $1.0 \mathrm{kH} / \mathrm{m}^{\wedge} 2$ )

Max power current ....... 24.0 amps

Max power voltage ....... 203.6 volts

Max power ........... 4878.6 watts

NORMALIZED CURUE DATA: (fill factor $=74.18$ ).

(for 40.0 deg $C$ and $1.0 \mathrm{kH} / \mathrm{m}^{\wedge} 2$ )

Max power current ...... 24.0 amps

Max power voltage ....... 187.8 volts

Max power ........... 4512.0 watts 
APPENDIX E

Mobil 1990

E-1 


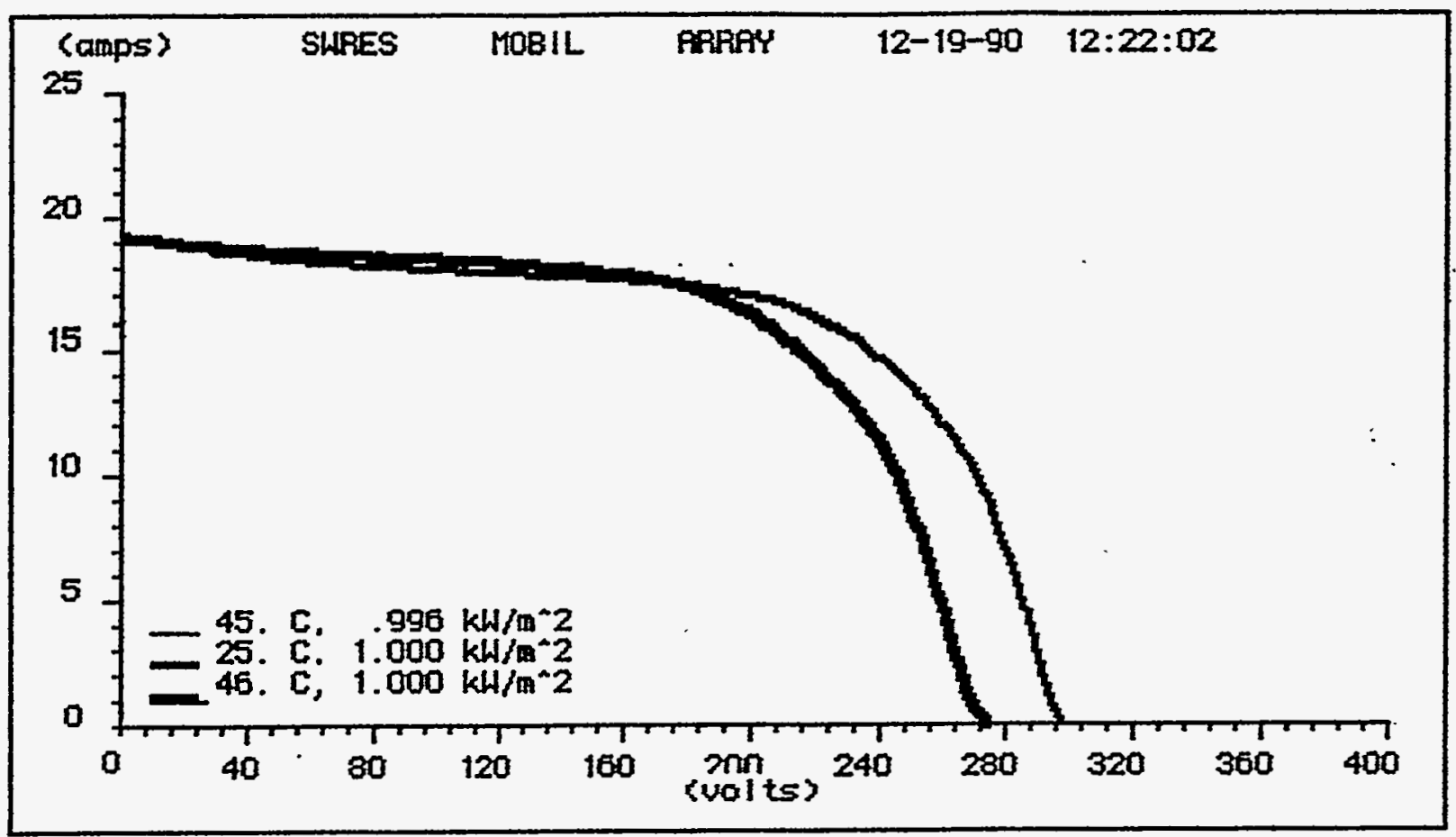

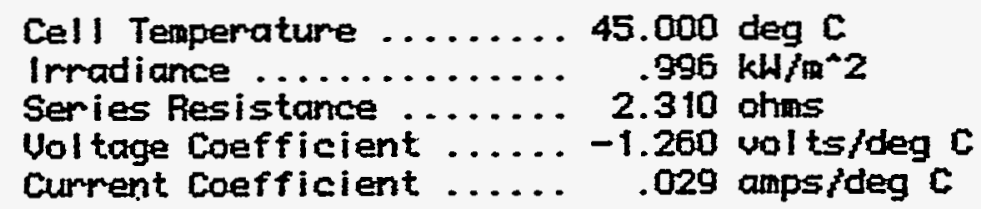

ACTUAL CURUE DATA: ( $\mathrm{fill}$ foctor $=62.18$ )

Short circuit current .... 19.2 aps

Open circuit vol tage ..... 274.4 volts.

Max power current ....... 15.9 amps

Max power vol tage ....... 206.3 volts

Max pomar ............ 3275.3 matts

NOPMALIZEO CURUE DATA: (fill factor $=63.68$ ) (for 25.0 deg. $C$ and $1.0 \mathrm{kH} / \mathrm{m}^{\star} 2$ )

Max pouner current....... 15.7 amps

Max power vol tage ....... 228.1 wolts

Max power .............. 3584.2 watts

MORTALIZED CUFUE DATA: (fill factor $=62.08$ )

(for 46.0 deg $C$ and $1.0 \mathrm{~kW} / \mathrm{ha}^{-2}$ )

Max powfer current........ 16.0 amps

Max pourer vol tage ....... 204.? wolts

?. Max power ............. 3273.1 watts 


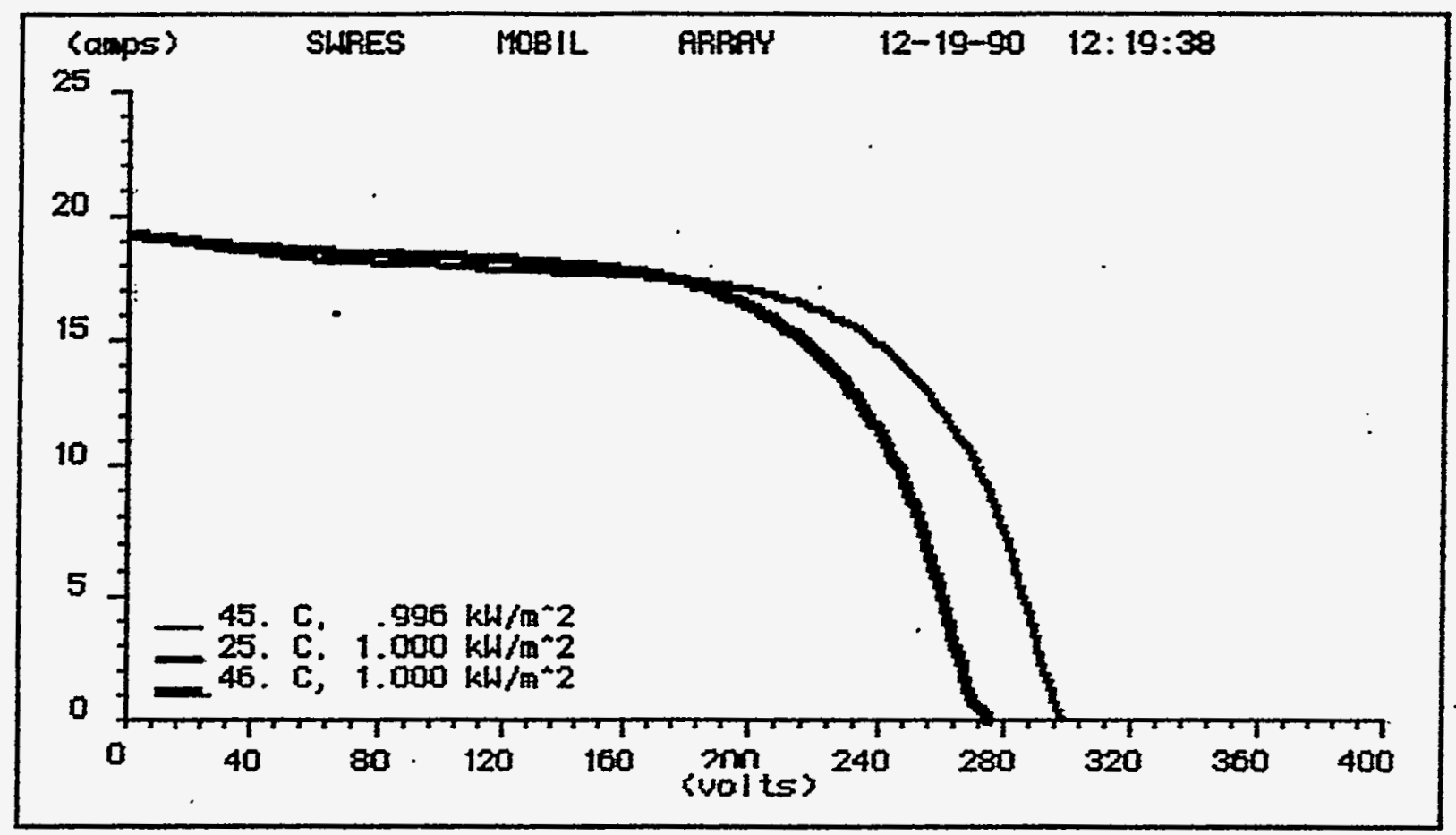

Call Temperature ....... 45.000 deg $C$

Irradiance ............. .995 kH/m²

Series Resistance ....... 2.310 ohms

Uoltage Coefficient ..... -1.260 wolts/deg c

Current coefficient ..... .029 coms/deg C

RCTUAL CURUE DATA: (fill factor $=62.3 \$$ )

Short circult ourrent .... 19.2 amps

Open circuit val tage ..... 275. 1 val ts

Max power current ....... 16.1 amps

Max power vol tage ....... 205.1 valts

Max power ........... 3297.7 watts

MORMAL IZED CURUE DATA: (fill factor $=63.98$ )

(for 25.0 deg $\mathrm{C}$ and $1.0 \mathrm{~kW} / \mathrm{m}^{-2} 2$ )

Max power current ....... 15.7 amps

Max pourer val tage ....... 230.3 volts

Max pouper ............ 3507.3 watts

MORMALIZEO CURUE DATA: (fill factor $=62.37$ )

(for $46.0 \mathrm{deg} C$ and $1.0 \mathrm{kH} / \mathrm{m}^{\wedge} 2$ )

Max power current ....... 16.2 anps

Mox poer voltage ....... 203.6 volts

Max power ............ 3295.1 watts 


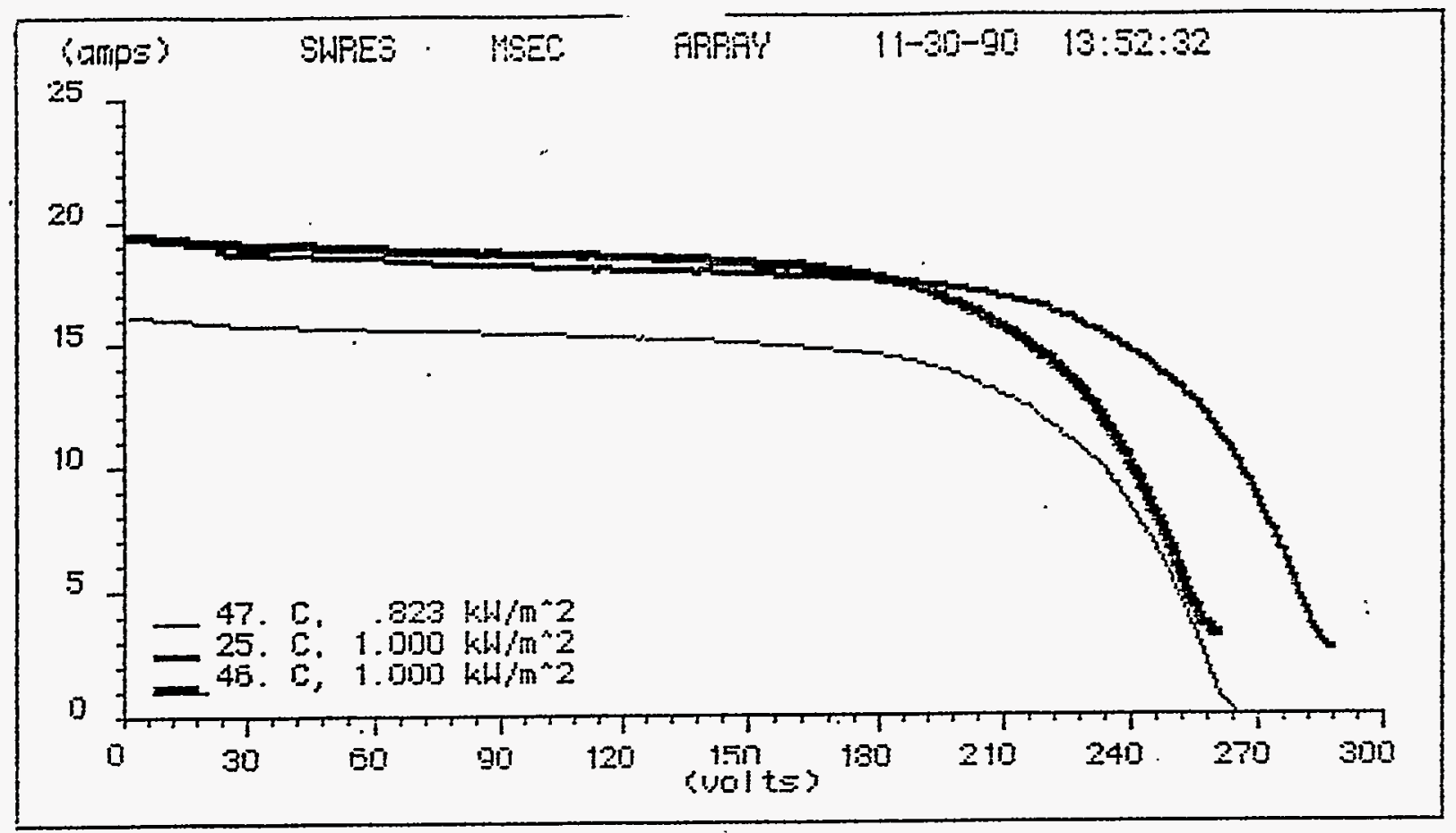

Cell Temperature ........

Irradiance .............

Ser i Es Resistance ....... 2.310 ohms

lioltage confficient. . . . - -1.250 wolts sidag 5

Current coefficient ..... . D2g ampsideg $C$

ACTUAL CURIE DATH: (fill factor $=63.6 \mathrm{~s}$

Short cirouit aurpent .... 15.1 amps

Open circuit vol tage .... 26E.Q volts

Mrox power gurrent ...... 13.3 amps

Max priner vol tage ....... 198.1 volts

Hox pougr ........... 2791.1 matts

HOPIIALIZED CURUE DATA: (fIIl factor $=67.08$ )

Sfor 25.0 deg 5 and $1.0 \mathrm{kH} / \mathrm{m}^{2} 2$ ?

Mox pourer atrment ....... . 15. o omps

Ma: puwer vol tage ....... 22E. E volts

Mras pouper ........... 3525.5 yntts

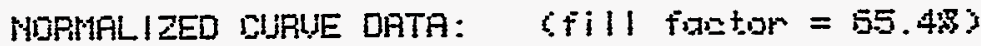

(for $40.0 \mathrm{deg}\left[\mathrm{and} 1.0 \mathrm{kH} / \mathrm{m}^{n} 2\right.$ )

Max poumer current. ...... 16.7 amps

Max pourer voltage ....... 198.4 volts

Max power ........... 3322.4 whtts 


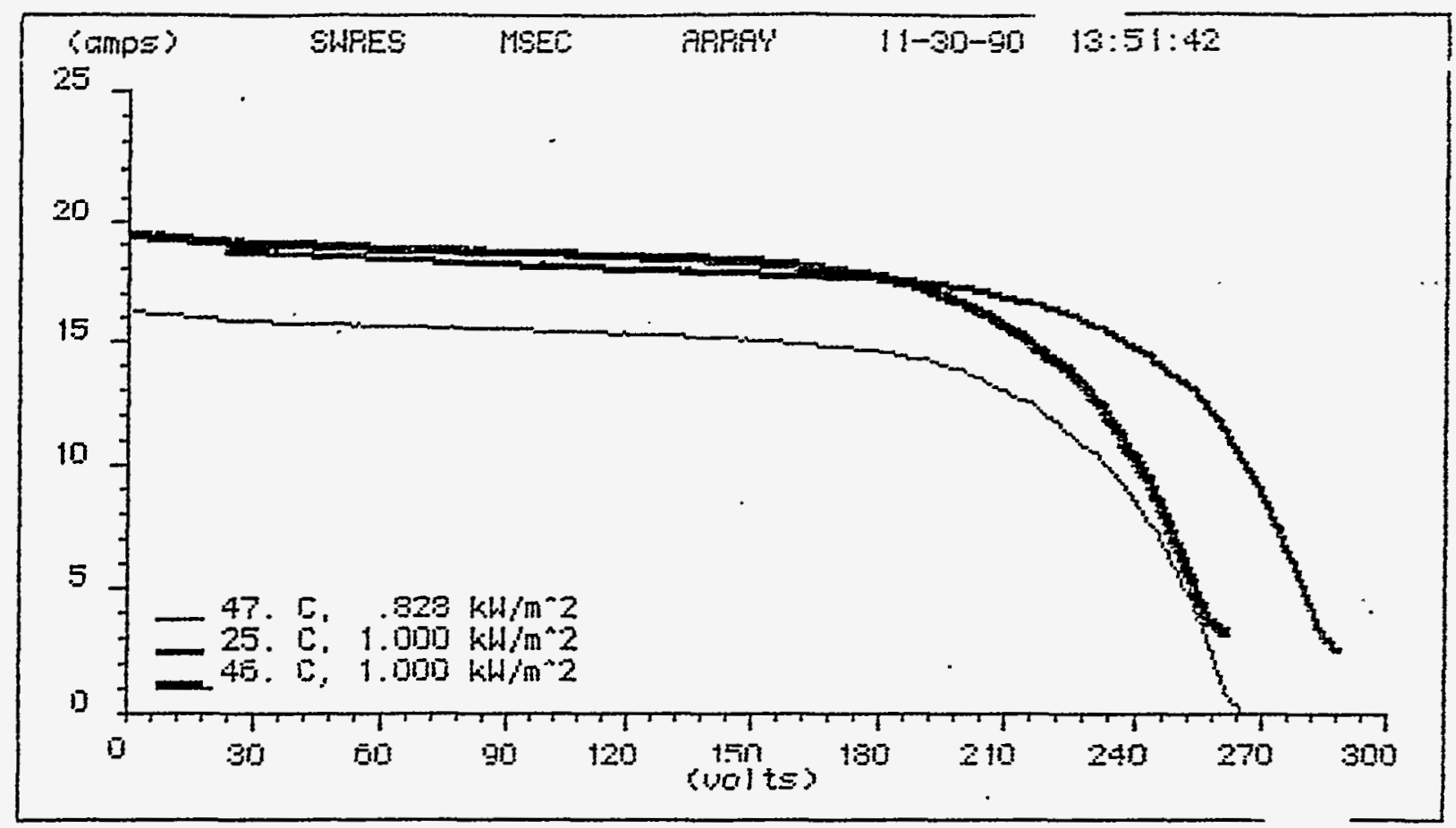

Cell Temperature .......47.000 deg $C$

Irradiance ......... .829 $\mathrm{kH}^{2} \mathrm{~m}^{\wedge} \mathrm{z}$

Series Resistance ........ 2.310 ohms

voltage coefficient ..... -1.250 wolts/deg c

Curpent Coefficient ..... .029 ampsideg 5

ACTUAL CUAUE DATA: (fill faetor $=63: \mathrm{EB}$ )

Shomi airat aurrent .... to. 2 amps

Gperr circuit voltage .... 266.5 volte

Wax power current. ...... 13.8 rmps

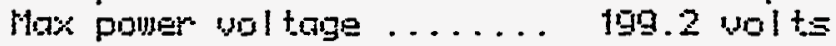

Mox pomer ........... 2753.2 matts

MOFHALIZED DURUE DATA: CAIll faOtor = 65.6\%

\{for 25.0 deg $\mathrm{C}$ and $1.0 \mathrm{kH} / \mathrm{m}^{\wedge} \mathrm{Z}$ ?

Max pouser atrm:ant. ...... 15.0 amps

Max pumer vel tage..$\ldots \ldots .225 .3$ valts

Max power . . . . . . 3529.3 matts

HORTILLIZED DURUE DATA: CAIII fGEtor = 5.5.3R ?

(for 40.0 deg $C$ and $1.0 \mathrm{kh} / \mathrm{m}^{2} 2$ )

Mox pourer gurpent ....... 15.5 amps

Max power voltage ....... 199.9 volts

Max poumer ............ 3.325.3 matts 


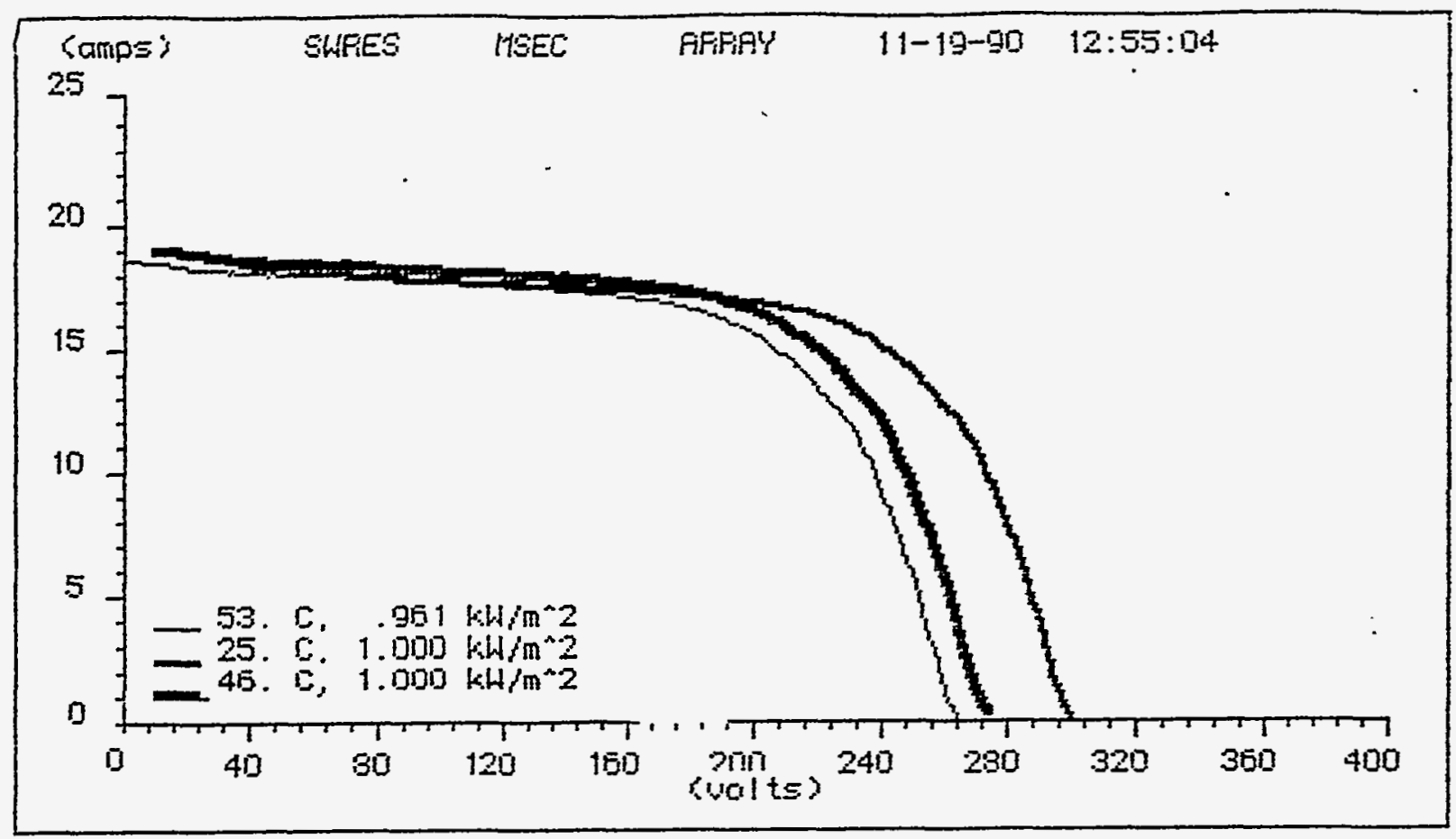

Cell Temperature ....... 53.000 deg $C$

Irradiance .......... . $351 \mathrm{kH}^{2} \mathrm{~m}^{-2}$

Series Resistance ...... 2.3to ohms

Uoltage coafficient .... -1.250 woltsideg 5

Current Coefficient. ..... .029 ompsiseg $\mathrm{C}$

ACTUAL CURUE DATA: (fill foctor $=0.3 .28$ )

Short aireuit aurpent .... 19.5 amps

Open sircuit voltage .... 265.4 volts

Max pomer current ...... 15.7 amps

Max pouer vol tage ....... 199.2 volts

Ma\% poum ........... 3125.8 matts

MOPITILIZED CURUE DRTA: (fill fGEtor = 55.7B)

(for 25.0 deg 0 and $1.0 \mathrm{kH} / \mathrm{m}^{*} 2$ ?

Max pouer gurment ....... 15.5 amps

Mas power val tage ...... 234.? wa! $t=$

Max pryer ............. 3551.5 wntts

HOPHALIZED CUPUE DATA: Gill FGOtor $=54.2 \% 3$

(for 40.0 Jeg $C$ and $1.0 \mathrm{kH}\left(\mathrm{m}^{\circ} \mathrm{2}\right)$

Max pouser current ....... 15.2 amps

Max power vol tage ....... 200.8 volts

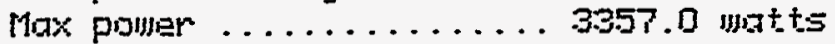



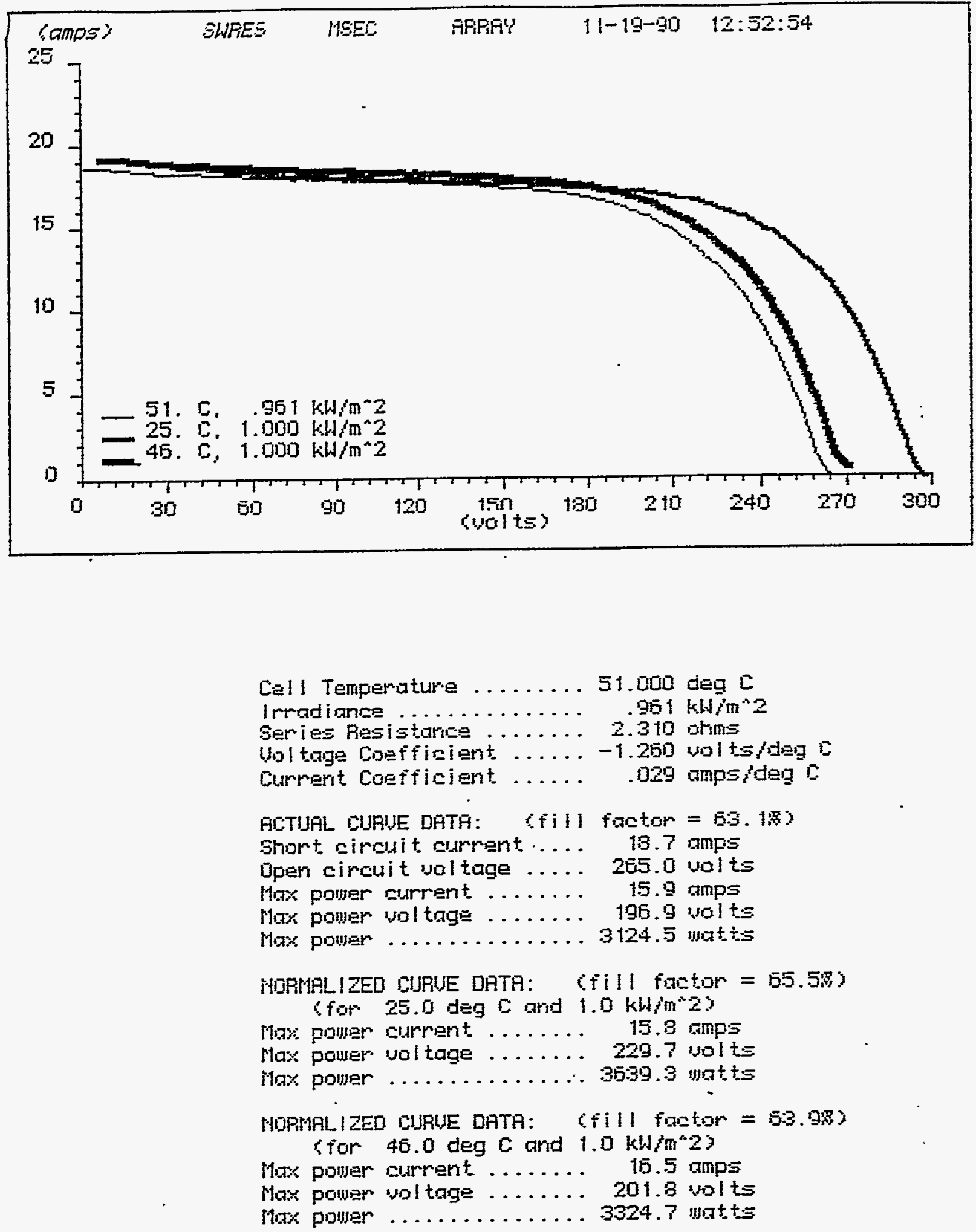


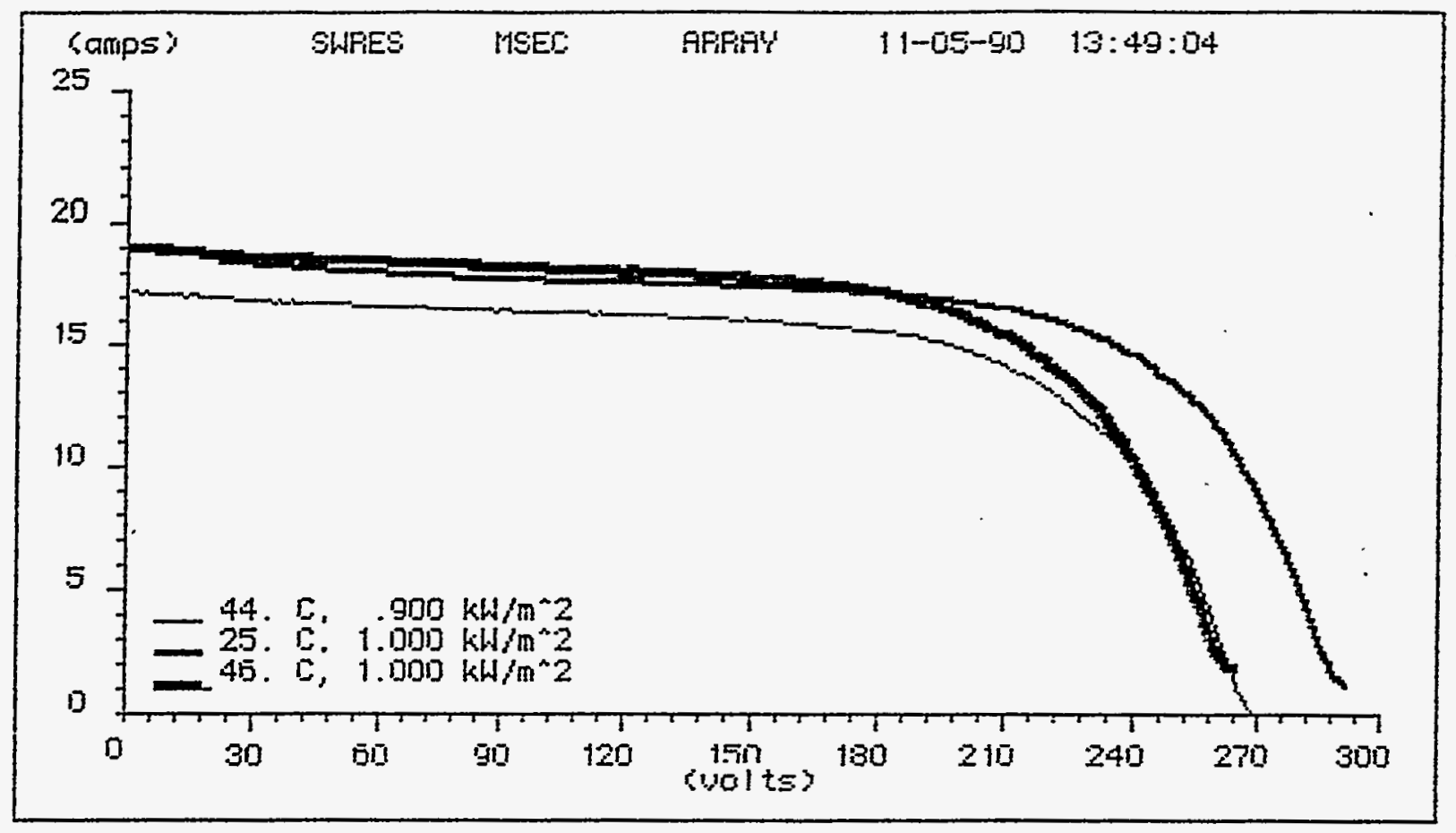

Cell Temperature ........44.000 deg 5

Irrodiance ........... .9no $4 H_{i} m^{* 2}$

Series Resistunoe ....... 2.3t0 ohms

Uoltage Coefficiant ..... -1.200 usltsideg

Curpent Coefficient. .... .029 anpsideg $\mathrm{C}$

FLTUAL CURUE DATA: (fill faCtor = 53. SB?

Short circuit current. ... 17.2 amps

Upen circuit vol tage .... 270.1 volt:

Max pouser'current ....... 14.4 amps

Max pouner wol tage ....... 205.3 volt.s

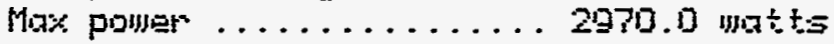

HORMALIZED CURUE DATA: Cfill factor $=55.2 \mathrm{k}$ :

(for 25.0 deg $\mathrm{C}$ and $1.0 \mathrm{khl} / \mathrm{m}^{2} \mathrm{2}$ )

Max pourer aurment ....... 15.9 ame:

Ma: power vol tage ...... 223.7 volt:

Max pouner ............ 3557.5 mat.

HORTALIZED CURUE DATA: 6fill Fotor $=64.793$

(for 40.0 deg $C$ and $1.0 \mathrm{kh} / \mathrm{m}^{2} \mathrm{2}$ )

Max poujer aurrent....... 15.3 umps

Max power voltage ....... 200.3 volts

Max pourer ........... 3252.3 untts 


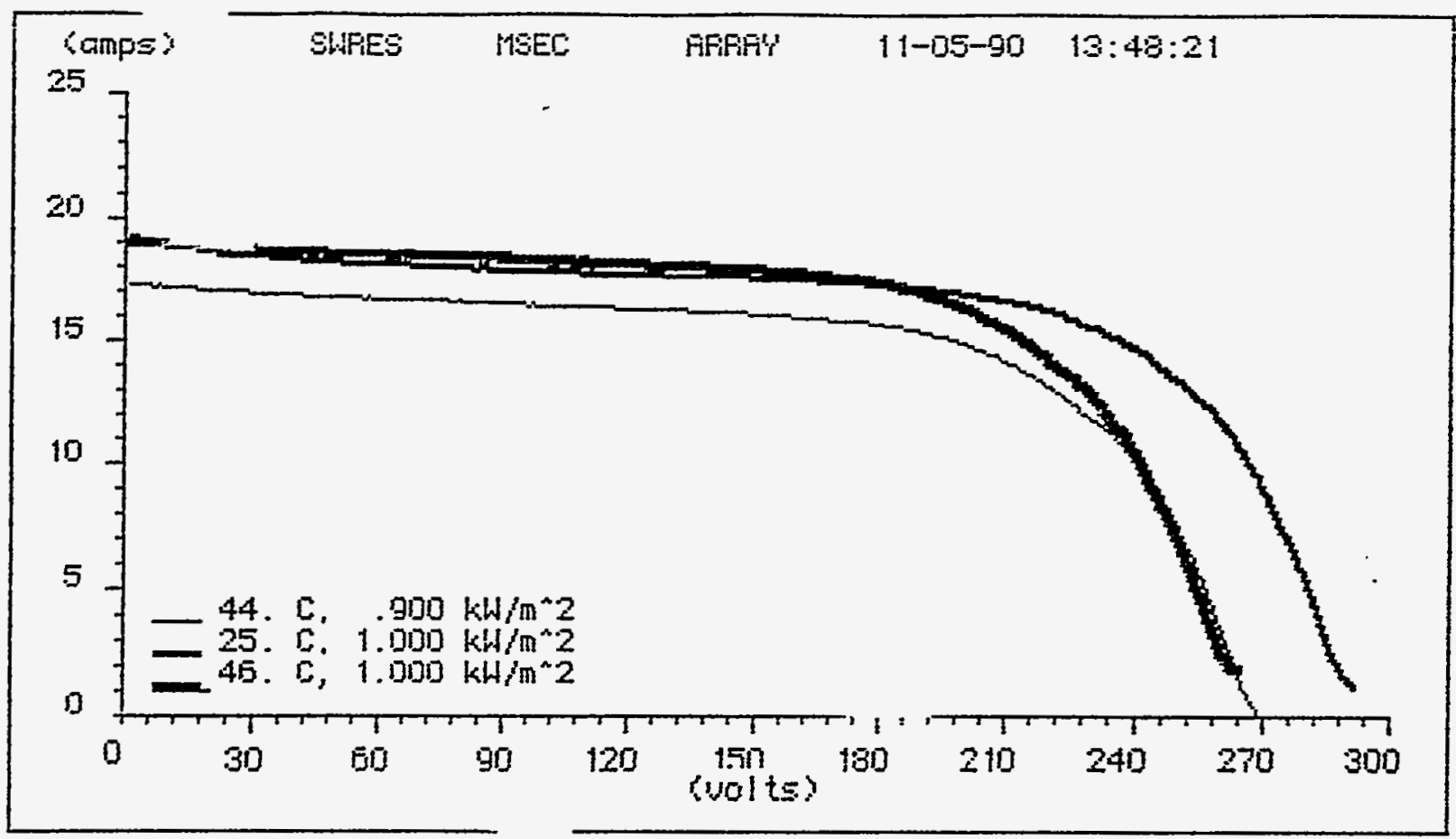

Cell Temperature ....... 44.000 deg $\mathrm{C}$

Irradiance ........... .900 kh/m

Series Resistance ...... 2.310 ohms

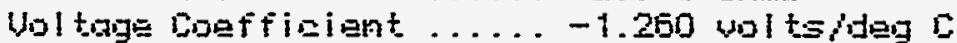

curment coefficient ...... .029 ampsideg $c$

FCTUAL LURUE DATH: ¿fill fGotor $=64.0 \%$ )

Short simeuit sumpant.... 17.2 amps

upen circuit vol tage ..... 270. 1 wal ts

Max pouner current ...... 14.5 amps

Max pouer vol tage ....... 20.5. 1 volts

Hax pounr ............ 2979.8 wht.

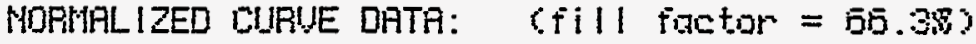

Sfor $25.0 \mathrm{deg} C$ ard $1.0 \mathrm{kH} / \mathrm{m}$ "

Max poumer current. ...... 15.8 Jmps

Max power. vol tage ....... 220.0 wolts

Max ponar .............. 3579.5 matts

MIDPIALLIZED CURUE DATA: $\quad$ fill fGotor $=54.78)$

(for $40.0 \mathrm{deg} C$ and $1.0 \mathrm{kH} / \mathrm{m}^{*} \mathrm{Z}$ )

Max poujer ctarrent ....... 15. 1 amps

Migx power altage . . . . . 202.7 volts

Mox pouler ............ 3271.2 mutts 


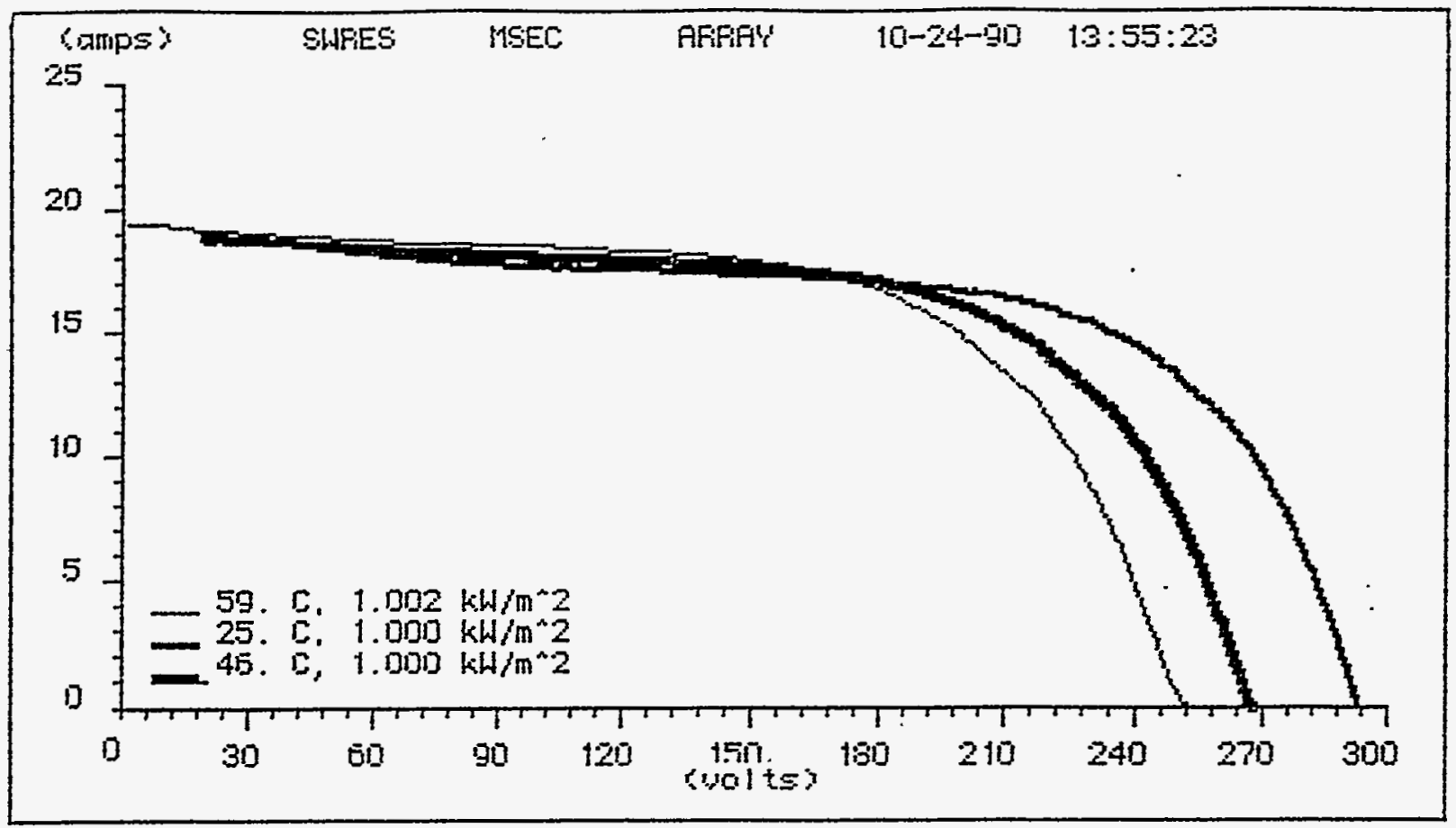

Cel 1 Temperature ........59.000 deg $\mathrm{C}$

Irradiance ............ $1.002 \mathrm{kH} \mathrm{im}^{* 2}$

Series Resistance ....... 2.310 ohms

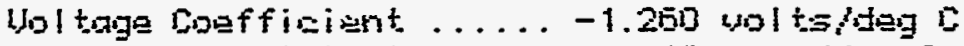

Curment Coeficiaient. .... . .029 ampstdeg 5

ACTUAL CUAVE DATA: (fill factor $=51.8 \mathrm{~S}$ )

Short circuit rumpent .... 19.4 amps

open circuit uol tage .... 253.0 wolts

Hox pouser surrent ....... . 15.10 smps

Max power voltage ....... 189.3 valts

Max pouner . . . . . . . . 3035.5 mats

IOPRTALIZED CURUE DATH: (fill fantor $=64.68$ )

(for $25.0 \mathrm{deg}$ [0 and $1.0 \mathrm{kH} / \mathrm{m}^{*} 2$ ?

Max pouser gurrent ....... 15.5 amps

Max power sol tage ....... 229.2 uol t:s

Hax pomer . . . . . . . 2542.5 mat:

NORIALIZED CURUE DATA: Cfill fBOtor $=53.0 \mathrm{~S}$ ) (for 40.0 deg $C$ and $1.0 \mathrm{kH} / \mathrm{m}^{\circ} 2$ )

Max power current...... 15.1 amps

Max pouser vol tage ....... 201.3 volts

Max pourar ............ 3234.2 matts 


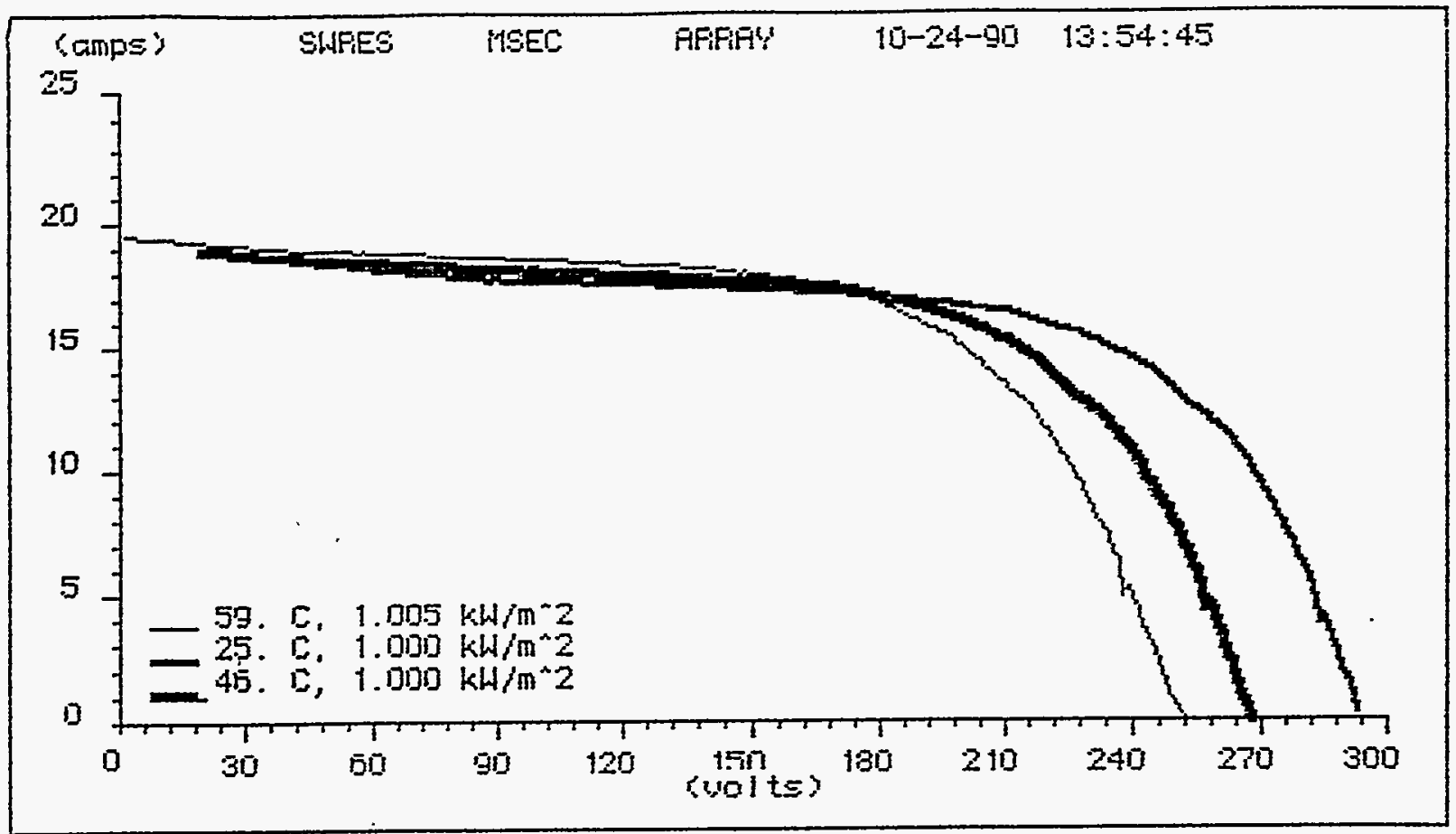

\footnotetext{
Cell Temperature ....... 59.000 deg $\mathrm{C}$

Irradiance .......... $1.005 \mathrm{kH} / \mathrm{m}^{2} \mathrm{z}$

Series Resistonce ........ 2.310 ohms

Uoltage coufficient .....-1.260 usltsideg $c$

Current Coefficient ..... .029 ampsideg C

RCTUAL CUAVE DATA: (fill faetor $=61.68$ )

Short circuit cumpent.... 19.5 amps

Upen circuit vol tage .... 253.3 volts

Max power current ....... i5. 3 amps

Max power voltage ....... 185.5 wolt

Max power . . . . . . . . . 3039.5 mot:ts

NOPMALIZED DURUE DATA: (Fill factor $=54.48$ )

(for 25.0 deg $C$ and $1.0 \mathrm{kH} / \mathrm{m}^{2} 23$

Max pomar aurrent ...... 15.7 amps

Max power vol tage ....... 225.8 volts

Max pomer . . . . . . . . 3530.7 matts

HORHFILIZED DURUE DATA: Cfill faotor = 62.3BS

(for 46.0 deg $C$ and $1.0 \mathrm{kh} / \mathrm{m}^{+2}$ )

Max power current ...... 15.8 amps

Max pouer voltage ...... 203.9 volts

Max poiner ........... 3223.0 unt.s
} 


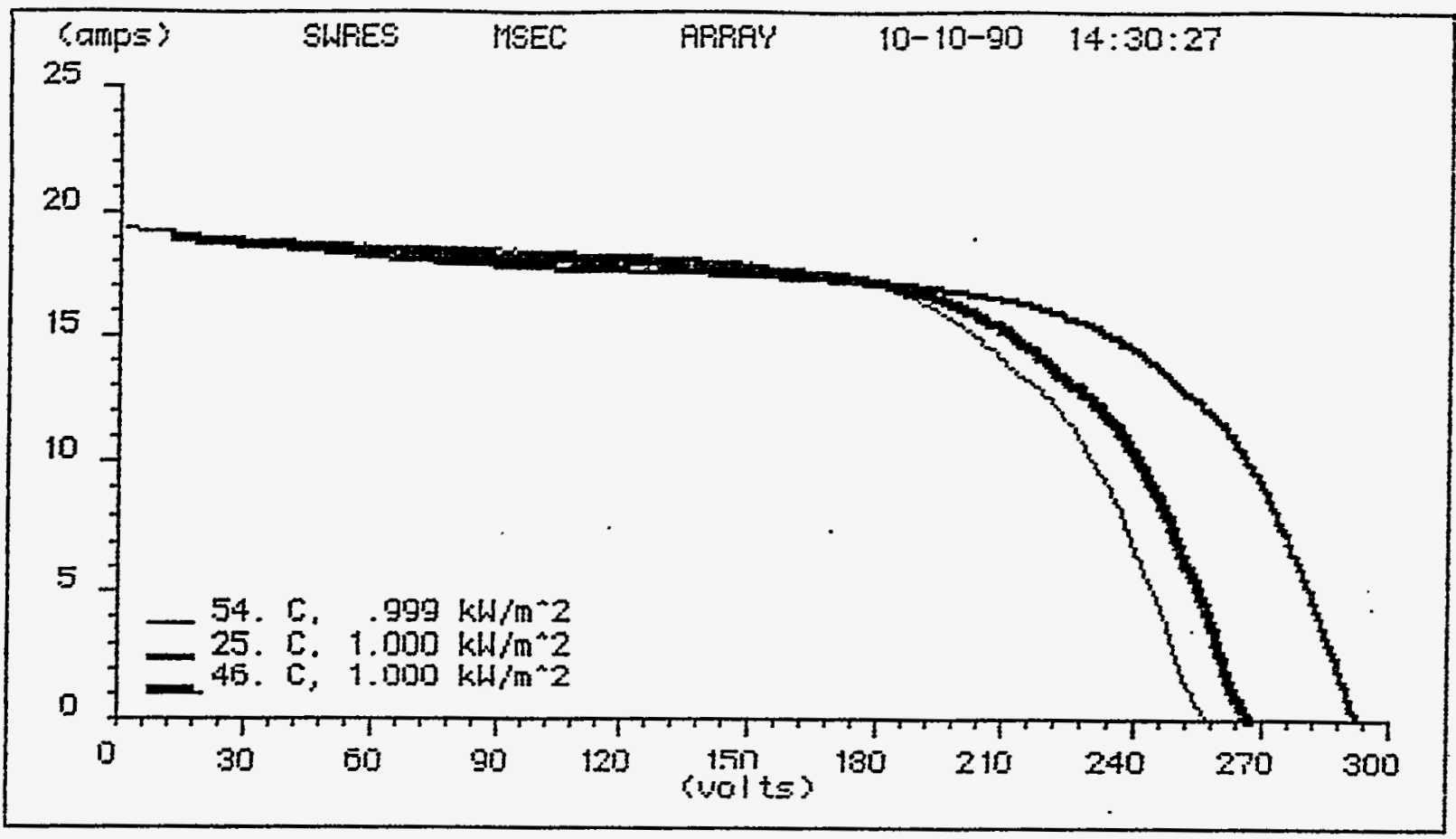

Cell Temperature .......5.54.000 deg $C$

Impadiance ........... $.999 \mathrm{kH} \mathrm{m}^{\circ} \mathrm{2}$

Series Resistance ....... 2.310 ohms

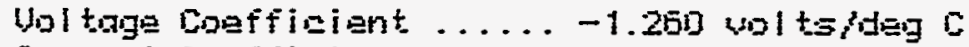

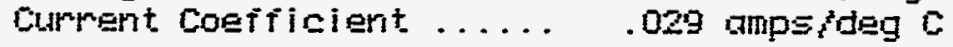

FITUAL CIIRIJE DATA: 6fill factor = E2.5\%)

Short cirauit aurrent .... 19.4 amps

Open eireuit ual tage ..... 253.0 val ts

Mrx poujer current. ...... 15.2 amps

Mox pouser vol tage ...... 192.2 wol t.

Mox punar ..........3121.2 matts

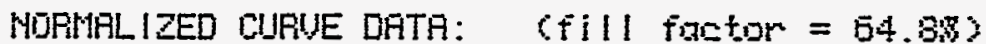

(for 25.0 deg $C$ ard $1.0 \mathrm{kH} \mathrm{im}^{2} 2$ ?

Mox pouser ourrant ....... 15.7 amps

Max power wol tage ....... 225.9 valts

Max pounar ............ 3558.0 matts

MDPMALIZED CIJAUE DATA: $\quad$ CIII fagtor $=63.2 \mathrm{~B} 3$

Sior 46.0 deg $C$ arid $1.0 \mathrm{kH} / \mathrm{m}^{\prime \prime 2}$;

Max pouler current ....... 16.0 amps

Max power woltage ...... 202.8 vol ts

Max pomer ........... 3249.7 matts 

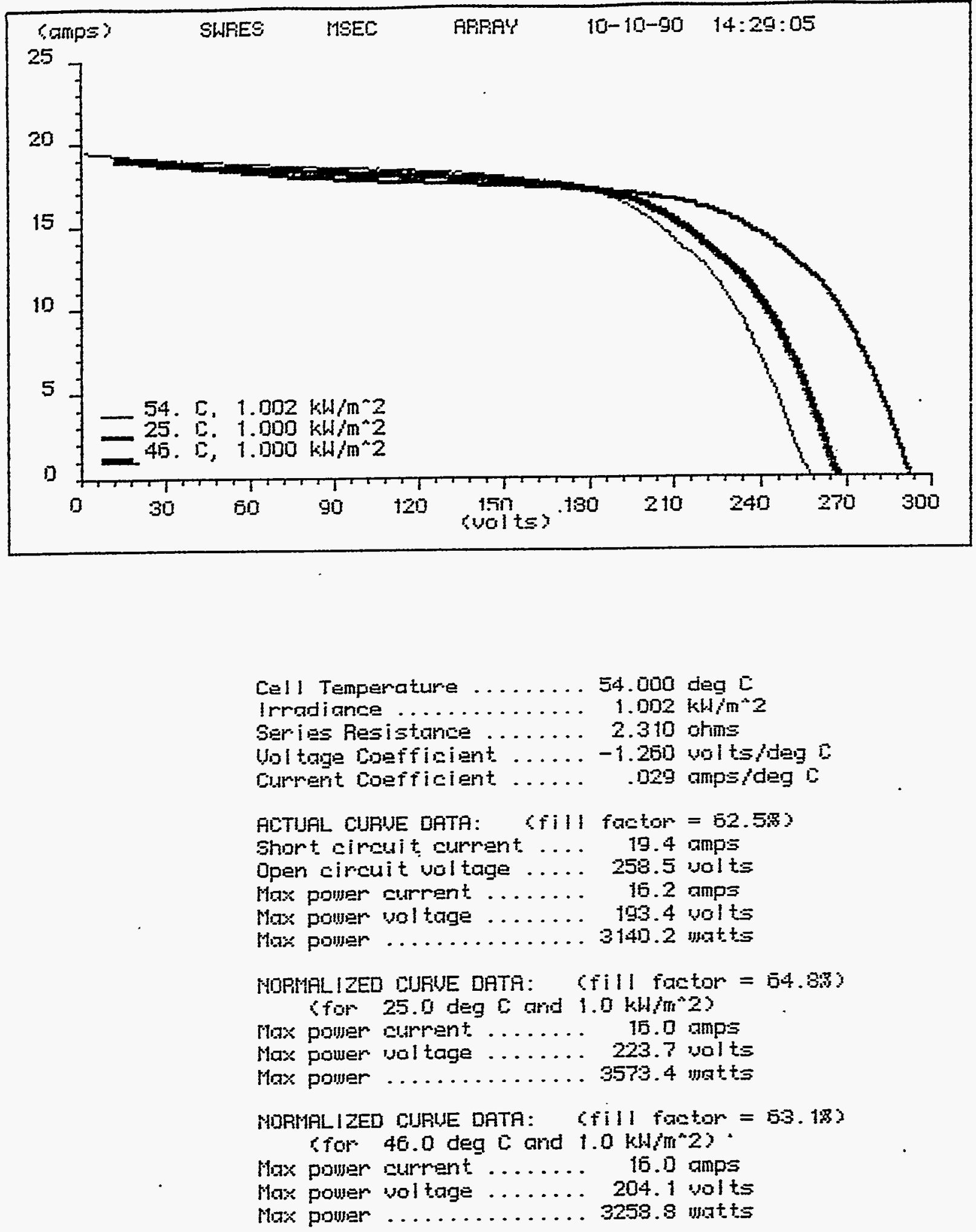


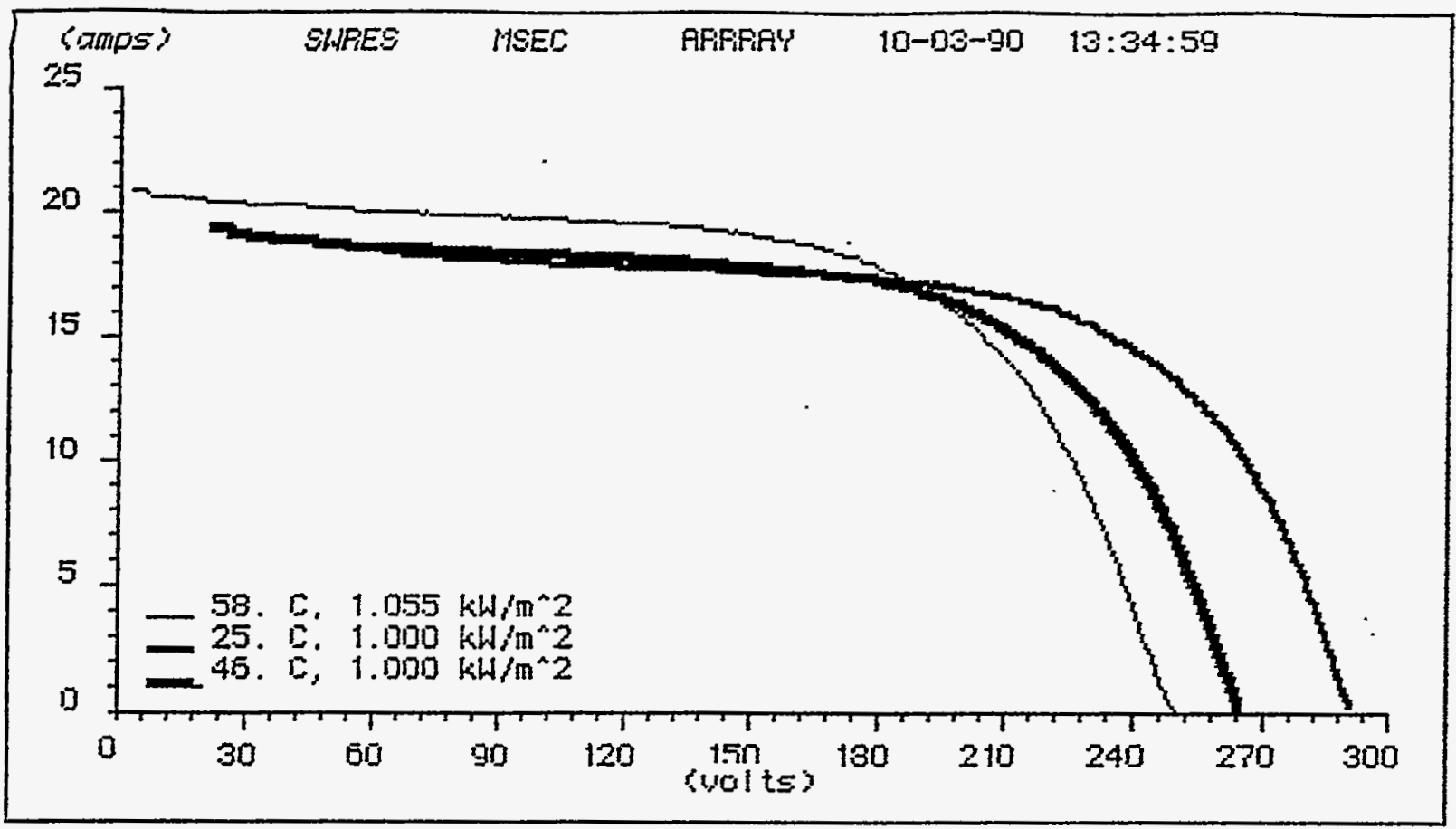

Cell Temperature .......58.000 deg $ᄃ$

Irradiance ........... $1.055 \mathrm{kH}^{2} \mathrm{~m}^{*} 2$

Series Resistance ....... 2.310 ohms

Uol toge coefficient ..... -1.250 uoltsideg c

Current Coefficient ..... .029 ampsideg $C$

ACTUAL CURUE DATA: Gill factor $=61.3 \mathrm{~m}$ \%

Short cirouit curpent .... 20.9 amps

open eircuit wal tage ..... 251.5 volts

Max power current. ...... 17.4 amps

Max pourer vol tage ...... 185.2 volts

Hax pourar ............ 3221.3 ungt.s

HOPHAL IZED CURUE DRTA: Sfill factor $=63.5 \% \mathrm{~s}$

(for 25.0 deg $C$ and $1.0 \mathrm{kH} / \mathrm{m}^{\circ} 2$ )

Max pouner Gurrent ....... 15.9 amps

Max power vol tage ....... 225.5 volts

Max pomer ............ 3581.5 urgtts

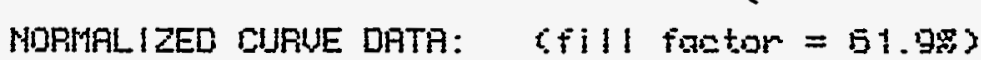

(for 40.0 deg $c$ and $1.0 \mathrm{kH} / \mathrm{m}^{\circ} 2$ ?

Mox pouser current ....... 15.5 amp:s

Max power voltage ...... 197.7 wolts

Max pouser ........... 3254.2 ugatts 

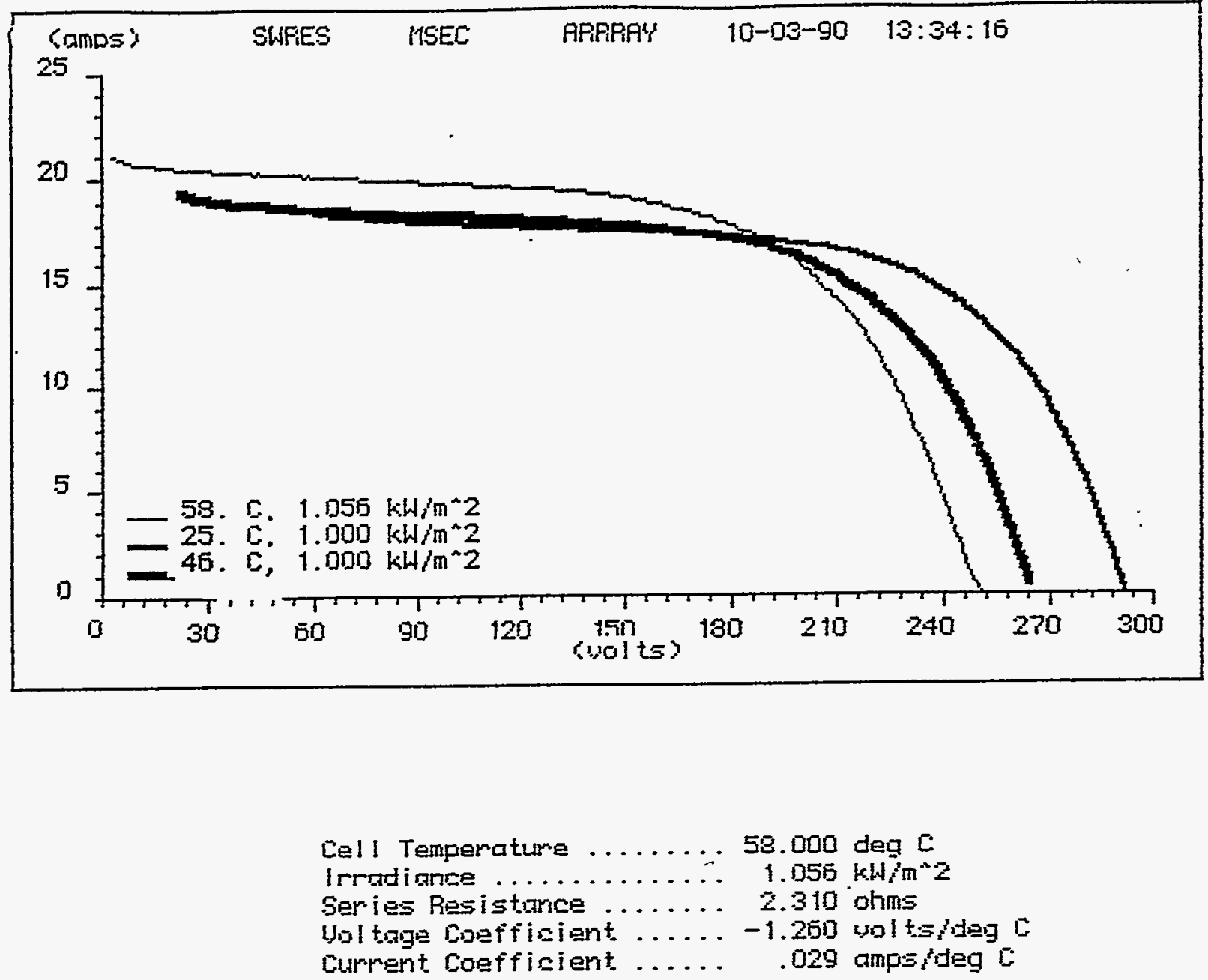

ACTUAL CUAUE DATA: Gill factor $=51.33$ )

Short cirauit surrent. . . 20.9 amps

Open circuit vol tage ..... 251.9 volts

Max power gurrent ....... 17.5 ramps

Hax power voltage ....... 185.2 volts

Max poumer ........... 3232.9 matts

NDFMALIZED CURUE DATA: (

ifor 25.0 deg 0 arod $1.0 \mathrm{kH} / \mathrm{m}^{2} 2$ ?

Mixe power current ...... 15.3 amps

Max power vol tage ...... 226. ? jol ts

Hax power ............ 3589.5 watts

HORMALIZED DUPUE DATA: Gill fontor $=51.982$

(for 40.0 deg $C$ and $1.0 \mathrm{kH} / \mathrm{m}^{\circ} 2$ )

Max power current ....... 15.4 amps

Max power vol tage ...... 198.9 volts

Max pouser ........... 3264.2 uratts 


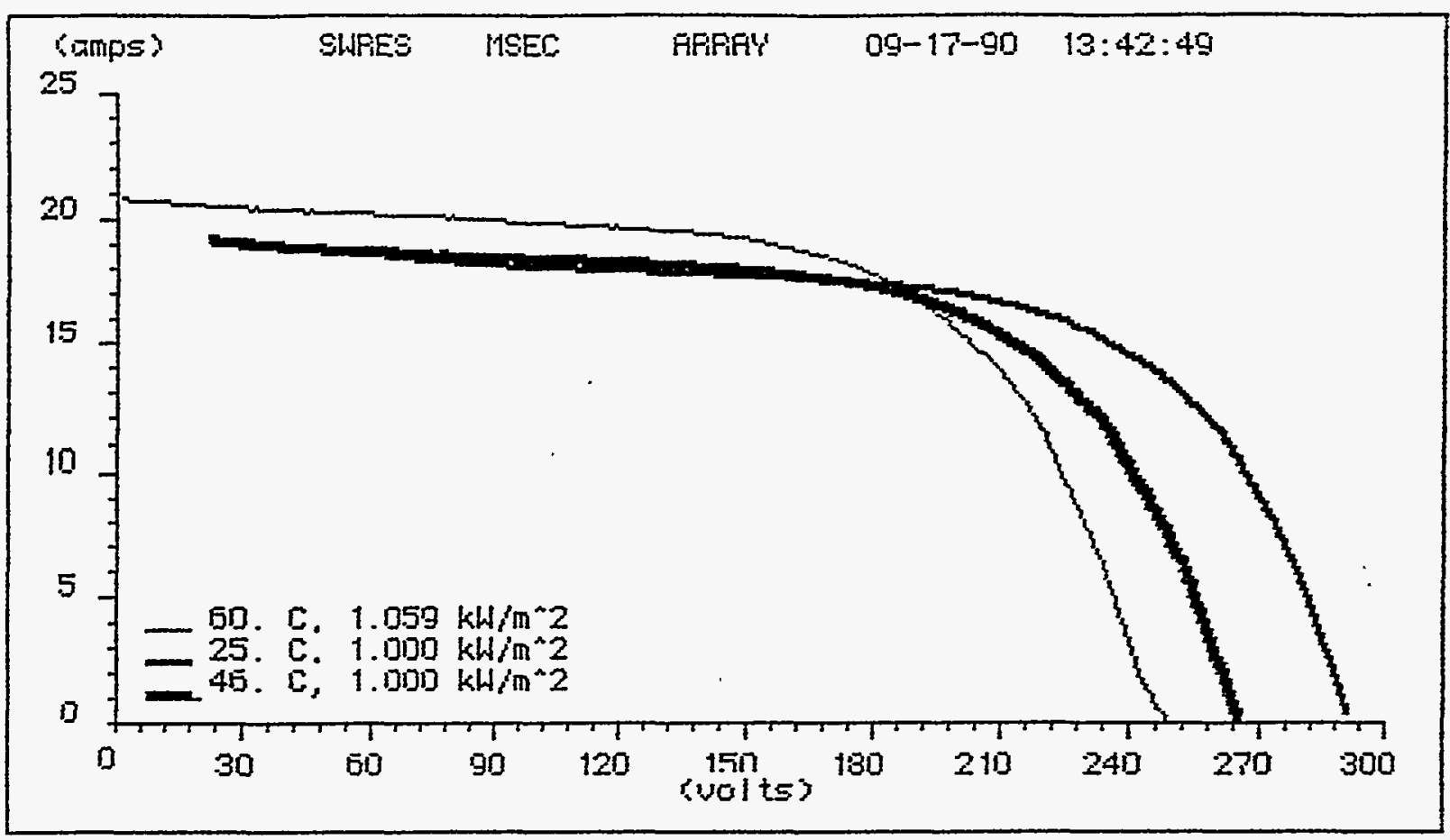

Cell Temperature ....... 60.000 deg C

Irradiance ........... $1.059 \mathrm{kH} / \mathrm{m}^{* 2} 2$

Series Resistance ....... 2,310 ohms

Uoltage coefficient ...... -1.250 woltsidag $c$

Current Coefficient ..... .029 ampsideg $c$

ACTURL CURUE DATA: (fill faetor $=61.48$ )

Short circuit current .... 20.9 amps

Open circuit voltage .... 249.9 volts

Mrax poimer current ....... 13.4 amps

Max power soltage ....... 134.0 volts

Max power ............. 3201.3 yatts

MORMALIZED DUPUE DATA: (fill factor $=54.0 \mathrm{~B})$

(for $25.0 \mathrm{deg} C$ ard $1.0 \mathrm{kH} / \mathrm{m}^{2} 2$ )

Max poujer sumpent ....... 15.9 amps

Max power vol tage ....... 224.8 volts

Mrox pouner ............. 3585.0 matts

MORMALIZED CIJRUE DATA: (fill factor $=52.23$ )

(for 40.0 deg $C$ and $1.0 \mathrm{kH} / \mathrm{m}^{\circ} 2$ ?

Max power gurrent ....... 16.1 amps

Max power voltage ....... 202.9 wolts

Max power ............. 3258.6 uratts 

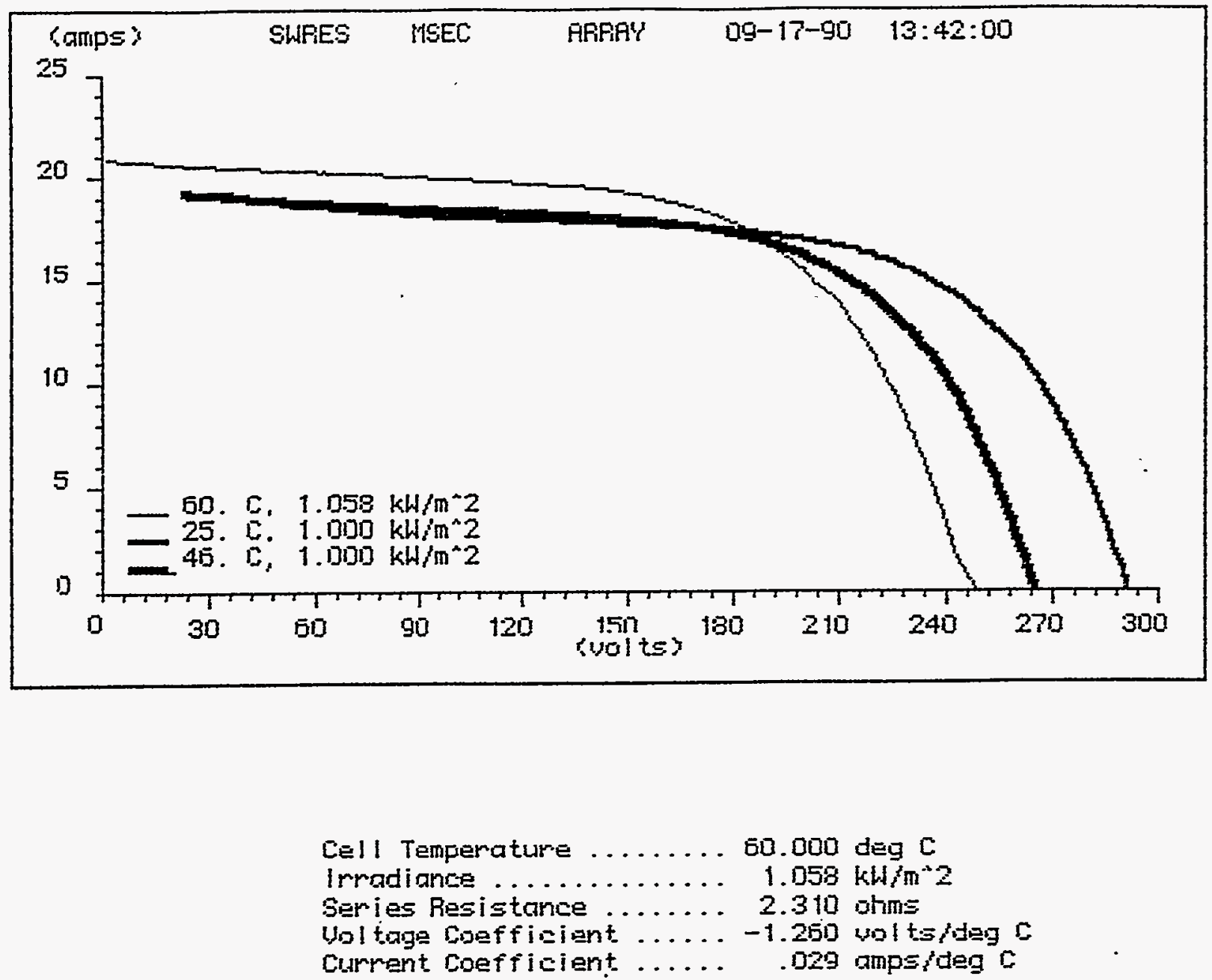

ACTUAL CURUE DATA: (Fill factor $=51.48$ )

Short sircuit current ... 20.9 amps Open circuit vol tage .... 249.5 valts

Max pouser current. ....... 17.3 amps

Max power vol tage ....... 195.2 wolts

Ma\% pourar . . . . . . . 3197.7 watt:s

HORHALIZED CURUE DATA: (fill figetor $=84.18$ ?

(for 25.0 deg $\left[\right.$ and $1.0 \mathrm{kH} / \mathrm{m}^{*} 2$ )

Mox pouser sumpent....... T5. D amps

Max power val tage ....... 224.7 valts

Maxe power ........... 3580.3 matts

NORMALIZED CURUE DATF: GFill fBotor $=62.3 \% 3$

(for $40.0 \mathrm{deg} C$ and $1.0 \mathrm{kH} / \mathrm{m}^{\circ} 2$ )

Max pouser current....... 15.2 amps

Max pourer voltage ....... 201.6 volts

Max pouser ............ 3260.9 watts 


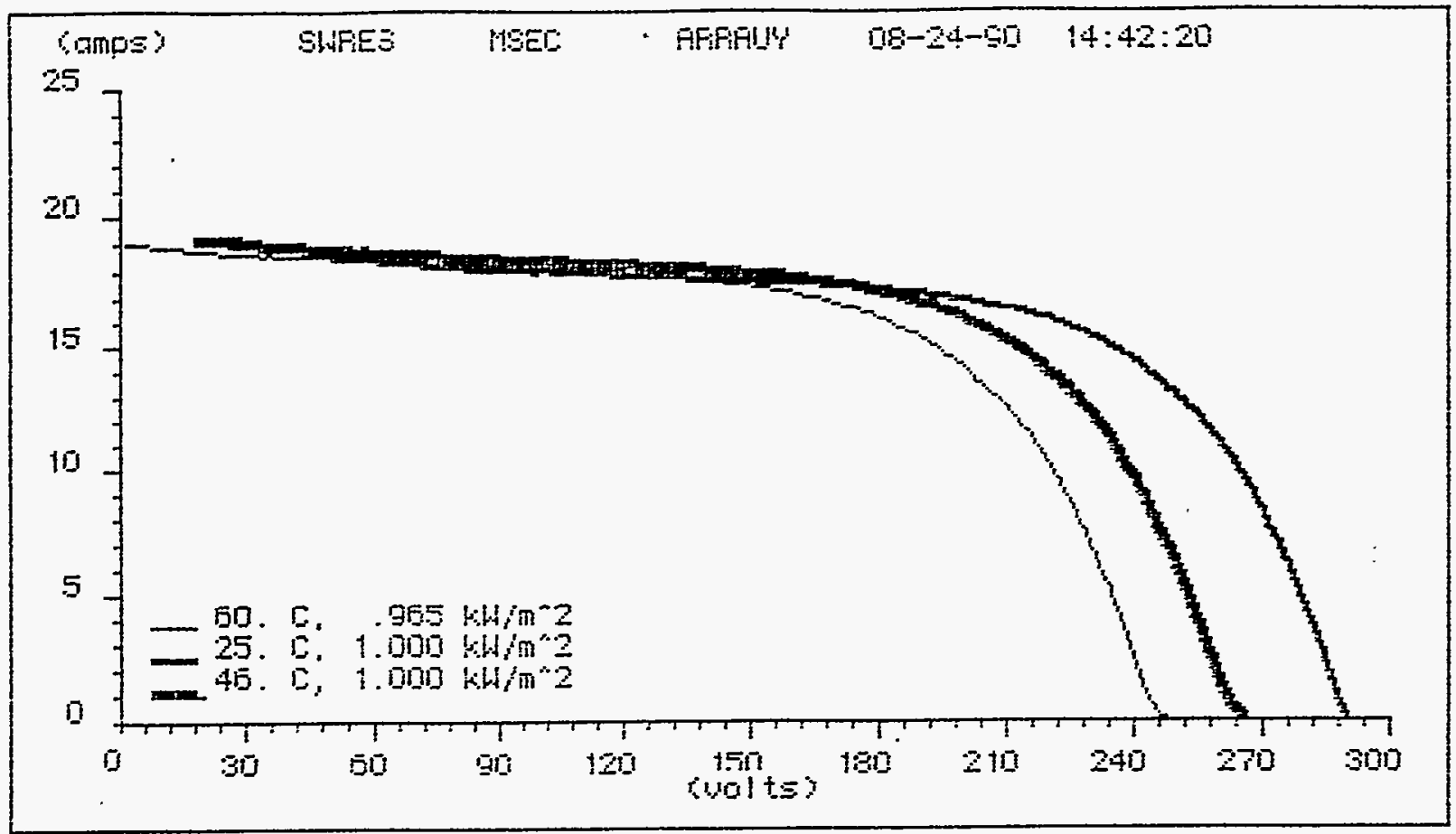

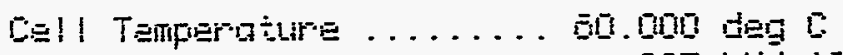

Irrodiance ........... . $95.5 \mathrm{kH} \mathrm{m}^{2} \mathrm{z}^{2}$

Sori

U⿻ 1 t

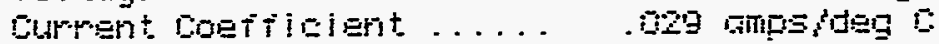

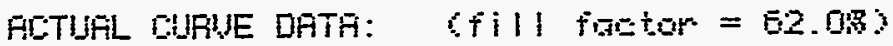

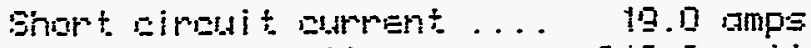

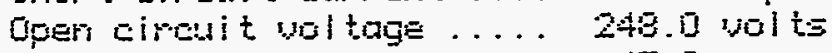

Max primer current. ...... 15.9 amps

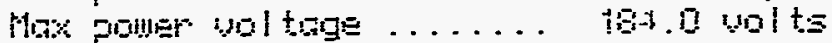

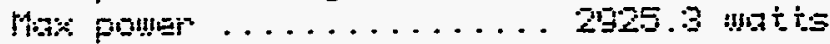

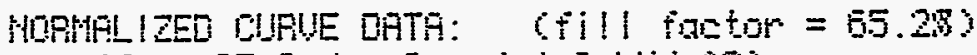

6to 25.0 deg 50 and $1.0+4 \mathrm{~m}^{*} 2$

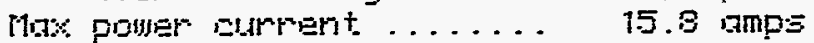

Max pumer wo! tage ...... . 225.4 vilts

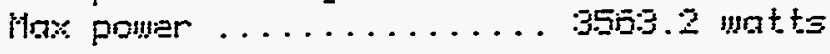

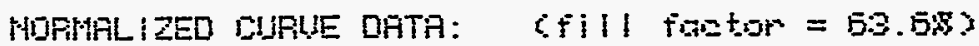

(for 45.0 deg $\mathrm{C}$ arid $1.0 \mathrm{kbl} / \mathrm{m}^{\circ} \mathrm{z}$ ?

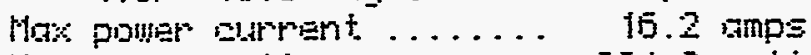

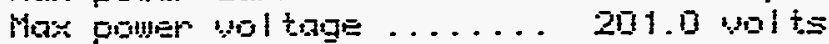

Toge pourer ........... 3249.9 unt.s 

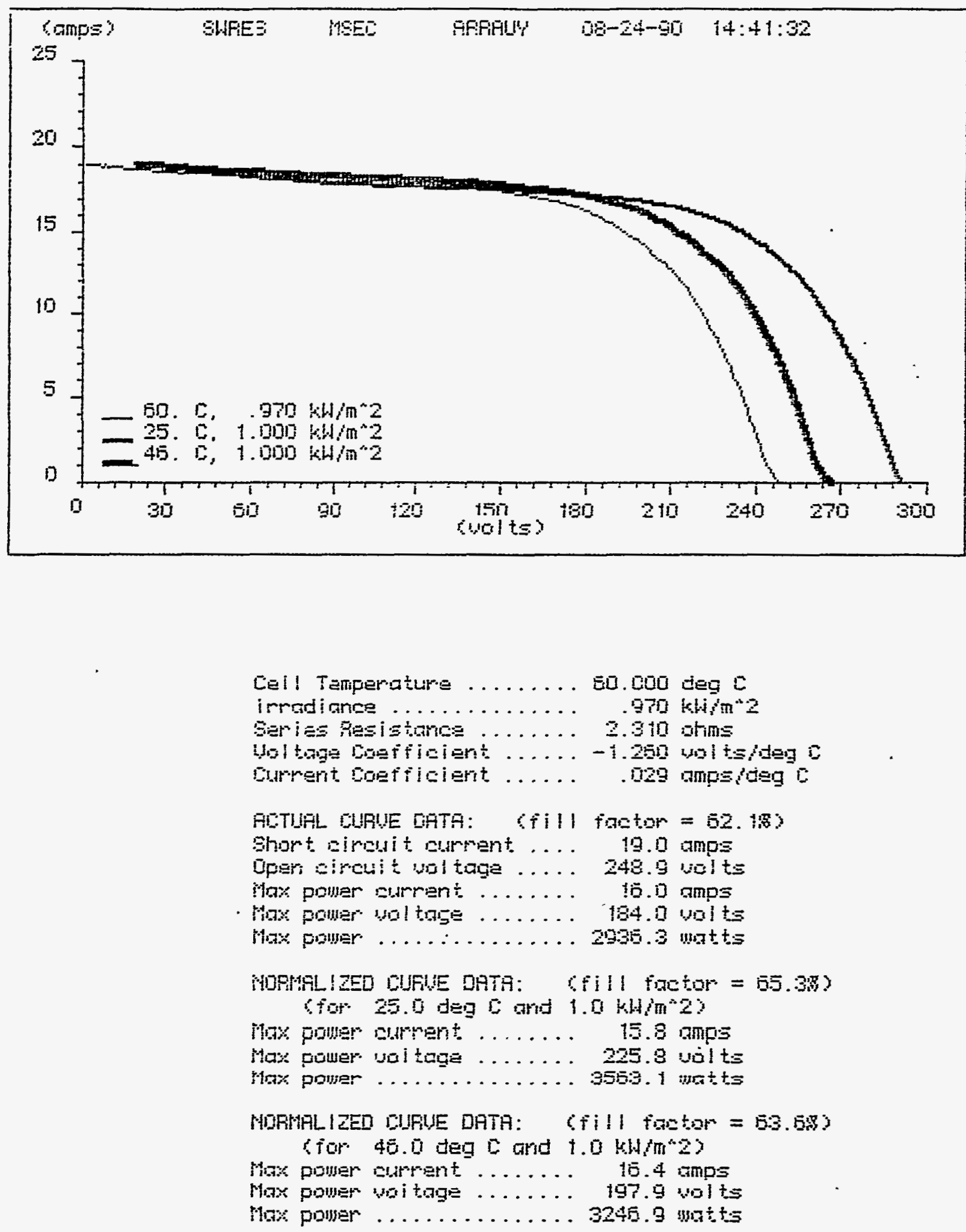


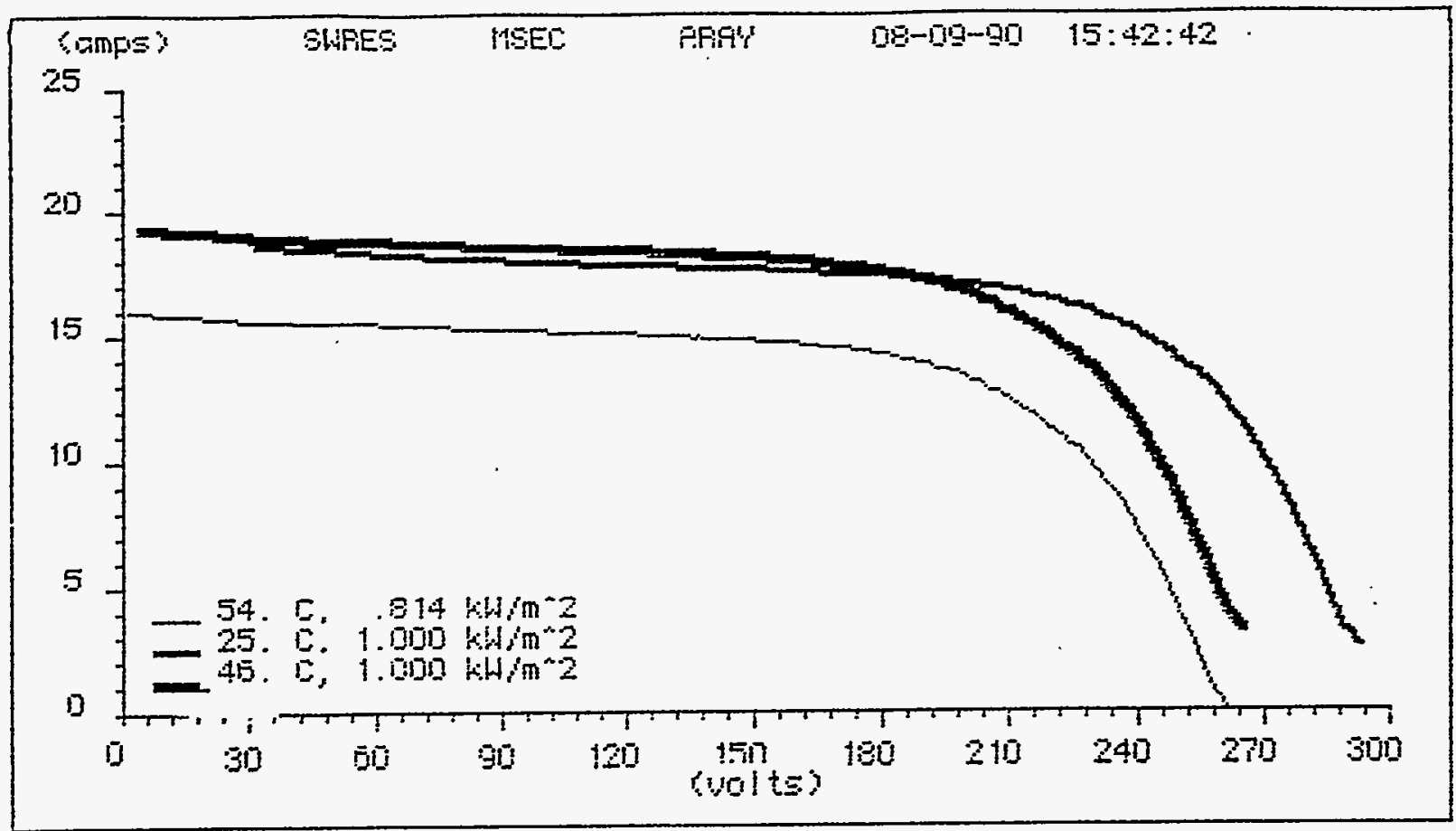

Cell Temperuture .......54.000 deg C

Irradiance ........... .814 kH/m²

Ser i

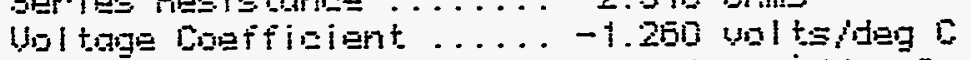

Curront Coefficient ..... .029 amp:ideg $C$

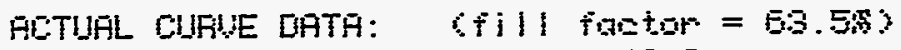

Short ciraut cumpent ... 16 . 0 amps

Goen eircuit uol tage .... 202.1 volts

Hox pnuer eturrent. ...... 13.5 amps

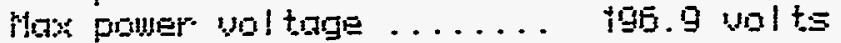

Mow Fumer .......... 2003.9 matts

NDFHFL IZED CUFUE DATA: CFII! figator $=67.48 \%$

ffor 25.0 deg and $1.0 \mathrm{kH} / \mathrm{m}^{2} 2 \mathrm{y}$

Hox powar cument ....... 15.0 amps

Max pawer wol tage ...... 229. ? volt

Max pomer ........... 3574.5 matts

HOFHALIZED DUPLE DATA: GIII fRotor = 55. Dos

(for $40.0 \mathrm{deg} C$ and $1.0 \mathrm{kH} / \mathrm{m} 2$ )

Mox pomer aurent ....... 15.5 amps

Max power voltage ...... 205.2 volts

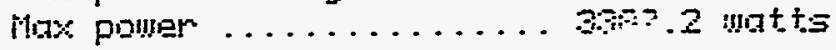



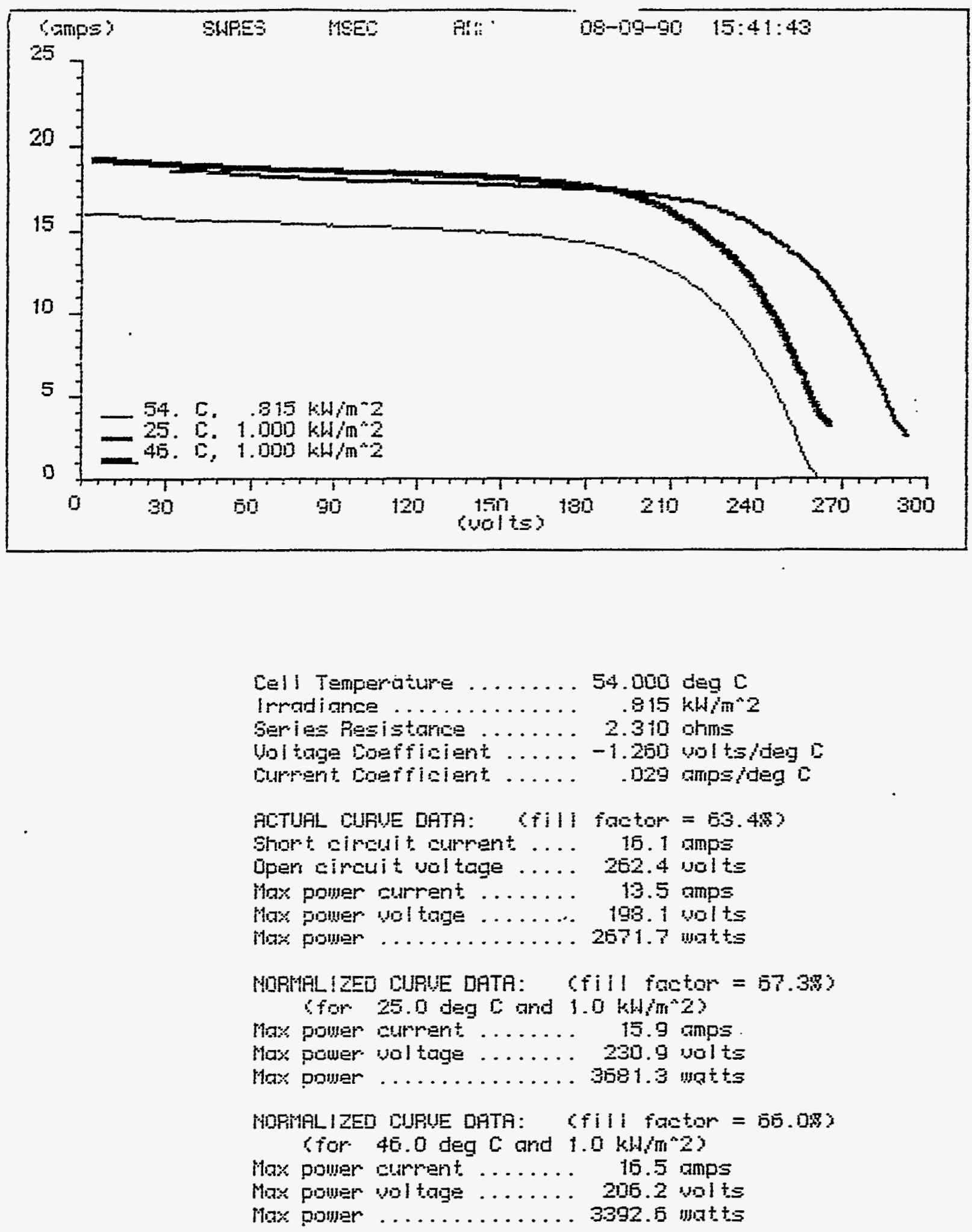


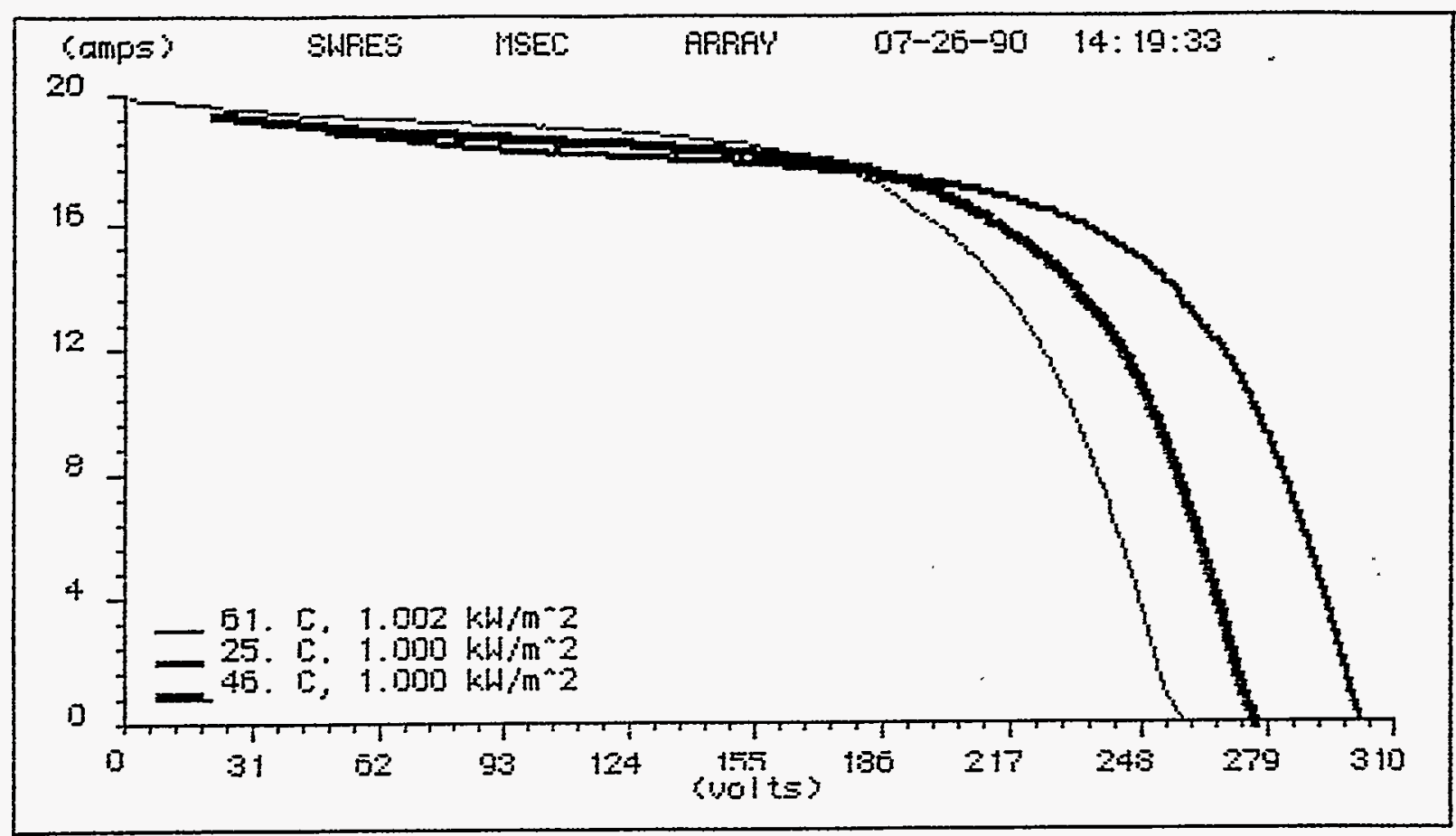

Sell Temperatire ....... 51.000 deg

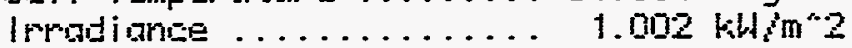
Sani is Piesistance ...... 2.310 onms

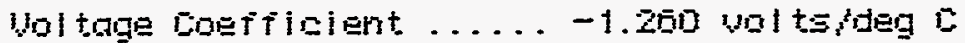
Durrent Doefficiant..... . 029 ginpsideg 5

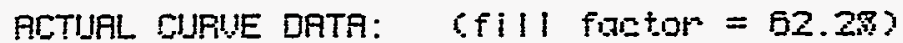

Short cimeuit cument .... 19.9 amp= Dpen girnut upl tage ..... 250. 1 uplts Hox piller cumpant ...... it. 5 amps Mox poiner ul tage ....... 19:.4 uglts Max poulder . ........... 3196.2 matts

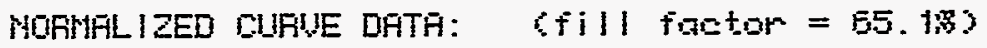
Cfir 25.0 deg $c$ and $1.0 \mathrm{khim} 23$

Hox puider current ....... 1E. 0 amps Hax priner ul tage ...... 2:34.2 ults Mox pouler ........... 3746.6 matts

HORMAL IZED DUAVE DHTA: (fill factor $=53.5 \% 3$ (for 46.0 deg $C$ and $1.0 \mathrm{kh} / \mathrm{m}^{2} 2 \mathrm{j}$

Max power aldryent ...... 1E. 4 amps

Max poujer uol toge ....... 209.8 volts Max pawer ............ 3432. 1 watts 


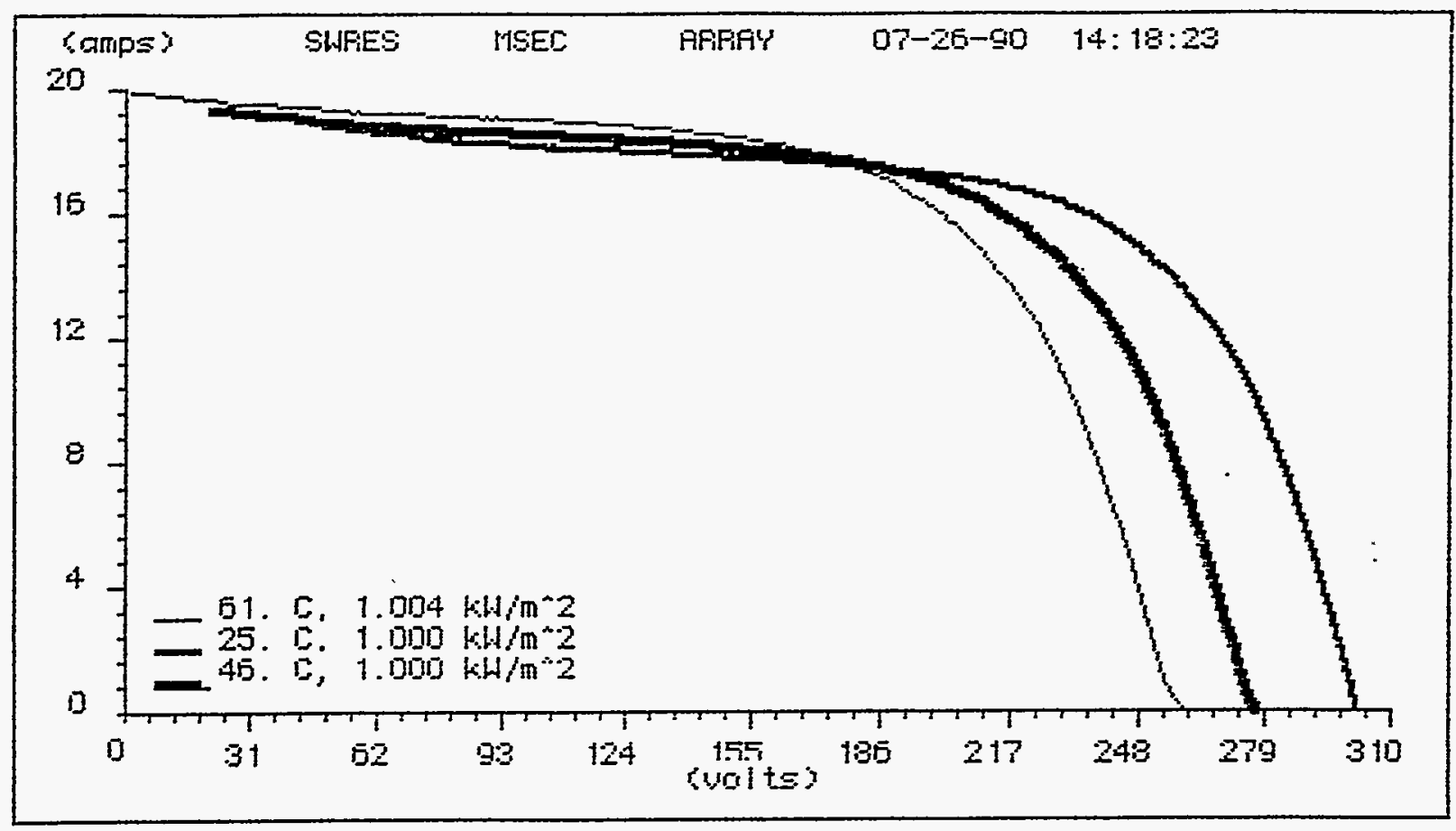

Cell Temperature ....... 51.000 deg $\mathrm{C}$

Irrodiance .......... $1.004 \mathrm{bH}^{\circ} \mathrm{m}^{\circ} 2$

Earias Posistance ...... 2.310 ohms

voltage coefficient ...... - 1.200 voltsideg $c$

Durrent Doefficient ..... .029 ampsideg $\mathrm{C}$

RCTURL DIURUE DRTA: (fill fartor $=62.3 \%$ )

Short circuit current .... 19.9 amp:

Dpen sirouit woltage .... 258.5 uplts

Max poumer curment. ....... 16.5 amp:

Max pourer uoltage ...... 194.5 uolt

Max power ........... $3213 . \overline{0}$ watts

HORHFLIZED CURUE DATA: GIIl factor = $65.2 \mathrm{~g} 3$

Efor 25.0 deg $c$ and $1.0 \mathrm{kH} / \mathrm{m}^{-2} \mathrm{z}$ ?

Max power current....... TE. 0 amps

Max power yol tage ....... 235.5 volfs

Max pulwar .......... 3750.3 watts

HURMALIZED CURUE UATH: 〈fill factor = 53. $5: 3$ ?

(for 46.0 deg $C$ and $1.0 \mathrm{~kg} / \mathrm{m}^{*} \mathrm{2}$ )

Mox power aurrent....... 15. 5 omps

Max pouper uoltage ...... 207.7 uolts

Ma* pawer ........... 3441.5 watts 


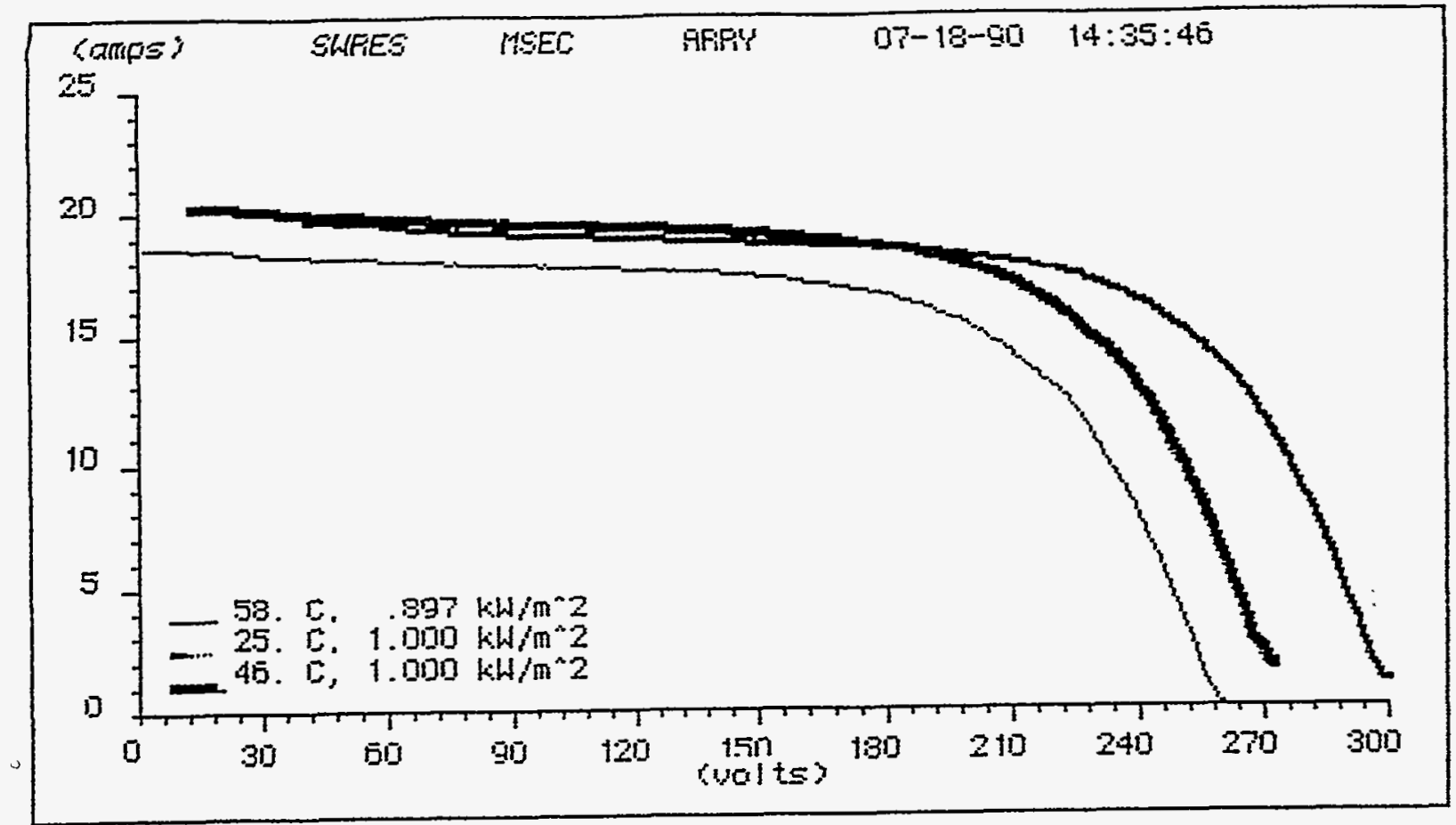

Cell Temperature .......58.01010 deg 5

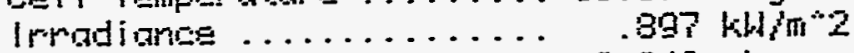

Sarias Pissistanas ....... 2.311 ohms

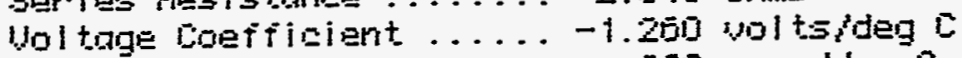

Curmont Doefficiant ..... . . 29 ampsideg c

ACTUAL CIIPUE DATA: Cfill factor $=63.03 \mathrm{y}$

Short Eircuit current ... 13.7 amps

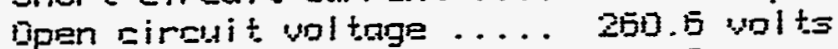

Max pouer ourpart. ...... 15.6 amps

How poujer usl toge ...... 10.

Max pour ........... 3001.5 watts

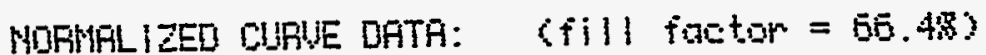

Cfor 25.0 deg 5 and $1.0 \mathrm{khm} \mathrm{m}^{2} \mathrm{y}$

Max pumer current ....... tE. amps

Mak power vol tage ...... 230.5 volts

Max pous ........... 3926.3 watts

MUFMFL IZEU CUFUE DATA: (fill factor $=04.985$

(fior 46.0 deg $C$ and $1.0 \mathrm{kh} / \mathrm{m}^{\circ 2}$ )

Max pouns curpent...... 17.3 amps

Max pouser woltoge ...... 207.9 uplts

Max power ........... 3598.5 watts 


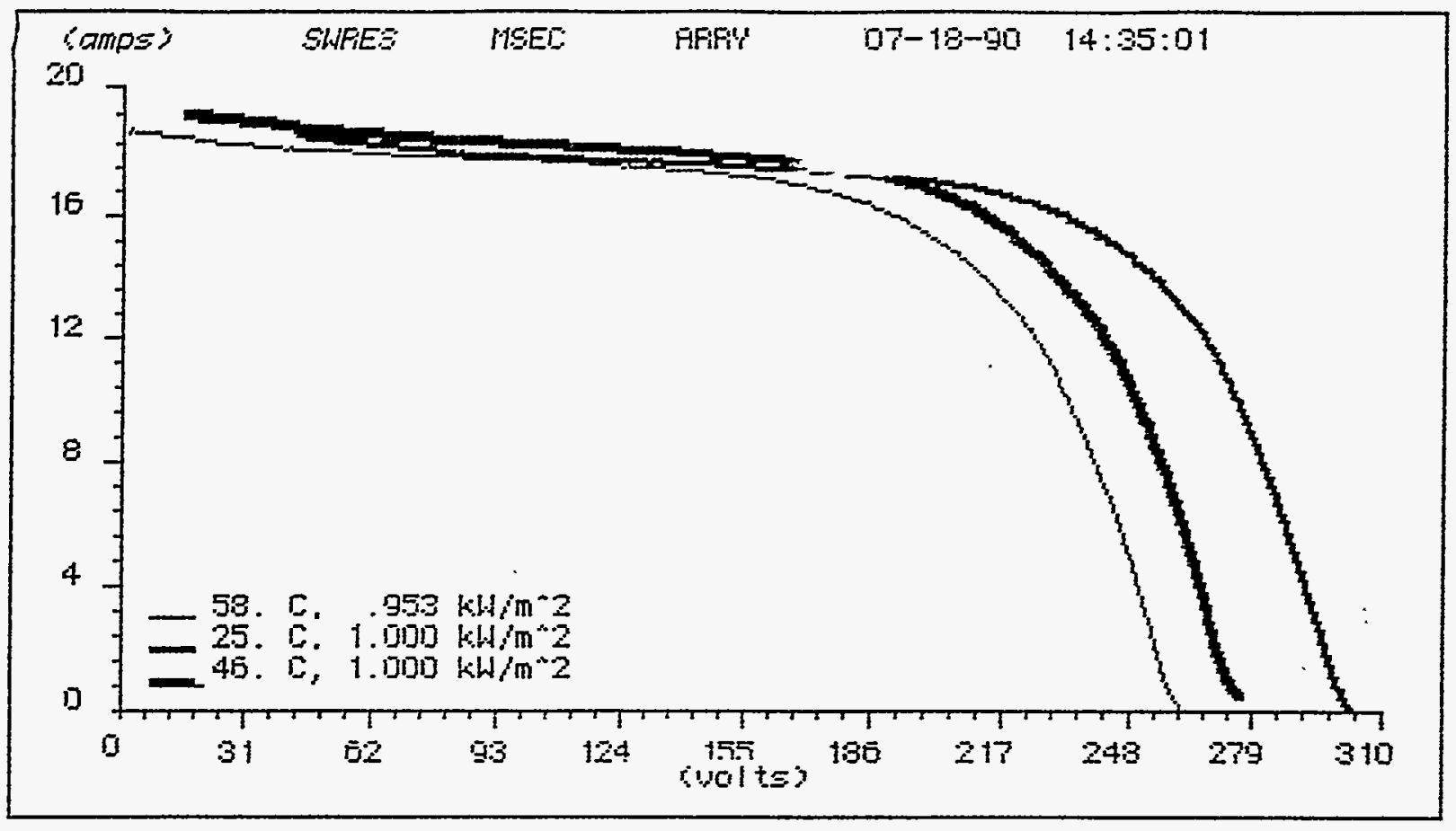

Sell Tempergture ....... 59.000 deg 5

Irradiance .......... .953 $4 \mathrm{H}_{\mathrm{i}} \mathrm{m}^{\circ 2}$

Series Resistanca ....... 2.310 ohms

Uoltage Coefficient ..... -1.250 voltsideg $c$

Curpent Cogfticiant ..... . .029 amps/deg 5

RCTURL CIJPUE DRTA: (fIII frator $=62.33$ )

Shor t cireuit ourment .... 18.8 umps

Dpen sirouit vol tage .... 261.2 volts

Mox power aurrent ...... 15.6 amps

Max pour uol tage ....... 190.9 yol t.s

Max power ........... 3079.2 watts

MORHAL IZED CURUE DATA: (fill factor $=55.78$ )

(for 25.0 Jeg $\left[\right.$ and $1.0 \mathrm{kH} / \mathrm{m}^{-2} \mathrm{z}$

Max pawer curyent ....... 15.8 amps

Max poyer unltage ...... 2:35.3 volts

Max pourer .......... 3717.6 mitts

HORMALIZED CIURUE DATA: (Fill factor $=54.2 \%$ )

Cfor 46.0 deg $C$ and $\left.1.0 \mathrm{k} / 4 / \mathrm{m}^{2} 2\right)$

Hox power gurrent ....... 15.2 amps

Max pouser uoltage ...... 210.7 uolts

Max power ........... 3413.5 watts 


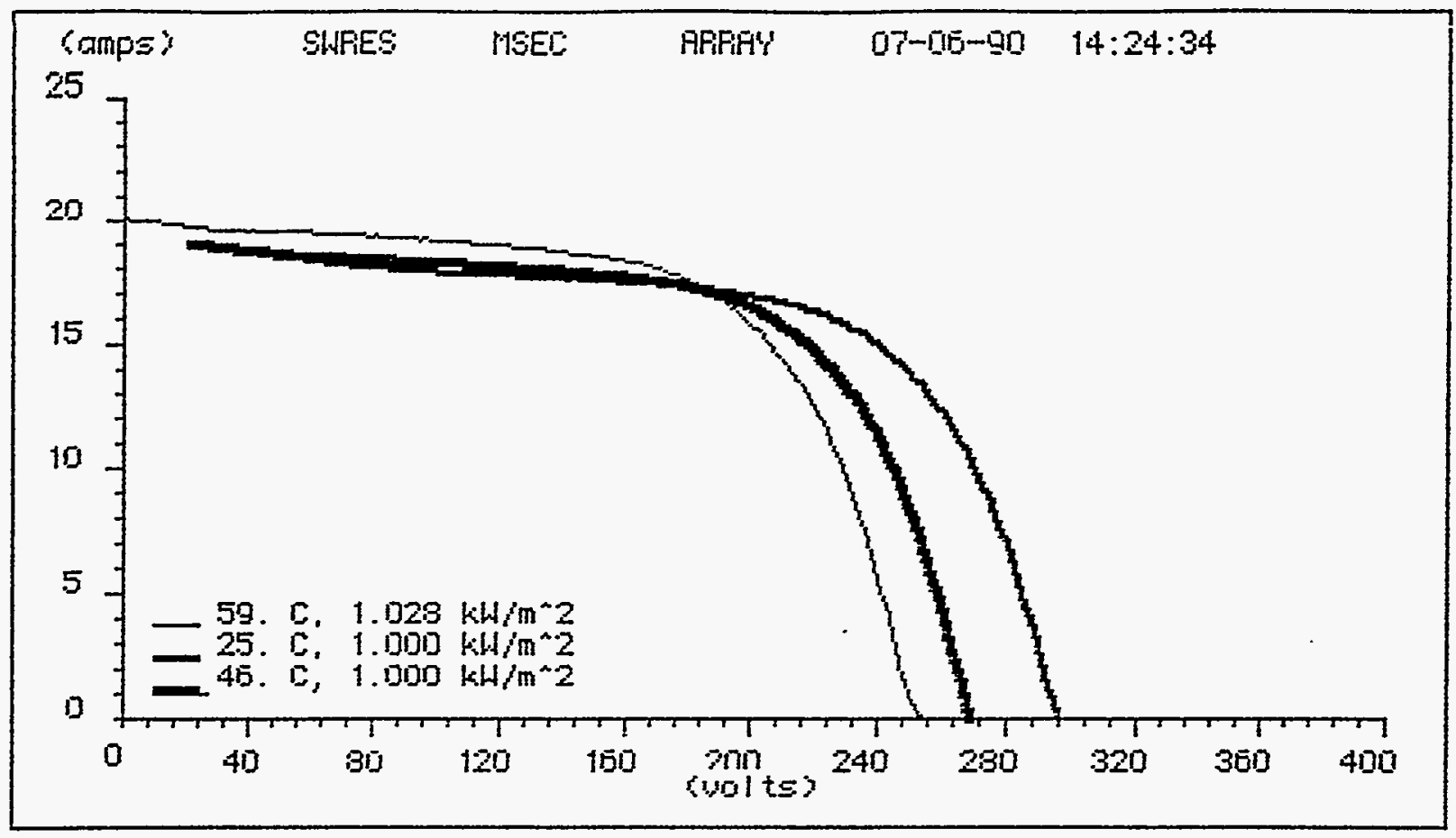

\footnotetext{
CEll Temperature ...... 59.000 deg $c$

Irradiance ............ $1.029 \mathrm{kH}^{2} \mathrm{~m}^{2} \mathrm{z}$

Series Resistarieg ....... 2.3 to ohms

Uoltage coafficiant ..... -1.250 usltsideg [

Currert Doefficient ..... .029 ampsideg i

FCTUAL CURUE DATA: (fill factor $=52.58$ )

Short siroult ourrant .... 20.1 umps

Upen circuit waltuge ..... 255.0 volts

Max pouer current ....... 17. 0 amps

Max power voltage ....... 158.7 volts

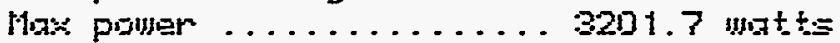

HOPITLLIZED CIJUE DATA: (

(for 25.0 deg 0 and $1.0 \mathrm{khtm} 2 \mathrm{~s}$

Max pouser gumpent. ...... 15.5 amps

Mase power val tage ...... 235.0 volts

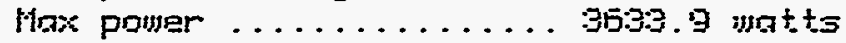

MORMALIZED DURUE DATA: (fill fantor = 53.4B?

(for $40.0 \mathrm{deg} C$ and $1.0 \mathrm{kH} / \mathrm{m}^{\wedge} 2$ )

Max pouser aurrent. ...... 15.1 omp:

Max power vol tage . . . . . 207.2 volts

Mox pomer ........... 3326.2 unats
} 


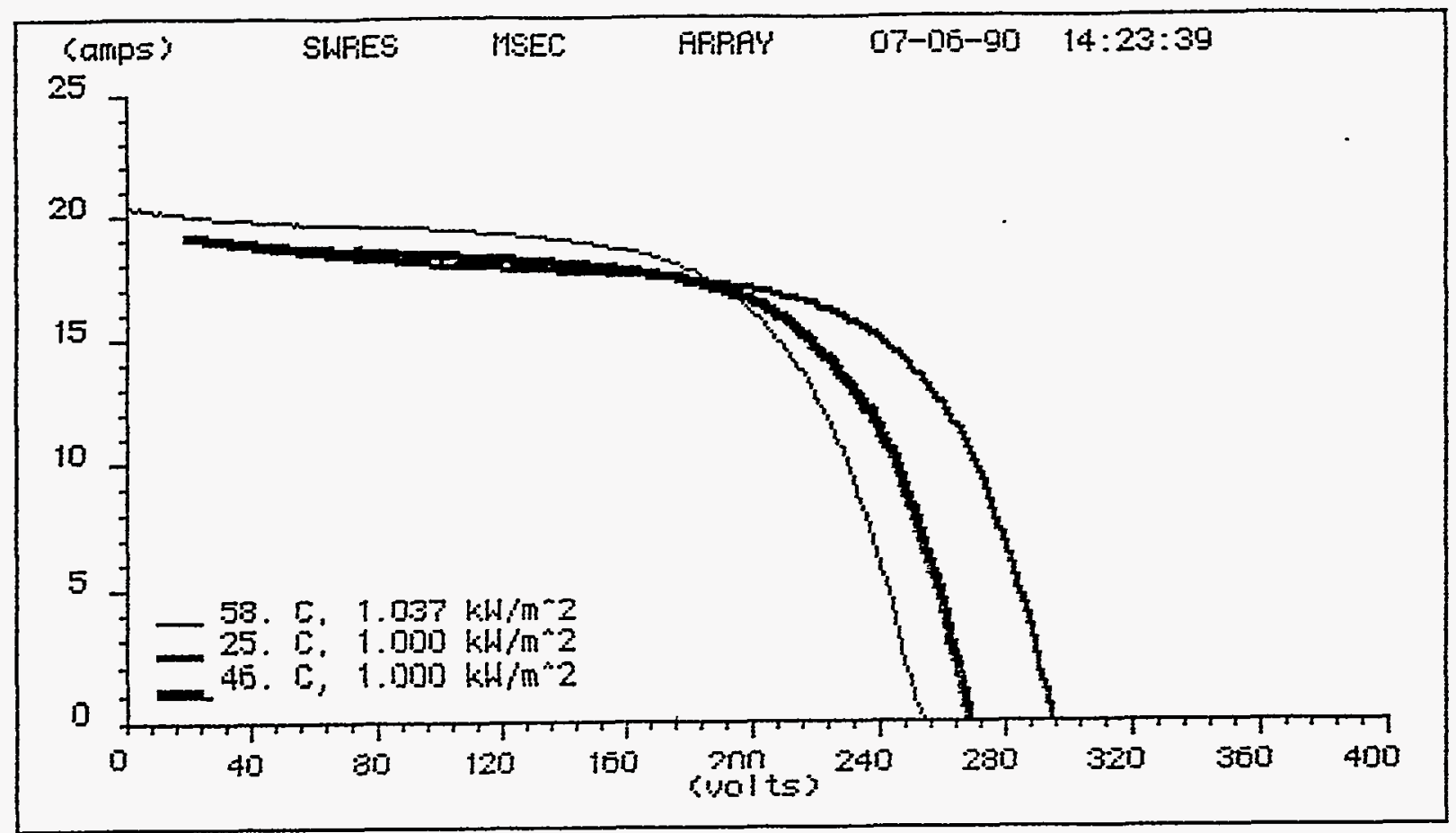

Cell Temperature ....... 58.000 deg $c$

Irradiance ........... $1.037 \mathrm{kH} / \mathrm{m}^{2} \mathrm{z}$

Series Resistariae ...... 2.310 ohms

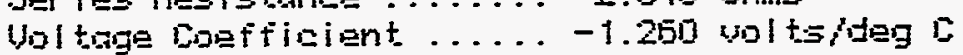

Dumpont coefficient ..... . D29 ampsideg 5

ACTUAL CUFIUE DATH: (fill figetor $=62.5 \%$ )

Short circuit current . ... 20.3 amps

Dpen circuit voltage .... 255.3 volts

Mas pouser current. ...... 17.0 amps

Max pouler voltage ......... 191.0 volts

Hax pomar . . . . . . . . 3247.3 matts

MDRMALIZED CIRUE DATA: 6fill footor = 64.8B)

(for 25.0 deg of and $1.0 \mathrm{kH} / \mathrm{m}+2$ ?

Fox poujer aurrent. ....... 15. 1 amps

Max power vol tage ....... 227.0 volts

Mrax pouer . . . . . . . . 3544.3 mntt:s

MORMALIZED CURUE DATA: (FIII faOtor = 53.2N)

sfor 40.0 deg $C$ and $1.0 \mathrm{kH}\left(\mathrm{m}^{\circ} \mathrm{Z}\right.$ )

Mox pouler curpment ....... 15.9 amps

Max poumer vol tage ....... 208.6 volts

Max pouser ............ 3324.s untts 

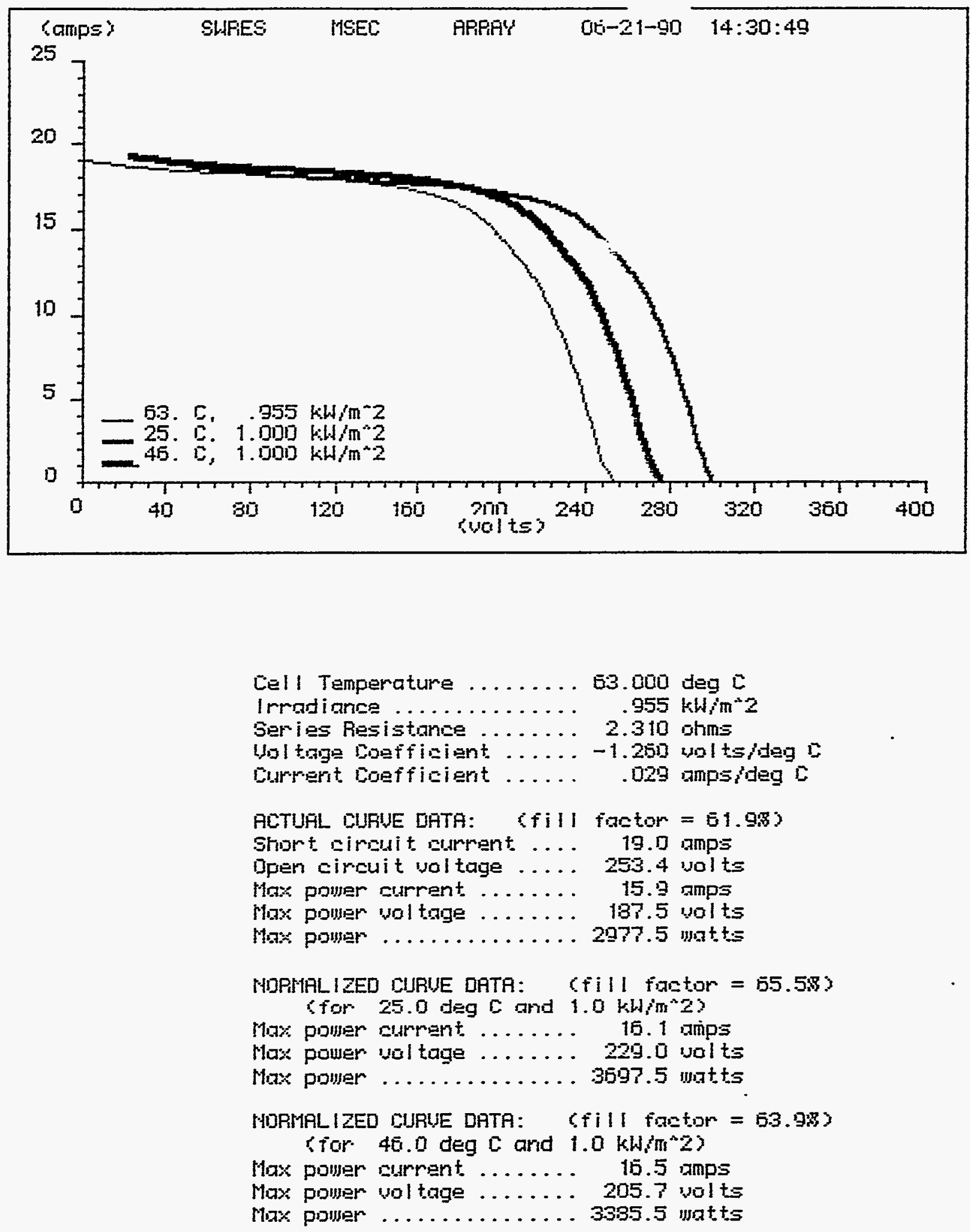


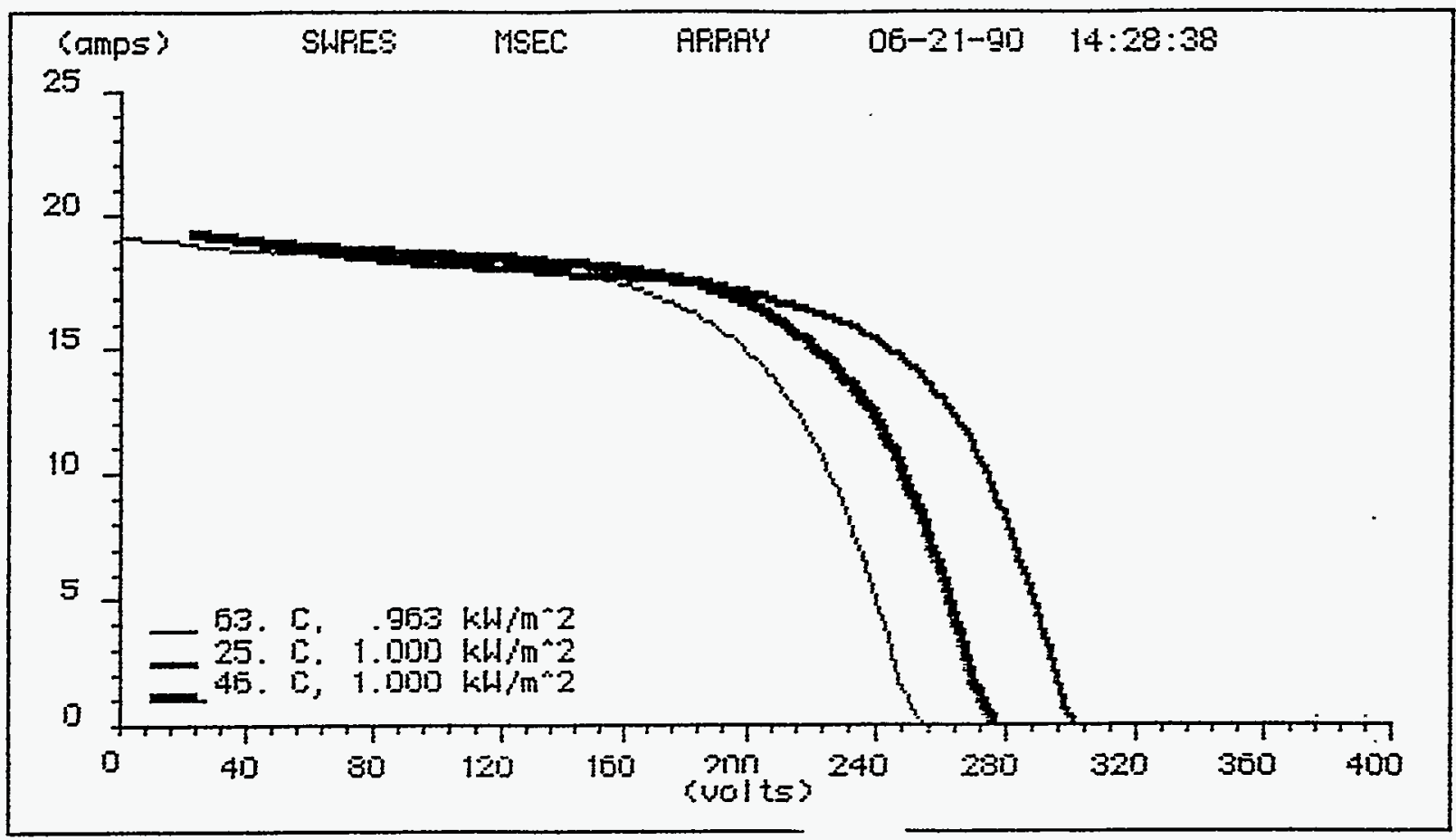

Cell Temperature ........ Gi3. Di. Jeg $C$

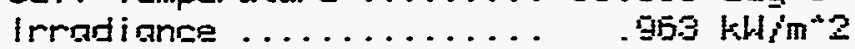

Series Resistunce ...... 2.310 ohms

Uoltage Doefficient .....-1.250 und tsideg 5

Current coefficient..... .029 ampsideg $C$

ACTURL CURUE DATA: (fill factor $=51.9 B$ )

Short Eirauit current .... 19.1 umps

Open circuit waltage .... 234.0 ual ts

Mres pouer gurrent...... 15.10 amps

Max pouner voltage ........ 158.7 volts

Max poumar .......... 3018.7 mati.s

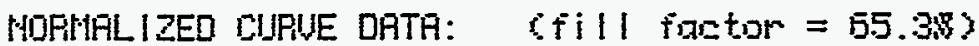

Cfor 25.0 deg $C$ and $1.0 \mathrm{kH} / \mathrm{m}^{\circ} \mathrm{2}$ )

Max pouer surrent ....... 15.0 anps

Max power val tage ....... 232.8 volts

Max pouner ............ 3715.9 undts

MDPMALIZED DURUE DATA: Cfill faztor = 53.783

(for 40.0 deg $C$ and $1.0 \mathrm{kH} / \mathrm{m} \cdot 2$ )

Max power current ....... 15.2 amps

Mar powser voltage ....... 209.5 wolts

Max polyer ........... 3400. 1 ugtts 


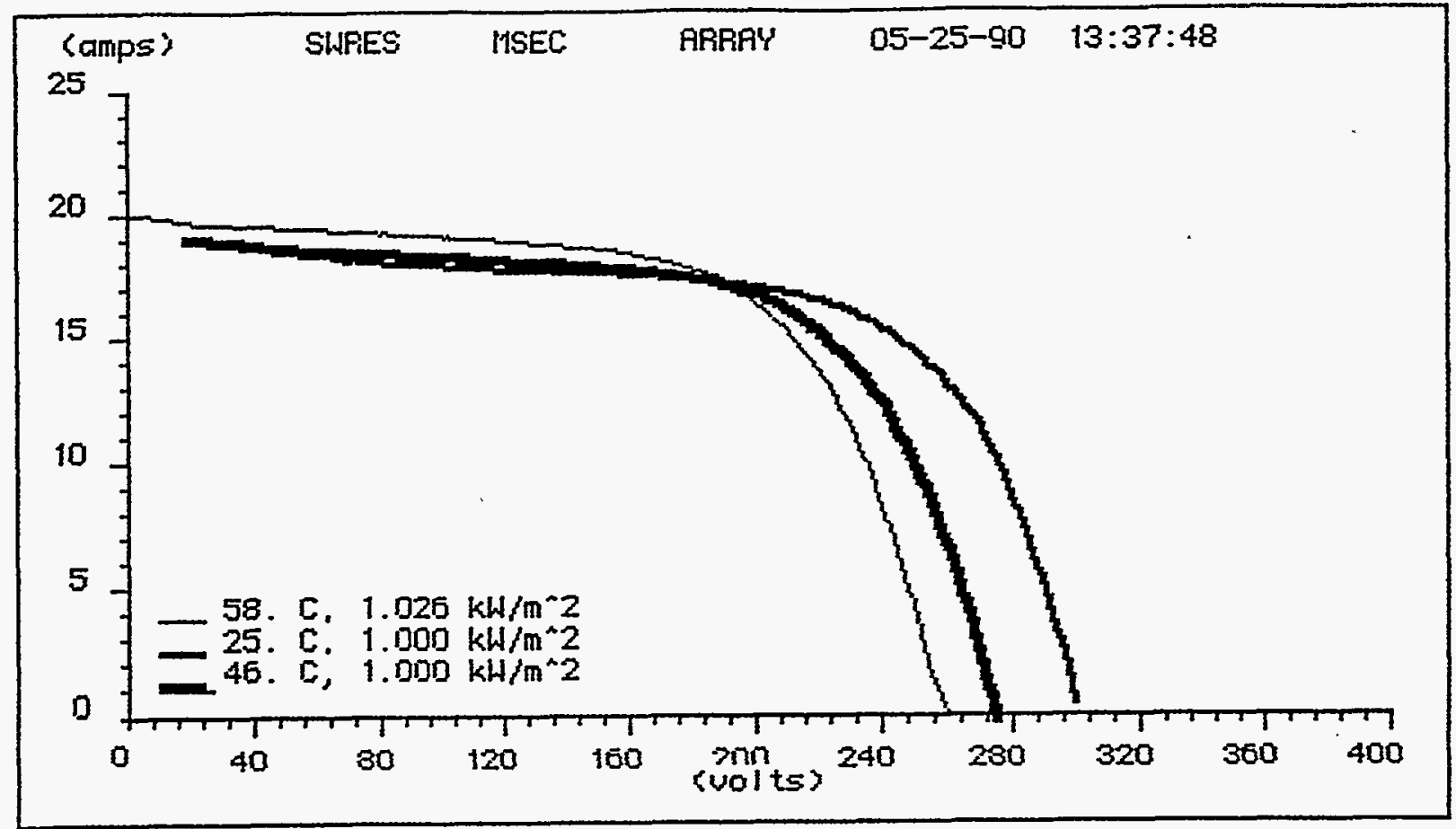

Cell Temperature .......58.000 deg $c$

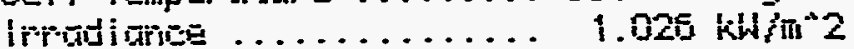

Series Resistance ....... 2.310 ohms

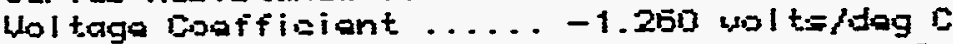

Current coefflcient ..... . . az9 ampsideg c

ACTUAL CURUE DATA: (fill factor $=52.78$ )

Short circuit ourrent. ... 20.0 amps Open circuit voltage ..... 261.3 volts Max pouer current ....... 15.7 amps Max pouser val tage ....... 195.7 vol ts Max poun ............ 3275.0 mat $\pm=$

MORMRLIZED CURUE DATA: (fill factor $=65.19$ )

(for 25.0 deg $C$ and $1.0 \mathrm{~kW} / \mathrm{m}^{\wedge} 2$ )

Max pouser aurrent ...... 15.8 amps

Max power vol tage ....... 234.7 volts

Max pouper ............ 3597.4 matts

NDRMALIZED CURUE DATA: (fill factor = 63.68s

sfor 46.0 deg $C$ and $1.0 \mathrm{kH} / \mathrm{m}^{\wedge} 2$ )

Max pouser curpent ....... 15.1 amps

Max power vol tage ...... 210.4 volts

Max powar ............ 3.386 .8 watts 

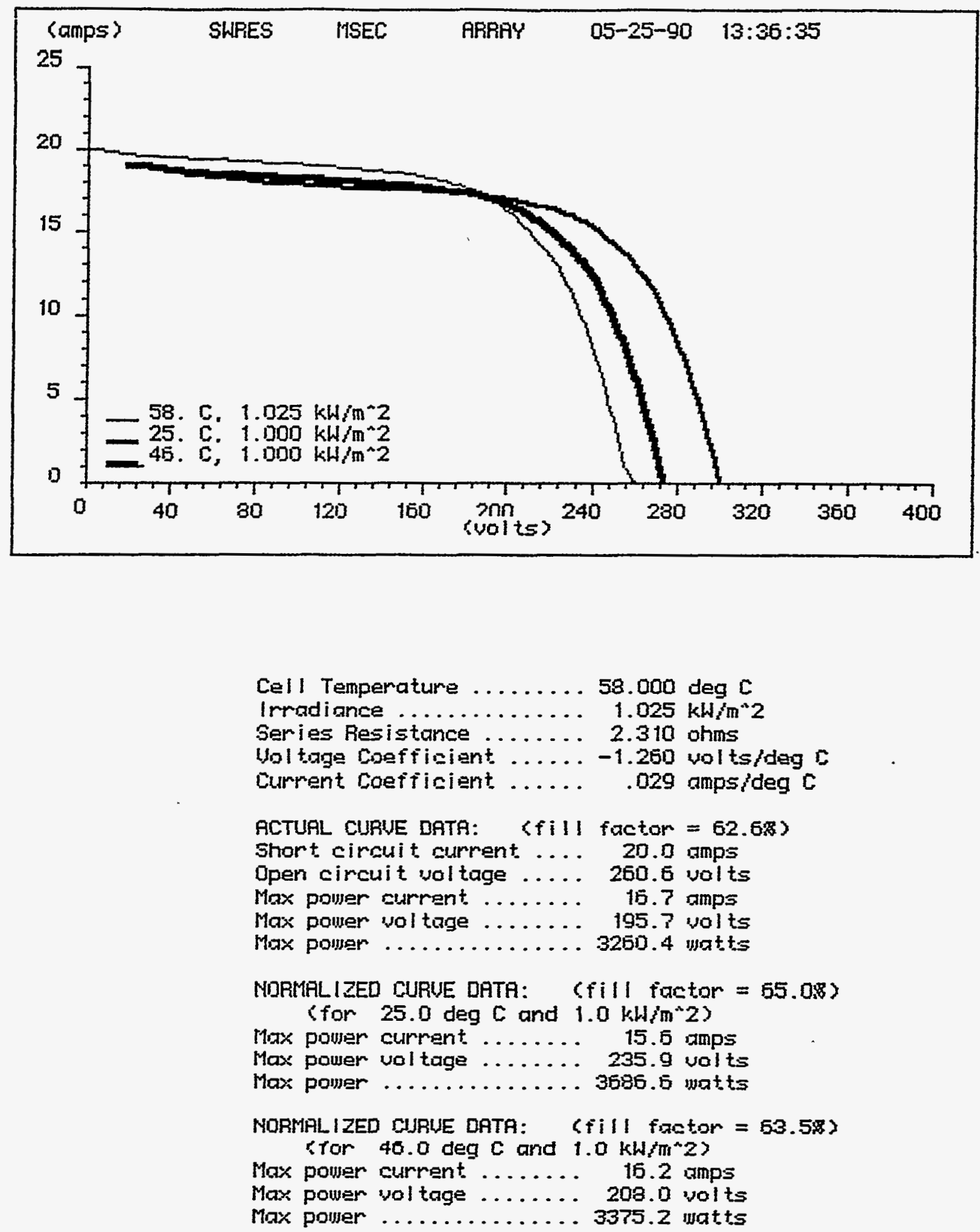


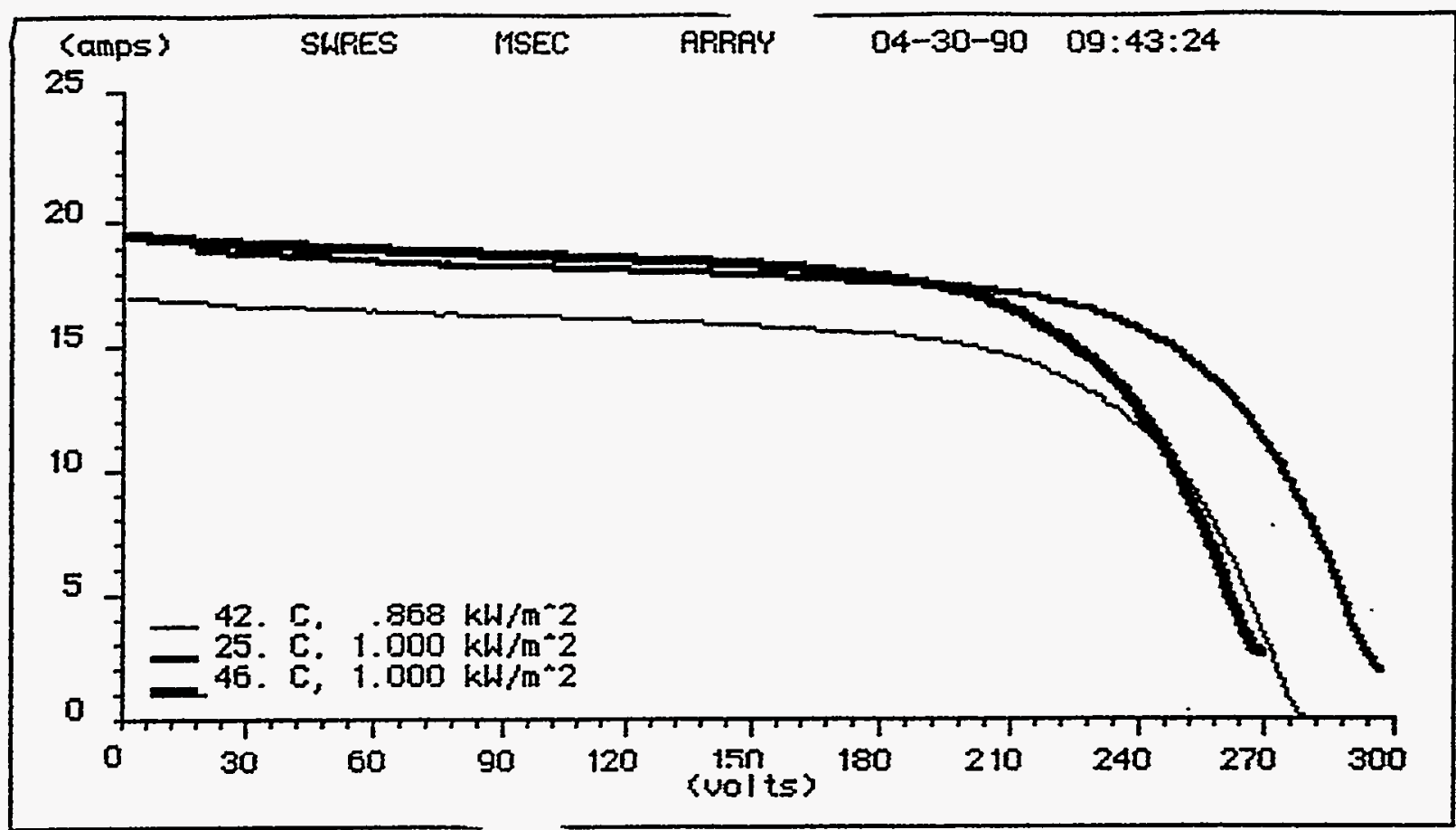

Cell Temperature ....... 42.000 deg $c$

Irrad iance ............ $.968 \mathrm{kH} / \mathrm{m}^{\wedge} 2$

Series Resistance ....... 2.310 ohms

Uoltage Coefficiant ..... -1.260 wolts/deg C

Current Coefficient ..... .029 ampsideg C

ACTUAL CURUE DATA: (fill factor $=64.98$ )

Short circuit current .... 17.0 amps Open cimcuit waltage ..... 279.3 volts

Max power current ....... 14.4 amps

Max power vol tage ....... 214.4 valts

Max power ............ 3079.5 watts

NORMALIZED CURUE DATA: ( $\mathrm{fi} / \mathrm{l}$ factor $=67.38$ )

(for 25.0 deg $C$ and $1.0 \mathrm{k} / \mathrm{H} / \mathrm{m}^{\circ} 2$ )

Max power current....... 16.1 amps

Max power vol tage ...... 234.7 volts

Max power .......... 3787.0 matts

NDRMALIZED CURUE DATA: (fill factor = 65.8\$)

(for $46.0 \mathrm{deg} C$ and $1.0 \mathrm{kH} / \mathrm{m}^{\circ} 2$ )

Max polyer current ....... 16.6 amps

Max power vol tage ....... 210.2 wolts

Max power ........... 3479.2 watts 


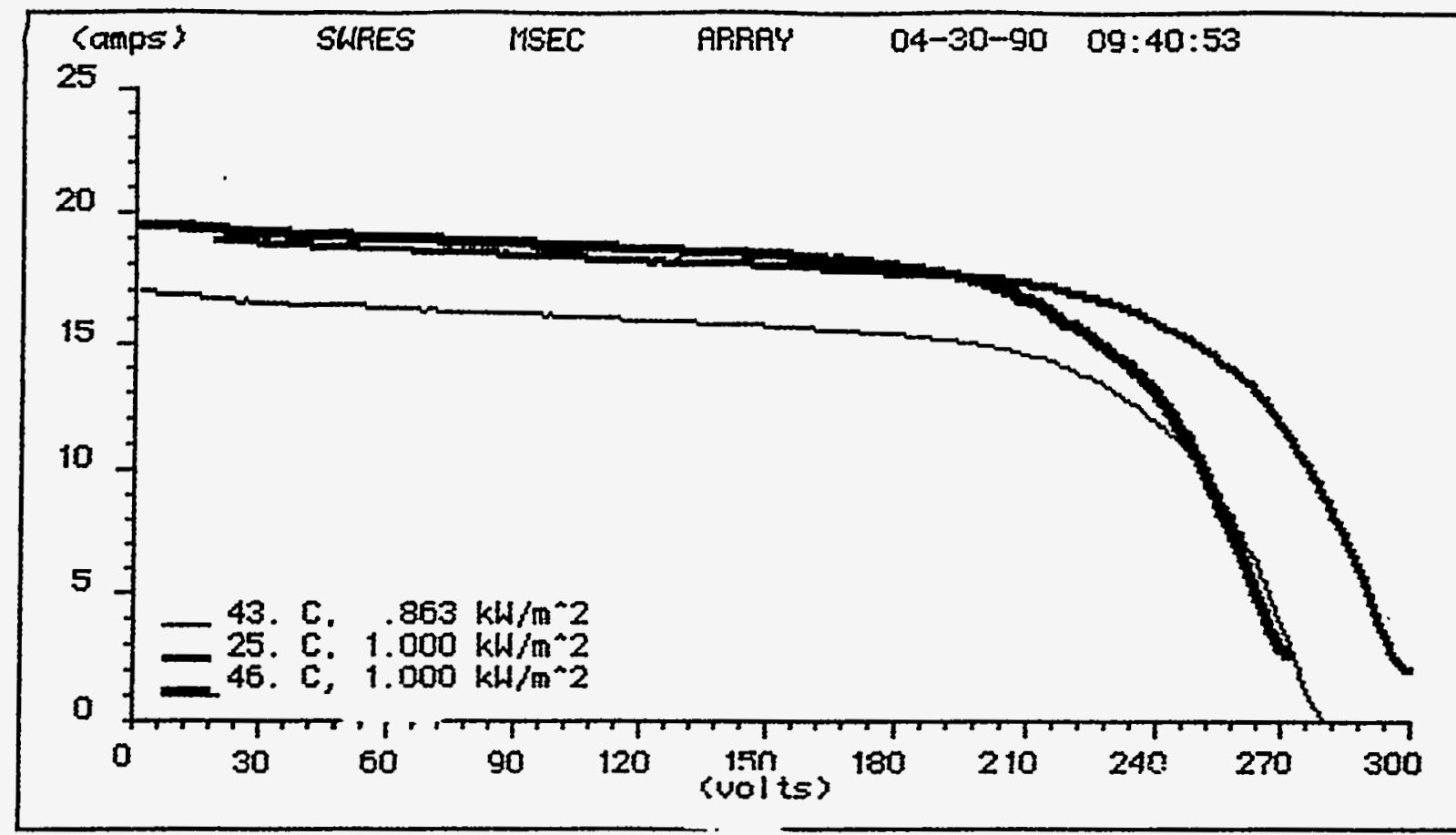

Cell Temperature ....... 43.000 deg C

Irradiance ............ $.863 \mathrm{kH} / \mathrm{m}^{\wedge} 2$

Series Resistance ....... 2.310 ohms

Uoltage Coefficient ..... -1.250 wolts/deg c

Current Coefficient ..... .029 ampsideg $c$

RCTUAL CURUE DATA: (fill factor $=64.88$ )

Short circuit curment .... 17.0 amps

Open circuit ual tage .... 281.1 valts

Max power current....... 14.3 amps

Max power vol tage ....... 215.6 vol.ts

Max power ............ 3087.8 watts

MORMRLIZED CURUE DATA: ( $f i l l$ factor $=67.38$ )

(for 25.0 deg $C$ and $1.0 \mathrm{~kW} / \mathrm{m}^{\wedge} 2$ )

Max power current ....... 16.2 amps

Max power vol tage ...... 237.0 volts

Max power ............ 3831.7 watts

NORMALIZED CURUE DATR: ( $\mathrm{f}$ ill factor = 60.

(for $46.0 \mathrm{deg} C$ and $1.0 \mathrm{kH} / \mathrm{m}^{\wedge} 2$ )

Max pouser current ....... 16.7 amps

Max power vol tage ........ 211.2 volts

Max power ........... 3530.6 matts 


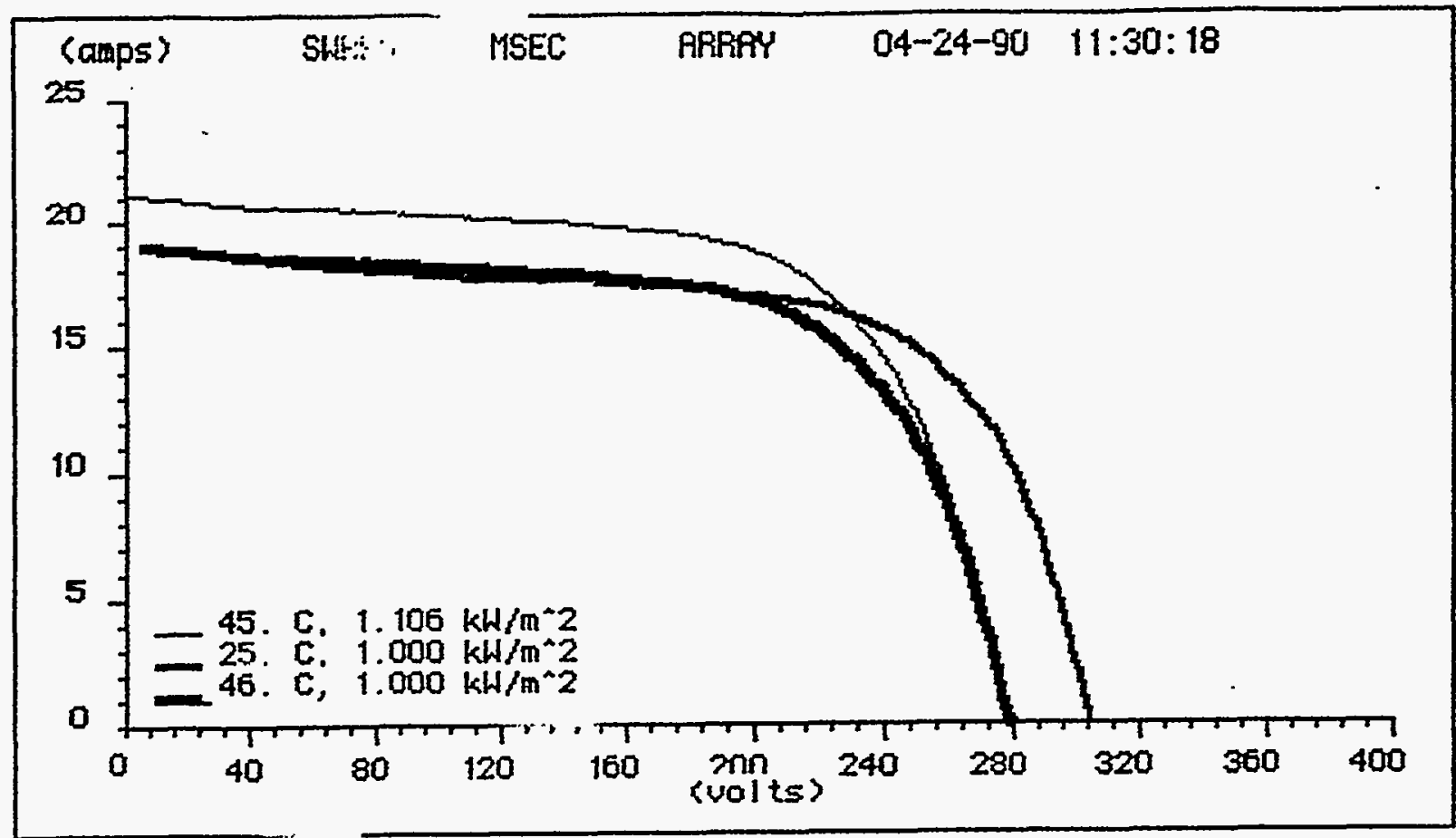

Cell Tenperature .......45.000 deg $c$

Irradiance ........... $1.106 \mathrm{kH} / \mathrm{m}^{*} 2$

Series Resistance ........ 2.310 ohms

Wol tage coefficient ...... - 1.260 wolts/deg $c$

Current Coefficient ..... .029 amps 1 deg $C$

ACTUAL CURUE DATA: (fill factor $=64.3 \%$ )

Short circuit current. ... 21.1 amps

Open circuit valtage ..... 281.1 wolts

Max power current ....... 17.7 amps

Max power vol tage $\ldots \ldots \ldots .215 .6$ volts

Max power ............ 3825.3 watts

MORMRLIZED CUFAE DATA: (fill factor $=64.98$ )

(for 25.0 deg $C$ and $1.0 \mathrm{kH} / \mathrm{m}^{\wedge} 2$ )

Max power current ....... 15.7 amps

Max pomer vol tage ....... 239.8 volts

Max power ............ 3757.4 watts.

MORMALIZED CURUE DATR: ( $\mathrm{fill}$ factor $=63.48$ )

(for 46.0 deg $C$ and $1.0 \mathrm{kH} / \mathrm{m}^{\wedge} 2$ )

Max power surrent ....... 15.1 amps

Max power vol tage ....... 214.3 valts

Max power ........... 3452.2 watts 


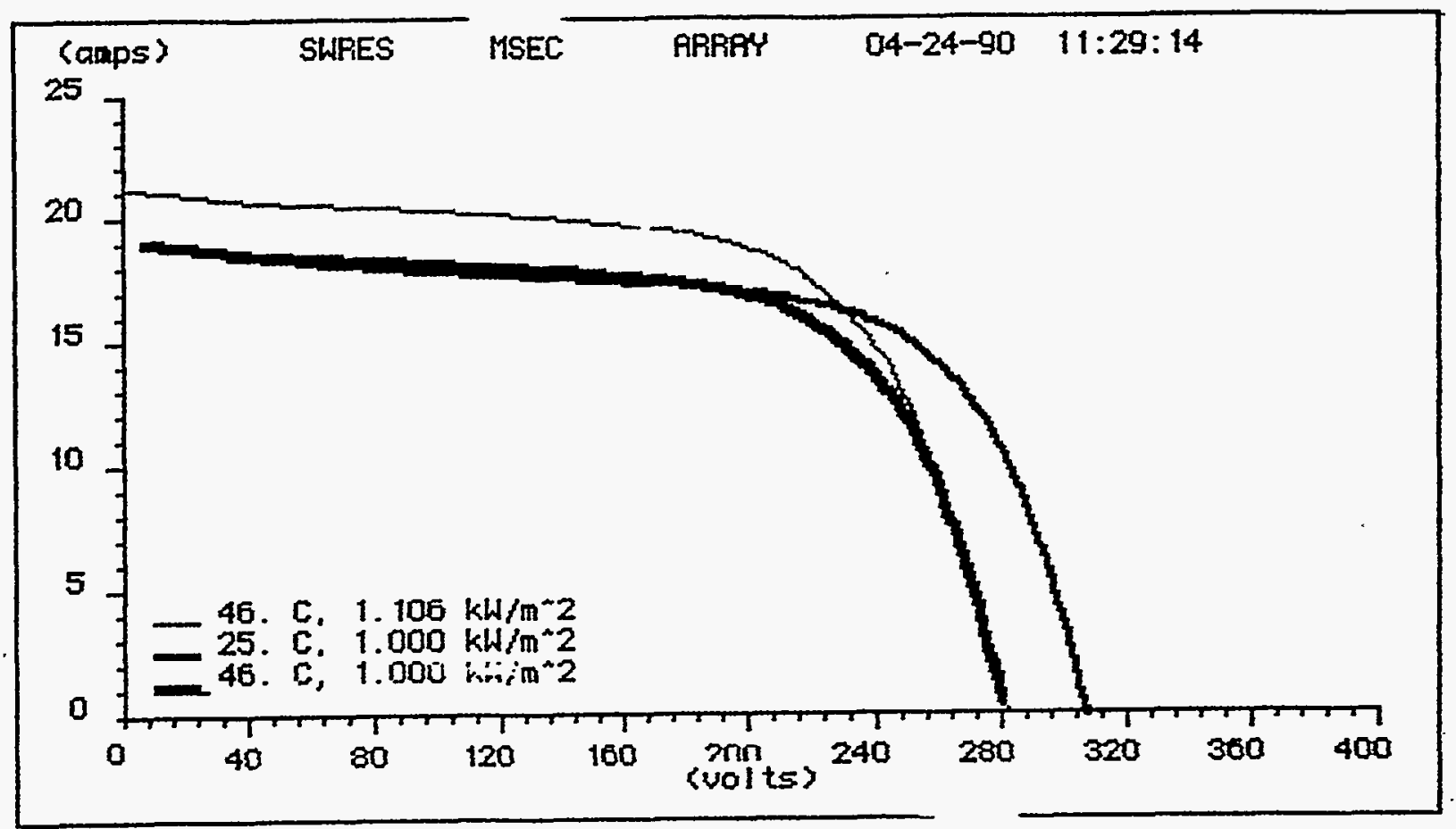

Cell Temperature .......46.000 icg C

Irradiance ........... $1.106 \mathrm{kH/m}-2$

Series Resistance ....... 2.310 ohms

Uol tage Coefficient ...... -1.260 wol ts/deg $c$

Current coefficient ..... .029 amps/deg C

RCTUAL CURIJE DATA: ( $\mathrm{fil}$ factor $=64.58$ )

Short circuit current.... 21.1 amps

Open circuit valtage .... 282.0 volts

Max power current. ...... 17.8 amps

Max power vol tage ....... 215.6 volts

Max power ........... 3842.5 matts

NORMALIZED CURUE DATA: ( $\mathrm{fill}$ factor $=65.08$ )

(for 25.0 deg $C$ and $1.0 \mathrm{kw} / \mathrm{m}^{+} 2$ )

Max power current ...... 15.6 amps

Max power vol tage ....... 243.3 volts

Max power ............ 3790.0 watts

NORMALIZED CURUE DATR: ( $\mathrm{fill}$ factor $=63.6 \%$ )

(for 46.0 deg $C$ and $1.0 \mathrm{~kW}\left(\mathrm{~m}^{\wedge} 2\right.$ )

Max pouser current ....... 16.0 amps

Max power vol tage ....... 218.0 volts

Max power ............ 3481.9 watts 


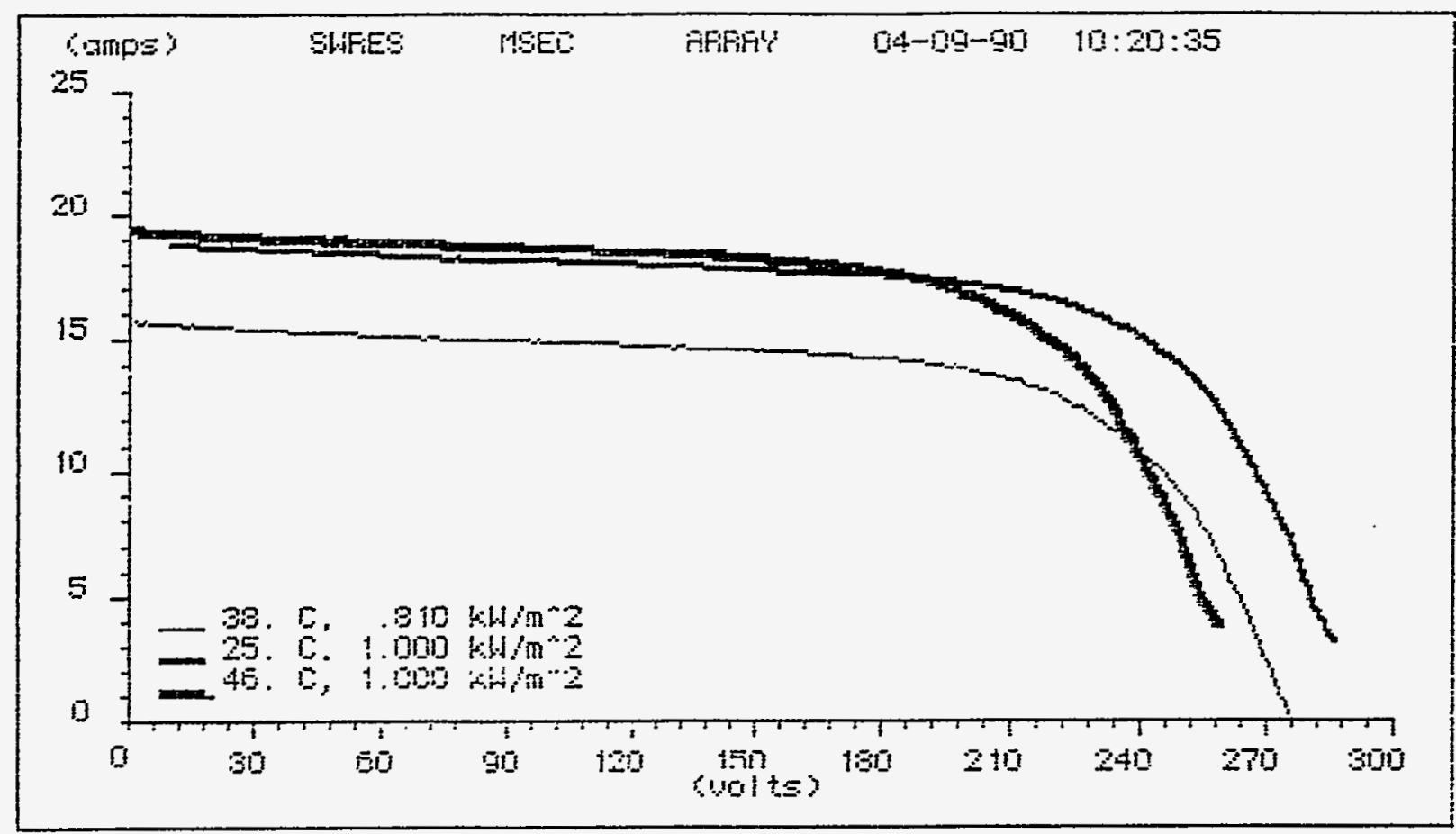

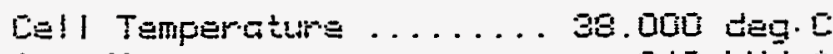

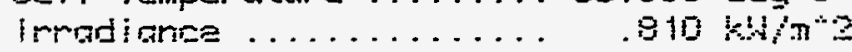

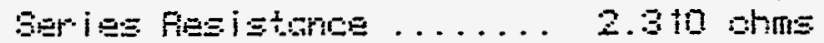

i.

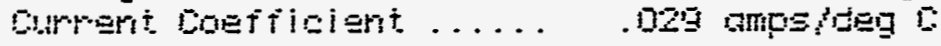

FITHL GHUE THTA:

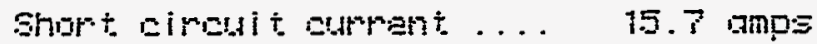

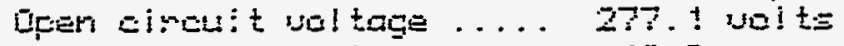

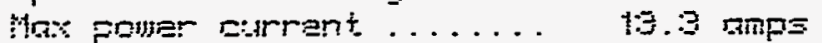

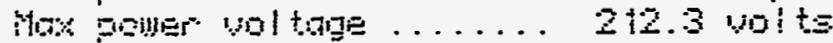

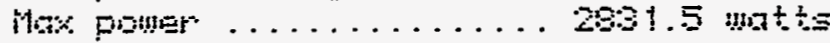

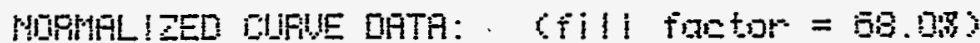

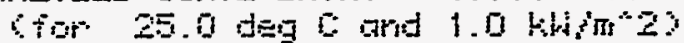

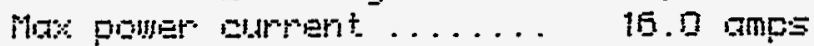

Mox puller vo: toge ....... 229.3 wol ts

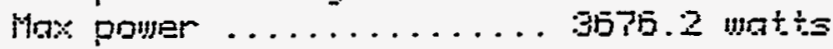

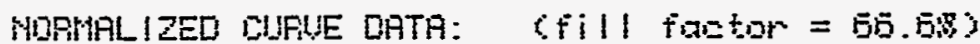

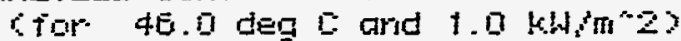

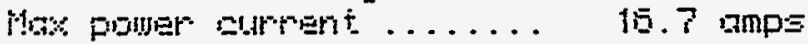

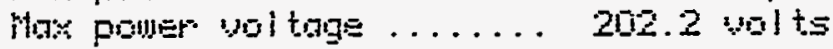

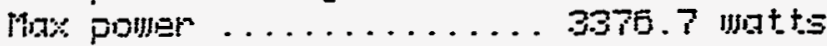




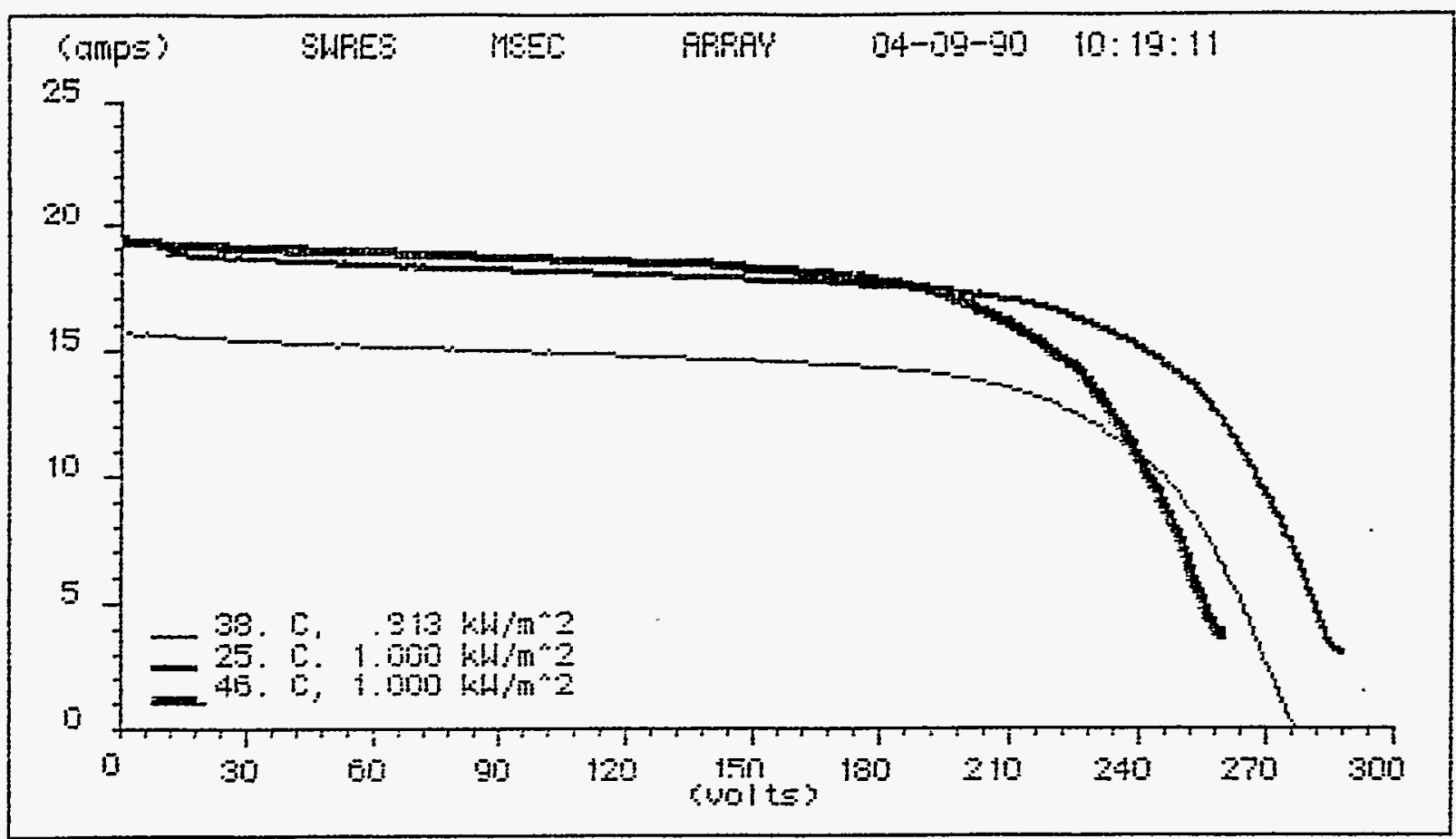

\footnotetext{
Ceil Temperature ....... Se.b00 deg $\mathrm{C}$

Irradianoe ......... .910 $4 \mathrm{H}^{2} \mathrm{~m}^{2}$

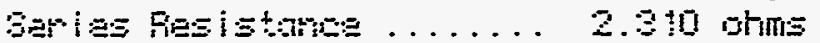

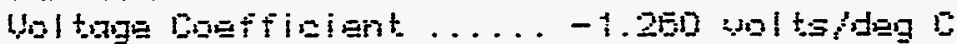

Durrent boefficiont. . . . . .029 amperieg $\mathrm{C}$

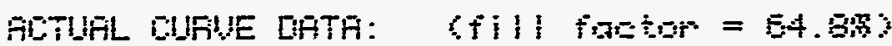

Short cimouit aurent ... 15.7 amps

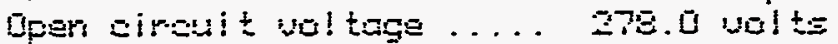

Whe pouper summent. ...... 13.2 amps

Max power woltage ...... 2t4.4 nolts

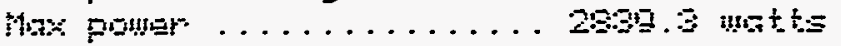

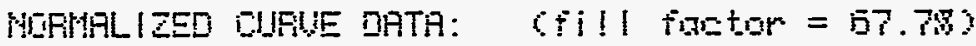

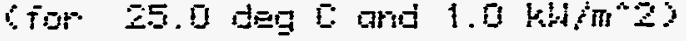

Mas pouner ourrent ...... 15.1 ames

Mo\% puluer vol tage . . . . . 223.2 volts

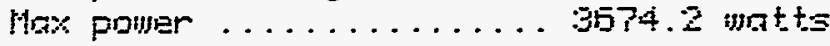

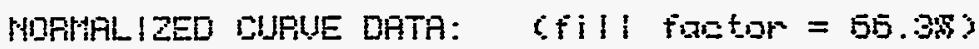

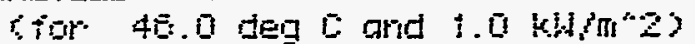

Mx pomer aurrent ...... 10.2 umps

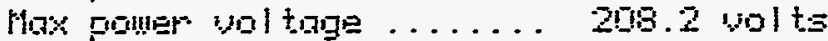

Mise pullar . . . . . . .
} 


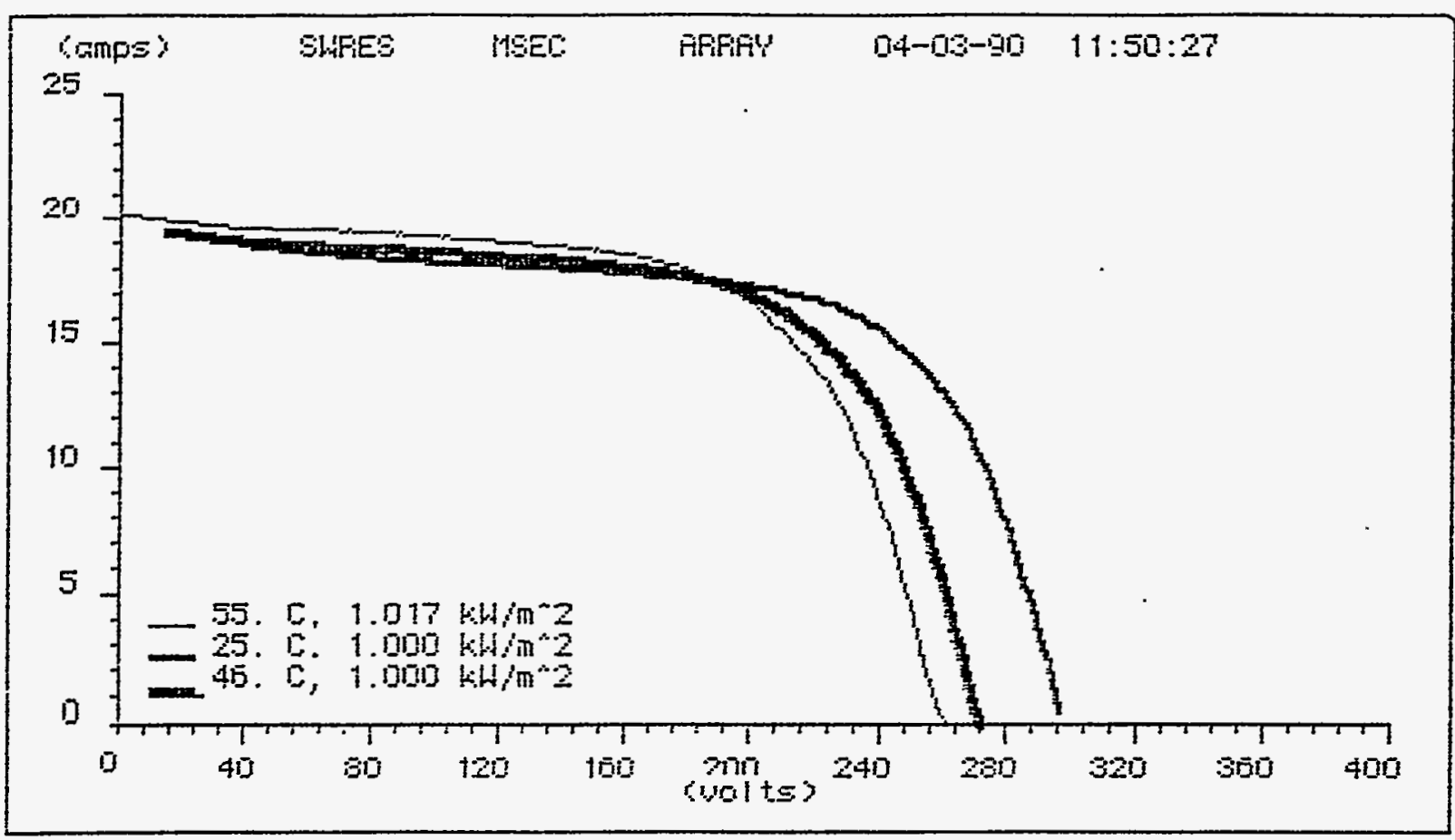

Lel I Temperature ........ 55.000 deg $C$

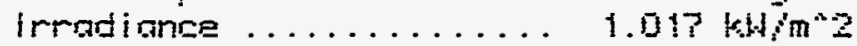

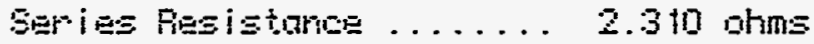

Lo It.

Diwpent Donfficient ..... . .029 ampsideg

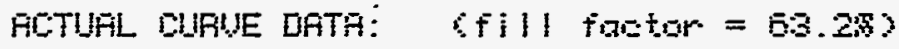

Ghort sirest surrent. . . . 20. 1 anps

Gpen Eirguit voltage ..... 2E2. 1 wol tE

Was power current ...... is is amps

Mox Fower voltoge ...... 197. n volts

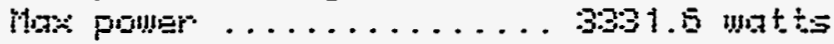

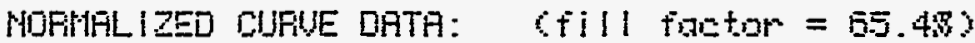

Cfor 25.0 deg 5 aru $1.0 k 4 m^{*} 23$

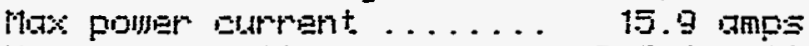

Max Fumer vuitage ....... 235. I voits

Hras power ............3744.5 wntts

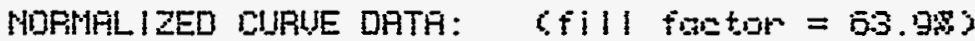

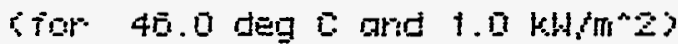

Mor poumar aturent. ...... 15.5 gmps

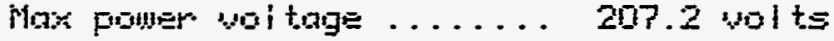

Mos puswer ........... 3425. 1 mats 


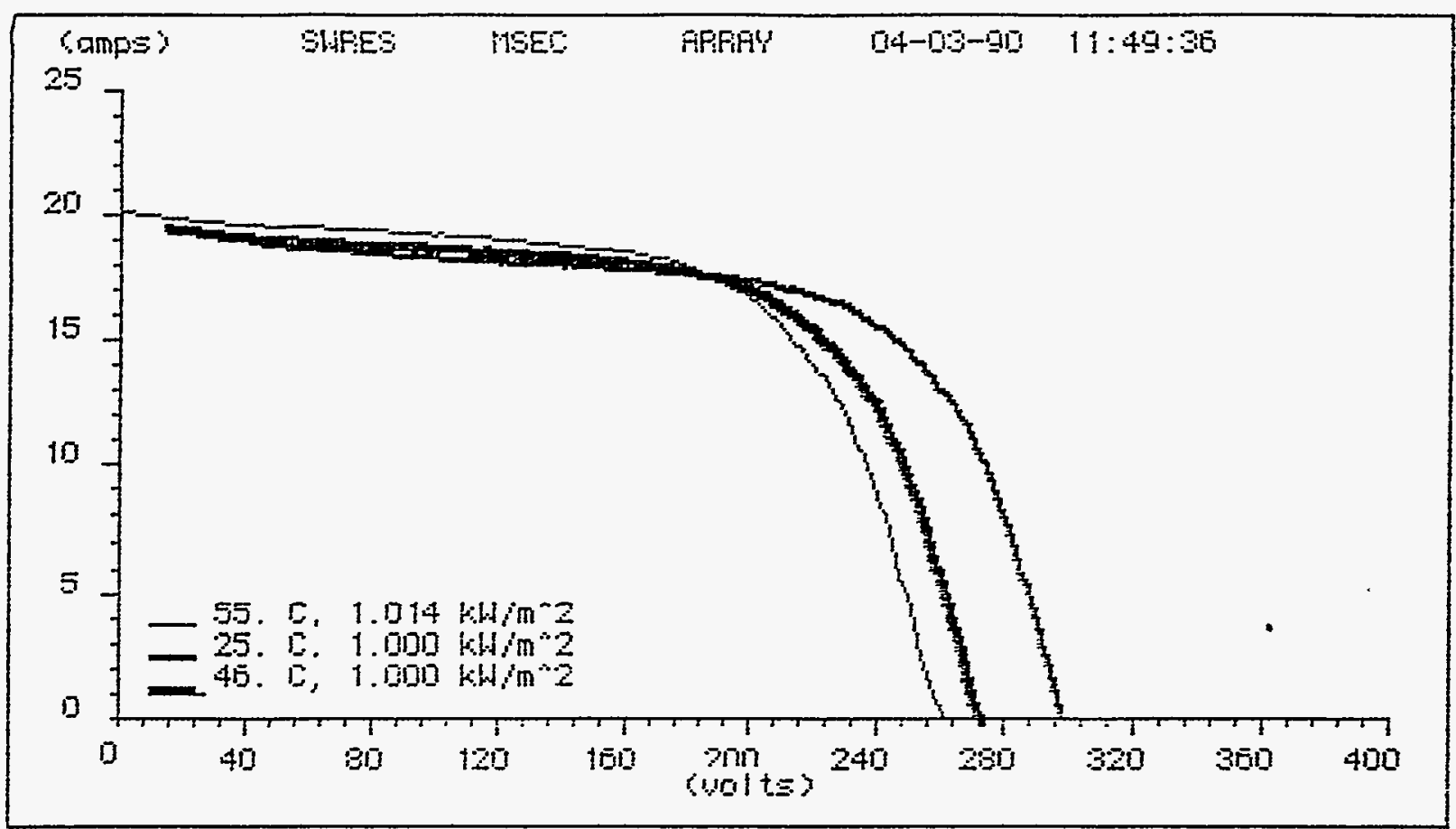

\footnotetext{
Cell Temperature ...... S5. Q00 deg [ irradiance ............. $1.014 \mathrm{kWH}^{2} \mathrm{~m}^{2}$ Saries Fesi itance ...... 2.310 bhts Uoltage coufficient ..... -1.260 uplts/deg 5

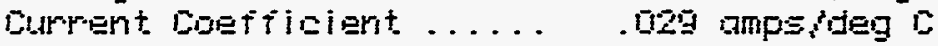

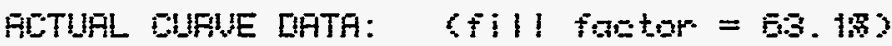

Short. aimosit athrant. ... 20.1 smp: Gpen circuit woltage .... 202. vo volts Mros pouser current. ...... 17.0 smps Hox pouler woltage . . . . . 195.7 bolts

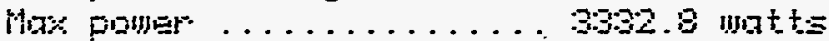

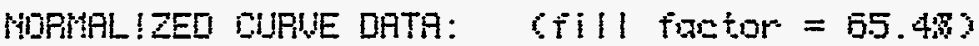
Cfor 25.0 deg 0 and 1.0 bh m 23

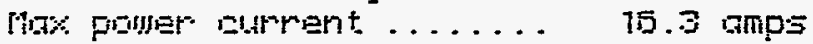

Max poluer voltage ....... 230.3 volts

Mas pomer ........... 3759.9 mot $\div 3$

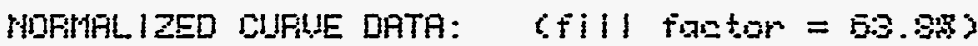
(ior 40.0 deg $C$ and $1.0 \mathrm{kH} / \mathrm{m}^{2} \mathrm{2}$ )

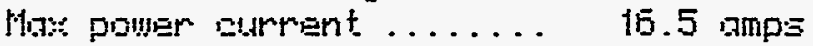
Mos pusurer vol tage ...... 208.3 volts

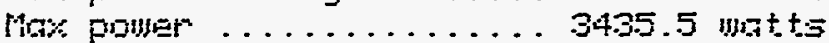




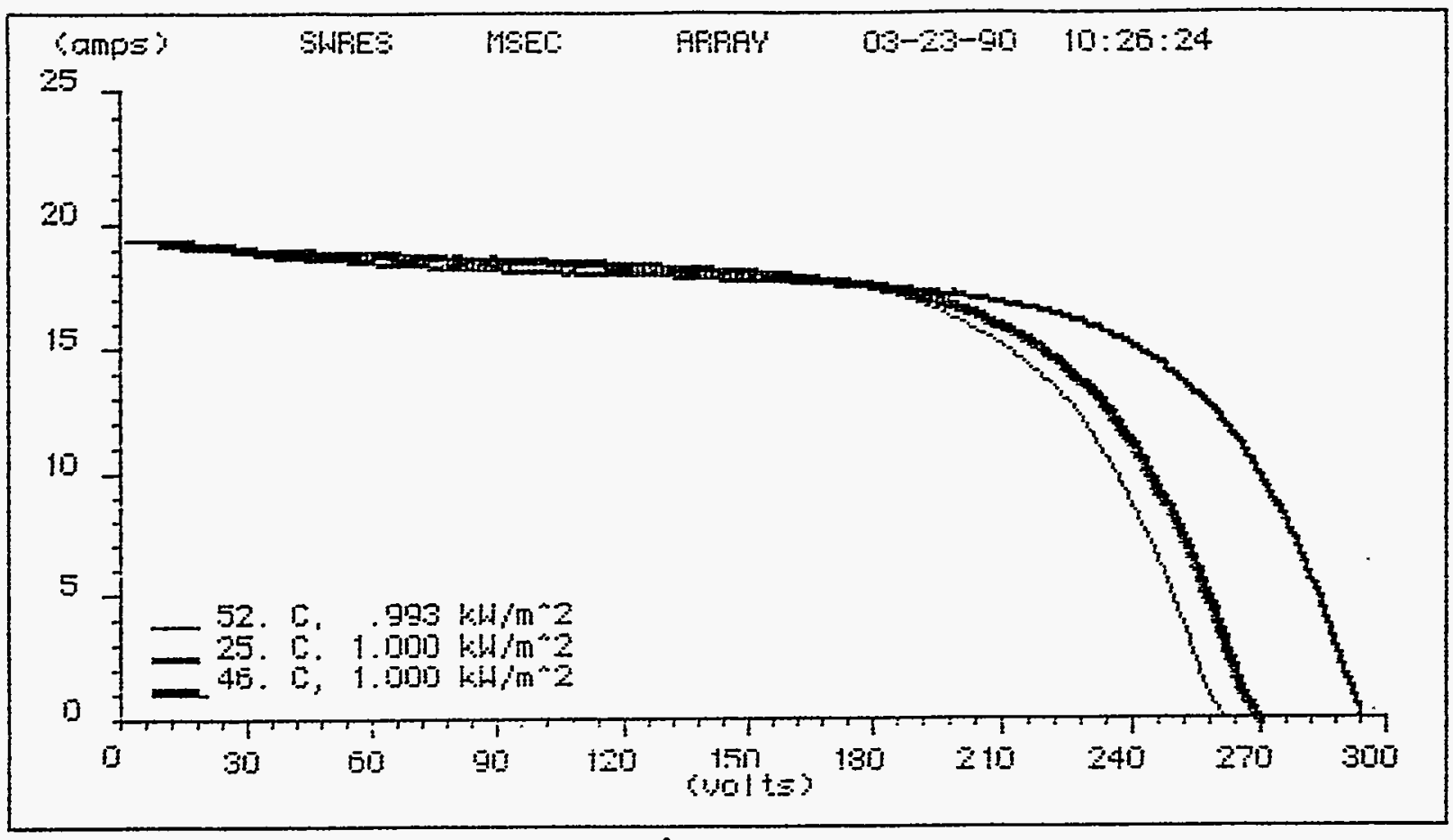

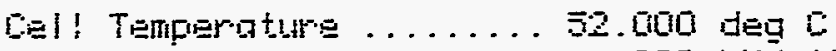

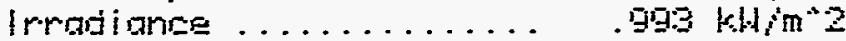

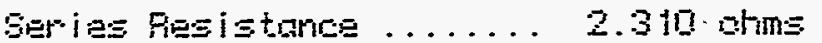

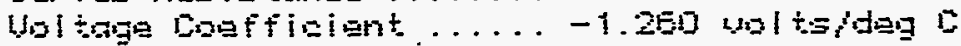

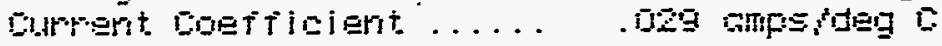

ALTUHL ELAIVE CATA:

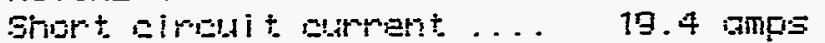
Dpers circuit ub! tage ..... 20. 20.4 30! ts

Max poimer ctirrant ...... 15.5 amps

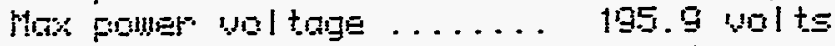

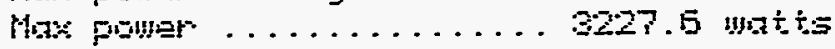

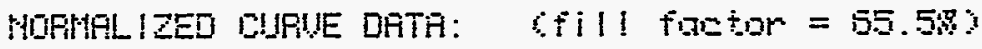

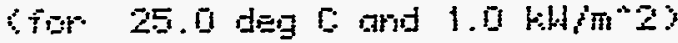

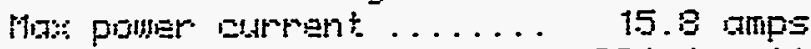

Max puwer vol tage ...... 231.4 ublts

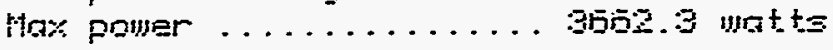

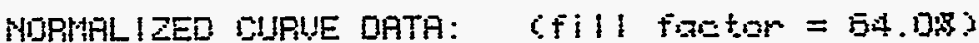

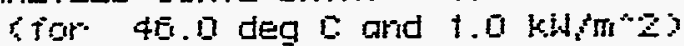

Mare poular gurrent ....... is. 4 smps

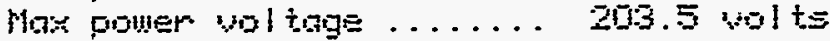

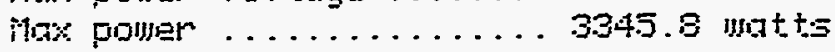




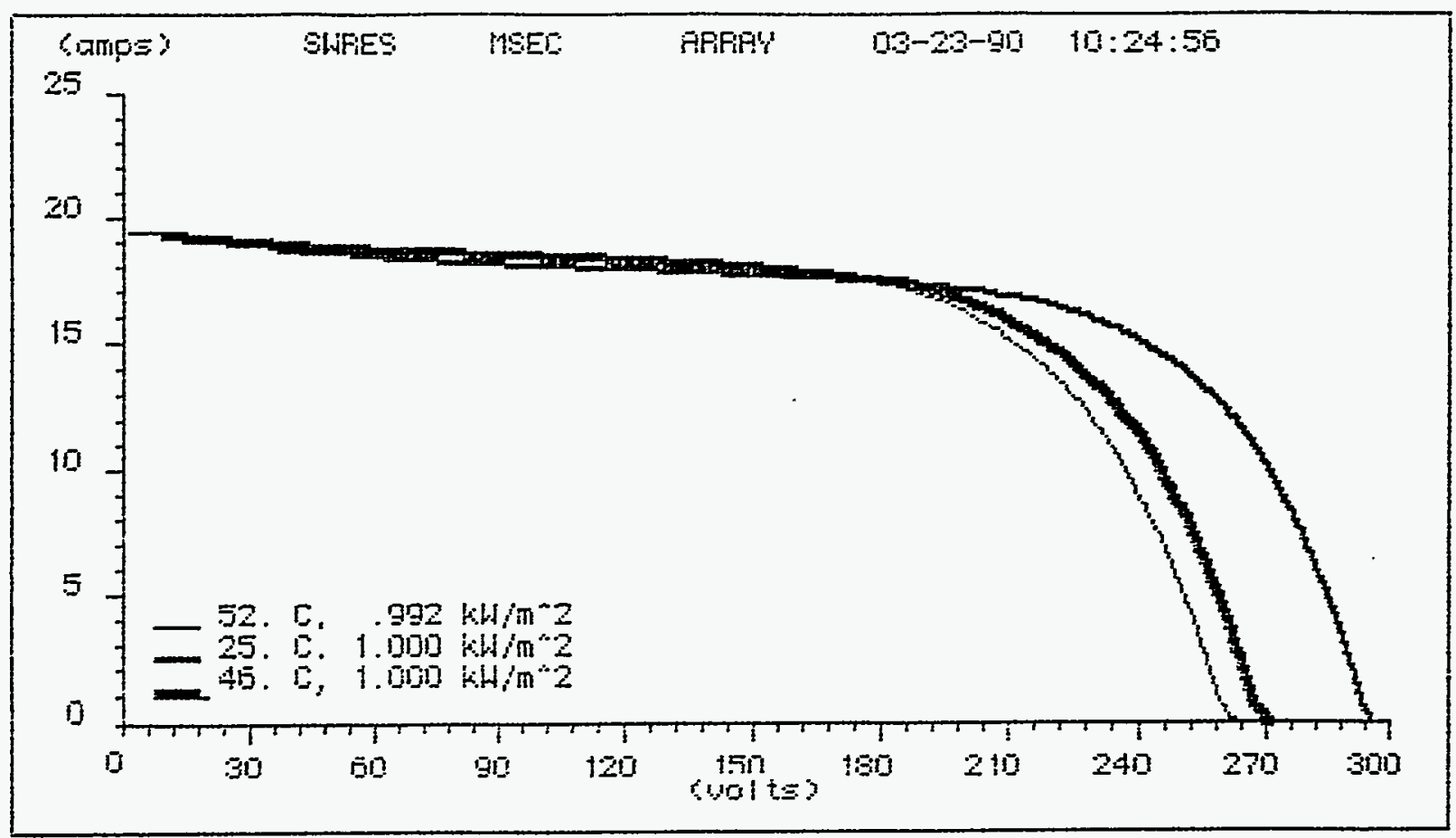

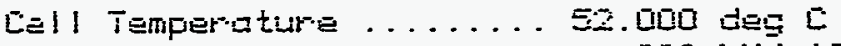

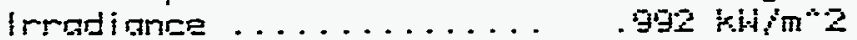

Ser-i

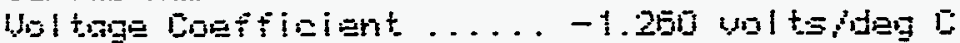

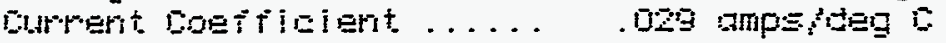

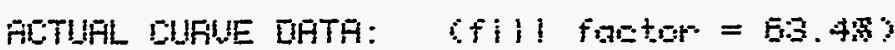

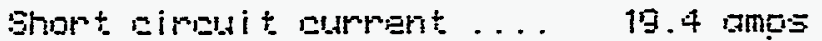

Upers eirauit jol tage ..... 20.3. 2 voli

hiox power current. ...... 15.2 amps

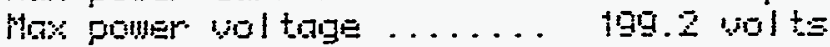

hox pomer ............ 3235.3 mat

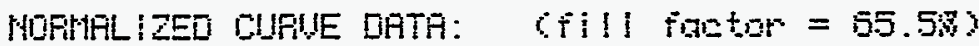

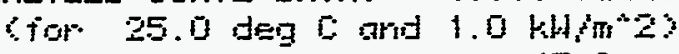

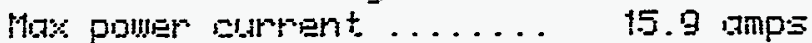

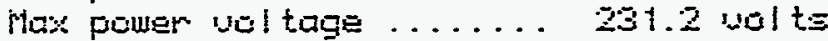

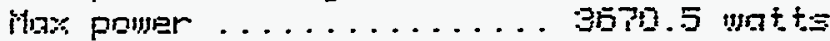

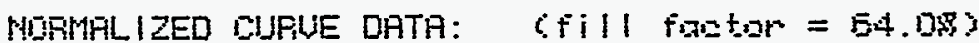

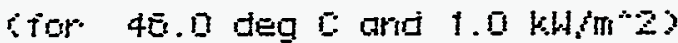

Mox primer gutrent. ...... 15.2 amps

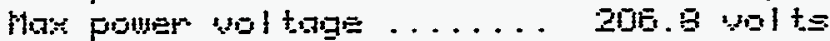

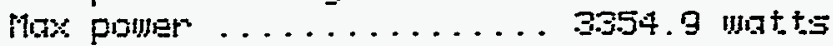



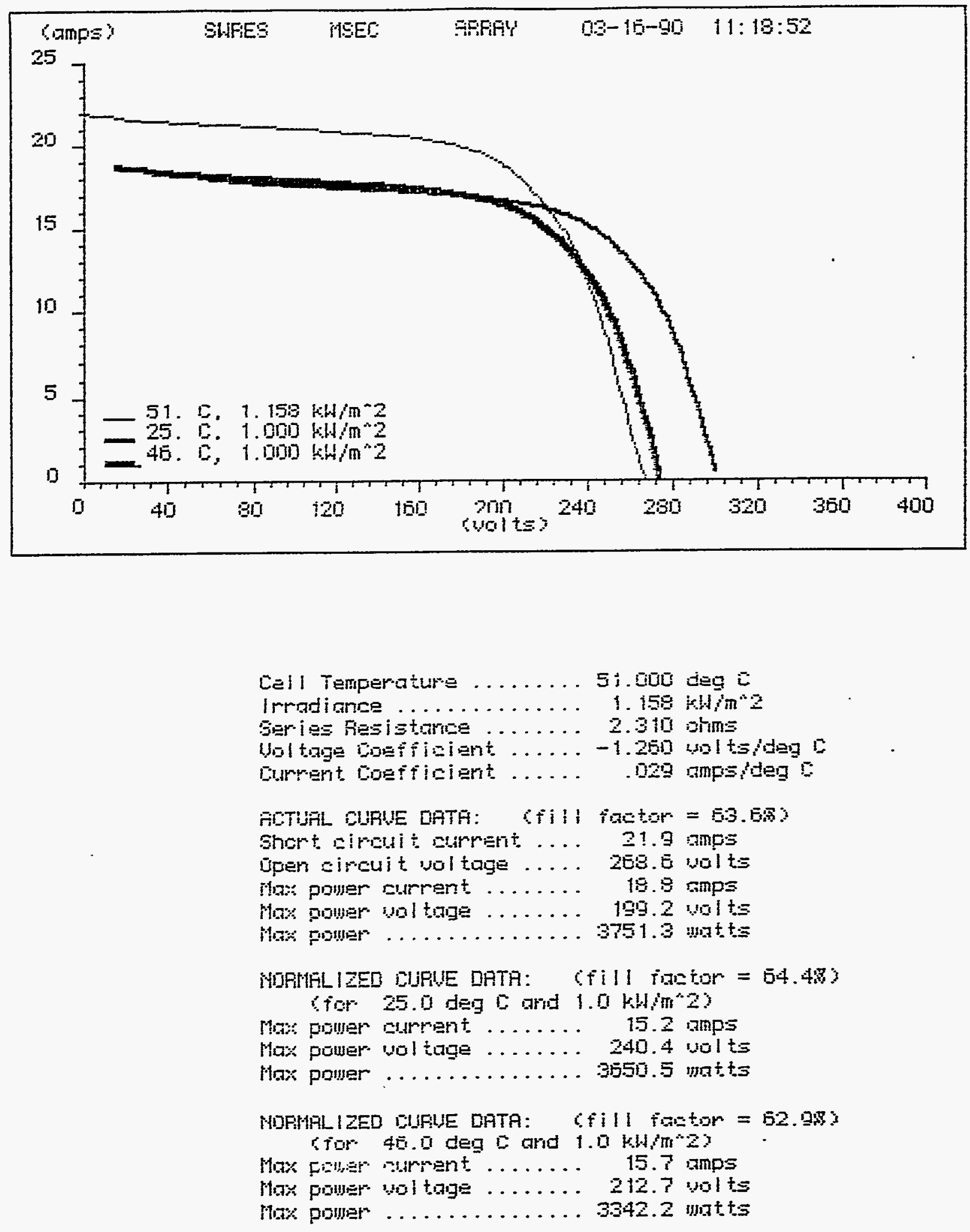


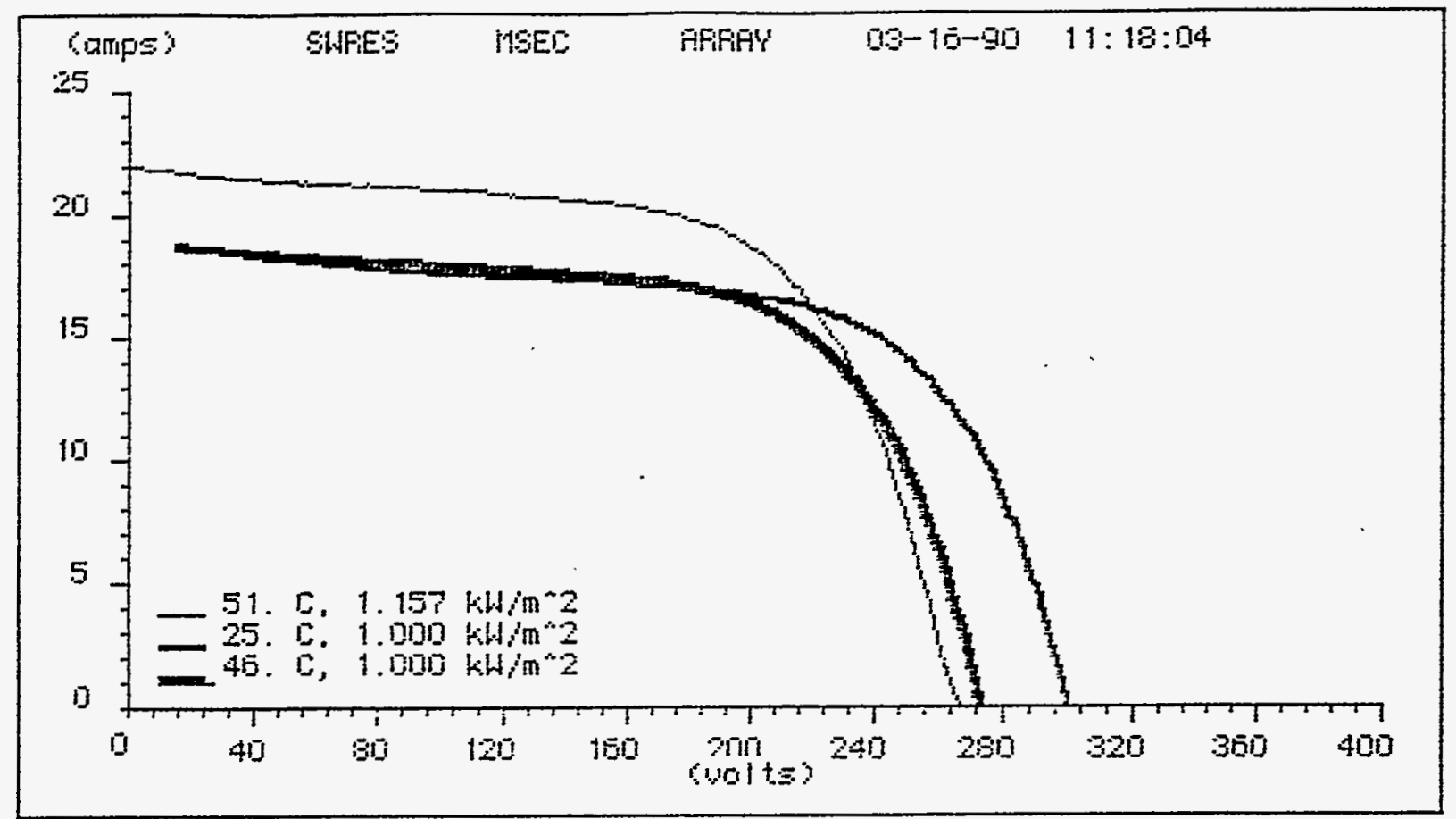

\footnotetext{
Lall Temperqture ....... 51. Dü deg $\mathrm{C}$ impadiance . . . . . . Sor ies Fesigtance ...... 2.310 ohms

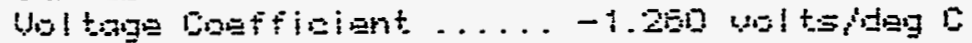

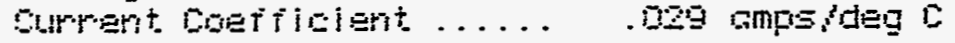

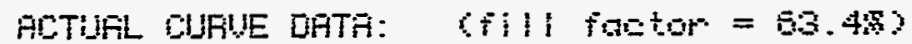
Ghort girouit ourpent ... 22. D amps Opers eincuit vol tage ..... 208.4 volts Wres power gurrent ....... 19.3 smps Mox poupr vol toge ...... 20.9 volts

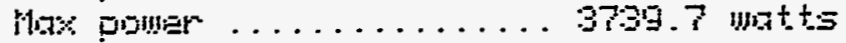

HOFMELIZED GUFIE DATA: GTII! faOtG $=64.23$

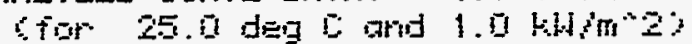

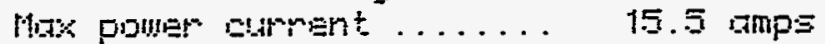

Max power val tage ....... 234.5 volt. inox power . . . . . . . 3543.5 matts

MURIALIZED CURUE DATA: CFill factor $=52.7 \%$ Sior $40.0 \mathrm{deg} C$ and $1.0 \mathrm{kH}\left(\mathrm{m}^{\circ} \mathrm{z}\right.$ )

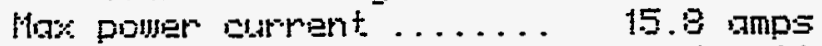
How puwer voltage ...... 211.5 volts

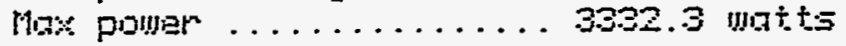




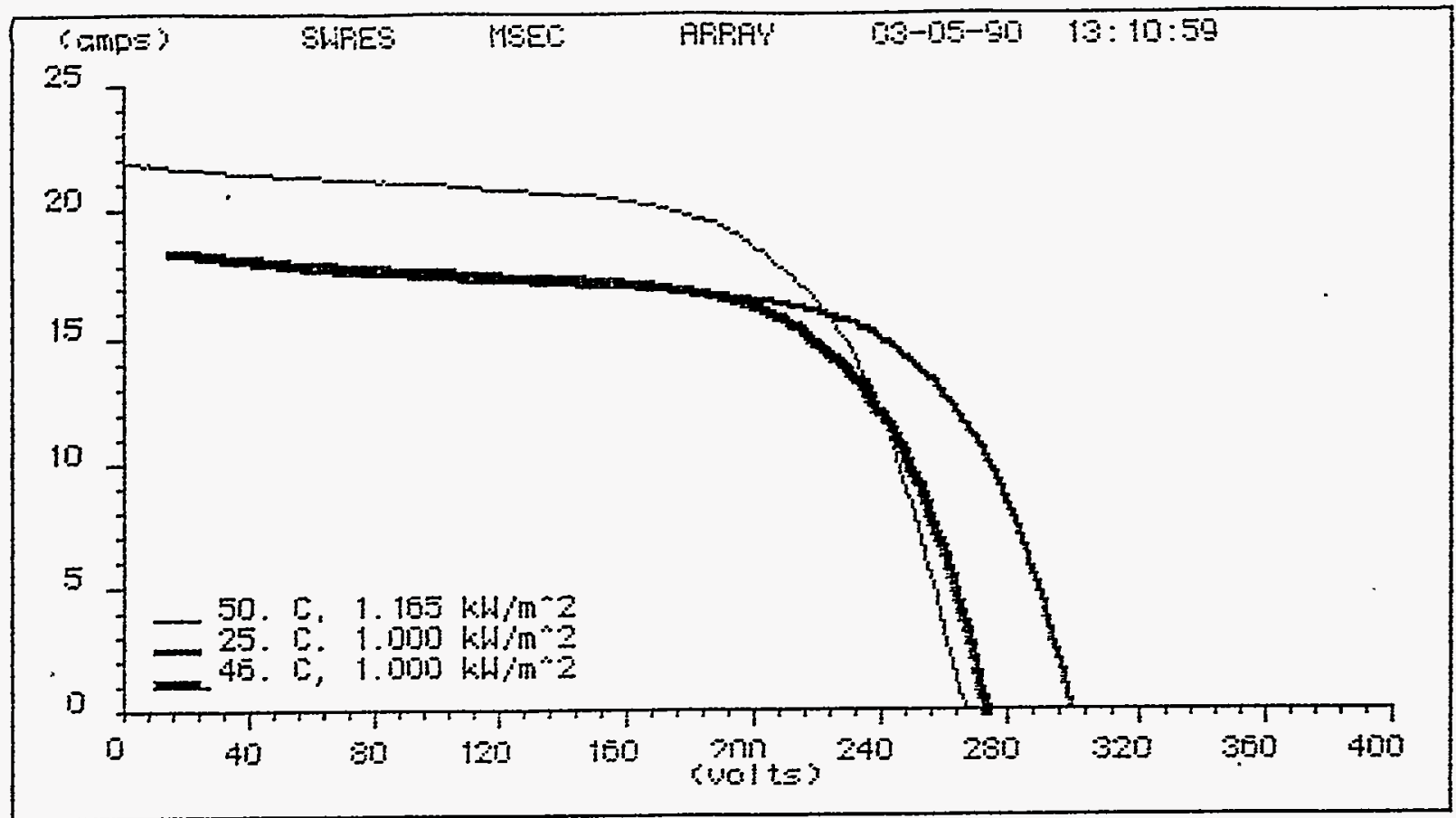

Cell Temperature ....... 50.000 deg $c$

irradiance ............ $1.165 \mathrm{kiH}^{2} \mathrm{~m}^{2}$

Sar i $=$ Resi

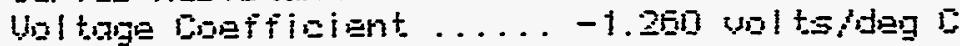

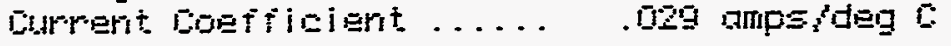

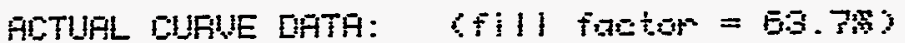

short airouit ourpent. ... 21.9 amps

Dpen circuit $v: 140 . \ldots .269 .1$ volts

Was pouer estrent. . . . . . 10.5 ramps

Nax bumer uol tage ...... 202.7 wolts

Mar poumer . . . . . . . 3742.4 matts

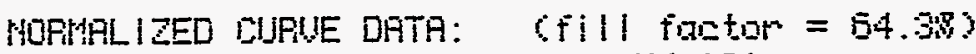

ffor 25.0 deg $C$ ard $1.04 H_{1}{ }^{2} 2$ ?

Wax pouser Gurpent ...... 15.3 amps

Max power voltage ...... 235.3 uslts

Hox poumer . . . . . . . 3505.9 motts

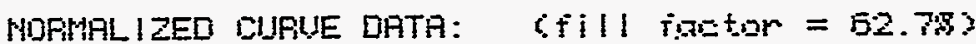

Sfor 40.0 deg $\left[\right.$ and $1.0 \mathrm{kH} / \mathrm{m}^{\circ} \mathrm{Z}$ )

Mage poumer aurrent. . . . . 15.8 amps

Mar puouser wol tage ....... 20s. 1 wolts

Mige pouser ........... 3291.6 udats 

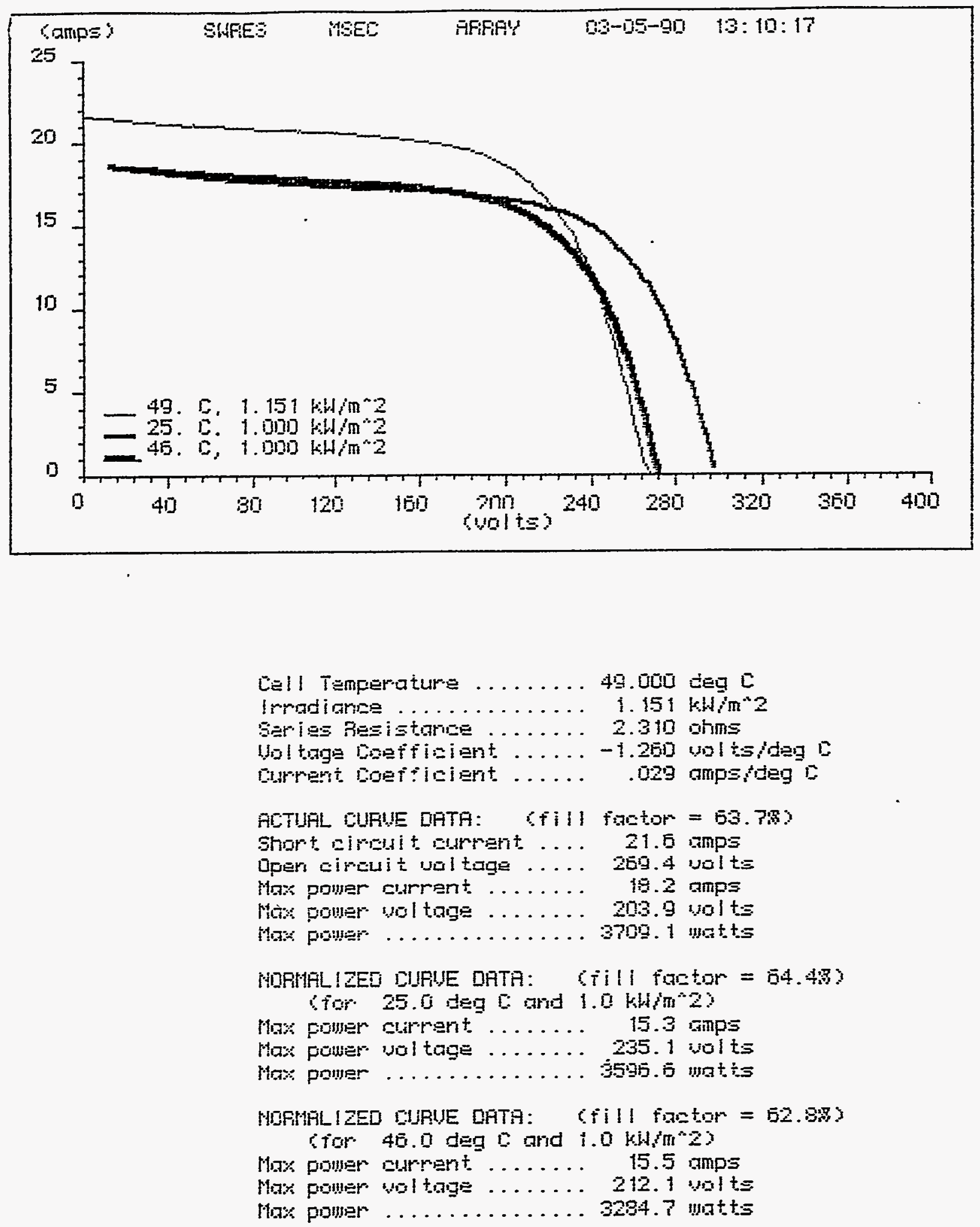


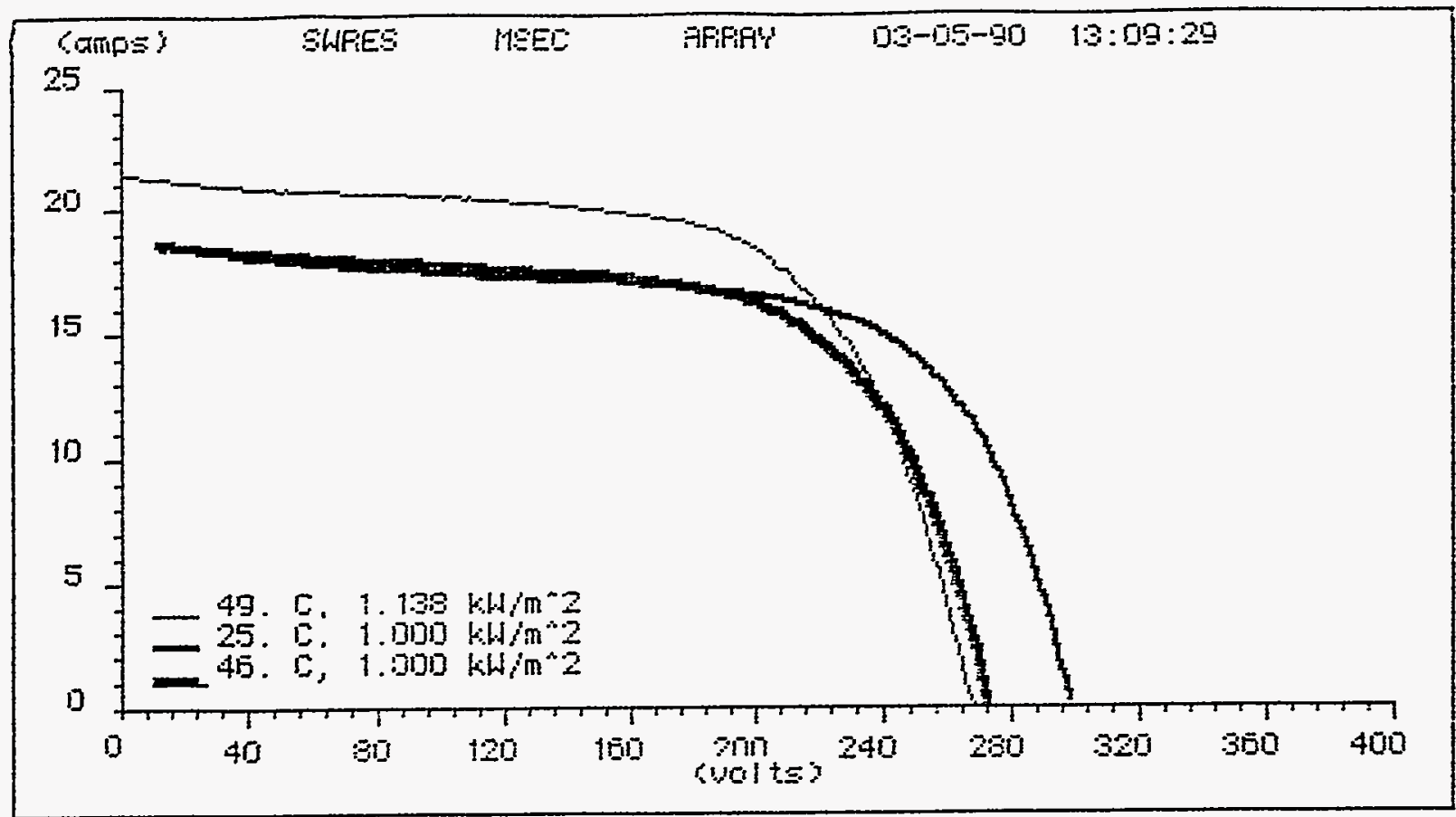

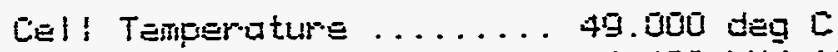

Imradianne ........... 1. $193 \mathrm{kH}$.

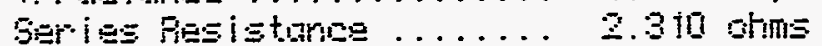

Us t.

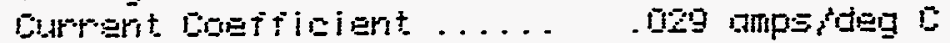

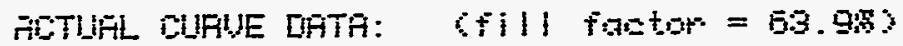

Shot sirust curnent. . . 21.4 smp:

Ufer aircuit vel tage ..... 2E9.8 volts

Mas pouner surrent ...... 13.2 amps

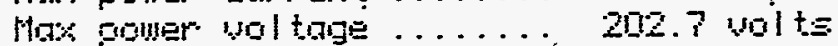

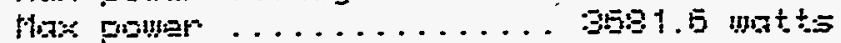

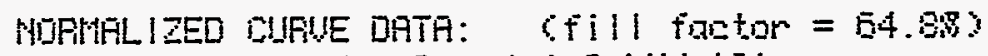

ifor 25.0 deg a and $1.04 h m+23$

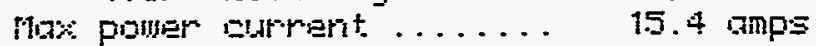

Hax pruer wol tage ....... 234.5 val ts

Mnx pomer ........... 3515.8 mats

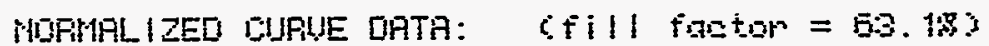

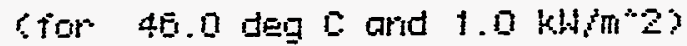

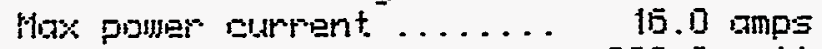

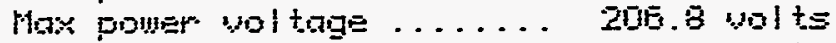

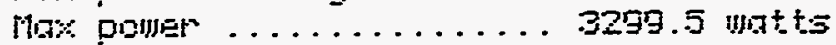




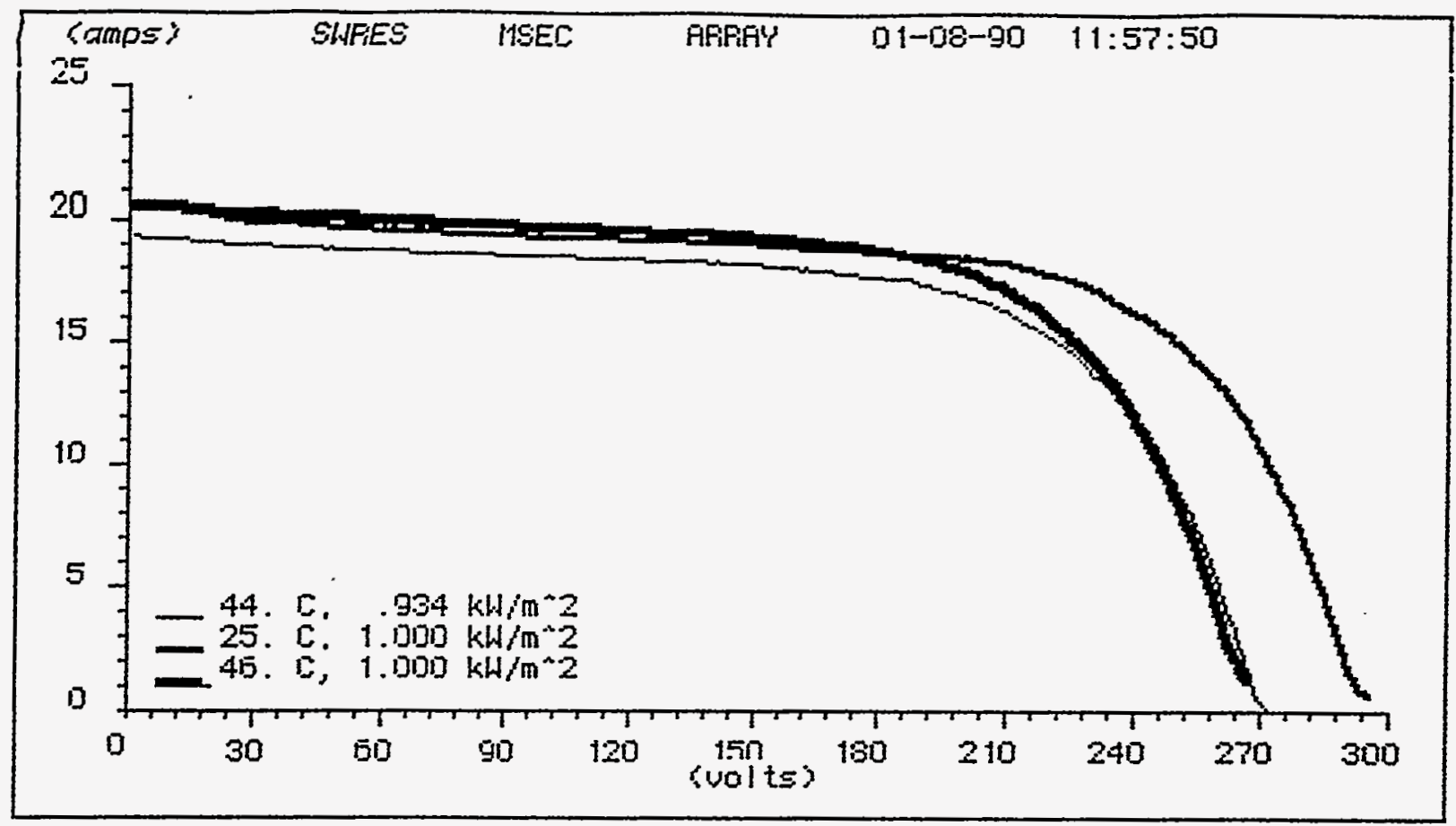

Cell Temperature .......44. aüü deg C Irradiance ........... . $3.34 \mathrm{kh} / \mathrm{m}^{* 2}$ Seri

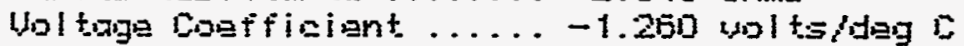
Burrerit Coneficient ..... .029 ampsideg $c$

ACTUAL CUAUE DATA: (fill factor $=64.3 \%$ )

Short aimosit asmrent. ... 19.3 amps Upen eircuit vol tage ..... 272.3 volts Max pouner cturrant ....... 15.2 anps Max poumer wal tage ....... 209.3 volts

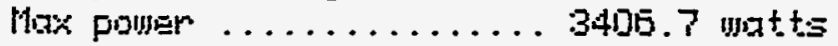

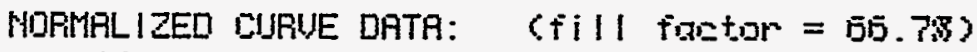
ifor 25.0 deg [0 and $1.0 \mathrm{kh} / \mathrm{m}^{\wedge} \mathrm{Z}$ ?

Mux powler curtent. ...... 17.0 amps

Max power vol tage ....... 231.9 wolts Max powner .............3 345.2 matts

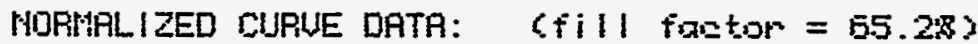
Sior 46.0 deg $C$ and $1.0 \mathrm{kH} / \mathrm{m}^{\circ} \mathrm{z}$ ?

Hax pouner sumpent ...... 17.7 gmps

Mox power woltigge ....... 204.0 volts Mox pomser ..............350.3.3 untts 

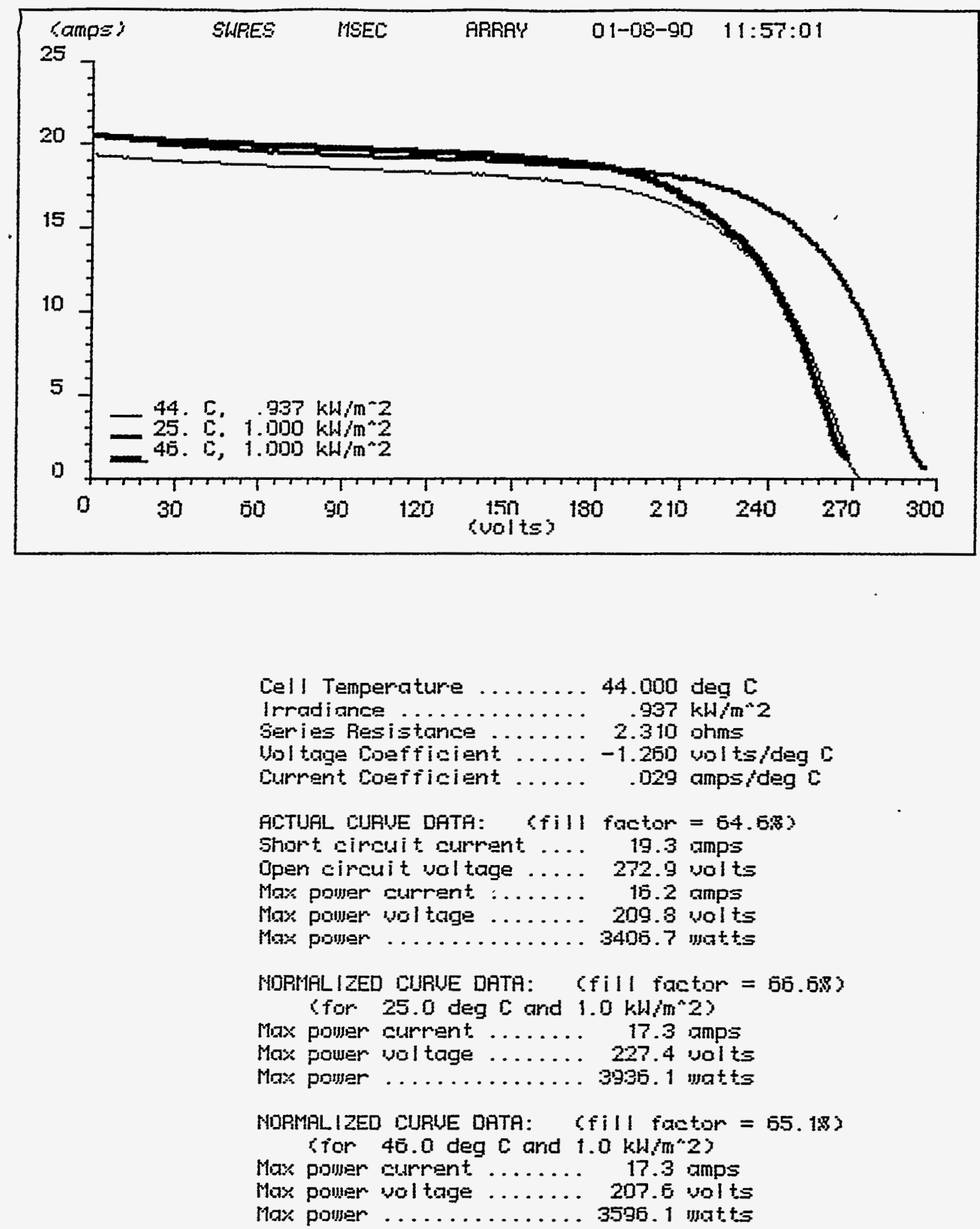


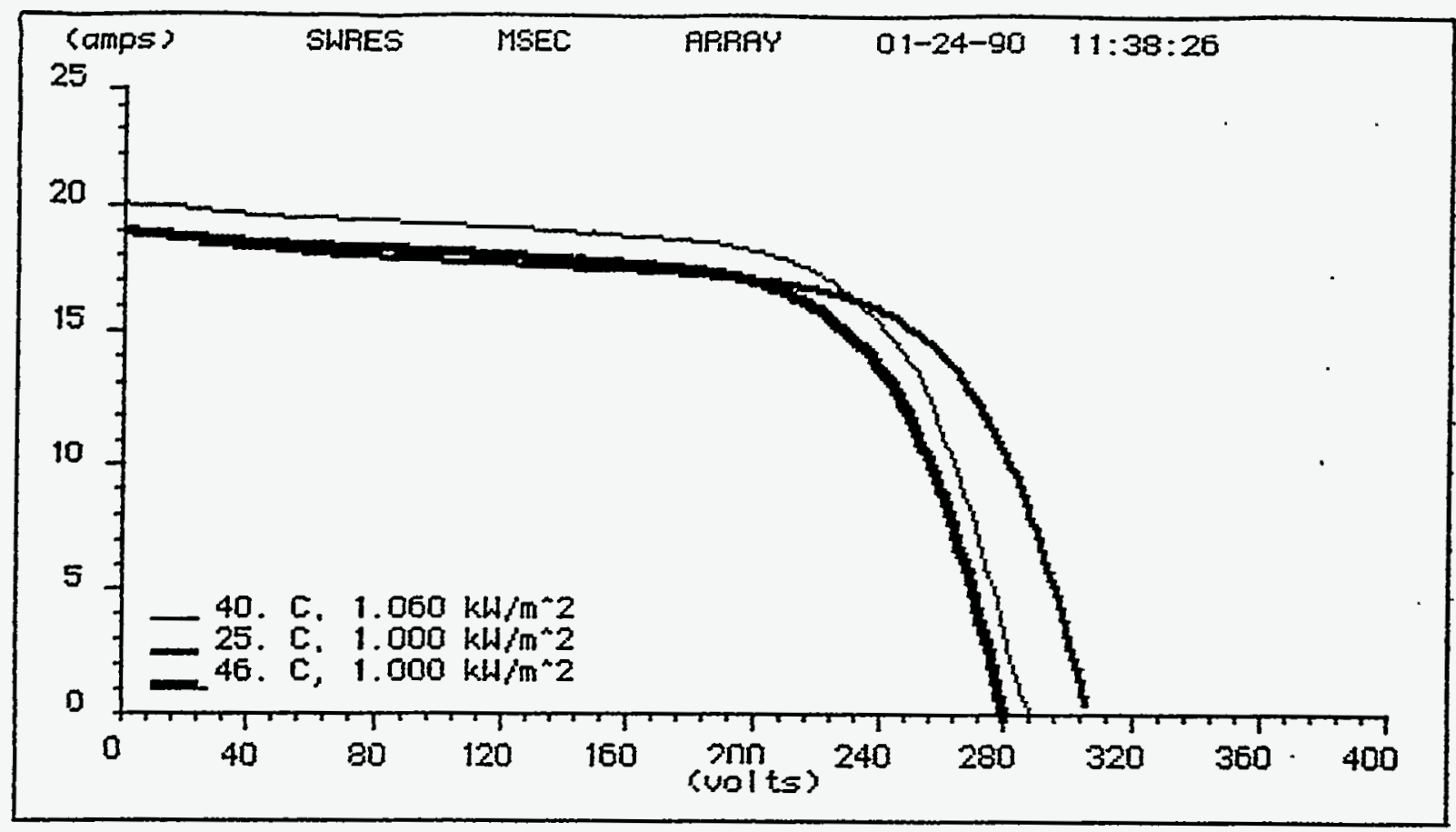

\footnotetext{
Cell Temperature .......440.000 deg $\mathrm{C}$

Irradiance ........... $1.060 \mathrm{kH}^{\circ} \mathrm{m}^{2} 2$

Series Fiesistance ....... 2.310 ohms

Uol tage Conefficient ..... -1.260 yol ts/deg $C$

Durpent Coefficient ..... . .029 umps/deg $\mathrm{C}$

ACTUAL CUPUE DATA: (fill fuctor = 65.48 )

Short circuit current .... 20.1 amps

Dpen circuit yoltage ..... 288.4 yolts

Max pouer cur.rent. ...... 17.1 amps.

Max power voltage ...... 221.5 volts

Max power ...........3791.7 untts

NORMAL IZED CURUE DATA: (fill factor $=65.98$ ) (for 25.0 deg 5 and $1.0 \mathrm{kw} / \mathrm{m}^{2} 2$ )

Max pounar current ....... 15.8 amps

Max pouner vol tuge ....... 240.5 volts

Max power ............ 3799.5 watts

NORMALIZEO CURUE DATA: (fill factor $=64.58$ )

(for 40.0 deg $c$ and $1.0 \mathrm{kl} / \mathrm{m}^{\wedge} 2$ )

Max power current ....... 16.1 amps

Max power vol tage ....... 215.2 wolts

Max pouner ........... 3490.4 watts.
} 


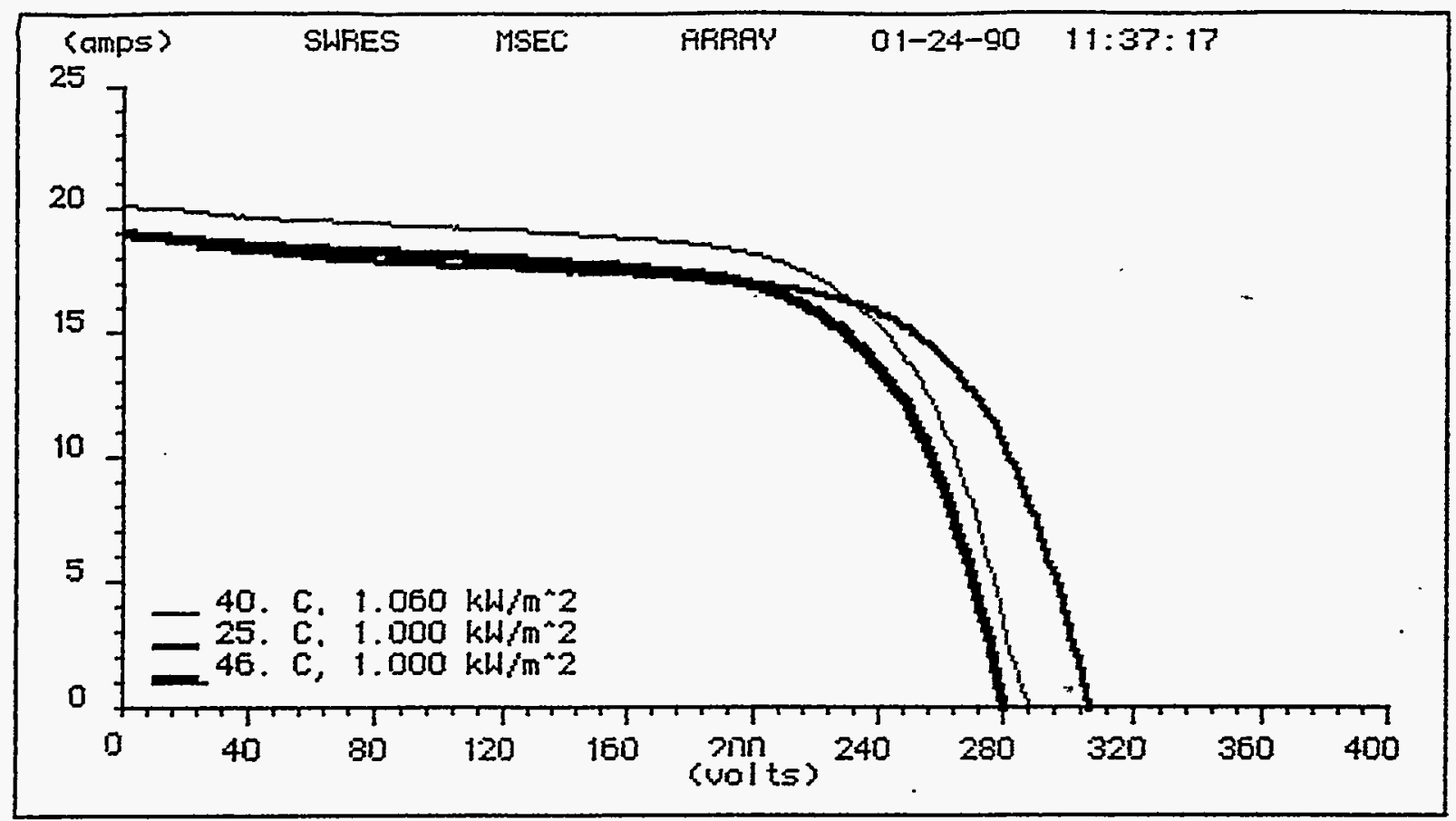

Cell Temperature .......40.000 deg C

Irradiance ............ $1.060 \mathrm{kH} / \mathrm{m}^{\wedge} 2$

Series Resistance ....... 2.310 ohms

Voltage Coefficient ...... -1.260 voltsideg $c$

Current Doefficient. ..... .029 ampsideg C

ACTUAL CURUE DATA: 〈 $\mathrm{i} i l$ factor $=65.48$ )

Short circuit current.... 20.1 amps

Dpen Eirouit voltage ..... 258.7 wolts

Max power surpent ....... 17.0 amps

Max power voltage ...... 223.8 valts

Max power ........... 3796.2 watts

MORMALIZED CURUE DATA: ( $\mathrm{fi}$ l factor $=65.98$ )

(fior 25.0 deg $C$ and $1.0 \mathrm{kH} / \mathrm{m}^{\wedge} 2$ )

Max power current ....... 15.8 amps

Max power vol tage ........ 240.6 volts

Max power ............ : 3804.3 matts

MORMAL IZED CURUE DATA: ifill factor $=64.48$ ) (for 45.0 deg $C$ and $1.0 \mathrm{kH} / \mathrm{m}^{\circ} 2$ )

Max power current....... 10.0 amps

Max pouer uoltage ....... 218.5 volts

Max power ............ 349.3.3 watts 